\title{
Europäische Rechnungslegungsharmonisierung: Vergleichende Analyse zwischen Finnland und Deutschland
}

\author{
Dissertation \\ zur Erlangung des wirtschaftswissenschaftlichen Doktorgrades \\ der Wirtschaftswissenschaftlichen Fakultät \\ der Georg-August-Universität Göttingen
}

vorgelegt von

Dipl.-Kffr. Petra Raderschall aus Braunschweig

Göttingen, 2003 
Erstgutachter:

Prof. Dr. Lothar Schruff

Zweitgutachter:

Prof. Dr. Wilhelm H. Wacker

Tag der mündlichen Prüfung: $\quad$ 05. Februar 2003 


\section{Inhaltsverzeichnis}

Inhaltsverzeichnis

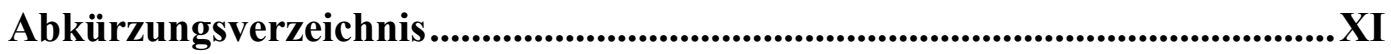

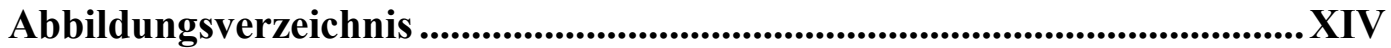

1 Gang der Untersuchung .................................................................. 1

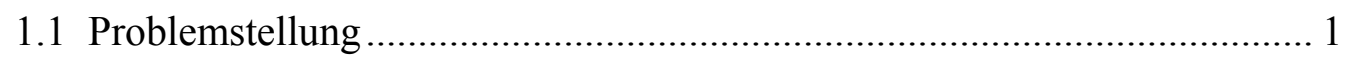

1.1.1 Heterogenität der Rechnungslegungssysteme ............................... 1

1.1.2 Harmonisierung von Einzelabschlüssen innerhalb der Europäischen Union.................................................................... 3

1.2 Zielsetzung und Methodik vorliegender Untersuchung ............................ 7

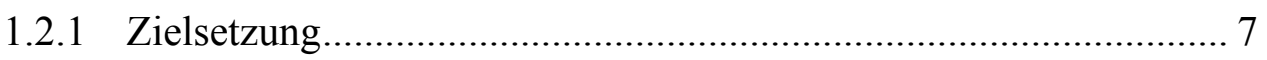

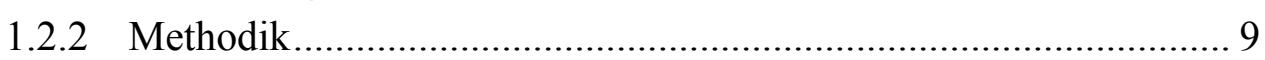

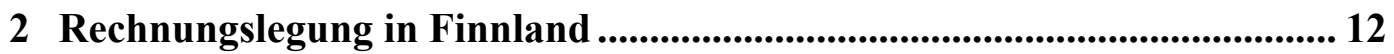

2.1 Entwicklung und Zäsuren der finnischen Rechnungslegung .................... 12

2.1.1 Einführung der doppelten Buchführung in Finnland ab 1850 ....... 12

2.1.2 Deutscher und skandinavischer Einfluss auf die finnische Rechnungslegung von 1920 bis 1950 ........................................... 14

2.1.2.1 Buchführungsgesetz von 1925 ..................................... 14

2.1.2.2 Buchführungsgesetz von 1945 ..................................... 16

2.1.3 Theoriegeprägte Rechnungslegung 1950 bis 1990 ....................... 19

2.1.3.1 Ausgaben-Einnahmen-Theorie der Buchführung............ 19

2.1.3.2 Buchführungsgesetz von 1973 ..................................... 24

2.1.4 Internationale Harmonisierung seit 1990 .................................. 27

2.1.4.1 Nordische Harmonisierungsbestrebungen...................... 27

2.1.4.2 Einfluss der Europäischen Union auf die finnische

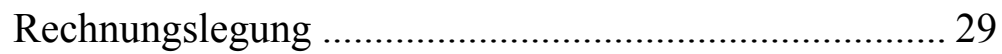

2.1.4.2.1 Buchführungsgesetz von 1992 ....................... 29

2.1.4.2.2 Buchführungsgesetz von 1997 ....................... 32

2.1.5 Zusammenfassender Überblick ................................................... 33

2.2 Normierung der finnischen Rechnungslegung ........................................ 35

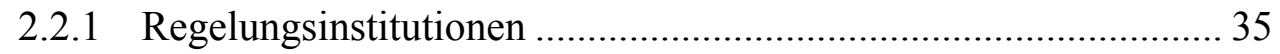

2.2.1.1 Gesetzgeber, Regierung, Ministerien und Behörden....... 35

2.2.1.2 Buchführungsausschuss................................................. 36

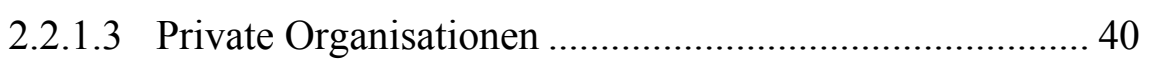

2.2.1.3.1 Organisationen wirtschaftsprüfender Berufsstände 40

2.2.1.3.2 Berufs- und Industrieverbände, Aktienmarkt und Börse, Wissenschaft ................................ 43

2.2.1.4 Zusammenfassender Überblick ..................................... 44 
2.2.2 Konzeption des Buchführungsgesetzes von 1997 . .45

2.2.2.1 Regelungsbereich, Anwendungsbereich und Struktur .... 45

2.2.2.2 Buchführungsgesetz, Aktiengesellschaftsgesetz und Wertpapiermarktgesetz. 46

2.2.2.2.1 Aktiengesellschaft 46

2.2.2.2.2 Buchführungsgesetz und Aktiengesellschaftsgesetz 49

2.2.2.2.3 Buchführungsgesetz und Wertpapiermarktgesetz 50

2.2.2.3 Buchführungsgesetz und Steuergesetzgebung ............... 51

2.2.2.4 Buchführungsgesetz und Wirtschaftsprüfungsgesetz..... 53

2.2.2.5 Zusammenfassender Überblick .................................... 55

3 Richtlinienkonformität der finnischen Rechnungslegung ........................ 56

3.1 Grundlagen der finnischen Rechnungslegung......................................... 56

3.1.1 Zielsetzung der finnischen Rechnungslegung ............................ 56

3.1.2 Instrumente der finnischen Rechnungslegung............................. 58

3.1.2.1 Bestandteile des Jahresabschlusses und ihre Aufgaben .. 58

3.1.2.2 Weitere Rechnungslegungsinstrumente ........................ 60

3.1.3 Anerkannte Grundsätze ordnungsmäßiger Buchführung ............. 61

3.1.3.1 Die Art guter Buchführung und ihre Ermittlung ............. 61

3.1.3.2 Kodifizierte Grundsätze ordnungsmäßiger

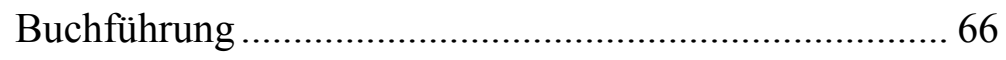

3.1.3.3 Nicht kodifizierte Grundsätze ordnungsmäßiger

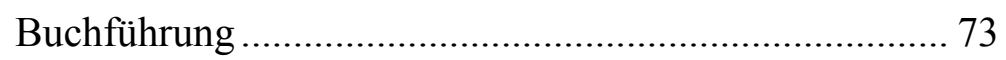

3.1.3.4 True and Fair View ...................................................... 75

3.1.3.4.1 Aufnahme des True and Fair View in finnisches Recht ............................................ 75

3.1.3.4.2 Finnisches Verständnis des True and Fair View ...................................................... 77

3.1.3.4.3 Verhältnis des True and Fair View zur Art guter Buchführung und zu den anderen Grundsätzen ordnungsmäßiger Buchführung . 81

3.1.3.4.4 Notwendige Zusatzangaben im Anhang ......... 82

3.1.3.4.5 True and Fair View-Override......................... 85

3.1.3.4.6 Zusammenfassender Überblick....................... 89

3.1.4 Inhalt, Dokumentation und Aufbewahrung der Buchführungsunterlagen......................................................... 90

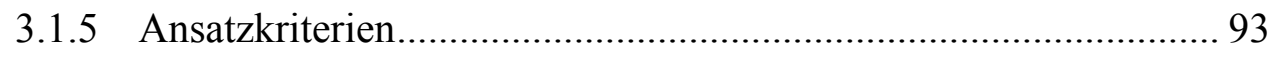

3.1.5.1 Erfassung von Erträgen und Aufwendungen.................. 93

3.1.5.2 Ansatz von Aktiva ..................................................... 95

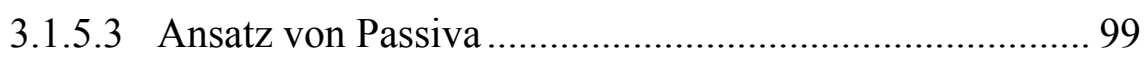


3.1.6 Bewertung von Aktiva und Passiva ........................................... 100

3.1.6.1 Bewertungsmaßstäbe ................................................... 100

3.1.6.2 Bewertung von Aktiva................................................ 102

3.1.6.2.1 Bewertung bei Zugang ................................. 102

3.1.6.2.1.1 Anschaffungskosten ........................ 102

3.1.6.2.1.2 Herstellungskosten .......................... 102

3.1.6.2.1.3 Behandlung von Zuschüssen............. 105

3.1.6.2.1.4 Behandlung von

Wiederbeschaffungsrückstellungen .. 106

3.1.6.2.2 Bewertung in Folgeperioden......................... 107

3.1.6.2.2.1 Planmäßige Abschreibungen............. 107

3.1.6.2.2.2 Andere als planmäßige

Abschreibungen................................. 110

3.1.6.2.2.3 Außerplanmäßige Abschreibungen auf das Anlagevermögen.................. 112

3.1.6.2.2.4 Außerplanmäßige Abschreibungen auf das Umlauf- und

Finanzumlaufvermögen.

3.1.6.2.2.5 Wertaufholung.................................. 116

3.1.6.2.2.6 Neubewertung ................................. 118

3.1.6.2.3 Zusammenfassender Überblick..................... 123

3.1.6.3 Bewertung von Passiva.............................................. 124

3.1.7 Fremdwährungsumrechnung einzelner Geschäftsvorfälle .......... 124

3.1.7.1 Vorschriften zur Fremdwährungsumrechnung............. 124

3.1.7.2 Zugangsbewertung und Behandlung unterjährig realisierter Umrechnungsdifferenzen ........................... 125

3.1.7.3 Bewertung von Fremdwährungspositionen am

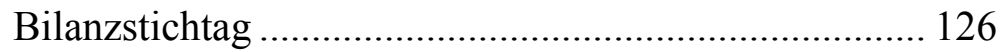

3.1.7.4 Behandlung und Ausweis nicht realisierter Umrechnungsdifferenzen am Bilanzstichtag................ 127

3.1.8 Behandlung von Bilanzierungsfehlern aus Vorperioden ............. 129

3.2 Ansatz und Bewertung einzelner Bilanzposten im Einzelabschluss ....... 130

3.2.1 Anlagevermögen .................................................................. 130

3.2.1.1 Immaterielle Vermögenswerte ................................... 130

3.2.1.1.1 Ansatz, Bewertung und Ausweis ................. 130

3.2.1.1.2 Gründungsausgaben ................................... 133

3.2.1.1.3 Forschungs- und Entwicklungsausgaben...... 135

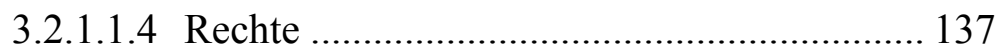

3.2.1.1.5 Geschäfts- oder Firmenwert......................... 138 
3.2.1.1.6 Ausgaben mit lang anhaltender Wirkung...... 139

3.2.1.1.6.1 Darlehensausgaben.....

3.2.1.1.6.2 Andere Ausgaben mit lang anhaltender Wirkung 141

3.2.1.2 Sachanlagen 142

3.2.1.2.1 Ansatz, Bewertung und Ausweis .... 142

3.2.1.2.2 Geringwertige Wirtschaftsgüter.................... 146

3.2.1.2.3 Bildung von Festwerten ............................. 147

3.2.1.2.4 Behandlung von Leasingverhältnissen ......... 148

3.2.1.3 Finanzanlagen ........................................................... 150

3.2.1.3.1 Ansatz, Bewertung und Ausweis ................. 150

3.2.1.3.2 Verbundene Unternehmen ........................... 151

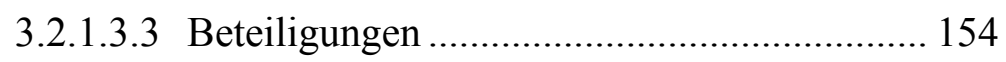

3.2.2 Umlaufvermögen .............................................................. 155

3.2.2.1 Ansatz, Bewertung und Ausweis des

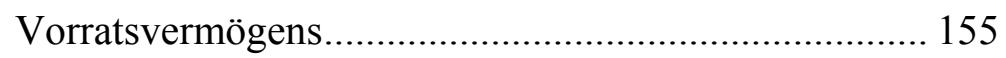

3.2.2.2 Bildung von Festwerten........................................... 158

3.2.2.3 Langfristige Auftragsfertigung ................................... 158

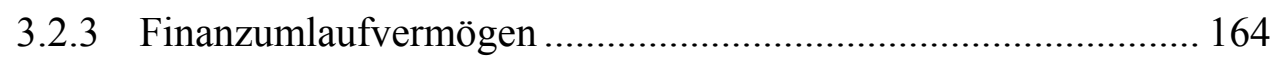

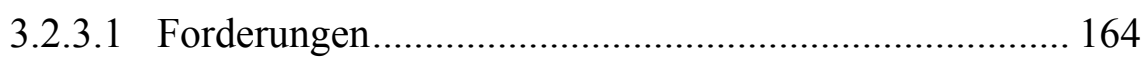

3.2.3.1.1 Ansatz, Bewertung und Ausweis ................. 164

3.2.3.1.2 Aktiver Rechnungsabgrenzungsposten......... 166

3.2.3.2 Wertpapiere des Finanzumlaufvermögens .................... 167

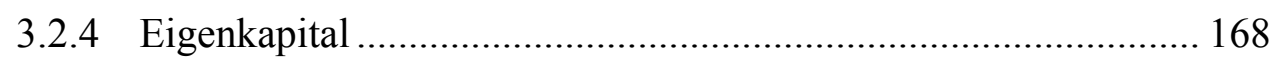

3.2.4.1 Gebundenes und nicht gebundenes Eigenkapital .......... 168

3.2.4.2 Eigenkapitalersetzende Rangrücktrittsdarlehen ............ 173

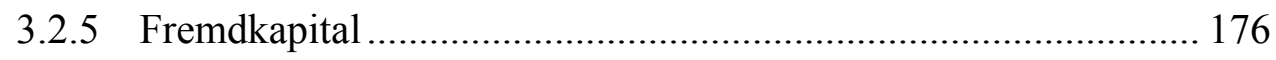

3.2.5.1 Steuerliche Ausgleichsposten .................................... 176

3.2.5.1.1 Abschreibungsunterschied .......................... 176

3.2.5.1.2 Freiwillige Rückstellungen mit Rücklageanteil.......................................... 178

3.2.5.2 Pflichtrückstellungen ............................................... 181

3.2.5.2.1 Ansatz, Bewertung und Ausweis ................. 181

3.2.5.2.2 Pensionsrückstellungen................................ 185

3.2.5.2.3 Steuerrückstellungen................................... 185

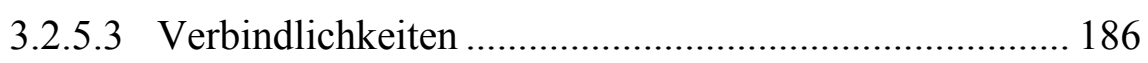

3.2.5.3.1 Ansatz, Bewertung und Ausweis .................. 186

3.2.5.3.2 Sonstige Verbindlichkeiten und passiver Rechnungsabgrenzungsposten ..................... 188

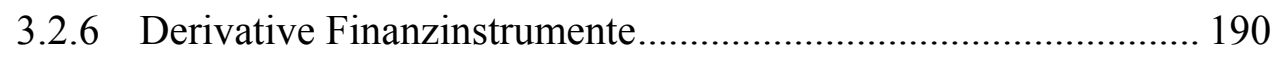

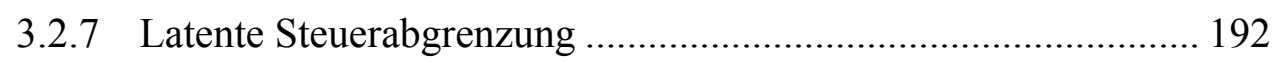


3.3 Ausgewählte Posten der Gewinn- und Verlustrechnung........................ 198

3.3.1 Umsatzerlöse....................................................................... 198

3.3.2 Außerordentliche Erträge und Aufwendungen ........................... 199

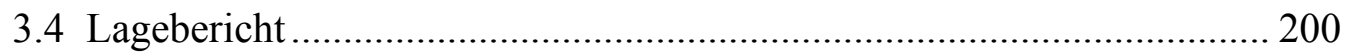

3.4.1 Stellung des Lageberichts und Anforderungen an den

Lagebericht ....................................................................... 200

3.4.2 Kapitalflussrechnung als Bestandteil des Lageberichts............... 202

3.5 Zusammenfassender Überblick ........................................................ 205

4 Harmonisierung der Rechnungslegung zwischen Finnland und

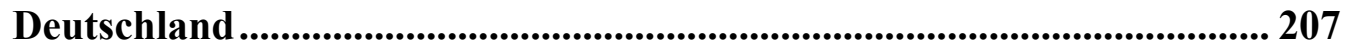

4.1 Deskriptiv-vergleichende Untersuchung der formalen

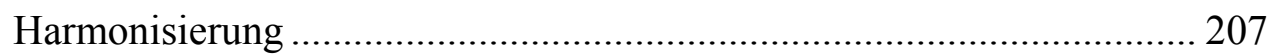

4.1.1 Normierung der Rechnungslegung ........................................... 207

4.1.1.1 Gesetzgebung mit Rechnungslegungsrelevanz ............ 207

4.1.1.1.1 Buchführungsgesetz und Handelsgesetzbuch207

4.1.1.1.2 Steuergesetzgebung..................................... 208

4.1.1.1.3 Gesetzgebung betreffend die

Wirtschaftsprüfung 209

4.1.1.2 Buchführungsausschuss und

Rechnungslegungsinstitut.......................................... 210

4.1.1.3 Wirtschaftsprüfende Berufsstände................................ 211

4.1.1.4 Kommentierung zur Rechnungslegung und

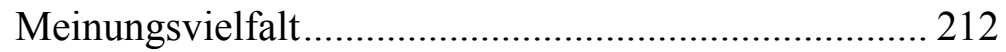

4.1.2 Grundlagen der Rechnungslegung........................................... 213

4.1.2.1 Zielsetzung und Adressaten der Rechnungslegung....... 213

4.1.2.2 Instrumente der Rechnungslegung ............................. 214

4.1.2.2.1 Bestandteile des Jahresabschlusses ............... 214

4.1.2.2.2 Gliederung der Bilanz ................................. 215

4.1.2.2.3 Gliederung der Gewinn- und

Verlustrechnung ............................................. 215

4.1.2.2.4 Abweichungen von der gesetzlich vorgegebenen Gliederung ............................ 216

4.1.2.2.5 Erleichterungen bei Erstellung des Jahresabschlusses ....................................... 216

4.1.2.2.6 Prüfungspflicht.............................................. 217

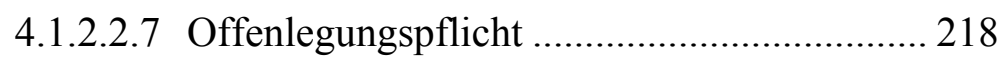

4.1.2.2.7.1 Jährliche Anforderungen................... 218

4.1.2.2.7.2 Erleichterungen ............................... 219

4.1.2.2.7.3 Sanktionen...................................... 220 
4.1.2.3 Grundsätze ordnungsmäßiger Buchführung. 221

4.1.2.3.1 Kodifizierte Grundsätze ordnungsmäßiger Buchführung............................................. 221

4.1.2.3.2 True and Fair View ...................................... 224

4.1.2.3.2.1 Charakter des True and Fair View .... 224

4.1.2.3.2.2 Notwendige Zusatzangaben im

Anhang. 224

4.1.2.3.2.3 True and Fair View-Override............ 225

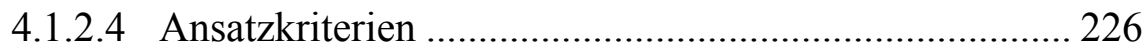

4.1.2.4.1 Ansatz von Vermögenswerten ...................... 226

4.1.2.4.2 Ansatz von Schulden................................... 226

4.1.2.5 Bewertung von Aktiva und Passiva............................. 227

4.1.2.5.1 Bewertungsmaßstäbe .................................. 227

4.1.2.5.2 Bewertung bei Zugang ................................ 227

4.1.2.5.2.1 Anschaffungskosten ........................ 227

4.1.2.5.2.2 Herstellungskosten ........................... 228

4.1.2.5.2.3 Behandlung von Zuwendungen......... 230

4.1.2.5.2.4 Behandlung von

Wiederbeschaffungsrückstellungen .. 230

4.1.2.5.3 Bewertung in Folgeperioden........................ 231

4.1.2.5.3.1 Planmäßige Abschreibungen............. 231

4.1.2.5.3.2 Andere als planmäßige

Abschreibungen. 233

4.1.2.5.3.3 Außerplanmäßige Abschreibungen auf das Anlagevermögen 233

4.1.2.5.3.4 Außerplanmäßige Abschreibungen auf das Umlaufvermögen .................. 234

4.1.2.5.3.5 Wertaufholung.................................. 235

4.1.2.5.3.6 Neubewertung ................................. 236

4.1.2.6 Fremdwährungsumrechnung ..................................... 236

4.1.2.7 Behandlung von Bilanzierungsfehlern aus Vorperioden .................................................................. 238

4.1.2.7.1 Anpassung von Vorjahreszahlen................... 238

4.1.2.7.2 Änderung der Bilanzpolitik........................... 238 
4.1.3 Ansatz und Bewertung einzelner Bilanzposten im

Einzelabschluss 239

4.1.3.1 Anlagevermögen

4.1.3.1.1 Immaterielle Vermögenswerte 239

4.1.3.1.1.1 Ansatz und Bewertung ..... 239

4.1.3.1.1.2 Gründungsausgaben und Aufwendungen für die Ingangsetzung und Erweiterung des

Geschäftsbetriebs 240

4.1.3.1.1.3 Forschungsausgaben 241

4.1.3.1.1.4 Entwicklungsausgaben..... 241

4.1.3.1.1.5 Geschäfts- oder Firmenwert 242

4.1.3.1.1.6 Ausgaben mit lang anhaltender Wirkung 242

4.1.3.1.2 Sachanlagen 244

4.1.3.1.2.1 Ansatz, Bewertung und Ausweis ...... 244

4.1.3.1.2.2 Geringwertige Wirtschaftsgüter ........ 245

4.1.3.1.2.3 Bildung von Festwerten 246

4.1.3.1.2.4 Behandlung von Leasingverhältnissen 246

4.1.3.1.3 Finanzanlagen 247

4.1.3.1.3.1 Ansatz und Bewertung. 247

4.1.3.1.3.2 Verbundene Unternehmen 248

4.1.3.1.3.3 Beteiligungen 248

4.1.3.1.3.4 Phasengleiche Vereinnahmung von Beteiligungserträgen 249

4.1.3.2 Umlaufvermögen 250

4.1.3.2.1 Ansatz des Vorratsvermögens... 250

4.1.3.2.2 Bewertung des Vorratsvermögens 251

4.1.3.2.2.1 Bewertungsmaßstäbe 251

4.1.3.2.2.2 Bewertungsvereinfachungsverfahren 251

4.1.3.2.2.3 Gängigkeitsabschreibungen ............. 252

4.1.3.2.3 Bildung von Festwerten .............................. 252

4.1.3.2.4 Langfristige Auftragsfertigung .................... 253

4.1.3.3 Finanzumlaufvermögen ........................................... 254

4.1.3.3.1 Forderungen ............................................... 254

4.1.3.3.2 Sonstige Vermögensgegenstände und aktive Rechnungsabgrenzungsposten ..................... 255

4.1.3.3.3 Geleistete Anzahlungen ............................... 256

4.1.3.3.4 Wertpapiere des Finanzumlaufvermögens.... 256

4.1.3.3.5 Eigene Anteile 256 


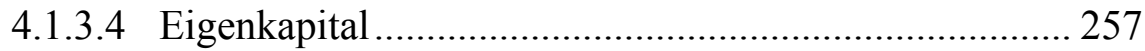

4.1.3.4.1 Gezeichnetes Kapital ................................... 257

4.1.3.4.2 Gesetzliche Rücklage................................. 257

4.1.3.4.3 Kapitalrücklage .......................................... 258

4.1.3.4.4 Neubewertungsrücklage............................... 258

4.1.3.4.5 Rücklage für eigene Anteile........................ 259

4.1.3.4.6 Eigenkapitalersetzendes

Rangrücktrittsdarlehen ............................... 259

4.1.3.5 Fremdkapital......................................................... 260

4.1.3.5.1 Steuerlich veranlasste Posten ........................ 260

4.1.3.5.2 Rückstellungen............................................ 261

4.1.3.5.2.1 Ansatz............................................. 261

4.1.3.5.2.2 Bewertung ........................................ 262

4.1.3.5.2.3 Pensionsrückstellungen .................... 263

4.1.3.5.2.4 Steuerrückstellungen ......................... 264

4.1.3.5.2.5 Sonstige Rückstellungen .................. 265

4.1.3.5.3 Verbindlichkeiten....................................... 269

4.1.3.5.3.1 Erhaltene Anzahlungen ..................... 269

4.1.3.5.3.2 Sonstige Verbindlichkeiten und passive Rechnungsabgrenzungsposten 269

4.1.3.6 Derivative Finanzinstrumente ....................................... 270

4.1.3.7 Latente Steuerabgrenzung .......................................... 270

4.1.4 Ausgewählte Posten der Gewinn- und Verlustrechnung ............. 271

4.1.4.1 Umsatzerlöse ............................................................ 271

4.1.4.2 Außerordentliche Posten ............................................. 271

4.1.5 Ausgewählte Anhangangaben ............................................... 272

4.1.5.1 Segmentinformationen............................................... 272

4.1.5.2 Angaben bezüglich nahestehender Unternehmen und Personen ........................................................... 273

4.1.5.3 Ergebnis je Aktie .................................................... 274

4.1.6 Lagebericht ...................................................................... 274

4.1.7 Zusammenfassung und kritische Würdigung der Ergebnisse...... 275 
4.2 Empirische Untersuchung der tatsächlich erreichten

Harmonisierung anhand ausgewählter Rechnungslegungsaspekte 278

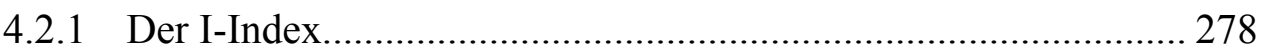

4.2.2 Messung des Harmonisierungsgrads mithilfe des I-Indexes ....... 282

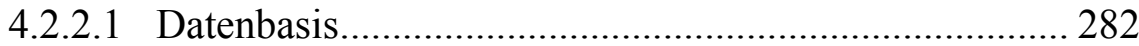

4.2.2.2 Empirische Messung des Harmonisierungsgrads ausgesuchter Jahresabschlusspositionen ....................... 284

4.2.2.2.1 Vorgehensweise

4.2.2.2.2 Ansatz eines derivaten Geschäfts- oder Firmenwerts

4.2.2.2.3 Ansatz von Forschungs- und Entwicklungskosten

4.2.2.2.4 Ansatz von immateriellen Vermögenswerten 288

4.2.2.2.5 Neubewertung 288

4.2.2.2.6 Wahl der Abschreibungsmethode 289

4.2.2.2.7 Ermittlung der Herstellungskosten beim Vorratsvermögen

4.2.2.2.8 Wahl des

Bewertungsvereinfachungsverfahrens

4.2.2.2.9 Ansatz passiver latenter Steuern

4.2.3 Zusammenfassung und kritische Würdigung der Untersuchungsergebnisse 293

5 Zusammenfassung der Ergebnisse und Möglichkeiten einer weiterführenden Betrachtung 


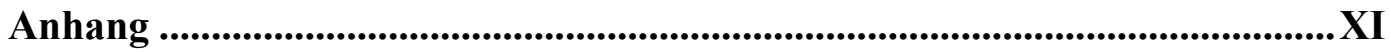

Anhang 1: Struktur des finnischen Buchführungsgesetzes ............................. II

Anhang 2: Finnische Spezialgesetze mit Rechnungslegungsrelevanz .......... XIV

Anhang 3: Allgemeingültige Anweisungen des KILA...................................

Anhang 4.1: Finnisches Bilanzgliederungsschema ..................................... XVI

Anhang 4.2: Finnisches vereinfachtes Bilanzgliederungsschema................XVIII

Anhang 5.1: Finnisches Gliederungsschema der Gewinn- und

Verlustrechnung nach Gesamtkostenverfahren ..................................... XIX

Anhang 5.2: Finnisches Gliederungsschema der Gewinn- und

Verlustrechnung nach Umsatzkostenverfahren .....................................XX

Anhang 6.1: Gliederungsschema Kapitalflussrechnung nach direkter

Methode gemäß KILA-Anweisung „,Kapitalflussrechnung“ ................ XXI

Anhang 6.2: Gliederungsschema Kapitalflussrechnung nach indirekter

Methode gemäß KILA-Anweisung „Kapitalflussrechnung“ .................XXII

Anhang 7: Ermittlung der latenten Steuern nach KILA-Anweisung

„Latente Steuern“ ........................................................................ XXIV

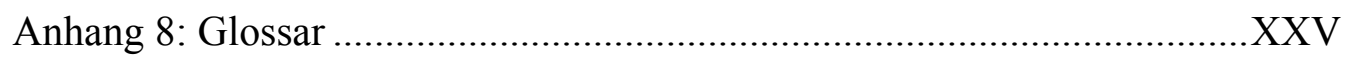

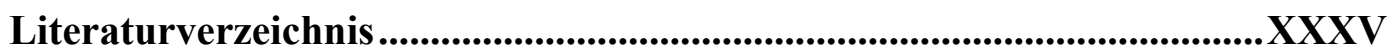

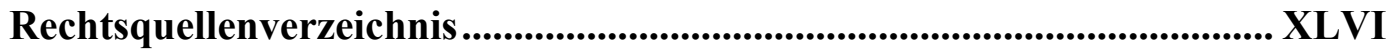




\section{Abkürzungsverzeichnis}

a. 0 . außerordentlich

$\mathrm{Ab}(\mathrm{Oy})$ finnische private Aktiengesellschaft

Abb.

Abbildung

ABl.

Amtsblatt der Europäischen Gemeinschaft

Abp (Oyj) finnische öffentliche Aktiengesellschaft

Abs.

Absatz

AfA

Absetzung für Abnutzung

AG Aktiengesellschaft

AktG

Aktiengesetz

AML

finnisches Wertpapiermarktgesetz

Art. Artikel

Aufl.

Auflage

AusInvG

Auslandsinvestitionsgesetz

Ay finnische Offene Handelsgesellschaft bearb. bearbeitete

bzw. beziehungsweise

ca. circa

d. h. das heißt

Diss. Dissertation

DRS Deutscher Rechnungslegungs Standard DRSC Deutsche Rechnungslegungs Standards Committee e.V. DSR Deutscher Standardisierungsrat e.V. eingetragener Verein

EDV Elektronische Datenverarbeitung

EG Europäische Gemeinschaft

EGHGB Einführungsgesetz zum HGB erh. erhalten(e)

EStG Einkommensteuergesetz

EStR Einkommensteuerrichtlinie

EU Europäische Union

EVL finnisches Gewerbesteuergesetz

EWG Europäische Wirtschaftsgemeinschaft

f. folgende FEE Fédération des Experts Comptables Européens

ff. fortfolgende

FIFO First in, first out GAAP Generally Accepted Accounting Principles gel. geleistet $(\mathrm{e})$

GenG Genossenschaftsgesetz 
ggü. gegenüber

$\mathrm{GmbH}$ Gesellschaft mit beschränkter Haftung $\mathrm{GmbHG}$ Gesetz betreffend die Gesellschaft mit beschränkter Haftung GoB Grundsätze ordnungsmäßiger Buchführung

$\mathrm{GuV}$ Gewinn- und Verlustrechnung

HE Regierungsvorlage an das finnische Parlament

HFA Hauptfachausschuss des IDW

HGB Handelsgesetzbuch

Hrsg. Herausgeber

HTM finnischer anerkannter Wirtschaftsprüfer i. Allg. im Allgemeinen

i. V.m. in Verbindung mit

IASB International Accounting Standards Board

IASC International Accounting Standards Committee IDW Institut der Wirtschaftsprüfer in Deutschland e.V.

IFAC International Federation of Accountants incl. inclusive

ISA International Standards on Auditing

Jg. Jahrgang JHTT finnischer Wirtschaftsprüfer im öffentlich-rechtlichen Bereich

Kap. Kapitel

KapAEG Kapitalaufnahmeerleichterungsgesetz

KapCoRiLiG Kapitalgesellschaften- und Co-Richtlinie-Gesetz KELA finnischer staatlicher Rentenversicherungsträger

kfr. kurzfristig

KHT autorisisierter Wirtschaftsprüfer

KILA finnischer Buchführungsausschuss

KonTraG Gesetz zur Kontrolle und Transparenz im Unternehmensbereich korr. korrigiert

KPA finnische Buchführungsverordnung

KPL finnisches Buchführungsgesetz

KTL finnischer Verband der privaten Buchführungsfirmen KWG Kreditwesengesetz Ky finnische Kommanditgesellschaft

lfd. laufend

lfr. langfristig

LIFO Last in, first out Mio. Million(en) $\mathrm{Nr}$.

o. Ä. Nummer

o. V. oder Ähnliches OECD ohne Verfasser Organization for Economic Cooperation and Development 
OKL finnisches Genossenschaftsgesetz

Oy $(\mathrm{Ab})$ finnische private Aktiengesellschaft Oyj (Abp) finnische öffentliche Aktiengesellschaft OYL finnisches Aktiengesellschaftsgesetz

p. a. pro anno

PublG Publizitätsgesetz

r.y. finnischer eingetragener Verein

S. Satz

$\mathrm{S}$. Seite S. siehe sonst. sonstige stl. steuerlich

TilintA finnische Wirtschaftsprüfungsverordnung TilintL finnisches Wirtschaftsprüfungsgesetz TVL finnisches Einkommensteuergesetz u. a. unter anderem

u. $\ddot{\text { A. }}$ und Ähnliche(s) überarb überarbeitete US(A) United States (of America) usw. und so weiter V. von v. a. vor allem VAG Versicherungsaufsichtsgesetz vgl. vergleiche z. B. zum Beispiel 


\section{Abbildungsverzeichnis}

Abb. 1: $\quad$ Einflüsse auf die finnische Rechnungslegung seit 1920 ............... 33

Abb. 2: $\quad$ Einflussnehmer auf die finnische Rechnungslegung.................... 44

Abb. 3: Anteil der Aktiengesellschaften an allen finnischen Unternemen. 47

Abb. 4: Regelungsbereiche der finnischen Gesetze mit Rechnungslegungsrelevanz .................................................... 55

Abb. 5: Veränderung finnischer Jahresabschlusszwecke im Zeitablauf .... 58

Abb. 6: Verhältnis des True and Fair View zur Art guter Buchführung und anderen Grundsätzen ordnungsmäßiger Buchführung ................ 89

Abb. 7: $\quad$ Bewertung von Vermögenswerten .......................................... 123

Abb. 8: $\quad$ Voraussetzungen für die Aktivierung immaterieller Vermögenswerte

Abb. 9: $\quad$ Abgrenzung von Rückstellungen, Verbindlichkeiten und Eventualverbindlichkeiten. 183 
Gang der Untersuchung

\subsection{Problemstellung}

\subsubsection{Heterogenität der Rechnungslegungssysteme}

Die Rechnungslegung dient der ziffernmäßigen Erfassung, Aufbereitung und Abbildung von betrieblichen Strukturen, Realprozessen und anderen Ereignissen, welche die Vermögens-, Finanz- und Ertragslage eines Unternehmens beeinflussen $^{1}$. Instrumente der Rechnungslegung sind Einzel- und Konzernabschluss mit ihren Bestandteilen Bilanz, Gewinn- und Verlustrechnung und Anhang sowie der Lagebericht und ergänzende Rechnungen.

An die im Einzel- und Konzernabschluss bereitzustellenden Informationen stellt das Unternehmensumfeld - Kapitalgeber, andere Marktteilnehmer, der nationale Gesetzgeber oder ein „Standard Setter“ - Anforderungen. Es gibt Rahmenbedingungen vor, die das Unternehmen entweder zwingend befolgen muss oder freiwillig beachten kann. Die Gesamtheit dieser von einem Unternehmen unter bestimmten Bedingungen zu beachtenden Regelungen wird als Rechnungslegungssystem bezeichnet. Aus dem Wechsel- und Zusammenspiel zwischen dem Unternehmensumfeld mit seinen unterschiedlichen Informationsinteressen und den Interessen der Unternehmen haben sich im Laufe der Zeit komplexe Rechnungslegungssysteme entwickelt, die im internationalen Vergleich Unterschiede aufweisen. Unterschiedliche nationale Normen bestehen in Bezug auf die Definition der rechnungslegenden Einheit und auf den Inhalt, den Umfang und die Form der Daten, die offen zu legen sind. Nationale Gepflogenheiten prägen auch die Detailliertheit der Rechnungslegungsnormen, ihre Auslegung und die hierfür zuständigen Institutionen. Relevant ist zudem, ob und wie die Einhaltung dieser Normen überwacht wird ${ }^{2}$. In den Rechnungslegungssystemen spiegeln sich geschichtliche, kulturelle und sozial-ökonomische Gegebenheiten wider ${ }^{3}$.

Als problematisch erweisen sich die Unterschiede zwischen den Rechnungslegungssystemen dann, wenn ein Bilanzleser Daten analysieren möchte, die aus einem ihm fremden Bilanzierungsraum stammen. Um die Zahlen zu beurteilen, zu werten und zu gewichten, muss der Leser sie zu einem aussagefähigen Vergleich mit Daten anderer Unternehmen aus ihm bekannten Bilanzierungsräumen heran-

\footnotetext{
Vgl. van der Tas (1988), S. 157 und vgl. Lappalainen (1999), S. 19.

Vgl. Pellens (1999), S. 1-2. Zur Überwachung von Normeneinhaltung vgl. beispielsweise Tielmann (2001).

Vgl. Nobes und Parker (2000), S. 16-27 und vgl. Glaum und Mandler (1996), S. 26.
} 
ziehen. Dies ist ihm nur eingeschränkt möglich, wenn er die erforderliche Kenntnis der Bilanzierungs- und Bewertungsgrundsätze und -vorschriften nicht vorweisen kann. Hinderlich sind auch mangelndes Wissen und unzureichende Erfahrung damit, wie genau und verlässlich die betreffenden Regelungen angewendet werden und welche Qualität den landesüblichen Jahresabschlussprüfungen beizumessen ist.

In Fremdsprachen verfasste Unternehmensinformationen bergen ein Sprachrisiko, das zu einer Fehlanalyse führen kann; selbst bei Übersetzungen besteht die Gefahr unterschiedlicher Interpretation von Begriffen ${ }^{4}$. Aus Rechnungslegungsdaten resultierende Missverständnisse und Fehlurteile wirken sich negativ aus, wenn die Daten die Grundlage für die Lösung von Entscheidungsfragen, die das Unternehmen betreffen, bilden. Auch kann das Anwerben ausländischer Kapitalgeber und das Agieren in internationalen Finanzmärkten beeinträchtigt werden, wenn die veröffentlichten Rechnungslegungsdaten nicht der Intention des Erstellers entsprechend ausgelegt werden ${ }^{5}$.

Zur Bewältigung der genannten Probleme können grundsätzlich drei Ansätze in Betracht gezogen werden:

- Der Bilanzleser kann versuchen, länderbezogene Spezialkenntnisse aufzubauen; bei der Vielzahl der existierenden Rechnungslegungssysteme erscheint diese Möglichkeit jedoch unrealistisch.

- Alternativ können die Ersteller dem Jahresabschluss Erläuterungen und zusätzliche Informationen beifügen, die es dem Leser erlauben, den Abschluss gedanklich in einen Abschluss anderer Vorgehensweise überzuleiten.

- Der dritte Ansatz - die aktuell vorherrschende Entwicklung - geht dahin, die unterschiedlichen nationalen Rechnungslegungssysteme einander auf internationaler Ebene anzugleichen ${ }^{6}$.

Vgl. Glaum und Mandler (1996), S. 31-32.

Vgl. Pellens (1999), S. 12-21. In der Literatur wird kritisch angemerkt, dass die Internationalisierung der Kapitalmärkte trotz der existierenden unterschiedlichen Rechnungslegungssysteme voranschreite. Hieraus könne geschlossen werden, dass die Informationseffizienz der Kapitalmärkte unabhängig von Rechnungslegungsdaten sei (vgl. Cronos (1991), S. 188 oder Goeltz (1991)). Es erhebt sich in diesem Zusammenhang die Frage, ob mit der Annäherung der Rechnungslegungssysteme zur Vermeidung von Missverständnissen und Fehlurteilen nicht auch die Internationalisierung der Kapitalmärkte schneller fortschreiten würde.

6 Vgl. Glaum und Mandler (1996), S. 33-37. Die Notwendigkeit der internationalen Harmonisierung wird in einigen Literaturbeiträgen infrage gestellt. Mit einer Harmonisierung sind erheblicher Arbeits- und Zeitaufwand verbunden. Anpassungskosten werden durch Änderungen im Rechtssystem verursacht. Die Abstimmung der Rechnungslegungssysteme aufeinander allein ist nicht ausreichend, da sie durch nicht harmonisierte Umfeldbedingungen stark relativiert würde: Es gilt, auch Steuergesetzgebung, Gesellschaftsrecht und Börsengesetze einander anzunähern. Kosten fallen zudem bei Bilanzerstellern und -adressaten an, sobald 


\subsubsection{Harmonisierung von Einzelabschlüssen innerhalb der Europäi- schen Union}

Rechnungslegungssysteme können einander durch Anpassung angeglichen werden. Dies geschieht, indem wesentliche Vorschriften und Methoden eines existierenden Rechnungslegungssystems von anderen übernommen werden ${ }^{7}$.

Der Annäherungsprozess kann aber auch durch Kooperation erfolgen: Zielvorstellungen unterschiedlicher Rechnungslegungssysteme werden auf dem Verhandlungswege einander angenähert und so ein für alle Beteiligten neues, gemeinsames Rechnungslegungssystem begründet ${ }^{8}$. Die Zielspektren einer Angleichung unterschiedlicher Rechnungslegungssysteme variieren zwischen Verringerung und völliger Aufhebung aller Unterschiede zwischen nationalen Rechnungslegungssystemen. Mit einer Verringerung der Unterschiede soll in einem ersten Schritt Vergleichbarkeit erreicht werden, die unterschiedliche Ausprägungen annehmen kann. Unter einer Vergleichbarkeit auf einem solchen Niveau, dass nationale Rechnungslegungssysteme aus gleichen Geschäftsvorfällen sich nicht wider-

diese sich und ihre Systeme auf das neue Bilanzrecht umstellen müssen (vgl. Glaum und Mandler (1996), S. 80--81). Harmonisierung sei deshalb nur dann als sinnvoll anzusehen, wenn ihre Vorteile aus gesamtwirtschaftlicher Sicht gegenüber den Nachteilen überwiegen. Offen bleibt indes, wie diese gemessen werden können (vgl. Pellens (1999), S. 365 und vgl. Glaum und Mandler (1996), S. 38 und die dort angegebene Literatur).

Im Fachschrifttum wird neben dem Kostenargument gegen eine Harmonisierung angeführt, Rechnungslegungssysteme seien historisch gewachsen und böten traditionell diejenigen Informationen an, die das betreffende finanzwirtschaftliche Umfeld nachfrage: Unter der Prämisse, dass weiterhin unterschiedliche ökonomische Welten - segmentierte Märkte - existieren, müssten diese ihren Ausdruck in unterschiedlich gestalteten Bilanzierungswelten finden (vgl. Pellens (1999), S. 366).

Diese Prämisse ist jedoch nicht haltbar, da weltweit die Integration der Märkte durch Liberalisierung und Deregulierung sowie verbesserte Kommunikationsmöglichkeiten durch eine sich entwickelnde Informationstechnologie voranschreitet.

Vor dem Hintergrund grenzüberschreitenden Handels, der Internationalisierung der Unternehmensstrukturen - verbunden mit der Pflicht zur Erstellung von „Weltabschlüssen“ - und der Globalisierung der Kapitalmärkte erscheint eine Harmonisierung erstrebenswert: Konzernen mit internationalem Aufbau bedeutet es eine kostensenkende Arbeitserleichterung, wenn sie bei der Erstellung ihrer Abschlüsse keine unterschiedlichen nationalen Bewertungsund Besteuerungseigenheiten berücksichtigen müssen. Zudem ist zu erwarten, dass sich die Qualität der Konzernabschlüsse erhöht und somit ihre Aussagefähigkeit verbessert, wenn nationale Gepflogenheiten entfallen. Börsennotierte Unternehmen werden durch die Anforderungen der nationalen Aufsichtsbehörden zum parallelen Erstellen mehrer Abschlüsse veranlasst; eine internationale Annäherung der Rechnungslegung muss aus Zeit- und Kostengründen in ihrem Interesse liegen. Auch Wirtschaftsprüfungsgesellschaften erleichtert eine länderübergreifende Harmonisierung die Beantwortung von Konsolidierungsfragen und den internationalen Einsatz ihrer Arbeitnehmer (vgl. Lappalainen (1999), S. 24, vgl. Pellens (1999), S. 365-366, vgl. Nobes und Parker (2000), S. 67 und vgl. Glaum und Mandler (1996), S. 38-78).

Dem in der Literatur aufgeführten Argument, der Harmonisierungsprozess sei wegen des Verlusts an nationaler politischer Souveränität abzulehnen, ist entgegenzuhalten, dass er de facto stattfindet, unabhängig davon, ob er befürwortet wird oder nicht. Es sollte deshalb im eigenen politischen Interesse versucht werden, ihn aktiv mitzugestalten.

Vgl. Glaum und Mandler (1996), S. 36.

Vgl. Glaum und Mandler (1996), S. 36. 
sprechende Informationen generieren oder durch Zusatzangaben die Überführung in die jeweils andere Verfahrensweise ermöglichen, wird Gleichwertigkeit verstanden ${ }^{9}$. Bei einer Standardisierung werden sämtliche Rechnungslegungsunterschiede eliminiert; ein einheitliches System wird geschaffen ${ }^{10}$.

Das Ausmaß der Unterschiedsreduzierung wird mit dem Harmonisierungsgrad gemessen. Er hängt ab von Normen, Jahresabschlussinhalten, von Informationsbreite und -tiefe sowie von Informationsinstrumenten ${ }^{11}$. Bei einer Harmonisierung der Rechnungslegung auf internationaler Ebene - gleichgültig, ob durch Anpassung oder durch Kooperation - ist von den beteiligten Staaten zu diskutieren, auf welcher Ebene und in welchem Ausmaß die Rechnungslegungsverfahren einander angeglichen werden sollen. Über den erforderlichen Harmonisierungsgrad müssen sich die Staaten - beispielsweise innerhalb der Europäischen Union - einig werden.

Die Harmonisierungsbestrebungen der EU basieren auf dem am 25. März 1957 in Rom geschlossenen „Vertrag zur Gründung der Europäischen Wirtschaftsgemeinschaft“. Er regelt, dass die Mitgliedstaaten ,[...] soweit erforderlich die Schutzbestimmungen koordinieren, die in den Mitgliedstaaten den Gesellschaften [...] im Interesse der Gesellschafter sowie Dritter vorgeschrieben sind, um diese Bestimmungen gleichwertig zu gestalten. “12 Hieraus wird deutlich, dass die Europäische Union nicht eine Standardisierung im Sinne einer Vereinheitlichung, sondern einer Gleichwertigkeit derjenigen gesellschaftsrechtlichen Vorschriften anstrebt, die auf den Schutz der Gesellschafter und Dritter gerichtet sind. Hierzu zählen auch die Rechnungslegungsvorschriften.

Mit einer Abstimmung der Rechnungslegungsnormen in den EU-Staaten wird das Ziel verfolgt, die Entwicklung des europäischen Binnenmarkts durch Niederlassungsfreiheit für Unternehmen zu fördern, indem ihnen und ihren Gläubigern in allen Mitgliedstaaten gleichwertiger Schutz zukommt. Auch sollen Handelsbeziehungen innerhalb der Mitgliedstaaten erleichtert werden. Eine Harmonisierung dient zudem der Förderung gleicher Wettbewerbsbedingungen für Unternehmen in den Mitgliedstaaten - konkurrierende Unternehmen sollten gleichwertige finanzielle Informationen offen legen. Diese Forderung gilt insbesondere für Kapi-

Vgl. Pellens (1999), S. 361-362.

10 Vgl. Pellens (1999), S. 362-363, vgl. Nobes und Parker (2000), S. 66-69, vgl. Choi und Mueller (1992), S. 257 und vgl. Goerdeler (1987), S. 122-123.

11 Vgl. Majala (1991), S. 184.

12 Art. 54 Abs. 3 (g) EG-Vertrag. 
talgesellschaften, da diese häufig international tätig sind und Dritten eine Sicherheit nur durch ihr Gesellschaftsvermögen bieten ${ }^{13}$.

Um diese Ziele zu erreichen, stehen der EU verschiedene Handlungsalternativen zur Rechtsangleichung zur Verfügung. Die wichtigste - weil wirksamste - von ihnen ist das Erlassen einer Richtlinie ${ }^{14}$. Richtlinien bedürfen innerhalb eines vorgeschriebenen Zeitraums der Umsetzung in das nationale Recht jedes einzelnen Mitgliedstaats. Den Staaten ist freigestellt, wie sie die Richtlinieninhalte in ihre nationale Gesetzgebung einbinden; die Regelungsziele allerdings sind ihnen verbindlich vorgeschrieben ${ }^{15}$.

Zur Harmonisierung der Rechnungslegung im Einzelabschluss sind von der EU bislang folgende gesellschaftsrechtliche Richtlinien erlassen worden, die unter dem Begriff „4. EG-Richtlinie“ zusammengefasst werden ${ }^{16}$ :

- Die 4. EG-Richtlinie vom 25. Juli 1978, in welcher die Vorschriften über Form und Inhalt sowie über Offenlegung und Prüfung von Jahresabschluss und Lagebericht von Kapitalgesellschaften koordiniert werden,

- die Mittelstandsrichtlinie vom 08. November 1990, welche die Bilanzierungsvorschriften für mittelständische Kapitalgesellschaften erleichtert,

- die GmbH\&Co-Richtlinie vom 08. November 1990, welche die 4. und 7. EGRichtlinie auf Personenhandelsgesellschaften ausdehnt, deren Komplementäre ausschließlich Kapitalgesellschaften sind und

- die Richtlinie vom 17. Juni 1999, welche die Größenkriterien zur Definition von kleinen und mittelgroßen Gesellschaften neu bestimmt.

Nach der Transformation dieser Richtlinien in nationales Recht muss dieses zwingend im Sinne des Richtliniengebers ausgelegt und interpretiert werden. Zur Unterstützung der Mitgliedstaaten, der Jahresabschluss-Leser und -Ersteller hat die Europäische Kommission 1998 eine Interpretationshilfe, ausgewählte Artikel der 4. EG-Richtlinie betreffend, veröffentlicht. Bei Unklarheiten, Unstimmigkeiten und Streitigkeiten obliegt die Definition bestimmter Begriffe und die Auslegung der Richtlinie letztlich dem Europäischen Gerichtshof ${ }^{17}$.

Vgl. Präambel der 4. EG-Richtlinie.

Andere rechtliche Instrumente stellen Verordnungen, Entscheidungen, Empfehlungen und Stellungnahmen dar. Vgl. van Hulle und van der Tas (2001), S. 781 und vgl. Pellens (1999), S. 377.

15 Vgl. van Hulle und van der Tas (2001), S. 781 und vgl. Pellens (1999), S. 377.

16 Außer Acht gelassen werden wegen ihrer Brachenbezogenheit im Folgenden die EGBankbilanzrichtlinie vom 08.12.1986 und die EG-Versicherungsbilanzrichtlinie vom 19.12.1991. Ausgeklammert wird ebenfalls die 7. EG-Richtlinie, da sich die vorliegende Untersuchung auf Einzelabschlüsse beschränkt.

17 Vgl. van Hulle und van der Tas (2001), S. 781. 
Grundsätzlich besteht in den Mitgliedstaaten und in der Fachliteratur Einigkeit darüber, dass auch nach Transformation der bisher bestehenden Richtlinien zum Einzelabschluss in die nationalen Gesetzgebungen der einzelnen Länder die Notwendigkeit $\mathrm{zu}$ weiterer Harmonisierung besteht. So klärt die 4. EG-Richtlinie wichtige Grundsatzfragen nicht, nennt weder Ziele noch Adressaten der Rechnungslegung und definiert die allgemeinen Jahresabschluss-Grundsätze nicht. Wichtige Bilanzierungsprobleme, wie die Behandlung von Leasingverträgen, die Währungsumrechnung, die Gewinnrealisierung bei langfristiger Auftragsfertigung oder die Ermittlung latenter Steuern, werden in ihr nicht explizit geregelt. Die 4. EG-Richtlinie setzt sich mehr mit formellen Fragen als mit materiellen Inhalten auseinander, die für die Vergleichbarkeit und Gleichwertigkeit von Jahresabschlüssen bedeutsamer sind. Hieraus wird der Kompromiss deutlich, der geschlossen worden ist, als die Traditionen verschiedener Staaten vereint werden sollten und bestimmte Unterschiede mit fundamentaler Bedeutung für die jeweiligen nationalen Wirtschaftssysteme bestehen bleiben mussten. Im Ergebnis dieses Kooperationsprozesses räumt die 4. EG-Richtlinie in 62 Artikeln 76 Wahlrechte sowohl Mitgliedstaaten- als auch Unternehmenswahlrechte - ein.

Es stellt sich die Frage, inwieweit durch die Implementierung der Vorschriften der 4. EG-Richtlinie die Heterogenität der Rechnungslegung wirklich verringert worden. Fraglich ist, ob die genannten Harmonisierungsbestrebungen tatsächlich zu einer Angleichung von Rechnungslegungsregeln geführt haben, ob die formale Harmonisierung fortgeschritten ist. Interessant ist auch die Frage nach dem derzeitigen Stand der materiellen, tatsächlich realisierten Harmonisierung, also die Frage, ob sich die Rechnungslegungspraxis der Unternehmen verschiedener Staaten einander genähert hat ${ }^{18}$. Seit der Transformation der Richtlinie können zahlreiche bisher länderspezifische Wahlrechte länderübergreifend ausgeübt werden. Zudem werden in der Richtlinie nicht erfasste bzw. nur vage formulierte Vorschriften von nationalen Einrichtungen - Behörden, Standard-Settern oder Wirtschaftsverbänden - geregelt. Es besteht in diesen Fällen die Gefahr, dass dies vor dem Hintergrund nationaler Denkweisen geschieht und die Heterogenität der Rechnungslegungssysteme so nicht beseitigt wird ${ }^{19}$. Seit 1996 verfolgt die EU deshalb die Strategie, ihre Rechnungslegungsvorschriften und die europäische Harmonisierung in die globale Harmonisierung, angestrebt durch das IASB, einzubinden $^{20}$.

Zum Begriffspaar ,Formale und materielle Harmonisierung“ vgl. van der Tas (1988), S. 158. Vgl. Pellens (1999), S. 381-385 und vgl. Glaum und Mandler (1996), S. 11.

Vgl. van Hulle und van der Tas (2001), S. 784-785. Die modifizierte 4. EG-Richtlinie wird permanent weiterentwickelt und ergänzt, die Auslegung ihrer Inhalte wandelt sich. Es wird das Ziel verfolgt, die Aussagekraft der Jahresabschlüsse zu erhöhen und ihre Vergleichbar- 


\subsection{Zielsetzung und Methodik vorliegender Untersuchung}

\subsubsection{Zielsetzung}

Aufgabe der vorliegenden Arbeit ist es, den momentanen Stand der formalen Harmonisierung zwischen Finnland und Deutschland sowie das tatsächlich realisierte Vergleichbarkeitsniveau von Einzelabschlüssen finnischer und deutscher Unternehmen zu ermitteln.

Deutschland hat als Gründungsmitglied der Europäischen Wirtschaftsgemeinschaft - der späteren Europäischen Gemeinschaft und der heutigen Europäischen Union - von Anfang an die Chance gehabt und genutzt, die Inhalte der 4. EGRichtlinie mitzubestimmen. So diente das deutsche Aktiengesetz 1965 dieser Richtlinie als Vorlage. Deutschlands Bilanzierungspraktiken hatten auch schon früher die Nachbarstaaten und Wirtschaftspartner beeinflusst. Deutschland selbst hat die 4. EG-Richtlinie 1985 mit dem Bilanzrichtlinien-Gesetz in nationales Recht umgesetzt ${ }^{21}$. Die Mittelstandsrichtlinie hat der deutsche Gesetzgeber 1994 übernommen. Mit dem Kapitalgesellschaften\&Co-Richtlinie-Gesetz vom 24. Februar 2000 hat er schließlich die EU-Vorgaben der GmbH\&Co-Richtlinie implementiert und gleichzeitig die Größenklassen der Gesellschaften für den Jahresabschluss und die Konzernabschlussbefreiung nach Maßgabe der Richtlinie vom 17. Juni 1999 angepasst.

Finnland ist erst seit 1995 EU-Mitglied und hat die modifizierte 4. EG-Richtlinie in zwei Schritten in seine Gesetzgebung transformiert: im Rahmen der Beitrittsverhandlungen teils mit der Modifizierung des alten Buchführungsrechts zum 01. Januar 1992, endgültig dann mit der Einführung des neuen Buchführungsgesetzes zum 31. Dezember 1997, das erstmals für in 1998 endende Geschäftsjahre zur Anwendung gekommen ist.

Sowohl Deutschland als auch Finnland haben die genannten Gesetzesänderungen vorgenommen, um die Unternehmen dazu zu veranlassen, ihre Bilanzierungspraktiken im Sinne des Richtliniengebers zu ändern und die Jahresabschlüsse von Unternehmen innerhalb ihres Staats und im Verhältnis zu denen anderer EU-Staaten

keit untereinander zu verbessern. Dies lässt vermuten, dass die angestrebte Gleichwertigkeit der Abschlüsse innerhalb der EU noch nicht erreicht ist, was durch wissenschaftliche Untersuchungen aus den Jahren 1992 und 1995 bestätigt wird. Vgl. Herrmann und Thomas (1995) und Emenyonu und Gray (1992).

21 Vgl. Gross und Schruff (1986), S. 17 ff. und vgl. Biener und Berneke (1986), welche die Transformation der 4. EG-Richtlinie und ihre Hintergründe darstellen. 
vergleichbarer zu gestalten ${ }^{22}$. Es wird im Folgenden angenommen, dass unternehmensindividuelle Bilanzierungspraktiken sowie nationale Eigenheiten sich nach Gesetzesänderungen nur langsam wandeln. Der Harmonisierungsprozess innerhalb eines Staats und zwischen den Staaten dauert mehrere Jahre, da sich unterschiedliche inhaltliche Auffassungen einzelner Agierender über die Bewertungsgrundsätze und zahlreiche Einzelregelungen aufeinander zu bewegen müssen ${ }^{23}$.

In der europäischen Fachliteratur existieren zahlreiche kritische Abhandlungen zu der Frage, wie Deutschland die Regelungen der modifizierten 4. EG-Richtlinie in nationales Recht transformiert hat und ob dies im Sinne des Richtliniengebers geschehen ist $^{24}$. Finnland ist in länderübergreifenden Rechnungslegungsvergleichen bislang kaum berücksichtigt worden. Dies mag daran liegen, dass es erst 1995 der EU beigetreten ist und als kleines Land international aus ökonomischer Sicht relativ unbedeutend ist. Zudem stellt die mangelnde Kenntnis der finnischen Sprache im Ausland ein Zugangshindernis zur finnischen Fachliteratur und zu finnischsprachigen Geschäftsberichten dar ${ }^{25}$.

Dieser Zustand hat sich trotz des Betritts Finnlands zur EU bislang nicht gebessert, obwohl finnische Unternehmen ihre Handels- und Investitionstätigkeiten im europäischen Ausland intensiviert haben. Finnland hat als bislang einziger nordeuropäischer Staat den Euro eingeführt ${ }^{26}$ und ist somit fester Bestandteil eines zusammenwachsenden Europas. Eine Auseinandersetzung mit der finnischen Rechnungslegung wird aus diesen Gründen als notwendig erachtet ${ }^{27}$.

Ein Ziel dieser Arbeit ist es deshalb, dem deutschsprachigen Leser das finnische Rechnungslegungssystem sowie das finnische Verständnis des True and Fair View, der zentralen Jahresabschlussgrundsätze und damit der Ansatz- und Bewertungsgrundsätze zu verdeutlichen. Untersucht wird,

- wie zwingend vorgeschriebene Richtlinien-Regelungen umgesetzt worden sind,

- wie Richtlinien-Wahlrechte von den Unternehmen der beiden Staaten ausgeübt werden dürfen bzw. müssen und

Vgl. HE 173/1997, Abschnitt 1.1.

Vgl. Lappalainen (1999), S. 13 und vgl. Ordelheide (1998), S. 22-23.

Hierauf soll deshalb in vorliegender Arbeit nicht primär eingegangen werden, sondern nur dort, wo es für das bessere Verständnis notwendig ist. Es wird auf das bereits vorhandene Schrifttum verwiesen. Vgl. z. B. Biener und Berneke (1986).

25 Vgl. Lappalainen (1999), S. 33.

26 Stand 01. Januar 2002.

27 Die Darstellungen von HEINO, KYKKÄNEN und SCHOLTISSEK zur finnischen Rechnungslegung basieren auf nicht geltendem Recht und sind für aktuelle Vergleiche nicht mehr anwendbar. Vgl. Heino (1995), vgl. Kykkänen (1986) und vgl. Scholtissek (1984). 
- welche nationalen Lösungen für die in der Richtlinie enthaltenen Regelungslücken gefunden worden sind.

Daneben ist das es Ziel dieser kritischen Auseinandersetzung mit der finnischen Rechnungslegung, eine deutschsprachige Basis zu schaffen, von der aus Finnland mit allen anderen EU-Mitgliedstaaten verglichen werden kann.

Diese Arbeit beschränkt sich auf Einzelabschlüsse. Die Anpassung unterschiedlicher nationaler Einzelabschlüsse an eine konzerneinheitliche Bilanzierung, Bewertung und Gliederung - sei es nach nationaler Gesetzgebung oder internationalen Standards - kann nur dann erfolgen, wenn Unterschiede und Gemeinsamkeiten der Rechnungslegungssysteme bekannt sind. Auch hierzu soll die vorliegende Arbeit im Hinblick auf deutsch-finnische bzw. finnisch-deutsche Konzerne beitragen.

\subsubsection{Methodik}

Um die Rechnungslegungssysteme verschiedener Staaten miteinander vergleichen zu können, sind in der Fachliteratur verschiedene Ansätze entwickelt worden. MAJALA stellt vier hierarchische Ebenen für die Untersuchung von Rechnungslegungspraktiken und ihren anschließenden Vergleich fest: die Kultur-Ebene, die Theorie-Ebene, die Regelwerk-Ebene sowie die Format-Ebene ${ }^{28}$.

Die Kultur-Ebene kann als Ausgangspunkt für die Analyse von Rechnungslegungssystemen dienen. In ihr sind die Rahmenbedingungen enthalten, die sich auf die Bilanzierung auswirken: die geschichtliche Entwicklung der Buchführung, das politische und wirtschaftliche System und die Akteure der Rechnungslegungsnormierung. Das Wissen um diese Einflussfaktoren bildet die Voraussetzung für das Verstehen eines Rechnungslegungssystems und für die Erklärung von Gemeinsamkeiten und Unterschieden verschiedener Systeme ${ }^{29}$. In Kap. 2.1 der vorliegenden Arbeit wird die geschichtliche Entwicklung, in Kap. 2.2 die Normierung der finnischen Rechnungslegung und ihre Akteure dargestellt.

Die Untersuchung der Theorie-Ebene dient der Feststellung, welche theoretischen Konzeptionen einem Rechnungslegungssystem zu Grunde liegen. In den seltensten Fällen basieren Bilanzierungspraktiken auf einer in sich geschlossenen Theo- 
rie $^{30}$; in den meisten Staaten stützt sich die Buchführungspraxis jedoch auf Grundsätze und Definitionen, die wissenschaftlichen Ursprungs und theoretischer Natur sind. Untersuchungen der Theorie-Ebene helfen bei der Erklärung und Interpretation von Jahresabschlussinhalten ${ }^{31}$. In Kap. 3 der vorliegenden Arbeit werden die Grundlagen des aktuellen finnischen Rechnungslegungssystems erläutert und vor dem Hintergrund der 4. EG-Richtlinie kritisch erörtert. Analysiert wird, ob wesentliche Widersprüche zur Intention des Richtliniengebers vorliegen. Die Untersuchung dieser Frage ist Bedingung für eine Beurteilung des erreichten Harmonisierungsgrads zwischen Deutschland und Finnland, da die modifizierte 4. EGRichtlinie das zwingende Referenzsystem für beide Staaten darstellt. Die Ergebnisse der Theorie-Ebene sollen einen Vergleich Finnlands mit allen anderen EUStaaten auf Regelwerk-Ebene ermöglichen.

Analysen der Regelwerk-Ebene beschäftigen sich mit der Frage, wie und bis zu welchem Grad ein Rechnungslegungssystem normiert ist: Gesetze, Erlasse, Gerichtsurteile, Standards und Anweisungen sowie ihre Einhaltung werden untersucht. Auf dieser Ebene kann die formale Harmonisierung zwischen Staaten ermittelt werden ${ }^{32}$. In Kap. 4 der vorliegenden Arbeit werden Grundlagen und Einzelfallregelungen des deutschen und des finnischen Rechnungslegungsrechts deskriptiv-vergleichend gegenübergestellt und der Stand der formalen Harmonisierung zwischen beiden Staaten dargestellt erarbeitet. Aufgezeigt wird, welche Gemeinsamkeiten und Unterschiede nach Transformation der modifizierten 4. EGRichtlinie in beiden Staaten bestehen. Hintergrund dieser Untersuchung ist die Frage nach dem Fortschritt der formalen Harmonisierung. Diese deskriptiv-vergleichende Darstellung soll der Identifizierung von alternativen Verfahren und Unterschieden dienen, um in der Empirie sinnvoll ausgewählte Bereiche vergleichen zu können.

In der Empirie - Kap. 4.2 dieser Arbeit - soll der materielle, tatsächlich realisierte Harmonisierungsgrad zwischen Deutschland und Finnland nach In-Kraft-Treten des Buchführungsgesetzes von 1997 in Finnland ermittelt werden. Untersuchungen auf der Format-Ebene basieren überwiegend auf veröffentlichten Jahresabschlussinformationen der Unternehmen. Hier werden Gemeinsamkeiten und Unterschiede der Bilanzierungspraktiken anhand von Bilanz, Gewinn- und Verlustrechnung, Anhang und Lagebericht deutlich. Der Grad der tatsächlich realisierten

30 Der Autorin sind zwei Staaten bekannt, in denen in der Vergangenheit die Buchführung auf einer geschlossenen theoretischen Konzeption basiert hat: die Niederlande mit der Buchführungstheorie Theodore Limpergs und Finnland mit der Ausgaben-Einnahmen-Theorie Martti Saarios. Vgl. Kap. 2.1.3.1 dieser Arbeit.

31 Vgl. Majala (1992), S. 327-328.

32 Vgl. Majala (1992), S. 328. 
Harmonisierung innerhalb eines Lands und zwischen Ländern lässt sich von hier aus ermitteln ${ }^{33}$. Es werden Geschäftsberichte der Jahre 1999 und 2000 herangezogen. Untersucht werden die Einzelabschlüsse der zehn größten börsennotierten Unternehmen beider Länder ${ }^{34}$.

Ausgehend von diesen vier Ebenen sind in der Literatur zahlreiche Klassifikationskataloge entworfen worden. Bei der Klassifizierung wird versucht, Faktoren, die einander stark ähneln, zu identifizieren. Die Systeme unterschiedlicher Länder können dann zu möglichst homogenen Gruppen hinsichtlich der Kultur, der Theorie oder des Regelwerks zusammengefasst werden. Innerhalb der Gruppen kann daraufhin der Harmonisierungsgrad ermittelt werden ${ }^{35}$. In dieser Arbeit soll im Weiteren nicht auf Klassifizierungen eingegangen werden ${ }^{36}$. Die Konzentration auf nur zwei Staaten - Deutschland und Finnland - erlaubt keine Klassifizierung, sondern lediglich das Aufzeigen von Gemeinsamkeiten und Unterschieden ${ }^{37}$.

33 Vgl. Majala (1992), S. 327 und vgl. Lappalainen (1999), S. 29-30.

34 Die Größe dieser Unternehmen wird gemessen an ihrem Marktkapitalisierungswert per 29. Dezember 2000. Banken und Versicherungen werden in dieser Untersuchung nicht berücksichtigt, da für sie sowohl in Deutschland als auch in Finnland rechnungslegungsspezifische Spezialvorschriften gelten und ihre Abschlüsse somit nicht mit denjenigen anderer Unternehmen vergleichbar sind.

35 Vgl. Pellens (1999), S. 30 und vgl. Nobes und Parker (2000), S. 54.

36 LAPPALAINEN hat auf Basis einer Abhandlung von AISBITT sämtliche Klassifizierungen zusammengestellt, in denen Finnland berücksichtigt worden ist, und sie kritisch kommentiert. Diese Klassifikationsuntersuchungen erfassen neben anderen europäischen und außereuropäischen Staaten immer auch Deutschland.

Klassifikationskataloge auf Kultur-Ebene, die Deutschland und Finnland beinhalten, sind von HOFSTEDE (1980) und GRAY (1986) aufgestellt worden. Deutschland findet sich bei beiden in der Gruppe der ,germanischen Staaten“ zusammen mit Österreich, der Schweiz und Israel wieder, während Finnland gemeinsam mit Dänemark, Schweden, Norwegen und den Niederlanden den ,,nordischen Staaten“ zugeordnet worden ist.

Auf Theorie-Ebene sind Klassifizierungen von DOUPNIK und SALTER (1992, 1993 und 1995) sowie von HOOGENDOORN (1996) vorgenommen worden. In diesen Klassifizierungsmodellen gehören Finnland und Schweden immer derselben Gruppe an; in den Analysen von 1995 und 1996 werden sie gar einer Gruppe zugeordnet, der außer ihnen keine anderen Staaten angehören.

LAPPALAINEN hat keine Klassifizierungsuntersuchungen auf Regelwerk- oder FormatEbene identifizieren können, in die Finnland einbezogen worden ist. Vgl. Lappalainen (1999).

$37 \quad$ Vgl. Nobes und Parker (2000), S. 54. 


\section{Rechnungslegung in Finnland}

\section{1}

\section{Entwicklung und Zäsuren der finnischen Rechnungslegung}

\subsubsection{Einführung der doppelten Buchführung in Finnland ab 1850}

Die Geschichte der Buchführung in Finnland geht zurück ins 14. und 15. Jahrhundert $^{38}$, die Anfänge der doppelten Buchführung liegen im 17. Jahrhundert ${ }^{39}$. Da Finnland von ca. 1100 bis 1809 Teil des schwedischen Königreichs und somit Schwedisch während dieser Zeit Amts- und Unterrichtssprache war, prägte das schwedische Verständnis von Buchführung die finnische Rechnungslegung ${ }^{40}$.

Infolge wiederholter Kriege zwischen Schweden und Russland verlor Schweden Finnland im Frieden von Tilsit 1809 an Zar Alexander I; danach blieb Finnland bis zur Unabhängigkeitserklärung 1917 autonomes Großherzogtum im Russischen Zarenreich. Auf Grund der Entscheidung des Zaren, Teile der schwedischen Gesetzgebung in Finnland beizubehalten und das Land nicht zu russifizieren, blieb das schwedische Gedankengut im wirtschaftlichen und kulturellen Leben in Finnland weitgehend erhalten ${ }^{41}$. Dennoch beeinflusste der zu dieser Zeit herrschende wirtschaftliche Liberalismus in Russland auch den Handel und die Industrie in Finnland: So war es der Landbevölkerung seit 1859 per Erlass gestattet, Einzelhandel zu betreiben, und seit 1879 wurde Handel generell erlaubt. Mit dem Aufschwung des Handels und dem Beginn der Industrialisierung in der zweiten Hälfte des 19. Jahrhunderts fand die Buchführung in Finnland breite Anwendung und wurde zunehmend zum Gegenstand von Theorie und Gesetzgebung ${ }^{42}$.

1862 erschien das erste finnischsprachige Lehrbuch zur Buchführung, von Augusti Lilius als „Praktisches Handbuch für die einfache Buchführung, speziell für

38 MAJALA unterteilt die Geschichte der finnischen Buchführung in vier Abschnitte, die im Folgenden zum besseren Verständnis der heutigen Rechnungslegungspraxis kurz erläutert werden. Bei den Abschnitten handelt es sich um

- Einführung der doppelten Buchführung in Finnland ab 1850 (Kap. 2.1.1 dieser Arbeit),

- $\quad$ Deutscher und skandinavischer Einfluss auf die finnische Rechnungslegung von 1920 bis 1950 (Kap. 2.1.2 dieser Arbeit),

- Theoriegeprägte Rechnungslegung von 1950 bis 1990 (Kap. 2.1.3 dieser Arbeit) und

- Internationale Harmonisierung seit 1990 (Kap. 2.1.4 dieser Arbeit). Vgl. Majala (2001 b), S. 957.

39 Vgl. Järvenpää (1996), S. 899.

$40 \quad$ Vgl. Majala (2001 b), S. 957.

$41 \quad$ Vgl. Majala (2001 b), S. 957.

$42 \quad$ Vgl. Näsi (1994), S. 493 f. 
Fabrikanten und Einzelunternehmer“"43 verfasst. Die von ihm ins Finnische übersetzten Fachausdrücke sind teilweise auch heute noch in Gebrauch. Die Buchführungsmethode, die LILIUS darlegt, stellt eine Mischung aus einfacher und doppelter Buchführung dar. Mit den Bestandteilen Anlagenbuch, Journal, Kassenbuch und Hauptbuch erinnert sie an die italienische Buchführung. LILIUS empfiehlt den Buchführenden, vorsichtig zu bilanzieren und den niedrigeren von historischem Anschaffungswert oder Marktwert zu wählen. Gebäude, Maschinen und Geschäftsausstattung sollen seiner Auffassung nach linear abgeschrieben werden $^{44}$. 1883 kam das erste Lehrbuch zur doppelten Buchführung, M. Lyytinens „Praktisches Handbuch der Handelsbuchführung, des Briefwechsels, der Wechsel-Geschäfte u.s.w. “45 auf den Markt ${ }^{46}$. Das erste Buchführungslehrbuch für produzierende Unternehmen, das auch Kosten- und Leistungsrechnung und die Problematik der Bewertung selbst erstellter Erzeugnisse einbezog, wurde 1915 von Ilmari Kaitila als „Lehrbuch für Buchhaltung und Bilanzen“47 veröffentlicht ${ }^{48}$. Dass der Rechnungslegung in dieser Zeit seitens der Wissenschaft Bedeutung beigemessen wurde, wird daraus ersichtlich, dass Buchführung sowie Kosten- und Leistungsrechnung bereits im ersten Vorlesungskommentar der 1911 gegründeten finnischsprachigen Handelshochschule Helsinki ${ }^{49}$ auf dem Lehrplan standen ${ }^{50}$.

Auch der Gesetzgeber erkannte die Notwendigkeit zur Rechnungslegung und sah es als seine Aufgabe an, die Buchführungspflicht zu regeln. 1864 wurden Aktiengesellschaften und Partnerschaftsunternehmen in Erlassen dazu verpflichtet, Bücher $\mathrm{zu}$ führen, und in der Konkursverordnung von 1868 wurden erstmals alle Gruppen von Gewerbetreibenden aufgelistet, die von der Buchführungspflicht betroffen waren. Gesetzlich verankert wurde die Buchführungspflicht für Aktiengesellschaften 1895 im Aktiengesellschaftsgesetz ${ }^{51}$. Dieses Aktiengesellschaftsgesetz spiegelt Finnlands Autonomie im russischen Zarenreich wider. Es wurde auf Finnisch, nicht auf Russisch veröffentlicht. Inhaltlich basierte es auf dem kurz zuvor verabschiedeten schwedischen Aktiengesellschaftsgesetz und nicht auf russischen Prinzipien. Das Aktiengesellschaftsgesetz verlangte, für jedes Geschäfts-

43 Der Titel des finnischen Originals lautet „Käytännöllinen opastus Yksinkertaisessa kirjanpidossa varsinkin Tehdastelioille ja Ammattilaisille“. Übersetzung ins Deutsche durch die Verfasserin.

$44 \quad$ Vgl. Majala (2001 b), S. 957.

45 Der Titel des finnischen Originals lautet „Käytännöllinen kauppakirjanpidon, kirjevaihdon, vekseli-asiain y.m. opastus“. Übersetzung ins Deutsche durch die Verfasserin.

$46 \quad$ Vgl. Majala (2001 b), S. 957.

47 Der Titel des finnischen Originals lautet „Kirjanpidon ja bilanssiopin oppikirja“. Übersetzung ins Deutsche durch die Verfasserin.

$48 \quad$ Vgl. Näsi (1994), S. 494 f.

49 Der finnische Name der Handelshochschule Helsinki lautet „Helsingin Kauppakorkeakoulu“. Übersetzung ins Deutsche durch die Verfasserin.

$50 \quad$ Vgl. Näsi (1994), S. 494.

51 Vgl. Majala (2001 b), S. 958. 
jahr einen Jahresabschluss zu erstellen, der Aktivposten und Verbindlichkeiten sowie Jahresüberschuss bzw. -fehlbetrag enthalten sollte. Für Genossenschaften folgte eine vergleichbare Regel im Genossenschaftsgesetz von $1901^{52}$. Diese ersten gesetzlichen Regelungen waren noch sehr allgemein gehalten und hatten relativ geringen Umfang. Grundsätzlich sollte durch sie lediglich sichergestellt werden, dass die Unternehmen eine Buchhaltung betreiben und deren Ergebnisse veröffentlichen. Die konkrete Ausgestaltung der Rechnungslegung war nicht geregelt $^{53}$.

Die 1911 gegründete Vereinigung der Wirtschaftsprüfer hielt fest, dass die Buchführung so vollständig sein sollte, dass aus ihr die täglichen Geschäftsvorfälle sowie Aktivposten, Verbindlichkeiten und Geschäftsergebnis nebst Haftungsverhältnissen und Eventualverbindlichkeiten hervorgehen ${ }^{54}$.

\subsubsection{Deutscher und skandinavischer Einfluss auf die finnische Rech- nungslegung von 1920 bis 1950}

\subsubsection{Buchführungsgesetz von 1925}

Nach der Unabhängigkeitserklärung von 1917 und dem anschließenden Bürgerkrieg benötigte der junge Staat Finnland ein eigenes nationales Rechtssystem, das die Regelungen aus der Zarenzeit und die noch älteren schwedischen Gesetzesteile ersetzen sollte. Im Rahmen dieser Gesetzesreformen wurde aus der Notwendigkeit heraus, Gläubiger zu schützen, die Selbstinformation der Eigentümer zu gewährleisten und über eine Grundlage für die Besteuerung zu verfügen, 1925 das erste Buchführungsgesetz ${ }^{55}$ verabschiedet $^{56}$.

Das Buchführungsgesetz von 1925 basierte auf deutschem wissenschaftlichen Gedankengut, war aber weniger genau und weniger formalistisch gestaltet als die deutschen Gesetze dieser Zeit ${ }^{57}$. Es berücksichtigte zusätzlich schwedische Ansätze, die ihrerseits von Deutschland beeinflusst waren ${ }^{58}$. Ein Grund für den starken deutschen Einfluss ist, dass Deutschland der wichtigste Handelspartner Finn-

Vgl. Järvenpää (1996), S. 900.

Vgl. Järvenpää (1996), S. 900 und vgl. Majala (2001 b), S. 957-958 und vgl. Artsberg (2000), S. 521.

54 Vgl. Sorsa (1998 b), S. 32.

55 Der finnische Titel des Buchführungsgesetzes von 1925 lautet „laki kirjanpitovelvollisuudesta“. Übersetzung ins Deutsche durch die Verfasserin.

56 Vgl. Kettunen (1993), S. 595.

57 Vgl. Järvenpää (1996), S. 900.

58 Zum traditionell vorhandenen schwedischen Einfluss auf Finnland vgl. Kap. 2.1.2 dieser Arbeit. 
lands war $^{59}$. Zudem hatten wegen fehlender inländischer Lehrtradition auf dem Gebiet der Betriebswirtschaft vor 1911 viele Professoren der Handelshochschule Helsinki zumindest einen Teil ihrer eigenen Ausbildung in Deutschland absolviert und pflegten enge Kontakte zu deutschen Wissenschaftlern. Dies hatte zur Folge, dass deutsche Lehrbücher in Finnland verwendet wurden und die deutsche Rechnungslegungsauffassung die universitäre Ausbildung im Bereich der Unternehmensrechnung prägte ${ }^{60}$.

Nach dem Buchführungsgesetz von 1925 waren alle im Handelsregister eingetragenen Unternehmen buchführungspflichtig. Das Gesetz war allgemein gehalten, Form und Inhalt der Jahresabschlüsse waren im Detail nicht vorgeschrieben. Die Buchhaltung sollte mit den allgemeinen Buchführungsgrundsätzen und mit der Art ordentlicher Kaufleute ${ }^{61}$ übereinstimmen sowie Guthaben, Verbindlichkeiten und deren Änderungen wiedergeben ${ }^{62}$. Das Eigentum sollte $\mathrm{zu}$ Anschaffungsbzw. Herstellungskosten oder zum niedrigeren Marktwert bewertet werden. Lag der Marktwert jedoch dauerhaft über den Anschaffungs- und Herstellungskosten, bestand auch die Möglichkeit der Bilanzierung zum Marktwert. In einem solchen Fall war die Wertsteigerung als eigener Rücklageposten innerhalb der Gruppe der Verbindlichkeiten auszuweisen. Gegenstände des Anlagevermögens sollten der Zeit oder dem Verbrauch nach oder aus einem anderen Grund abgeschrieben werden. Forderungen mussten auf ihre Werthaltigkeit hin überprüft und gegebenenfalls wertberichtigt werden ${ }^{63}$.

Wie bereits erwähnt, war ein Zweck der Rechnungslegung auch der, eine Grundlage für die Besteuerung zu haben. Die neu gewonnene Unabhängigkeit und speziell der Bürgerkrieg im Frühjahr 1918 waren kostenintensiv; die Regierung musste ihren Bedarf an Finanzmitteln durch die Erhebung von Steuern decken. Die Frage nach dem Zusammenhang eines neu zu bildenden Steuersystems und der Unternehmensrechnungslegung wurde diskutiert. Der Gesetzgeber übernahm das Maßgeblichkeitsprinzip und das Prinzip der umgekehrten Maßgeblichkeit

Vgl. Majala (2001 b), S. 958 .

Vgl. Näsi (1994), S. 495 f. und vgl. Artsberg (2000), S. 518. Bei den zwischen 1910 und 1930 an der Handelshochschule Helsinki als Lehrmaterial verwendeten Schriften und Büchern handelte es sich u. a. um Balduin Penndorf: „, Fabrikbuchhaltung im Zusammenhang mit Kalkulation und Statistik“ (Berlin 1924), Albert Calmas: „Der Fabrikbetrieb, die Organisation, die Buchhaltung und die Selbstkostenrechnung industrieller Betriebe“ (Leipzig, 4. Auflage 1916), M.R. Lehmann: „Die Wirtschaftlichkeitsmessung des Betriebs“ (Zeitung für Betriebswirtschaft 1926, S. 573-653), Eugen Schmalenbach: „Grundlagen dynamischer Bilanzlehre“ (Leipzig 1925) sowie Eugen Schmalenbach: „Grundlagen der Selbstkostenrechnung“ (Leipzig 1925). Vgl. Näsi und Näsi (1996), S. 203 und vgl. Näsi (1994), S. 495.

61 Im finnischen Buchführungsgesetz von 1925 werden die allgemeinen Buchführungsgrundsätze ,yleiset kirjanpidon periaatteet“ und die Art ordentlicher Kaufleute „hyvä kauppiastapa“ genannt. Übersetzung ins Deutsche durch die Verfasserin.

62 Vgl. Kettunen (1993), S. 595.

63 Vgl. Sorsa (1998 b), S. 33. 
deutscher Prägung $^{64}$ : Demnach bildete der nach Buchführungsgesetz erstellte Jahresabschluss die Grundlage für die steuerliche Gewinnermittlung (Grundsatz der Maßgeblichkeit). Bestimmte Steuervergünstigungen konnten jedoch nur in Anspruch genommen werden, wenn die entsprechenden Bilanzierungs- und Bewertungsmethoden auch im Jahresabschluss angewendet wurden (Grundsatz der umgekehrten Maßgeblichkeit) ${ }^{65}$.

Ab 1919 mussten die zur Buchführung verpflichteten Unternehmen ihrer Einkommensteuererklärung eine Kopie ihrer Jahresabschlüsse und eine Aufstellung der getätigten Abschreibungen beifügen. Das erste Einkommensteuergesetz Finnlands wurde 1920 verabschiedet, das zweite folgte schon 1924. Der sich aus der Buchhaltung ergebende Jahresüberschuss wurde Bemessungsgrundlage für die Einkommensteuer, wenn es auch in der Einleitung zum Buchführungsgesetz von 1925 in Bezug auf die Besteuerung heißt, dass der Jahresüberschuss nicht direkt als besteuerbares Einkommen angesehen werden könne, und dass es sinnvoll erscheine, weitergehende Regelungen für Steuerzwecke zu entwerfen ${ }^{66}$. Aus dieser engen Verbindung zwischen Steuerrecht und Rechnungslegung ist zu erklären, dass die Steuerbehörden Anstrengungen unternahmen, um die Qualität der externen Rechnungslegung zum Zweck einer ordnungsmäßigen Steuererhebung zu verbessern. Handels- und steuerrechtliche Vorschriften ergänzten sich und ergänzen sich noch heute ${ }^{67}$.

\subsubsection{Buchführungsgesetz von 1945}

Die Umstände des Zweiten Weltkriegs erforderten die Regulierung der Wirtschaft und die Kontrolle der Marktpreise durch die Regierung. Um für Käufer und Verkäufer faire Preise am Markt festzulegen und über eine Verhandlungsbasis für die Ausgestaltung militärischer Verträge zu verfügen, benötigte die Regierung Informationen über die Zusammensetzung und Höhe der Herstellungskosten der Produkte sowie die Kostenstruktur und Profitabilität der Unternehmen. Die Anforderungen an die Finanzbuchhaltungen der produzierenden Unternehmen stiegen und im Jahre 1943 wurde erstmals in Finnland ein Erlass verabschiedet, der eine Kostenrechnung für selbst erstellte Güter vorschrieb ${ }^{68}$.

Vgl. Artsberg (2000), S. 525.

Vgl. auch Kap. 2.2.2.3 dieser Arbeit.

Vgl. Järvenpää (1996), S. 900 f.

Auf die wesentlichen Auswirkungen der Steuergesetzgebung auf die Rechnungslegung wird in dieser geschichtlichen Darstellung im Folgenden nur eingegangen, wenn es dem besseren Verständnis der Entwicklung dient. Zur Beziehung zwischen dem Buchführungsgesetz und der Steuergesetzgebung heute vgl. Kap. 2.2.2.3 dieser Arbeit. 
Die Waffenstillstandsvereinbarungen von 1944 sahen Reparationszahlungen von Finnland an die Sowjetunion in Höhe von 300 Millionen US-Dollar vor. Diese sollten in Form von Rohstoffen und Waren wie Holz, Papier, Zellulose, Schiffen und Maschinen verschiedenster Art geleistet werden. Die Preise für diese Güter wurden auf Basis des amerikanischen Golddollars von 1938 kalkuliert. Die speziell gegründete „Kommission für Kriegsreparations-Branchen“69 veröffentlichte am 27. März 1945 Anweisungen an die Industrie für die Bewertung selbst erstellter Güter ${ }^{70}$.

Vor diesem Hintergrund wurde 1945 ein neues Buchführungsgesetz verabschiedet, das 1947 in Kraft trat. Die Veränderungen gegenüber dem Gesetz von 1925 lagen weniger in den Informationsbedürfnissen der finnischen Unternehmen als vielmehr in der Aufgabe der Regierung begründet, die Wirtschaft zu regulieren. Grundlegende Idee des neuen Gesetzes war es, die Jahresabschlüsse detaillierter und einheitlicher zu gestalten, um Vergleiche zwischen Unternehmen und Branchen zu ermöglichen. Die Hauptaufgabe von Jahresabschlüssen lag in der Gewinnermittlung, die Bilanz wurde zur Schätzung des Eigenkapitals benötigt ${ }^{71}$. Der Schwerpunkt des Jahresabschlusses lag auf der Bilanz. Das Jahresergebnis wurde kalkuliert, indem die Veränderung des Eigenkapitals im Vergleich zum Vorjahr ermittelt wurde. Hierbei war die Bewertung von Vermögensgegenständen und Verbindlichkeiten von wesentlicher Bedeutung. Die Gewinn- und Verlustrechnung wurde lediglich als Kontrollinstrument angesehen, das zeigen sollte, dass die Veränderung im Eigenkapital der Bilanz nach der Methode der doppelten Buchführung korrekt ermittelt wurde ${ }^{72}$.

Die buchführungspflichtigen Unternehmen wurden in vier Gruppen unterteilt, an die hinsichtlich der Buchführung unterschiedliche Anforderungen gestellt wur$\operatorname{den}^{73}$ :

- Einzelhändler, Handwerker ${ }^{74}$

- Gewerbetreibende, Handwerksgewerbetreibende, Gaststätteninhaber ${ }^{75}$

- Aktiengesellschaften, Genossenschaften, Offene Handelsgesellschaften, Kommanditgesellschaften ${ }^{76}$

69 Der finnische Name der Kommission lautet „Sotakorvausteollisuuden valtuuskunta“. Übersetzung ins Deutsche durch die Verfasserin.

70 Vgl. Näsi (1994), S. 499.

71 Vgl. Kettunen (1993), S. 596 f.

72 Vgl. Majala (1994), S. 74, der sich auf Virtaneva beruft.

73 Vgl. Sorsa (1998 b), S. 35.

74 Im Buchführungsgesetz von 1945 wird der Einzelhänder „kauppias“, der Handwerker „käsityöläinen" genannt. Übersetzung ins Deutsche durch die Verfasserin.

75 Im Buchführungsgesetz von 1945 werden Gewerbetreibende „liikkeenharjoittajat”, Handwerksgewerbetreibende „käsityöliikkeenharjoittajat” und Gaststätteninhaber „ravintoloitsijat" genannt. Übersetzung ins Deutsche durch die Verfasserin.

76 Im Buchführungsgesetz von 1945 werden - wie heute auch noch - die Aktiengesellschaft „osakeyhtiö“, die Genossenschaft „osuuskunta“, die Offene Handelsgesellschaft „,avoin yhtiö“ und die Kommanditgesellschaft „kommandiittiyhtiö“ genannt. 
- Industrieunternehmen ${ }^{77}$ unabhängig von ihrer Rechtsform, wenn bestimmte Größenkriterien bei der Produktion überschritten wurden.

Es galten die Art guter Buchführung und die Grundsätze ordnungsmäßiger Buchführung; Realisations- und Vorsichtsprinzip wurden explizit genannt ${ }^{78}$.

Eine bemerkenswerte Neuerung hinsichtlich der Bewertung gegenüber dem Gesetz von 1925 war die Verpflichtung aller Unternehmen zur Durchführung einer Kostenrechnung, die bei wesentlichen Änderungen ihrer zu Grunde liegenden Annahmen und Daten angepasst werden und mit den Unterlagen der Buchhaltung abstimmbar sein musste ${ }^{79}$.

Erstmals wurden Ausweisfragen für Bilanz und Gewinn- und Verlustrechnung geregelt. In der Gewinn- und Verlustrechnung einzeln aufgeführt werden sollten die verschiedenen Aufwandsarten wie Löhne und Gehälter, Mieten, Zinsen, Abschreibungen und Steuern sowie außerordentliche, nicht dem Betriebszweck zugehörige Aufwendungen; ebenso die Erträge, wie Umsatzerlöse, Dividenden und Zinsen sowie außerordentliche Erträge. Die Aktivseite der Bilanz wies die Hauptgruppen Finanzen, Umlauf- und Anlagevermögen, aktivisch abgesetzte Wertberichtigungen, Abgrenzungsposten und gegebenenfalls Verlust aus. Die Passivseite zeigte das Fremdkapital, untergliedert in kurz- und langfristiges Fremdkapital, sowie passivisch abgesetzte Wertberichtigungen, Abgrenzungsposten und Eigenkapital, das gegebenenfalls den Jahresüberschuss enthielt ${ }^{80}$.

Im Steuergesetz von 1943 wurde ein verändertes Verständnis der Vorratsbewertung deutlich. Von einer Bewertung zum Marktwert wurde jetzt abgesehen. Eine steuerlich abzugsfähige Unterbewertung der Vorräte um bis zu 100 Prozent des Stichtagsmarktwerts wurde handelsrechtlich erlaubt, um die Innenfinanzierungskraft der Unternehmen zu stärken und den Unternehmen eine Reservenbildung für künftige negative Ereignisse zu ermöglichen ${ }^{81}$. Steuerlich nicht zulässig war jedoch eine Änderung der einmal gewählten Unterbewertungsmethode, sodass die Manipulation des Jahresergebnisses immerhin eingeschränkt wurde ${ }^{82}$.

Obwohl in der Öffentlichkeit diskutiert, enthielt das Buchführungsgesetz 1945 keine Bewertungsregeln für Inflationszeiten. Diese Regelungslücke erwies sich in den inflationären Zeiten nach dem Zweiten Weltkrieg als problematisch ${ }^{83}$. Verur-

\footnotetext{
77 Im Buchführungsgesetz von 1945 wird ein Industrieunternehmen ,teollinen yritys“ genannt. Übersetzung ins Deutsche durch die Verfasserin.

78 Zur Art guter Buchführung und den Grundsätzen ordnungsmäßiger Buchführung vgl. Kap. 3.1.3 dieser Arbeit.

79 Vgl. Kirjanpitolaki (1945) 2 luku 2 und vgl. Kirjanpitoasetus (1945) 6 luku $3 \S$.

$80 \quad$ Vgl. Sorsa (1998 b), S. 34 f.

81 Zur Anwendung des Maßgeblichkeitsprinzips und seiner Umkehrung in Finnland vgl. auch Kap. 2.1.2.1 und Kap. 2.2.2.3 dieser Arbeit.

82 Vgl. Järvenpää (1996), S. 902.

83 Vgl. Majala (2001 b), S. 958.
} 
sacht durch Inflation und hohe Steuern im Jahrzehnt nach dem Zweiten Weltkrieg und dem Aufschwung der Wirtschaft in den 60er Jahren des 20. Jahrhunderts gewann die Ermittlung des Jahresüberschusses immer mehr an Bedeutung. Das bilanzorientierte Konzept des Buchführungsgesetzes von 1945 mit seinen Änderungen von 1954 konnte die Forderungen der Praxis nach einer dynamischeren Rechnungslegungsmethode nicht erfüllen: Die geltenden Gesetze erwiesen sich wegen ihrer Betonung der Bilanz und der Vernachlässigung der Gewinn- und Verlustrechnung als unbefriedigend. Die zwischen 1940 und 1960 von Professor Martti Saario entwickelte Ausgaben-Einnahmen-Theorie der Buchführung (,Kirjanpidon meno-tulo-teoria" $)^{84}$ bot eine interessante Alternative ${ }^{85}$. Sie fand zunehmend Anklang in der Praxis und soll auf Grund ihres großen Einflusses auf die finnische Rechnungslegung im Folgenden skizziert werden.

\subsubsection{Theoriegeprägte Rechnungslegung 1950 bis 1990}

\subsubsection{Ausgaben-Einnahmen-Theorie der Buchführung}

Basierend auf der Feststellung, dass die meisten vor dem Obersten Verwaltungsgerichtshof Finnlands verhandelten Steuerstreitigkeiten die Abschreibung von Anlagevermögen betrafen, begann Martti Saario ${ }^{86}$ seine Forschung, um eine logische, einfache und zweckgerichtete Abschreibungstheorie zu entwickeln ${ }^{87}$.

SAARIOs theoretische Überlegungen über die Unternehmung gingen von den Interessen zweier Gruppen aus: von den Eigentümern, die das Ziel verfolgten, ausschüttungsfähigen Gewinn zu erwirtschaften und von den Finanzbehörden, die an diesem Gewinn partizipieren wollten ${ }^{88}$. Anders als die meisten anderen Betriebs-

84 Im Finnischen wird die Ausgaben-Einnahmen-Theorie der Buchführung „Kirjanpidon menotulo-teoria" genannt. Übersetzung ins Deutsche durch die Verfasserin.

85 Vgl. Majala (1994), S. 75.

86 Martti Saario (1906 bis 1988) schloss seine wirtschaftswissenschaftlichen Studien 1929 mit dem Diplom („,Kauppatieteiden Maisteri“; der finnische Abschluss „Kauppatieteiden Maisteri“ entspricht in etwa dem deutschen Diplom-Kaufmann) an der Handelshochschule Helsinki ab. Von 1929 bis 1939 unterrichtete er an der Handelsschule in Viipuri/Vyborg. Im Zweiten Weltkrieg, von 1942 bis 1944, fungierte er als Chef-Revisor der finnischen Regierung. Von 1943 bis 1971 lehrte er Rechnungslegung an der Handelshochschule Helsinki, seit 1948 als Professor; von 1964 bis 1973 agierte er zusätzlich als Professor an der Handelshochschule Turku. Vgl. Pihlanto und Lukka (1993), S. 251-253.

$87 \quad$ Vgl. Lukka und Pihlanto (1994), S. 61.

88 Vgl. Lukka und Pihlanto (1994), S. 61. Das dynamische, einkommensorientierte Verständnis der Rechnungslegung, das SAARIO maßgeblich, aber sehr kritisch auf die Arbeiten der deutschsprachigen Betriebswirte SCHMALENBACH, FISCHER, WALB, RIEGER und KOSIOL stützte (vgl. Pihlanto und Lukka (1993), S. 253 und Lukka und Pihlanto (1994), S. 61-65), legte er in Grundzügen erstmals 1945 in seiner Dissertation „Realisationsprinzip und Abschreibung von Gegenständen des Sachanlagevermögens“ („Realisointiperiaate ja käyttöomaisuuden poistot tuloslaskennassa") dar und baute es bis 1961 weiter aus (vgl. Saario, Martti: Realisointiperiaate ja käyttöomaisuuden poistot tuloslaskennassa, Helsinki 1945, vgl. Saario, Martti: Kirjanpidon meno-tulo-teoria, Keuruu 1959 und vgl. Saario, 
wirtschaftler seiner Zeit - unter ihnen SCHMALENBACH - sah SAARIO die Aufgabe der Ergebnisrechnung nicht darin, den Unternehmensgewinn zu ermitteln, sondern den Gewinn, den der Eigentümer aus der Tätigkeit seines Unternehmens erzielt ${ }^{89}$.

Einen wesentlichen Eckpunkt der Ausgaben-Einnahmen-Theorie bildet das Realisationsprinzip, aus dem sich nach SAARIO alles andere ableiten lässt und das er wie folgt definiert: „Nur wenn Kaufkraft in liquiden Mitteln erzielt wurde, und nur wenn diese Steigerung der Kaufkraft tatsächlich in den Händen des Unternehmens liegt, dann existiert Gewinn, und nur dann kann er gezählt werden; er beträgt natürlich Gewinn $=$ Kaufkraft erzielt beim Verkauf + Kaufkraft verloren beim Einkauf. Wir nennen diese Art der Gewinnkalkulation oder Gewinnermittlungsmethode das Realisationsprinzip." "90 Die strikte Anwendung des Realisationsprinzips bildete für SAARIO die zentrale Grundlage der Gewinnermittlung und der gesamten Buchführung ${ }^{91}$. SAARIO hat das Realisationsprinzip nicht erfunden, war aber der Erste und bis heute der Einzige, der es in eine in sich geschlossene Buchführungstheorie eingebaut hat ${ }^{92}$.

Gewinn wird dem Realisationsprinzip entsprechend aus Werten basierend auf liquiden Mitteln (Kasse), Ausgaben und Einnahmen berechnet. SAARIO definiert liquide Mittel als im Unternehmen vorhandene Kaufkraft. Sie umfassen neben der Kasse im eigentlichen Sinne auch Forderungen aus Lieferungen und Leistungen sowie - als Abgrenzungsposten - Verbindlichkeiten aus Lieferungen und Leistungen. Dass erlangte wie auch verlorene Kaufkraft oftmals zu einem anderen Zeitpunkt realisiert werden als die Zahlungsvorgänge stattfinden, ist bei dieser Definition unwesentlich (Grundsatz der leistungsabhängigen Buchführung). Keine der innerhalb der liquiden Mittel stattfindenden Transaktionen kann das Unternehmensergebnis beeinflussen; deshalb könnten sie aus dem Standpunkt der Gewinnermittlung betrachtet alle auf ein und demselben Konto gebucht werden ${ }^{93}$. Aus Gründen der notwendigen Kontrolle über Eigentumsverhältnisse müssen sie jedoch voneinander getrennt ausgewiesen werden.

Unter Ausgaben versteht SAARIO die ergebniswirksame Minderung von liquiden Mitteln, unter Einnahmen ihre ergebniswirksame Mehrung. Der Wert der Ausgaben und Einnahmen kann sich nach ihrem Realisationszeitpunkt noch ändern,

Martti: Poistojen pääoma-arvo ja oikea-aikaisuus, in: Mercurialia MCMLXI, Kokoelma kirjoituksia Kauppakorkeakoulun täyttäessä 50 vuotta, Helsinki 1961.) Seine AusgabenEinnahmen-Theorie prägte in den 60er Jahren die Lehre und Praxis der Buchführung in Finnland und wurde, um gesetzlich zu bestätigen, was praktisch schon durchgeführt wurde, schließlich Grundlage für das Gewerbe-Einkommensteuergesetz („Elinkeinotuloverolaki“) von 1968 und das Buchführungsgesetz von 1973 (vgl. Lukka und Pihlanto (1994), S. 69).

Vgl. Pihlanto und Lukka (1993), S. 256.

Saario (1965), S. 30. Übersetzung ins Deutsche durch die Verfasserin.

Vgl. Pihlanto und Lukka (1993), S. 256.

Vgl. Lukka und Pihlanto (1994), S. 64.

Vgl. Pihlanto und Lukka (1993), S. 257. 
z. B. durch Forderungsausfall oder Währungsdifferenzen, sodass unter Umständen Korrekturbuchungen notwendig werden ${ }^{94}$.

Um den Gewinn zu ermitteln, müssen zuerst die monetären Ströme einer Unternehmung ermittelt werden. Aus dieser Anforderung ergeben sich für die Rechnungslegung zwei Aufgaben:

Eine der beiden Aufgaben der Buchführung ist es, die monetären Ströme in einem Unternehmen, nämlich

- Ausgaben für Waren und Dienstleistungen,

- Einnahmen aus dem Verkauf von Waren und Dienstleistungen und

- Zahlungen

zu identifizieren. Andere Transaktionen als die drei genannten existieren nicht. Für die Buchführung bedeutet dies, dass die Konten in Ausgabenkonten, Einnahmenkonten und Zahlungskonten unterteilt werden. Ausgaben werden getätigt, um zukünftig Einnahmen zu erzielen. Liquide Mittel und Kapital werden allein dazu benötigt, den zeitlichen Unterschied zwischen Ausgaben und Einnahmen zu überbrücken. Mithilfe der doppelten Buchführung werden für jeden Geschäftsvorfall die Mittelherkunft und die Mittelverwendung registriert ${ }^{95}$. Buchführung wird vornehmlich als Abbildung von Geschäftsvorfällen und monetären Strömen aufgefasst, nicht primär als Abbildung von Vermögenswerten ${ }^{96}$.

Die zweite Aufgabe der Buchführung ist es, das Unternehmensergebnis zu berechnen. Unter dem „Gesamt-Unternehmensergebnis “97 wird das Ergebnis gemessen über die Lebenszeit des Unternehmens verstanden; es kann auf zwei verschiedene Arten ermittelt werden:

- als Veränderung der liquiden Mittel während der Lebensdauer des Unternehmens oder

- als Differenz von Gesamt-Einnahmen und Gesamt-Ausgaben.

Das Gesamt-Unternehmensergebnis kann in Perioden-Unternehmensergebnisse aufgeteilt werden. Alle Periodenergebnisse addiert müssen wiederum das GesamtUnternehmensergebnis ergeben. Analog zum Gesamt-Unternehmensergebnis kann das Periodenergebnis auf zwei verschiedene Arten berechnet werden,

- als Veränderung der liquiden Mittel während der Periode oder

- als Differenz von Einnahmen und Ausgaben einer Periode.

Mit Bezug auf das Realisationsprinzip kommt bei der Ermittlung des Periodenergebnisses für SAARIO jedoch nur die zweite Variante in Betracht: Das Periodenergebnis wird als Differenz zwischen Einnahmen der Periode und Ausgaben, die

Vgl. Pihlanto und Lukka (1993), S. 257.

Vgl. Lukka und Pihlanto (1994), S. 63.

Vgl. Majala (2001 b), S. 959.

SCHMALENBACH nennt dieses Gesamt-Unternehmensergebnis „Totalerfolg“. Vgl. Schmalenbach (1953), S. 49-51. 
zur Erzielung dieser Einnahmen notwendig waren, definiert ${ }^{98}$. Aufwand ist die der Periode zugeordnete Ausgabe oder ein Teil von ihr; analog ist Ertrag eine der Periode zugeordnete Einnahme oder ein Teil von ihr.

Folglich müssen die kumulierten Ausgaben einer Unternehmung in zwei Gruppen unterteilt werden $^{99}$,

- einerseits in die Ausgaben, denen bereits Einnahmen gegenüberstehen und die deshalb bereits in die Gewinn- und Verlustrechnung eingegangen sind (Aufwendungen) und

- andererseits in die restlichen angefallenen Ausgaben, aus denen noch Einnahmen erwartet werden. Diese Ausgaben bilden zusammen mit Finanzwerten die Aktivseite der Bilanz. Die Passivseite der Bilanz zeigt lediglich, wie die Aktivseite finanziert wird. Folglich besteht die Bilanz nur aus Residualgrößen und hat keinen Einfluss auf die Ermittlung des Unternehmensergebnisses. Ausgaben, denen keine erwarteten Einnahmen mehr gegenüberstehen, werden erfolgswirksam ausgebucht ${ }^{100}$.

Das größte Problem bei der Berechnung des Periodenergebnisses ist die Frage der Zuordnung der Ausgaben zur Gewinn- und Verlustrechnung oder zur Bilanz, d. h. die Frage nach dem Zeitpunkt der Gewinnrealisierung. Bei der Lösung dieses Problems sieht SAARIO in der Ausgaben-Einnahmen-Theorie folgenden Ansatz vor:

Im Allgemeinen wird das Gesamt-Unternehmensergebnis über die Lebensdauer einer Unternehmung gesehen positiv sein, da andernfalls kein vernünftig handelnder Mensch in die Unternehmung investieren würde (homo oeconomicus) ${ }^{101}$. Da das Gesamtergebnis positiv ist und die Summe aller Periodenergebnisse bildet, sollte grundsätzlich kein Periodenergebnis als Verlust dargestellt werden.

Dies gelingt, indem sowohl bereits realisierte Einnahmen als auch erst in Zukunft anfallende Einnahmen, die beide zusammengenommen die Ausgaben inklusive der Abschreibungen decken sollen, über die Höhe der Perioden-Abschreibungen gesteuert werden. Eine Perioden-Abschreibung sollte - sofern noch eine Gewinnerwartung besteht - somit nicht durchgeführt werden, wenn durch sie ein negatives Periodenergebnis entsteht. Umgekehrt rechtfertigt ein hohes Periodenergebnis vor Abschreibung eine hohe Abschreibung. Vermögensgegenstände werden der Theorie nach also nicht auf Basis ihrer Anschaffungs- oder Herstellungskosten und auch nicht auf Basis physikalischer Gesichtspunkte, sondern auf Basis des Periodenergebnisses abgeschrieben, jedoch maximal in Höhe der Anschaffungsoder Herstellungskosten. Diese Realisationsabschreibung ${ }^{102}$ ist so begründbar,

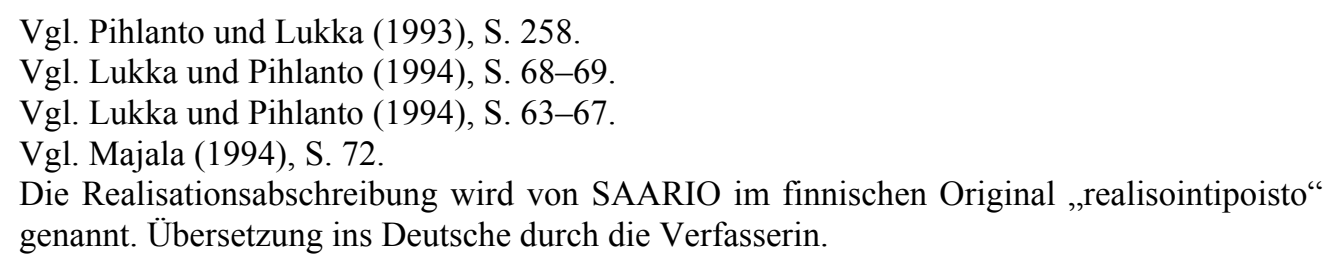


dass beim Kauf einer Produktionsanlage das aus dieser Anlage erwartete künftige Einkommen erworben wird. Die jährliche Abschreibung entspricht somit dem Anschaffungspreis des jeweiligen korrespondierenden jährlichen Einkommens. Oder mathematisch ausgedrückt: Die jährliche Abschreibung entspricht dem erwarteten jährlichen Einkommen, abgezinst auf den Anschaffungszeitpunkt ${ }^{103}$.

SAARIO war sich durchaus bewusst, dass er mit dieser Abschreibungsmethode den anderen Theoretikern seiner Zeit widersprach. Dennoch sah er sich durch die Abschreibungspraxis der Unternehmen, die seiner Theorie entsprach, ausreichend bestätigt ${ }^{104}$.

SAARIO bezieht in seiner Ausgaben-Einnahmen-Theorie Steuerzahlungen, Zinsaufwendungen und Dividendenzahlungen nicht in die Ausgaben mit ein, da ihnen keine konkreten, direkt zuzuordnenden Einnahmen gegenüberstehen. Diese Zahlungen sind seiner Meinung nach ein Teil der Gewinnverwendung bzw. der Gewinnausschüttung. Durch das Konzept der Abschreibungen und die Nichtberücksichtigung von Steuern, Zinsen und Dividenden bei den Ausgaben, kann ein Periodenverlust nach SAARIO nur in zwei Fällen auftreten:

- wenn die Differenz von Periodeneinnahmen und -ausgaben negativ ist

- wenn die Differenz von Periodeneinnahmen und -ausgaben kleiner ist als der Buchwert des Anlagevermögens in der Periode, ab der dem Anlagevermögen keine Gewinnerwartung mehr gegenübersteht ${ }^{105}$.

Aus diesem Verständnis des Jahresergebnisses heraus wird ersichtlich, dass der Gewinn- und Verlustrechnung eine weit größere Bedeutung beigemessen wird als der Bilanz, die nach Auffassung SAARIOs nur sehr eingeschränkt die tatsächlichen Unternehmenswerte widerspiegelt - nämlich nur in dem Maß, wie die historischen Anschaffungs- und Herstellungskosten mit den Marktwerten übereinstimmen. Die Bilanz wird der Theorie zufolge lediglich als zahlenmäßige Verbindung zwischen zwei Geschäftsjahren verstanden ${ }^{106}$.

\footnotetext{
103 Vgl. Majala (1994), S. 72.

104 Vgl. Pihlanto und Lukka (1993), S. 261.

105 Vgl. Pihlanto und Lukka (1993), S. 261.

106 Vgl. Majala (2001 b), S. 959.
} 


\subsubsection{Buchführungsgesetz von 1973}

Die Ausgaben-Einnahmen-Theorie SAARIOs wurde an den finnischsprachigen Handelshochschulen seit den 50er Jahren des 20. Jahrhunderts unterrichtet. Die „,neue Generation“ diplomierter Bilanzierer setzte ihr theoretisches Wissen in den 60er und 70er Jahren in die Praxis um. Parallel hierzu sorgte SAARIO mit vielen Veröffentlichungen in Fachzeitschriften dafür, dass seine Theorie in Finnland weiter bekannt wurde. Durch die immer breitere Anwendung dieser Theorie entwickelte sich allmählich eine immer größere Diskrepanz zwischen der neuen Rechnungslegungspraxis einerseits und dem Buchführungsgesetz andererseits. Die Wirtschaft ging erstmals einen komplett neuen Weg der Rechnungslegung, der ganz offensichtlich im Widerspruch zum geltenden Recht stand. Es fand ein Paradigmenwechsel in der Bilanzierung statt, ausgehend von der Praxis, nicht vom Gesetzgeber ${ }^{107}$.

Die drastischen Änderungen in der Rechnungslegungspraxis sorgten für gegensätzliche Meinungen und Diskussionen in der Fachwelt, die sich in zwei Lager teilte. Auf der einen Seite standen die Praktiker und Wissenschaftler, die davor warnten, einer neuen Theorie zu folgen und sich so von den Rechnungslegungsgepflogenheiten der nordischen Nachbarstaaten und Deutschlands zu entfernen und somit traditionell enge Bindungen an wichtige Handelspartner aufzugeben. Auf der anderen Seite stand SAARIO mit seinen Anhängern, den Managern der jüngeren Generation. Sie gewannen die Auseinandersetzung um die Richtung der Rechnungslegungspraxis und erreichten sogar, dass die Ausgaben-EinnahmenTheorie 1968 Basis des neuen Steuergesetzes und 1973 schließlich des reformierten Buchführungsgesetzes wurde ${ }^{108}$.

Die Voraussetzungen für die Entwicklungen zu Gunsten der AusgabenEinnahmen-Theorie wurden FLOWER zufolge durch drei Faktoren geschaffen:

Zum einen war - und ist auch heute noch - Finnland ein bevölkerungsarmes Land, in dem persönliche Kontakte wichtig waren. Es herrschte ein Umfeld, in

107 Vgl. Lukka und Pihlanto (1994), S. 69. Zur Ermittlung von Grundsätzen ordnungsmäßiger Buchführung (vgl. Kap. 3.1.3 dieser Arbeit) kann die induktive oder die deduktive Methode herangezogen werden. Bei der induktiven Methode werden die Ansichten der ordentlichen und ehrenwerten Kaufleute als maßgebende Quelle der Grundsätze ordnungsmäßiger Buchführung betrachtet (vgl. Schmalenbach (1933), S. 225-233). Die deduktive Methode geht bei der Ermittlung der GoB von den vom Gesetzgeber beabsichtigten handelsrechtlichen Jahresabschlusszwecken aus (vgl. Döllerer (1959), S. 1217-1221). Durch die AusgabenEinnahmen-Theorie und ihre Anwendung wurden in Finnland seinerzeit GoB induktiv ermittelt.

108 Vgl. Majala (1994), S. 76. Mit der Einbindung der Ausgaben-Einnahmen-Theorie in das Steuergesetz von 1968 gewann die Theorie wegen des seinerzeit geltenden Grundsatzes der umgekehrten Maßgeblichkeit an Einfluss auf den Jahresabschluss. Zur Beziehung zwischen Buchführungsgesetz und Steuergesetzgebung vgl. auch Kap. 2.1.2.1 und Kap. 2.2.2.3 dieser Arbeit. 
dem es durch geschickten Aufbau von Netzwerken möglich war, dass eine einzelne Person diejenigen beeinflussen konnte, welche die relevanten Schlüsselpositionen innehielten ${ }^{109}$.

Zum anderen war Finnland ein relativ isoliertes Land, geographisch gesehen kein Durchgangsland, mit einer Sprache, die kaum ein Ausländer erlernte. Aus wirtschaftlicher Sicht war es mit kleinem und staatlich restriktiv reguliertem Kapitalmarkt für potenzielle ausländische Investoren bis in die 80er Jahre des 20. Jahrhunderts hinein wenig interessant ${ }^{110}$.

Begünstigt wurde die Ausdehnung der Ausgaben-Einnahmen-Theorie in Finnland auch dadurch, dass sie im Einklang mit den Zielen der Bilanzierenden einerseits und der Gesetzgebung andererseits stand ${ }^{111}$. Die Unternehmen waren der Theorie gegenüber positiv eingestellt, weil die flexible Argumentationsweise ihnen besonders in Bezug auf die einkommensorientierten Abschreibungen entgegenkam. Sie ermöglichte einerseits, das Periodenergebnis durch hohe Abschreibungen zu senken und so den zu versteuernden Gewinn niedrig zu halten. Andererseits ließ sich in weniger erfolgreichen Jahren durch niedrige Abschreibungen das Jahresergebnis zur Zufriedenheit der Aktionäre positiv darstellen. Die Periodenergebnisse im Zeitablauf glätten zu können, erschien vielen Unternehmern attraktiv ${ }^{112}$.

Die Steuerbehörden verfolgten den Ansatz, durch die Gesetzesänderung von 1968 der vorhandenen Praxis zuzustimmen und sie ins Steuergesetz zu integrieren, statt sich gegen die Praxis zu stellen. Die Glättung der Periodenergebnisse wurde als nicht gravierend empfunden, da sie lediglich eine Zeitverschiebung in den Steuereinnahmen - eine Steuerstundung - bedeutete, nicht aber ihren Verlust ${ }^{113}$.

Die Politik stimmte der Theorie zu, weil die Unternehmen ausreichend Mittel einbehalten konnten, um sich selbst zu finanzieren. Die Regierungen der 50er bis 70er Jahre verfolgten das Ziel, den Wiederaufbau nach dem Zweiten Weltkrieg schnell voranzutreiben, Investitionen zu fördern und den Konsum einzuschrän$\operatorname{ken}^{114}$.

So kam es, dass die Ausgaben-Einnahmen-Theorie schließlich allgemeine Anerkennung fand und mindestens bis Anfang der 90er Jahre in Finnland tief verwurzelt blieb.

Theoriegerecht dienten Jahresabschlüsse nach dem Buchführungsgesetz von 1973 in Finnland in erster Linie dem Selbstinformationszweck des Unternehmers und waren weniger an potenzielle Investoren adressiert. Ziel war die Ermittlung des ausschüttungsfähigen Gewinns, auch als Basis für die Besteuerung ${ }^{115}$. Im Buch-

\footnotetext{
Vgl. Flower (1994), S. 244.

Vgl. Flower (1994), S. 244.

Vgl. Kettunen (1993), S. 598-599 und vgl. Flower (1994), S. 244.

Vgl. Järvenpää, S. 903 und vgl. Flower (1994), S. 243.

Vgl. Flower (1994), S. 243.

Vgl. Järvenpää (1996), S. 903 und vgl. Flower (1994), S. 244.

Vgl. Järvenpää (1996), S. 904.
} 
führungsgesetz von 1973 wurde hinsichtlich der Abschreibungen lediglich verlangt, dass Vermögensgegenstände des Sachanlagevermögens über die Dauer ihrer Nutzung abzuschreiben sind. Bei der Erstellung von Jahresabschlüssen orientierte sich die Praxis an den steuerlichen Vorgaben, die im Steuergesetz von 1968 enthalten waren ${ }^{116}$ :

- Die Abschreibungsrate durfte maximal 30 Prozent p. a. des Buchwerts betragen $^{117}$.

- Wenn die Nutzungsdauer eines Vermögensgegenstands weniger als drei Jahre betrug, durfte der Gegenstand im Jahr seiner Anschaffung voll abgeschrieben werden ${ }^{118}$, und

- die degressive Abschreibung für Gebäude wurde vorgesehen ${ }^{119}$.

Die Vorratsbewertung beruhte auf dem Anschaffungs- und Herstellungskostenprinzip; Gemeinkosten durften nicht berücksichtigt werden ${ }^{120}$. Um die Existenz der Unternehmen durch Innenfinanzierung zu sichern, wurde nach Steuergesetz 1968 und nach Buchführungsgesetz 1973 eine so genannte erfolgswirksame „Vorratsrückstellung“ erlaubt. Die Vorratsrückstellung durfte maximal 50 Prozent der Anschaffungs- und Herstellungskosten des Vorratsbestands am Stichtag betragen. Das Vorratsvermögen wurde durch sie aus betriebswirtschaftlicher Sicht steuerlich wirksam unterbewertet. Die Existenz der Vorratsrückstellung hatte zur Folge, dass die Unternehmen dazu neigten, sich hohe Vorratsbestände zuzulegen. Das Jahresergebnis wurde durch die Möglichkeit zur Bildung umfangreicher stiller Reserven manipulierbar ${ }^{121}$. Dadurch, dass diese stillen Reserven aber offen von den Vorräten abgesetzt wurden, blieb dem Leser die Chance zur gedanklichen Korrektur der Jahresabschlussaussage.

Um neben den produzierenden Unternehmen auch den Unternehmen personalintensiver Branchen eine ähnliche Reservenbildung $\mathrm{zu}$ ermöglichen und so die Steuergerechtigkeit zu wahren, wurde 1978 die „Betriebsrückstellung“ eingeführt: Anfangs durften bis zu zwei Prozent der Löhne und Gehälter des Vorjahrs zurückgestellt werden, bis 1987 stieg diese Rate auf 30 Prozent $^{122}$. Unternehmen, die wuchsen und investierten, konnten ihre zu versteuernden Gewinne stark senken und hohe nicht versteuerte Reserven als Puffer für wirtschaftlich schlechte Jahre aufbauen. Unternehmen, denen es wirtschaftlich schlecht ging, hatten irgendwann kein Abschreibungspotenzial mehr. Sie konnten keine zusätzlichen Aufwendun-

116 Zur Anwendung des Maßgeblichkeitsprinzips und des Prinzips der umgekehrten Maßgeblichkeit in Finnland vgl. Kap. 2.1.2.1 und Kap. 2.2.2.3 dieser Arbeit.

117 Vgl. Järvenpää (1996), S. 904.

Vgl. Järvenpää (1996), S. 904.

Vgl. Järvenpää (1996), S. 904.

Vgl. Järvenpää (1996), S. 904.

Vgl. Järvenpää (1996), S. 904.

Vgl. Järvenpää (1996), S. 903-905. 
gen durch Vorratsaufbau oder Zahlung hoher Löhne und Gehälter mehr kreieren und mussten hohe Steuern zahlen ${ }^{123}$.

Weitere Merkmale des Buchführungsgesetzes von 1973 waren,

- dass Finanzleasing-Gegenstände vom Leasinggeber bilanziert werden mussten $^{124}$,

- dass Beteiligungen nicht nach der Equity-Methode, sondern nach der Cost-Methode in der Bilanz ausgewiesen wurden ${ }^{125}$,

- dass nicht durch Tilgungsfonds besicherte Pensionsverpflichtungen nicht in der Bilanz ausgewiesen wurden ${ }^{126}$ und

- dass bei langfristiger Auftragsfertigung die Completed Contract-Methode anzuwenden war. Drohende Verluste durften während des laufenden Projekts nicht zurückgestellt werden ${ }^{127}$.

Hinsichtlich der Verbindung zum Steuerrecht ${ }^{128}$ ist zu erwähnen, dass seit 1968 Rückstellungen für Drohverluste ${ }^{129}$ und Garantie gebildet werden mussten und auch steuerlich anerkannt wurden. Neu war ebenfalls die steuerliche Anerkennung von Verlustvorträgen. Eine Dividendenausschüttung minderte nach neuer Sichtweise den zu versteuernden Gewinn ${ }^{130}$.

\subsubsection{Internationale Harmonisierung seit 1990}

\subsubsection{Nordische Harmonisierungsbestrebungen}

Trotz der Tatsache, dass sich die nationalen Rechnungslegungssysteme in den nordischen Staaten Dänemark, Schweden, Norwegen, Finnland und Island v. a. wegen des Einflusses der jeweiligen Steuergesetzgebung individuell unterschiedlich entwickelten und entwickeln, kooperieren die mit der Rechnungslegung befassten Berufsstände und die Gesetzgeber seit den 20er Jahren des 20. Jahrhunderts miteinander ${ }^{131}$.

Vgl. Kettunen (1993), S. 599.

Vgl. Räty (1992), S. 415-416.

Vgl. Sorsa (1998 b), S. 35.

Vgl. Räty (1992), S. 416.

Vgl. Sorsa (1998 b), S. 36.

Zur Anwendung des Maßgeblichkeitsprinzips und des Prinzips der umgekehrten Maßgeblichkeit in Finnland vgl. Kap. 2.1.2.1 und Kap. 2.2.2.3 dieser Arbeit.

Eine Ausnahme hiervon bildeten Projekte langfristiger Auftragsfertigung.

Vgl. Järvenpää (1996), S. 905.

1925 wurde die Nordische Föderation der Wirtschaftsprüfer („Pohjoismaiden tilintarkastusliitto“ bzw. „Nordiska Revisorsförbundet“) gegründet, die auch heute noch existiert. Darüber hinaus setzt das Nordic Auditing Committee Maßstäbe für die Durchführung der Wirtschaftsprüfung in den nordischen Ländern und entsendet einen Vertreter an die IFAC. Vgl. Elling (1993), S. 581. 
Bereits ab 1934 bemühten sich die Gesetzgeber der nordischen Länder, ihre Unternehmensgesetzgebung zu vereinheitlichen. Ziel war die Schaffung eines nordischen Wirtschaftsmarkts. 1942 veröffentlichten die Staaten einen gemeinsamen Gesetzesentwurf, der aber wegen der schwierigen politischen und wirtschaftlichen Lage während des Zweiten Weltkriegs nie verabschiedet wurde ${ }^{132}$.

Der nächste Versuch einer Harmonisierung wurde 1962 begonnen. Der Entwurf für ein gemeinsames Unternehmensgesetz entstand bis 1969, die in ihm enthaltenen Buchführungsgrundsätze waren allgemein gehalten und berücksichtigten nationale Interessen ${ }^{133}$. Der Entwurf konnte deshalb einen nur geringen Harmonisierungsgrad zur Folge haben, bewirkte aber immerhin Gesetzesänderungen und auch -modernisierungen in den nordischen Staaten ${ }^{134}$.

Finnland hatte sich aus den Verhandlungen um den Gesetzesentwurf - vermutlich unter dem Druck der Sowjetunion - zurückgezogen ${ }^{135}$. Mit dem Buchführungsgesetz von 1973 und der Ausgaben-Einnahmen-Theorie SAARIOs ging Finnland seinen eigenen Weg, übernahm aber dennoch den nordischen Harmonisierungsentwurf 1978 in sein Gesetz für Kapitalgesellschaften, das 1980 in Kraft trat. Es wurden erstmals Regeln zur Konsolidierung und für Konzernabschlüsse eingefügt. Konzernabschlüsse inklusive Kapitalflussrechnung mussten von den Mutterunternehmen für den Kreis der Konzerngesellschaften erstellt werden, der börsennotiert war. Methoden zur Kapitalkonsolidierung waren nicht festgelegt. Eigenkapital wurde gegliedert in ausschüttungsfähiges und nicht ausschüttungsfähiges Eigenkapital $^{136}$.

Da sich neben Finnland auch Dänemark 1981 aus dem Harmonisierungsbestreben der nordischen Länder verabschiedete, um im Rahmen seiner EG-Mitgliedschaft das dänische Unternehmensrecht an die Anforderungen der 4. EG-Richtlinie anzupassen, wurde der nordische Harmonisierungsversuch durch die weiter greifende europäische Harmonisierung überholt ${ }^{137}$.

Vgl. Artsberg (2000), S. 521.

Vgl. Elling (1993), S. 582.

Er wurde 1973 von Dänemark in nationales Recht umgesetzt, Schweden folgte 1975, Norwegen 1976 und Island 1978.

Vgl. Flower (1994), S. 240.

Vgl. Majala (2001 a), S. 997.

Vgl. Artsberg (2000), S. 522. 


\subsubsection{Einfluss der Europäischen Union auf die finnische Rechnungsle- gung}

\subsection{Buchführungsgesetz von 1992}

Die Jahresabschlüsse finnischer Unternehmen bzw. Konzernabschlüsse finnischer Konzerne waren nach dem Scheitern der nordischen Harmonisierungsbestrebungen und Finnlands Alleingang bezüglich der Ausgaben-Einnahmen-Theorie SAARIOs nicht mit denen anderer international operierender Gesellschaften vergleichbar $^{138}$. Einer Untersuchung des IASC $^{139}$ von 1988 folgend, waren die finnische Buchführungspraxis und die finnischen Abschlüsse im Vergleich zu den Praktiken 53 anderer Staaten am wenigsten IAS-konform ${ }^{140}$.

Nach finnischem Recht erstellte Abschlüsse wurden von den internationalen Finanzmärkten nicht akzeptiert. Unternehmen, die Investoren auf dem internationalen Kapitalmarkt suchten, waren gezwungen, neben den finnischen Abschlüssen zusätzlich internationalen Regeln entsprechende Abschlüsse z. B. nach den Standards des IASB oder nach US-GAAP zu erstellen oder ihre finnischen Abschlüsse um Zusatzinformationen zu ergänzen. Da diese Situation für alle Beteiligten unbefriedigend war, beauftragte das Ministerium für Handel und Industrie Finnlands im Januar 1989 ein „Komitee zur Novellierung des Buchführungsgesetzes“،141 mit der Ausarbeitung eines Entwurfs für ein international akzeptables Gesetz betreffend die Einzel- und Konzernabschlüsse ${ }^{142}$.

Als Finnland 1992 dem Europäischen Wirtschaftsraum beitrat, sollten die finnischen Rechnungslegungsregeln im Buchführungsgesetz, im Gesetz für Kapitalgesellschaften und im Genossenschaftsgesetz zumindest in den Kernpunkten den Anforderungen der 4. und 7. EG-Richtlinie angepasst werden. Das Komitee und der Gesetzgeber hofften, mit nur wenigen, absolut notwendigen Gesetzesänderungen die an eine internationale Vergleichbarkeit gestellten Minimalanforderungen erfüllen zu können ${ }^{143}$.

Problematisch und in der Fachwelt umstritten zeigte sich die Aufrechterhaltung der Ausgaben-Einnahmen-Theorie als im Buchführungsgesetz verankerte Grundlage der Buchführung. Die Theorie betonte die geringe Bedeutung der Bilanz im Jahresabschluss im Verhältnis zur Gewinn- und Verlustrechnung. Hierin wurde

138 Vgl. Adams et al. (1993), S. 471-492.

139 Im Folgenden soll der Begriff „IASC“ für Sachverhalte, die den Zeitraum von 1973 bis 2000 betreffen, „IASB“ für Sachverhalte, die den Zeitraum ab 2001 betreffen, verwendet werden.

140 Vgl. IASC (1988).

141 Der Name des Komitees lautet im Finnischen „Kirjanpitolainsäädännön uudistamiskomitea“, kurz: KOM. Übersetzung ins Deutsche durch die Verfasserin.

142 Vgl. Pirinen (1996), S. 27-35 und vgl. Räty (1992), S. 413-415.

143 Vgl. KOM (1990) und HE 1992/111. 
ein Widerspruch zur Forderung gesehen, im Jahresabschluss ein den tatsächlichen Verhältnissen entsprechendes Bild der Vermögenslage zu vermitteln ${ }^{144}$. Die Ausgaben-Einnahmen-Theorie blieb dennoch theoretische Grundlage des Buchführungsgesetzes von 1992. Es wurde die Auffassung vertreten, dass mithilfe einer soliden Theorie, die jahrelang in der Praxis getestet war, auftretende Problemstellungen leichter gelöst werden könnten ${ }^{145}$.

So wurde das Buchführungsgesetz nicht grundlegend erneuert, sondern ergänzt und modifiziert. Gegenüber den bisherigen Gesetzen nahm das neue Gesetz an Umfang zu. Während die bisherigen Buchführungsgesetze als Mantelgesetze nicht sehr detailliert ausgestaltet waren und wegen der Gültigkeit der Theorie viele Fragen nicht explizit gesetzlich geregelt waren, galt es nun, die 62 Artikel der 4. EGRichtlinie und die 51 Artikel der 7. EG-Richtlinie in nationales Recht umzuset$z^{146}{ }^{146}$. Dies hatte zur Folge, dass die Rechnungslegung nicht mehr so eindeutig wie vorher durch die Ausgaben-Einnahmen-Theorie gestützt wurde. Es gab jetzt Abweichungen von der Theorie ${ }^{147}$.

Wo die EG-Richtlinien Regelungslücken aufwiesen, orientierte sich der Gesetzgeber an den Standards des IASB, so bei der Bewertung von langfristigen Aufträgen (IAS 11 (revised 1993)), der Währungsumrechnung (IAS 21 (revised 1993)), der Behandlung latenter Steuern (IAS 12 (revised 1996)) und der Kapitalflussrechnung (IAS 7 (revised 1992)). Jetzt standen nicht mehr nur die Selbstinformation des Unternehmers, die Gewinnermittlung für Besteuerungszwecke und der Gläubigerschutz im Vordergrund, sondern auch potenzielle Investoren sowie die Gesellschaft als Ganzes sollten mit dem Jahresabschluss über Vermögens-, Finanzund Ertragslage eines Unternehmens informiert werden. Ziel des Jahresabschlusses wurde v. a. die Ermittlung des ausschüttungsfähigen Jahresüberschusses ${ }^{148}$.

Im Buchführungsgesetz von 1992 wurden folgende Sachverhalte eingeführt bzw. geändert $^{149}$ :

- Das Konzept des True and Fair View wurde eingeführt ${ }^{150}$.

- Bei der Vorratsbewertung wurde die Vollkostenrechnung (Einbeziehung der fixen Kosten in die Herstellungskosten) als Alternative erlaubt, ob-

144 Vgl. Sorsa (1998 b), S. 36 und Järvenpää (1996), S. 906.

145 Vgl. Kettunen (1993), S. 600 und Järvenpää (1996), S. 906.

146 Vgl. Flower (1994), S. 265.

147 Vgl. Kettunen (1993), S. 600 f. und vgl. Majala (2001 b), S. 962 und vgl. Järvenpää, (1996), S. 906.

148 Vgl. Järvenpää (1996), S. 906.

149 Vgl. Räty (1992), S. 414-420.

150 Vgl. KPL (1992) 3:9.2. 
wohl die Teilkostenrechnung weiterhin als „Benchmark-Methode“6151 angegeben wurde ${ }^{152}$.

- Für die Bewertung langfristiger Aufträge wurde die Percentage of Completion-Methode erlaubt ${ }^{153}$.

- Anschaffungs- und Herstellungskosten sollten planmäßig, nicht mehr einkommensorientiert abgeschrieben werden ${ }^{154}$.

- Neben einer Erstellung der Gewinn- und Verlustrechnung nach Gesamtkostenverfahren wurde jetzt auch das Umsatzkostenverfahren gestattet ${ }^{155}$.

- Die Berechnung von latenten Steuern in Konzernabschlüssen wurde erlaubt, aber nicht Pflicht. Für den Einzelabschluss war die Bildung latenter Steuern wegen der engen Verbindung zwischen Steuer- und Rechnungslegungsrecht nach wie vor nicht vorgesehen ${ }^{156}$.

- Es bestand die Verpflichtung zur Rückgängigmachung von Neubewertungen bei Wegfall der Gründe für die Neubewertungen ${ }^{157}$.

- Es bestand erstmals explizit die Verpflichtung zur Bildung von Pensionsrückstellungen. Pensionsrückstellungen stellen in finnischen Abschlüssen meist eine unwesentliche Größe dar, da ein Großteil der Pensionszahlungen über das staatliche Sozialsystem ${ }^{158} \operatorname{erfolgt}^{159}$. Einzelne Unternehmen haben jedoch mit ihren Mitarbeitern Pensionsvereinbarungen getroffen, deren bilanzielle Behandlung bis 1992 nicht geregelt war. Bis dahin wurden potenzielle Pensionszahlungen als Eventualverbindlichkeiten betrachtet und im Allgemeinen im Anhang ausgewiesen ${ }^{160}$.

- Rückstellungen in freiwillige Rückstellungen und Pflichtrückstellungen unterteilt $^{161}$.

- Hinsichtlich des Ausweises entsprachen Bilanz sowie Gewinn- und Verlustrechnung jetzt der Richtlinie ${ }^{162}$.

- Die Anhangangaben wurden um zusätzliche Informationen erweitert ${ }^{163}$.

151 Unter der „Benchmark-Methode“ wird die vom Gesetzgeber bevorzugte und empfohlene Bilanzierungs- bzw. Bewertungsmethode verstanden, neben der eine alternativ zulässige Methode existiert.

Vgl. KPL (1992) 3:16.2.

Vgl. KPL (1992) 3:16.4.

Vgl. KPA (1992) 1-6.

156 Vgl. KPL (1992) 3a:22c.4. Zur Anwendung des Maßgeblichkeitsprinzips und des Prinzips der umgekehrten Maßgeblichkeit in Finnland vgl. Kap. 2.1.2.1 und Kap. 2.2.2.3 dieser Arbeit.

158 Innerhalb des staatlichen Sozialsystems ist der staatliche Rentenversicherungsträger „Kansaneläkelaitos", kurz: KELA, für die Pensionszahlungen zuständig.

159 Vgl. Räty (1992), S. 418.

160 Vgl. KPL (1992) 3:16a.

161 Vgl. KPA (1992) 7 und KPL (1992) 3:20.

162 Vgl. KPA (1992) 1-7.

163 Vgl. KPL (1992) 3:21. 
- Die Währungsumrechnung erfolgte zum Stichtagskurs oder alternativ zum historischen Kurs, wenn die Laufzeit der Position mehr als ein Jahr be$\operatorname{trug}^{164}$.

Parallel zur grundlegenden Änderung des Rechnungslegungsrechts wurde 1992 eine Steuerreform durchgeführt, die sich auch auf den Jahresabschluss ausgewirkt hat $^{165}$.

Vorrats- und Betriebsrückstellung wurden steuerrechtlich verboten. Bis 1992 bereits gebildete Rückstellungen mussten in einer Übergangsphase bis spätestens 1997 erfolgswirksam aufgelöst werden, sodass sie heute aus den handelsrechtlichen Abschlüssen verschwunden sind ${ }^{166}$.

Hinsichtlich der Bewertung des Anlagevermögens wurden die Abschreibungsregeln geändert: Die Abschreibungsrate von Gebäuden betrug vier bis sieben Prozent p. a.; Nutzungsdauer und Abschreibungsrate waren nicht länger an das Material und die Bauweise des Gebäudes gebunden, sondern an dessen Zweck. Während Abschreibungen steuerlich auch degressiv möglich waren, waren handelsrechtlich nur lineare Abschreibungen vorgesehen. Die sich ergebende Differenz wurde in der Handelsbilanz als Abschreibungsunterschied unter dem kumulierten Abgrenzungsunterschied zwischen handelsrechtlich und steuerrechtlich veranlassten Posten auf der Passivseite der Bilanz ${ }^{167}$ berücksichtigt ${ }^{168}$.

\subsection{Buchführungsgesetz von 1997}

Im Vorfeld des Beitritts Finnlands zur Europäischen Union führte die EU-Kommission im Sommer 1993 einen detaillierten Vergleich zwischen dem finnischen Buchführungsgesetz von 1992 einerseits und den 4. und 7. EG-Richtlinien andererseits durch. Das Ergebnis war ein Katalog mit Änderungsforderungen ${ }^{169} .1995$ wurde Finnland Mitglied der Europäischen Union. Eine vollständige Übernahme der EG-Richtlinien bzw. der von der Kommission geforderten Änderungen in das nationale Recht wurde obligatorisch, sodass ein neues Buchführungsgesetz geschaffen werden musste.

Mit der Ausarbeitung eines Entwurfs für dieses neue Buchführungsgesetz beauftragte das Ministerium für Handel und Industrie am 29. März 1995 die so genann-

\footnotetext{
164 Vgl. KPL (1992) 3:15.2.

165 Zur Anwendung des Maßgeblichkeitsprinzips und des Prinzips der umgekehrten Maßgeblichkeit in Finnland vgl. Kap. 2.1.2.1 und Kap. 2.2.2.3 dieser Arbeit.

166 Vgl. Järvenpää (1996), S. 908.

167 Vgl. Kap. 3.2.5.1.1 dieser Arbeit.

168 Vgl. KPL (1992) 3:16.6 und 3:20.

169 Vgl. Räty (1998 a), S. 31.
} 
te „Arbeitsgruppe von 1995 zur Erneuerung des Buchführungsgesetzes“"170. Die Arbeitsgruppe hatte im Wesentlichen drei Aufgaben: Sie sollte klären, wie das Buchführungsgesetz und die auf seiner Grundlage verabschiedeten Verordnungen weiterzuentwickeln seien, um alle in der modifizierten 4. EG-Richtlinie enthaltenen, zwingend umzusetzenden Artikel in finnisches Recht zu transformieren. Zudem sollte die Arbeitsgruppe vorschlagen, wie das bestehende Buchführungsgesetz weiterzuentwickeln sei, um die Vergleichbarkeit zwischen finnischen und internationalen Abschlüssen zu verbessern. Außerdem sollte die Arbeitsgruppe untersuchen, wie die mit dem Buchführungsgesetz verbundene Gesetzgebung Gesellschaftsrecht und Wirtschaftsprüfungsgesetz - zu ändern sei, um die Forderungen des Richtliniengebers zu erfüllen und Widersprüche zwischen den Einzelgesetzen sowie Regelungslücken zu vermeiden. Die Arbeitsgruppe hat ihren Bericht am 25. März 1996 veröffentlicht ${ }^{171}$. Das auf ihren Vorschlägen basierende Gesetz trat am 31. Dezember 1997 in Kraft.

Wie dieses aktuelle Gesetz die 4. EG-Richtlinie umsetzt, und ob es eine internationale Vergleichbarkeit der Abschlüsse gewährleistet, ist Gegenstand der vorliegenden Arbeit.

\subsubsection{Zusammenfassender Überblick}

Folgende Graphik gibt zusammenfassend einen Überblick über die Denkansätze, die im Zeitablauf maßgebend auf die finnische Rechnungslegung eingewirkt haben und noch einwirken:

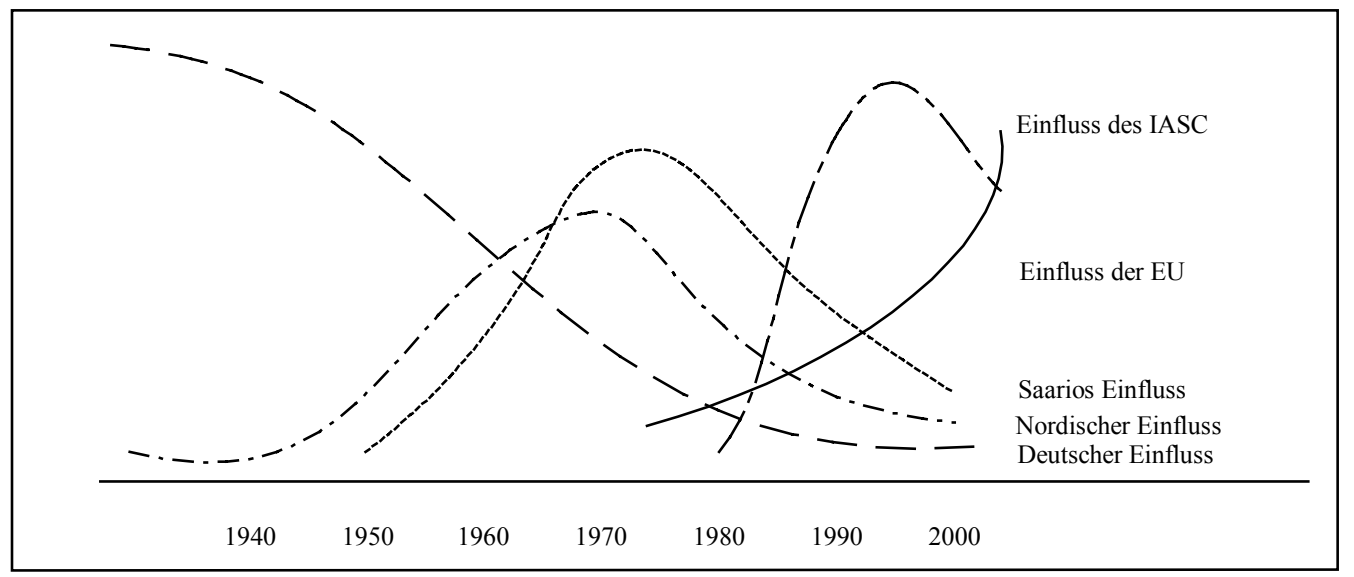

Abb. 1: Einflüsse auf die finnische Rechnungslegung seit $1920^{172}$

170 Der Name der Arbeitsgruppe lautet im Finnischen ,Kirjanpitolain uudistamistyöryhmä 1995“, im Folgenden kurz: KPL-Arbeitsgruppe 1995. Übersetzung ins Deutsche durch die Verfasserin.

171 Vgl. KPL-Arbeitsgruppe (1995), S. 13-19.

172 Graphik in Anlehnung an Flower (1994), S. 274. 
Der Einfluss der EU auf die finnische Rechnungslegung ist mit den Vorbereitungen zur Transformation der 4. und 7. EG-Richtlinien seit Ende der 80er Jahre stark gestiegen. Seinen vorläufigen Höhepunkt hat er mit In-Kraft-Treten des Buchführungsgesetzes von 1997 erreicht. Da Finnland als EU-Mitglied an die Vorgaben der 4. und 7. EG-Richtlinien zwingend gebunden ist, wird der Einfluss der EU weiterhin hoch bleiben. Zur Schließung von Regelungslücken, welche die 4. und 7.EG-Richtlinie aufweisen, hat sich der finnische Gesetzgeber an den Standards des IASB orientiert und diese an nationale Gegebenheiten angepasst ${ }^{173}$. Der Gesetzgeber empfiehlt zudem, sich bei der Weiterentwicklung der Rechnungslegung an den Standards des IASB zu orientieren ${ }^{174}$. Auch das Accounting Advisory Forum ${ }^{175}$ der EU erachtet eine Orientierung an diesen Standards für sinnvoll $^{176}$. Da die EU zudem beschlossen hat, dass ab dem Jahr 2005 auf europäischer Ebene Konzernabschlüsse börsennotierter Unternehmen nach den Standards des IASB zu erstellen sind, kann davon ausgegangen werden, dass der Einfluss des IASB künftig weiter steigen wird.

175 Das Accounting Advisory Forum der EU wurde durch EFRAG ersetzt. Im Folgenden soll der Begriff „Accounting Advisory Forum der EU“ verwendet werden, da alle zitierten Empfehlungen und Stellungnahmen aus der Zeit stammen, als dieser Name noch aktuell war. 


\subsubsection{Regelungsinstitutionen}

\subsubsection{Gesetzgeber, Regierung, Ministerien und Behörden}

Zentrale Bedeutung für die Rechnungslegung kommt den Gesetzen, Verordnungen und Anordnungen zu. Wesentlichen Einfluss auf die Rechnungslegung üben somit die Institutionen aus, die diese Regelungen erlassen.

Die Ministerien bereiten Gesetzes- und Verordnungsentwürfe vor. In den Fachgebieten Rechnungslegung, Besteuerung und Gesellschaftsrecht fällt diese Aufgabe dem Ministerium für Handel und Industrie zu. Das Parlament als Gesetzgeber verabschiedet die Gesetze ${ }^{177}$. Der Gesetzgeber regelt Buchführungs- und Rechnungslegungsfragen im Buchführungsgesetz KPL als Mantelgesetz (lex generalis). Zusätzlich hat er ergänzende Regelungen in den jeweiligen Spezialgesetzen für einzelne Gesellschaftsformen oder bestimmte Branchen erfasst ${ }^{178}$. Für börsennotierte Unternehmen gelten spezielle Rechnungslegungsanforderungen gemäß Wertpapiermarktgesetz $A M L{ }^{179}$. Der Gesetzgeber übt auch wegen des geltenden Maßgeblichkeitsprinzips und seiner Umkehrung starken Einfluss auf die Rechnungslegung aus. Hervorzuheben sind hier das Gewerbesteuergesetz EVL und das Einkommensteuergesetz TVL ${ }^{180} .1995$ hat der Gesetzgeber das Wirtschaftsprüfungsgesetz TilintL verabschiedet, das die Anforderungen an persönliche und fachliche Eigenschaften eines Wirtschaftsprüfers sowie die Überwachung seiner Arbeit regelt. Die an die Wirtschaftsprüfer gestellten Aufgaben wirken sich indirekt auf die Rechnungslegung aus ${ }^{181}$.

Die Regierung kann Verordnungen verfügen, die den Gesetzen untergeordnet sind, da sie nicht vom Parlament verabschiedet werden. Für die Buchführung gilt die Buchführungsverordnung KPA, die Vorschriften zur Gliederung von Bilanz sowie Gewinn- und Verlustrechnung enthält und das Buchführungsgesetz in Einzelfragen zur Bewertung und Bilanzierung ergänzt.

Die Ministerien können auf Forderung des Gesetzgebers Anordnungen erteilen, um mit ihnen Gesetze und Verordnungen verbindlich zu konkretisieren. Mit Hin-

177 Die im Zuge der Gesetzesentwicklung formulierten Gesetzesbegründungen bilden die wesentliche Basis für eine spätere Gesetzesauslegung. Die Gerichte interpretieren die Gesetze in ihrer Rechtsprechung; besonders der Oberste Verwaltungsgerichtshof („Korkein hallinto-oikeus“) kann mit seinen Urteilen die Praxis der Rechnungslegung beeinflussen.

178 Vgl. Kap. 2.2.2.2 der vorliegenden Arbeit.

179 Vgl. Kap. 2.2.2.2 dieser Arbeit.

180 Vgl. Järvenpää (1996), S. 899-914 und vgl. Kap. 2.2.2.3 dieser Arbeit.

181 Zur Beziehung von Buchführungsgesetz und Wirtschaftsprüfungsgesetz vgl. Kap. 2.2.2.4, zum Berufsstand der Wirtschaftsprüfer S. Kap. 2.2.1.3.1 dieser Arbeit. 
blick auf die Rechnungslegung sind hier das Ministerium für Handel und Industrie, das Finanzministerium für börsennotierte und für mit Aktien handelnde Unternehmen, die Bankenaufsicht für Banken sowie das Sozial- und Gesundheitsministerium für Versicherungen aktiv ${ }^{182}$. Die Einhaltung dieser Anordnungen soll vom Ministerium für Handel und Industrie überwacht werden. Da das Ministerium zu diesem Zweck bisher keine eigene Abteilung gebildet hat und die Überwachung auch nicht anderweitig organisiert hat, kommt die Überwachungsfunktion in der Praxis Wirtschaftsprüfern und Betriebsprüfern $\mathrm{zu}^{183}$.

Trotz des seit Beginn der 1990er Jahre steigenden Detaillierungsgrads sind Gesetze, Verordnungen und Anordnungen relativ allgemein gehalten und nehmen nicht zu allen Rechnungslegungsfragen Bezug ${ }^{184}$. Das Buchführungsgesetz verweist auf die Art guter Buchführung als Generalnorm ${ }^{185}$, sodass nicht alle denkbaren Fragestellungen durch gesetzliche Bestimmungen gelöst werden müssen, sondern vielmehr auch außerhalb der Gesetzgebung eine Normierung und Entwicklung stattfinden kann. Zur Unterstützung eines solchen Prozesses werden staatliche und private Einrichtungen herangezogen, und der Gesetzgeber lässt der Praxis eine wichtige Rolle bei der Weiterentwicklung der Rechnungslegung zukommen ${ }^{186}$. Es ist hierbei Aufgabe von Rechnungslegungspraxis, Wissenschaft und Rechtsprechung, die Art guter Buchführung anhand der vom Gesetzgeber beabsichtigten Jahresabschlusszwecke zu ermitteln (deduktive Methode zur Gewinnung von Grundsätzen ordnungsmäßiger Buchführung) ${ }^{187}$.

\subsubsection{Buchführungsausschuss}

Im Jahr 1974 wurde der finnische Buchführungsausschuss KILA gegründet ${ }^{188}$. Im Gegensatz zu den Accounting Boards in den meisten anderen Staaten ist der KILA keine private, sondern eine staatliche Einrichtung, die dem Ministerium für Handel und Industrie untergeordnet ist. Sie genießt eine relativ hohe Autorität, da sie von der Regierung mit einem Mandat ausgestattet wurde.

Die Aufgaben des KILA liegen darin, Stellungnahmen und Kommentare zu rechnungslegungsspezifischen Fragen zu liefern, die bestehenden Gesetze zu interpre-

182 Das derzeit aktuelle Buchführungsgesetz fordert an sieben Stellen Anordnungen vom Ministerium für Handel und Industrie.

183 Vgl. Majala (1994), S. 79.

184 Für die Zukunft ist zu erwarten, dass das Buchführungsgesetz und die anderen die Rechnungslegung betreffenden Gesetze durch die Verdrängung der Ausgaben-EinnahmenTheorie von EG-Richtlinien und internationalen Standards sowohl an Umfang als auch inhaltlich noch weiter an Bedeutung zunehmen werden, als es bisher der Fall war. Vgl. Flower (1994), S. 265.

185 Vgl. Kap. 3.1.3.1 dieser Arbeit.

186 Vgl. Artsberg (2000), S. 534.

187 Vgl. auch Fußnote 107 und Kap. 3.1.3.1 dieser Arbeit.

188 Die Aufgaben des KILA und seine Zusammensetzung sind in KPL 8:2 und KPL 8:3 geregelt. 
tieren und zu erklären und die Handhabung der Regeln für die Praxis zu verdeutlichen. Der KILA ist aber nicht dazu ermächtigt, neue Regeln zu entwerfen und zu bestimmen; er ist kein Standard Setter.

Das Ziel ist v. a. die Definition und Weiterentwicklung der Art guter Buchführung und der Grundsätze ordnungsmäßiger Buchführung ${ }^{189}$. Zu diesem Zweck kann der KILA allgemeingültige Anweisungen hinsichtlich der Auslegung von Gesetzen und der anzuwendenden Verfahrensweisen veröffentlichen ${ }^{190}$. Zu betonen ist hierbei, dass es sich um Anweisungen und nicht um Standards handelt. Diese Anweisungen hat der KILA in der Vergangenheit meist getroffen, ohne vorher Entwürfe zu veröffentlichen oder Stellungnahmen der betroffenen Interessengruppen zu erbitten $^{191}$.

Die Verlautbarungen vom KILA haben einen niedrigeren rechtlichen Stellenwert als die bestehenden Gesetzesvorschriften, weil sie nicht direkt vom Parlament gebilligt werden. Ihnen fehlt die rechtliche Verbindlichkeit. Es hat sich jedoch gezeigt, dass sich die Gerichte in ihrer Entscheidungsfindung an den KILA-Verlautbarungen orientieren.

Allgemeingültige Anweisungen sind von allen Buchführungspflichtigen zu befolgen. Der KILA hat in den Jahren, in denen die Ausgaben-Einnahmen-Theorie in Finnland alleiniger Maßstab der Rechnungslegung war, nur zwei allgemeingültige Anweisungen erlassen ${ }^{192}$. Seit 1990 stieg die Anzahl der allgemeingültigen Anweisungen sprunghaft $a^{193}$. In der Literatur wird vermutet, dass die Begründung hierfür in dem Abwenden von einer alles erklärenden Ausgaben-EinnahmenTheorie hin zu den EG-Richtlinien, die Regelungslücken beinhalten, zu suchen $\operatorname{sei}^{194}$.

Bei einer Änderung von Gesetzen und Erlassen behalten die allgemeingültigen Anweisungen grundsätzlich ihre Gültigkeit, auch wenn sie auf altem Recht basieren. Dies kann zu Widersprüchen zwischen den Anweisungen des KILA und dem geltenden Recht führen. Das Nebeneinander von überholten KILA-Anweisungen und neuem Recht führt unter Umständen zu Verwirrung in der Praxis ${ }^{195}$.

189 Vgl. hierzu Kap. 3.1.3.1 der vorliegenden Arbeit.

190 Vgl. Järvinen et al. (2000), S. 54-55.

191 Vgl. Flower (1994) S. 245.

192 Diese Anweisungen sind 1981 zum Thema Konzernabschlüsse und 1983 zur Kapitalflussrechnung erlassen worden.

193 Eine Übersicht über die vom KILA veröffentlichten allgemeingültigen Anweisungen ist in Anhang 3 dieser Arbeit enthalten.

194 Vgl. Majala (1994), S. 83-85.

195 Vgl. Majala (2001 b), S. 965. 
Behörden, Kommunen, Zivil- und Finanzgerichte erhalten ebenso wie private Organisationen und einzelne Unternehmen vom KILA Auskunft bei Rechnungslegungsproblemen. Anfragen sind schriftlich und formlos an den KILA zu richten und müssen notwendige und ausreichende Angaben zu dem Sachverhalt beinhalten, den der KILA beurteilen soll. Der KILA äußert sich auf solche Anfragen in Form von Gutachten, Entscheidungen und Erlaubnissen, von der Regel abzuweichen ${ }^{196}$. Seit seiner Gründung hat der KILA mehr als 1600 Gutachten und Entscheidungen veröffentlicht ${ }^{197}$.

In seinen Gutachten nimmt der KILA unter Berücksichtigung der Art guter Buchführung Stellung zu einzelnen Buchführungs- und Jahresabschlussfragen. Gutachten werden veröffentlicht und gelten nicht nur für den Fragesteller. Auch andere Buchführungspflichtige müssen sich bei analogen Sachverhalten an ihnen orientieren.

Entscheidungen trifft der KILA, wenn einzelne Unternehmen oder Industrieverbände stellvertretend für ganze Branchen den Antrag stellen, von einer bestehenden Regel abweichen zu dürfen. Ein solcher Antrag kann den Zeitpunkt der Jahresabschlusserstellung oder einzelne Ausweisfragen für Bilanz, Gewinn- und Verlustrechnung sowie Anhang betreffen. Der KILA unterliegt bei der Entscheidungsfindung zwei Einschränkungen: Erstens ist der KILA nach KPL 8:1.3 nicht zuständig für Unternehmen des Finanzsektors, kann also diesen Unternehmen keine Abweichung von bestehenden Regeln erlauben. Zweitens hat der KILA nicht die Befugnis, eine Abweichung von den Regeln zu gestatten, die in den EGRichtlinien zwingend vorgegeben sind. Eine einmal vom KILA getroffene Entscheidung kann vom Antragsteller nicht angefochten werden. Erteilt der KILA die Erlaubnis zur Abweichung von bestehenden Regeln, gilt diese Erlaubnis nur für den Antragsteller und ist zeitlich begrenzt ${ }^{198}$. Die zeitliche Begrenzung ist teilweise in der Entscheidung genannt. Mit In-Kraft-Treten des neuen Buchführungsgesetzes zum 31. Dezember 1997 sind alle auf Basis des vorherigen Gesetzes genehmigten Ausnahmen verfallen ${ }^{199}$.

Gemäß KPL 8:2 setzt der KILA sich aus einem Sprecher, seinem Stellvertreter und wenigstens sechs, höchstens zehn weiteren Mitgliedern und ihren jeweiligen Stellvertretern zusammen ${ }^{200}$. Die Mindest-Qualifikation der KILA-Mitglieder

196 Vgl. Leppiniemi und Leppiniemi (1997), S. 22-24.

197 Vgl. www.vn.fi $/ \mathrm{ktm} /$ kirjanpitolautakunta, Stand 01. September 2001, wo alle Anweisungen, Gutachten und genehmigten Ausnahmen in finnischer oder schwedischer Sprache abrufbar sind.

198 Vgl. Leppiniemi und Leppiniemi (1997), S. 20-28 und Järvinen et al. (2000), S. 548-561.

199 Vgl. KILA 1529/1998.

200 Momentan setzt der KILA sich wegen vieler Anfragen und dem gestiegenen Erklärungsbedarf nach Verabschiedung des neuen Buchführungsgesetzes aus der Maximalbesetzung von Sprecher, stellvertretendem Sprecher und zehn anderen Mitgliedern zusammen. 
wird im Gesetz nur vage beschrieben: Während ein Mitglied und sein Stellvertreter Rechtsassessoren (Magister Legum) sein müssen, wird von allen anderen lediglich verlangt, mit Buchführungsfragen vertraut $\mathrm{zu}$ sein ${ }^{201}$.

Da die Regierung die KILA-Mitglieder in ihre Funktion beruft, hat sie neben der Legislative, dem Parlament, einen beträchtlichen Einfluss auf die Entwicklung der Rechnungslegung. Sie bestimmt die Mitglieder des KILA für jeweils drei Jahre ${ }^{202}$. Die Mitgliedschaft ist zeitlich nicht begrenzt. Seit Gründung des KILA herrschte nur eine geringe Fluktuation ${ }^{203}$.

Der KILA selbst sieht es als Ziel an, dass seine Mitglieder über eine hohe fachliche Qualifikation und Berufserfahrung verfügen. Die Zusammensetzung sollte derart gestaltet sein, dass das Wissen und die Erfahrung der Mitglieder möglichst viele Teilgebiete der Buchführung und Rechnungslegung abdecken und auch fachübergreifende Aspekte nicht außer Acht gelassen werden. So wurde der KILA in der Praxis von Anfang an nicht nach parteipolitischen oder lobbyistischen Gesichtspunkten zusammengesetzt, sondern die Mitglieder wurden auf Grund ihrer fachlichen Qualifikation bestimmt. Besonders in den Jahren bis 1992 hat der KILA es v. a. als seine Aufgabe angesehen, die Ausgaben-Einnahmen-Theorie zu erklären und auf neu auftretende Situationen anzuwenden, sie Kritikern gegenüber zu verteidigen und zu unterstützen. Dieses Ziel konnte nur mit Fachleuten erreicht werden, die fundierte Kenntnisse der Theorie besaßen ${ }^{204}$.

Ob die Mitglieder aus Interessengruppen stammen sollen, ist ebenfalls nicht festgelegt. Beamte und Wissenschaftler waren in den ersten 20 Jahren nach Gründung des KILA in der Mehrheit, nicht Vertreter von Industrieunternehmen, Verbänden oder aus der Wirtschaftsprüfung. Dieses Verhältnis von Theorie zu Praxis scheint sich seit Anfang der 90er Jahre zu Gunsten der Praxis, also der privaten Organisationen und Interessengruppen zu wandeln ${ }^{205}$.

KILA-Mitglieder üben ihr Mandat nebenberuflich aus. Sie treffen sich etwa alle zwei Wochen, um Anfragen zu bearbeiten. Der KILA ist entscheidungsfähig, sobald neben dem Sprecher oder dem stellvertretenden Sprecher wenigstens die Hälfte der anderen Mitglieder - unter ihnen wenigstens ein Rechtsassessor - an-

$201 \quad$ Vgl. KPL 8:2.4.

202 Die momentan laufende Amtsperiode umfasst den Zeitraum 01. Januar 2001 bis 31. Dezember 2003.

203 So hatte beispielweise der erste Sprecher, Bror Wahlroos, seinen Posten von 1974 bis 1992 inne. Bror Wahlroos war darüber hinaus von 1969 bis 1992 Leiter des Ministeriums für Handel und Industrie. Vgl. Pirinen (1996) Appendix 3: Compositions of the Accounting Board.

204 Vgl. Flower (1994), S. 245.

205 Vgl. Majala (1994), S. 79-81, vgl. Flower (1994), S. 245 und S. 260 und vgl. Kap. 2.2.1.3 dieser Arbeit. 
wesend ist. Daneben werden allgemeingültige Anweisungen in Arbeitsgruppen erarbeitet. Der KILA besitzt keine Forschungsabteilung, die ihm zuarbeitet. Er hat zwar die Möglichkeit, bei Problemen externe Experten zu Rate zu ziehen, hat diese in der Vergangenheit jedoch nur selten genutzt ${ }^{206}$.

\subsubsection{Private Organisationen}

\subsection{Organisationen wirtschaftsprüfender Berufsstände}

Vergleichbar mit Deutschland, gibt es auch in Finnland den Berufsstand der Wirtschaftsprüfer, nicht den der „Accountants“ anglo-amerikanischen Typs.

Eine Besonderheit im europäischen Vergleich ist die Tatsache, dass in Finnland alle Gesellschaften, die buchführungspflichtig sind, dazu verpflichtet sind, einen oder mehrere Abschlussprüfer zu bestellen, und das unabhängig von Rechtsform und Größ $\mathrm{e}^{207}$. Unterschieden wird in Wirtschaftsprüfer für Betriebe des privaten Sektors einerseits und des öffentlichen Sektors andererseits.

Für den privaten Sektor gilt nach dem Wirtschaftsprüfungsgesetz von $1995^{208}$, dass autorisierte, von der Zentralhandelskammer zertifizierte KHT-Wirtschaftsprüfer Unternehmen sämtlicher Rechtsformen und Größen prüfen dürfen. Von den lokalen Handelskammern anerkannte HTM-Wirtschaftsprüfer hingegen sind nicht dazu ermächtigt, Kapitalgesellschaften und an der Börse notierte Gesellschaften selbstständig zu prüfen. Hinsichtlich der Prüfungstätigkeit bei kleinen und mittelgroßen Gesellschaften sind HTM-Wirtschaftsprüfer durch bestimmte Größenkriterien eingeschränkt ${ }^{209}$. Begründet wird dies mit den höheren Anforderungen an die Ausbildungsart und -dauer, Weiterbildung und Berufserfahrung, die an die KHTWirtschaftsprüfer gestellt werden. Die Mindestanforderungen der 8. EGRichtlinie werden von beiden Berufsgruppen erfüllt ${ }^{210}$.

206 Vgl. Majala (1994), S. 81 und vgl. Flower (1994), S. 245.

207 Vgl. TilintL 3:9 i. V.m. rechtsformspezifischer Gesetzgebung und vgl. Suomela et al. (1995),

S. 59-60. Seit dem Geschäftsjahr 2000 sind Abschlussprüfungen Wirtschaftsprüfern mit entsprechender Berufsqualifikation vorbehalten. In früheren Geschäftsjahren war es kleinen Gesellschaften und Stiftungen mit einer Bilanzsumme bis $€ 336.000,00$, Umsatzerlösen bis $€ 673.000,00$ und mit max. zehn Arbeitnehmern erlaubt, so genannte „Laien-Wirtschaftsprüfer“ zu beauftragen. Von diesem Recht wurde auch Gebrauch gemacht, v. a., weil die Prüfung durch qualifizierte KHT- oder HTM-Prüfer mit vergleichsweise höheren Kosten verbunden war. Vgl. TilintL (1995) 11 und vgl. Quick, Hemeier und Parthe (1999), S. 229. Vgl. TilintL 12 und vgl. Kap. 2.2.2.4 dieser Arbeit.

HTM-Wirtschaftsprüfer dürfen börsennotierte Unternehmen und solche Unternehmen, die zwei der drei aufgeführten Kriterien überschreiten, nur gemeinsam mit einem KHTWirtschaftsprüfer prüfen: Bilanzsumme $>€ 25$ Mio., Umsatzerlös $>€ 50$ Mio. und Arbeitnehmerzahl $>300$. Vgl. TilintL (1995) 11 bis 14 . 
Als Prüfer können neben Einzelprüfern auch Wirtschaftsprüfungsgesellschaften zugelassen werden, wenn sie bestimmten Anforderungen genügen. Wie die KHTWirtschaftsprüfer werden die KHT-Wirtschaftsprüfungsgesellschaften von der finnischen Zentralhandelskammer ernannt ${ }^{211}$. HTM-Wirtschaftsprüfungsgesellschaften werden von den jeweiligen lokalen Handelskammern autorisiert.

Weder KHT- noch HTM-Wirtschaftsprüfern ist es gestattet, Unternehmen des öffentlichen Sektors zu prüfen. Diese Aufgabe ist den JHTT-Wirtschaftsprüfern des öffentlich-rechtlichen Bereichs vorbehalten, die ihrerseits Unternehmen des privaten Sektors nicht prüfen dürfen. Diese Tatsache erstaunt, weil Betriebe des öffentlichen Sektors heute ihre Abschlüsse nicht mehr als kameralistische Buchführung, sondern nach den für Privatunternehmen geltenden Regeln erstellen müssen.

Die Berufsstände der Wirtschaftsprüfer sind in Verbänden organisiert ${ }^{212}$. Die Berufsverbände überwachen die Arbeitsqualität ihrer Mitglieder, welche einem verbindlichen Qualitäts-Zertifizierungsprogramm unterliegen. Ziel ist es, die Kenntnisse und Fähigkeiten der Prüfer zu festigen und weiterzuentwickeln. Die Berufsverbände organisieren zu diesem Zweck Weiterbildungsseminare, Examensvorbereitungskurse und veröffentlichen Literatur zu aktuellen Sachverhalten. Die Zeitschrift „Tilintarkastus/Revision“, herausgegeben vom Berufsverband der KHTWirtschaftsprüfer, erscheint sechsmal jährlich, „HTM/GRM-Info“ acht- bis zehnmal im Jahr. Darüber hinaus entwickeln die Berufsverbände - orientiert an internationalen Standards - Grundsätze ordnungsmäßiger Abschlussprüfung, die von ihren Mitgliedern angewendet werden müssen ${ }^{213}$.

Die Vereinigungen sehen es als ihre Aufgabe, die Interessen ihrer Mitglieder untereinander und nach außen hin zu vertreten sowie ihre Mitglieder anzuhalten, den an sie gestellten berufsethischen Anforderungen gerecht zu werden ${ }^{214}$.

Der Berufsverband der KHT-Wirtschaftsprüfer nimmt mit zahlreichen Veröffentlichungen mit erklärendem oder ergänzendem Inhalt aktiv an der Rechnungslegungsentwicklung teil, wobei der Verband selbst bislang keine eigenen Standards erstellt, sondern seine Verlautbarungen und Empfehlungen im Wesentlichen auf die IAS stützt ${ }^{215}$. Die vom KHT-Wirtschaftsprüferverband entworfenen und be-

211 Die so genannten „Big Four“-Wirtschaftsprüfungsgesellschaften werden in Finnland durch solche Gesellschaften vertreten.

212 Der Berufsverband der KHT-Wirtschaftsprüfer KHT-yhdistys - Föreningen CGR r.y. wurde 1925 gegründet, der Berufsverband der HTM-Wirtschaftsprüfer HTM-tilintarkastajat r.y. 1951. Vgl. www.htm.fi/english.html.

213 Vgl. Saarikivi (1999), S. 520-521.

214 Vgl. Nieminen (1995), S. 275.

215 Interessant ist in diesem Zusammenhang die historische Entwicklung der Einflussnahme der Wirtschaftsprüfer auf die Rechnungslegung:

Obwohl sie eine fundierte rechnungslegungsspezifische Ausbildung vorweisen konnten, beeinflussten die finnischen Wirtschaftsprüfer die Konzeption und Weiterentwicklung der 
reits in mehreren Auflagen erschienenen Titel „Jahresabschlussmodell für Aktiengesellschaften“216, der „Jahresabschluss von offenen Handelsgesellschaften und Kommanditgesellschaften“‘217 sowie der „Jahresabschluss von Vereinen und Stiftungen“6218 finden in der Praxis breite Anwendung ${ }^{219}$.

Rechnungslegung von den 60ern bis in die 90er Jahre des 20. Jahrhunderts hinein nur vergleichsweise gering im Verhältnis zu den Wirtschaftsprüfern in anderen Staaten. Trotz der Tatsache, dass sie in den Vorbereitungskommissionen zu Gesetzesänderungen in den Bereichen Rechnungslegung, Wirtschaftsprüfung und Besteuerung regelmäßig durch wenigstens eines ihrer Mitglieder vertreten waren, und Mitglieder des Berufsstands regelmäßig in den KILA berufen wurden, wurden Vorschläge der Berufsverbände der Wirtschaftsprüfer weitgehend ignoriert (Vgl. Flower (1994), S. 246). Dieses Phänomen liegt in nachstehenden Faktoren begründet:

Die interne Uneinigkeit in den 70er Jahren darüber, ob der nordische Harmonisierungsversuch oder die Ausgaben-Einnahmen-Theorie zu favorisieren sei, führte dazu, dass der KHTWirtschaftsprüferverband nach außen nicht geschlossen auftrat (Vgl. Flower (1994), S. 246). Seit 1976 Mitglied im IASC (bzw. heute IASB), zeigte sich der KHT-Wirtschaftsprüferverband in den 80er Jahren dann als Gegner der Ausgaben-Einnahmen-Theorie und befürwortete im Hinblick auf die internationale Vergleichbarkeit der Abschlüsse von Großunternehmen die Einführung der Standards des IASC. Anders als der KILA hält der KHT-Wirtschaftsprüferverband kein offizielles Mandat inne und besitzt nicht das Recht, verbindliche Anweisungen, Standards oder Empfehlungen zu verabschieden. Da die multinationalen Unternehmen anfangs den Wandel zu den International Accounting Standards wegen der nicht absehbaren Folgen für die Besteuerung ablehnten, außerdem die mit der Rechnungslegung befasste Fachwelt die IAS als unpatriotisch ansah und deshalb die Ausgaben-Einnahmen-Theorie präferierte, blieben die Internationalisierungsforderungen der Wirtschaftsprüfer weitgehend unbeachtet (Vgl. Flower (1994), S. 246-247).

Darüber hinaus wurde der KHT-Wirtschaftsprüferverband lange Zeit als Konkurrent des KILA angesehen, obwohl eigentlich eine strenge Kompetenzteilung zwischen beiden Organisationen vorlag. Der KILA erteilte Anweisungen, und die Mitglieder des KHT-Wirtschaftsprüferverbands hatten in ihrem Berufsalltag die Aufgabe, die Einhaltung dieser Anweisungen bei ihren Mandanten zu prüfen und zu gewährleisten. Es war deshalb nicht erwünscht, dass der KHT-Wirtschaftsprüferverband versuchte, die Entwicklung der Anweisungen zu beeinflussen (Vgl. Majala (1994), S. 85).

Der Einfluss der Wirtschaftsprüfer nahm erst mit dem Bestreben einer immer größeren Anzahl von Unternehmen zu, ausländische Kapitalmärkte zu erschließen. Nun erreichten der KHT-Wirtschaftsprüferverband und die großen Wirtschaftsprüfungsgesellschaften mit Stellungnahmen zu IAS und US-GAAP die Praxis. Dieses Vorgehen war effektiver als die vorherigen Versuche, Gesetzgebung und den KILA von der Notwendigkeit der Internationalisierung zu überzeugen (Vgl. Majala (1994), S. 86-88). Seit Beginn der 90er Jahre arbeiten der KILA und der KHT-Wirtschaftsprüferverband enger zusammen.

216 Der Titel des finnischen Originals lautet „Osakeyhtiön tilinpäätösmalli“. Übersetzung ins Deutsche durch die Verfasserin.

217 Der Titel des finnischen Originals lautet „Avoimen yhtiön ja kommandiittiyhtiön tilinpäätös“. Übersetzung ins Deutsche durch die Verfasserin.

218 Der Titel des finnischen Originals lautet „Yhdistyksen ja säätiön tilinpäätös“. Übersetzung ins Deutsche durch die Verfasserin.

219 Vgl. Järvinen et al. (2000), S. 55. 


\subsection{Berufs- und Industrieverbände, Aktienmarkt und Börse, Wissen- schaft}

Die Buchhaltungsfirmen, die als Externe die Bücher kleiner und mittelgroßer Unternehmen führen und deren Jahresabschlüsse erstellen, sind organisiert im privaten „Verband der Buchhaltungsfirmen“ KTL. Die Mitgliedsfirmen sind hinsichtlich ihrer Qualität vom Verband geprüft und weisen einen relativ hohen Standard auf, staatlich anerkannt sind die Buchhaltungsfirmen jedoch nicht ${ }^{220}$.

Seit Beginn der 90er Jahre beteiligt sich der KTL aktiv mit Stellungnahmen an der Entwicklung der Rechnungslegung in Finnland. Der Einfluss des KTL auf die Berichterstattung kleiner und mittelständischer Betriebe ist groß. Der Verband gilt darüber hinaus als Fürsprecher dieser Unternehmensgruppen in Debatten um Gesetzesänderungen $^{221}$.

Die Industrieverbände und die großen, international tätigen Unternehmen sind in die Entwicklung der Rechnungslegungsmethoden formell mit Vertretern im KILA involviert und nehmen darüber hinaus in Gesetzgebungsprozessen Stellung. Informell verfügen die Großunternehmen auf Grund ihrer herausragenden Bedeutung für die relativ kleine finnische Volkswirtschaft und Gesellschaft über eine relativ große Macht, die sich auch auf die Gesetzesentwicklung auswirkt - wie groß dieser Einfluss ist, lässt sich allerdings kaum messen. International agierende Konzerne nehmen seit Anfang der 90er Jahre neben dem Berufsstand der Wirtschaftsprüfer und den Universitäten eine Vorreiterrolle bei der Verbreitung international üblicher Rechnungslegungspraktiken wie US-GAAP und IAS ein ${ }^{222}$.

Der Aktienmarkt beeinflusst die Normierung der Rechnungslegung insofern, als dass immer häufiger verlangt wird, dass Jahres- und Konzernabschlüsse nützliche Daten für Investitionsentscheidungen zur Verfügung stellen. Die Intermediäre des Aktienmarkts, die Finanzanalysten und Börsenmakler sowie andere Jahresabschlussadressaten, beteiligen sich jedoch bisher nicht aktiv an der Klärung von Rechnungslegungsproblemen ${ }^{223}$.

Die Börse Helsinki Stock Exchange hat seit Ende der 80er Jahre mehrere Empfehlungen hinsichtlich der Konzernabschlüsse börsennotierter Unternehmen ausge-

\footnotetext{
220 Vgl. Majala (1994), S. 71.

221 Vgl. Pirinen (1996), S. 42-52 und S. 162-176. PIRINEN hat den Einfluss verschiedener Interessengruppen auf die Entstehung des Buchführungsgesetzes von 1992 untersucht und aufgezeigt. Es wird hier angenommen, dass dieselben Interessengruppen auch auf das Buchführungsgesetz von 1997 eingewirkt haben, da sich ihre Zusammensetzung zwischen 1992 und 1997 kaum geändert hat und die Art des Gesetzgebungsverfahrens beibehalten worden ist.

222 Vgl. Majala (1994), S. 85-88.

223 Vgl. Artsberg (2000), S. 530 und vgl. Majala (1994), S. 71.
} 
sprochen, bislang aber keine eigenen Rechnungslegungsstandards verabschie$\operatorname{det}^{224}$.

In der Vergangenheit hat die Wissenschaft mit der Ausgaben-Einnahmen-Theorie SAARIOs v. a. durch die Präsenz der Wissenschaftler im KILA die gesetzliche und außergesetzliche Normierung der Rechnungslegung in Finnland stark beeinflusst. Auch heute finden die Ansichten der Wissenschaftler durch Veröffentlichungen und Lehre breites Gehör. Das Ausmaß ihrer Einflussnahme auf die Rechnungslegung scheint seit Beginn der 90er Jahre abzunehmen, ist jedoch nur schwer zu messen ${ }^{225}$.

\subsubsection{Zusammenfassender Überblick}

Folgende Graphik zeigt die Einflussfaktoren der finnischen Rechnungslegung:

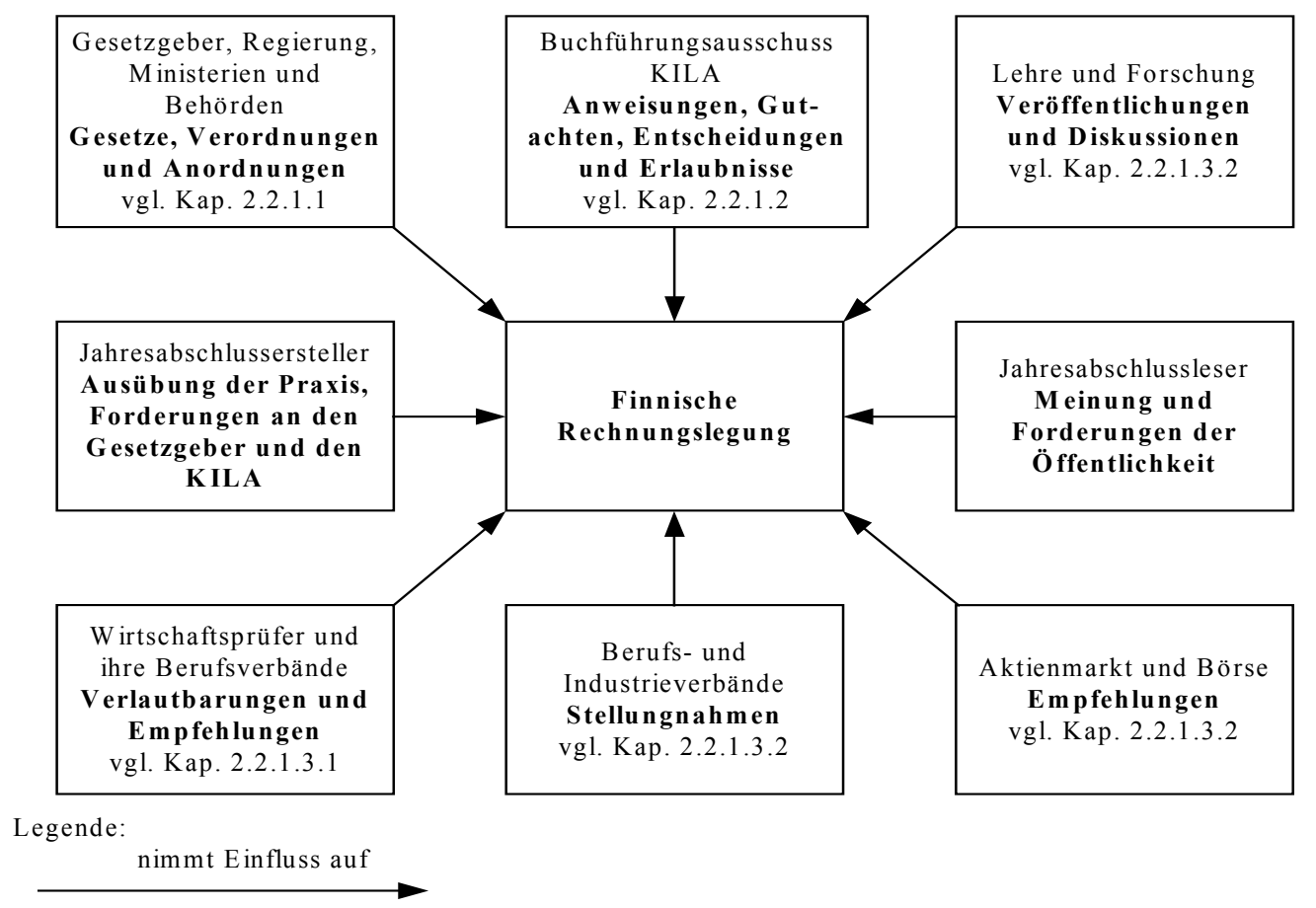

Abb. 2: $\quad$ Einflussfaktoren der finnischen Rechnungslegung

$224 \quad$ Vgl. Artsberg (2000), S. 530 f.

225 Vgl. Majala (1994), S. 82. 


\subsubsection{Konzeption des Buchführungsgesetzes von 1997}

\subsubsection{Regelungsbereich, Anwendungsbereich und Struktur}

Das Buchführungsgesetz von 1997 regelt den Einzel- und den Konzernabschluss. Die 4. und 7. EG-Richtlinie sind in ihm sowie in der ergänzenden Buchführungsverordnung und in rechtsform- und branchenspezifischen Spezialgesetzen in finnisches Recht transformiert. Die Mindestanforderungen der 4. EG-Richtlinie sind in Finnland für Aktiengesellschaften, Offene Handelsgesellschaften und Kommanditgesellschaften relevant. Um aber auch die Vergleichbarkeit finnischer Abschlüsse untereinander zu gewährleisten, hat der finnische Gesetzgeber die Richtlinie so in nationales Buchführungsrecht implementiert, dass ihre Vorschriften in weiten Teilen für alle Buchführungspflichtigen gelten. Die Umsetzung der 8. EGRichtlinie wurde bereits durch die Verabschiedung des Wirtschaftsprüfungsgesetzes 1995 vollzogen ${ }^{226}$.

Das Buchführungsgesetz als lex generalis ist von allen Buchführungspflichtigen anzuwenden. Buchführungspflichtig ist nach KPL 1:1.1 jede Person, die eine gewerbliche Tätigkeit ausübt oder Handel betreibt. Darüber hinaus sind bestimmte Personen unabhängig von ihrer Tätigkeit auf Grund ihrer Rechtsform dazu verpflichtet, Bücher zu führen. Zu ihnen gehören Aktiengesellschaften, Genossenschaften, Offene Handelsgesellschaften, Kommanditgesellschaften, Vereine und andere Gemeinschaften, Stiftungen, Pensionsstiftungen, Versicherungskassen, Versicherungsunternehmen, Versicherungsvereine, Investmentfonds, Personalfonds, Spar- und ähnliche Fonds sowie Effektenanteilssysteme.

Explizit von der Buchführungspflicht befreit sind nach KPL 1:1.2 öffentlichrechtliche Unternehmen im Eigentum von Staat und Gemeinde, die Skandinavische Investitionsbank, der Skandinavische Projektexportfonds sowie Landwirte, sofern letztere nicht die Rechtsform der Gemeinschaft oder Stiftung angenommen haben.

Neben dem KPL sind von den Buchführungspflichtigen - je nach Rechtsform oder Branche - auch andere die Rechnungslegung betreffende Spezialgesetze oder Vorschriften zu beachten, die ihren Ursprung in der 4. EG-Richtlinie haben (lex specialis derogat lex generalis) ${ }^{227}$.

226 Vgl. Kap. 2.2.2.4 der vorliegenden Arbeit.

227 Eine Übersicht über die Spezialgesetze mit Rechnungslegungsrelevanz ist im Anhang dieser Arbeit enthalten. Auf Grund ihrer besonderen Bedeutung wird die Beziehung vom Buchführungsgesetz zum Aktiengesellschaftsgesetz in Kap. 2.2.2.2 dargestellt. 
Die Vorschriften des Buchführungsgesetzes von 1997 sind teils ausschließlich national begründet, teils beruhen sie auf der Umsetzung von Gemeinschaftsrecht oder den Standards des IASB. Das Gesetz beinhaltet insgesamt 92 Paragraphen $^{228}$ :

Vorschriften zur Gliederung der Gewinn- und Verlustrechnung sowie der Bilanz und Vorschriften, die auch in den Gemeinschaftsrichtlinien einem permanenten Wandel unterliegen, befinden sich in der Buchführungsverordnung ${ }^{229}$.

\subsubsection{Buchführungsgesetz, Aktiengesellschaftsgesetz und Wertpapier- marktgesetz}

\subsection{Aktiengesellschaft}

Das finnische Gesellschaftsrecht kennt keine mit der deutschen GmbH vergleichbare Rechtsform. Einzige Form der Kapitalgesellschaft in Finnland ist die Aktiengesellschaft. Sie ist die mit Abstand am weitesten verbreitete Gesellschaftsform in Finnland ${ }^{230}$. Die Bevorzugung der Aktiengesellschaft gegenüber der Personengesellschaft ${ }^{231}$ resultiert aus der beschränkten Haftung der Gesellschafter und ihrer steuerrechtlichen Behandlung ${ }^{232}$.

228 Übersetzung der Kapitel- und Paragraphenüberschriften ins Deutsche durch die Verfasserin. Die vollständige Struktur des Buchführungsgesetzes mit Inhaltsverzeichnis aller Paragraphen wird im Anhang dieser Arbeit aufgezeigt.

229 Hierzu zählen die Gliederungsschemata von Bilanz und Gewinn- und Verlustrechnung (Kap. 1 der KPA), erforderliche Anhangangaben und Angaben im Lagebericht (Kap. 2 der KPA) sowie die Aufgliederung einzelner Bilanz- und Anhangangaben (Kap. 5 der KPA). Die Buchführungsverordnung lässt sich im Bedarfsfall einfacher und schneller ändern als das Gesetz.

230 Es wird geschätzt, dass Anfang des Jahres 2000 mehr als 100.000 Unternehmen dieser Rechtsform registriert waren. Vgl. www.tilastokeskus.fi. Die letzten exakten Zahlen datieren aus dem Jahr 1996. Damals wurden 191.173 Aktien- und 72.470 Personengesellschaften in Finnland gezählt. Vgl. Hagert (1997), S. 605.

231 An dieser Stelle folgt eine kurze Erläuterung zur Offenen Handelsgesellschaft und Kommanditgesellschaft, da für diese Gesellschaften neben der Aktiengesellschaft die Anwendung der 4. EG-Richtlinie zwingend vorgeschrieben ist:

Die Offene Handelsgesellschaft und die Kommanditgesellschaft werden als Personengesellschaften durch Vereinbarung natürlicher oder juristischer Personen gegründet. Es besteht Eintragungspflicht im Handelsregister. Offene Handelsgesellschaft und Kommanditgesellschaft sind juristische Personen, die Verträge eingehen können, Eigentum innehalten, klagen und verklagt werden können. Jeder Gesellschafter kann für die Gesellschaft verbindliche Verträge eingehen. Die Gesellschafter haften gemeinsam, im Falle der Offenen Handelsgesellschaft unbeschränkt mit ihrem Privatvermögen. Bei der Kommanditgesellschaft haftet wenigstens ein Gesellschafter unbeschränkt mit seinem Privatvermögen, wenigstens ein Gesellschafter haftet nur in Höhe seiner Kapitaleinlage.

Im Verhältnis zum Buchführungsgesetz ist das Gesetz über Offene Handelsgesellschaften und Kommanditgesellschaften AKYL Spezialgesetz. Es stellt in Kap. $9 \S 3$ spezielle Anforderungen an den Ausweis von Forderungen und Verbindlichkeiten gegenüber Gesellschaftern. Auch werden Angaben zu Pfandsicherheiten und der Gesamtsumme der anderen (Kredit-)Sicherheiten und Haftungsverhältnisse verlangt, die das Unternehmen zugunsten der Gesellschaften eingegangen ist. Diese Anforderungen basieren auf der 4. EG-Richtlinie. Alle anderen für Offene Handelsgesellschaften und Kommanditgesellschaften geltenden Buchfüh- 


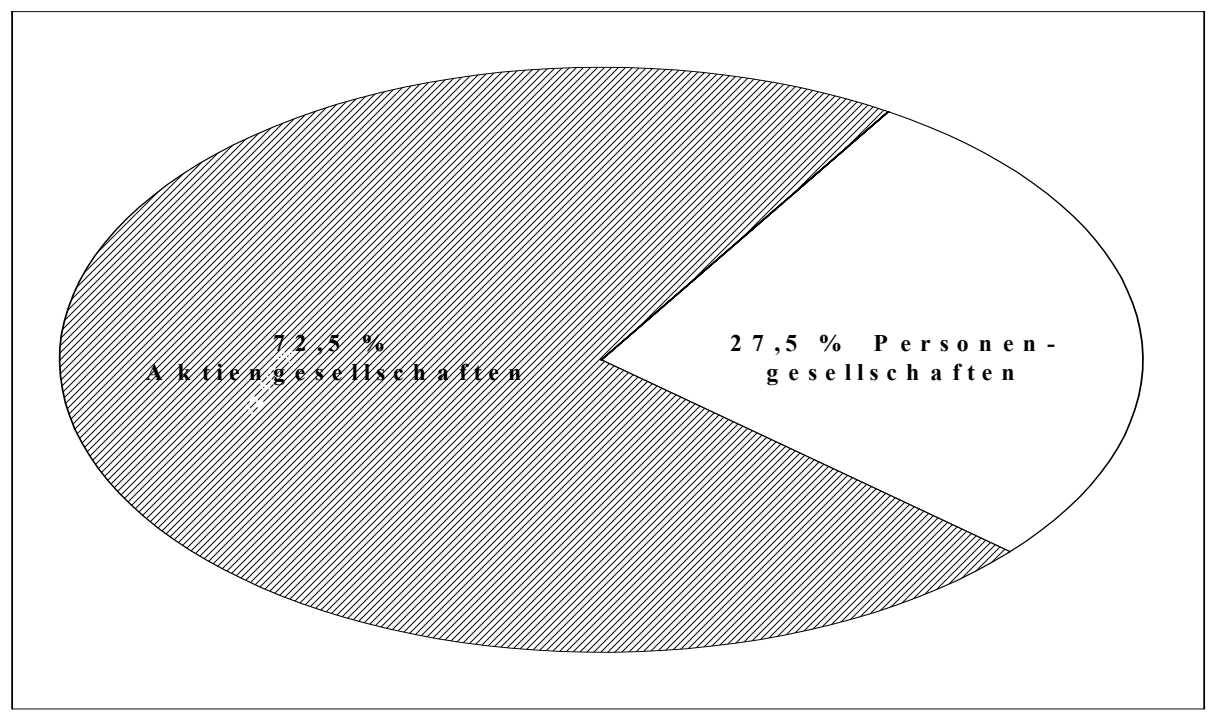

Abb. 3: Anteil der Aktiengesellschaften an allen finnischen Unternemen

Die Aktiengesellschaft ist im Aktiengesellschaftsgesetz OYL von 1978 erfasst, das 1997 in weiten Teilen novelliert wurde, um es mit den entsprechenden EGRichtlinien in Einklang zu bringen. Eine Aktiengesellschaft kann von einer oder mehreren natürlichen oder juristischen Personen gegründet werden. Von den Gründungsmitgliedern muss ein Gründungsvertrag und ein Gesellschaftsvertrag erstellt und unterschrieben werden. Anschließend muss das gezeichnete Kapital als Geldwert oder Sacheinlage, deren Wert ein Sachverständiger und ein unabhängiger anerkannter Rechnungsprüfer festgestellt haben muss, in voller Höhe einbezahlt werden ${ }^{233}$. Erst dann kann die staatliche Genehmigung zur Pflichteintragung ins Handelsregister erfolgen. Erst nach Eintragung ins Handelsregister entsteht die Aktiengesellschaft als juristische Person. Werden bereits vorher Aktivitäten im Namen der Aktiengesellschaft durchgeführt, haften die Aktionäre persönlich ${ }^{234}$.

Differenziert wird seit der Gesetzesnovelle von 1997 zwischen privaten Aktiengesellschaften und öffentlichen Aktiengesellschaften. Private und öffentliche Aktiengesellschaften unterscheiden sich in zwei wesentlichen Punkten: in der Börsen-

rungsregeln sind Bestandteil des KPL, sodass auf eine gesonderte Darstellung der Vorschriften für diese Rechtsformen in dieser Arbeit verzichtet wird.

232 Die Aktiengesellschaft ist körperschaftsteuerpflichtig. Die finnische Körperschaftsteuer wird nicht in einem eigenen Gesetz geregelt, sondern im Einkommensteuergesetz TVL sowie im Gewerbesteuergesetz EVL. Der Körperschaftsteuersatz beträgt seit dem 01. Januar 2000 pauschal 29 Prozent (vgl. Uusitalo und Collan (2000), G 314). Daneben bilden die Aktien die Grundlage für die Vermögensbesteuerung, weshalb die Wertfestsetzung der Aktien bei der Veranlagung eine zentrale Bedeutung hat (vgl. Uusitalo und Collan (2000), G 345).

233 Vgl. OYL 2:9. Die 2. EG-Richtlinie (77/91/EEC) fordert in Art. 9 lediglich, dass mindestens 25 Prozent des gezeichneten Kapitals eingezahlt sein müssen.

234 Vgl. OYL 2:10. 
zulassungsfähigkeit, die den öffentlichen Aktiengesellschaften vorbehalten ist, und in der Höhe des Mindestkapitals, das für private Aktiengesellschaften $€ 8.000,00$ und für öffentliche Aktiengesellschaften $€ 80.000,00$ beträgt. Hinsichtlich der Rechnungslegungs- und Publizitätspflichten unterliegt die öffentliche Aktiengesellschaft strengeren Bestimmungen als die private ${ }^{235}$. Ziel der Gesetzesnovelle war eine verbesserte Gleichbehandlung der Aktionäre sowie ein effektiverer Gläubigerschutz ${ }^{236}$.

Wichtiges Organ einer Aktiengesellschaft ist die Hauptversammlung die innerhalb von sechs Monaten nach Ablauf eines Geschäftsjahrs einberufen werden muss $^{237}$. Die Themen einer ordentlichen Hauptversammlung umfassen die Bestätigung von Gewinn- und Verlustrechnung und Bilanz des Einzelabschlusses sowie von Gewinn- und Verlustrechnung und Bilanz des Konzernabschlusses, wenn es sich bei der Aktiengesellschaft um ein Mutterunternehmen handelt ${ }^{238}$; auffällig ist, dass die weiteren Pflichtbestandteile finnischer Jahres- und Konzernabschlüsse - Anhang und Lagebericht - nicht zu den gesetzlich verankerten Pflichtthemen einer ordentlichen Hauptversammlung zählen ${ }^{239}$. Es wird über die Gewinnverteilung sowie die Entlastung des Vorstands, des Aufsichtsrats und des Geschäftsführers für das abgelaufene Geschäftsjahr entschieden. Darüber hinaus werden andere Themen gemäß Aktiengesellschaftsgesetz oder Gesellschaftsvertrag diskutiert $^{240}$. Sieht der Gesellschaftsvertrag nichts anderes vor, entscheidet die einfache Mehrheit. Eine Ausnahme hiervon bildet der Gesellschaftsvertrag selbst, der nur mit 2/3-Mehrheit geändert werden kann ${ }^{241}$. Eine außerordentliche Hauptversammlung kann unter Angabe ihres Zwecks von Aktionären, die mehr als zehn Prozent der Anteile am Unternehmen repräsentieren, oder vom Wirtschaftsprüfer einberufen werden ${ }^{242}$. Zudem muss seitens des Vorstands unverzüglich eine außerordentliche Hauptversammlung wegen Liquidation einberufen werden, wenn das Eigenkapital die Hälfte des gezeichneten Kapitals unterschreitet. Kommt der Vorstand

235 Vgl. Hagert (1997), S. 605-606.

236 Vgl. Blummé et al. (1997), S. 23-27.

237 Vgl. OYL 9:5.

238 Von der Pflicht zur Aufstellung eines Konzernabschlusses ist ein Mutterunternehmen nach KPL 6:1.3 dann befreit, wenn der konsolidierte Abschluss nicht mehr als eines der folgenden Kriterien überschreitet: Umsatzerlös von $€ 16,8$ Mio., Bilanzsumme von $€ 8,4$ Mio. und Arbeitnehmerzahl von 250 im Jahresdurchschnitt.

Der Pflicht zur Aufstellung eines Konzernabschlusses wird ein Mutterunternehmen nach KPL 6:1.4 weiterhin dann enthoben, wenn sein Mutterunternehmen einen befreienden Konzernabschluss erstellt. Dieses ist dann möglich, wenn das befreiende Mutterunternehmen seinen Sitz in einem EU-Mitgliedstaat hat und wenigstens 90 Prozent der Anteile am zu befreienden Mutterunternehmen hält (KPL 6:1.4,1), wenn die anderen Eigentümer des zu befreienden Mutterunternehmens ihre Zustimmung zur Nicht-Erstellung des Konzernabschlusses erteilt haben (KPL 6:1.4,2) und wenn der befreiende Konzernabschluss offen gelegt wird (KPL 6:1.4,3).

239 Vgl. OYL 9:6.

240 Vgl. OYL 9:5.2,1-4.

241 Vgl. OYL 9:15.

242 Vgl. OYL 9:6. 
dieser Pflicht nicht nach, kann dieses Versäumnis neben der gesellschaftsinternen Haftung auch zu einer persönlichen Haftung des Vorstands gegenüber Dritten führen ${ }^{243}$.

Ein weiteres Organ der Aktiengesellschaft ist der Vorstand, der von der Hauptversammlung gewählt wird. Er besteht aus mindestens drei Mitgliedern, wenn das gezeichnete Kapital wenigstens $€ 80.000,00$ beträgt $^{244}$. Er zeichnet verantwortlich für alle Geschäftsaktivitäten und unterschreibt den Jahresabschluss ${ }^{245}$. Die Berufung eines Geschäftsführers ist für Unternehmen mit einem gezeichneten Kapital ab $€ € 80.000,00$ Pflicht $^{246}$. Der Geschäftsführer ist gemäß der vom Vorstand aufgestellten Bestimmungen für die Verwaltungsarbeiten des Alltags verantwortlich und überwacht die Übereinstimmung der Unternehmensführung mit den gesetzlichen Regelungen ${ }^{247}$.

Ein Aufsichtsrat kann nur gegründet werden, wenn das gezeichnete Kapital mindestens $€ 80.000,00$ beträgt. Ihm gehören wenigstens fünf Mitglieder an. Er kontrolliert den Vorstand und benennt den Geschäftsführer ${ }^{248}$.

\subsection{Buchführungsgesetz und Aktiengesellschaftsgesetz}

Neben den Minimalanforderungen des Buchführungsgesetzes und -erlasses gelten für Buchführung, Rechnungslegung und Offenlegung von Abschlüssen der Aktiengesellschaften die Bestimmungen des Kap. 11 des Aktiengesellschaftsgesetzes OYL, das die Überschrift „Einzelabschluss und Konzernabschluss“ trägt. Wichtige Regeln betreffen die Eigenkapital-Bilanzposten ${ }^{249}$ und die Meldung der Anzahl eigener Aktien und der Aktien des Mutterunternehmens, die sich im Besitz des bilanzierenden Unternehmens befinden ${ }^{250}$. Auch wird die Angabe von Darlehen, die den Mitgliedern der Führungsorgane und den Mitgliedern des ,inneren Krei-

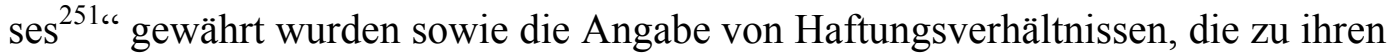
Gunsten eingegangen wurden, verlangt ${ }^{252}$. Weitere Regelungen betreffen die

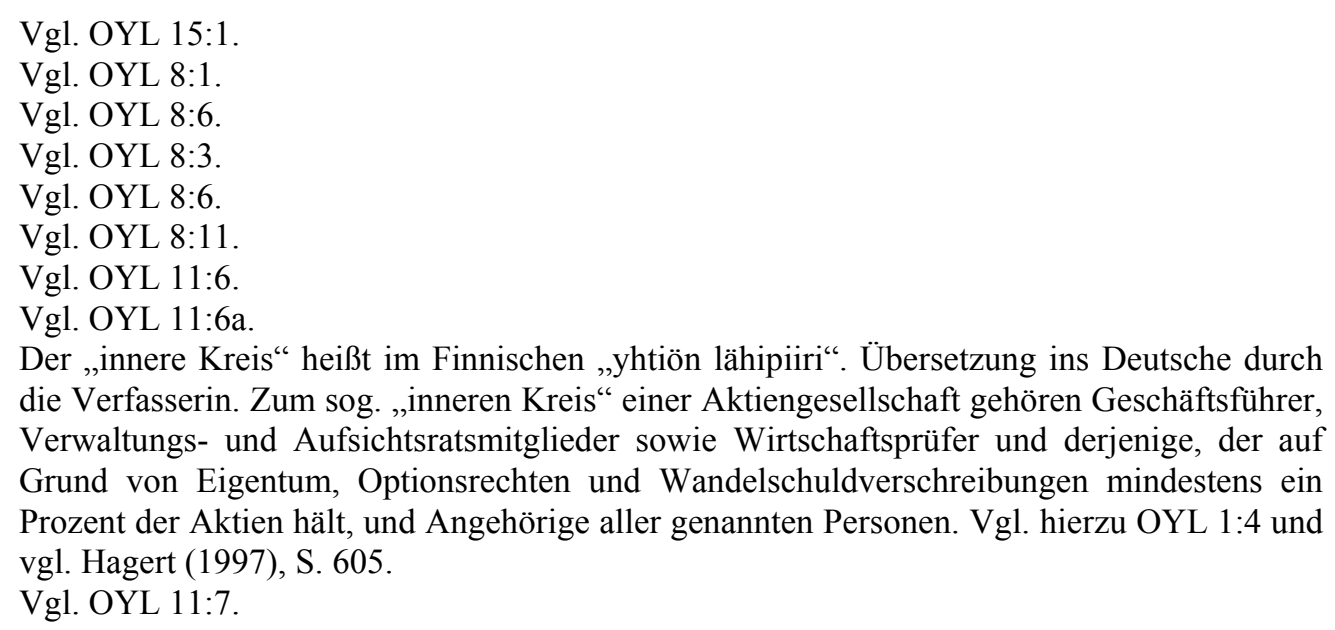
die Verfasserin. Zum sog. ,inneren Kreis“ einer Aktiengesellschaft gehören Geschäftsführer, Verwaltungs- und Aufsichtsratsmitglieder sowie Wirtschaftsprüfer und derjenige, der auf Grund von Eigentum, Optionsrechten und Wandelschuldverschreibungen mindestens ein Prozent der Aktien hält, und Angehörige aller genannten Personen. Vgl. hierzu OYL 1:4 und vgl. Hagert (1997), S. 605.

Vgl. OYL 11:7. 
Meldung der Eigenkapitalstruktur, Optionsrechte und Wandelschuldverschreibungen $^{253}$, den Inhalt des Lageberichts und die Pflicht zur Darstellung einer Kapitalflussrechnung ${ }^{254}$, den Konzernabschluss ${ }^{255}$ sowie den Zwischenbericht ${ }^{256}$. Hinsichtlich der Darstellung des Eigenkapitals und der Unterteilung in gebundenes und freies Eigenkapital gelten die Regeln des Kap. 12 OYL.

\subsection{Buchführungsgesetz und Wertpapiermarktgesetz}

Börsennotierte öffentliche Aktiengesellschaften unterliegen neben den Vorschriften des Buchführungsgesetzes und Aktiengesellschaftsgesetzes den strengen Publizitätsverpflichtungen des Wertpapiermarktgesetzes AML. Kap. 2 dieses Gesetzes regelt Veröffentlichungsfristen, Mindestanforderungen an den Inhalt und die Darstellungsweise des Inhalts von Zwischenberichten, vorläufigem Jahresabschluss und Jahresabschluss ${ }^{257}$ :

Gemäß AML 2:5 müssen Zwischenberichte ein richtiges und ausreichendes Bild des Ergebnisses und der ökonomischen Lage widerspiegeln ${ }^{258}$ sowie nach Art guter Buchführung erstellt werden ${ }^{259}$. Die Zwischenberichterstattung erfolgt entweder pro Quartal oder für die ersten acht Monate eines Geschäftsjahres. AML 2:6a verlangt, eine Vorabmitteilung über den Jahresabschluss mit seinen Hauptaussagen unverzüglich nach Fertigstellung zu veröffentlichen. Ein Mutterunternehmen muss dann auch über den Konzern informieren. Nach AML 2:6 hat der Jahresabschluss ein richtiges und ausreichendes Bild des Ergebnisses und der ökonomischen Lage zu liefern sowie die den Aktienwert beeinflussenden Faktoren aufzuzeigen. Der Jahresabschluss muss - so wie auch der zugehörige Prüfungsbericht - spätestens eine Woche vor der Hauptversammlung, auf der er festgestellt werden soll, veröffentlicht werden, spätestens jedoch drei Monate nach Ablauf des Geschäftsjahres.

Die Gesetzesregelungen für börsennotierte öffentliche Aktiengesellschaften wurden durch Entscheidungen des Finanzministeriums konkretisiert ${ }^{260}$.

\footnotetext{
$253 \quad$ Vgl. OYL 11:8.

254 Vgl. OYL 11:9 und 9a.

255 Vgl. OYL 11:10 und 11.

256 Vgl. OYL 11:12.

257 Vgl. AML 2.

258 Vgl. Kap. 3.1.3.4 dieser Arbeit.

259 Vgl. Kap. 3.1.3.1 dieser Arbeit. Die Art guter Buchführung umfasst Gesetzestreue und die Beachtung der Grundsätze ordnungsmäßiger Buchführung.

260 Die Entscheidungen 905/1994, 879/1995 und 390/1999 listen detailliert und katalogartig den Inhalt und die Darstellungsweise von Zwischenbericht, vorläufigem Jahresabschluss und Jahresabschluss auf. Sie gelten verbindlich für alle börsennotierten Unternehmen. Die Entscheidung 390/1999 wurde darüber hinaus vom KILA in einer Allgemeingültigen Anweisung konkretisiert. Vgl. hierzu KILA-Anweisung „Erstellung von Jahresabschluss, vorläufigem Abschluss und Zwischenbericht".
} 


\subsubsection{Buchführungsgesetz und Steuergesetzgebung}

Die 4. EG-Richtlinie erlaubt ein Abweichen von handelsrechtlichen Bewertungsgrundsätzen nach Art. 35 Abs. 1(d) für das Anlagevermögen und Art. 39 Abs. 1 (e) für das Umlaufvermögen zu Gunsten nationaler steuerlicher Regelungen nur, wenn die Inanspruchnahme steuerlicher Vergünstigungen hiervon abhängt. Die Transformation der 4. EG-Richtlinie in finnisches Recht führte zu einer Debatte über die Verknüpfung von externer Rechnungslegung und steuerlicher Gewinnermittlung ${ }^{261}$.

Der Jahresabschluss in Finnland wird nach Maßgabe des Buchführungsgesetzes, nicht nach Maßgabe der Steuergesetze, erstellt. Er ist aber prinzipiell als Grundlage für die steuerliche Gewinnermittlung heranzuziehen, sowohl im Hinblick auf den Ansatz als auch auf die Bewertung von Vermögensgegenständen. Zur Ermittlung des steuerlichen Ergebnisses sind die Jahresabschlussposten der Bilanz und der Gewinn- und Verlustrechnung gegebenenfalls nach steuerrechtlichen Vorschriften zu korrigieren. Die Inanspruchnahme bestimmter Steuervergünstigungen ist jedoch davon abhängig, ob die entsprechenden Bewertungs- und Bilanzierungsmaßnahmen auch im Jahresabschluss durchgeführt wurden. Anders ausgedrückt bedeutet diese Verknüpfung von Buchführungsgesetz und Steuergesetzgebung nach HELENIUS, dass bestimmte Steuergesetzänderungen eine Änderung der Grundsätze ordnungsmäßiger Buchführung nach sich ziehen. Diese Aussage betrifft besonders die Bewertungsgrundsätze von Anlage- und Umlaufvermögen, die Währungsumrechnung und bestimmte Rückstellungen ${ }^{262}$. Dieser Grundsatz der Maßgeblichkeit des Jahresabschlusses für die steuerliche Gewinnermittlung und seine Umkehrung sind in den jeweils relevanten Steuergesetzen verankert ${ }^{263}$. Der finnische Gesetzgeber hat diese Grundsätze deutscher Prägung im Jahr 1919 übernommen und im Zeitablauf unterschiedlich stark ausgeprägt bis heute beibehalten $^{264}$.

Diskutiert wurde bei der Umsetzung der 4. EG-Richtlinie v. a. das mögliche Scheitern internationale Harmonisierungsbemühungen auf Grund nationaler steuerlicher Einflüsse und nationaler Interessen der (Steuer-)Politiker sowie die mit der Aufstellung von getrennter Handels- und Steuerbilanz verbundene Mehrarbeit für Unternehmen ${ }^{265}$. Im Rahmen einer Umfrage ist auch die Meinung der Vertre-

261 Diese Debatte wurde bei der Transformation der 7. EG-Richtlinie nicht geführt, da auch in Finnland Konzernabschlüsse steuerlich nicht relevant sind. Zur Debatte über die Transformation der 4. EG-Richtlinie vgl. Järvenpää (1996), S. 905-907, vgl. Helenius (1990), S. 182184 und vgl. Troberg (1995), S. 125. Vgl. Helenius (1990), S. 182-184.

Vgl. Järvenpää (1996), S. 899-905.

264 Vgl. Artsberg (2000), S. 527, vgl. Hoogendoorn (1996), S. 786-793 und vgl. Järvenpää (1996), S. 899-905.

265 Diese Diskussion wird in Deutschland ebenfalls geführt. Vgl. beispielsweise Broer, Frank Daniel: Maßgeblichkeitsprinzip und Harmonisierung der Rechnungslegung, Baden-Baden 
ter mittelgroßer und großer Kapitalgesellschaften ermittelt worden, von denen sich 80 Prozent für eine Trennung von Handels- und Steuerrecht aussprachen ${ }^{266}$. Die mit dem Entwurf des Buchführungsgesetzes 1992 beauftragte Arbeitsgruppe hat schließlich vorgeschlagen, die Eliminierung steuerlicher Einflüsse im Konzernabschluss, nicht aber im Jahresabschluss zu erlauben.

Infolge dieser Diskussion wird die Verbindung zwischen Buchführungsgesetz und Steuergesetzgebung seit 1993 sukzessiv gelockert. Dort, wo eine Beibehaltung von Maßgeblichkeit und umgekehrter Maßgeblichkeit Aussagekraft und Entwicklung der Rechnungslegung zu beeinträchtigen schienen, sind Durchbrechungstatbestände eingeräumt worden: Die Unternehmen müssen die steuerlichen Werte nicht mehr in allen Fällen in den Jahresabschluss übernehmen, um Steuervergünstigungen geltend machen zu dürfen ${ }^{267}$.

Steuerliche Aspekte beeinflussen die Jahresabschlussaussage noch in folgenden wesentlichen Fällen:

Ausgeprägt sind die Wirkungen steuerlicher Vorschriften in den Bereichen freiwilliger Rückstellungen ${ }^{268}$ und so genannter ,,anderer als planmäßiger Abschreibungen““269. Diese dürfen überhaupt nur dann im Jahresabschluss vorgenommen werden, wenn sie auch steuerlich akzeptiert werden. Diese Posten, die nur aus steuerlichen Gesichtspunkten im Jahresabschluss ausgewiesen werden, können isoliert betrachtet werden, da sie als Bilanzposition unter den Passiva als ,steuerliche Ausgleichsposten“" zusammengefasst werden ${ }^{270}$.

Der steuerliche Höchstsatz für Abschreibungen kann nur geltend gemacht werden, wenn auch im handelsrechtlichen Jahresabschluss wenigstens in seiner Höhe abgeschrieben wird.

Existieren hinsichtlich eines Sachverhalts Wahlrechte sowohl nach Buchführungsgesetz als auch nach Steuergesetz, muss zum Zweck der Steuerakzeptanz oft dieselbe Alternative ausgeübt werden ${ }^{271}$. Besteht nach Buchführungsgesetz ein Ansatz- oder Bewertungswahlrecht, wird in der Bilanzierungspraxis zumeist diejenige Alternative, die mit den Steuervorschriften einhergeht, gewählt, auch wenn sie handelsrechtlich nicht immer die beste Lösung darstellt ${ }^{272}$.

2001 oder vgl. Dziadkowski, Dieter: Die Diskussion über das Verhältnis von Handels- und Steuerbilanz: Alter Wein in neuen Schläuchen?, in: Deutsche Steuer-Zeitung, Bd. 89 (2001), 1/2, S. 9-16.

266 Vgl. Järvenpää (1996), S. 904.

267 Vgl. Artsberg (2000), S. 527, die ihre Aussage auch auf Schweden bezieht.

268 Vgl. EVL 46 a.

269 Vgl. EVL 54.2.

270 Vgl. Kap. 3.1.6.2.2.2 dieser Arbeit zu „anderen als planmäßigen Abschreibungen“ und vgl. Kap. 3.2.5.1.1 zum Abschreibungsunterschied. In Kap. 3.2.5.1.2 wird die Kontroverse um eine Passivierung freiwilliger, nicht steuerlich abzugsfähiger Rückstellungen dargestellt.

271 Als Beispiel für solche Fälle dienen die Ermittlung der Anschaffungs- und Herstellungskosten nach EVL 14.1 und die Gewinnrealisierung bei langfristigen Projekten nach EVL 19.2. Vgl. Leppiniemi und Leppiniemi (1997), S. 13-14.

272 Vgl. Leppiniemi und Leppiniemi (1997), S. 14. 
Zusätzlich zu diesen Fällen der Maßgeblichkeit und umgekehrter Maßgeblichkeit mit (temporärer) Ergebnisauswirkung beinhaltet die Steuergesetzgebung auch Vorschriften hinsichtlich der Buchführung, die von denen des Buchführungsgesetzes abweichen und die Unternehmenspraxis beeinflussen. Es handelt sich hierbei um die Buchführungspflicht sowie die Dokumentation und den Aufbewahrungszeitraum von Unterlagen, die steuerlich relevante Informationen enthalten ${ }^{273}$.

\subsubsection{Buchführungsgesetz und Wirtschaftsprüfungsgesetz}

Am 01. Januar 1995 traten das Wirtschaftsprüfungsgesetz TilintL, die Wirtschaftsprüfungsverordnung TilintA sowie auf Grund des Wirtschaftsprüfungsgesetzes notwendig gewordene Änderungen der Gesellschaftsgesetze in $\mathrm{Kraft}^{274}$.

Dass 70 Jahre nach Gründung des Berufsverbands der KHT-Wirtschaftsprüfer ${ }^{275}$ gesetzliche Vorschriften für und über den Berufsstand eingeführt wurden, hat mehrere Gründe:

Seit 1983 müssen KHT-Wirtschaftsprüfer nicht mehr zwangsläufig Mitglied im Berufsverband sein. Die Arbeitsqualität der Nicht-Mitglieder sollte deshalb von anderen Organen gewährleistet und überwacht werden ${ }^{276}$.

Ende der 80er und Anfang der 90er Jahre des 20. Jahrhunderts führte der wirtschaftliche Abschwung in Finnland, verbunden mit Inhaber- und Generationenwechsel sowie der Entlassung erfahrener Mitarbeiter in vielen Unternehmen, zu zahlreichen Konkursen. Daraufhin wurde eine Debatte in der Öffentlichkeit ausgelöst, in deren Verlauf sich zeigte, dass von der Arbeit eines Wirtschaftsprüfers andere Ergebnisse erwartet wurden als damals gesetzlich vorgesehen waren ${ }^{277}$. Diese Erwartungslücke führte im Jahr 1992 zu neuen Prüfungsstandards vom KHT-Wirtschaftsprüferverband. 1993 erfolgte die Gründung einer für Qualität zuständigen Arbeitsgruppe des Berufsverbands und 1994 die Einführung eines Ethik-Komitees im Berufsverband, das die Einhaltung der Art guter Wirtschaftsprüfung überwachen sollte. Zudem wurde verstärkt auf Öffentlichkeitsarbeit Wert gelegt $^{278}$.

\footnotetext{
273 Vgl. Järvinen et al. (2000), S. 585-586.

274 Das Wirtschaftsprüfungsgesetz ist ein Mantelgesetz. Rechtsformspezifische Regelungen in Bezug auf die Wirtschaftsprüfung finden sich im jeweiligen Gesellschaftsgesetz als Spezialgesetz (lex specialis derogat lex generalis). Vgl. Suomela et al. (1995), S. 22-25.

275 Vgl. Kap. 2.2.1.3.1 dieser Arbeit.

276 Vgl. Nieminen (1995), S. 283.

277 Vgl. Nieminen (1995), S. 281-282 und vgl. Saarikivi (1999), S. 522.

278 Vgl. Nieminen (1995), S. 283.
} 
Zeitgleich musste die 8. gesellschaftsrechtliche EG-Richtlinie in nationales Recht umgesetzt werden. Außerdem wurde gefordert, internationale Prüfungsstandards gesetzlich festzuschreiben, obwohl diese auf Grund der jahrzehntelangen Mitgliedschaft vom KHT-Wirtschaftsprüferverband in internationalen Berufsverbänden ohnehin in der Praxis angewendet wurden.

Das Wirtschaftsprüfungsgesetz enthält grundlegende und generelle Vorschriften in 48 Paragraphen, die acht Kapiteln zugeordnet sind. Sie regeln die Art guter Wirtschaftsprüfung, Prüfungstechnik und -methoden, Anforderungen an Unabhängigkeit und andere persönliche Eigenschaften der Wirtschaftsprüfer sowie ihre Zulassungs-, Bestellungs- und Überwachungsverfahren. Das Gesetz erfüllt die Mindestanforderungen der 8. EG-Richtlinie und geht teilweise über diese hinaus $^{279}$.

Unter den Anwendungsbereich des Wirtschaftsprüfungsgesetzes fallen nach TilintL 1:1 alle Prüfungen von Unternehmen und Stiftungen, die nach KPL 1:1 buchführungspflichtig sind ${ }^{280}$. Zudem regelt KPL 3:12, dass Prüfungsberichte dem Handelsregister zu überreichen sind und gemeinsam mit dem Jahresabschluss veröffentlicht werden müssen, sofern Buchführungspflichtige nach Wirtschaftsprüfungsgesetz prüfungspflichtig sind.

Im Ministerium für Handel und Industrie existiert der staatliche Wirtschaftsprüfungsausschuss TILA, der - analog dem KILA das Buchführungsgesetz betreffend - folgende Aufgaben hat: Der Wirtschaftsprüfungsausschuss gibt Anweisungen und Stellungnahmen zum Wirtschaftsprüfungsgesetz und zur Wirtschaftsprüfungsverordnung, unterbreitet Vorschläge und Kommentare zur Entwicklung prüfungsrelevanter Vorschriften und kümmert sich um die allgemeine Anleitung, Entwicklung und Beaufsichtigung im Wirtschaftsprüfungswesen ${ }^{281}$.

Der Prüfungsausschuss der Zentralhandelskammer beaufsichtigt die KHT-Wirtschaftsprüfer und stellt sicher, dass sie die Zulassungsvoraussetzungen weiterhin erfüllen sowie die Prüfungsnormen beachten ${ }^{282}$. Er kann Disziplinarmaßnahmen verhängen $^{283}$.

279 Vgl. Saarikivi (1999), S. 519.

280 Spezialvorschriften zur Prüfung einzelner Rechtsformen sind in den jeweiligen Gesellschaftsgesetzen enthalten. Bei möglichen Unstimmigkeiten haben die Spezialvorschriften Vorrang (lex specialis derogat lex generalis). Vgl. Järvinen et al. (2000), S. 360 und Suomela et al. (1995), S. 22-25.

281 Vgl. TilintL 28:1.1-3.

282 Vgl. TilintL 38:1.

283 Diese Disziplinarmaßnahmen sind der Vermerk (TilintL 35:1 und 39:2), die Verwarnung oder der Ausschluss aus dem Berufsstand (TilintL 38:1). 


\subsubsection{Zusammenfassender Überblick}

In folgender Graphik sind die Regelungsbereiche der finnischen Gesetze mit Rechnungslegungsrelevanz aufgezeigt:

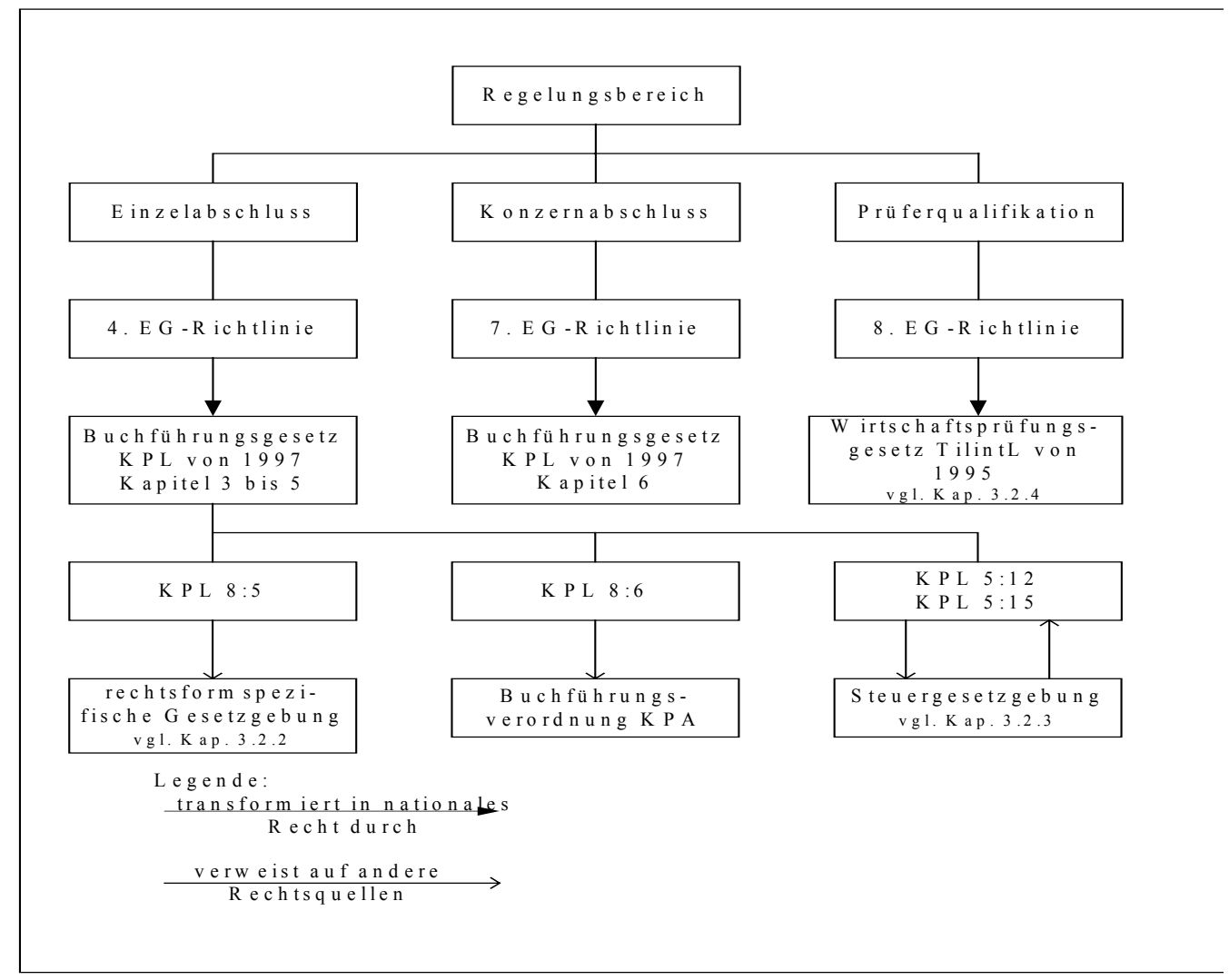

Abb. 4: Regelungsbereiche der finnischen Gesetze mit Rechnungslegungsrelevanz 


\section{Grundlagen der finnischen Rechnungslegung}

\subsubsection{Zielsetzung der finnischen Rechnungslegung}

Mit Ausnahme der Forderung, der Jahresabschluss habe ein richtiges und ausreichendes Bild der Ertragslage und der ökonomischen Lage zu bieten ${ }^{284}$, werden die Ziele und Zwecke des Jahresabschlusses und seine Adressaten im Buchführungsgesetz von 1997 nicht explizit genannt.

Die mit dem Entwurf des Buchführungsgesetzes beauftragte KPL-Arbeitsgruppe 1995 erklärt in ihrem Bericht, dass die Ermittlung des ausschüttungsfähigen Gewinns weiterhin primäres Ziel des Jahresabschlusses sei. Sie stellt jedoch gleichzeitig klar, dass die Intention für den Gesetzesentwurf darin liege, die 4. und 7. EG-Richtlinie in nationales Recht zu transformieren, um „die den Jahresabschluss betreffenden Regelungen mit der europäischen Unternehmensgesetzgebung zu vereinen“'285. Es wird betont, dass vom primären Jahresabschlussziel der Gewinnermittlung künftig öfter als bisher zu Gunsten der anderen in der 4. EGRichtlinie genannten Ziele abzuweichen sei. Für den finnischen Einzelabschluss gelten die Ziele der 4. EG-Richtlinie, wie sie in der Präambel genannt werden. Neben der Vermittlung des True and Fair View handelt es sich hierbei um Gesellschafterschutz und Gläubigerschutz sowie um die Vergleichbarkeit und Gleichwertigkeit der Abschlüsse verschiedener Mitgliedstaaten ${ }^{286}$.

Die KPL-Arbeitsgruppe 1995 konkretisiert diese Ziele in ihrem Bericht über den Gesetzesentwurf des Buchführungsgesetzes von 1997. Betont wird die Entwicklung der internationalen Finanzmärkte: Es wird das Ziel der Informationsgewährung hinsichtlich der Einzelabschlüsse börsennotierter Unternehmen (und der Konzernabschlüsse) verfolgt ${ }^{287}$. Empirischen Studien zufolge treffen finnische Privatanleger ihre Investitionsentscheidungen selten auf der Grundlage von Jahresabschlüssen, somit kommen vor dem Hintergrund dieses Ziels als Adressaten überwiegend Finanzanalysten sowie (potenzielle) institutionelle Anleger in Betracht ${ }^{288}$. Das Ziel, anlegerorientiert zu informieren, ist ein Novum in der finnischen Rechnungslegung ${ }^{289}$.

\footnotetext{
Vgl. KPL 3:2.

Vgl. KPL-Arbeitsgruppe 1995, Teil I (1996), S. 17

Vgl. Präambel der 4. EG-Richtlinie und vgl. KPL-Arbeitsgruppe 1995, Teil I (1996), S. 19.

Vgl. KPL-Arbeitsgruppe 1995, Teil I (1996), S. 13-19.

Vgl. Majala (2001 b), S. 967, der sich auf eine Studie von Ikäheimo bezieht.

Vgl. HE 173/1997, Abschnitt 1.2.
} 
Als weiteres Ziel dieser auf internationale Kapitalmärkte ausgerichteten $\mathrm{Ab}$ schlüsse wird in der Regierungsvorlage an das finnische Parlament die Vergleichbarkeit zwischen finnischen Jahresabschlüssen und „internationalen Berichten“ genannt $^{290}$. Da Richtlinienkonformität angestrebt wird, sind unter dem Begriff „internationale Berichte“ die Abschlüsse von Unternehmen der EU-Mitgliedstaaten zu verstehen und nicht nach den Standards des IASB oder nach US-GAAP erstellte Abschlüsse. Die Gesetzesmaterialien berücksichtigen, dass Richtlinien und IAS in einigen Fällen übereinstimmen. Es wird aber ausdrücklich darauf hingewiesen, dass nur in den Fällen, in denen die Richtlinien Wahlrechte gestatten oder Regelungslücken enthalten, die Standards des IASB als Orientierungshilfe bei der Lösung von Einzelfallproblemen angewendet werden dürfen ${ }^{291}$.

Als Ziele des Jahresabschlusses gelten, teils finnischer Tradition, teils der 4. EGRichtlinie entsprechend,

- die Dokumentation des Unternehmensgeschehens,

- die Selbstinformation der Eigentümer zum Zwecke des Gesellschafterschutzes sowie die Bestimmung von Dividenden (Ausschüttungsbemessungsfunktion),

- die Information der Kreditoren zum Zwecke des Gläubigerschutzes und

- die Information der Steuerbehörden (Maßgeblichkeitsprinzip) ${ }^{292}$.

Während nach allgemeiner Auffassung das Hauptinteresse der Eigentümer und der Steuerbehörden auf die Gewinn- und Verlustrechnung gerichtet ist, dürften sich die Kreditoren auf die Bilanz konzentrieren, da die Gesellschaften Dritten eine Sicherheit nur durch ihr Gesellschaftsvermögen bieten. Der Schwerpunkt des finnischen Jahresabschlusses liegt - der Tradition entsprechend - auf der Gewinnund Verlustrechnung, auch wenn die Bilanz durch die Umsetzung der 4. EGRichtlinie an Bedeutung gewonnen hat ${ }^{293}$.

Traditionelle wirtschaftspolitische Ziele, die mit den Buchführungsgesetzen von 1945 und 1973 noch verfolgt wurden ${ }^{294}$, werden in der Gesetzesbegründung zum Buchführungsgesetz von 1997 nicht mehr erwähnt ${ }^{295}$.

290 Vgl. HE 173/1997, Abschnitt 1.2.

291 Vgl. KPL-Arbeitsgruppe 1995, Teil I (1996), S. 29.

292 Vgl. Artsberg (2000), S. 541 f., die diese Ziele und Zwecke nicht nur auf die Rechnungslegung Finnlands, sondern auch aller skandinavischen Staaten bezieht.

293 Vgl. Määttä (2000), S. 24.

294 Bei den traditionellen wirtschaftspolitischen Zielen handelt es sich um Innenfinanzierung, Investitionsförderung und den Ausgleich von Wirtschafts- und Branchenzyklen. Vgl. Kap. 2.1.2.2 bis 2.1.3.2 dieser Arbeit.

295 Vgl. HE 173/1997 und vgl. Artsberg (2000), S. 541 f. 
Folgende Graphik zeigt die Veränderung der Gewichtung von Zielen und Zwecken, die mit den Buchführungsgesetzen im Zeitablauf verfolgt wurden:

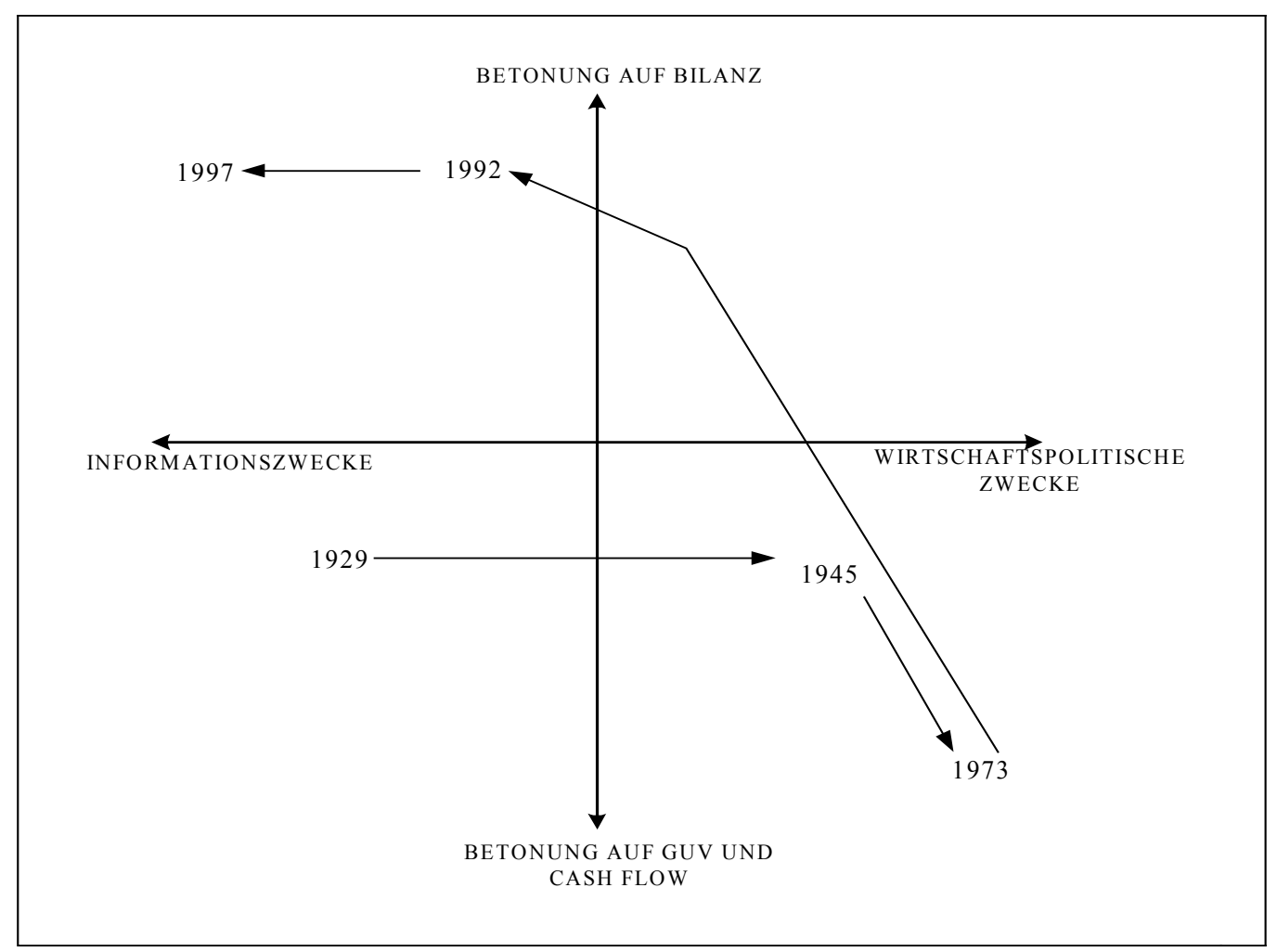

Abb. 5: Veränderung finnischer Jahresabschlusszwecke im Zeitablauf ${ }^{296}$

\subsubsection{Instrumente der finnischen Rechnungslegung}

\subsubsection{Bestandteile des Jahresabschlusses und ihre Aufgaben}

Der Jahresabschluss besteht nach Art. 2 Abs. 1 der 4. EG-Richtslinie aus der Bilanz, der Gewinn- und Verlustrechnung sowie dem Anhang, die eine Einheit bilden. Er ist klar und übersichtlich aufzustellen ${ }^{297}$.

Nach KPL 3:1.1 umfasst der finnische Jahresabschluss neben Gewinn- und Verlustrechnung, Bilanz und Anhang auch den Lagebericht. Diese Bestandteile des Jahresabschlusses sind zu veröffentlichen. Vollständig ist der Jahresabschluss erst, wenn auch die nicht veröffentlichungspflichtige Aufgliederung der Bilanzposten und des Anhangs erstellt und der Jahresabschluss unterschrieben ist. Anders als nach den Vorschriften der 4. EG-Richtlinie ist der Lagebericht in Finnland für alle Buchführungspflichtigen Bestandteil des Jahresabschlusses und unterliegt somit hinsichtlich der Vermittlung des True and Fair View, der Offenle- 
gungspflicht und der Wirtschaftsprüfung denselben Anforderungen wie die anderen Jahresabschluss-Bestandteile ${ }^{298}$.

In KPL 3:1.1 definiert der finnische Gesetzgeber die Bestandteile des Jahresabschlusses, indem er deren Aufgaben nennt:

Die Gewinn- und Verlustrechnung bildet die Zusammensetzung des Ergebnisses $\mathrm{ab}^{299}$. Die Bilanz zeigt die wirtschaftliche Lage am Stichtag auf ${ }^{300}$. Der Anhang enthält grundsätzliche Informationen zur Gewinn- und Verlustrechnung und zur Bilanz $^{301}$.

Der Lagebericht informiert über die Entwicklung der wichtigsten Angelegenheiten, welche die Tätigkeit des Buchführungspflichtigen betreffen. Er hat demnach Informationen über Sachverhalte und Tatbestände mit Einfluss auf den True and Fair View zum Inhalt, außerdem wichtige Ereignisse des Geschäftsjahres, die voraussichtliche Entwicklung des Unternehmens sowie den Bereich Forschung \& Entwicklung. Private wie öffentliche Aktiengesellschaften müssen gemäß OYL 11:9, 9a zusätzlich im Lagebericht über Veränderungen in der Konzernstruktur und Eigenkapitalstruktur informieren und den Gewinnverwendungsvorschlag des Vorstands nennen.

Der 4. EG-Richtlinie entsprechend, haben die Jahresabschlussunterlagen nach KPL 3:1.3 „klar“ zu sein. Gewinn- und Verlustrechnung, Bilanz, Anhang und Lagebericht sind eigenständige Teile des Jahresabschlusses, die jeder für sich korrekt erstellt sein müssen und den Leser nicht irreführen dürfen. So darf beispielsweise der Anhang nicht eventuell in Gewinn- und Verlustrechnung oder Bilanz enthaltene Fehler korrigieren oder im Lagebericht getroffene Falschaussagen verbessern $^{302}$. Eine alternative Darstellung von Sachverhalten in Gewinn- und Verlustrechnung, Bilanz oder Anhang ist nur dort erlaubt, wo Gesetz oder Verord-

298 Dies gilt seit dem Buchführungsgesetz von 1997.

299 Vgl. KPL 3:1.1,1. Zulässige Gliederungsschemata der Gewinn- und Verlustrechnung sind in KPA 1:1-5 geregelt, sie finden sich im Anhang dieser Arbeit. S. auch Fußnote 300 dieser Arbeit.

300 Vgl. KPL 3:1.1,2. Bilanzschemata sind in KPA 1:6-7 enthalten. Sie finden sich im Anhang dieser Arbeit. Die in der Buchführungsverordnung genannten Gliederungsschemata von Gewinn- und Verlustrechnung sowie Bilanz beruhen auf Art. 23 und 25 sowie Art. 9 der 4. EGRichtlinie und sind verbindlich von allen Buchführungspflichtigen anzuwenden (abweichend gelten rechtsform- und größenspezifische (vereinfachende) Regeln). Der KILA darf keine Abweichung von diesen Schemata erlauben (KPL 8:2.2). Gewinn- und Verlustrechnung und Bilanz müssen nach KPL 3:1.2 neben den Zahlen des Geschäftsjahres die Vergleichszahlen des Vorjahrs beinhalten. Bei Änderungen in der Darstellungsweise sind die Vorjahreszahlen möglichst anzupassen.

Vgl. KPL 3:1.1,3. Anhangangaben sind in KPL 3:2, den rechtsform- und branchenspezifischen Spezialgesetzen, KPA 2:2-11, den Entscheidungen des Ministeriums für Handel und Industrie und des Finanzministeriums sowie in den KILA-Verlautbarungen geregelt. 
nung es ausdrücklich gestatten ${ }^{303}$. Wenn die Vermittlung des True and Fair View es erfordert, können einzelne Gewinn- und Verlustrechnungs- oder Bilanzposten der Klarheit wegen zusammengefasst oder saldiert werden. Eine Aufgliederung dieser Posten ist dann im Anhang anzugeben ${ }^{304}$. Für den Fall, dass Gewinn- und Verlustrechnung und Bilanz nicht ausreichen, um einen True and Fair View zu vermitteln, sind Zusatzangaben im Anhang zwingend erforderlich ${ }^{305}$.

Der Jahresabschluss ist in finnischer oder schwedischer Sprache zu erstellen. Er muss in Euro aufgestellt werden. Wird zusätzlich in ausländischen Währungen informiert, ist der angewendete Umrechnungskurs zu nennen ${ }^{306}$.

\subsubsection{Weitere Rechnungslegungsinstrumente}

Öffentliche Aktiengesellschaften - und, sofern sie bestimmte Größenkriterien ${ }^{307}$ überschreiten, auch private Aktiengesellschaften - haben die Pflicht, eine Kapitalflussrechnung zu erstellen und zu veröffentlichen, die sich nach KILA-Anweisung im Aufbau und in der Darstellungsart an IAS 7 (revised 1992) orientiert ${ }^{308}$. Auch der Berufsverband der KHT-Wirtschaftsprüfer basiert seine Empfehlung zum Aufbau einer Kapitalflussrechnung auf IAS 7.

Börsennotierte, öffentliche Aktiengesellschaften unterliegen den strengen Publizitätspflichten des Wertpapiermarktgesetzes hinsichtlich der Zwischenberichterstattung, der Vorabmitteilung über den Jahresabschluss und den Veröffentlichungsfristen für den Jahresabschluss ${ }^{309}$. Außerdem müssen börsennotierte Aktiengesellschaften nach allgemeingültiger Anweisung vom KILA bestimmte Gewinn- und Verlustrechnungs- sowie Bilanzkennzahlen, aktienbezogene Kennzahlen und Kennzahlensysteme angeben ${ }^{310}$.

Von den meisten Unternehmen wird jährlich auf freiwilliger Basis ein Geschäftsbericht erstellt, der die Tätigkeit des Unternehmens, seine Entwicklung, Zukunftspläne, die Unternehmensorganisation, Aufgaben einzelner Abteilungen u. Ä. zum Inhalt hat. Wenn Bestandteile des Jahresabschlusses im Geschäftsbericht abge-

303 Dies ist z. B. in OYL 11:6a (Anzahl eigener Aktien), 11:7 (Angabe von eingegangenen Haftungsverhältnissen zu Gunsten der Führungsorgane) oder 11:8 (Meldung der Eigenkapitalstruktur) der Fall.

304 Vgl. KPA 1:10 und vgl. KILA-Anweisung „Darstellung von GuV und Bilanz“.

305 Vgl. KPL 3:2.1 und vgl. Art. 2 Abs. 4 der 4. EG-Richtlinie.

306 Vgl. KPL 3:5.

307 Größenkriterien gem. KPL 3:9.2, von denen mindestens zwei überschritten werden: Umsatzerlös $€ 3,4$ Mio., Bilanzsumme $€ 1$ 1,7 Mio. oder 50 Mitarbeiter.

308 Vgl. KILA-Anweisung „Kapitalflussrechnung“.

309 Vgl. Kap. 2.2.2.2 dieser Arbeit.

310 Vgl. KILA-Anweisung „Erstellung von Jahresabschluss, vorläufigem Abschluss und Zwischenberichten". 
druckt werden, sind diese als Ganzheit wiederzugeben und deutlich von den freiwillig gemachten Angaben abzuheben.

Überwiegend international tätige sowie große Unternehmen veröffentlichen zusätzlich Umweltberichte ${ }^{311}$, Personalbilanzen und Sozialbilanzen ${ }^{312}$. Auch werden „duale Abschlüsse“ aufgestellt, die sowohl finnische als auch IAS-Normen erfüllen sollen. Hier werden Wahlrechte des finnischen Buchführungsgesetzes in Übereinstimmung mit IAS-Grundsätzen ausgeübt ${ }^{313}$. Dies ist kritisch zu sehen, da einige Standards des IASB nicht mit dem finnischen Buchführungsgesetz vereinbar $\operatorname{sind}^{314}$.

\subsubsection{Anerkannte Grundsätze ordnungsmäßiger Buchführung}

\subsubsection{Die Art guter Buchführung und ihre Ermittlung}

Die Buchführung dient der systematischen Erfassung der Geschäftsvorfälle und liefert somit die Ausgangsdaten für den zu erstellenden Jahresabschluss ${ }^{315}$.

Es ist dem Gesetzgeber unmöglich, sämtliche auftretenden Buchführungs- und Rechnungslegungsprobleme durch kodifizierte Regelungen abzudecken ${ }^{316}$. Zudem unterliegen die auftretenden Probleme und die sie betreffenden Lösungskonzepte einem permanenten Wandel, die der Gesetzgeber nicht immer vorhersehen oder zeitgenau berücksichtigen $\mathrm{kann}^{317}$. Um durch allgemein gehaltene Formulierung möglichst viele Tatbestände zu erfassen, hat der finnische Gesetzgeber das allgemeine und unbestimmte Normierungskonzept der Art guter Buchführung ${ }^{318}$ geschaffen, das in KPL 1:3 enthalten ist ${ }^{319}$ :

311 Vgl. Nieminen und Niskanen (1998) und vgl. Mätäsaho (1999).

312 Vgl. Artsberg (2000), S. 548-549.

313 Eine Umfrage vom KHT-Wirtschaftsprüferverband im Jahr 1995 ergab, dass von 74 befragten Unternehmen sieben einen dualen Abschluss für das Geschäftsjahr 1994 erstellt hatten (drei davon wiesen eine Überleitung des Jahresüberschusses von finnischen Vorschriften zu IAS-Regeln aus, vier eine Überleitung des Eigenkapitals). Neun weitere Unternehmen hatten zusätzlich zum finnischen Abschluss einen gesonderten IAS-Abschluss erstellt; vier Jahre zuvor, 1990, waren es noch 33. Aus dem Vergleich der Jahre 1990 zu 1994 kann gefolgert werden, dass nach Einführung des Buchführungsgesetzes von 1992 in der Praxis weitgehend die Meinung vertreten wurde, dass finnische und IAS-Abschlüsse derart kompatibel seien, dass auf die Erstellung von dualen und gesonderten IAS-Abschlüssen verzichtet werden könne. Vgl. KHT-yhdistys (1995), S. 97-98.

314 Zur Problematik der Vermittlung des True and Fair View bei dualen Abschlüssen vgl. Kap. 3.1.3.4.2 dieser Arbeit. Duale Abschlüsse, die gleichzeitig finnische Vorschriften und US-GAAP erfüllen sollen, werden nur sehr vereinzelt erstellt. Diese Abschlüsse sind jedoch ähnlich kritisch wie duale Abschlüsse finnischer Normen und IASB-Standards zu sehen. Vgl. Baetge, Kirsch und Thiele (2001), S. 2-3.

316 Vgl. Baetge, Kirsch und Thiele (2001), S. 92.

317 Vgl. Baetge, Kirsch und Thiele (2001), S. 93.

318 Die Art guter Buchführung steht in vorliegender Arbeit für den finnischen Ausdruck ,hyvä kirjanpitotapa“. Der Begriff „hyvä kirjanpitotapa“ wird in den Fachwörterbüchern teilweise 
Der Buchführungspflichtige hat die Art guter Buchführung zu befolgen.

Es gilt, den unbestimmten Rechtsbegriff „Art guter Buchführung“ zu konkretisieren. In der Regierungsvorlage zum Buchführungsgesetz von 1997 wird festgelegt, dass die Art guter Buchführung ihren Inhalt aus der Buchführungspraxis und theorie erhält ${ }^{320}$. Sie zu befolgen heißt nach allgemeiner Auffassung, in der Buchführung

- die Gesetzgebung,

- die zugehörigen Gesetzesmaterialien,

- allgemeingültige Anweisungen, Gutachten, Entscheidungen und Erlaubnisse des KILA,

- die Grundsätze ordnungsmäßiger Buchführung (GoB) sowie darüber hinaus

- Stellungnahmen der wirtschaftsprüfenden und anderer mit der Rechnungslegung befassten Berufsstände sowie der Börse

zu befolgen ${ }^{321}$.

Auf Grund ihrer Stellung in Kap. 1 des Buchführungsgesetzes, den allgemeingültigen Regeln, ist die Art guter Buchführung auf das gesamte Gesetz zu beziehen. Sie soll einerseits Hilfestellung bei der Gesetzesauslegung geben. Andererseits soll sie die Einzelvorschriften ergänzen und als Richtschnur für die Weiterentwicklung der Rechnungslegung dienen ${ }^{322}$. Diese Aufgabe kommt maßgeblich den GoB zu, die im Folgenden ausführlich beschrieben werden.

Da die GoB einem ständigen Entwicklungsprozess unterworfen sind, hat der finnische Gesetzgeber vor 1997 bewusst darauf verzichtet, einzelne GoB in das kodifizierte Recht aufzunehmen oder zu definieren ${ }^{323}$. Er wurde aber 1993 im Vorfeld des EU-Beitritts von der EU-Kommission dazu aufgefordert ${ }^{324}$, die allgemeinen Bilanzierungs- und Bewertungsgrundsätze des Art. 31 Abs. 2 der 4. EG-Richtlinie explizit ins Buchführungsgesetz aufzunehmen ${ }^{325}$. Dies ist in zentraler Stellung in Kap. 1 des KPL geschehen. Die kodifizierten GoB sind daher auf alle Elemente der Rechnungslegung zu beziehen. Neben der Buchführung, aus auch mit „Buchführungsanstand“ oder „Grundsätzen ordnungsmäßiger Buchführung“ (GoB) übersetzt. Der Begriff GoB soll hier, um Missverständnissen vorzubeugen, nicht verwendet werden, da das Konzept der Art guter Buchführung umfassender ist als die GoB nach deutschem Handelsrecht.

319 Übersetzung KPL 1:3 ins Deutsche durch die Verfasserin.

320 Vgl. HE $173 / 1997$ vp., S. 8.

321 Vgl. Järvinen et al. (2000), S. 49, vgl. Leppiniemi (2000 a), S. 18 und vgl. KPLArbeitsgruppe 1995, Teil I (1996), S. 36-37.

322 Vgl. Järvinen et al. (2000), S. 49.

323 Vgl. KPL-Arbeitsgruppe 1995, Teil I (1996) S. 13.

324 Vgl. KPL-Arbeitsgruppe 1995, Teil I (1996) S. 13 f. und (Teil II), S. 59-60.

325 Bei diesen Grundsätzen handelt es sich um den Grundsatz der Fortführung der Unternehmenstätigkeit, den Grundsatz der Stetigkeit, den Grundsatz der Vorsicht, das Realisationsprinzip, das Imparitätsprinzip, den Grundsatz der leistungsabhängigen Buchung, den Grundsatz der Einzelbewertung und den Grundsatz der Bilanzkontinuität. 
der Rechnungslegung zu beziehen. Neben der Buchführung, aus der Bilanz sowie Gewinn- und Verlustrechnung generiert werden, sind sie auch auf den Lagebericht anzuwenden. Die allgemeinen Bilanzierungs- und Bewertungsgrundsätze gehörten auch in Finnland zu den GoB, waren ohne gesetzliche Fixierung verbindliche Rechtsnorm. Da sie nach Aufnahme in das kodifizierte Recht nicht näher durch den Gesetzgeber definiert wurden, konnte die Flexibilität der GoB trotz Kodifizierung beibehalten werden. Der Inhalt dieser Grundsätze kann sich nach wie vor wandeln ${ }^{326}$.

Die Konkretisierung der unbestimmten Rechtsbegriffe „Art guter Buchführung“ und „GoB“ erfordert somit die Ermittlung von GoB. Nur mithilfe konkretisierter GoB kann der Buchführungspflichtige die gesetzliche Anforderung, die Art guter Buchführung zu befolgen, erfüllen ${ }^{327}$. Trotz der gewünschten Weiterentwicklung, der Dynamik der Rechnungslegungsnormierung, autorisieren die GoB die Buchführungspraktiker nicht zu Verstößen gegen rechtliche Regelungen. Die GoB sind vielmehr deduktiv zu ermitteln ${ }^{328}$. Bilanzierungspraxis, Wissenschaft und Rechtsprechung müssen ausgehend von den buchführungsrechtlichen Jahresabschlusszwecken bei der Gewinnung der GoB einen gesetzesentsprechenden Konsens oder Kompromiss erzielen $^{329}$. Die Bilanzierungspraxis wird von vielen Agierenden gestaltet. Die zentrale Rolle bei der Entwicklung der GoB nimmt der KILA ein ${ }^{330}$.

Von der Reaktionsgeschwindigkeit des KILA und seiner Sensibilität für zukünftige Entwicklungen hängt es ab, auf welche Weise die Buchführungspflichtigen die GoB einhalten. Können sie neue Entwicklungen schon bei der Erstellung eines Jahresabschlusses berücksichtigen oder erfahren sie erst danach, wie sie hätten handeln sollen ${ }^{331}$ ? Neben den trendbestimmenden Unternehmen ändern auch viele

326 Vgl. Järvinen et al. (2000), S. 324. Auf die seit dem 31. Dezember 1997 als allgemeine Bilanzierungs- und Bewertungsgrundsätze kodifizierten GoB sowie ihre Beziehung zu den anderen GoB und dem True and Fair View wird in Kap. 3.1.3.2 und 3.1.3.4.3 dieser Arbeit eingegangen.

In der Theorie existieren diverse Systeme, die zur Konkretisierung von GoB angewendet werden könnten. Hierzu zählen die v. a. in Deutschland bekannten Systeme von Leffson, Moxter und Baetge (vgl. z. B. Ballwieser, Wolfgang (1987), S. 3-24). In Finnland sind aus den Jahresabschlusszwecken des Buchführungsgesetzes abgeleitete GoB-Systeme nicht bekannt; GoB-Systeme finden in Finnland keine Anwendung. Zur deduktiven und induktiven Ermittlung von GoB vgl. Fußnote 107 und Kap. 2.2.1.1 dieser Arbeit. Vgl. Järvinen et al. (2000), S. 49 f. Der KILA kann neue Entwicklungen durch Veröffentlichen von Allgemeinen Anweisungen, Gutachten oder Entscheidungen beschleunigen und in geregelte Bahnen lenken. Vgl. KPLArbeitsgruppe 1995, Teil I (1996), S. 27; vgl. Järvinen et al. (2000), S. 54 f. und vgl. Artsberg (2000), S. 534-538 sowie Kap. 2.2.1.2 dieser Arbeit.

331 Mit Hinsicht auf Stetigkeit, Vergleichbarkeit und Rechtssicherheit hat LEPPINIEMI, heutiger Sprecher des KILA, 1993 die Meinung geäußert, der KILA solle aktiv GoB entwickeln und bei bereits getroffenen Bilanzierungsentscheidungen als Richter fungieren. Er hat außerdem einen möglichst hohen Konkretisierungsgrad der vom KILA erteilten Anweisungen gefordert, da eine zu große Abstraktion die Gefahr unterschiedlicher Interpretation durch Buch- 
andere Unternehmen ihre Rechnungslegung erst dann, wenn eine Stellungnahme vom KILA vorliegt ${ }^{332}$.

Auch die Wertpapierbörse und die mit Buchführung und Rechnungslegungspraxis befassten Berufsstände - v. a. die Wirtschaftsprüfer - nehmen Einfluss auf die GoB, wenn sie eigene Stellungnahmen zu bestimmten Sachverhalten veröffentlichen und von ihren jeweiligen Berufsangehörigen die Beachtung derselben for$\operatorname{dern}^{333}$.

Die von den Großunternehmen praktizierte Bilanzierung wird regelmäßig deutlich, wenn der Berufsverband der KHT-Wirtschaftsprüfer eine Umfrage zur Rechnungslegung der Unternehmen namens „Rechnungslegungspraxis in Finnland“6334 durchführt und die Ergebnisse veröffentlicht ${ }^{335}$.

In Finnland liegen die Quellen für die Ermittlung der GoB in erster Linie in der Gesetzgebung einschließlich der Grundsätze der 4. EG-Richtlinie und in den Standards des IASB ${ }^{336}$. Ministerien, Behörden, der KILA, die Börse und die mit der Rechnungslegung befassten Berufsstände, welche Empfehlungen aussprechen, haben die diesen Quellen zu Grunde liegende Intention bei der Ermittlung der GoB zu beachten. Die den Jahresabschluss erstellenden Unternehmen minimieren ihr Risiko, wenn sie das bereits vorhandene Buchführungsregelwerk befolgen und sich in Zweifelsfragen an den KILA richten ${ }^{337}$.

Auf Grund der erforderlichen Konsensbildung sind die GoB und mit ihnen die Art guter Buchführung nicht unbedingt logisch ableitbar.

Die Gesetzesmaterialien verweisen darauf, dass der Inhalt der Art guter Buchführung neben der Buchführungspraxis, die sich im Wesentlichen im Verständnis der GoB widerspiegelt, auch aus der Buchführungstheorie zu konkretisieren sei ${ }^{338}$. Offen bleibt, um welche Theorie es sich konkret handelt.

führungspflichtige berge und in einem solchen Fall die GoB unterschiedlich angewendet würden. Vgl. Väyrynen (1993), S. 8, im Gespräch mit Leppiniemi.

332 Vgl. Artsberg (2000), S. 534-538, die ihre Ausführungen auf alle nordischen Staaten, nicht speziell auf Finnland, bezieht und vgl. Järvinen et al. (2000), S. $54 \mathrm{f}$.

333 Vgl. KPL-Arbeitsgruppe 1995, Teil I (1996), S. 36 f. und vgl. Järvinen et al. (2000), S. 55.

334 Der Titel des finnischen Originals lautet „Tilinpäätöskäytäntö Suomessa“. Übersetzung ins Deutsche durch die Verfasserin.

335 Zuletzt wurde eine solche Untersuchung 1995 für das Geschäftsjahr 1994 durchgeführt. Vgl. KHT-yhdistys (1995).

336 Vgl. KPL-Arbeitsgruppe 1995, Teil I (1996), S. 35-37, vgl. Järvinen et al. (2000), S. 52 f. und vgl. Sorsa (1998 a), S. 163.

337 Vgl. Leppiniemi und Leppiniemi (1997), S. 21.

338 Vgl. HE 173/1997, S. 8. 
Nach der Regierungsvorlage HE $111 / 1992^{339}$ und der Auffassung der KPL-Arbeitsgruppe 1995 ist die Ausgaben-Einnahmen-Theorie SAARIOs nach wie vor die zentrale Erklärungs- und Ausgangsbasis der Buchführung. Von dieser sei allerdings, so die Arbeitsgruppe, künftig zu Gunsten anderer Grundsätze und der in der 4. EG-Richtlinie genannten Jahresabschlusszwecke abzuweichen ${ }^{340}$.

Es herrscht in Finnland Einigkeit darüber, dass die Ausgaben-Einnahmen-Theorie durch die Implementierung der 4. EG-Richtlinie weitgehend an Bedeutung verloren hat ${ }^{341}$. Die Ausgaben-Einnahmen-Theorie sei dennoch als umfassendste Buchführungstheorie immer noch gültig für Analyse und Interpretation von Buchführungsregel ${ }^{342}$. Da mithilfe einer Theorie irreführende und in sich widersprüchliche Normen überhaupt erst wahrgenommen würden, könne die Theorie immer noch die Art guter Buchführung beeinflussen. In Zeiten des Umbruchs nach Umsetzung der Richtlinie fordern v. a. Praktiker eine Auslegungsbasis ${ }^{343}$.

$\mathrm{Zu}$ prüfen ist, ob die Anwendung einer Buchführungstheorie richtlinienkonform sein kann, da sich die 4. EG-Richtlinie selbst nicht aus einer Theorie ableitet. Die Richtlinie bezieht sich vielmehr auf allgemeine, der Buchführungspraxis entstammende Bilanzierungs- und Bewertungsgrundsätze ${ }^{344}$.

Die 4. EG-Richtlinie konzentriert sich vorrangig auf die Erstellung des Jahresabschlusses und nicht auf die Buchführung. Ein Jahresabschluss, der auf einer theoriegeprägten Buchführung beruht, muss nicht automatisch den Grundsätzen der Richtlinie widersprechen, sofern bei der Erstellung des Jahresabschlusses die Regeln der Richtlinie eingehalten werden. Angesichts der Fülle und der Qualität der Quellen zur Ermittlung der Art guter Buchführung kann im Problemfall regelmäBig eine Lösung gefunden werden, bevor die Theorie zur Anwendung kommt.

Dennoch kann nicht ausgeschlossen werden, dass die Ausgaben-EinnahmenTheorie auch künftig berücksichtigt wird. Zu beachten ist zudem, dass in der Vergangenheit vom KILA erteilte, auf der Ausgaben-Einnahmen-Theorie beruhende Anweisungen auch heute noch ihre Gültigkeit behalten, sofern sie nicht im Widerspruch zur aktuellen Rechtslage stehen ${ }^{345}$.

339 Die HE 111/1992 ist in der Vorlage HE 173/1997 zum derzeit gültigen Buchführungsgesetz in diesem Punkt nicht korrigiert worden, behält demnach weiterhin Gültigkeit.

340 Vgl. KPL-Arbeitsgruppe 1995, Teil I (1996), S. 17

341 Vgl. Majala (2001 b), S. 964 und vgl. Järvinen et al. (2000), S. 50.

342 Vgl. Järvinen et al. (2000), S. 50 f. und vgl. Sorsa (1998 a), S. 163.

343 Vgl. Lappalainen (1999), S. 104.

344 Vgl. hierzu Majala (2001 b), S. 964, der die Auffassung vertritt, die Richtlinie würde eine Buchführungstheorie verbieten.

345 Vgl. Järvinen et al. (2000), S. 51 und vgl. Majala (2001 b), S. 965. 
Verstöße gegen die Art guter Buchführung können strafrechtliche, handelsrechtliche, gesellschaftsrechtliche und steuerrechtliche Konsequenzen zur Folge haben ${ }^{346}$.

\subsubsection{Kodifizierte Grundsätze ordnungsmäßiger Buchführung}

Die EU-Kommission hat 1993 den finnischen Gesetzgeber dazu aufgefordert, die in Art. 31 der 4. EG-Richtlinie genannten Bewertungs- und Bilanzierungsgrundsätze explizit in nationales Recht aufzunehmen. Sie hat dies mit dem Richtlinienziel der verbesserten Vergleichbarkeit und der Umsetzung des Art. 31 in den anderen Mitgliedstaaten der Europäischen Union begründet ${ }^{347}$.

Da ein Großteil der international anerkannten Bilanzierungs- und Bewertungsgrundsätze bereits seinerzeit Bestandteil der finnischen Buchführungspraxis gewesen ist, wird ihre Kodifizierung als Überregulierung beurteilt, die keine wirkliche Änderung der Rechtslage zur Folge hat ${ }^{348}$. Aus diesem Grund hat die mit dem Entwurf des Buchführungsgesetzes von 1997 beauftragte KPL-Arbeitsgruppe 1995 vorgeschlagen, nur diejenigen GoB zu kodifizieren, die in Art. 31 genannt werden. Außerdem sollte die Aufnahme der GoB nach Auffassung der KPLArbeitsgruppe 1995 nicht in Kap. 1 „Allgemeingültige Regeln“, sondern in Kap. 3

346 Nach finnischem Strafgesetzbuch RL 30:9 und 30:10 können Buchführungspflichtige oder ihre gesetzlichen Vertreter mit einer Geldstrafe oder Haft (bei Fahrlässigkeit bis zu zwei Jahre, bei vorsätzlichem Handeln bis zu drei Jahre) bestraft werden, wenn sie die Buchung von Geschäftsvorfällen oder die Erstellung des Jahresabschlusses ganz oder teilweise unterlassen, wenn sie in der Buchführung falsche oder irreführende Informationen vermerken oder wenn sie Buchführungsunterlagen nicht archivieren, sie verbergen oder beschädigen und so wesentlich den True and Fair View der Ertrags- oder ökonomischen Lage des Unternehmens beeinträchtigen.

Handelsrechtlich liegt ein Verstoß gegen Buchführungsvorschriften nach KPL 8:4 dann vor, wenn die Fristen zur Buchung von Geschäftsvorfällen nach KPL 2:4.2 nicht eingehalten werden, wenn die Aufbewahrungspflichten des KPL 2:10 verletzt werden, wenn Jahresabschluss oder Konzernabschluss so erstellt werden, dass sie keinen True and Fair View im Sinne des KPL 3:2 abbilden, oder wenn den Offenlegungspflichten nicht nachgekommen wird. Sind die Verstöße nicht wesentlich, werden sie gerichtlich nicht verfolgt; andernfalls wird im Einzelfall durch das zuständige Gericht über das Strafmaß entschieden.

Verstöße gegen Buchführungsregeln, die sich auf steuerliche Sachverhalte auswirken, können Geld- oder Haftstrafen nach sich ziehen (vgl. RL 17:8 i. V. m. den jeweils relevanten Steuergesetzen, z. B. verohallintoL 17, verotusmenettelyL 86, ennakkoperintöL 54 und 55, perintö- ja lahjaveroL 58 und varainsiirtoveroL 54).

347 Vgl. KPL-Arbeitsgruppe 1995, Teil I (1996), S. 34. Auch VAN HULLE betonte die Notwendigkeit der Umsetzung von Art. 31: „In the area of valuation, there is much to say in favour of an explicit inclusion of the general valuation principles (going concern, prudence,...) in the law. Even if these principles belong to general accounting practice, they may not be legally enforceable unless they are included in the law." Van Hulle (1994), S. 235.

Da die GoB in den verschiedenen Mitgliedstaaten der EU durchaus unterschiedlich interpretiert und gewichtet werden können, sollen Verständnis und Anwendung der kodifizierten GoB in Kap. 3.1.3.2 und der nicht kodifizierten GoB in Kap. 3.1.3.3 der vorliegenden Arbeit dargestellt werden. Wegen seiner besonderen Bedeutung wird das True and Fair ViewKonzept in Kap. 3.1.3.4 diskutiert. 
„Jahresabschluss“ erfolgen, da die 4. EG-Richtlinie primär auf den Jahresabschluss und nicht auf die Buchführung zu beziehen sei ${ }^{349}$.

Der finnische Gesetzgeber hat Art. 31 mit folgendem Wortlaut in KPL 3:3 implementiert $^{350}$ :

Allgemeine Jahresabschlussgrundsätze

(1) $\mathrm{Zu}$ den beim Erstellen des Jahresabschlusses und bei Eröffnungsbuchungen anzuwendenden Grundsätzen gehören:

- die Annahme über die Fortführung der Unternehmenstätigkeit;

- die Anwendung von Bilanzierungsgrundsätzen und -methoden von einem Geschäftsjahr zum nächsten;

- vom Ergebnis des Geschäftsjahrs unabhängige Vorsicht;

- die Eröffnungsbilanz beruht auf der Schlussbilanz des vorherigen Geschäftsjahres;

- Einbeziehung von zum Wirtschaftsjahr gehörenden Erträgen und Aufwendungen unabhängig vom Tag der zugehörigen Zahlungen; sowie

- Einzelbewertung von Erzeugnissen und Waren und anderen in der Bilanz zu vermerkenden Posten.

(2) Die in Abs. 1 Nr. 3 gemeinte Vorsicht setzt insbesondere voraus, dass im Jahresabschluss berücksichtigt werden:

- nur im Geschäftsjahr bewahrheitete Gewinne; sowie

- alle Abschreibungen und Wertminderungen der Aktiva sowie der Anstieg der Verbindlichkeiten ebenso wie alle dem zu Ende gehenden Geschäftsjahr oder früheren Geschäftsjahren zuzuordnende, vorhersehbare Risiken und mögliche Schäden, auch wenn sie erst nach dem Abschluss-Stichtag bekannt werden.

(3) Von den in Abs. 1 und Abs. 2 genannten Grundsätzen darf nur aus besonderen Gründen abgewichen werden, sofern ein Abweichen nicht auf gesetzlichen Vorschriften oder aus auf diesen gesetzlichen Vorschriften entstammenden Regelungen beruht. Im Anhang ist eine Erläuterung zum Grund der Abweichung und eine Berechnung der Ergebnisauswirkung und des Einflusses auf die wirtschaftliche Lage anzugeben.

Nach Auffassung der KPL-Arbeitsgruppe 1995 spielt die Reihenfolge, in der die Grundsätze genannt werden, keine Rolle für ihre Gewichtung ${ }^{351}$. Während die KPL-Arbeitsgruppe 1995 darüber hinaus die Meinung vertritt, dass kodifizierte und nicht kodifizierte GoB als gleichwertig anzusehen seien ${ }^{352}$, wird in der Literatur die Auffassung vertreten, dass im Falle eines Widerspruchs zwischen kodifizierten und nicht kodifizierten GoB den kodifizierten GoB der Vorrang zu geben sei. Dies sei auch Ziel der 4. EG-Richtlinie, die mit der Kodifizierung bestimmter GoB eine Stärkung eben dieser GoB erreichen wolle ${ }^{353}$. 
$\mathrm{Zu}$ prüfen ist, welcher Auffassung zu folgen ist. Der Richtliniengeber stuft kodifizierte GoB untereinander sowie kodifizierte und nicht kodifizierte GoB hierarchisch nicht $\mathrm{ab}$, bildet somit keine Rangordnung zwischen ihnen ${ }^{354}$. Die Ausgewogenheit zwischen den Grundsätzen ist erforderlich, weil der Richtliniengeber nicht bestimmte Interessen von Adressaten einseitig berücksichtigen will. Sein Ziel ist vielmehr, einen Ausgleich zwischen den verschiedenen am Jahresabschluss interessierten Gruppen derart zu schaffen, dass der Jahresabschluss einem angemessenen Schutz aller Adressaten dienen soll ${ }^{355}$. Dennoch wird die Bedeutung des Vorsichtsprinzips betont, was aus den Gesetzesmaterialien und dem Umstand folgt, dass dieses Prinzip als Einziges konkretisiert wird ${ }^{356}$.

Der finnische Gesetzgeber hat als einzigen Bilanzierungs- und Bewertungsgrundsatz das Vorsichtsprinzip präzisiert ${ }^{357}$, welches durch das Realisationsprinzip, das Imparitätsprinzip und das Wertaufhellungsprinzip konkretisiert wird ${ }^{358}$. Die Auslegung sämtlicher Prinzipien erfolgt durch die Buchführungspraxis, Berufsstände und den KILA, welcher bereits Gutachten bezüglich der Jahresabschlussgrundsätze erstellt hat ${ }^{359}$.

Im finnischen Fachschrifttum besteht Einigkeit darüber, wie die einzelnen Grundsätze zu interpretieren sind. Die KPL-Arbeitsgruppe 1995 hat in ihrem Bericht die allgemeinen Bilanzierungsregeln kurz definiert.

Der Grundsatz der Fortführung der Unternehmenstätigkeit - an sich in der 4. EGRichtlinie nicht definiert ${ }^{360}$ - wird von der KPL-Arbeitsgruppe 1995 wie folgt aufgefasst $^{361}$ :

Grundsatz der Fortführung der Unternehmenstätigkeit (Going Concern)

Es wird angenommen, dass das Unternehmen seine Tätigkeit für die gesamte denkbare Zukunft fortsetzen wird.

354 Vgl. van Hulle und van der Tas (2001), S. 820.

355 Vgl. Baetge, Kirsch und Thiele (2001), S. 90-92.

356 Vgl. van Hulle und van der Tas (2001), S. 820.

357 Daneben hat der finnische Gesetzgeber in KPL 2:6 einen die Organisation der Buchführung betreffenden Grundsatz definiert, den Grundsatz der Nachprüfbarkeit (,kirjausketju“, Audit trail): „Geschäftsvorfälle sind so in der Buchführung zu vermerken, dass der Weg der Buchung von Beleg in die Neben- und die Hauptbuchhaltung sowie von der Hauptbuchhaltung in die Gewinn- und Verlustrechnung und Bilanz nach KPL 3:1 problemlos nachgewiesen werden kann." (Übersetzung ins Deutsche durch die Verfasserin). Vgl. auch Kap. 3.1.4 dieser Arbeit.

358 Vgl. Pärssinen (1998), S. 181. Einen die Definition der allgemeinen Jahresabschlussgrundsätze betreffenden Rechtsstreit hat es bislang noch nicht gegeben.

359 Vgl. Järvinen et al. (2000), S. 318 und S. 324.

360 Vgl. Art. 31 Abs. 1 a) der 4. EG-Richtlinie und vgl. van Hulle und van der Tas (2001), S. 821 .

361 Definition durch KPL-Arbeitsgruppe 1995, Teil I (1996), S. 43. Übersetzung ins Deutsche durch die Verfasserin. 
„Die gesamte denkbare Zukunft“ wird nicht näher erläutert. In der kommentierenden Fachliteratur wird davon ausgegangen, dass sie mit „,bis auf Weiteres“ gleichzusetzen sei $^{362}$. Betont wird in der Literatur die besondere Bedeutung dieses Grundsatzes für die Bewertung des Anlagevermögens. Die Obergrenze des Ansatzes bilden hier die erwarteten zukünftigen Einnahmen, nicht der wahrscheinliche Veräußerungswert des Vermögensgegenstands ${ }^{363}$. Umgekehrt sei bei absehbarer Beendigung der Unternehmenstätigkeit oder der Tätigkeit einzelner Teilbereiche unter Berücksichtigung des Vorsichtsprinzips der Markt- und Liquidationswert anzusetzen ${ }^{364}$.

Der Grundsatz der Stetigkeit, vom Richtliniengeber nicht definiert ${ }^{365}$, wird von der KPL-Arbeitsgruppe 1995 folgendermaßen interpretiert ${ }^{366}$ :

Grundsatz der Stetigkeit (Consistency)

Eine einmal hinsichtlich der Buchführung oder der Erstellung des Jahresabschlusses gewählte Methode ist fortwährend beizubehalten, es sei denn, es gibt einen gewichtigen Grund, zu einer anderen Methode zu wechseln.

Die mit der Erstellung des Jahresabschlusses verbundenen Zuordnungs-, Bemessungs-, Bewertungs- und Kostenverteilungsprobleme sollen in allen Geschäftsjahren grundsätzlich identisch behandelt werden, um die Vergleichbarkeit der Abschlüsse verschiedener Geschäftsjahre zu gewährleisten ${ }^{367}$. Ein Wechsel der Grundsätze und Methoden ist nur aus besonderem Grund möglich. Zu nennen sind hier Gesetzesänderungen, Änderungen in der Buchführungspraxis, Veränderung im Kreis verbundener Unternehmen oder eine mit dem Wechsel verbundene wesentliche Verbesserung der Informationsgewährung im Hinblick auf den True and Fair View ${ }^{368}$.

Das Vorsichtsprinzip wird, der 4. EG-Richtlinie entsprechend ${ }^{369}$, vom Gesetzgeber mithilfe des Realisationsprinzips, des Imparitätsprinzips und des Wertaufhellungsprinzips konkretisiert. Die KPL-Arbeitsgruppe 1995 definiert Vorsichts- und

362 Vgl. Leppiniemi (2000 a), S. 43.

363 Vgl. Pärssinen (1998), S. 174, vgl. Järvinen et al. (2000), S. 319, vgl. KPL-Arbeitsgruppe 1995, Teil I (1996), S. 43 und vgl. HE 173/1997, S. 15-16. Vgl. auch Kap. 3.1.6.1 dieser Arbeit.

364 Vgl. Pärssinen (1998), S. 174 und vgl. Leppiniemi (2000 a), S. 43-44.

365 Vgl. Art. 31 Abs. 1 b) der 4. EG-Richtlinie und vgl. van Hulle und van der Tas (2001), S. 821.

366 Definition durch KPL-Arbeitsgruppe 1995, Teil I (1996), S. 43, Übersetzung ins Deutsche durch die Verfasserin.

367 Vgl. Pärssinen (1998), S. 174, vgl. KPL-Arbeitsgruppe 1995, Teil I (1996), S. 44 und vgl. HE 173/1997, S. 16.

368 Vgl. Pärssinen (1998), S. 174, vgl. KPL-Arbeitsgruppe 1995, Teil I (1996), S. 44, vgl. Järvinen et al. (2000), S. 319 und vgl. Kap. 3.1.3.4 vorliegender Arbeit.

369 Vgl. Art. 31 Abs. 1 c) der 4. EG-Richtlinie und vgl. van Hulle und van der Tas (2001), S. $820-821$. 
Realisationsprinzip, nicht aber das Imparitätsprinzip und das Wertaufhellungs$\operatorname{prinzip}^{370}$ :

Grundsatz der Vorsicht (Prudence)

Die im Jahresabschluss enthaltenen Erträge und das Eigentum sind nicht überbewertet, Aufwendungen und Verbindlichkeiten nicht unterbewertet.

Realisationsprinzip (Realization Principle)

Nur realisierte Erträge und Aufwendungen werden in der Buchführung berücksichtigt.

Auffällig ist, dass im Fachschrifttum der Forderung, dass der Grundsatz der Vorsicht unabhängig vom Ergebnis zu beachten ist, anhand von Beispielen und Erläuterungen Bedeutung beigemessen wird ${ }^{371}$. Es ist zu vermuten, dass diese Vorgehensweise auf die einkommensorientierte Realisationsabschreibung der Ausgaben-Einnahmen-Theorie, welche nicht mehr zulässig ist, zurückzuführen ist ${ }^{372}$.

In der Vergangenheit wurde die Bildung stiller Reserven oder die steuerlich motivierte Gewinnminimierung häufig unter dem Deckmantel des Vorsichtsprinzips durchgeführt $^{373}$. Das Realisationsprinzip und das Prinzip der sachlichen Abgrenzung standen unter der Dominanz dieses so verstandenen Vorsichtsprinzips ${ }^{374}$.

Da ein solches Verhalten im Widerspruch zum Konzept des True and Fair View und zum Grundsatz periodengerechter Erfolgsermittlung steht, wird es im Fachschrifttum abgelehnt ${ }^{375}$. In der Buchführungspraxis findet seit Anfang der 90er Jahre eine Entwicklung vom steuerlich geprägten Vorsichtsprinzip zum angloamerikanischen Verständnis hin statt. Stille Reserven sollen grundsätzlich vermieden und auf Grund des Vorsichtsprinzips lediglich wahrscheinlich eintretende Risiken des Unternehmens in der Bilanz erfasst werden ${ }^{376}$. Die Betonung dieser periodengerechten Erfolgsermittlung zu Lasten des Vorsichtsprinzips wirkt sich wie folgt aus:

Die KPL-Arbeitsgruppe 1995 hat in ihrem Bericht empfohlen, das Realisationsprinzip nicht explizit in finnisches Recht umzusetzen, da es sowohl im nicht kodifizierten Grundsatz periodengerechter Abgrenzung als auch im kodifizierten Grundsatz leistungsabhängiger Buchführung enthalten sei ${ }^{377}$. Der finnische Ge-

370 Übersetzung ins Deutsche durch die Verfasserin.

371 Vgl. Pärssinen (1998), S. 175, Järvinen et al. (2000), S. 320 und KPL-Arbeitsgruppe 1995, Teil I (1996), S. 44.

372 Vgl. Kap. 2.1.3.1 dieser Arbeit.

373 Vgl. Majala (2001 b), S. 968.

374 Vgl. Artsberg (2000), S. 544.

375 Vgl. KPL-Arbeitsgruppe 1995, Teil I (1996), S. 44.

376 Vgl. KPL-Arbeitsgruppe 1995, Teil I (1996), S. 45, vgl. Artsberg (2000), S. 544 und vgl. Järvinen et al. (2000), S. 320. JÄRVINEN ET AL. weisen darauf hin, dass der schwedische Gesetzgeber in diesem Zusammenhang von „,angemessener Vorsicht“ (,rimligt försiktighet") spricht, die auch der finnische Gesetzgeber meint, ohne sie jedoch explizit zu nennen.

377 Vgl. KPL-Arbeitsgruppe 1995, Teil I (1996), S. 44. 
setzgeber hat das Prinzip dennoch in finnisches Recht implementiert, ausgehend vom Wortlaut der englischen Version der 4. EG-Richtlinie, ,profits made“. Die finnische Übersetzung dieses Begriffs lautet „toteutuneet voitot“, im Deutschen gleichzusetzen mit „bewahrheitete Gewinne“. Im finnischen Schrifttum wird „bewahrheitet“ und „realisiert“ inhaltlich gleichgesetzt. Offen bleibt, ob eventuell doch bewahrheitete, sichere Gewinne bereits vor ihrer Realisierung ergebniswirksam berücksichtigt werden dürfen ${ }^{378}$.

Der Grundsatz der Bilanzkontinuität ${ }^{379}$ (Correspondence of Balance Sheets) wird in Finnland allgemein akzeptiert, sodass er von der KPL-Arbeitsgruppe 1995 nicht definiert worden ist ${ }^{380}$. In der Literatur wird darauf hingewiesen, dass Fehler aus der Vergangenheit nicht über eine Korrektur des Eigenkapitals zu beseitigen, sondern vielmehr in der Periode ergebniswirksam zu buchen seien, in der sie entdeckt würden ${ }^{381}$. Zudem beinhalte der Grundsatz der Bilanzkontinuität auch das Saldierungsverbot, welches nicht explizit im KPL genannt ist, sondern in KPA 1:10.

Auch der Grundsatz der leistungsbezogenen Abgrenzung ${ }^{382}$ - zentraler Bestandteil der Ausgaben-Einnahmen-Theorie und seit langem angewendete Praxis - wird grundsätzlich befolgt, sodass er nach Meinung der Fachwelt nicht im Gesetz verankert sein muss ${ }^{383}$. Die KPL-Arbeitsgruppe 1995 definiert ihn folgendermas$\operatorname{sen}^{384}$ :

Grundsatz der leistungsbezogenen Abgrenzung (Accrual Basis)

Als Realisationszeitpunkt eines Ertrags wird der Erbringungszeitpunkt der Leistung (Übergabezeitpunkt) betrachtet, als Realisationszeitpunkt eines Aufwands gilt grundsätzlich die Übernahme des Produktionsfaktors.

Oft können diese Realisationszeitpunkte durch Lieferscheine, Materialentnahmescheine o. Ä. dokumentiert und belegt werden ${ }^{385}$.

Der Grundsatz der leistungsabhängigen Buchung gilt nur für den Jahresabschluss, nicht jedoch für die laufende Buchführung, auf die nach KPL 2:3.2 auch der Grundsatz der zahlungsabhängigen Buchführung angewendet werden darf. Im

378 Dies ist in Schweden der Fall, wo die Frage aufgeworfen wurde, ob ,bewahrheitet” und „realisiert” von identischer Bedeutung seien. Vgl. Pärssinen (1998), S. 175 und vgl. Järvinen et al. (2000), S. 323.

379 Vgl. Art. 31 Abs. 1 f) der 4. EG-Richtlinie und vgl. van Hulle und van der Tas (2001), S. 823 .

380 Vgl. auch Pärssinen (1998), S. 176 und Järvinen et al. (2000), S. 324

381 Vgl. KPL-Arbeitsgruppe 1995, Teil I (1996), S. 44, vgl. Pärssinen (1998), S. 176 und vgl. Järvinen et al. (2000), S. 320.

382 Vgl. Art. 31 Abs. 1 d) der 4. EG-Richtlinie und vgl. van Hulle und van der Tas (2001), S. 822.

383 Vgl. Järvinen et al. (2000), S. 321.

384 Übersetzung ins Deutsche durch die Verfasserin. Vgl. KPL-Arbeitsgruppe 1995, Teil I (1996), S. 44.

385 Vgl. Leppiniemi (2000 a), S. 57. 
Fall von Aus- und Einzahlungen als Grundlage der Buchungen müssen die Forderungen und Verbindlichkeiten aus Lieferungen und Leistungen aber jederzeit ermittelbar sein. Sowohl nach KPL 3:3, KPL 3:4 als auch nach der Regierungsbegründung zum Gesetzesentwurf HE 173/1997 sind auf Basis des Grundsatzes zahlungsabhängiger Buchungen geführte Konten bzw. Posten zu korrigieren, um den Jahresabschluss unter Beachtung des Grundsatzes der leistungsabhängigen Buchführung erstellen zu können ${ }^{386}$.

Zwei richtlinienkonforme Ausnahmen vom Grundsatz der leistungsbezogenen Abgrenzung sind in Finnland für den Jahresabschluss gesetzlich verankert. KPL 5:4 gestattet die Anwendung der Percentage of Completion-Methode bei der Bewertung langfristiger Projekte ${ }^{387}$, KPL 5:14 fordert die Bildung von Drohverlustrückstellungen ${ }^{388}$.

Der KILA weist zudem auf Ausnahmen vom Grundsatz der leistungsbezogenen Abgrenzung hin, welche nach finnischem Verständnis neu sind, da sie nicht im Einklang mit der Ausgaben-Einnahmen-Theorie stehen. Zum einen sind seit Einführung des Buchführungsgesetzes von 1997 Steuerzahlungen als Aufwand zu interpretieren, obwohl ihnen keine direkte Gegenleistung zugerechnet werden $\mathrm{kann}^{389}$. Zum anderen werden Zinszahlungen als Aufwand behandelt ${ }^{390}$. Steuerund Zinszahlungen waren bisher nach SAARIOs Ausgaben-Einnahmen-Theorie der Gewinnverwendung zugerechnet ${ }^{391}$.

Der Grundsatz der Einzelbewertung (Individual Valuation Principle) verlangt, alle Vermögensgegenstände unabhängig voneinander zu erfassen. Eine Überbewertung eines Vermögensgegenstands mit der Begründung, ein anderer Vermögensgegenstand sei unterbewertet, wird ebenso ausgeschlossen wie eine Unterbewertung von Verbindlichkeiten wegen unzutreffender Unterbewertung des Eigenkapitals $^{392}$. Von diesem Grundsatz ausgenommen ist in Übereinstimmung mit Art. 38 der 4. EG-Richtlinie die mögliche Bildung eines Festwerts gem. KPL 5:6.3.

Von den gesetzlich verankerten Jahresabschlussgrundsätzen darf Art. 31 Abs. 2 der 4. EG-Richtlinie entsprechend gemäß KPL 3:3.3 nur aus besonderen Gründen

386 Vgl. HE 173/1997, S. 16-17, vgl. Leppiniemi (2000 a), S.57. Wortlaut des KPL 3:4, Übersetzung ins Deutsche durch die Verfasserin: „Vor Jahresabschlusserstellung sind zahlungsabhängige Buchungen [...] zu korrigieren und haben den Grundsatz leistungsabhängiger Buchführung zu erfüllen.“ sion gestattet sie in XVI/7009/97.

Drohverlustrückstellungen sind in Art. 31 Abs. 1 c) bb) und Art. 20 Abs. 1 der 4. EG-Richtlinie verankert.

Vgl. KILA 1542/1998.

Vgl. KILA 1317/1995.

Vgl. Kap. 2.1.3.1 dieser Arbeit.

Vgl. KPL-Arbeitsgruppe 1995, Teil I (1996), S. 45, vgl. Järvinen et al. (2000), S. 322, die auf KILA 1603/2000 verweisen und vgl. Pärssinen (1998), S. 178. 
abgewichen werden, es sei denn, eine Abweichung ist gesetzlich vorgeschrieben. Im Falle einer Abweichung von den kodifizierten Grundsätzen ordnungsmäßiger Buchführung müssen diese Abweichung, deren Grund sowie ihre Ergebnisauswirkung im Anhang erläutert werden ${ }^{393}$.

\subsubsection{Nicht kodifizierte Grundsätze ordnungsmäßiger Buchführung}

Die in Finnland allgemein anerkannten, nicht kodifizierten GoB wurden teils in der Regierungsvorlage HE 111/1992 zum Buchführungsgesetz 1992, teils im Bericht der KPL-Arbeitsgruppe 1995 aufgelistet und von der KPL-Arbeitsgruppe 1995 definiert $^{394}$.

Die Interpretation des Grundsatzes der periodengerechten Abgrenzung ${ }^{395}$ befindet sich in Finnland derzeit im Wandel ${ }^{396}$ :

Grundsatz der periodengerechten Abgrenzung (Matching)

Aufwendungen sind der Gewinn- und Verlustrechnung derjenigen Periode zuzu-

rechnen, in der die entsprechenden Erträge anfallen.

Unter dem Grundsatz der periodengerechten Abgrenzung ist bis Anfang der 90er Jahre eine Zurechnung der Aufwendungen zu realisierten Erträgen bzw. die Erfassung eines sicher eintretenden Verlusts verstanden worden. Verluste werden heute auch dann berücksichtigt, wenn es lediglich wahrscheinlich ist, dass keine entsprechenden Erträge mehr anfallen ${ }^{397}$.

Auf eine inhaltliche Auseinandersetzung mit weiteren nicht kodifizierten GoB soll an dieser Stelle verzichtet werden, da in diesen Fällen das finnische dem international üblichen Verständnis entspricht. Im Folgenden sind der Vollständigkeit halber die von der KPL-Arbeitsgruppe 1995 gelieferten Definitionen aufgeführt ${ }^{398}$ :

Grundsatz der Richtigkeit und Willkürfreiheit (Reliability)

Eine Jahresabschlussinformation darf keine bemerkenswerten Fehler oder Lücken beinhalten.

Grundsatz der Vollständigkeit (Completeness)

In Buchführung und Jahresabschluss sind alle den Buchführungspflichtigen betreffenden Geschäftsvorfälle sowie Eigentum und Verbindlichkeiten enthalten.

393

Vgl. HE 173/1997, S. 16.

Vgl. KPL-Arbeitsgruppe 1995, Teil I (1996), S. 28 und S. 36-37.

Der Grundsatz der periodengerechten Abgrenzung wird nicht explizit in der 4. EG-Richtlinie aufgeführt. Er findet jedoch in einer Vielzahl ihrer Vorschriften Anwendung. So reflektieren das Aktivierungsgebot für bestimmte Ausgaben oder die Forderung nach planmäßiger Abschreibung bestimmter Werte des Anlagevermögens eindeutig den Grundsatz der periodengerechten Abgrenzung. Vgl. van Hulle und van der Tas (2000), S. 822.

Vgl. KPL-Arbeitsgruppe 1995, Teil I (1996), S. 28. Übersetzung ins Deutsche durch die Verfasserin.

Vgl. Majala (2001 b), S. 968.

Vgl. KPL-Arbeitsgruppe 1995, Teil I (1996), S. 28 und S. 36-37. 
Grundsatz der Wesentlichkeit (Materiality)

Aus Informationsgesichtspunkten können unwesentliche Posten dahingehend vernachlässigt werden, dass sie nicht mit der für wesentliche Posten erforderlichen Genauigkeit behandelt werden.

Grundsatz der vertretbaren Kosten (Balance Between Benefit and Costs)

Die Bereitstellung von Informationen darf keine übermäßigen Kosten verursachen.

Grundsatz der sachlichen Richtigkeit (Substance Over Form)

Bei der Auswahl der Darstellungsart von Jahresabschlussinformationen wird die wirtschaftliche Betrachtungsweise zu Grunde gelegt, nicht die rechtliche.

Grundsatz der Vergleichbarkeit (Comparability)

Die Jahresabschlüsse mehrerer Geschäftsjahre eines Buchführungspflichtigen müssen untereinander und nach Möglichkeit auch mit den Jahresabschlüssen anderer Buchführungspflichtiger vergleichbar sein.

Grundsatz der zeitnahen Berichterstattung (Timeliness)

Die geschäftsjährliche Berichterstattung hat ausreichend zeitnah zu erfolgen.

Grundsatz der Betriebseinheit mit eigener Buchführung (Entity Principle)

Die buchführende Wirtschaftseinheit bildet eine gesonderte Ganzheit.

Grundsatz des stabilen Geldwerts: Anschaffungs- bzw. Herstellungskostenprinzip (Historical Cost)

Alle in der Buchführung vermerkten Geldeinheiten sind untereinander gleichwertig.

Grundsatz der Bedeutsamkeit (Relevance)

Die Information ist nach Inhalt und Darstellungsweise so gestaltet, dass der Jahresabschlussleser die Möglichkeit hat, auf ihrer Basis die vergangene Entwicklung zu beurteilen und mit ihrer Hilfe die zukünftige Entwicklung einzuschätzen.

Grundsatz der Objektivität (Objectivity)

Ein unabhängiger Berufsangehöriger muss im Bedarfsfall bestätigen können, dass die gegebene Information verlässlich und korrekt ist.

Grundsatz der Periodisierung (Periodicity)

Die Tätigkeit der Betriebseinheit wird auf Basis der Kalenderzeit in Rechnungsperioden unterteilt.

Grundsatz der Offenlegung (Disclosure)

Der Jahresabschluss und die ihm zu Grunde liegenden Erstellungsgrundsätze werden veröffentlicht. 


\subsubsection{True and Fair View}

\subsection{Aufnahme des True and Fair View in finnisches Recht}

In ihrer Präambel und ihrem Art. 2 Abs. 3 und Abs. 4 schreibt die 4. EG-Richtlinie vor, dass

„der Jahresabschluss ein den tatsächlichen Verhältnissen entsprechendes Bild der Vermögens-, Finanz- und Ertragslage der Gesellschaft zu vermitteln hat. Reicht die Anwendung dieser Richtlinie nicht aus, um ein den tatsächlichen Verhältnissen entsprechendes Bild zu vermitteln, so sind zusätzliche Angaben zu machen.“

Die Stellung der Vorschrift innerhalb der Richtlinie und die gleichlautende Forderung schon in ihrer Präambel verdeutlichen den Stellenwert dieser Norm als Zielvorschrift $^{399}$.

Der finnische Gesetzgeber hat zur Realisierung der von ihm beabsichtigten Jahresabschlusszwecke das Konzept der Art guter Buchführung und Einzelvorschriften zur konkreten Ausgestaltung eines Einzelabschlusses erlassen. Parallel zu diesen Vorschriften hat er aber mit KPL 3:2.1 eine Generalnorm geschaffen, die vom Jahresabschlussersteller fordert, dass

„der Einzelabschluss richtige und ausreichende Informationen über die Ertragslage und die ökonomische Lage der Tätigkeiten des Buchführungspflichtigen zu vermitteln hat (ein richtiges und ausreichendes Bild). ${ }^{4} 400$

Das Konzept des True and Fair View ist für die meisten Mitgliedstaaten der EU relativ neu und hat erst mit der Transformation der 4. EG-Richtlinie Eingang in die jeweiligen nationalen Rechnungslegungsgesetzgebungen gefunden ${ }^{401}$.

So auch in Finnland, wo der True and Fair View 1992 ins Buchführungsgesetz aufgenommen wurde ${ }^{402}$. Umstritten war bei der Übernahme des True and Fair View in finnisches Recht die Übersetzung. Der True and Fair View im Sinne des Art. 2 Abs. 3 der 4. EG-Richtlinie ist in Finnland mit der Übersetzung ,oikeat ja

399 Vgl. Präambel und Art. 2 Abs. 3 und 4 der 4. EG-Richtlinie und Großfeld (1986), S. 195.

400 Übersetzung von Kap. $3 \S 2$ KPL ins Deutsche durch die Verfasserin.

401 Vgl. van Hulle (1997), S. 716.

402 Bereits 1986 hat der Gesetzgeber mit $§ 38$ Abs. 2 eine Änderung in das Buchführungsgesetz eingefügt, die besagt hat, dass die wesentliche Beeinträchtigung des richtigen und ausreichenden Bildes über das wirtschaftliche Ergebnis oder die wirtschaftliche Lage des Buchführungspflichtigen durch vorsätzliches oder fahrlässiges Handeln strafbar sei. Bei der Novellierung des Strafgesetzbuchs 1990 ist dieser Paragraph aus dem Buchführungsgesetz in das 30. Kapitel des Strafgesetzbuchs verschoben worden, sodass der Begriff des richtigen und ausreichenden Bildes im Buchführungsgesetz nicht mehr enthalten war. Vgl. Riistama (1992), S. 6-8, vgl. KPL-Arbeitsgruppe 1995, Teil I (1996), S. 30 und vgl. Järvinen et al. (2000), S. 313. 
riittävät tiedot“" in das Buchführungsgesetz 1992 aufgenommen worden, was in deutscher Sprache ,richtige und ausreichende Informationen“403 bedeutet.

Die Wortwahl löste eine Debatte in der Fachwelt aus, wie sie ähnlich auch in Deutschland geführt worden ist ${ }^{404}$. Eine Kontroverse hat der Versuch verursacht, den Inhalt des Begriffs True and Fair View durch die Wortwahl bei der Übersetzung richtlinientreu zu konkretisieren. Fraglich ist, ob der Begriff „Informationen“ oder „Bild“ Sinn und Zweck der Richtlinie besser widerspiegelt ${ }^{405}$. Der Diskussion wurde ein Ende gesetzt, als im Buchführungsgesetz von 1997 der Begriff „oikeat ja riittävät tiedot“ (richtige und ausreichende Informationen) um den in Klammern gesetzten Ausdruck „oikea ja riittävä kuva“ (ein richtiges und ausreichendes Bild) ergänzt wurde. Dass im Buchführungsgesetz von 1997 beide Begriffe nebeneinander aufgeführt werden, hat seine Ursache in gesetzestechnischen Gründen $^{406}$. Die Gesetzesmaterialien verweisen in aller Deutlichkeit darauf, dass der finnische Gesetzgeber inhaltlich nicht von den europäischen Vorgaben abwei-

403 Übersetzung ins Deutsche durch die Verfasserin.

404 Vgl. beispielsweise Niehus, Rudolf: „True and Fair View“ - In Zukunft auch Bestandteil der deutschen Rechnungslegung, Der Betrieb 1997, S. 221 oder vgl. Großfeld und Junker: Die Prüfung des Jahresabschlusses im Zuge der 4. EG-Richtlinie, Zeitschrift für betriebswirtschaftliche Forschung 1980, S. 251.

405 EKHOLM und TROBERG vertreten die Meinung, dass „Informationen“ inhaltlich Fakten und Regeln über die wirtschaftliche Wahrheit stellten. Diese Autoren schlagen den Ausdruck „Bild“ vor, weil dieser den Grundsatz Substance Over Form betone und deshalb die Intention der Richtlinie genauer wiedergebe. Vgl. Ekholm und Troberg (1995), S. 343 f.

Dieser Meinung hat sich die mit dem Entwurf des Buchführungsgesetzes von 1997 vom Ministerium für Handel und Industrie beauftragte KPL-Arbeitsgruppe 1995 angeschlossen. Der Ausdruck „Informationen“ habe eine eingeschränktere und formellere, technischere Bedeutung als „Bild“; deshalb spiegele „Bild“ den Sinn und Zweck der Richtlinie besser wider als „Informationen“. Zudem sei bei der Beurteilung von Richtigkeit und Angemessenheit die Jahresabschlussaussage immer unter Berücksichtigung der gesetzten Ziele, der Zielgruppe, der Tätigkeit und der Struktur des Buchführungspflichtigen zu definieren. Es könne sich also beim True and Fair View nicht um einen absoluten Begriff, wie „Informationen“ ihn darstellt, handeln, sondern nur um einen relativen Begriff wie „Bild“.

Außerdem blieben einzelne Aussagen nach dem Willen der Richtlinie nicht als Einzelinformationen stehen, sondern sollen in ihrer Summe zu einem Gesamtbild führen. Darüber hinaus sei der True and Fair View als dynamischer Begriff aufzufassen, sodass sowohl Inhalt als auch Teilfaktoren einem Änderungsprozess unterlägen und der Wissenszuwachs im Zeitablauf zu neuen Interpretationen führen könne. Während Informationen immer Informationen blieben, könne das Bild über sie sich ändern. Vgl. KPL-Arbeitsgruppe 1995, Teil I (1996), S. 38-41.

Für MÄKINEN stellen „Informationen“ und „Bild“ zwei grundverschiedene Aussagen dar. Er beurteilt den Begriff „Informationen“ als problematisch, da diese auch Detailwissen enthielten, das den Leser das Gesamtbild vergessen lasse, ihn eher verwirrten und zu falschen Schlussfolgerungen verleiten könnten. Vgl. Mäkinen (1996).

RIISTAMA hingegen befürwortet „Informationen“, da seiner Meinung nach exakt so viele Bilder über die Vermögens-, Finanz- und Ertragslage eines Unternehmens entstehen könnten, wie es Jahresabschlussleser gäbe. Der Jahresabschluss sei aber nur eine einzige und unveränderliche Zusammensetzung verschiedener Angaben, deren Aufgabe es sei, Informationen über das Ergebnis und die ökonomische Lage eines Unternehmens zu vermitteln. Der Ersteller des Abschlusses könne nicht verantwortlich sein für alle unterschiedlichen Bilder, die sich in den Köpfen der Adressaten entwickelten. Vgl. Riistama (1996) S. 15 f. 
chen wollte, sondern vielmehr durch Richtlinienkonformität das Harmonisierungsziel zu erreichen suchte ${ }^{407}$.

Interessant ist die Tatsache, dass der finnische Gesetzgeber einen True and Fair View der Ertrags- und der ökonomischen Lage fordert. Die Begriffe Vermögensund Finanzlage werden, anders als in der 4. EG-Richtlinie, nicht explizit genannt. Die mit der Gesetzesnovellierung beauftragte Arbeitsgruppe begründet dies damit, dass die ökonomische Lage sowohl die Vermögens- als auch die Finanzlage umfasse $^{408}$. Ein allgemein gültiges Rangverhältnis zwischen der Ertrags- und der ökonomischen Lage hinsichtlich ihrer Bedeutung gibt es, nach finnischer wie nach deutscher ${ }^{409}$ Auffassung, nicht. Vermögens-, Finanz- und Ertragslage sind gleichrangig nebeneinander zu stellen. Das Bild aller drei Lagen ist gleichermaßen in einer den tatsächlichen Verhältnissen entsprechenden Weise zu vermitteln.

Letztendlich ist die Wortwahl bei der Übersetzung allein nicht wesentlich. Den Begriff True and Fair View mit seinem Bedeutungsinhalt wörtlich in eine andere Sprache zu übersetzen, dürfte unmöglich sein, auch wenn die meisten Mitgliedstaaten sich redlich um eine wortgetreue Übersetzung bemüht haben ${ }^{410}$. Der vom Verfasser der Richtlinie beabsichtigte Gesellschafter- und Gläubigerschutz ist gewährleistet, wenn der Einzelabschluss das geforderte Einblicksgebot erfüllt. Die sprachliche Konkretisierung der Formulierung True and Fair View hilft dem Interpreten inhaltlich nicht weiter. Entscheidend ist vielmehr, das hinter diesen Worten stehende Konzept zu verstehen ${ }^{411}$.

\subsection{Finnisches Verständnis des True and Fair View}

Der True and Fair View fordert, dass der vom Jahresabschluss vermittelte Gesamteindruck vom Zustand und den Entwicklungstendenzen des Unternehmens zutreffen muss. Diskutiert werden in Finnland Fragen zu Inhalt und Philosophie des True and Fair View, zum Informationsgehalt des Jahresabschlusses, zum Verhältnis zwischen dem True and Fair View und der Wirtschaftsprüfung sowie zu praktischen Erfahrungen von Unternehmen im Umgang mit dem True and Fair View.

407 Vgl. KPL-Arbeitsgruppe 1995, Teil I (1996) und HE 173/1997.

408 Vgl. KPL-Arbeitsgruppe 1995 (Teil II), S. 48. Da hierüber im finnischen Schrifttum keine Diskussion stattgefunden hat, kann vermutet werden, dass in diesem Punkt Einigkeit herrscht.

409 Vgl. ADS § 264 Anm. 60 und vgl. Küting und Weber (1995), § 264 Anm. 14.

410 So auch van Hulle (1993), S. 100, mit Beispielen und Hinweis auf Alexander und seinen Versuch, inhaltliche Unterschiede im Verständnis der Staaten durch Übersetzungsunterschiede zu erklären.

411 Vgl. Beine (1995), S. 471. 
Wie in den anderen europäischen Staaten, definiert auch der finnische Gesetzgeber den Begriff True and Fair View nicht ${ }^{412}$. Die mit dem Entwurf des Buchführungsgesetzes von 1997 beauftragte KPL-Arbeitsgruppe 1995 weist lediglich darauf hin, dass der True and Fair View kein statisches, sondern ein dynamisches Konzept sei, dessen Inhalt sich mit wachsendem Wissen über ein Unternehmen ändere. Der True and Fair View als Norm sei immer dann heranzuziehen, wenn Zweifel bei der Auslegung einzelner Vorschriften entstehen oder Lücken in der gesetzlichen Regelung zu schließen sind ${ }^{413}$. Bei der Beurteilung des True and Fair View sei immer die Zielgruppe des betreffenden Jahresabschlusses, die Branche, der das Unternehmen angehört, sowie die Struktur des Unternehmens zu beachten. Einzelfälle seien immer in Bezug auf das Ganze zu sehen ${ }^{414}$.

Im Hinblick auf die Auslegung einzelner Vorschriften und die Schließung von Gesetzeslücken wird gefordert, dass der KILA in Einzelfällen darüber zu befinden habe, wie zu verfahren sei, um den True and Fair View zu erreichen. Es besteht die Gefahr, dass der True and Fair View als leere Worthülse stehen bleibe und nicht mit Inhalt gefüllt werde, wenn der KILA seiner Aufgabe hinsichtlich der

412 Aufschluss könnte das britische Verständnis des True and Fair View geben, da die Formulierung des Art. 2 Abs. 3 und 4 der 4. EG-Richtlinie ursprünglich dem britischen Rechtsprinzip des True and Fair View entstammt, das nach dem Beitritt Großbritanniens zur EG 1973 in die europäischen Richtlinien zur Rechnungslegung implementiert wurde. Der Begriff des True and Fair View wurde erstmals 1844 in einem Gerichtsurteil verwendet, seine Kodifizierung in den britischen Companies Act geht auf das Jahr 1948 zurück. Mit dem True and Fair View handelt es sich auch im britischen Recht um einen unbestimmten Rechtsbegriff; die Analyse seiner Ursprünge erweist sich bei der Analyse seiner Bedeutung als wenig hilfreich (vgl. Alexander (1993), S. 61). „Doch auch wenn die britische Literatur uns näher über die Bedeutung von True and Fair View aus britischer Sicht aufklären könnte, würde dennoch das allgemeine Prinzip gelten, dass eine abschließende Erklärung eines in einer Gemeinschaftsrichtlinie vorkommenden Begriffes nur vom europäischen Gerichtshof abgegeben werden kann.“ (Van Hulle und van der Tas (2001), S. 797). Beine weist darauf hin, dass auch in Großbritannien seit der Umsetzung der europäischen Richtlinie in das nationale Recht ein Umorientierungsprozess eingesetzt habe, bei der Interpretation von auf Gemeinschaftsrecht beruhenden Normen die Auslegungskriterien des Europäischen Gerichtshofs anzuwenden, vgl. Beine (1995), S. 469.

Die Definition und Interpretation des „,europäischen True and Fair View“ obliegt also letztlich dem Europäischen Gerichtshof, der bislang noch nicht die Gelegenheit hatte, über die Bedeutung von ,true and fair“ zu entscheiden. Fraglich ist indes, ob es einen „europäischen True and Fair View“, der von allen Mitgliedstaaten identisch interpretiert wird, überhaupt geben kann.

ALEXANDER vertritt die Meinung, dass dies unwahrscheinlich und nicht wünschenswert sei, da Rechnungslegung in Ausübung und Anwendung kulturspezifisch sei. Ein einheitliches Verständnis von ,true and fair“ könne somit nur erreicht werden, wenn Europa eine einzige Einheitskultur entwickele (vgl. Alexander (1993)).

Auch VAN HULLE ist der Ansicht, dass es wegen der sozio-ökonomischen, kulturellen und rechtssystematischen Unterschiede zwischen den einzelnen Mitgliedstaaten durchaus möglich sei, dass Jahresabschlüsse, in einem Mitgliedstaat als „,true and fair“ angesehen, in einem anderen Staat eben nicht als solche interpretiert würden. Die Abschlüsse seien dennoch vor dem Hintergrund, vor dem sie entstünden, „true and fair“; Leser anderer Mitgliedstaaten benötigten jedoch Zusatzangaben im Anhang, um die Abschlüsse ebenfalls als „true and fair“ ansehen zu können (vgl. van Hulle (1993), S. 100).

413 Vgl. KPL-Arbeitsgruppe 1995, Teil I (1996), S. 38.

414 Vgl. KPL-Arbeitsgruppe 1995, Teil I (1996), S. 41. 
Entwicklung des True and Fair View nicht nachkomme ${ }^{415}$. Der KILA muss demnach beim Erteilen von Anweisungen, Gutachten und Ausnahmeerlaubnissen genau den Grundsatz beachten, den er andererseits für finnische Bedürfnisse definieren soll. Bislang hat der KILA den Begriff des True and Fair View nicht erläutert.

$\mathrm{Zu}$ untersuchen ist, wie der True and Fair View von der finnischen Fachwelt inhaltlich aufgefasst wird und inwieweit sich durch seine Aufnahme in finnisches Recht materielle Änderungen für die Bilanzierung ergeben.

Vielfach wird die Auffassung vertreten, die Einführung des True and Fair View verändere die Grundzüge des finnischen Jahresabschlusses nicht ${ }^{416}$. Grundlage der Buchführung sei nach wie vor die Ausgaben-Einnahmen-Theorie, primäres Ziel des Jahresabschlusses die Ermittlung des ausschüttungsfähigen Gewinns ${ }^{417}$. Der Schwerpunkt des Jahresabschlusses liege demzufolge auf der Gewinn- und Verlustrechnung, nicht auf der Bilanz. Als Beleg dieser Auffassung gilt, dass der True and Fair View der Ertragslage und der ökonomischen Lage gefordert wird, und dass der Gesetzgeber bei der Umsetzung des Konzepts die Ertrags- vor die ökonomische Lage gestellt hat. Der Gesetzgeber weicht hiermit von der Reihenfolge der Begriffe in der Richtlinie ab. Aber auch die Vertreter dieser Auffassung konstatieren, dass mit der Übernahme des True and Fair View in finnisches Recht die Stellung der Bilanz innerhalb des Jahresabschlusses gestärkt worden ist ${ }^{418}$.

Die Frage, welche Gruppe der Jahresabschlussadressaten - Gesellschafter, Einflussnehmer auf dem Kapitalmarkt, Gläubiger und/oder Steuerbehörden - den Abschluss als „richtig und ausreichend“ ansehen solle, ist bisher kaum diskutiert worden $^{419}$. Es wird von einigen Vertretern der Fachwelt betont, dass es nicht im Bereich des Möglichen liegen könne, die subjektiven Informationsbedürfnisse aller Leser in allen Beziehungen $\mathrm{zu}$ erfüllen ${ }^{420}$. Andere Autoren weisen darauf hin, dass die Interessen aller Jahresabschlussleser zu berücksichtigen seien, die den Jahresabschluss zur Entscheidungsfindung verwenden ${ }^{421}$.

Einige Autoren vertreten die Meinung, dass neben den Informationsbedürfnissen einzelner Gruppen innerhalb eines Lands auch zwischen denen in- und ausländischer Jahresabschlussleser zu unterscheiden sei. In den Geschäftsberichten des

415 Vgl. Väyrynen (1993), S. 9, im Gespräch mit Leppiniemi.

416 Vgl. beispielsweise Riistama (1992), S. 6-8 und vgl. Sorsa (1998 b), S. 32 ff.

417 Vgl. Kap. 3.1.3.1 dieser Arbeit.

418 Vgl. Määttä (2000), S. 24 und vgl. Sorsa (1998 a), S. 163.

419 Vgl. Määttä (2000) S. 29-31. MÄ̈̈TTÄ hat mehr als 50 im Zusammenhang mit dem True and Fair View in den Fachzeitschriften „Tilintarkastus“ und ,Tilisanomat“ erschienene Artikel aus den Jahren 1986 bis 1998 analysiert, die unterschiedlichen Gedanken und Auffassungen zusammenfassend dargestellt sowie neue Fragen aufgeworfen.

420 Vgl. Riistama (1996), S. 16.

421 Vgl. Tuokko bei Määttä (2000), S. 31. 
Jahres 1994 weisen einige finnische Großkonzerne duale Abschlüsse ${ }^{422}$ oder sogar Abschlüsse aus, die gleichzeitig die EG-Richtlinien, die Standards des IASB und das - seinerzeit noch nicht in allen Punkten richtlinienkonforme - finnische Recht befolgen sollen. Da aber das finnische Recht und die Standards des IASB in Teilbereichen widersprüchlich seien, könne, so die Kritik, der True and Fair View bei solchen Abschlüssen zumindest aus Sicht einiger Jahresabschlussleser nicht mehr gewährleistet sein. Das Erstellen von getrennten Jahresabschlüssen unterschiedlicher Normen nebeneinander sei trotz des damit verbundenen Mehraufwands notwendig, um den True and Fair View des finnischen Abschlusses zu gewährleis$\operatorname{ten}^{423}$.

Es stellt sich die Frage, ob hinsichtlich der Beurteilung richtiger und ausreichender Angaben zwischen kleinen, mittelgroßen und großen Unternehmen zu unterscheiden sei. Fraglich ist, ob bei Einhaltung des Grundsatzes der sachlichen Richtigkeit der True and Fair View auch dann von kleinen Unternehmen erreicht werden könne, wenn diese von den Vereinfachungsregeln Gebrauch machten ${ }^{424}$. Da ab dem Geschäftsjahr 2000 die Unternehmen aller Rechtsformen und Größen von KHT- oder HTM-Wirtschaftsprüfern geprüft werden müssen, üben die Wirtschaftsprüfer eine immer wichtigere Rolle hinsichtlich der Einhaltung des True and Fair View von kleinen Unternehmen aus ${ }^{425}$. Es wird erwartet, dass sich der Informationsgehalt der Jahresabschlüsse kleiner Unternehmen künftig weiter verbessert $^{426}$.

In der Literatur wird - auch im Hinblick darauf, dass der Lagebericht in Finnland Bestandteil des Jahresabschlusses ist ${ }^{427}$ - die Frage aufgeworfen, welche Informationen über ein Unternehmen der True and Fair View im Sinne der 4. EG-Richtlinie verlange, die der „herkömmliche“, unter Beachtung der GoB erstellte Jahresabschluss nicht leiste. Verwiesen wird auf die nicht bilanzierten immateriellen Vermögensgegenstände sowie die ökologische Situation des Unternehmens und die Corporate Governance. Es wird erörtert, ob es tatsächlich die Intention der 4. EG-Richtlinie sei und im Interesse der Jahresabschlussadressaten liege, den True and Fair View nur unter der Beachtung der GoB zu sehen, ihn aber nicht aus

422 Unter „dualen Abschlüssen“ werden solche verstanden, die sowohl finnische als auch IASNormen erfüllen sollen.

423 Vgl. Saarikivi und Räty (1996), S. 27-30.

424 Vgl. Sipola (1991), S. 13-17.

425 Laien-Wirtschaftsprüfer sind seit dem Geschäftsjahr 2000 nicht mehr zugelassen.

426 Vgl. Määttä (2000), S. 32 f.

427 Vgl. Määttä (2000), S. 27-29. Er vertritt die Auffassung, die Qualität der Lageberichte sei durch die Einführung des True and Fair View gestiegen. Die Wirtschaftsprüfer müssten auch die Einschätzung der zukünftigen Entwicklung durch die Geschäftsführung beurteilen, wodurch der Informationsgehalt des Jahresabschlusses zugenommen habe. 
betriebswirtschaftlichen, volkswirtschaftlichen, juristischen oder sozialen Gesichtspunkten zu beurteilen $^{428}$.

Thematisiert wurde bisher noch nicht, ob der True and Fair View in Finnland auf Grund der Verbindung zwischen Buchführungsgesetz und Steuerrecht ${ }^{429}$ überhaupt erreicht werden kann. Diese Frage ist deshalb kritisch zu stellen, weil die Bilanzierenden bei der Beurteilung einzelner Sachverhalte ihren Jahresabschluss an der Steuerbilanz ausrichten müssen, sofern sie bestimmte steuerliche Wahlrechte ausüben wollen. Durch diese so genannte „umgekehrte Maßgeblichkeit“ können Werte im Jahresabschluss angesetzt werden, die weder mit den GoB noch mit den Jahresabschlusszwecken vereinbar sind und folglich nicht mit dem True and Fair View in Einklang stehen.

\subsection{Verhältnis des True and Fair View zur Art guter Buchführung und zu den anderen Grundsätzen ordnungsmäßiger Buchführung}

Wegen des Harmonisierungsziels der Vergleichbarkeit muss sichergestellt sein, dass die gemachten Angaben nicht nur richtig (,true“) sind, sondern auch noch so ausreichend (,fair") bemessen werden, dass die Bilder, die in den Lesern hervorgerufen werden, nicht zu weit voneinander und von den tatsächlichen Verhältnissen abweichen. Aus dieser Überlegung resultiert die Frage: Wie viel ist ,ausreichend ${ }^{4430}$ ?

Im finnischen Schrifttum werden Informationen in ihrer Gesamtheit als ausreichend angesehen, wenn sie neben den Anforderungen der Gesetze, Anordnungen und Empfehlungen auch noch einen allgemein anerkannten Umfang haben und in einer allgemein anerkannten Form präsentiert werden. Abgestellt wird auf die Vertreter des Fachgebiets ${ }^{431}$.

Der allgemein anerkannte Umfang und die allgemein anerkannte Form der Informationen ergeben sich aus der Art guter Buchführung. Nach herrschender Meinung im finnischen Schrifttum ist der True and Fair View Teil dieser Art guter Buchführung ${ }^{432}$. Dass der Gesetzgeber dieser Auffassung zustimmt, wird u. a. aus

428 Vgl. Määttä (2000), S. 35-44. Eine Diskussion zu diesem Thema in Finnland wird erwartet, und es ist denkbar, dass sich künftig das Verständnis vom True and Fair View als Teil der GoB zum True and Fair View als umfassendem Konzept wandelt. Es bleibt ebenfalls abzuwarten, wie eine solche Diskussion in anderen europäischen Staaten - z.B. Deutschland vor dem Hintergrund des derzeit mangelnden Vertrauens in die Finanzmärkte geführt wird. Vgl. Kap. 2.2.2.3 dieser Arbeit.

430 Mit dieser Frage hat sich v. a. Riistama auseinander gesetzt. Vgl. Riistama (1996).

431 Als Vertreter des Fachgebiets werden in der Litertatur Jahresabschlussersteller, Wirtschaftsprüfer, öffentliche Erklärungen abgebende Analysten und nicht mit der Geschäftsführung betraute Eigentümer angesehen. Vgl. Riistama (1996), S. 18.

432 Vgl. Järvinen et al. (2000), S. 314 und die dort angegebene Literatur. 
dem Gesetzesaufbau ersichtlich: Die Art guter Buchführung mit den GoB befinden sich in Kap. 1 des Buchführungsgesetzes, den allgemeingültigen Regeln, die für alle nachfolgenden Kapitel gelten. Der True and Fair View ist im 3. Kapitel über den Jahresabschluss zu finden und nur für dieses Kapitel relevant. So hat auch die 4. EG-Richtlinie primär für die Rechnungslegung ihre Gültigkeit und nicht für die laufende Buchhaltung ${ }^{433}$. Somit ist der True and Fair View Bestandteil der Art guter Buchführung und den GoB gleichzusetzen. Der Art nach unterscheidet er sich aber von den anderen GoB. Der True and Fair View betrifft nur die Rechnungslegung, ist eine Zielvorstellung, welche die anderen GoB bei der Erstellung des Jahresabschlusses ergänzt ${ }^{434}$. Zudem besteht der Jahresabschluss nicht nur aus Zahlen-Informationen, die der Buchhaltung entstammen und den GoB unterliegen. V. a. der Lagebericht, in Finnland Bestand des Jahresabschlusses, enthält auch Text, Kennzahlen, Graphiken, Zeichnungen und Bilder ${ }^{435}$. Diese müssen nicht primär der Buchführung entspringen, aber aus ihr ableitbar sein. So betrachtet, bildet die Art guter Buchführung die Generalnorm für die Buchführung und der True and Fair View die Generalnorm für den Jahresabschluss ${ }^{436}$.

Der Gesetzesaufbau bedingt, dass das geforderte Bild lediglich im Kontext mit den GoB gefordert wird, d. h. unter der Beachtung der (einschränkenden) Bilanzierungs- und Bewertungsgrundsätze. Der True and Fair View als Teil der GoB ist als Relativierung zum Zwecke der Objektivierung des Bildes zu verstehen. Es soll verhindert werden, dass übertriebene Anforderungen an das geforderte Bild gestellt werden. Der Gesetzgeber stellt auf die reale Rechnungslegungspraxis ab und nicht auf ein letztlich nicht ,vollständig konkretisierbares, schon ins Philosophische reichendes, idealistisches Konzept ${ }^{\star 437}$. Mit den GoB orientiert er sich am Informationsgewinnungsprozess und am Jahresabschlussersteller, und nicht am Informationsergebnis mit Hinblick auf die Jahresabschlussadressaten ${ }^{438}$. „In einer auf Konsens und Kooperation ausgerichteten Kultur fällt es ohne Zweifel leichter zu entscheiden, was „gute Praxis“ darstellt, als herauszufinden, welche Rechnungslegungsmethode als true and fair zu bezeichnen ist. ““39

\subsection{Notwendige Zusatzangaben im Anhang}

Der vom Jahresabschluss vermittelte Gesamteindruck über den Zustand und die Entwicklungstendenzen eines Unternehmens muss zutreffend sein. Ein in Über-

433 Vgl. Järvinen et al. (2000), S. 313.

434 Vgl. KPL-Arbeitsgruppe 1995, Teil I (1996), S. 35.

435 Vgl. KPL-Arbeitsgruppe 1995, Teil I (1996), S. 35 ff.

436 Vgl. KPL-Arbeitsgruppe 1995, Teil I (1996), S. 35 ff.

437 Artsberg (2000), S. 544, die ihre Aussage auch auf Schweden bezieht.

438 Vgl. Artsberg (2000), S. 544, die diese Aussage auf Finnland und Schweden bezieht.

439 Artsberg (2000), S. 544, die diese Aussage auf Finnland und alle skandinavischen Länder bezieht. 
einstimmung mit den GoB erstellter Jahresabschluss - bestehend aus Gewinnund Verlustrechnung, Bilanz, Anhang und Lagebericht - erfüllt nach herrschender finnischer Auffassung regelmäßig diesen Anspruch ${ }^{440}$. Der finnische Gesetzgeber bringt mit der Beurteilung, der True and Fair View sei Teil der GoB, zum Ausdruck, dass ein Widerspruch zwischen GoB bzw. Einzelnormen und dem True and Fair View nicht entstehen könne. Der Jahresabschlussersteller, der den GoB Folge leistet, könne nicht in Konflikt mit dem True and Fair View geraten. Konsequenterweise bezieht der Gesetzgeber deshalb den True and Fair View v. a. auf seine eigene Arbeit ${ }^{441}$. Er hat ihn bei der Interpretation der Richtlinien anzuwenden und beim Entwurf der Einzelnormen zu beachten ${ }^{442}$.

Wenn auf Grund besonderer Umstände der Jahresabschluss trotz Anwendung der gesetzlichen Vorschriften und der GoB nicht die von ihm erwartete Aussagekraft erreicht, greift KPL 3:2.1 ${ }^{443}$ :

„Um dieses [das richtige und ausreichende Bild] zu erreichen, sind notwendige Zusatzangaben im Anhang zu machen.“

In den Gesetzesmaterialien heißt es hierzu, dass der „Einfluss subjektiver Elemente auf das Informationsergebnis möglichst gering “444 sein solle. Der finnische Gesetzgeber überlässt die Entscheidung über die Notwendigkeit von Zusatzangaben nicht dem Jahresabschlussersteller, sondern fällt sie selbst.

Die Notwendigkeit von Zusatzangaben vor dem Hintergrund des True and Fair View ist im Buchführungsgesetz von 1997 in sieben Fällen geregelt, welche den Konzernabschluss betreffen. In der Buchführungsverordnung 1997 finden sich sechs vergleichbare Regeln, die sowohl auf den Einzel- als auch auf den Konzernabschluss zu beziehen sind ${ }^{445}$. Von diesen 13 Regeln erlauben zehn die Abweichung von Einzelvorschriften unter Berufung auf den True and Fair View. Voraussetzung ist, dass im Anhang zusätzliche Angaben gemacht werden ${ }^{446}$. In diesen Fällen gibt der Gesetzgeber das Ermessen über die Notwendigkeit von $\mathrm{Zu}$ satzangaben an die Buchführungspflichtigen weiter. In drei anderen Fällen ist

$440 \quad$ Vgl. Kap. 3.1.3.4.2 dieser Arbeit.

441 Vgl. Artsberg (2000), S. 545, die ihre Aussage auch auf Schweden, Norwegen und Finnland ausdehnt.

442 Ebenso muss der KILA ihn bei der Erstellung von allgemeingültigen Anweisungen, Gutachten und Entscheidungen beachten.

443 KPL 3:2.1 ist die Transformation von Art. 2 Abs.4 der 4. EG-Richtlinie in finnisches Recht. Übersetzung von Kap. $3 \S 2$ Abs. 1 KPL ins Deutsche und Ergänzung des Paragraphen um die in eckigen Klammern stehende Erläuterung durch die Verfasserin.

444 Vgl. HE 111/1992, S. 4.

445 Den Einzelabschluss betreffend sind dies folgende Regeln: KPA 1:3.3, KPA 1:10.3 und 4, KPA 2:3.2, KPA 2:4.5, KPA 2:5.4 und KPA 2:9.3.2.

446 Einige Rechtsformen, für welche die Vorschriften der 4. EG-Richtlinie nicht zwingend gelten, dürfen diese Angaben unterlassen. 
Ergänzung von Einzelnormen um Zusatzangaben im Anhang auf Grund des True and Fair View zwingend.

$\mathrm{Zu}$ untersuchen ist, ob diese Umsetzung des Art. 2 Abs. 4 der 4. EG-Richtlinie in finnisches Recht eine Abweichung von den Richtlinienvorgaben darstellt oder eine Klarstellung. Nach dem Willen des Richtliniengebers sind zusätzliche Angaben im Anhang zwingend erforderlich, wenn das Ergebnis der Anwendung von Einzelvorschriften und das „richtige und ausreichende Bild“ als Zielvorstellung für den Abschluss eine Diskrepanz aufweisen, die erheblich für die Gesamteinschätzung des Unternehmens ist ${ }^{447}$.

Zahlreiche finnische Einzelvorschriften enthalten die Verpflichtung zur Anhangangabe $^{448}$. Praktische Fälle, in denen auch bei Erfüllung dieser Anhangangaben eine Diskrepanz zwischen der Aussage des Jahresabschlusses und dem zu vermittelnden Bild der Ertrags- und ökonomischen Lage besteht, treten relativ selten ein, zumal Umstände, welche die Zukunft beeinflussen, im Lagebericht zu erläutern sind. Es ist dennoch nicht nachzuvollziehen, warum sich die Zusatzangaben auf die vom Gesetzgeber ausdrücklich benannten Ausnahmen beschränken sollten. Der Oberste Finnische Verwaltungsgerichtshof hat unter Berufung auf den True and Fair View entschieden, dass auch solche Sachverhalte im Anhang aufzuführen sind, die vom Gesetzgeber nicht explizit geregelt sind ${ }^{449}$.

Obwohl sowohl der Richtliniengeber als auch der finnische Gesetzgeber die notwendigen Zusatzangaben ausdrücklich im Anhang fordern, hat der KILA eine andere Auffassung vertreten. Werden im Lagebericht notwendige Zusatzangaben platziert, dürfe dem falschen Ort des Ausweises keine ausschlaggebende Bedeutung beigemessen werden. Der Jahresabschluss könne dennoch einen True and Fair View vermitteln ${ }^{450}$.

447 Der Richtliniengeber geht im Grundsatz davon aus, dass bei Erfüllung der Einzelnormen auch der True and Fair View erfüllt wird. Er sieht das Abweichen von Einzelnormen nur für Ausnahmefälle vor. Vgl. Art. 2 Abs. 5 der 4. EG-Richtlinie.

448 Hierzu ist - nicht nur auf Finnland, sondern auch auf andere Staaten bezogen - kritisch anzumerken, dass ein Anhang nicht alle vorangegangenen Versäumnisse beheben kann. Dies ist insbesondere dann zu bedenken, wenn seine Textteile in einer Sprache wie der Finnischen verfasst sind, die den meisten ausländischen Jahresabschlusslesern fremd sein dürfte.

Vgl. KHO 2953/1997. Im konkreten Fall hat der Oberste Verwaltungsgerichtshof entschieden, dass eine Aktiengesellschaft, die in den Räumen einer Kreditanstalt angesiedelt ist, nicht über eigenes Personal verfügt und hauptsächlich durch die Kreditanstalt finanziert wird, zum Konsolidierungskreis der Kreditanstalt zu zählen ist. Die Ergebnisauswirkung von Wertpapiergeschäften, die zwischen der Aktiengesellschaft und der Kreditanstalt durchgeführt werden, ist im Anhang des Einzelabschlusses der Kreditanstalt anzugeben. 


\title{
3.1.3.4.5 True and Fair View-Override
}

Art. 2 Abs. 5 der 4. EG-Richtlinie verlangt, in Ausnahmefällen von den Einzelvorschriften abzuweichen, wenn das nach Abs. 3 geforderte Bild nicht vermittelt wird:

\begin{abstract}
„Ist in Ausnahmefällen die Anwendung einer Vorschrift dieser Richtlinie mit der in Abs. 3 vorgesehenen Verpflichtung unvereinbar, so muß von der betreffenden Vorschrift abgewichen werden, um sicherzustellen, daß ein den tatsächlichen Verhältnissen entsprechendes Bild im Sinne des Abs. 3 vermittelt wird. Die Abweichung ist im Anhang anzugeben und hinreichend zu begründen; ihr Einfluß auf die Vermögens-, Finanz- und Ertragslage ist darzulegen. Die Mitgliedstaaten können die Ausnahmefälle bezeichnen und die entsprechende Ausnahmeregelung festlegen."
\end{abstract}

Um subjektiv geprägte Verhaltensweisen und nicht richtlinienkonforme Rechnungslegungspraktiken zu unterbinden, hat die mit der Gesetzesnovellierung beauftragte Arbeitsgruppe dem Gesetzgeber vorgeschlagen, einen True and Fair View-Override von einer (eventuellauch erst nachträglich erteilten) Ausnahmeerlaubnis des KILA abhängig zu machen und mit dem True and Fair View-Override eine Meldepflicht an die Behörden zu verbinden ${ }^{451}$. Der Gesetzgeber ist dieser Empfehlung nicht vollständig gefolgt, sondern hat den Artikel mit folgendem Wortlaut in KPL 3:2.2 in das Buchführungsgesetz von 1997 aufgenommen $^{452}$ :

\footnotetext{
„Das Ministerium für Handel und Industrie entscheidet darüber, wann und wie von den Einzelnormen abgewichen werden darf, um ein richtiges und ausreichendes Bild zu vermitteln.“
}

$\mathrm{Zu}$ untersuchen ist, ob der finnische Gesetzgeber das Overriding Principle richtlinienkonform in nationales Recht umgesetzt hat. Der Wortlaut des KPL 3:2.2 ,,.. wann und wie von den Einzelnormen abgewichen werden darf..." lässt darauf schließen, dass es sich bei der Anwendung des Overrides in Finnland um ein Wahlrecht handelt. Nach dem Willen des Richtliniengebers ist der Override jedoch eine zwingend zu befolgende Vorschrift ${ }^{453}$.

Der finnische Gesetzgeber ist allerdings der Auffassung, der True and Fair View sei Bestandteil der Art guter Buchführung ${ }^{454}$. Einen Widerspruch zwischen den GoB bzw. den Einzelnormen und dem True and Fair View kann es deshalb - wie nach herrschender Auffassung in Deutschland auch - nicht geben. Das Abweichen von Einzelvorschriften vor dem Hintergrund des True and Fair View kann somit nicht zwingend erforderlich sein. Der Override ist folglich überflüssig. Es erscheint inkonsequent, dass der finnische Gesetzgeber - anders als der deutsche - das Overriding Principle überhaupt in nationales Recht transformiert hat. Aus

451 Vgl. KPL-Arbeitsgruppe 1995, Teil I (1996), S. 39-41.

452 Übersetzung ins Deutsche durch die Verfasserin.

453 Vgl. Art. 2 Abs. 5 der 4. EG-Richtlinie.

454 Vgl. Kap. 3.1.3.4.3 dieser Arbeit. 
den Gesetzesmaterialien geht eindeutig hervor, dass der Gesetzgeber Richtlinientreue angestrebt hat. Es kann vermutet werden, dass der Override eben wegen dieser Richtlinientreue der Vollständigkeit halber in nationales Recht aufgenommen wurde.

Der finnische Gesetzgeber bezieht Art. 2 Abs. 5 der 4. EG-Richtlinie auf seine eigene Arbeit ${ }^{455}$. Er begründet dies in erster Linie mit der Härte der strafrechtlichen Folgen und eventuell zu leistenden Schadensersatzzahlungen, die ein nicht korrekt erstellter Jahresabschluss nach sich ziehe ${ }^{456}$. Außerdem weist er darauf hin, dass ein individuell gestalteter True and Fair View-Override das Jahresabschlussziel der Vergleichbarkeit beeinträchtige. Nur durch eine Vorgabe des Ministeriums für Handel und Industrie könne eine irreführende Anwendung des Overrides unter dem Deckmantel des True and Fair View ausgeschlossen wer$\operatorname{den}^{457}$.

Der Gesetzgeber schreibt dem Ministerium für Handel und Industrie nicht vor, wann ein True and Fair View-Override zu genehmigen ist. Er führt lediglich an, dass sich bei Unternehmen bestimmter Branchen Preisschwankungen auf dem Rohstoffmarkt stark auf das Jahresergebnis auswirken können ${ }^{458}$. Mit Hinblick auf das richtige und ausreichende Bild könne diesen Unternehmen eine Bewertungsmethode wie „Last in, first out“ (LIFO) erlaubt werden, um ein getreueres Bild von Ergebnis und ökonomischer Lage zu erhalten, obwohl nach finnischem Recht sonst nur „First in, first out“ (FIFO) und die Durchschnittsmethode angewendet werden dürfen ${ }^{459}$.

Der True and Fair View-Override nach finnischem Recht entbindet also weder den KILA noch die mit der Rechnungslegung befassten Berufsstände und Buchführungspflichtigen von der Einhaltung der gesetzlichen Einzelvorschriften ${ }^{460}$.

Das Ministerium für Handel und Industrie hat noch keine Entscheidung darüber veröffentlicht, wann und wie im Einzelabschluss von den Einzelvorschriften abgewichen werden darf ${ }^{461}$. Daraus folgt, dass - obwohl er ausdrücklich umgesetzt

455 Vgl. Kap 3.1.3.4.3 und 3.1.3.4.4 dieser Arbeit.

456 Vgl. HE 173/1997, S. 15. Zu strafrechtlichen, handelsrechtlichen und steuerrechtlichen Konsequenzen bei Verstößen gegen die Art guter Buchführung, GoB und Einzelvorschriften, die zur Folge haben, dass kein True and Fair View vermittelt wird, vgl. Fußnote 346 und Kap. 3.1.3.1 dieser Arbeit.

457 Vgl. auch KPL-Arbeitsgruppe 1995, Teil I (1996), S. 39.

458 Als Beispiel für Branchen mit Preisschwankungen auf dem Rohstoffmarkt nennt der Gesetzgeber die Metallindustrie.

459 Vgl. HE 173/199, S. 15 und vgl. Leppiniemi und Leppiniemi (1997), S. 49.

460 Vgl. HE 173/1997, S.15.

461 Für den Konzernabschluss hat das Ministerium bislang in einem Fall entschieden, dass von Einzelvorschriften abgewichen werden darf, sofern der True and Fair View erreicht wird: Finnische Konzerne dürfen ihren Abschluss nach den Standards des IASB aufstellen und müssen finnisches Recht nicht mehr zwingend anwenden. Vgl. KTM 766/1998. 
worden ist - der True and Fair View-Override de facto in Finnland (noch) nicht existiert $^{462}$.

Kontrovers wird im europäischen Fachschrifttum diskutiert, wie der letzte Satz des Art. 2 Abs. 5 der 4. EG-Richtlinie zu verstehen sei, ob also Finnland ${ }^{463}$ den True and Fair View-Override korrekt in nationales Recht umgesetzt habe:

„Die Mitgliedstaaten können die Ausnahmefälle bezeichnen und die entsprechende Ausnahmeregelung festlegen.“

Zu prüfen ist, ob durch dieses Wahlrecht nach dem Willen des Richtliniengebers nur der nationale Gesetzgeber oder nicht doch der einzelne Jahresabschlussersteller oder gar beide dazu ermächtigt sind, den Override des True and Fair View anzuwenden.

In die Richtlinie eingefügt worden ist dieser Satz auf Wunsch der italienischen Delegation, welche die Möglichkeiten der Unternehmen, den True and Fair ViewOverride auszüben, möglichst weit einschränken wollte. Andere Autoren vertreten die Auffassung, dass es ursprüngliche Intention des Richtliniengebers gewesen sei, einzelne Jahresabschlussersteller und nicht die nationalen Gesetzgeber zum True and Fair View-Override zu ermächtigen ${ }^{464}$.

Der True and Fair View-Override müsse in Bezug auf eine einzelne Unternehmung angewendet werden und nicht in Bezug auf alle Unternehmen oder eine Kategorie von Unternehmen. Es sei für eine Norm nicht möglich, ein Ausnahmefall zu sein. Eine vom Gesetzgeber vorgegebene Regelung, die von der Richtlinie abweiche, könne nicht durch Art. 2 Abs. 5 der 4. EG-Richtlinie gerechtfertigt wer$\operatorname{den}^{465}$.

Andererseits könnte nach Auffassung anderer Autoren eine durch den Gesetzgeber eingeführte Ausnahmeregelung die Verlässlichkeit und Nützlichkeit eines Jahresabschlusses und die Vergleichbarkeit der Jahresabschlüsse untereinander erhöhen, indem sie die Anwendung verschiedener denkbarer Möglichkeiten eines True and Fair View-Override bei gleichen oder ähnlichen Sachverhalten verhindert ${ }^{466}$.

462 Ob der KILA mit der Erteilung von Ausnahmeerlaubnissen nicht doch schon in einigen Fällen einen True and Fair View-Override ermöglicht hat, ist fraglich. Auf eine nähere Untersuchung dieser Frage wird angesichts der Fülle der vom KILA getroffenen Entscheidungen an dieser Stelle jedoch verzichtet.

463 Diese Frage bezieht sich auch auf Schweden, das den True and Fair View-Override ähnlich wie Finnland auffasst.

464 Vgl. van Hulle (1997), S. 714.

465 Vgl. Kontaktausschuss der EU, Accountancy November 1990, zitiert bei Ordelheide (1993), S. 87.

466 Vgl. Alexander (1996), S. 491, der auf S. 490 auch darauf hinweist, dass die Praxis in Großbritannien ihre Meinung dahingehend wandelt, dass der True and Fair View-Override nicht nur von den Unternehmen, sondern auch vom Gesetzgeber anzuwenden sei. 
Das Konzept des True and Fair View ziele v. a. auf die Standard Setter in Europa ab, nicht aber auf die Unternehmen ${ }^{467}$.

Die EU-Kommission hat im Jahre 1997 betont, dass die Mitgliedstaaten keine Einzelnormen oder solche Wahlrechte verabschieden dürften, die der 4. EGRichtlinie widersprächen ${ }^{468}$. Sie hat den Mitgliedstaaten einerseits verboten, den True and Fair View-Override für Einzelfälle als generelle Norm zuzulassen. Andererseits dürfen die Mitgliedstaaten den Override für bestimmte Branchen und/oder Sachverhalte gestatten. VAN HULLE argumentiert, dass - obwohl nicht ursprüngliche Intention des Richtliniengebers - der Wortlaut der Richtlinie dahingehend interpretiert werden könne, dass der True and Fair View-Override durch den nationalen Gesetzgeber anzuwenden sei. Den Mitgliedstaaten sei nur deshalb gestattet, Ausnahmefälle zu erlauben und Ausnahmeregelungen zu definieren, um sich gegen den Missbrauch des True and Fair View-Overrides zu schützen ${ }^{469}$.

Der finnische Gesetzgeber hat somit den True and Fair View-Override hinsichtlich der angesprochenen Fragestellung richtlinienkonform in finnisches Recht implementiert. Nicht explizit umgesetzt hat er die Forderung des Richtliniengebers nach Angabe und Begründung des True and Fair View-Overrides im Anhang. Diese Forderung muss - so sieht es auch die Gesetzesbegründung vor - das Ministerium für Handel und Industrie bei der Entscheidung darüber, wann und wie von Einzelvorschriften abgewichen werden darf, stellen ${ }^{470}$.

467 Vgl. Burlaud (1993), S. 98.

Die EU-Kommission verlautet im englischen Original: „In the interest of harmonization, Member States may not use the last sentence of Article 2 (5) in order to introduce an accounting rule of a general nature, which is contrary to provisions of the Directive, nor can they use this sentence to create additional options allowing for accounting treatments which are not in conformity with the Directive." Vgl. EU-Kommission XV/7009/97 und Zitat dieser Stelle bei van Hulle (1997), S. $713 \mathrm{f}$.

Vgl. Van Hulle (1997), S. 714. Van Hulle hat hiermit seine 1994 geäußerte Meinung geändert, der True and Fair View-Override beziehe sich auf Einzelunternehmen und nicht auf Gesetzgeber: „This true and fair view override must be stated in the law. I should also stress that the override can only be applied by companies individually and that standard setters cannot invoke this principle in order to permit or to require accounting practices which are not in conformity with the Directive. Vgl. Van Hulle (1994), S. 235. 


\subsection{Zusammenfassender Überblick}

Der finnische Gesetzgeber hat den True and Fair View als Forderung nach ,richtigen und ausreichenden Informationen“ bzw. nach einem ,richtigen und ausreichenden Bild“ der Ertrags- und ökonomischen Lage in finnisches Recht aufgenommen. Er sucht hiermit das Harmonisierungsziel zu erreichen. Den Begriff des True and Fair View definiert der finnische Gesetzgeber nicht. Dies hat zur Folge, dass die Fachwelt den True and Fair View erörtert ${ }^{471}$. Er stellt herrschender Auffassung zufolge ein dynamisches Konzept dar, das bei der Jahresabschlusserstellung zu berücksichtigen ist. Der Jahresabschluss eines Unternehmens hat in Umfang und Form allgemein anerkannt zu sein. Umfang und Form ergeben sich aus der Art guter Buchführung; der True and Fair View ist ein Teil eben dieser. Er ist den GoB gleichzusetzen, ergänzt diese im Hinblick auf den zu vermittelnden Gesamteindruck über ein Unternehmen und auf die Teile des Jahresabschlusses, die nicht primär der Buchhaltung entstammen. Gemeint ist hier v. a. der Lagebericht.

Gleichzeitig wird der True and Fair View von den GoB dahingehend eingeschränkt, dass die an ihn gestellten Anforderungen relativiert werden ${ }^{472}$.

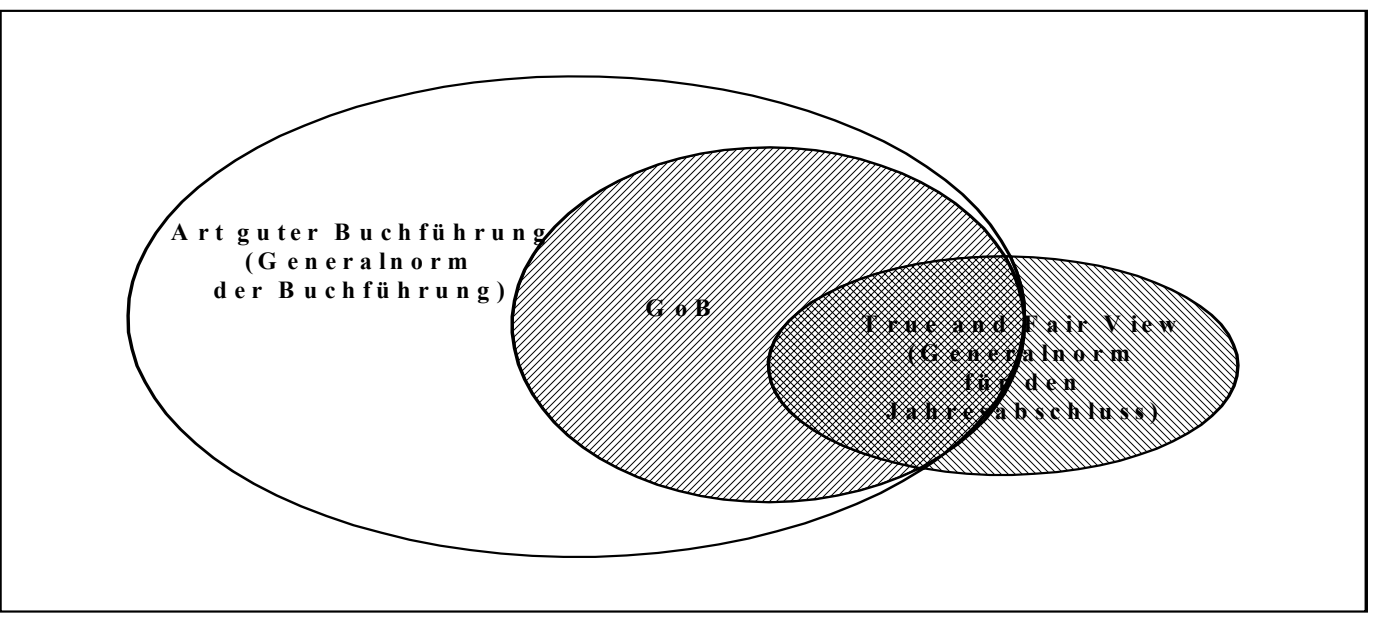

\section{Abb. 6: Verhältnis des True and Fair View zur Art guter Buchführung und anderen Grundsätzen ordnungsmäßiger Buchführung}

Nach herrschender Auffassung kann ein Widerspruch zwischen GoB und True and Fair View nicht entstehen. Der True and Fair View, wenn er mit Inhalt gefüllt werden soll, ist daher v. a. vom Gesetzgeber und vom KILA bei der Interpretation der Richtlinien anzuwenden und im Gesetzgebungsverfahren bzw. bei der Erstellung von Anweisungen, Gutachten oder Entscheidungen zu berücksichtigen. So lässt sich erklären, dass nicht der einzelne Jahresabschlussersteller sich mit der 
Frage nach der Notwendigkeit von Zusatzangaben im Anhang auseinander zu setzen hat, sondern der Gesetzgeber diese für Einzelfälle regelt ${ }^{473}$.

Der finnische Gesetzgeber bezieht auch den True and Fair View-Override auf seine eigene Arbeit. Der Override wird in Finnland nicht vom einzelnen Jahresabschlussersteller auf den Sachverhalt bezogen angewendet, sondern vom Ministerium für Handel und Industrie gestattet. Das Ministerium kann den Override in Einzelfällen zulassen, um einer missbräuchlichen Anwendung des Overrides durch einzelne Buchführungspflichtige vorzubeugen ${ }^{474}$.

\subsubsection{Inhalt, Dokumentation und Aufbewahrung der Buchführungsunterlagen}

Weder die 4. EG-Richtlinie noch die Standards des IASB enthalten Vorschriften zur Buchführung, Buchführungsmethoden oder zum Kontenrahmen. Sie regeln ausschließlich die Aufstellung des Jahresabschlusses und seine Darstellungsweise und setzen damit implizit die Anwendung adäquater Buchführungssysteme voraus $^{475}$.

Der finnische Gesetzgeber hat mit Kap. 2 des KPL (1997) „Buchung von Geschäftsvorfällen und Buchführungsunterlagen“"von früheren Buchführungsgesetzen erheblich abweichende Normen geschaffen, die nicht auf der Umsetzung von Gemeinschaftsrecht oder internationalen Regeln beruhen. Die neuen Vorschriften basieren auf der Notwendigkeit, die elektronische Datenverarbeitung für die Buchhaltung zu ermöglichen und zu regeln. Auch zentrale traditionelle Grundsätze ordnungsmäßiger Buchführung sind geändert worden ${ }^{476}$. Der Gesetzgeber hat von der Möglichkeit, modernste Buchführungsvorschriften im Hinblick auf elektronische Datenverarbeitung einzuführen, nicht konsequent Gebrauch gemacht. Er hat vielmehr zeitlose Regeln kodifiziert, damit sich diese GoB in Zukunft ohne Gesetzesänderung wandeln können ${ }^{477}$.

Verantwortlich für die Ordnungsmäßigkeit von Buchführung (und Jahresabschluss) zeichnet der Buchführungspflichtige selbst. Unter „Buchführungspflichtigen“" sind bei Personengesellschaften der oder die Gesellschafter ${ }^{478}$, bei Aktien-

Vgl. Kap. 3.1.3.4.4 dieser Arbeit.

Vgl. Kap. 3.1.3.4.5 dieser Arbeit.

Anders z. B. die Situation in Frankreich, das als Mitgliedstaat der EU seinen Unternehmen einen einheitlichen Standardkontenrahmen vorschreibt.

476 Vgl. Ilkka (1998), S. 287 und S. 298.

477 Vgl. Ilkka (1998), S. 287.

478 Geregelt ist dies in den jeweiligen rechtsformspezifischen Spezialgesetzen. 
gesellschaften der Vorstand und der Geschäftsführer zu verstehen ${ }^{479}$. Für die Überwachung der Einhaltung aller Buchführungsvorschriften zuständige Aufsichtsbehörden sind das Ministerium für Handel und Industrie, das Patent- und Registeramt sowie das Aufsichtsamt für die Finanzmärkte ${ }^{480}$, zudem hinsichtlich der Anwendung und Weiterentwicklung der Art guter Buchführung der KILA ${ }^{481}$. Diese Organe können gegebenenfalls die Einleitung von Gerichtsverfahren gegen den Buchführungspflichtigen veranlassen, die strafrechtliche, handelsrechtliche und steuerrechtliche Konsequenzen in Form von Geld- oder Haftstrafen zur Folge haben können ${ }^{482}$.

In seiner Buchführung hat der Buchführungspflichtige Ausgaben, Einnahmen, Finanztransaktionen sowie ihre Korrektur- und Abgrenzungsposten zu erfassen ${ }^{483}$. Ein Standard-Kontenrahmen wird durch den Gesetzgeber nicht vorgegeben. Manche Wirtschaftsverbände empfehlen ihren Mitgliedern, von ihnen entworfene Kontenrahmen anzuwenden, um die Vergleichbarkeit von Unternehmen einer Branche zu verbessern und das Zusammenstellen statistischen Datenmaterials zu erleichtern $^{484}$.

Buchungen haben grundsätzlich bei Leistungserbringung $\mathrm{zu}$ erfolgen. Es ist jedoch auch erlaubt, einen Geschäftsvorfall zum Zeitpunkt des Zahlungsvorgangs in den Büchern zu vermerken, sofern jederzeit der aktuelle Stand der Forderungen und Verbindlichkeiten aus Lieferungen und Leistungen ermittelbar ist ${ }^{485}$.

Geschäftsvorfälle sind in chronologischer Reihenfolge im Grundbuch und nach systematischen Gesichtspunkten geordnet im Hauptbuch festzuhalten. Hierbei ist zu beachten, dass Finanztransaktionen täglich, alle anderen Geschäftsvorfälle mindestens einmal monatlich zu erfassen sind ${ }^{486}$.

Grundlage einer jeden Buchung ist der Beleg, auf dem die Daten der Belegerstellung und der Leistungserbringung, die Belegnummer, Namen des Leistungserbringers und -empfängers, der Inhalt des Geschäftsvorfalls sowie der Geldbetrag ver-

479 Vgl. OYL 8:6.2: „Der Vorstand hat dafür zu sorgen, dass die Überwachung von Buchführung und Vermögenssicherung sachgerecht geregelt sind. Der Geschäftsführer hat dafür Sorge zu tragen, dass die Buchführung der Gesellschaft dem Gesetz entsprechend und die Vermögenssicherung zuverlässig geregelt sind." (Übersetzung ins Deutsche durch die Verfasserin).

$480 \quad$ Vgl. KPL 8:1.

481 Vgl. KPL 8:2.1

482 Vgl. auch Fußnote 346 und Kap. 3.1.3.1 dieser Arbeit.

483 Vgl. KPL 2:1 und KPL 2:2.

484 Vgl. Järvinen et al. (2000), S. 99-112, die Beispiele für Kontenrahmen aufzeigen und vgl. Majala (2001 b), S. 968. Studien darüber, ob und wie viele Unternehmen diese Kontenrahmen anwenden, stehen aus. 
merkt sein müssen ${ }^{487}$. Buchungen sind eindeutig und dahingehend dauerhaft zu dokumentieren, dass sie weder unbemerkt verändert werden können noch im Zeitablauf unlesbar werden ${ }^{488}$.

Der Grundsatz der Nachprüfbarkeit (Audit trail) muss für jeden Sachverhalt gewährleistet $\operatorname{sein}^{489}$, wobei die Buchführung grundsätzlich in klarer Schriftform zu erfolgen hat. Eine Ausnahme bildet der Gebrauch von computerisierten Buchungssystemen, den das Gesetz in KPL 2:8 gestattet und der durch eine KILAAnweisung vom 22. März 2000 geregelt wird ${ }^{490}$. Hiernach dürfen elektronische Datenträger zur Aufbewahrung genutzt werden, sofern die auf ihnen hinterlegten Informationen bei Bedarf in klare, geschriebene Form transformiert werden können. Seit In-Kraft-Treten des KPL (1997) ist es dem Buchführungspflichtigen erlaubt, Belege und durch sie begründete Buchungen gleichzeitig auf denselben elektronischen Datenträgern zu speichern. Ein Exemplar des Bilanzbuchs muss jedoch immer in Papierform vorliegen.

Das Bilanzbuch enthält gemäß KPL 3:8

- den Jahresabschluss des Buchführungspflichtigen (Bilanz, Gewinn- und Verlustrechnung, Anhang und Lagebericht),

- ein Verzeichnis über die Bücher des Buchführungspflichtigen (Hauptbuch, Grundbuch, Auflistung aller Nebenbücher) mit der Angabe über ihre Form (Loseblattsammlung, gebundenes Buch, Karteikarten, Computerausdruck u. Ä.) und

- ein Verzeichnis über die Belegarten des Buchführungspflichtigen (Jahresabschlussbelege, Belege über Geschäftsvorfälle Einkauf, Belege über Geschäftsvorfälle Verkauf, Zahlungsbelege, Kassenbelege, Bankbelege u. Ä.) mit Erklärung darüber, wie die Belege aufbewahrt werden (Papierform, Speicherung auf Datenträgern u. Ä.).

Die Seitenzahlen des Bilanzbuchs müssen vollständig nummeriert sein, und es muss ersichtlich sein, wie viele Seiten das Bilanzbuch insgesamt beinhaltet. Zweck des Bilanzbuchs ist es, die Prüfung der Buchführung zu erleichtern ${ }^{491}$.

Belege sind sechs Jahre, Grund-, Haupt- und Nebenbücher zehn Jahre aufzubewahren $^{492}$. Physischer Aufbewahrungsort bis zur Feststellung des Jahresabschlusses ist Finnland; nach der Feststellung dürfen die Buchführungsunterlagen auch in einem anderen OECD-Staat gelagert werden, sofern von Finnland aus jederzeit

\footnotetext{
Vgl. KPL 2:5.

Vgl. KPL 2:7 und Turja (1998), S. 155.

Vgl. KPL 2:6 und vgl. Fußnote 357 sowie Kap. 3.1.3.2 dieser Arbeit.

Vgl. KILA-Anweisung „Informationstechnologie in der Buchführung“.

Vgl. KPL 3:8 und vgl. KILA-Anweisung „Informationstechnologie in der Buchführung“.

Vgl. KPL 2:10.
} 
ein Zugriff auf die Daten möglich ist und die Original-Unterlagen in angemessenem Zeitrahmen nach Finnland transportiert werden können ${ }^{493}$.

\subsubsection{Ansatzkriterien}

\subsubsection{Erfassung von Erträgen und Aufwendungen}

Bei der Periodisierung von Einnahmen und Ausgaben stellt sich die Frage, in welchem Geschäftsjahr Einnahmen als Erträge und Ausgaben als Aufwendungen zu erfassen oder aber zu bilanzieren sind: der Realisationszeitpunkt ${ }^{494}$.

Dem Grundsatz der leistungsbezogenen Abgrenzung entsprechend ist hinsichtlich der Einnahmen der Zeitpunkt der Leistungserbringung des Bilanzierenden für eine erfolgswirksame Erfassung im Jahresabschluss relevant ${ }^{495}$.

Bei den Ausgaben ist der Übernahmezeitpunkt eines Produktionsfaktors für die Beurteilung der Frage, ob eine Ausgabe als Aufwand in der Gewinn- und Verlustrechnung oder als zu aktivierender Betrag in der Bilanz zu betrachten ist, maßgeb$\operatorname{lich}^{496}$. Es gilt, das Vorsichtsprinzip zu beachten, um Erträge und aktivierte Beträge im Jahresabschluss nicht zu hoch und Aufwendungen nicht zu niedrig auszuweisen $^{497}$.

Der Realisationszeitpunkt von Erträgen bzw. Aufwendungen unter Berücksichtigung des Vorsichtsprinzips ist in Finnland dann erreicht, wenn folgende Bedingungen erfüllt sind:

- Ein Vertrag über eine Lieferung oder Leistung muss abgeschlossen sein.

- Eine Lieferung oder Leistung muss erbracht worden sein.

- Der Gegenstand des Vertrags muss den Verfügungsbereich des liefernden oder leistenden Unternehmens verlassen haben.

Zusätzlich zum Grundsatz der leistungsbezogenen Abgrenzung schreibt der finnische Gesetzgeber einzelne Ansatz- und Bewertungskriterien in KPL 5 vor. Die zentrale Ansatzvorschrift des KPL 5:1 regelt allgemein und grundsätzlich die Periodisierung von Ausgaben, Verlust und Einnahmen. Sie legt Kriterien dafür fest, was generell als Ertrag bzw. Aufwand anzusehen ist und somit prinzipiell in der Gewinn- und Verlustrechnung erfasst werden darf und muss. Die übrigen, diese

493 Vgl. KPL 2:9 und vgl. Turja (1998), S. 157.

494 Zum mit dem Realisationszeitpunkt verbundenen Grundsatz leistungsabhängiger Buchführung vgl. Kap. 3.1.3.2 dieser Arbeit und die dort aufgeführte Literatur.

495 Vgl. KPL 2:3.1.

496 Vgl. KPL 2:3.1 und vgl. KPL-Arbeitsgruppe 1995, Teil I (1996), S. 37.

497 Vgl. Leppiniemi und Leppiniemi (1997), S. 70. 
Kriterien nicht erfüllenden Werte sind grundsätzlich in der Bilanz als Aktiv- oder Passivwerte anzugeben (Ausschlussprinzip).

Die allgemeine Ansatzregel nach KPL 5:1 lautet wie folgt ${ }^{498}$ :

Die Einnahmen eines Geschäftsjahrs werden als Erträge erfolgswirksam in der Gewinn- und Verlustrechnung gebucht. Von den Erträgen werden als Aufwendungen diejenigen Ausgaben abgezogen, denen voraussichtlich keine zukünftigen Einnahmen mehr gegenüberstehen, ebenso die Verluste. Andere Ausgaben dürfen den nachfolgenden Paragraphen entsprechend aktiviert werden.

Nach diesem Verständnis ist jede Einnahme, die auf einer Leistungserbringung im Geschäftsjahr beruht, gleichzeitig ein Ertrag. Ist eine Einnahme nicht dem Geschäftsjahr zuzuordnen, handelt es sich bei ihr um einen transitorischen Rechnungsabgrenzungsposten, der in der Bilanz auszuweisen ist ${ }^{499}$.

Die Frage nach der Zuordnung von Ausgaben zu Aufwendungen eines Geschäftsjahrs einerseits oder zu einem zu aktivierenden Betrag andererseits erweist sich als kompliziert.

Der Gesetzgeber definiert in KPL 5:1 Aufwendungen zum einen als diejenigen Ausgaben, die im Rahmen der gewöhnlichen Geschäftstätigkeit anfallen und denen voraussichtlich keine zukünftigen Einnahmen mehr gegenüberstehen.

Zum anderen versteht der finnische Gesetzgeber unter Aufwendungen „Verluste“. Im finnischen Schrifttum werden als Beispiele für solche Verluste Verpflichtungen aus übernommenen Bürgschaften oder eingegangenen Sicherungsverhältnissen genannt, ebenso der Abgang von Vermögensgegenständen oder finanziellen Mitteln aus dem Unternehmen durch Feuer und ähnliche Schadensfälle, Diebstahl oder Unterschlagung ${ }^{500}$.

Grundsätzlich darf aktiviert werden, was nicht dem Aufwand zugerechnet werden muss.

Zusätzlich - und implizit in KPL 5:1 enthalten - ist der Grundsatz der periodengerechten Abgrenzung bei der Erfassung von Aufwendungen und Erträgen zu befolgen: Aufwendungen sind der Gewinn- und Verlustrechnung derjenigen Periode zuzurechnen, in der die in direktem Zusammenhang mit ihnen stehenden entsprechenden Erträge anfallen.

498 Übersetzung ins Deutsche durch die Verfasserin.

499 Vgl. Järvinen et al. (2000), S. 412.

500 Vgl. Järvinen et al. (2000), S. 416. 


\subsubsection{Ansatz von Aktiva}

Ebenso wie der Richtliniengeber liefert der finnische Gesetzgeber keine Legaldefinition des Begriffs „Vermögen“ bzw. ,Vermögenswert“.

Der Gesetzgeber unterteilt in KPL 5:1 Ausgaben in Aufwendungen und solche Ausgaben, denen voraussichtlich zukünftige Einnahmen gegenüberstehen. Diese Ausgaben sind grundsätzlich als Vermögenswerte zu aktivieren.

Vermögenswerte in finnischen Abschlüssen sind nicht gleichzusetzen mit Vermögensgegenständen in deutschen, der handelsrechtlichen Konzeption entsprechenden Abschlüssen. Nach finnischem Verständnis ist eine Ressource zu aktivieren, über die ein Unternehmen verfügt und die als zukünftige Einnahmequelle angesehen wird. Es wird der Zufluss eines wirtschaftlichen Nutzens in Form von Zahlungsmitteln oder Zahlungsmitteläquivalenten verursacht durch diese Ressource erwartet (Aktivierungsgrundsatz) ${ }^{501}$. Wirtschaftlicher Nutzen in Form von Kosteneinsparungen oder anderen Vorteilen der internen Nutzung darf nicht aktiviert werden $^{502}$.

Die Festlegung der künftigen Einnahmenerwartung ist im Regelfall dann relativ einfach, wenn einer Ausgabe eine einmalige Einnahme gegenübersteht. Sind mit einer Ausgabe jedoch mehrmalige Einnahmen, eventuellüber mehrere Geschäftsjahre verteilt, verbunden, erweist sich die Einnahmenplanung oft als schwierig ${ }^{503}$. Zudem können bei Änderung der Einnahmenerwartung in früheren Geschäftsjahren aktivierte Ausgaben nicht mehr rückwirkend geändert werden ${ }^{504}$.

Auch schreiben Einzelvorschriften des KPL 5 vom Aktivierungsgrundsatz abweichende Verfahren, beispielsweise planmäßige Abschreibungen, vor ${ }^{505}$.

Die Grundsatzregel des KPL 5:1 lässt den Erwartungen des Buchführungspflichtigen hinsichtlich der künftigen Entwicklung und der Unternehmensstrategie und -

501 Vgl. Järvinen et al. (2000), S. 411-419 und vgl. Leppiniemi (2000 a), S. 137.

502 Zum besseren Verständnis der Beziehung zwischen Aktivierungsfähigkeit und Einnahmenerwartung wird in der Literatur folgendes Beispiel aufgeführt:

Einer Ausgabe von $€ 60,00$ steht insgesamt die Einnahmenerwartung von $€ 100,00$ gegenüber. $€ 50,00$ dieser Erwartung (= 50 Prozent) sind bereits realisiert worden; folglich dürfen im Jahresabschluss noch max. $€ 30,00$ (= 50 Prozent der ursprünglichen Ausgabe) in der Bilanz aktiviert sein, mindestens $€ 30,00$ müssen als Aufwand in der Gewinn- und Verlustrechnung diesen Geschäftsjahres oder früherer Geschäftsjahre erfasst sein. Nun wird die künftige Einnahmenerwartung nach unten korrigiert; es werden nur noch $€ 30,00$ erwartet. Die Gesamt-Einnahmenerwartung $\mathrm{zu}$ der getätigten Ausgabe von $€ 60,00$ beträgt nicht mehr $€ 100,00$, sondern nur noch $€ 80,00$. Als Konsequenz dürfen max. 60/80 des in der Bilanz stehenden Teilbetrags der Ausgabe aktiviert werden: $€ 30 * 60 / 80=€ 22,50$. Mit der Verringerung der Einnahmenerwartung muss sich der Aktivwert der Ausgabe in gleichem Maße verringern. Dieses Beispiel verdeutlicht die Problematik der Anwendung dieses Aktivierungsgrundsatzes für die Praxis. Vgl. Järvinen et al. (2000), S. 413-414. 
planung sowie der Budgetierung eine große Bedeutung zukommen. Eingeschränkt wird die Freiheit der Buchführungspflichtigen jedoch durch die Art guter Buchführung ${ }^{506}$, die eine dem Sachverhalt gerecht werdende Vorsicht (KPL 3:3) bei der Abgrenzung von Aufwand und Aktivierungswert verlangt. Diese Vorsicht hat unabhängig vom Jahresergebnis zu sein. Es ist generell eine umso größere Vorsicht geboten, je unsicherer und schwieriger die Bewertung der zukünftigen Einnahmen ist. Übertriebene Vorsicht sollte jedoch im Sinne des True and Fair View vermieden werden ${ }^{507}$.

SAARIOs Ausgaben-Einnahmen-Theorie der Buchführung bildet zwar nach Verdrängung durch die 4. EG-Richtlinie nicht mehr die wesentliche Grundlage geltenden finnischen Buchführungsrechts, aber ihr historisch bedingter Einfluss auf die Buchführung wird im Konzept einer dynamischen Bilanz ersichtlich ${ }^{508}$. Auch wird die traditionell stärkere Gewichtung der Gewinn- und Verlustrechnung im Verhältnis zur Bilanz schon durch den Wortlaut des Aktivierungsgrundsatzes deutlich, der sich auf den Aufwand konzentriert und den Aktivwert als Restgröße sieht, nicht aber den Begriff des Vermögenswerts definiert.

Die Bilanzgliederung des KPA 1:6 zählt die Vermögenswerte auf, die in der Bilanz anzusetzen sind.

Daneben hat der finnische Gesetzgeber in KPL 4:3 den Art. 15 Abs. 1 und Abs. 2 der 4. EG-Richtlinie umgesetzt und das Anlage- und Umlaufvermögen definiert $^{509}$ :

Vermögenswerte der Aktivseite in der Bilanz werden auf Basis ihrer Zweckbestimmung in Anlage- und Umlaufvermögen unterteilt. Zum Anlagevermögen zählen diejenigen Posten, die in mehreren Geschäftsjahren Einnahmen erzielen sollen. Alle anderen Posten sind Bestandteil des Umlaufvermögens.

Zudem besteht mit KPL 4:4 eine Vorschrift, die für die Aktivseite der Bilanz anders als in anderen europäischen Staaten der Fall - eine Dreiteilung in Anlagevermögen, Umlaufvermögen und Finanzumlaufvermögen vorsieht ${ }^{510}$ :

$506 \quad$ Vgl. Kap. 3.1.3.1 dieser Arbeit.

507 Vgl. Järvinen et al. (2000), S. 415.

508 Ebenso wird hier der Einfluss von SCHMALENBACH auf SAARIO und seine AusgabenEinnahmen-Theorie deutlich. Auch SCHMALENBACH definiert den Zugang von Vermögensgegenständen als Ausgaben, die noch nicht Aufwand sind. Er sieht die Bilanz als Konto aller „schwebenden Posten“, die alle Elemente aufnimmt, die erst in Folgejahren in der Gewinn- und Verlustrechnung zu verrechnen sind. Vgl. hierzu Schmalenbach (1953), S. 51-53. Übersetzung ins Deutsche durch die Verfasserin. Mit der Unterscheidung in Anlage- und Umlaufvermögen differieren der Richtliniengeber und der finnische Gesetzgeber von der Auffassung des IASB. Nach IAS 1 (revised 1997) beispielsweise sind langfristige Forderungen und Vorräte, die nicht innerhalb der ,normalen“ Umschlagszeit eines Unternehmens realisiert (veräußert) werden, dem Anlagevermögen zuzuordnen, während sie nach Richtlinie als Umlaufvermögen auszuweisen sind. Vgl. van Hulle und van der Tas (2001), S. 804.

Übersetzung ins Deutsche durch die Verfasserin. 
Das Anlagevermögen besteht aus Gegenständen, einzeln verwertbaren Rechten und anderen Gütern, welche die Kriterien des KPL 4:3 erfüllen.

Zum Umlaufvermögen zählen solche Güter, die im Rohzustand oder veredeltem Zustand dazu gedacht sind, entweder an Dritte veräußert oder aber konsumiert zu werden.

Dem Finanzumlaufvermögen sind Zahlungsmittel, Forderungen oder Zahlungsmitteläquivalente zuzurechnen.

Zum Anlagevermögen zählen - richtlinienkonform - nach KPL 4:3 Posten, die dazu bestimmt sind, in mehr als nur einem Geschäftsjahr Einnahmen zu erzielen. Diese Posten sind im Regelfall einzeln verwertbare Vermögensgegenstände, müssen es jedoch nicht sein. Ausschlaggebend für eine Aktivierung ist nach KPL 4:4 - anders als nach der handelsrechtlichen Konzeption in Deutschland - die Einnahmenerwartung, nicht die selbstständige Verwertbarkeit. So können KILA-Entscheidungen zufolge Ausgaben wie Forschungskosten, Schulungskosten, Werbekosten, Modernisierungskosten ${ }^{511}$ oder Beratungskosten ${ }^{512}$ aktiviert werden, sofern eine Einnahmenerwartung mit ihnen verbunden ist, die ihnen eindeutig zugeordnet werden $\operatorname{kann}^{513}$. Ausgewiesen werden solche Posten unter dem Oberbegriff „Immaterielle Vermögensgegenstände“ als „Andere Ausgaben mit lang anhaltender Wirkung“.

In der Praxis wird eine Einnahmenerwartung für solche Posten nur schwer zu dokumentieren und zu quantifizieren sein. Der KILA betont deshalb, dass eine erfolgswirksame Erfassung der genannten Kosten als Aufwendungen in der Gewinn- und Verlustrechnung immer der Art guter Buchführung entspreche und immer mit dem Vorsichtsprinzip in Einklang stehe ${ }^{514}$. Für immaterielle Vermögensgegenstände existiert somit ein Aktivierungswahlrecht, keine Aktivierungspflicht.

Hauptkriterium für den Ansatz von Gegenständen des Umlaufvermögens ist die nach KPL 4:3 mit ihnen verbundene, nicht über ein Geschäftsjahr hinausgehende Einnahmenerwartung. Der Gesetzgeber konkretisiert in KPL 4:4, dass der Buchführungspflichtige die Intention haben müsse, diese Gegenstände - gleichgültig, in welcher Produktionsstufe sie sich zum Bilanzstichtag befänden - zu veräußern oder zu verbrauchen. Neben materiellen Gegenständen des Umlaufvermögens können auch solche immaterieller Art bilanziert werden. Die Fachwelt nennt hier Wertpapiere, Dienstleistungen, Planungsarbeiten oder andere Ausgaben, denen als künftige Einnahmequelle kein materieller Vermögensgegenstand diene, deren Gegenwert aber als Leistung veräußert werden könne ${ }^{515}$.

Das Finanzumlaufvermögen besteht nach KPL 4:4 aus Zahlungsmitteln, Forderungen und Zahlungsmitteläquivalenten. Mit letzteren können beispielsweise Ak-

$511 \quad$ Vgl. KILA 1588/1999.

512 Vgl. KILA 1534/1998.

513 Vgl. Järvinen et al. (2000), S. 381. Auszugehen ist hier vom einzelnen Vermögenswert, nicht von seiner Cash-generating unit.

514 Vgl. KILA 1579/1999.

515 Vgl. Järvinen et al. (2000), S. 383. 
tien gemeint sein, die als Zahlungsmittel für erbrachte Leistungen entgegengenommen worden sind ${ }^{516}$. Das Finanzumlaufvermögen unterscheidet sich in seinem Charakter wesentlich von Anlagevermögen und Umlaufvermögen, weil mit ihm keine künftigen Einnahmeerwartungen verknüpft sind. Die Umwandlung von Anlagevermögen oder Umlaufvermögen in Finanzumlaufvermögen erfolgt ausschließlich durch Veräußerung eines Gegenstands aus diesen beiden Gruppen. Das Finanzumlaufvermögen bildet also eine vom Gesetzgeber vorgesehene Ausnahme zum Aktivierungsgrundsatz ${ }^{517}$. Aus dieser Ausnahme, der dreigeteilten Gliederung der Aktivseite in Anlage-, Umlauf- und Finanzumlaufvermögen und der Definition dieser Begriffe wird abermals die sich bis heute auswirkende Bedeutung der Ausgaben-Einnahmen-Theorie ersichtlich ${ }^{518}$.

Bei Erfüllung der Ansatzkriterien des Aktivierungsgrundsatzes besteht Aktivierungspflicht. Eine Ausnahme hiervon bildet lediglich das Aktivierungswahlrecht für immaterielle Vermögensgegenstände ${ }^{519}$. Ein Verbot über eine Aktivierung von Nicht-Aufwand existiert in Finnland nicht. Dennoch sind neben den Kriterien des Aktivierungsgrundsatzes und der Legaldefinition von Anlage-, Umlauf- und Finanzumlaufvermögen teilweise abweichende gesetzliche Vorschriften zu beachten. Dies wird auch durch den Wortlaut des KPL 5:1.3 deutlich, der besagt, dass ,andere Ausgaben den nachfolgenden Paragraphen entsprechend aktiviert werden dürfen“. Ergänzungen $\mathrm{zu}$ und Ausnahmen vom Aktivierungsgrundsatz des KPL 5:1 hinsichtlich Ansatz und/oder Bewertung finden sich in

- KPL 5:4 Percentage of Completion-Methode

- KPL 5:5 Periodisierung der Anschaffungs- oder Herstellungskosten des Anlagevermögens

- KPL 5:6 Periodisierung der Anschaffungs- oder Herstellungskosten des Umlaufvermögens

- KPL 5:7 Periodisierung von Gründungsausgaben

- KPL 5:8 Periodisierung von Entwicklungsausgaben

- KPL 5:9 Periodisierung eines Geschäfts- oder Firmenwerts

- KPL 5:11 Periodisierung anderer Ausgaben mit lang anhaltender Wirkung

\footnotetext{
Vgl. KILA 1054/1989 und vgl. Järvinen et al. (2000), S. 385.

Vgl. Järvinen et al. (2000), S. 379-380.

Vgl. Järvinen et al. (2000), S. 379.

Vgl. Leppiniemi (2000 a), S. 137.
} 


\subsubsection{Ansatz von Passiva}

Auf der Passivseite der Bilanz sind das Eigenkapital, steuerliche Ausgleichsposten und als Schulden die Pflichtrückstellungen sowie die Verbindlichkeiten auszuweisen $^{520}$. Das Eigenkapital bildet die Residualgröße aus dem Saldo der Vermögenswerte, steuerliche Ausgleichsposten und Schulden. Eine Legaldefinition des Begriffs „Schulden“ existiert nicht. Auch hat der finnische Gesetzgeber für den Ansatz von Schulden keinen Grundsatz der Passivierung konkretisiert.

Der Passivierungsgrundsatz ergibt sich implizit aus den Ansatzvorschriften für Pflichtrückstellungen nach KPL 5:14 sowie aus dem Grundsatz der leistungsbezogenen Abgrenzung gemäß KPL 3:3 und seiner Abgrenzung zum Grundsatz der zahlungsabhängigen Buchführung gemäß KPL $3: 4^{521}$.

Aus KPL 5:14.1 wird folgende Forderung des Gesetzgebers ersichtlich ${ }^{522}$ :

\section{Zukünftige Aufwendungen und Verluste sowie Pflichtrückstellung}

(1) Die Erträge sind um die in Zukunft aus Verbindlichkeiten hervorgehenden Ausgaben und Verluste zu mindern, wenn:

- diese das Geschäftsjahr oder frühere Geschäftsjahre betreffen;

- das Eintreten dieser Ausgaben und Verluste bei Erstellen des Jahresabschlusses als sicher oder wahrscheinlich gilt;

- diesen sicher oder wahrscheinlich keine Einnahme mehr zuzuordnen ist; und

- $\quad$ diese auf dem Gesetz oder auf bindenden, vom Buchführungspflichtigen mit Dritten abgeschlossenen Verträgen beruhen.

Ein Sachverhalt ist demnach in der Bilanz als Schuld anzusetzen (Passivierungsgrundsatz $^{523}$, wenn

- eine Verpflichtung des Bilanzierenden vorliegt,

- mit der Verpflichtung eine wirtschaftliche Belastung für den Bilanzierenden verbunden ist und

- die wirtschaftliche Belastung quantifizierbar ist.

Besteht das Passivierungsgebot für einen Sachverhalt, stellt sich die Frage, wie er auszuweisen ist: als Pflichtrückstellung oder als Verbindlichkeit? Die Bilanzierung der Schuld als Pflichtrückstellung oder als Verbindlichkeit erfolgt je nach Sicherheit bzw. Unsicherheit über Realisationszeitpunkt und/oder Höhe der Verpflichtung. Unsicherheit über den Eintritt der Verpflichtung ist für die Unterschei-

$520 \quad$ Vgl. KPA 1:6 und KPA 1:7.

521 Bei der Ermittlung des Passivierungsgrundsatzes ist zu berücksichtigen, dass der Gesetzgeber in KPL 4:6 Rechnungsabgrenzungsposten als Bestandteil der Verbindlichkeiten definiert und nach KPL 4:7 die Unterscheidung zwischen kurz- und langfristigen Verbindlichkeiten vorgibt.

522 Übersetzung ins Deutsche durch die Verfasserin.

523 Vgl. Järvinen et al. (2000), S. 407-408 und vgl. Majala (2001 b), S. 975. 
dung nicht wesentlich ${ }^{524}$. Ist zumindest eines der Kriterien „Realisationszeitpunkt" oder „Höhe“ unsicher, so handelt es sich bei der Verpflichtung um eine Pflichtrückstellung. Sind beide sicher, liegt eine Verbindlichkeit vor ${ }^{525}$.

\subsubsection{Bewertung von Aktiva und Passiva}

\subsubsection{Bewertungsmaßstäbe}

Die Anschaffungs- oder Herstellungskosten stellen nach Art. 32 und 35 Abs. 2 der 4. EG-Richtlinie den Bewertungsmaßstab für Vermögenswerte dar. Von ihm darf bzw. muss zwingend unter Beachtung anderer einzelner Vorschriften der Richtlinie abgewichen werden.

Das finnische Buchführungsgesetz nennt vier grundlegende Bewertungsmaßstäbe, die im Jahresabschluss zur Anwendung kommen können bzw. müssen. Es handelt sich dabei um die historischen Anschaffungs- und Herstellungskosten, den Marktwert im Sinne von Wiederbeschaffungskosten, den Marktwert im Sinne eines (wahrscheinlich) realisierbaren Betrags sowie den Barwert ${ }^{526}$.

Die Bestandteile der historischen Anschaffungs- oder Herstellungskosten legt der Gesetzgeber in KPL 4:5 fest. Unter ihnen kann der Betrag verstanden werden, der für Vermögenswerte zum Zeitpunkt ihres Erwerbs aufgewendet worden ist. Sie stellen auch den Betrag dar, den das bilanzierende Unternehmen erhalten hat, als es eine Schuld eingegangen ist; Ausnahmen von diesem Grundsatz existieren jedoch, beispielsweise das Disagio ${ }^{527}$.

Der Marktwert im Sinne von Wiederbeschaffungskosten bezeichnet den Betrag, der zur Wiederbeschaffung eines entsprechenden oder gleichartigen Vermögenswerts zum Zeitpunkt der Jahresabschlusserstellung ausgegeben werden müsste

524 Die KPL-Arbeitsgruppe 1995 hat in ihrem Bericht ursprünglich gefordert, erwartete Ausgaben und Verluste nur dann als Verbindlichkeiten auszuweisen, wenn in Bezug auf Eintritt, Höhe und Eintrittsdatum Sicherheit herrsche; bei Unsicherheit hinsichtlich des Eintritts solle eine Rückstellung ausgewiesen werden, selbst wenn Höhe und Eintrittsdatum eindeutig seien. Vgl. hierzu KPL-Arbeitsgruppe 1995, Teil I (1996), S. 66-67 und vgl. Räty (1998 b), S. 64 .

525 Vgl. Räty (1998 b), S. 65 und vgl. Leppiniemi und Leppiniemi (1999), S. 115. Ein weiterer Unterschied zwischen Pflichtrückstellung und Verbindlichkeit besteht darin, dass bei einer Verbindlichkeit der Gläubiger eine entsprechende Forderung in seiner Bilanz ausweist. Vgl. Järvinen et al. (2000), S. 431. Zur weiteren Abgrenzung von Rückstellungen und Verbindlichkeiten vgl. Kap. 3.2.5.2 und Kap. 3.2.5.3 dieser Arbeit.

526 Diese Bewertungsmaßstäbe entsprechen den vier Maßstäben, die das IASB im Framework 100 nennt: Historical cost (F. 100 a), Current cost (F. 100 b), Realisable (settlement) value (F. 100 c) und Present value (F. 100 d). Neben diesen grundlegenden Wertmaßstäben existieren noch weitere, in einzelnen Standards geregelte Maßstäbe. So definiert IAS 16 (revised 1993) den beizulegenden Zeitwert (fair value) und den erzielbaren Betrag (recoverable amount), die der finnische Gesetzgeber nicht vorsieht.

527 Vgl. Leppiniemi (2000 a), S. 140-142. 
bzw. der zur Begleichung einer Schuld zu diesem Zeitpunkt erbracht werden müsste ${ }^{528}$.

Der Marktwert im Sinne eines (wahrscheinlich) realisierbaren entspricht dem Betrag, der bei Veräußerung eines Vermögenswerts im Moment der JahresabschlussErstellung erzielt werden könnte bzw. für die Begleichung einer Schuld gezahlt werden müsste ${ }^{529}$.

Der auf einer Einnahmenerwartung beruhende Barwert ist gleichzusetzen mit dem Wert aller künftigen Nettoein- bzw. -auszahlungen am Bilanzstichtag ${ }^{530}$.

Der finnische Gesetzgeber folgt vorrangig dem Prinzip der historischen Anschaffungs- oder Herstellungskosten. Da grundsätzlich das Niederstwertprinzip gilt, ist das Anschaffungskostenprinzip in Verbindung mit den anderen genannten Bewertungsmaßstäben anzuwenden. Welcher Maßstab im konkreten Fall für einen bestimmten Jahresabschlussposten heranzuziehen ist, wird in KPL 5 geregelt, dessen einzelne Vorschriften Bewertungsmaßstäbe für bestimmte Sachverhalte teils zwingend vorsehen, teils aber auch Wahlrechte erlauben. So kommt der Marktwert als Wiederbeschaffungswert oder realisierbarer Betrag unter Beachtung des Niederstwertund Vorsichtsprinzips überwiegend dann zwingend zur Anwendung, wenn er niedriger ist als die ursprünglichen Anschaffungs- oder Herstellungskosten. Beispielhaft kann hier das Umlaufvermögen nach KPL 5:6 angeführt werden. Grundstücke, Wasserflächen und Wertpapiere des Finanzanlagevermögens dürfen auch mit dem die Anschaffungskosten übersteigenden realisierbaren Betrag bewertet werden (KPL 5:17) ${ }^{531}$. Ein Ansatz zum Barwert anstelle der Anschaffungsoder Herstellungskosten kann dann notwendig werden, wenn bei der Bewertung von Aktiva besondere Vorsicht geboten ist. Zu nennen sind hier Gründungsaufwendungen (KPL 5:7), Entwicklungsaufwendungen (KPL 5:8) oder Darlehensaufwendungen (KPL 5:10) ${ }^{532}$.

$528 \quad$ Vgl. Leppiniemi (2000 a), S. 141.

$529 \quad$ Vgl. Leppiniemi (2000 a), S. 141.

530 Vgl. Leppiniemi (2000 a), S. 141.

531 Vgl. Leppiniemi (2000 a), S. 141-142; vgl. zur Neubewertung auch Kap. 3.1.6.2.2.6 dieser Arbeit.

532 Vgl. Leppiniemi (2000 a), S. 141. 


\subsubsection{Bewertung von Aktiva}

\subsection{Bewertung bei Zugang}

\subsection{Anschaffungskosten}

Der finnische Gesetzgeber definiert in KPL 4:5.1 die Anschaffungskosten wie folgt $^{533}$ :

Zu den Anschaffungs- oder Herstellungskosten zählen die bei Anschaffung und Fertigung verursachten variablen Ausgaben.

Die Anschaffungskosten stellen in Übereinstimmung mit Art. 32 der 4. EGRichtlinie den Bewertungsmaßstab für erworbene Vermögenswerte dar. Der Begriff „,variable Kosten“ umfasst Einkaufspreis und Nebenkosten. Die Anschaffungskosten setzen sich neben dem eigentlichen Anschaffungspreis auch aus Frachtkosten, Einfuhrzöllen und Installationskosten sowie allen weiteren Kosten zusammen, die sich dem Anschaffungsvorgang direkt zurechnen lassen und durch die der Vermögenswert in einen betriebsbereiten Zustand versetzt wird. Abzuziehen sind Anschaffungspreisminderungen wie Mengenrabatte oder Preisnachlässe. Ebenfalls nicht den Anschaffungskosten zuzurechnen ist die Umsatzsteuer, sofern sie erstattungsfähig ist ${ }^{534}$.

\subsection{Herstellungskosten}

Die Bestandteile der Herstellungskosten als Ausgangswert für die Bewertung fertiger und unfertiger Erzeugnisse des Umlaufvermögens sowie selbst erstellter Vermögenswerte des Anlagevermögens legt der finnische Gesetzgeber in KPL 4:5.1-3 fest $^{535}$ :

Anschaffungs- oder Herstellungskosten

(1) Zu den Anschaffungs- oder Herstellungskosten eines Gegenstands zählen die bei Anschaffung und Fertigung verursachten variablen Ausgaben.

(2) Ist der mit Anschaffung und Fertigung des Gegenstands verbundene Betrag an fixen Ausgaben im Verhältnis zu den in Abs. 1 gemeinten Anschaffungs- oder Herstellungskosten wesentlich, darf er anteilsmäßig den Anschaffungs- oder Herstellungskosten zugerechnet werden.

(3) Wenn die Zinsen für Fremdkapital, das zur Finanzierung der Herstellung von Gegenständen des Anlagevermögens für den Zeitraum der Herstellung benötigt wird, und der in Abs. 2 gemeinte Fixkostenanteil zusammen im Verhältnis zu den in

533 Übersetzung ins Deutsche durch die Verfasserin. Wie aus der Regierungsbegründung zum Gesetzesentwurf HE 173/1997 hervorgeht, wird mit KPL 4:5.1 das Ziel verfolgt, Art. 35 Abs. 2 der 4. EG-Richtlinie korrekt und im Sinne des Richtliniengebers in finnisches Recht umzusetzen. Vgl. Järvinen et al. (2000), S. 387.

534 Vgl. KILA 1588/1999 und vgl. Järvinen et al. (2000), S. 387.

535 Übersetzung ins Deutsche durch die Verfasserin. 
Abs. 1 definierten Anschaffungs- oder Herstellungskosten wesentlich sind, dürfen neben den Fixkosten des Abs. 2 auch die Zinsen für das Fremdkapital anteilsmäßig zu den Anschaffungs- oder Herstellungskosten gerechnet werden.

In die Herstellungskosten sind verpflichtend alle variablen Kosten einzubeziehen. Mit „variablen Kosten“ sind den Gesetzesmaterialien zufolge solche Kosten gemeint, die innerhalb einer Periode leistungsbezogen in Abhängigkeit von der Produktionsmenge anfallen ${ }^{536}$.

Die Vorschrift verlangt implizit vom Buchführungspflichtigen die Einrichtung einer funktionsfähigen Kostenrechnung, die in der Lage ist, angefallene Kosten in ihren variablen und ihren Fixkostenanteil zu trennen. Die Forderung nach einer solchen Kostenrechnung ist im Gesetz in KPL 5:5.2 für die Bewertung von Gegenständen des Anlagevermögens, in KPL 5:6.2 für Gegenstände des Umlaufvermögens kodifiziert. Während in der Kostenrechnung im betriebswirtschaftlichen Sinne auch kalkulatorische Kosten berücksichtigt werden, werden unter variablen Kosten im Sinne dieses Gesetzes nur tatsächlich realisierte Kosten verstanden ${ }^{537}$. Sie entstehen aus den Anschaffungskosten der Roh-, Hilfs- und Betriebsstoffe und den dem einzelnen Erzeugnis direkt zurechenbaren $\operatorname{Kosten}^{538}$.

Das KPL schreibt, der Richtlinie entsprechend, den Ansatz der Herstellungskosten lediglich in Höhe der variablen Kosten zwingend vor (Teilkostenansatz). Eine Aktivierung zu Vollkosten - also unter Einbeziehung der Fixkosten - ist gemäß KPL 4:5.2 unter bestimmten Voraussetzungen möglich:

Gemäß Art. 35 Abs. 3 (b) der 4. EG-Richtlinie dürfen den Herstellungskosten angemessene Teile der dem einzelnen Erzeugnis nur mittelbar zurechenbaren Kosten, welche auf den Zeitraum der Herstellung entfallen, hinzugerechnet werden. Dieses Wahlrecht schränkt der finnische Gesetzgeber dadurch ein, dass er die Aktivierung eines angemessenen Fixkostenanteils nur dann erlaubt, wenn dieser im Verhältnis zu den variablen Kosten wesentlich ist ${ }^{539}$.

Fixkosten werden dabei als solche Kosten definiert, die unabhängig von der Produktionsmenge einer Periode bzw. von der Kapazitätsgrenze und der Auslastung anfallen $^{540}$. Zu ihnen gehören typischerweise Gehälter, Mietaufwand für Gebäude oder Versicherungszahlungen, nicht jedoch Planungs-, Forschungs- und Entwicklungsaufwendungen oder allgemeine Verwaltungs- und Vertriebsaufwendungen $^{541}$.

536 Vgl. HE 111/1992, S. 15-16 und vgl. HE 173/1997.

537 Vgl. Järvinen et al. (2000), S. 388.

538 Der finnische Gesetzgeber hat mit KPL 4:5.1 den Art. 35 Abs. 3 (a) der 4. EG-Richtlinie der Intention des Richtliniengebers folgend umgesetzt. Vgl. Järvinen et al. (2000), S. 389.

539 Vgl. Räty (1998 b), S. 62.

540 Vgl. Leppiniemi (2000 a), S. 145.

541 Vgl. Järvinen et al. (2000), S. 391 und vgl. KILA-Anweisung „Fixkosten als Bestandteil der Anschaffungs- oder Herstellungskosten“, Abschnitt 2. Auch nach Art. 39 Abs. 2 der 4. EGRichtlinie ist die Einbeziehung von Vertriebskosten in die Herstellungskosten explizit verboten. 
Wesentlichkeit wird weder durch den Gesetzgeber definiert noch wird ein Anhaltswert aus den Gesetzesmaterialien ersichtlich. Der KILA hat auf Anfrage entschieden, dass angefallene Fixkosten in Höhe von zwölf Prozent der variablen Kosten ein wesentlicher Betrag seien, der aktiviert werden dürfe ${ }^{542}$. Fixkostenanteile, die über zwölf Prozent der variablen Kosten liegen, dürfen demnach immer aktiviert werden ${ }^{543}$.

Der finnische Gesetzgeber erschwert mit dieser Regelung die Bewertung von Vermögenswerten zu Vollkosten zu Gunsten des Teilkostenansatzes und unterscheidet sich darin vom Richtliniengeber.

Bei den Herstellungskosten von Vermögensgegenständen des Anlagevermögens kommt unter bestimmten Voraussetzungen eine Einbeziehung von Fremdkapitalzinsen in Betracht. Aktivierungsfähige Fremdkapitalzinsen werden im Fachschrifttum beschrieben als der Zinsaufwand, der ohne eine entsprechende Anschaffung vermieden worden wäre ${ }^{544}$. Hierbei ist der tatsächlich realisierte Zinssatz heranzuziehen, kein kalkulatorischer ${ }^{545}$.

Erfolgt die Fremdkapitalaufnahme speziell für einen bestimmten Vermögenswert, dann sind die für sie tatsächlich angefallenen Fremdkapitalzinsen aktivierungsfähig, sofern sie gemeinsam mit den Fixkosten einen wesentlichen Betrag im Verhältnis zu den variablen Kosten darstellen ${ }^{546}$. Ist die Fremdkapitalaufnahme nicht zweckgebunden, ergeben sich die aktivierungsfähigen Fremdkapitalzinsen für den infrage kommenden Vermögenswert anteilig unter Berücksichtigung der Wesentlichkeit aus dem Anteil vom Fremdkapital am aufgewendeten Gesamtkapital für die getätigten Investitionen einer Periode ${ }^{547}$.

Was Wesentlichkeit bei Einbeziehung von Fremdkapitalzinsen bedeutet, ist in den Gesetzesmaterialien nicht erläutert worden. Der KILA betont, dass Fremdkapitalzinsen in jedem Fall dann einbezogen werden dürfen, wenn die Fixkosten allein einbezogen werden könnten ${ }^{548}$. Die Praxis entscheidet über gemeinsame Wesentlichkeit von Fixkosten und Fremdkapitalzinsen einzelfallbezogen und unterstellt in der Regel 10 bis 20 Prozent für beide Positionen ${ }^{549}$. Mit der Forderung nach Wesentlichkeit weicht der finnische Gesetzgeber abermals von der 4. EG-Richtlinie $a b$.

542 Vgl. KILA 1222/1993.

543 Vgl. Leppiniemi (2000 a), S. 145. Über die Wesentlichkeitsfrage bei geringeren Fixkostenanteilen hatte der KILA bislang nicht zu entscheiden (Stand September 2000).

544 Die Gesetzesmaterialien und das Fachschrifttum beziehen sich bei dieser Definition auf IAS 23.13. Vgl. Sipilä (1998), S. 186 und vgl. Järvinen et al. (2000), S. 398.

545 Vgl. Räty (1998 b), S. 68 und vgl. KILA-Anweisung „Fixkosten als Bestandteil der Anschaffungs- oder Herstellungskosten“, Abschnitt 5.2.

546 Vgl. HE 173/1997, S. 20 und vgl. Sipilä (1998), S. 186.

547 Vgl. HE 173/1997, S. 20 und vgl. KILA-Anweisung „Fixkosten als Bestandteil der Anschaffungs- oder Herstellungskosten“, Abschnitt 5.2

548 Vgl. KILA-Anweisung „Fixkosten als Bestandteil der Anschaffungs- oder Herstellungskosten“, Abschnitt 5.1.

549 Vgl. Sipilä (1998), S. 186. 
Wird die Herstellung der fraglichen Vermögenswerte unterbrochen oder verzögert sich ihre Inbetriebnahme, so ist die Aktivierung von Fremdkapitalzinsen für diesen Zeitraum auszusetzen ${ }^{550}$.

Die Aktivierung von Verzugszinsen ist nicht gestattet ${ }^{551}$.

\subsection{Behandlung von Zuschüssen}

Die buchhalterische Behandlung staatlicher Zuschüsse wird vom Richtliniengeber nicht vorgeschrieben. Aus Art. 43 Abs. 1 der 4. EG-Richtlinie folgt, dass im Anhang aufzuzeigen ist, wie die Zuschüsse im Jahresabschluss ausgewiesen werden.

Das Accounting Advisory Forum nennt zwei Ansätze, die für die Buchung in Betracht kommen. Der erfolgsneutrale Ansatz folgt der Auffassung, das Unternehmen habe den Zuschuss nicht aus eigener Kraft verdient, dieser sei deshalb direkt in das Eigenkapital zu buchen. Der erfolgswirksame Ansatz geht davon aus, dass in das Eigenkapital von außen nur Einlagen der Gesellschafter einzustellen sind und fordert deshalb eine Buchung des Zuschusses in der Gewinn- und Verlustrechnung, wodurch die zugehörige Aufwandsbuchung korrigiert wird. Das Accounting Advisory Forum favorisiert den erfolgswirksamen Ansatz, der brutto oder netto vorgenommen werden kann. Bei der Bruttomethode wird der Zuschuss abgegrenzt und erfolgswirksam über die Nutzungsdauer des zugehörigen Vermögenswerts verteilt. Bei der Nettomethode wird der Vermögenswert sofort nach Erhalt um den Betrag des Zuschusses gekürzt. Nach Auffassung des Forums ist die Anwendung der Bruttomethode zu bevorzugen ${ }^{552}$.

Hinsichtlich der Behandlung erhaltener Zuschüsse für Vermögenswerte des Anlagevermögens empfiehlt der KILA, den Wertansatz des entsprechenden Vermögenswerts um den Betrag des Zuschusses zu korrigieren. Der Abschreibungsplan ist bei dieser Vorgehensweise ausgehend vom gekürzten Anschaffungs- oder Herstellungswert zu erstellen. Der Zuschuss wird über die durch den niedrigeren Wertansatz reduzierten Abschreibungsbeträge im Laufe der Nutzungsdauer erfolgswirksam erfasst ${ }^{553}$.

Alternativ erlaubt der KILA, den Zuschuss als passiven Rechnungsabgrenzungsposten im Einzelabschluss zu berücksichtigen ${ }^{554}$. Der Vermögenswert wird zu

550 Vgl. Järvinen et al. (2000), S. 400 und vgl. KILA-Anweisung „Fixkosten als Bestandteil der Anschaffungs- oder Herstellungskosten", Abschnitt 5.2.

551 Vgl. Räty (1998 b), S. 69 und vgl. Sipilä (1998), S. 186 und vgl. KILA-Anweisung „Fixkosten als Bestandteil der Anschaffungs- oder Herstellungskosten“, Abschnitt 5.2.

552 Vgl. van Hulle und van der Tas (2001), S. 835.

553 Vgl. KILA-Anweisung „Planmäßige Abschreibungen“.

554 Vgl. KILA-Anweisung „Planmäßige Abschreibungen“. Vgl. auch IAS 20 (reformatted 1994), auf den der KILA sich bezieht. 
vollen Anschaffungs- oder Herstellungskosten aktiviert und planmäßig abgeschrieben, der Rechnungsabgrenzungsposten wird parallel über die Nutzungsdauer des Vermögenswerts erfolgswirksam aufgelöst ${ }^{555}$.

\subsection{Behandlung von Wiederbeschaffungsrückstellungen}

Das finnische Gewerbesteuerrecht gestattet unter bestimmten Voraussetzungen die Bildung von Wiederbeschaffungsrückstellungen als freiwillige Rückstellungen mit Rücklagencharakter für Vermögenswerte des Anlagevermögens ${ }^{556}$, beispielsweise für eine Maschine, die durch Feuer oder einen ähnlichen Schadensfall zerstört worden ist. Rücklagenähnliche, freiwillige Rückstellungen, werden steuerlich nur dann anerkannt, wenn das Unternehmen sie auch im handelsrechtlichen Abschluss ausweist. Streitig ist, ob solche freiwilligen Rückstellungen von Aktiengesellschaften nach geltendem Recht handelsrechtlich noch gebildet werden dürfen, da sie für Unternehmen dieser Rechtsform steuerlich nicht mehr akzeptiert werden $^{557}$.

Der Ausweis einer Wiederbeschaffungsrückstellung erfolgt im steuerlichen Ausgleichsposten. Hat ein Unternehmen die Anschaffungs- oder Herstellungskosten eines zum Anlagevermögen gehörenden Vermögenswerts durch eine solche Rückstellung ganz oder teilweise gedeckt, werden die Anschaffungs- oder Herstellungskosten bei Zugang in voller Höhe angesetzt und planmäßig abgeschrieben, während die Rückstellung erfolgswirksam aufgelöst wird ${ }^{558}$.

Vgl. IAS 20.26 .

Vgl. EVL 32 und 43.

557 Vgl. Kap. 3.2.5.1.2 dieser Arbeit

558 Vgl. Leppiniemi (2000 b), S. 18-19 und vgl. KILA-Anweisung „Planmäßige Abschreibungen", Abschnitt 2.4.

Soll die Auflösung der Rückstellung nicht in voller Höhe im Geschäftsjahr, in dem die Investition getätigt wird, ergebniswirksam zu Buche schlagen, kann in ihrer Höhe eine Zunahme des Abschreibungsunterschieds als Bestandteil des steuerlichen Ausgleichspostens in der Bilanz erfolgen. Die Auflösung dieses Abschreibungsunterschieds wirkt sich in späteren Perioden ertragswirksam aus, wenn die planmäßigen Abschreibungen die Jahresabschreibungen des Vermögenswertes übersteigen.

Diese Aussage soll anhand eines Beispiels verdeutlicht werden.:

Jahr $t_{1}$ : Die Maschine eines Buchführungspflichtigen mit einem Restbuchwert von $€ 4.000,00$ wird zerstört. Der Buchführungspflichtige erhält von der Versicherung $€ 5.000,00$ erstattet, von denen er $€ 1.000,00$ in die Wiederbeschaffungsrückstellung einstellt.

Jahr $\mathrm{t}_{2}$ : Der Buchführungspflichtige schafft eine neue Maschine für $€ 10.000,00$ an. Er lässt die für die Anschaffung gebildete Wiederbeschaffungsrückstellung in Höhe von $€ 1.000,00$ auf und stellt $€ 1.000,00$ in den Abschreibungsunterschied ein. Die Auflösung des Abschreibungsunterschieds erfolgt dann in späteren Perioden.

Die Buchungssätze lauten:

Jahr $\mathrm{t}_{1}$ : 1. Außerplanmäßige Abschreibung $€ 4.000,00$ an Sachanlagevermögen $€ 4.000,00$.

2. Bank $€ 5.000,00$ an Ertrag aus Versicherungserstattung $€ 5.000,00$. 3. Aufwand aus Einstellung in die Wiederbeschaffungsrückstellung $€ 1.000,00$ an Wiederbeschaffungsrückstellung $€ 1.000,00$. Die Ergebnisauswirkung dieser drei Buchungen beläuft sich auf Null. 


\subsection{Bewertung in Folgeperioden}

\subsection{Planmäßige Abschreibungen}

Art. 35 Abs. 1 (b) der 4. EG-Richtlinie schreibt vor, bei Gegenständen des Anlagevermögens mit zeitlich begrenzter Nutzungsdauer die Anschaffungs- oder Herstellungskosten planmäßig über die Nutzungsdauer um Wertberichtigungen $\mathrm{zu}$ vermindern. Ins finnische Recht in KPL 5:5.1 umgesetzt, lautet diese Vorschrift ${ }^{559}$ :

\section{Periodisierung der Anschaffungs- oder Herstellungskosten des Anlagevermögens}

Die Anschaffungs- oder Herstellungskosten des Anlagevermögens sind zu aktivieren und über ihre Nutzungsdauer planmäßig abzuschreiben.

Obwohl diese Regel dem Wortlaut nach auf alle Vermögenswerte des Anlagevermögens anzuwenden ist, geht aus Gesetzesmaterialien und der kommentierenden Literatur hervor, dass hinsichtlich der Forderung planmäßiger Abschreibungen dem Grundsatz nach lediglich abnutzbare, in ihrer Nutzung zeitlich begrenzte Vermögenswerte des Anlagevermögens angesprochen sind ${ }^{560}$. Im Normalfall unterliegen Grundstücke und Wasserflächen, Werte des Finanzanlagevermögens sowie geleistete Anzahlungen keinen planmäßigen Abschreibungen ${ }^{561}$. Ausdrücklich ausgenommen von der Pflicht zur planmäßigen Abschreibung sind nach KPL 5:5.3 geringwertige Wirtschaftsgüter, die periodengleich als Aufwand erfasst werden dürfen, sowie solche Vermögenswerte, für die ein Festwert gebildet worden ist ${ }^{562}$. Die Vorschrift des KPL 5:5.1 gilt auch für diejenigen aktivierten Ver-

Jahr $t_{2}$ : 1. Sachanlagevermögen $€ 10.000,00$ an Bank $€ 10.000,00$. 2. Wiederbeschaffungsrückstellung $€ 1.000,00$ an Ertrag aus der Auflösung der Wiederbeschaffungsrückstellung $€ 1.000,00$. 3. Veränderung des Abschreibungsunterschieds $€ 1.000,00$ an Abschreibungsunterschied $€ 1.000,00$. Die Ergebnisauswirkung dieser drei Buchungen beläuft sich auf Null. In späteren Perioden wird der bilanzielle Abschreibungsunterschied dann ertragswirksam aufgelöst (und mindert so die Jahresabschreibungen auf die Maschine). Zu Jahresabschreibungen vgl. auch Kap. 3.1.6.2.2.2 dieser Arbeit. Vgl. KILA-Anweisung „Planmäßige Abschreibungen“, Anhang 2.

Übersetzung ins Deutsche durch die Verfasserin.

Vgl. Järvinen et al. (2000), S. 459, vgl. Leppiniemi, (2000 b), S. 15 und vgl. KILA-Anweisung „Planmäßige Abschreibungen“, Abschnitt 1.3.

561 Ausnahmen bilden dem KILA zufolge solche Grundstücke und Wasserflächen, die wirtschaftlich derart genutzt werden, dass ihr Wert sich regelmäßig mindert (z. B. im Abbau befindliche Mineralgruben). Ausnahmen stellen auch Wertpapiere des Finanzanlagevermögens dar, für die zu einem in der Zukunft liegenden Zeitpunkt bereits ein Kaufvertrag abgeschlossen worden ist, in dem der vereinbarte Verkaufspreis unter den Anschaffungskosten liegt. Es stellt sich die Frage, ob solche Wertpapiere der Intention des Richtliniengebers folgend statt im Anlagevermögen nicht besser im Umlaufvermögen auszuweisen sind. Vgl. KILAAnweisung „Planmäßige Abschreibungen“ Abschnitt 3.3.

562 Vgl. KILA-Anweisung „Planmäßige Abschreibungen“, Abschnitte 2.6 und 2.7, vgl. Ivaska (2000), S. 23. 
mögenswerte, für die ein Aktivierungswahlrecht besteht ${ }^{563}$, d. h. für immaterielle Vermögenswerte $^{564}$ und Ausgaben für Erweiterung und Verbesserung ${ }^{565}$.

Vor bzw. bei Zugang eines abnutzbaren Vermögenswerts ist ein Abschreibungsplan zu erstellen, aus dem das Abschreibungsvolumen, die Nutzungsdauer, der Abschreibungsbeginn und die Abschreibungsmethode hervorgehen ${ }^{566}$. Der Abschreibungsplan soll mit Hinblick auf den True and Fair View den tatsächlichen Nutzungsverlauf des Vermögenswerts möglichst exakt widerspiegeln. Seine Ausgangsbasis bildet die mit dem Vermögenswert verbundene Einnahmenerwartung. Die Einnahmenerwartung berücksichtigt die Zeitspanne, für die mit Einnahmen gerechnet wird - die wirtschaftliche Nutzungsdauer - sowie die Höhe der Einnahmen insgesamt und im Zeitablauf ${ }^{567}$.

Die Einnahmenerwartung für den einzelnen Vermögenswert ist nicht immer quantifizierbar. Die 4. EG-Richtlinie schreibt den Grundsatz der Einzelbewertung vor, legt aber keine Verfahrensweise für den Fall fest, dass eine Einzelbewertung nicht möglich ist. Der KILA verweist auf IAS 36 (approved 1998) und empfiehlt, die Einnahmenerwartung an die Cash-generating unit zu knüpfen. Diese wird vom IASB definiert als ,... the smallest identifiable group of assets that generates cash inflows from continuing use that are largely independent of the cash inflows from other assets or groups of assets. ${ }^{\text {“568 }}$. Unter ihnen wird somit die kleinste erkennbare Gruppe von Vermögenswerten, die Einzahlungen durch ihre fortlaufende Nutzung generiert und weitestgehend unabhängig von den Einzahlungen anderer Vermögenswerte ist, verstanden. Kann eine zukünftige Einnahme für einen einzelnen Vermögenswert nicht ermittelt werden, so ist sie für die Cash-generating unit des Vermögenswerts zu bestimmen.

Das Abschreibungsvolumen ergibt sich aus den Anschaffungs- oder Herstellungskosten abzüglich eines eventuell erwarteten Restwerts ${ }^{569}$. Der Restwert ist auf Basis von eigenen oder Erfahrungswerten der Branche unter Beachtung des Vorsichtsprinzips ${ }^{570}$ zu schätzen. Bei Ermittlung des Abschreibungsvolumens sollte kein Restwert angesetzt werden, wenn ein Verkauf des Vermögenswerts am Ende seiner betrieblichen Nutzungsdauer unsicher erscheint oder der Betrag des Rest-

563 Vgl. Leppiniemi, (2000 b), S. 15 und KILA-Anweisung „Planmäßige Abschreibungen“, Abschnitt 1.2.

564 Vgl. Kap. 3.2.1.1 dieser Arbeit.

565 Vgl. Kap. 3.2.1.2.1 dieser Arbeit

566 Vgl. Leppiniemi (2000 b), S. 15; einen Abschreibungsplan zu dokumentieren, wird nicht von Freiberuflern, Immobiliengesellschaften, Vereinen und Verbänden sowie Stiftungen verlangt.

567 Vgl. Leppiniemi (2000 b), S. 20, und vgl. KILA-Anweisung „Planmäßige Abschreibungen“, Abschnitt 6.

568 Vgl. IAS 36.5.

569 Das finnische Gewerbesteuergesetz EVL sieht bei der Ermittlung des Abschreibungsvolumens keinen Restwert vor; hieraus können Abschreibungsunterschiede resultieren. Vgl. Kap. 3.1.6.2.2.2 dieser Arbeit.

570 Vgl. KPL 3:1.3. 
werts im Verhältnis zu den Anschaffungs- oder Herstellungskosten unwesentlich ist $^{571}$. Zieht die Anschaffung oder Nutzung eines Vermögenswerts am Ende seiner Nutzungsdauer wesentliche Kosten für Abbruch, Abtransport oder Rekultivierung nach sich, dürfen diese Kosten bei der Festlegung des Restwerts nicht berücksichtigt werden. Sie können vor ihrer Realisation nur erfolgswirksam erfasst werden, indem sie bis zum Ende der wirtschaftlichen Nutzungsdauer des Vermögenswerts als Pflichtrückstellung oder Rechnungsabgrenzungsposten angesammelt wer$\operatorname{den}^{572}$.

Die wirtschaftliche Nutzungsdauer eines Vermögenswerts ist abhängig von der mit ihm verbundenen Einnahmenerwartung. Sie umfasst den Zeitraum, für den der Buchführungspflichtige die Erzielung von Einnahmen voraussieht. Die Einnahmenerwartung entspricht somit der individuellen Planung und Budgetierung des Unternehmens und richtet sich auch nach seiner Instandhaltungs- und Wiederbeschaffungspolitik. Die Bestimmung der wirtschaftlichen Nutzungsdauer hat auf dem Vorsichtsprinzip zu beruhen, sollte zudem Erfahrungswerte des Unternehmens oder der Branche berücksichtigen ${ }^{573}$. Das Buchführungsgesetz bestimmt keine standardisierten Nutzungsdauern, sieht jedoch Abschreibungshöchstdauern für immaterielle Vermögenswerte vor, welche zwischen fünf und 20 Jahren liegen $^{574}$.

Der Abschreibungszeitraum beginnt mit der tatsächlichen Inbetriebnahme des Vermögenswerts im normalen Produktionsprozess, nach Beendigung der Testläufe, die eine angemessene Zeitspanne nicht überschreiten dürfen ${ }^{575}$. Es ist nicht gestattet, Abschreibungen schon ab dem Zeitpunkt der Betriebsbereitschaft vorzunehmen und auch nicht, sie erst ab dem Zeitpunkt der erstmaligen Einnahmenerzielung durchzuführen ${ }^{576}$. Bei unterjähriger Inbetriebnahme ist grundsätzlich pro rata temporis abzuschreiben. Halbjahres-Abschreibungen sind nur dann mit der Art guter Buchführung vereinbar, wenn durch sie die planmäßigen Abschreibungen der Periode in ihrer Gesamtheit nicht wesentlich verfälscht werden ${ }^{577}$.

Der finnische Gesetzgeber schreibt im Buchführungsgesetz keine Abschreibungsmethode vor. Erlaubt ist folglich jede Abschreibungsmethode, die unter dem Ge-

571 Vgl. KILA-Anweisung „Planmäßige Abschreibungen“, Abschnitt 2.3, vgl. Leppiniemi (2000 b), S. 19-20 und vgl. Ivaska (2000), S. 22.

572 Vgl. KPL 5:14 und vgl. Leppiniemi (2000 b), S. 20.

573 Vgl. KILA-Anweisung „Planmäßige Abschreibungen“, Abschnitt 3.1.1

574 Vgl. KPL 5:7 bis 5:11. Vgl. auch Kap. 3.2.1.1 dieser Arbeit.

575 Vgl. KILA-Anweisung „Planmäßige Abschreibungen“, Abschnitt 3.1.2 und vgl. Ivaska (2000), S. 24.

576 Vgl. KILA-Anweisung „Planmäßige Abschreibungen“, Abschnitt 3.1.2.

577 Vgl. KILA-Anweisung „Planmäßige Abschreibungen“, Abschnitt 3.1.2, vgl. Ivaska (2000), S. 24 und vgl. Järvinen et al. (2000), S. 466. 
sichtspunkt des tatsächlichen Nutzungsverlaufs begründbar ist ${ }^{578}$. In der Praxis ist die lineare Abschreibungsmethode am Weitesten verbreitet, da sie nach herrschender Meinung eine bessere Vergleichbarkeit der Geschäftsjahre untereinander gewährleistet als andere Methoden ${ }^{579}$. Die steuerlich akzeptierte degressive Abschreibungsmethode findet in ihren beiden Ausprägungen (geometrisch-degressive Methode und arithmetisch-degressive Methode) ebenfalls Anwendung. Auch die Leistungsabschreibung und die progressive Abschreibung sind denkbar, jedoch äußerst selten ${ }^{580}$.

Die der Abschreibung zu Grunde liegende Nutzungsdauer und Abschreibungsmethode sind regelmäßig zu überprüfen. Ergeben sich wesentliche Abweichungen von den im Abschreibungsplan getroffenen Annahmen, so ist der Abschreibungsplan zu korrigieren. Die Abschreibungsbeträge des laufenden und künftiger Geschäftsjahre sind an die neuen Erkenntnisse anzupassen ${ }^{581}$.

\subsection{Andere als planmäßige Abschreibungen}

Der finnische Gesetzgeber regelt in KPL 5:12.1 ${ }^{582}$ :

Andere als planmäßige Abschreibungen

Der Buchführungspflichtige darf den Abschreibungsplan übersteigende Abschreibungen vornehmen, wenn hierfür ein besonderer Grund vorliegt.

Einen besonderen Grund liefert nach Ansicht der Regierungsbegründung zum Gesetzesentwurf ${ }^{583}$ und des finnischen Fachschrifttums ${ }^{584}$ das Gewerbesteuerrecht, nach dem steuerlich höchstzulässige Abschreibungen nur dann abzugsfähig sind, wenn sie auch handelsrechtlich durchgeführt werden ${ }^{585}$.

Art. 35 Abs. 1 (d) der 4. EG-Richtlinie sieht für den Fall, dass bei einem Gegenstand des Anlagevermögens steuerlich motivierte außerordentliche Wertberichtigungen vorgenommen werden, vor, dass der Betrag im Anhang zu erwähnen und hinreichend zu begründen sei. Art. 43 Abs. 1 (10) verlangt zudem, das Ausmaß der Anwendung steuerlicher Vorschriften auf das Jahresergebnis im Anhang anzugeben.

578 Vgl. KILA-Anweisung „Planmäßige Abschreibungen“, Abschnitt 3.2, vgl. Ivaska (2000), S. 23-24, vgl. Leppiniemi (2000 b), S. 21-22 und vgl. Järvinen et al. (2000), S. 464.

579 Vgl. Leppiniemi (2000 b), S. 22.

580 Vgl. KILA-Anweisung „Planmäßige Abschreibungen“, Abschnitt 3.2.

581 Vgl. KILA-Anweisung „Planmäßige Abschreibungen“, Abschnitt 5, vgl. Ivaska (2000), S. 24 und vgl. Järvinen et al. (2000), S. 463-465.

582 Übersetzung ins Deutsche durch die Verfasserin.

583 Vgl. HE 173/1997.

584 Vgl. Leppiniemi (2000 a), S. 236, vgl. Ivaska (2000), S. 24, vgl. Leppiniemi (2000 b), S. 24 und vgl. Järvinen et al. (2000), S. 485.

585 Vgl. EVL 54.2. 
Die mit dem Gesetzesentwurf beauftragte KPL-Arbeitsgruppe 1995 und der finnische Gesetzgeber vertreten die Ansicht, es sei wesentlich übersichtlicher und informativer, steuerlich veranlasste Posten im Jahresabschluss statt im Anhang direkt in der Bilanz und Gewinn- und Verlustrechnung auszuweisen ${ }^{586}$. Deshalb umfasst die erfolgswirksame Abschreibung des Jahres nicht nur die planmäßigen, handelsrechtlichen, meist linear vorgenommenen Abschreibungen auch die vom Abschreibungsplan abweichenden, steuerlich veranlassten, meist degressiv durchgeführten Abschreibungen ${ }^{587}$.

Der Gesetzgeber hat auf der Passivseite der Bilanz in der Gruppe der ,steuerlichen Ausgleichsposten“ den Posten „Abschreibungsunterschied“ eingefügt ${ }^{588}$. Dieser setzt sich kumulativ aus den Differenzen zwischen planmäßiger Abschreibung und Jahresabschreibung des laufenden Jahres sowie früherer Geschäftsjahre zusammen $^{589}$. Auf der Aktivseite der Bilanz werden die fortgeführten Anschaffungs- oder Herstellungskosten des Anlagevermögens ausgewiesen, die steuerliche Mehr- oder Minderabschreibung wird auf der Passivseite von diesem handelsrechtlich zutreffenden Wert abgesetzt ${ }^{590}$.

Während die planmäßigen Abschreibungen in der Gewinn- und Verlustrechnung Bestandteil des Betriebsergebnisses sind, wird der steuerlich veranlasste Teil der Jahresabschreibung nach dem „Jahresergebnis vor Steuerausgleichsposten und Steuerzahlungen“ als „Änderung des Abschreibungsunterschieds“ ausgewiesen ${ }^{591}$. Die Änderung des Abschreibungsunterschieds wird gebildet aus den steuerlichen Mehr- oder Minderabschreibungen der Periode sowie der Auflösung eines Abschreibungsunterschieds bei Abgang eines Vermögenswerts ${ }^{592}$. In der Regel sind die Änderung des Abschreibungsunterschieds (Ausweis in der Gewinn- und Verlustrechnung) und die bilanzielle Differenz zwischen dem Abschreibungsunterschied zu Beginn und Ende einer Periode betragsmäßig identisch ${ }^{593}$.

Die dargestellte Vorgehensweise führt dazu, dass Gliederungen von Bilanz und Gewinn- und Verlustrechnung in Finnland von den in der 4. EG-Richtlinie vorgeschlagenen Schemata abweichen. Wegen der verbesserten Qualität der Jahresabschlussaussage hat die von Finnland zu diesem Thema befragte EU-Kommission die Auffassung vertreten, dass gegen einen gesonderten Ausweis steuerlicher Ab-

\footnotetext{
586 Vgl. KPL-Arbeitsgruppe 1995, Teil I (1996), S. 178-179.

587 Vgl. KILA-Anweisung „Planmäßige Abschreibungen“, Abschnitt 1.3.

588 Vgl. Bilanzgliederungsschemata des KPA 1:6 und 1:7.

589 Vgl. KILA-Anweisung „Planmäßige Abschreibungen“, Abschnitt 1.3.

590 Vgl. hierzu Kap. 3.2.5.1.1 dieser Arbeit.

591 Vgl. Gliederungsschemata der Gewinn- und Verlustrechnung nach KPA 1:1 und 1:2.

592 Vgl. KILA-Anweisung „Planmäßige Abschreibungen“, Abschnitt 1:3.

593 Vgl. KILA-Anweisung „Planmäßige Abschreibungen“, Abschnitt 1.3.
} 
schreibungsunterschiede in Bilanz und Gewinn- und Verlustrechnung nichts einzuwenden sei ${ }^{594}$.

Die Jahresabschreibung ist regelmäßig in den ersten Nutzungsjahren eines Vermögenswerts höher als die planmäßige Abschreibung. In späteren Geschäftsjahren kehrt sich das Verhältnis dann um. Nach KILA-Anweisung darf die Jahresabschreibung für einen Vermögenswert überhaupt nur dann geringer als die planmäBige Abschreibung ausfallen, wenn sie in früheren Geschäftsjahren die planmäßige Abschreibung überstiegen hat ${ }^{595}$.

\subsection{Außerplanmäßige Abschreibungen auf das Anlagevermögen}

Die 4. EG-Richtlinie sieht für Finanzanlagen ein Wahlrecht ${ }^{596}$, für andere Vermögenswerte des Anlagevermögens die Pflicht ${ }^{597}$ zur Wertberichtigung auf den am Abschluss-Stichtag niedrigeren beizulegenden Wert vor, sofern die Wertminderung von Dauer ist (gemildertes Niederstwertprinzip). Der finnische Gesetzgeber dagegen fordert für alle Vermögenswerte des Anlagevermögens die Einhaltung des gemilderten Niederstwertprinzips. Er geht somit über die Mindestanforderungen der Richtlinie hinaus ${ }^{598}$. Mit KPL 5:13 setzt der finnische Gesetzgeber Art. 35 Abs. 1 (c) (aa) und (bb) der 4. EG-Richtlinie mit folgendem Wortlaut in nationales Recht um ${ }^{599}$ :

\section{Außerplanmäßige Abschreibung}

Ist die einem Wert des Anlagevermögens zuzuordnende in Zukunft erzielbare Einnahme dauerhaft niedriger als der noch nicht wertberichtigte Teil der Anschaffungsoder Herstellungskosten, so ist der Differenzbetrag als außerplanmäßige Abschreibung unmittelbar im Aufwand zu erfassen.

Während der Richtliniengeber den ,niedrigeren beizulegenden Wert“ nicht definiert, versteht der finnische Gesetzgeber unter ihm die zukünftige Einnahmenerwartung, die unter den fortgeführten Anschaffungs- oder Herstellungskosten liegt. Bei der Prüfung der Erwartung über in Zukunft erzielbare Erträge ist das Vorsichtsprinzip zu beachten ${ }^{600}$. Sind erwartete Einnahmen nicht eindeutig einem

594 Vgl. KPL-Arbeitsgruppe 1995, Teil I (1996), S. 179.

595 Vgl KILA-Anweisung „Planmäßige Abschreibungen“, Abschnitt 4.

596 Vgl. Art. 35 Abs. 1 (c) (aa) der 4. EG-Richtlinie.

597 Vgl. Art. 35 Abs. 1 (c) (bb) der 4. EG-Richtlinie.

598 Vgl. KPL 5:13 und das Fachschrifttum, z. B. Järvinen et al. (2000), S. 487. Steuerlich wird die Wertberichtigung von Wertpapieren des Finanzanlagevermögens nur dann anerkannt, wenn eines der folgenden Kriterien erfüllt ist: 1 . Andauernde Verluste des Tochterunternehmens, 2. Laufendes Vergleichsverfahren oder Insolvenz des Tochterunternehmens, 3. Kapitalherabsetzung beim Tochterunternehmen, 4. Vergabe eines eigenkapitalersetzenden Darlehens des Mutterunternehmens an das Tochterunternehmen oder 5. Einbruch der Ertragserwartungen, vgl. hierzu Leppiniemi (2000 a), S. 162. 
Vermögenswert zuzuordnen, so ist auf die Cash-generating unit, der er angehört, zurückzugreifen $^{601}$. Unter der Prämisse der Unternehmensfortführung ist grundsätzlich auszugehen vom Marktwert als Wiederbeschaffungswert. Auch eine Bewertung auf Basis des wahrscheinlichen Verkaufspreises des Vermögenswerts ist möglich $^{602}$.

Außerplanmäßige Abschreibungen sind immer unmittelbar als Aufwand zu erfas$\operatorname{sen}^{603}$.

Zur Abgrenzung planmäßiger und außerplanmäßiger Abschreibungen verweist das Fachschrifttum darauf, dass KPL 5:13 primär auf diejenigen Vermögenswerte anzuwenden sei, deren Nutzungsdauer unbegrenzt ist. KPL 5:13 gilt jedoch auch für Vermögenswerte, die zwar planmäßig abgeschrieben werden, aber auf Grund eines nicht vorhersehbaren, einmaligen Ereignisses ursprünglich erwartete Einnahmen nicht mehr erzielen können. Ein solches Ereignis stellt beispielsweise eine Beschädigung oder Zerstörung durch Feuer oder Explosion dar. Eine Wertminderung kann auch aus einer langfristigen Stillstandszeit resultieren ${ }^{604}$.

Planmäßige Abschreibungen und außerplanmäßige Abschreibungen auf immaterielle Vermögenswerte und Vermögenswerte des Sachanlagevermögens beeinflussen das Betriebsergebnis. Außerplanmäßige Abschreibungen auf das Finanzanlagevermögen sind Bestandteil des Finanzergebnisses ${ }^{605}$.

601 Vgl. KILA-Anweisung „Planmäßige Abschreibungen“, Abschnitt 6, in dem auf IAS 36 (approved 1998) verwiesen wird.

602 Vgl. Järvinen et al. (2000), S. 488.

603 Wird es jedoch notwendig, eine in früheren Jahren durchgeführte Neubewertung rückgängig zu machen, ist die für sie gebildete Neubewertungsrücklage erfolgsneutral aufzulösen. Vgl. KILA-Anweisung „Planmäßige Abschreibungen“, Abschnitt 2.2, vgl. OYL 12:3b und vgl. Järvinen et al. (2000), S. 513.

604 Vgl. Leppiniemi (2000 b), S. 16-17, KILA-Anweisung „Planmäßige Abschreibungen“, Abschnitt 6 und vgl. Ivaska (2000), S. 25.

605 Vgl. Gliederungsschemata der Gewinn- und Verlustrechnung KPA 1:1 und 1:2. Ein möglicher Ausweis außerplanmäßiger Abschreibungen im außerordentlichen Ergebnis ist in der Regierungsbegründung zum Gesetzesentwurf wegen des einmaligen Charakters der außerordentlichen Posten abgelehnt worden. Vgl. hierzu HE 173/1997, S. 19. 


\subsection{Außerplanmäßige Abschreibungen auf das Umlauf- und Finanz- umlaufvermögen}

Nach Art. 39 Abs. 1 (b) der 4. EG-Richtlinie sind bei Gegenständen des Umlaufvermögens Wertberichtigungen vorzunehmen, um diese Gegenstände mit dem niedrigeren Marktpreis oder mit einem anderen niedrigeren Wert anzusetzen, der ihnen am Bilanzstichtag beizulegen ist.

In finnisches Recht implementiert ist diese Vorschrift mit KPL 5:6.1 für Vermögenswerte des Umlaufvermögens (Roh-, Hilfs- und Betriebsstoffe sowie halbfertige und fertige Erzeugnisse) und mit KPL 5:2.1-2 für Vermögenswerte des Finanzumlaufvermögens (Forderungen, Zahlungsmittel und Zahlungsmitteläquivalente).

KPL 5:6.1 lautet wie folgt ${ }^{606}$ :

Periodisierung der Anschaffungs- oder Herstellungskosten des Umlaufvermögens

Die Anschaffungs- oder Herstellungskosten des am Bilanzstichtag noch vorhandenen Umlaufvermögens sind zu aktivieren. Ist der wahrscheinliche Wiederbeschaffungswert oder Veräußerungswert des Umlaufvermögens am Stichtag niedriger als die Anschaffungs- oder Herstellungskosten, so ist der Unterschiedsbetrag als Aufwand zu erfassen.

Ist die mit einem Vermögenswert des Umlaufvermögens verbundene Einnahmenerwartung am Stichtag niedriger als sein voraussichtlicher Veräußerungspreis, so ist der Wert außerplanmäßig auf den niedrigeren Veräußerungspreis abzuschreiben ${ }^{607}$. Hierbei ist unter der Prämisse der Unternehmensfortführung vom Veräußerungspreis im gewöhnlichen Geschäftsverkehr auszugehen, es sei denn, eine Auflösung des Lagers oder die Still-Legung ganzer Betriebszweige befinden sich bereits in Planung. In einem solchen Fall ist die Bewertung am Bilanzstichtag anhand des einmaligen Realisationspreises durchzuführen ${ }^{608}$.

Eine außerplanmäßige Abschreibung hat zu erfolgen, wenn der Wiederbeschaffungspreis eines im Umlaufvermögen bilanzierten Vermögenswerts unter seinen ursprünglichen Anschaffungs- oder Herstellungskosten liegt. Basierend auf dem Vorsichtsprinzip ist diese Abschreibung auch dann vorzunehmen, wenn zu erwarten ist, dass der Vermögenswert oder sein Endprodukt verlustfrei veräußert werden können ${ }^{609}$.

606 Übersetzung ins Deutsche durch die Verfasserin.

607 Vgl. Järvinen et al. (2000), S. 470 und vgl. Räty (1998 b), S. 71. Diese Forderung des Buchführungsgesetzes entspricht auch der gewerbesteuerrechtlichen Vorgehensweise nach EVL 28.

608 Vgl. Järvinen et al. (2000), S. 471.

609 Vgl. Järvinen et al. (2000), S. 470 und vgl. Räty (1998 b), S. 71. Diese Forderung des Buchführungsgesetzes entspricht auch der gewerbesteuerrechtlichen Vorgehensweise des EVL 28. 
KPL 5:2.1-2 für Vermögenswerte des Finanzumlaufvermögens heißt ${ }^{610}$ :

\section{Forderungen, liquide Mittel und Verbindlichkeiten}

In der Bilanz sind zu erfassen:

- Forderungen zum Nennwert, höchstens jedoch zu ihrem wahrscheinlichen Wert

- zum Finanzumlaufvermögen gehörende Wertpapiere und andere Zahlungsmitteläquivalente in Höhe ihres Anschaffungswerts oder, sofern niedriger, in Höhe ihres wahrscheinlichen Veräußerungspreises am Bilanzstichtag.

$[\ldots]$

Wie Vermögenswerte des Umlaufvermögens, sind auch die Werte des Finanzumlaufvermögens erfolgswirksam auf den am Stichtag niedrigeren beizulegenden Wert abzuschreiben. Forderungen sind demnach zu ihrem Nennwert oder ihrem niedrigeren wahrscheinlich realisierbaren Betrag anzusetzen. V. a. das Risiko eines Kundenausfalls ist hier in Betracht zu ziehen. Wertpapiere und Zahlungsmitteläquivalente sind zu Anschaffungskosten oder ihrem wahrscheinlich niedrigeren Veräußerungspreis - z. B. dem Börsenwert am Bilanzstichtag - anzusetzen. Verluste aus Fremdwährungsumrechnung können sich bei der Bewertung von Vermögenswerten des Finanzumlaufvermögens wesentlich auswirken.

Für Vermögenswerte des Umlauf- und Finanzumlaufvermögens gilt das strenge Niederstwertprinzip. Es ist unabhängig davon auf den niedrigeren beizulegenden Wert abzuschreiben, ob es sich um eine dauerhafte oder nur um eine vorübergehende Wertminderung handelt.

In der Regierungsbegründung zum Gesetzesentwurf wird im Zusammenhang mit Wertberichtigungen des Umlauf- und Finanzumlaufvermögens explizit der Grundsatz der Einzelbewertung genannt. Die Wertminderung eines Vermögenswerts darf nicht durch die Wertsteigerung eines anderen Vermögenswerts kompensiert werden ${ }^{611}$.

Bei Jahresabschlusserstellung sind nach KPL 3:3.2,2 auch Wertminderungen zu berücksichtigen, die ihre Ursache im laufenden Geschäftsjahr oder früheren Jahren haben, aber erst nach dem Stichtag bekannt werden (wertaufhellende Informationen) $)^{612}$.

610 Übersetzung ins Deutsche durch die Verfasserin.

611 Vgl. HE 173/1997, S. 16.

612 Diese Vorgehensweise steht im Einklang mit dem Vorsichtsprinzip nach Art. 31 der 4. EGRichtlinie. Vgl. auch van Hulle und van der Tas (2001), S. 831. 
Buchungstechnisch muss in der Bilanz die Abwertung in jedem Fall direkt im Umlauf- bzw. Finanzumlaufvermögen stattfinden. Eine Möglichkeit, den Unterschiedsbetrag als (Drohverlust-)Rückstellung zu passivieren, besteht nicht ${ }^{613}$.

In der Gewinn- und Verlustrechnung fließen Abschreibungen auf das Umlaufvermögen in das Betriebsergebnis ein. Auf Risiken des normalen Geschäftsbetriebs beruhende Wertberichtigungen - z. B. mangelnde Marktgängigkeit des Vorratsbestands - werden als „Bestandsveränderung der Vorräte“ gezeigt. Aus besonderen Ereignissen - wie Schadensfällen - resultierende Abwertungen werden unter dem Posten „Außergewöhnliche Abwertungen des Umlaufvermögens“ ausgewiesen. Abschreibungen auf Forderungen sind als „sonstiger betrieblicher Aufwand" ebenfalls Bestandteil des Betriebsergebnisses, Korrekturen des Finanzumlaufvermögens hingegen finden sich im Finanzergebnis ${ }^{614}$.

\subsection{Wertaufholung}

Die 4. EG-Richtlinie verlangt eine Wertaufholung, wenn die Umstände, die in früheren Perioden zur außerplanmäßigen Abschreibung von Vermögenswerten des Anlagevermögens ${ }^{615}$ oder des Umlaufvermögens ${ }^{616}$ geführt haben, nicht mehr vorliegen.

In Finnland hat sich die mit dem Gesetzesentwurf befasste Arbeitsgruppe mangels EU-Vorgaben zum Wertaufholungsverfahren an den Standards des IASB orientiert $^{617}$.

Sie hat in Anlehnung an IAS 25 (reformatted 1994) empfohlen, Wertaufholungen erfolgswirksam $\mathrm{zu}$ buchen $^{618}$. Mit Hinweis auf IAS 16 (revised 1993) hat die KPL-Arbeitsgruppe 1995 zudem vorgeschlagen, dass der Betrag der Wertaufholung unter Berücksichtigung der planmäßigen Abschreibungen, die inzwischen vorgenommen worden wären, zu ermitteln sei ${ }^{619}$.

Im Buchführungsgesetz von 1997 ist die Vorschrift zur Wertaufholung mit KPL 5:16 erstmals in finnisches Recht eingeführt worden ${ }^{620}$ :

Wertaufholung

613

614

615

616

617

618

Vgl. Leppiniemi (2000a), S. 167.

Vgl. Gliederungsschemata der Gewinn- und Verlustrechnung in KPA 1:1 und KPA 1:2.

Vgl. Art. 35 Abs. 1 (c) (dd) der 4. EG-Richtlinie.

Vgl. Art. 39 Abs. 1 (d) der 4. EG-Richtlinie.

Vgl. KPL-Arbeitsgruppe 1995, Teil I (1996), S. 91.

Gewerbesteuerrechtlich sind Wertaufholungen in Finnland seit 1993 ertragswirksam vorzunehmen und unterliegen der Steuerpflicht. Das Buchführungsrecht ist in diesem Fall nicht maßgeblich für die steuerliche Gewinnermittlung. Vgl. EVL 5a.

Vgl. KPL-Arbeitsgruppe 1995, Teil I (1996), S. 91-93.

Übersetzung ins Deutsche durch die Verfasserin. 
Erweist sich eine Aufwandsbuchung, die gemäß KPL 5:2 im Finanzumlaufvermögen, gemäß KPL 5:6.1 im Umlaufvermögen oder gemäß KPL 5:13 im Anlagevermögen vorgenommen wurde, spätestens am Abschluss-Stichtag als gegenstandslos, so ist die Aufwandsbuchung zu korrigieren.

Eine Wertaufholung ist in Finnland dann vorzunehmen, wenn sich die in Vorjahren außerplanmäßig zum Nachteil des Unternehmens korrigierten Einnahmenerwartungen wieder verbessern ${ }^{621}$. Ist in der Vergangenheit nicht nur ein einzelner Vermögenswert, sondern seine Cash-generating unit wertberichtigt worden, so ist das Wertaufholungspotenzial proportional auf die einzelnen Buchwerte dieser Cash-generating unit zu verteilen ${ }^{622}$.

Ein Unternehmen hat zu jedem Bilanzstichtag zu prüfen, ob die Gründe für eine in Vorjahren durchgeführte außerplanmäßige Abschreibung noch existieren. Werden Tatsachen, die das Geschäftsjahr oder frühere Jahre betreffen und eine Wertaufholung fordern, erst nach dem Abschluss-Stichtag bekannt (Wertaufhellung), so müssen diese berücksichtigt werden, wertbegründende Tatsachen hingegen nicht $^{623}$.

Fraglich ist, in welchem Posten der Gewinn- und Verlustrechnung Wertaufholungen auszuweisen sind. Die Mitgliedstaaten der EU gehen hier durchaus unterschiedlich vor. In Finnland werden Wertaufholungen für Vermögenswerte des Anlagevermögens in der Regel unter dem Posten ausgewiesen, unter dem auch die ursprüngliche Aufwandsbuchung vermerkt worden ist. Wertaufholungen von Vermögenswerten des Umlaufvermögens sind hingegen grundsätzlich in der Bestandsveränderung der Vorräte zu erfassen. Sind die Wertaufholungsbeträge im Verhältnis zum Jahresergebnis wesentlich, so dürfen sie auch als außerordentliche Erträge in der Gewinn- und Verlustrechnung gezeigt werden ${ }^{624}$. Korrigiert die Wertaufholung die Wertberichtigungen mehrerer - zurückliegender - Geschäftsjahre, so ist sie immer Bestandteil des außerordentlichen Ergebnisses ${ }^{625}$.

Wertaufholungen sind im Anhang einerseits beim entsprechenden Bilanzposten zu erläutern ${ }^{626}$, andererseits - sofern dort ausgewiesen - beim außerordentlichen Ergebnis aufzugliedern ${ }^{627}$.

$621 \quad$ Vgl. Räty (1998 b), S. 74

622 Vgl. KILA-Anweisung „Planmäßige Abschreibungen“, Abschnitt 6, die hinsichtlich der Cash-generating unit auf IAS 36 (approved 1998) verweist. S. auch Kap. 3.1.6.2.2.3 dieser Arbeit.

623 Vgl. Järvinen et al. (2000), S. 505.

624 Vgl. KPL-Arbeitsgruppe 1995, Teil I (1996) S. 93, vgl. Järvinen et al. (2000) S. 505-506, vgl. Leppiniemi (2000 a), S. 169 und vgl. Räty (1998 b), S. 75.

625 Vgl. Räty (1998 b), S. 74.

626 Vgl. KPA 2:4.

627 Vgl. KPA 2:3.2. 


\subsection{Neubewertung}

Mit Art. 33 Abs. 1 der 4. EG-Richtlinie gesteht der Richtliniengeber den Mitgliedstaaten das Wahlrecht zu, allen oder einzelnen Gruppen von Gesellschaften

- die Bewertung von abnutzbaren Vermögenswerten des Sachanlagevermögens und der Vorräte auf Basis des Wiederbeschaffungspreises ${ }^{628}$,

- die Bewertung aller Jahresabschlussposten unter Berücksichtigung der Inflation $^{629}$ und/oder

- die Neubewertung der Sachanlagen und der Finanzanlagen ${ }^{630}$

zu gestatten oder vorzuschreiben. In solchen Fällen sind Inhalt, Anwendungsbereich und Verfahren der Bewertungsmethoden durch den nationalen Gesetzgeber festzulegen ${ }^{631}$; zudem sind Vorschriften zur Bildung und Behandlung einer Neubewertungsrücklage ${ }^{632}$, zur Überprüfung der Neubewertung ${ }^{633}$ sowie zu Anhangangaben $^{634}$ vom Richtliniengeber vorgesehen und in nationales Recht umzusetzen.

Die Möglichkeit zur Neubewertung von Werten des Sachanlagevermögens besteht in der finnischen Rechnungslegung seit dem Buchführungsgesetz von $1925^{635}$. Ihre Einführung ist ursprünglich damit begründet worden, dass sie im Ausland üblich sei ${ }^{636}$. Die Neubewertung hat immer im Gegensatz zu den Basisprinzipien des jeweilig geltenden Buchführungsrechts gestanden. $\mathrm{Zu}$ Zeiten SAARIOs Ausgaben-Einnahmen-Theorie widersprach sie der vorrangigen Aufgabe der Rechnungslegung, die monetären Ströme in einem Unternehmen - Ausgaben, Einnahmen und Finanztransaktionen - abzubilden, da ihr keine Mittelherkunft oder -verwendung zu Grunde lag ${ }^{637}$. Heute stellt sie eine Ausnahme zur geltenden Vorschrift des KPL 2:1 dar, wonach der Buchführungspflichtige in seiner Buchführung Geschäftsvorfälle als Ausgaben, Einnahmen, Zahlungsvorgänge sowie deren Korrektur- und Abgrenzungsposten zu vermerken hat ${ }^{638}$. Eine Neubewertung bildet keinen Geschäftsvorfall ab. Sie zeigt stille Reserven im Anlagevermögen auf, ohne dass diese ertragswirksam realisiert werden. Sie bildet so erfolgsneutral künftige Gewinne im Jahresabschluss $a b^{639}$.

Bei der Umsetzung von Art. 33 in finnisches Recht hat sich die Forderung der Richtlinie, neben den fortgeführten Anschaffungs- oder Herstellungskosten auch

\footnotetext{
Vgl. Art. 33 Abs. 1 (a) der 4. EG-Richtlinie.

Vgl. Art. 33 Abs. 1 (b) der 4. EG-Richtlinie.

Vgl. Art. 33 Abs. 1 (c) der 4. EG-Richtlinie.

Vgl. Art. 33 Abs. 1 S. 2 der 4. EG-Richtlinie.

Vgl. Art. 33 Abs. 2 der 4. EG-Richtlinie.

Vgl. Art. 33 Abs. 3 der 4. EG-Richtlinie.

Vgl. Art. 33 Abs. 4 der 4. EG-Richtlinie.

Vgl. Sorsa (1998 b), S. 33 und vgl. Kap. 2.1.2.1 dieser Arbeit.

Vgl. Räty (1998 b), S. 44.

Vgl. Kap. 2.1.3.1 dieser Arbeit.

Vgl. KPL 2:1 und die kommentierende Literatur, z. B. Räty (1998 b), S. 44 und Järvinen et al. (2000), S. 507.

Vgl. Järvinen et al. (2000), S. 507.
} 
den neubewerteten Teil von Werten des Sachanlagevermögens planmäßig abzuschreiben, als problematisch erwiesen. Das Verbuchen nicht realisierter Ausgaben als Aufwand widerspricht nicht nur dem Realisationsprinzip, ein solches Vorgehen hätte auch umfangreiche Änderungen im Steuerrecht hinsichtlich der Abzugsfähigkeit von Abschreibungen oder andere Unterschiede zwischen Handels- und Steuerrecht zur Folge gehabt. Auch hätten Teile des Aktienrechts mit Hinblick auf die Ausschüttungsfähigkeit des Eigenkapitals novelliert werden müssen ${ }^{640}$. Der Gesetzgeber hat deshalb auf die Umsetzung von Art. 33 Abs. 1 (a) und (b) der 4. EG-Richtlinie in finnisches Recht verzichtet und die Neubewertung nach Art. 33 Abs. 1 (c) so implementiert, dass sie auf diejenigen Vermögenswerte des Sach- und Finanzanlagevermögens beschränkt wird, die nicht planmäßig abgeschrieben werden ${ }^{641}$.

KPL 5:17 lautet ${ }^{642}$ :

\section{Neubewertung}

(1) Wenn der wahrscheinliche Veräußerungspreis von zum Anlagevermögen gehörenden Grundstücken, Wasserflächen oder Wertpapieren am Abschluss-Stichtag dauerhaft und wesentlich über den ursprünglichen Anschaffungskosten liegt, darf höchstens der Unterschiedsbetrag in der Bilanz als Neubewertung vermerkt werden. Ein der Neubewertung entsprechender Betrag ist als Neubewertungsrücklage im Eigenkapital auszuweisen. Sind die der Neubewertung zu Grunde liegenden Annahmen nicht mehr gegeben, so ist sie aufzulösen.

(2) Wenn die in Abs. 1 gemeinte Neubewertung Wertpapiere oder Anteile eines Mutterunternehmens am Tochterunternehmen zum Gegenstand hat, sind bei der Durchführung der Neubewertung diejenigen Wertpapiere oder Anteile, die das Tochterunternehmen seinerseits am Mutterunternehmen hält, als wertlos zu betrachten.

Der finnische Gesetzgeber lässt den Buchführungspflichtigen das Wahlrecht zur Neubewertung. Folgende Kriterien müssen kumulativ erfüllt sein:

- Die neubewerteten Vermögenswerte müssen dem Anlagevermögen angehören.

- Bei den neubewerteten Vermögenswerten handelt es sich um Grundstücke, Wasserflächen oder Wertpapiere.

- Am Abschluss-Stichtag liegt der wahrscheinliche Veräußerungspreis über den ursprünglichen Anschaffungskosten.

- Der Unterschiedsbetrag ist von Dauer.

- Der Unterschiedsbetrag ist wesentlich.

Das Fachschrifttum ist sich einig, diese Kriterien inhaltlich eng auszulegen, da die Neubewertung zum Ausweis nicht realisierter Werte führe ${ }^{643}$.

640 Vgl. Räty (1998 b), S. 45-46.

641 Vgl. HE 173/1997, S. 27 und vgl. KILA-Anweisung „Planmäßige Abschreibungen“, Abschnitt 2.2 .

642 Übersetzung ins Deutsche durch die Verfasserin.

643 Vgl. Leppiniemi (2000 a), S. 295, vgl. Järvinen et al. (2000), S. 508-509 und vgl. Räty (1998 b), S. 45-46. 
Es stellt sich die Frage nach der Bedeutung des Begriffs ,wahrscheinlicher Veräußerungspreis“. Dem Wortlaut nach handelt es sich hierbei um den auf dem Absatzmarkt realisierbaren Preis. Kritiker weisen zu Recht darauf hin, dass bei Vermögenswerten des Anlagevermögens keine Verkaufsintention bestehe und es unter der Prämisse der Unternehmensfortführung Ziel der Neubewertung sei, Informationen über den Wiederbeschaffungspreis zu vermitteln ${ }^{644}$.

Es kann sich bei Vermögenswerten des Anlagevermögens durchaus um Werte handeln, die nicht oder nur schwierig ermittelbar sind, weil für sie kein Marktpreis existiert. Für solche Fälle wird das Einholen eines Sachverständigen-Gutachtens empfohlen $^{645}$.

Die Forderung, der wahrscheinliche Veräußerungspreis habe über den ursprünglichen - nicht den fortgeführten - Anschaffungskosten zu liegen, basiert einzig auf finnischer Tradition und nicht auf einer Vorschrift der Richtlinie. Sie ist überflüssig, da bei Wegfall der Gründe für eine in früheren Geschäftsjahren durchgeführte Wertberichtigung ein Wertaufholungsgebot besteht ${ }^{646}$. Eine Neubewertung von nicht abnutzbaren Werten des Anlagevermögens, die grundsätzlich nicht planmäßig abgeschrieben werden, muss deshalb immer die ursprünglichen Anschaffungskosten überschreiten.

Als Neubewertung zu aktivieren ist höchstens der Unterschiedsbetrag zwischen ursprünglichen Anschaffungskosten und wahrscheinlichem Veräußerungspreis. Der Gesetzgeber sieht somit eine Aktivierungsobergrenze vor und lässt dem Buchführungspflichtigen einen Bewertungsspielraum zwischen Anschaffungskosten und dieser Aktivierungsobergrenze. Dies kann dem Ziel der Neubewertung, ein korrektes Bild der Kapitalstruktur eines Unternehmens zu erzeugen, entgegenstehen.

Kritiker dieser Regelung vertreten die Auffassung, die Festlegung einer Aktivierungsobergrenze bedeute implizit das Verbot, wahrscheinliche oder kalkulatorische Kosten, die bei Abbau oder Transport des auf Basis des Veräußerungspreises neu zu bewertenden Vermögenswerts entstehen, zu aktivieren. Diese Kosten seien vielmehr bei der Neubewertung von der Obergrenze abzuziehen ${ }^{647}$.

Andere Autoren sind dagegen der Ansicht, ein solcher Realisierungsaufwand müsse nicht vom Unterschiedsbetrag abgezogen werden, da keine Verkaufsintention bestehe. Eine Aktivierung zu einem niedrigeren Betrag als dem Höchstbetrag

644 Vgl. Leppiniemi (2000 a), S. 295.

645 Vgl. Järvinen et al. (2000), S. 509.

646 Vgl. KPL 5:16 und Kap. 3.1.6.2.2.5 dieser Arbeit. Es ist allerdings zu beachten, dass die Wertaufholung im Gegensatz zu einer Neubewertung erfolgswirksam geschieht.

647 Vgl. Järvinen et al. (2000), S. 509. 
entspräche aber immer dem Vorsichtsprinzip als Bestandteil der Art guter Buchführung ${ }^{648}$.

Die vom Gesetzgeber geforderte Dauerhaftigkeit interpretiert die kommentierende Literatur als „absehbare Zukunft“. Andernfalls sei die getroffene Regelung über die Rücknahme der Neubewertung bei Nicht-Fortbestehen der ihr zu Grunde liegenden Annahmen nicht notwendig ${ }^{649}$. Aus dem Gebot der Dauerhaftigkeit folgt, dass es sich bei den neu zu bewertenden Grundstücken nicht um solche handeln kann, die aktuell oder in Zukunft dem Abbau von Substanzen dienen, da sie einer laufenden Wertveränderung unterliegen ${ }^{650}$. Auch für die vom Gesetzgeber verlangte Wesentlichkeit existiert keine Legaldefinition ${ }^{651}$. Die Praxis nimmt Beträge, die zehn Prozent der ursprünglichen Anschaffungskosten übersteigen, als wesentlich $a^{652}$. Der KILA hat noch auf Basis des alten Buchführungsgesetzes die Empfehlung ausgesprochen, im Zuge einer Neubewertung Dauerhaftigkeit und Wesentlichkeit des Unterschiedsbetrags durch externe Gutachter bestätigen zu lassen $^{653}$.

Anders als nach IAS muss in Finnland nicht für die gesamte Gruppe, zu der der neu bewertete Vermögenswert zählt, eine Neubewertung vorgenommen werden, sondern nur für den einzelnen Vermögensgegenstand ${ }^{654}$.

Die Werthaltigkeit einer in früheren Jahren durchgeführten Neubewertung ist jährlich im Rahmen der Jahresabschlusserstellung zu überprüfen ${ }^{655}$. Hierbei ist die notwendige Vorsicht geboten.

Buchungstechnisch wird die Neubewertung dem jeweiligen Vermögenswert zugeordnet und gemeinsam mit seinen ursprünglichen Anschaffungskosten der Summe ausgewiesen. In Höhe des aktivierten Unterschiedsbetrags ist die unrealisierte Wertsteigerung erfolgsneutral in die Neubewertungsrücklage als Bestandteil des gebundenen Eigenkapitals einzustellen ${ }^{656}$. Die Neubewertungsrücklage von Aktiengesellschaften kann auf Beschluss der Hauptversammlung auch in gezeich-

648 Vgl. Leppiniemi (2000 a), S. 295.

649 Vgl. Järvinen et al. (2000), S. 510 und vgl. Räty (1998 b), S. 45.

650 Vgl. Järvinen et al. (2000), S. 508.

651 KILA wurde bislang nicht aufgefordert, eine Aussage zu treffen (Stand September 2001).

652 Vgl. Järvinen et al. (2000), S. 510 und vgl. Leppiniemi (2000 a), S. 73-74. Auch in Schweden existiert die Aktivierungsvoraussetzung, der Unterschiedsbetrag habe wesentlich zu sein. Wesentlichkeit wird in Schweden vom Gesetzgeber mit 20 Prozent der fortgeführten Anschaffungs- oder Herstellungskosten definiert. Die finnische Fachwelt weist zwar auf die schwedische Auffassung hin, übernimmt sie aber nicht. Vgl. KILA 1427/1996 und KILA 1453/1997, vgl. auch Leppiniemi (2000 a), S. 73-74.

654 Vgl. Järvinen et al. (2000), S. 514.

655 Vgl. Räty (1998 b), S. 47.

656 Zur Neubewertungsrücklage vgl. auch Kap. 3.2.4.1 dieser Arbeit. 
netes Kapital umgewandelt werden ${ }^{657}$. Die Neubewertungsrücklage unterliegt unabhängig vom Ausweis stets einer Ausschüttungssperre.

Eine erfolgsneutrale Rücknahme der Neubewertung und der Neubewertungsrücklage ist zwingend dann vorgeschrieben, wenn die Voraussetzungen für die Aktivierung nicht mehr gegeben sind. Diese Situation kann nach einer Änderung der allgemeinen Marktlage oder der betrieblichen Rahmenbedingungen wie beispielsweise der Umweltgesetzgebung eintreten ${ }^{658}$. Die Neubewertungsrücklage ist auch dann aufzulösen, wenn die in sie eingestellten Beträge durch den Abgang des Vermögenswerts realisiert worden sind ${ }^{659}$.

Es kann sein, dass die Neubewertungsrücklage zwischenzeitlich ganz oder teilweise in das gezeichnete Kapital eingestellt worden ist und deshalb nicht ausreicht, um die Neubewertung rückgängig zu machen. Dann ist das nicht gebundene Eigenkapital in Höhe des Differenzbetrags aufzulösen, auch, wenn das nicht gebundene Eigenkapital bei diesem Vorgang negativ wird. Mit der Neubewertung verbundene Vorgänge (Einstellung oder Auflösung) sind in jedem Fall erfolgsneutral zu behandeln ${ }^{660}$.

Im Anhang sind die mit der Neubewertung verbundenen Grundsätze, die Ermittlung und die Höhe des wahrscheinlichen Veräußerungspreises sowie die Wertbestimmungsmethode anzugeben $^{661}$.

657 Vgl. OYL 12:3b.

658 Vgl. Järvinen et al. (2000), S. 511.

659 Vgl. OYL 12:3b.

660 Vgl. Järvinen et al. (2000), S. 512.

661 Vgl. KPA 2:4.1 Nr. 6. 


\subsection{Zusammenfassender Überblick}

Folgende Abbildung zeigt die Alternativen zur Bewertung von Vermögenswerten nach finnischem Buchführungsgesetz auf:

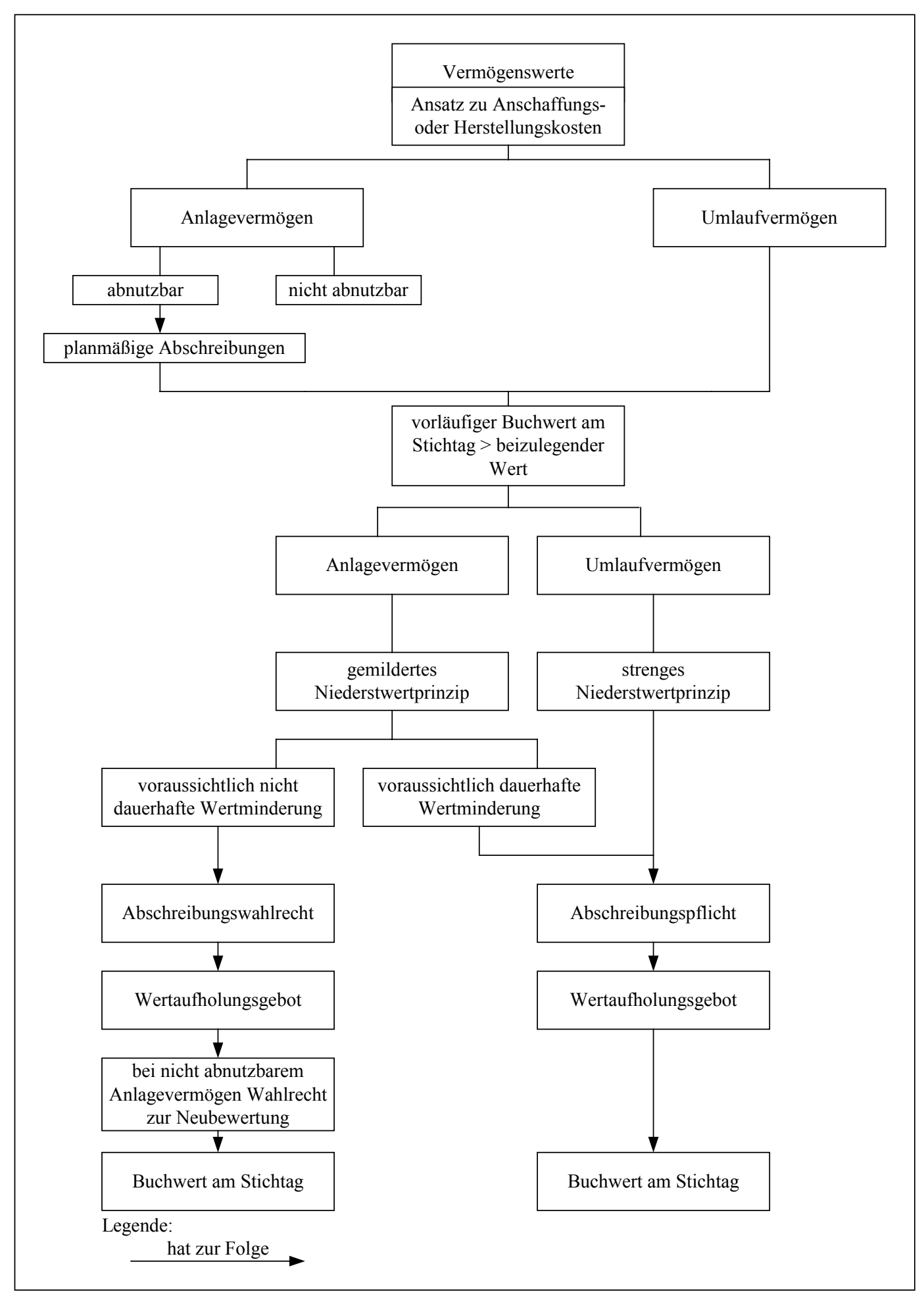

Abb. 7: Bewertung von Vermögenswerten 


\subsubsection{Bewertung von Passiva}

Nach KPL 5:2.3 sind Verbindlichkeiten mit ihrem Rückzahlungsbetrag anzusetzen, also mit dem Betrag, der für die Tilgung der entsprechenden Schuld benötigt wird $^{662}$ :

Forderungen, liquide Mittel und Verbindlichkeiten

In der Bilanz werden angesetzt:

[...] 3. Verbindlichkeiten mit ihrem Nennwert oder, wenn die Schuld an einen Index oder sonstigen Vergleichswert gebunden ist, mit dem auf dem Vergleichswert beruhenden höheren Wert als dem Nennwert.

Passiviert wird der (voraussichtliche) Wert der wirtschaftlichen Belastung zu dem Zeitpunkt, an dem die Verpflichtung entsteht oder bekannt wird. Dies betrifft auch zukünftige Aufwendungen und Verluste.

Pflichtrückstellungen sind nach KPL 5:14 mit dem Betrag anzusetzen, der nach vernünftiger kaufmännischer Beurteilung erforderlich ist. Dies ist der Teil der Ausgaben, der die zukünftige Einnahmenerwartung übersteigt. Nach KPL 5:14.4 - im Einklang mit Art. 42 der 4. EG-Richtlinie - ist bei der Bewertung das Vorsichtsprinzip zu beachten.

Rückstellungen dürfen nach KPL 5:14.5 - basierend auf Art. 20 Abs. 3 der 4. EGRichtlinie - keine Wertberichtigungen zu Aktivposten darstellen ${ }^{663}$.

Auf Einzelfragen zur Bewertung der Schulden wird im Folgenden in Kap. 3.2.5 bei der Erläuterung der jeweiligen Bilanzposten näher eingegangen.

\subsubsection{Fremdwährungsumrechnung einzelner Geschäftsvorfälle}

\subsubsection{Vorschriften zur Fremdwährungsumrechnung}

Die 4. EG-Richtlinie enthält explizit keine Bewertungsvorschriten zur Fremdwährungsumrechnung ${ }^{664}$. Die Richtlinienverfasser nennen jedoch in Art. 31 grundlegende Bewertungsregeln, die es auch bei der Währungsumrechung zu beachten gilt. Hervorzuheben ist hier neben Vorsichts-, Realisations- und Imparitätsprinzip die Forderung, dass alle Abschreibungen und Wertberichtigungen ungeachtet der Frage, ob das Jahresergebnis positiv oder negativ ist, zu erfassen sind (Vollstän-

662 Übersetzung ins Deutsche durch die Verfasserin.

663 Übersetzung ins Deutsche durch die Verfasserin.

664 Die Mitgliedstaaten der Europäischen Union haben unter Beachtung der genannten grundlegenden Bewertungsregeln entscheiden können, ob und wie sie die Fremdwährungsumrechnung vorschreiben. Eine Untersuchung der FEE aus dem Jahr 1991 hat große Unterschiede in der Fremdwährungsbewertung zwischen den einzelnen Staaten ergeben. Vgl. FEE Survey of Published Accounts 1991, in dem Jahresabschlüsse europäischer Unternehmen für das Geschäftsjahr 1989 untersucht werden, in: FEE (1992). 
digkeitsgebot). Es gilt die Regel, dass alle Erträge und Aufwendungen des Geschäftsjahrs unabhängig vom Zahlungstag zu buchen sind (Pagatorik).

Art. 41 der 4. EG-Richtlinie erlaubt, bei Verbindlichkeiten den Unterschiedsbetrag zwischen erhaltener Zahlung und höherem zurückzuzahlendem Betrag zu aktivieren. In einem solchen Fall muss der Unterschiedsbetrag über die Restlaufzeit der Verbindlichkeit erfolgswirksam aufgelöst werden.

Die Umrechnungsprinzipien sind gemäß Art. 43 der 4. EG-Richtlinie im Anhang anzugeben $^{665}$.

Anders als in Deutschland, wo keine gesetzlichen Vorschriften zur Währungsumrechnung im Einzelabschluss existieren, greift der finnische Gesetzgeber mangels EU-Vorschriften auf IAS 21 (revised 1993) zurück. Auf ihm basiert die in KPL 5:3 geregelte Währungsumrechnung für die Abschlusserstellung. Die unterjährige Währungsumrechnung in der laufenden Buchhaltung - Zugangsbewertung und Behandlung unterjährig realisierter Umrechnungsdifferenzen - ist Thema der allgemeingültigen Anweisung vom 15. November $1999^{666}$, die auch die gesetzliche Vorschrift des KPL 5:3 auslegt und somit die Art guter Buchführung für die Fremdwährungsumrechnung insgesamt festlegt ${ }^{667}$.

\subsubsection{Zugangsbewertung und Behandlung unterjährig realisierter Um- rechnungsdifferenzen}

Bei Erstverbuchung erfolgt die Umrechnung von Geschäftsvorfällen in Fremdwährung grundsätzlich mit dem von der Europäischen Zentralbank bzw. von Finnlands Zentralbank notierten Mittelkurs am Zugangstag ${ }^{668}$.

Aus Vereinfachungsaspekten kann die Einbuchung auch zu festen internen Kursen erfolgen, wenn der Unterschied zum Mittelkurs betragsmäßig nicht von Bedeutung ist (Grundsatz der Wesentlichkeit). Anstelle des Mittelkurses dürfen auch andere von Finnlands Zentralbank bzw. der Europäischen Zentralbank veröffent-

665 Da trotz dieser allgemeinen, anwendbaren Grundsätze ein erheblicher Spielraum bei der Fremdwährungsumrechnung besteht, welche die Vergleichbarkeit und Gleichwertigkeit der Abschlüsse verschiedener Mitgliedstaaten untereinander gefährdet, hat die EU-Kommission das Accounting Advisory Forum zu einer Stellungnahme aufgefordert. Das Forum diskutiert momentan einen Entwurf, der sich mit dem anzuwendenden Umrechnungskurs und der Behandlung von Währungsumrechnungsdifferenzen auseinander setzt. Vgl. van Hulle und van der Tas (2001), S. 836-839.

Vgl. KILA-Anweisung „Währungsumrechnung“.

667 Zudem hat der KILA in vier Anweisungen die Umstellung auf den Euro und damit verbunden Umrechnungsprobleme für die Übergangsphase vom 01. Januar 1999 bis 31. Dezember 2001 und darüber hinaus normiert. Vgl. KILA 1489/1997, KILA 1520/1998, KILA 1528/1998 und KILA 1532/1998.

Vgl. KILA-Anweisung „Währungsumrechnung“, Abschnitt 2. 
lichte Kurse oder die Geld- und Briefkurse der Geschäftsbanken verwendet werden, wobei das Vorsichtsprinzip beachtet werden muss ${ }^{669}$.

Werden unterjährig Kursgewinne oder -verluste aus Lieferungs- und Leistungsgeschäften realisiert, sind die ursprünglichen Zugangsbuchungen um die jeweiligen Beträge zu korrigieren.

Unterjährig realisierte Umrechnungsdifferenzen aus Finanzgeschäften werden in der Gewinn- und Verlustrechnung gesondert unter „Andere Zinsen und Erträge“ bzw. „Zinsen und andere Aufwendungen“" ausgewiesen.

Bei der Bewertung von Gegenständen des Anlagevermögens mit einem Anschaffungspreis in ausländischer Währung ist grundsätzlich der Zahlungstag - nicht der Anschaffungstag - für die Umrechnung maßgeblich. Ausnahme von dieser Regel ist der Kauf auf Kredit, bei dem der Anschaffungspreis durch den Wechselkurs zum Zeitpunkt der Anschaffung bestimmt wird. Bei Vermögensgegenständen des Anlagevermögens mit langfristiger Fertigungsdauer können jedoch Kursgewinne oder -verluste aus Darlehen, die für die Zeitdauer der Fertigstellung aufgenommen wurden, den Anschaffungspreis korrigieren ${ }^{670}$.

Finanztransaktionen sind gemäß KPL 2:4 täglich zu verbuchen, andere in Fremdwährung stattfindende Geschäftsvorfälle wenigstens einmal monatlich ${ }^{671}$. Buchungen im Ausland befindlicher Niederlassungen oder Betriebsstätten dürfen in Fremdwährung durchgeführt werden, sofern sie einmal im Monat in Euro umgerechnet werden ${ }^{672}$.

\subsubsection{Bewertung von Fremdwährungspositionen am Bilanzstichtag}

Die Bewertung von Fremdwährungspositionen im Jahresabschluss erfolgt grundsätzlich zum Stichtagsmittelkurs ${ }^{673}$. Seit Einführung des Euro zum 01. Januar 1999 gelten in Finnland die von der Europäischen Zentralbank veröffentlichten Wechselkurse von Fremdwährungen gegenüber dem Euro.

Ist zur Ausschaltung von Wechselkursänderungen der Kurs vertraglich oder anderweitig fixiert, werden die hiervon betroffenen Fremdwährungspositionen zum Bilanzstichtag mit dem fixierten Kurs bewertet ${ }^{674}$. Im Fall geleisteter oder erhal-

669 Das Vorsichtsprinzip fordert, dass die Umrechnung der Forderungen mit Geldkurs, die Umrechnung der Verbindlichkeiten mit Briefkurs erfolgen muss. Vgl. KILA-Anweisung ,Währungsumrechnung“, Abschnitt 2.

670 Vgl. KILA-Anweisung „Währungsumrechnung“, Abschnitt 2.

671 Vgl. Kap. 3.1.4 dieser Arbeit.

672 Vgl. KILA-Anweisung „Währungsumrechnung“, Abschnitt 2.

673 Vgl. KPL 5:3.1 und vgl. KILA-Anweisung ,Währungsumrechnung“, Abschnitt 3.1.

674 Vgl. KPL 5:3.1,2. 
tener Anzahlungen gilt bei der Bewertung für den Jahresabschluss der Zugangs-, nicht der Stichtagskurs ${ }^{675}$.

Die im Jahresabschluss angewendeten Umrechnungsprinzipien sind im Anhang anzugeben, sofern die Umrechnung nicht zum Stichtagsmittelkurs erfolgt ist ${ }^{676}$.

\subsubsection{Behandlung und Ausweis nicht realisierter Umrechnungsdiffe- renzen am Bilanzstichtag}

Nicht realisierte Umrechnungsdifferenzen aus Forderungen, Verbindlichkeiten oder Finanztransaktionen am Bilanzstichtag werden grundsätzlich so behandelt wie unterjährig realisierte Kursgewinne oder -verluste. Die Umsatzerlöse werden am Stichtag um nicht realisierte Umrechnungsdifferenzen aus Forderungen erfolgswirksam berichtigt, Aufwendungen oder Anschaffungskosten werden um nicht realisierte Umrechnungsdifferenzen aus Verbindlichkeiten erfolgswirksam korrigiert und nicht realisierte Umrechnungsdifferenzen aus Finanztransaktionen gehen erfolgswirksam in „Zinsen und ähnliche Erträge“ oder ,Zinsen und ähnliche Aufwendungen“ ein. Diese Vorgehensweise trifft sowohl auf nicht realisierte Kursgewinne als auch auf nicht realisierte Kursverluste zu. Aus dem Vorsichtsprinzip heraus müssen nicht realisierte Kursverluste erfolgswirksam verbucht werden, nicht realisierte Kursgewinne dürfen laut KILA-Anweisung jedoch nur dann verbucht werden, wenn ihr Eintritt wahrscheinlich ist. Der Begriff ,wahrscheinlich“" wird in diesem Zusammenhang vom KILA nicht näher definiert ${ }^{677}$.

Sind die am Bilanzstichtag zu bewertenden Forderungen und Verbindlichkeiten langfristig, d. h. beträgt ihre Restlaufzeit noch länger als ein Jahr, kann auf eine erfolgswirksame Verbuchung der Umrechnungsdifferenzen zu diesem Stichtag verzichtet werden. In die Gewinn- und Verlustrechnung müssen solche Umrechnungsdifferenzen erst in dem Geschäftsjahr eingehen, in dem sie tatsächlich realisiert werden. Für den Fall, dass sie nicht sofort erfolgswirksam verbucht werden, sind Kursverluste als aktive Rechnungsabgrenzung zu berücksichtigen und über die Restlaufzeit der zugehörigen Forderung bzw. Verbindlichkeit aufzulösen. Kursgewinne müssen analog als passive Rechnungsabgrenzung ausgewiesen und ebenfalls über die Restlaufzeit des zu Grunde liegenden Sachverhalts aufgelöst werden $^{678}$.

\footnotetext{
675 Vgl. KILA-Anweisung, „Währungsumrechnung“, Abschnitt 3.1.

676 Vgl. KPA 2:2.1,7.

677 Vgl. KILA-Anweisung, Währungsumrechnung“, Abschnitt 3.2.

678 Vgl. KILA-Anweisung „Währungsumrechnung“, Abschnitt 3.2. Sind die in der aktiven und/oder passiven Rechnungsabgrenzung enthaltenen Posten aus Währungsumrechnung wesentlich, so sind sie im Anhang gesondert anzugeben (KPA 2:4.1,11).
} 
Devisentermingeschäfte, die nicht zum Zweck der Kurssicherung abgeschlossen worden sind, werden im Jahresabschluss differenziert berücksichtigt. Unrealisierte Kursverluste werden erfolgswirksam gebucht, während unrealisierte Kursgewinne nicht erfasst werden. Handelt es sich um eine geschlossene Devisenposition, bei der bei Wechselkursänderung das Ergebnis nicht mehr beeinflusst werden kann, sind Kursgewinne und -verluste in dem Geschäftsjahr als Erträge oder Aufwendungen zu erfassen, in dem die Position geschlossen wurde ${ }^{679}$.

Zur Behandlung von Umrechnungsdifferenzen bei Kurssicherungsgeschäften am Bilanzstichtag verweist der KILA auf IAS 39. Der KILA erläutert, dass mit dem Ziel der Kurssicherung angeschaffte Forderungen, Verbindlichkeiten und Eventualverbindlichkeiten dem Grundsatz nach mit dem Stichtagsmittelkurs umzurechnen seien, ebenso die Forderungen und Verbindlichkeiten, die gesichert werden sollen $^{680}$.

Für die Bewertung von Kurssicherungsgeschäften gilt, dass unrealisierte Kursgewinne auf Grund des Vorsichtsprinzips maximal in der Höhe ausgewiesen werden dürfen, in denen ihnen unrealisierte Kursverluste gegenüberstehen ${ }^{681}$.

Bei Devisentermingeschäften, die zum Zweck der Kurssicherung abgeschlossen worden sind, werden unrealisierte Kursverluste in die aktive Rechnungsabgrenzung, unrealisierte Kursgewinne in die passive Rechnungsabgrenzung eingestellt und über die Restlaufzeit des Geschäfts erfolgswirksam aufgelöst ${ }^{682}$.

Die Bewertungs- und Abgrenzungsmethoden für die im Jahresabschluss enthaltenen Umrechnungsdifferenzen sind im Anhang zu erläutern ${ }^{683}$. Die Angabe von Eventualverbindlichkeiten im Anhang hat in Euro zu erfolgen, als Umrechnungskurs ist der Stichtagsmittelkurs anzuwenden ${ }^{684}$. 


\subsubsection{Behandlung von Bilanzierungsfehlern aus Vorperioden}

Die 4. EG-Richtlinie regelt nicht explizit, wie Bilanzierungsfehler aus Vorperioden zu behandeln sind. Auch der Kontaktausschuss der EU-Kommission hat sich zu dieser Frage bislang nicht geäußert. Mitglieder des Kontaktausschusses haben sich dafür ausgesprochen, kumulierte Unterschiedsbeträge aus der Änderung von Bilanzierungs- und Bewertungsmethoden erfolgswirksam zu erfassen. Diese sind einer Korrektur von Bilanzierungsfehlern aus Vorperioden vergleichbar ${ }^{685}$.

Über den Umgang mit Bilanzierungsfehlern aus Vorperioden - sei es, dass sie bewusst oder unbewusst aus Rechenfehlern oder falscher Interpretation von Bilanzierungsvorschriften resultieren - findet sich weder im KPL noch im KPA eine Regelung. Der KILA hat festgelegt, dass solche Fehler im Abschluss der Periode, in der sie bekannt werden, erfolgswirksam zu korrigieren sind ${ }^{686}$.

Beeinflusst die Korrektur das Jahresergebnis der laufenden Periode wesentlich, so ist sie unter dem Posten ,außerordentliche Erträge“ bzw. ,außerordentliche Aufwendungen“" auszuweisen, sonst unter demjenigen Posten, dem sie bei korrekter Buchung zuzuordnen gewesen wäre. Zudem ist bei wesentlicher Ergebnisauswirkung eine Korrektur im Anhang anzugeben und zu erläutern ${ }^{687}$.

685 Vgl. van Hulle und van der Tas (2001), S. 839.

686 Vgl. KILA 1344/1995.

687 Vgl. KILA 1344/1995 und vgl. Leppiniemi (2000 a) S. 56 und S. 219. 

schluss $^{688}$

\section{Anlagevermögen}

\subsubsection{Immaterielle Vermögenswerte}

\subsection{Ansatz, Bewertung und Ausweis}

Eine Legaldefinition des Begriffs ,,immaterieller Vermögenswert“ existiert nicht. Nach Auffassung des finnischen Fachschrifttums ist ein immaterieller Vermögensgegenstand ein identifizierbarer, nicht monetärer Wert ohne physische Form $^{689}$. Er soll dem Unternehmen in mehreren Geschäftsjahren als Einnahmequelle dienen $^{690}$.

Fraglich ist, was unter dem Begriff ,identifizierbarer Wert" verstanden wird. Ein hinreichendes, aber nicht zwingend notwendiges Kriterium für Identifizierbarkeit liegt in der selbstständigen Verwertbarkeit des Werts. Es können jedoch auch solche Werte aktiviert werden, die zwar eine Einnahmenerwartung verkörpern, aber nicht einzeln verwertbar sind: beispielsweise Ausgaben für Werbung ${ }^{691}$.

Ein weiteres wichtiges, aber ebenfalls nicht zwingendes Indiz für Identifizierbarkeit kann in der Kontrolle des Unternehmens über den Vermögenswert liegen. Kontrolle ist dann gegeben, wenn das Unternehmen auf die künftigen Einnahmen aus dem immateriellen Wert mit Sicherheit zugreifen kann, notfalls vor Gericht seine Rechte geltend machen kann. Es dürfen jedoch auch solche Werte aktiviert werden, über die das Unternehmen keine Kontrolle innehat. So sind beispielsweise Ausgaben für Schulungen der Mitarbeiter aktivierbar, obwohl diese das Unternehmen jederzeit verlassen und ihm die Möglichkeit entziehen können, auf ihr in den Schulungen erworbenes Wissen zuzugreifen ${ }^{692}$.

Ein immaterieller Wert ist nach finnischem Verständnis dann identifizierbar und somit aktivierungsfähig, wenn die erwarteten Einnahmen in Form von Zahlungs-

${ }_{688}$ Im Folgenden werden die einzelnen Bilanzposten sowie ausgewählte Posten der Gewinnund Verlustrechnung dargestellt und untersucht. Finnischer Denkweise entsprechend müsste die Gewinn- und Velustrechnung vorangestellt werden; hier wird, um dem deutschen Leser das Verständnis zu erleichtern, zunächst auf die Bilanz eingegangen. Bei den Bilanzposten werden die korrespondierenden Posten der Gewinn- und Verlustrechnung weitgehend behandelt.

Vgl. Järvinen et al. (2000 a), S. 379.

690 Vgl. KPL 4:3.

691 Vgl. Leppiniemi (2000 a), S. 252, vgl. Järvinen et al. (2000), S. 483 und vgl. Kap. 3.1.5.2 dieser Arbeit.

${ }^{692}$ Vgl. HE 89/1996, S. 128, wo die Aktivierung von Schulungskosten für den BasisMitarbeiterstamm als Gründungsausgabe für zulässig erachtet wird. Vgl. auch Kap. 3.2.1.1.2 dieser Arbeit. 
mitteln oder Zahlungsmitteläquivalenten dokumentierbar sind und dem Vermögenswert eindeutig zugeordnet werden können ${ }^{693}$. Auf seine Cash-generating unit darf wegen des geltenden Vorsichtsprinzips nicht abgestellt werden. Außerdem müssen die mit der Erlangung des immateriellen Werts verbundenen Kosten verlässlich messbar sein ${ }^{694}$. Diese Aktivierungskriterien gelten unabhängig davon, ob der Vermögenswert selbst erstellt oder von Dritten erworben wird.

Die für einen Vermögenswert aufgebrachten Mittel sind regelmäßig relativ zuverlässig ermittelbar. Eine Einnahmenerwartung zu dokumentieren, zu quantifizieren und einem Vermögenswert eindeutig zuzuordnen, erweist sich in den meisten Fällen als problematisch. Der KILA weist wegen dieser Unsicherheit explizit darauf hin, dass eine erfolgswirksame Verbuchung der immateriellen Vermögenswerte immer der Art guter Buchführung entspräche und immer mit dem Vorsichtsprinzip in Einklang stehe ${ }^{695}$. Für sämtliche immaterielle Vermögenswerte gleichgültig, ob selbstständig verwertbar, kontrollierbar oder nicht - besteht ein Aktivierungswahlrecht, keine Aktivierungspflicht ${ }^{696}$.

Dieses Aktivierungswahlrecht ist nicht ausdrücklich im Gesetz verankert. KPL 5:5.1 verlangt zwingend, materielle Vermögenswerte zu aktivieren und planmäßig über ihre voraussichtliche Nutzungsdauer abzuschreiben, ohne die immateriellen Vermögenswerte zu erwähnen - sie unterliegen diesem Zwang somit nicht. Da der Gesetzgeber aber in KPL 5:7 ff. die Periodisierung immaterieller Vermögenswerte regelt und somit ihre Aktivierung voraussetzt, vertreten sowohl der KILA als auch die übrige Fachwelt einhellig die Meinung, dass ein Aktivierungswahlrecht existiere, keine -pflicht ${ }^{697}$.

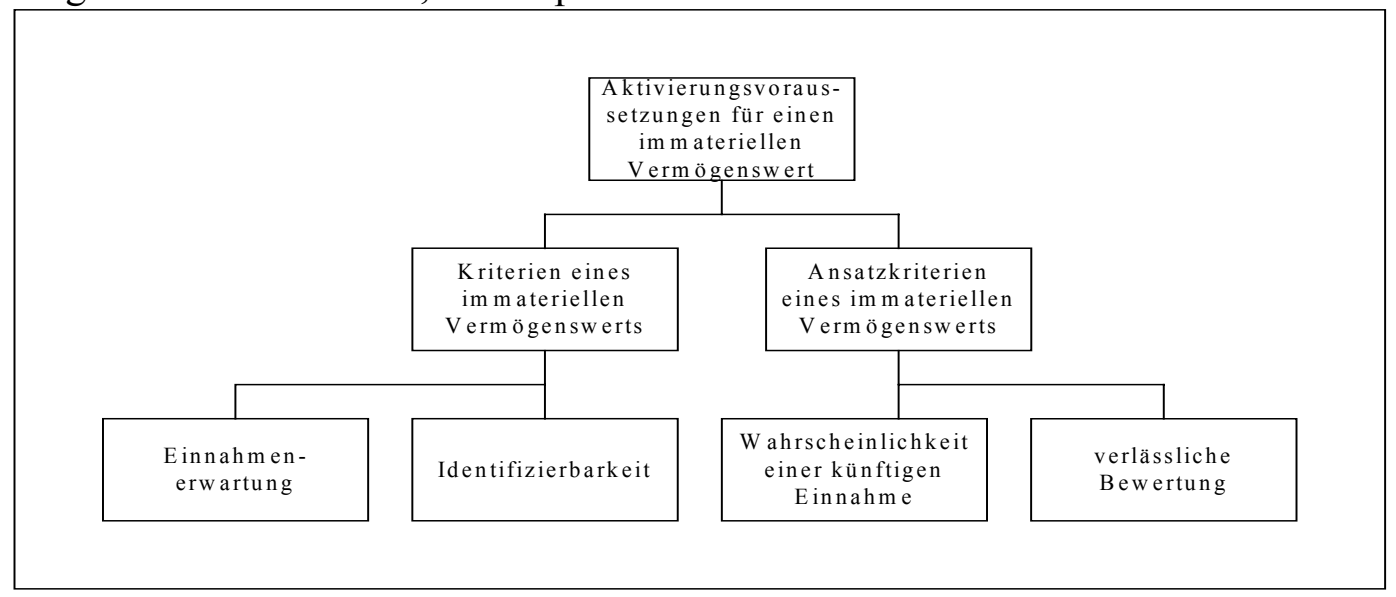

\section{Abb. 8: Voraussetzungen für die Aktivierung immaterieller Vermögens- werte}

\footnotetext{
693 Vgl. Leppiniemi (2000 a), S. 156.

694 Vgl. KPL 5:5.2 und vgl. Järvinen et al. (2000), S. 468-469.

695 Vgl. KILA 1579/1999.

696 Vgl. KILA 1579/1999.

697 Vgl. Leppiniemi und Leppiniemi (1999), S. 85, vgl. Leppiniemi (2000), S. 243 und vgl. Järvinen et al. (2000), S. 460.
} 
Der Gesetzgeber verlangt „besondere Vorsicht“ bei der Aktivierung von Gründungs- ${ }^{698}$ sowie Forschungs- und Entwicklungsausgaben ${ }^{699}$, allerdings, ohne diesen Begriff zu definieren. Außerdem sieht der Gesetzgeber eine Ausschüttungssperre für das nicht gebundene Eigenkapital in Höhe der aktivierten Beträge für Gründungs- ${ }^{700}$, Forschungs-, Teile der Entwicklungsausgaben ${ }^{701}$ und Disagios ${ }^{702}$ bei Darlehen vor ${ }^{703}$.

Aktivierte immaterielle Vermögenswerte sind bei ihrem Zugang mit Anschaffungs- oder Herstellungskosten zu bewerten ${ }^{704}$. Sonderregelungen für die Behandlung von Zuwendungen und von durch Rückstellungen gedeckte Anschaffungsoder Herstellungskosten sind auch bei diesen Werten zu beachten ${ }^{705}$.

Die Abschreibungshöchstdauer der planmäßig abzuschreibenden Werte ist unabhängig von der tatsächlichen Nutzungsdauer gesetzlich festgelegt ${ }^{706}$. Zusätzlich sind andere als planmäßige Abschreibungen möglich ${ }^{707}$. Zu jedem Periodenende ist ein Niederstwerttest durchzuführen. Stellt sich heraus, dass die fortgeführten Anschaffungs- oder Herstellungskosten über der Erwartung künftiger Einnahmen liegen, ist die Differenz als außerplanmäßige Abschreibung zu erfassen und der Wertansatz des immateriellen Vermögenswerts zu korrigieren. Dieser Niederstwerttest ist nach Meinung der kommentierenden Literatur für diejenigen Vermögenswerte besonders gewissenhaft zu dokumentieren, für die der Gesetzgeber den Ansatz unter ,besonderer Vorsicht“" verlangt ${ }^{708}$. Ebenso ist für diese immateriellen Vermögenswerte die Restnutzungsdauer jedes Jahr neu zu überprüfen ${ }^{709}$. Entfallen die Gründe für eine in der Vergangenheit durchgeführte außerordentliche Abschreibung, so besteht ein Wertaufholungsgebot ${ }^{710}$.

Die Gliederung einer finnischen Bilanz sieht die Unterteilung der immateriellen Vermögenswerte in verschiedene Positionen vor.

- Gründungsausgaben

- Forschungsausgaben

- Entwicklungsausgaben

- Immaterielle Rechte

$698 \quad$ Vgl. KPL 5:7.

699 Vgl. KPL 5:8.

700 Vgl. Kap. 3.2.1.1.2 dieser Arbeit.

701 Vgl. Kap. 3.2.1.1.3 dieser Arbeit.

702 Vgl. Kap. 3.2.1.1.6.1 dieser Arbeit.

703 Vgl. OYL 12:2.1.

704 Vgl. Kap. 3.1.6.2.1.1 und 3.1.6.2.1.2 dieser Arbeit.

705 Vgl. Kap. 3.1.6.2.1.3 und 3.1.6.2.1.4 dieser Arbeit.

706 Vgl. Kap. 3.1.6.2.2.1 dieser Arbeit.

707 Für die unterschiedlichen immateriellen Werte gelten die KPL 5:7 ff. Vgl. Kap. 3.2.1.1.2 bis 3.2.1.1.6 dieser Arbeit.

708 Vgl. Leppiniemi (2000 a), S. 101-102 und Järvinen et al. (2000), S. 475.

709 Vgl. Leppiniemi (2000 a), S. 157.

710 Vgl. Kap. 3.1.6.2.2.5 dieser Arbeit. 
- Geschäfts- oder Firmenwert

- Andere Ausgaben mit lang anhaltender Wirkung

- Geleistete Anzahlungen für immaterielle Vermögenswerte

Die Zuordnung der immateriellen Vermögenswerte zu den einzelnen Positionen und ihr Ausweis sind wegen der vom Gesetzgeber geforderten ,besonderen Vorsicht" und der lediglich für Gründungs-, Forschungs- sowie bestimmte Entwicklungsausgaben geltenden Ausschüttungssperre von wesentlicher Bedeutung.

Inhalte dieser Positionen werden im Folgenden dargestellt ${ }^{711}$. Auf eine Erläuterung der geleisteten Anzahlungen für immaterielle Vermögenswerte wird an dieser Stelle verzichtet, da sie wie Forderungen behandelt werden.

\subsection{Gründungsausgaben}

Der Richtlinienverfasser gestattet den nationalen Gesetzgebern, Gründungsausgaben im Rahmen der Vorgaben der 4. EG-Richtlinie selbst zu definieren. Zu beachten ist, dass Gründungsausgaben keine Ausgaben enthalten dürfen, die im Sinne der Richtlinie anderen Bilanzposten zuzuordnen sind.

Nach Auffassung des Kontaktausschusses gehören zu den Gründungsausgaben sowohl im Zusammenhang mit der Unternehmensgründung angefallene Kosten als auch Kosten für Aktienemissionen und Erweiterungs- und Restrukturierungs$\operatorname{kosten}^{712}$.

Untersucht wird, ob der finnische Gesetzgeber die Periodisierung von Gründungsausgaben mit KPL 5:7 richtlinienkonform regelt ${ }^{713}$ :

\section{Periodisierung von Gründungsausgaben}

Ausgaben in Zusammenhang mit der Unternehmensgründung (Gründungsausgaben) dürfen unter Beachtung besonderer Vorsicht aktiviert werden. Aktivierte Gründungsausgaben sind innerhalb von maximal fünf Jahren planmäßig abzuschreiben.

Mit dieser Vorschrift setzt der finnische Gesetzgeber Art. 34 Abs. 1 (a) der 4. EGRichtlinie in nationales Recht um. Die in Art. 34 Abs. 1 (b) geforderte Ausschüttungssperre für nicht gebundenes Eigenkapital in Höhe der aktivierten Beträge ist im finnischen Aktiengesellschaftsgesetz OYL 12:2.1 verankert und demnach von

111 Vgl. Kap. 3.2.1.1.2 bis 3.2.1.1.6 dieser Arbeit.

712 Vgl. van Hulle und van der Tas (2001), S. 808.

713 Übersetzung ins Deutsche durch die Verfasserin. 
allen finnischen Kapitalgesellschaften, aber nicht von allen Buchführungspflichtigen zu beachten ${ }^{714}$.

Zum Inhalt der Gründungsausgaben verweist der Gesetzgeber auf die Buchführungspraxis $^{715}$. Die Auslegung des Inhalts obliegt den mit der Rechnungslegung befassten Berufsständen. Diese wiederum orientieren sich an der Regierungsbegründung HE 89/1996 zum Entwurf des Aktiengesellschaftsgesetzes, in der Gründungsausgaben definiert werden. Die Praxis wendet diese Definition analog für alle Rechtsformen an ${ }^{716}$. Demnach zählen zu den Gründungsausgaben neben Rechtsanwalts- und Notargebühren für den Gründungsvorgang sowie Gebühren für die Eintragung ins Handelsregister beispielsweise auch Kosten für die Entwicklung der Geschäftsidee, für die Standortsuche, für die Planung der Unternehmensstruktur oder die Rekrutierung und Schulung des Basis-Mitarbeiterstamms $^{717}$.

$\mathrm{Ob}$ den Gründungsausgaben in Finnland - wie der Kontaktausschuss dies vorschlägt - auch die Erweiterung des Geschäftsbetriebs bzw. eine Abspaltung, Umwandlung, Restrukturierung oder Neugründung subsumiert werden kann, geht aus der Literatur nicht eindeutig hervor. Die Wortwahl des Gesetzgebers „Gründungsausgaben“ lässt vermuten, dass die Aktivierung lediglich auf den eigentlichen Vorgang der Unternehmensgründung beschränkt ist ${ }^{718}$. Die Frage bleibt offen, da der KILA bislang noch nicht entschieden hat ${ }^{719}$.

Die Nutzungsdauer von Gründungsausgaben beträgt längstens fünf Jahre. Gründungsausgaben sind über ihre Nutzungsdauer planmäßig abzuschreiben.

714 Fraglich ist, ob eine Ausschüttungssperre für andere Rechtsformen sinnvoll sein würde. Häufigste Rechtsform in Finnland ist die Aktiengesellschaft. Auf Grund der Ausstrahlungswirkung auf andere Rechtsformen kann vermutet werden, dass die Ausschüttungssperre von Gesellschaften anderer Rechtsformen berücksichtigt wird.

715 Vgl. HE 173/1997, S. 23.

716 Vgl. HE 89/1996, S. 128.

717 Vgl. Järvinen et al. (2000), S. 474.

718 Diese Interpretation würde auch der britischen Interpretation der „formation expenses“ und den „frais d'établissement“ in Frankreich entsprechen. Anders stellt sich die Lage in Deutschland dar, wo die ,Aufwendungen für Ingangsetzung und Erweiterung des Geschäftsbetriebs" auch die Aktivierung derjenigen Ausgaben zulässt, die nach dem Ende der Anlaufphase auf Grund von räumlicher oder sonstiger Erweiterung des Betriebs getätigt werden. Der Grundsatz der Vorsicht fordert, den Begriff „Erweiterung“ eng auszulegen. 


\subsection{Forschungs- und Entwicklungsausgaben}

Art. 37 Abs. 1 der 4. EG-Richtlinie lässt den Mitgliedstaaten das Wahlrecht, die Aktivierung von Forschungs- und Entwicklungskosten zu gestatten ${ }^{720}$. Der Richtliniengeber empfiehlt in einem solchen Fall eine Abschreibungshöchstdauer von fünf Jahren und eine Ausschüttungssperre des nicht gebundenen Eigenkapitals in Höhe der aktivierten Beträge, schreibt diese jedoch nicht zwingend vor.

KPA 1:6 sieht in der Bilanzgliederung zwar Forschungsausgaben als eigene Bilanzposition vor, der Gesetzgeber regelt aber weder Inhalt noch Nutzungsdauer von aktivierungsfähigen Forschungsausgaben explizit. Sie gehören zur Gruppe der „Ausgaben mit lang anhaltender Wirkung““721. Forschungsausgaben sind typischerweise Kosten für die Erlangung neuer Kenntnisse und für das Erforschen ihrer Anwendungsmöglichkeiten, für das Erforschen von Produkt- und Prozessalternativen sowie für die Planung neuer oder verbesserter Produkt- und Prozessalternativen $\mathrm{zu}$ verstehen ${ }^{722}$.

Die inhaltliche Abgrenzung von Forschungs- zu Entwicklungsausgaben ist von besonderer Bedeutung. Für aktivierte, noch nicht vollständig abgeschriebene Forschungsausgaben hat der Gesetzgeber in OYL 12:2.1 eine Ausschüttungssperre verfügt. Derjenige Teil der aktivierten Entwicklungsausgaben, der die Aktivierungskriterien des KTM:p 50/1998 erfüllt, führt hingegen nicht zu einer Ausschüttungssperre.

Die Ausschüttungssperre für Forschungsausgaben wird teilweise als Empfehlung interpretiert, Forschungsausgaben nicht $\mathrm{zu}$ aktivieren ${ }^{723}$. So vertritt die KPLArbeitsgruppe 1995 die Auffassung, Forschungsausgaben seien immer im Jahr ihrer Entstehung im Aufwand zu erfassen ${ }^{724}$.

Werden Forschungsausgaben aktiviert, sind sie beim Zugang mit Herstellungskosten zu bewerten. In der Folge sind sie wie „Ausgaben mit lang anhaltender Wirkung“ gemäß KPL 5:11 innerhalb von maximal fünf Jahren planmäßig abzuschreiben, sofern nicht eine längere Nutzungsdauer, längstens jedoch 20 Jahre, aus besonderem Grund mit der Art guter Buchführung vereinbar ist.

720 Die 4. EG-Richtlinie unterscheidet sich hierin von den Standards des IASB. Nach IAS 9 (revised 1993) Abs. 15 dürfen Forschungskosten nicht aktiviert werden. Entwicklungskosten hingegen müssen aktiviert werden, sofern die Bedingungen des IAS 9 (revised 1993) Abs. 16 und 17 erfüllt sind. Untersagt ein Mitgliedstaat der EU den in ihm ansässigen Unternehmen die Aktivierung von Entwicklungskosten, differiert er hierin von den IAS; die Erstellung dualer Abschlüsse ist nicht möglich. Vgl. Kontaktausschuss (1998), S. 14.

721 Vgl. Kap. 3.2.1.1.6 dieser Arbeit.

722 Vgl. KPL-Arbeitsgruppe 1995, Teil I (1996), S. 86.

723 Vgl. Leppiniemi (2000), S. 244.

724 Vgl. KPL-Arbeitsgruppe 1995, Teil I (1996), S. 86. 
Der finnische Gesetzgeber hat folglich Art. 37 Abs. 1 i. V. m. Art. 34 Abs. 1 (b) der 4. EG-Richtlinie korrekt in nationales Recht umgesetzt.

Anders als für die Forschungsausgaben, legt der finnische Gesetzgeber die Behandlung von Entwicklungsausgaben in KPL 5:8 explizit fest ${ }^{725}$ :

\section{Periodisierung von Entwicklungsausgaben}

(1) Entwicklungsausgaben dürfen unter Beachtung besonderer Vorsicht aktiviert werden. Aktivierte Entwicklungsausgaben sind planmäßig innerhalb von maximal fünf Jahren abzuschreiben, es sei denn, dass eine längere Nutzungsdauer, die jedoch 20 Jahre nicht überschreiten darf, aus besonderen Gründen mit der Art guter Buchführung vereinbar ist.

(2) Über die Aktivierungsvoraussetzungen von Entwicklungsausgaben entscheidet im Einzelnen das Ministerium für Handel und Industrie.

$\mathrm{Zu}$ den Entwicklungsausgaben zählen nach einem Entscheid des Ministeriums für Handel und Industrie folgende Ausgaben ${ }^{726}$ :

- Ausgaben für die Durchführung der Bewertung neuer oder verbesserter Produkt- und Prozessalternativen,

- für die Planung, Fertigstellung und den Test von Modellen und Prototypen für ein Produkt,

- für die Planung, Fertigstellung und Erprobung von Werkzeugen, Modellen und Formen, die auf neuen Technologien beruhen sowie

- für die Planung, den Bau und die Einführung des Versuchszentrums bzw. der Versuchsabteilung

Mit KPL 5:8 hat der finnische Gesetzgeber die Intention verfolgt, die Aktivierung und Periodisierung von Entwicklungsausgaben auf Basis von IAS 9 (revised 1993) so zu regeln, dass sie nicht im Widerspruch zu Art. 37 Abs. 1 der 4. EGRichtlinie stehen ${ }^{727}$. Bei In-Kraft-Treten des Gesetzes 1997 waren die in IAS 9 (revised 1993) genannten fünf Ansatzkriterien in Finnland maßgeblich ${ }^{728}$. Seit IAS 9 (revised 1993) aufgehoben und zum 01. Juli 1999 durch IAS 38 (approved 1998) ersetzt worden ist, gelten nach KPL 5:8.2 die Ansatzkriterien des Ministeriums für Handel und Industrie ${ }^{729}$ :

- Der Buchführungspflichtige hat eine schriftliche Planung zum Projekt und ausreichende Ressourcen für die Vollendung des Projekts sowie für seine Vermarktung oder für seine interne Nutzung vorzuweisen.

- Der Vermögenswert ist klar und eindeutig abzugrenzen.

- Der Buchführungspflichtige kann die mit der Entwicklung verbundenen Kosten verlässlich ermitteln.

\footnotetext{
725 Übersetzung ins Deutsche durch die Verfasserin.

726 Vgl. KTM:p 50/1998 § 2 .

727 Vgl. Tuominen (1998), S. 30.

728 Vgl. Tuominen (1998), S. $32 \mathrm{ff}$.

729 Vgl. KTM:p 50/1998 $§ 4$.
} 
- Zukünftige Wirtschaftlichkeit und Rentabilität des Vermögenswerts können als wahrscheinlich nachgewiesen werden.

- Der aktivierte Betrag ist nicht höher als der unter Vorsichtsprinzipien aus einer Ertragskalkulation ermittelte zukünftige kumulierte Deckungsbeitrag.

Werden diese Kriterien kumulativ erfüllt, kann der immaterielle Vermögenswert als Entwicklungsausgabe aktiviert werden. Das nicht gebundene Eigenkapital unterliegt dann keiner Ausschüttungssperre ${ }^{730}$.

Entwicklungsausgaben umfassen die Herstellungskosten gemäß KPL 4:5; von den Personalaufwendungen ist nur der variable Teil in die Herstellungskosten einzubeziehen $^{731}$.

Auch für die Aktivierung von Entwicklungsausgaben erfüllt das finnische Buchführungsgesetz die Mindestanforderungen der 4. EG-Richtlinie.

\subsection{Rechte}

Die 4. EG-Richtlinie beinhaltet keine speziellen Ansatz- und Bewertungsvorschriften für Rechte. Der EU-Kommission zufolge sind erworbene Rechte zwingend zu aktivieren, selbst erstellte Rechte hingegen nur dann, wenn die nationale Gesetzgebung es gestattet ${ }^{732}$.

Finnlands Gesetzgeber schreibt keine spezielle, ausschließlich Rechte betreffende Regel vor. Sie sind nach KPA 1:6 als eigener Bilanzposten auszuweisen, unterliegen aber sonst der Vorschrift des KPL 5:11 für ,,andere Ausgaben mit lang anhaltender Wirkung“.

Zur Gruppe der Rechte zählen Lizenzen, Patente, Markenrechte und Konzessionen, die selbstständig verwertbar sind und für die das Unternehmen eine direkt zuzuordnende Einnahmenerwartung budgetiert und dokumentiert hat ${ }^{733}$.

Sind mit erworbener EDV-Software auch EDV-Gebrauchslizenzen verbunden, ist es aus Gründen der Wesentlichkeit gestattet, diese Lizenzen gemeinsam mit der Software unter „Ausgaben mit lang anhaltender Wirkung“ auszuweisen ${ }^{734}$.

\footnotetext{
$730 \quad$ Vgl. KTM:p 50/1998 § 3.

731 Vgl. KTM:p 50/1998 § 3 .

732 Vgl. van Hulle und van der Tas (2001), S. 825.

733 Vgl. Leppiniemi und Leppiniemi (1999), S. 89-90.

734 Vgl. KILA 1604/2000.
} 


\subsection{Geschäfts- oder Firmenwert}

Art. 37 Abs. 2 i. V. m. Art. 34 Abs. 1 (a) der 4. EG-Richtlinie ist mit KPL 5:9 wie folgt in finnisches Recht umgesetzt worden ${ }^{735}$ :

Periodisierung des Geschäfts- oder Firmenwerts

Die Anschaffungskosten des Geschäfts- oder Firmenwerts sind innerhalb von maximal fünf Jahren planmäßig abzuschreiben, oder, sofern die tatsächliche Nutzungsdauer länger ist, innerhalb dieser Nutzungsdauer, längstens jedoch in 20 Jahren.

Weder im Gesetz noch in den zugehörigen Gesetzesmaterialien wird der Geschäfts- oder Firmenwert für den Einzelabschluss definiert.

Das finnische Fachschrifttum vertritt - der Kommentierung zur 4. EG-Richtlinie folgend $^{736}$ - einhellig die Meinung, dass es sich bei ihm nur um den derivativen Geschäfts- oder Firmenwert handeln kann ${ }^{737}$. Er entspricht der Differenz zwischen dem Kaufpreis für ein Unternehmen und dem Saldo der bilanzierten Beträge der übernommenen Vermögenswerte und Schulden. Er drückt zum einen den nicht bilanzierungsfähigen Wert des Unternehmens, bestehend z. B. aus Kundenstamm oder Management-Qualität aus, zum anderen enthält er den über die fortgeführten Anschaffungs- oder Herstellungskosten hinausgehenden Zeitwert der Vermögenswerte und Schulden ${ }^{738}$.

Ein selbstgeschaffener, originärer Firmenwert darf nach Auffassung der finnischen Fachwelt nicht aktiviert werden. Ein negativer Geschäfts- oder Firmenwert ist ebenfalls nicht bilanzierungsfähig ${ }^{739}$.

Der Geschäfts- oder Firmenwert ist planmäßig längstens in den folgenden fünf Jahren oder über den Zeitraum seiner tatsächlichen Nutzung abzuschreiben. Eine Abschreibung über die tatsächliche Nutzungsdauer ist nach KPA 2:4 im Anhang zu begründen. Eine solche Begründung kann in der Praxis nur auf der mit dem Geschäfts- oder Firmenwert verbundenen Einnahmenerwartung basieren, da Ausgaben grundsätzlich nur in der Höhe aktiviert werden dürfen, in der ihnen auch künftige Einnahmen gegenüberstehen. Die Einnahmen aus dem Geschäftsoder Firmenwert sind regelmäßig weder eindeutig zurechenbar noch quantifizierbar, da sie einem Teil der Gesamteinnahmenerwartung entsprechen. Der Geschäfts- oder Firmenwert ist nur bei Weiterverkauf des übernommenen Unternehmens wertmäßig verifizierbar ${ }^{740}$. Wegen dieser Problematik wird die Nutzungsdauer eines Geschäfts- oder Firmenwerts selten länger als fünf Jahre

\footnotetext{
Übersetzung ins Deutsche durch die Verfasserin.

Vgl. Van Hulle und van der Tas (2001), S. 825.

Vgl. z. B. Järvinen et al. (2000), S. 480-481.

Vgl. Järvinen et al. (2000), S. 480-481.

Vgl. KILA 886/1986.

Vgl. Järvinen et al. (2000), S. 481.
} 
schäfts- oder Firmenwerts selten länger als fünf Jahre angesetzt, obwohl für Aktiengesellschaften eine Nutzungsdauer von 20 Jahren zulässig ist ${ }^{741}$.

\subsection{Ausgaben mit lang anhaltender Wirkung}

\subsection{Darlehensausgaben}

Nach Art. 41 Abs. 1 und Abs. 2 der 4. EG-Richtlinie darf der Unterschiedsbetrag zwischen Ausgabebetrag einer Verbindlichkeit und ihrem höheren Rückzahlungsbetrag aktiviert werden ${ }^{742}$.

Während der deutsche Gesetzgeber dieses Disagio als Rechnungsabgrenzungsposten auffasst und seine Aktivierung mit $\S 250$ Abs. 3 HGB ins deutsche Recht implementiert hat, geht der finnische Gesetzgeber davon aus, dass es sich bei ihm um einen immateriellen Vermögenswert handelt.

$\mathrm{Zu}$ untersuchen ist, wie ein Disagio dem Wesen nach zu beurteilen ist. Sowohl in Deutschland $^{743}$ als auch in Finnland ${ }^{744}$ wird die Auffassung vertreten, ein Disagio habe den Charakter einer einmaligen Vorabzinszahlung an den Kreditgeber und sei demnach auf Basis des Grundsatzes der Abgrenzung der Sache und der Zeit nach auf die Laufzeit des Darlehens zu verteilen. Das Disagio stellt eine Ausgabe der abgelaufenen Rechnungsperiode dar, die Aufwand für eine bestimmte Zeit nach dem Abschluss-Stichtag ist.

Auf das Disagio trifft daher in Deutschland die Definition des transitorischen aktiven Rechnungsabgrenzungspostens nach $\S 250$ Abs. 1 S. 1 HGB zu. Gemäß $\S 250$ Abs. 3 HGB besteht für Unterschiedsbeträge zwischen dem Ausgabe- und dem Rückzahlungsbetrag eines Darlehens ein Aktivierungswahlrecht.

In Finnland erfüllt das Disagio die - den deutschen entsprechenden - Voraussetzungen für einen transitorischen Rechnungsabgrenzungsposten nach KPL 4:6.1 ${ }^{745}$. Bei einem immateriellen Vermögenswert handelt es sich nach finnischem Verständnis um einen aktivierungsfähigen, identifizierbaren Wert, dessen erwartete

741

742

Das IASB hat die Prinzip und dem Matching-Gedanken lässt sich jedoch ableiten, dass die Erfassung über die Laufzeit der zu Grunde liegenden Verbindlichkeit erfolgen muss. Es besteht Aktivierungspflicht. (IAS 23.5 (b) i. V. m. IAS 23.7). Vgl. KPMG (1999), S. 126 und vgl. Baetge, Kirsch und Thiele (2001), S. 476.

743 Vgl. Baetge, Kirsch und Thiele (2001), S. 340.

744 Vgl. Järvinen et al. (2000), S. 481-483 und vgl. Leppiniemi (2000 a), S. 252.

745 Zur Definition von aktiven Rechnungsabgrenzungsposten in Finnland vgl. Kap. 3.2.3.1 dieser Arbeit. 
Einnahmen in Form von Zahlungsmitteln dokumentierbar sind und dem Vermögenswert eindeutig zugeordnet werden können ${ }^{746}$. Diese Forderungen werden vom Disagio erfüllt: Sein Wert ist identifizierbar, die mit ihm erwartete Einnahme der Darlehensbetrag - ist vertraglich geregelt, somit dokumentierbar und dem Disagio eindeutig zuzuordnen. Ein Disagio könnte in Finnland demnach sowohl als aktiver Rechnungsabgrenzungsposten als auch als immaterieller Vermögenswert betrachtet werden. Fraglich ist, warum es von Gesetzgeber, Wissenschaft und Praxis als immaterieller Vermögenswert aufgefasst wird. Dieses ist vermutlich auf den Grundsatz der Vorsicht zurückzuführen ${ }^{747}$ : Während transitorische aktive Rechnungsabgrenzungsposten nach KPL 4:6.1 aktivierungspflichtig sind, besteht für immaterielle Vemögenswerte ein Aktivierungswahlrecht.

KPL 5:10 lautet wie folgt ${ }^{748}$ :

Unterschiedsbetrag zwischen Ausgabebetrag einer Verbindlichkeit und ihrem höheren Rückzahlungsbetrag

Ausgaben, die aus dem Unterschied zwischen Rückzahlungsbetrag von aufgenommenem Darlehen und erhaltenem Betrag herrühren, dürfen unter Beachtung besonderer Vorsicht aktiviert werden.

Der aktivierte Betrag ist über die Laufzeit des Darlehens planmäßig abzuschreiben, wenigstens jedoch im selben Verhältnis, wie auch das Darlehen zurückgezahlt wird.

KPA 1:6 sieht den Ausweis des Unterschiedsbetrags unter „Anderen Ausgaben mit lang anhaltender Wirkung“ vor. Bei einer solchen Bilanzierung ist die Höhe des Betrags gesondert im Anhang zu nennen. KPA 1:9 gestattet jedoch auch den gesonderten Ausweis des Disagios in der Bilanz, sofern dies zu einem besseren Verständnis der Vermögenslage beiträgt.

Der Unterschiedsbetrag ist durch planmäßige Abschreibungen zu tilgen, die auf die Laufzeit der Verbindlichkeit verteilt werden. Die Tilgung des Unterschiedsbetrags kann im selben Verhältnis erfolgen, wie die Verbindlichkeit getilgt wird ${ }^{749}$. Es ist aber auch möglich, das Disagio degressiv abzuschreiben ${ }^{750}$. Eine Ausnahme bilden Kredite mit endfälliger Rückzahlung. Hier erfolgt die Abschreibung des Unterschiedsbetrags linear über die Restlaufzeit des Kredits ${ }^{751}$.

\footnotetext{
746 Vgl. auch Kap. 3.2.1.1.1 dieser Arbeit.

747 Vgl. Järvinen et al. (2000), S. 482 und vgl. Leppiniemi (2000 a), S. 252-253.

748 Übersetzung ins Deutsche durch die Verfasserin.

749 Vgl. HE 173/1997, S. 24.

750 Vgl. Järvinen et al. (2000), S. 482.

751 Vgl. HE 173/1997, S. 24 und vgl. Järvinen et al. (2000), S. 482.
} 


\subsection{Andere Ausgaben mit lang anhaltender Wirkung}

Der finnische Gesetzgeber regelt ,,andere Ausgaben mit lang anhaltender Wirkung“" in KPL 5:11 ${ }^{752}$ :

Periodisierung anderer Ausgaben mit lang anhaltender Wirkung

Wenn andere als die in den KPL 5:7 bis 5:10 gemeinten Ausgaben mit lang anhaltender Wirkung aktiviert sind, sind sie innerhalb von maximal fünf Jahren planmäBig abzuschreiben, sofern nicht eine längere Nutzungsdauer, längstens jedoch 20 Jahre, aus besonderem Grund mit der Art guter Buchführung vereinbar ist.

Diese Vorschrift ist auf alle aktivierten immateriellen Vermögenswerte anzuwenden, die weder den Gründungsausgaben ${ }^{753}$, den Entwicklungsausgaben ${ }^{754}$, dem Geschäfts- oder Firmenwert ${ }^{755}$ noch den aktivierungsfähigen Unterschiedsbeträgen aus Darlehen ${ }^{756}$ zugeordnet werden können.

$\mathrm{Zu}$ solchen immateriellen Vermögenswerten zählen

- diejenigen Entwicklungsausgaben, die nicht die Ansatzkriterien des KPL 5:8 i. V. m. KTM:p 50/1998 erfüllen,

- EDV-Software,

- Ausgaben für solche Rechte, Patente und Lizenzen, die nicht selbstständig verwertbar sind,

- Kosten für Werbekampagnen,

- Ausgaben für die Beschaffung von Know-how sowie

- Kosten für Asphaltierungsarbeiten oder ähnliche Veränderungen auf gemieteten oder gepachteten Grundstücken und

- andere Ausgaben, mit denen Einnahmenerwartung verbunden ist, die diesen Ausgaben eindeutig zugeordnet werden kann und die quantifizierbar ist $^{757}$.

Eine mehr als fünfjährige Nutzungsdauer wird in der Praxis nur selten angenommen. Sie kann beispielsweise in Betracht kommen, wenn Ausgaben für Asphaltierungsarbeiten auf einem Grundstück getätigt worden sind, für das der Miet- oder Pachtvertrag noch eine Restlaufzeit hat, die fünf Jahre überschreitet ${ }^{758}$.

Übersetzung ins Deutsche durch die Verfasserin.

Vgl. KPL 5:7.

Vgl. KPL 5:8.

Vgl. KPL 5:9.

Vgl. KPL 5:10.

Vgl. Järvinen et al. (2000), S. 483 und vgl. Leppiniemi (2000 a), S. 252.

Vgl. Järvinen et al. (2000), S. 484. 


\subsubsection{Sachanlagen}

\subsection{Ansatz, Bewertung und Ausweis}

Der Richtliniengeber definiert den Begriff „Sachanlagevermögen“ nicht. Welche Werte er ihm zuordnet, geht jedoch aus Art. 15 Abs. 1 und 2 der 4. EG-Richtlinie, der Unterscheidung zwischen Anlage- und Umlaufvermögen, sowie aus den Bilanzgliederungsschemata der Art. 9 und 10 hervor. Richtlinienkonform zählen nach finnischem Verständnis zum Sachanlagevermögen solche materiellen Vermögenswerte, mit denen über mehrere Jahre eine Einnahmenerwartung verbunden ist. Sie sind dazu bestimmt, dem Betriebszweck dauerhaft zu dienen, sei es bei der Herstellung oder Lieferung von Gütern und Dienstleistungen oder auch bei Vermietung oder Verwaltung ${ }^{759}$.

Für aktivierungsfähige Vermögenswerte des Sachanlagevermögens besteht ein Aktivierungsgebot ${ }^{760}$. Sie sind im Zeitpunkt ihrer Erstverbuchung grundsätzlich zu ihren Anschaffungs- oder Herstellungskosten anzusetzen ${ }^{761}$.

Einen Sonderfall bildet die Aktivierung von Ausgaben für die grundlegende Verbesserung eines Vermögenswerts. Dem Unternehmen können für einen bereits aktivierten Vermögenswert des Sachanlagevermögens im Laufe seiner Nutzungsdauer weitere Ausgaben für seine Erweiterung oder Verbesserung entstehen. Diese können als nachträgliche Anschaffungs- oder Herstellungskosten aktiviert werden, wenn

- die nachträglichen Veränderungen die wirtschaftliche Nutzungsdauer verlängern oder die Kapazität erhöhen oder

- nach Veränderung des Vermögenswerts die Ausbringungsqualität wesentlich verbessert ist oder

- $\quad$ eine wesentliche Reduzierung der Betriebskosten ermöglicht wird ${ }^{762}$.

Trifft keines dieser Kriterien zu, wird für das Unternehmen kein zusätzlicher künftiger Nutzen in Form einer Einnahmenerwartung geschaffen. Die getätigten Ausgaben sind dann erfolgswirksam als Aufwand zu erfassen ${ }^{763}$.

Für Ausgaben, die eines dieser Kriterien erfüllen, besteht ein Aktivierungswahlrecht. Sie dürfen auch periodengleich als Aufwand behandelt werden. Entscheidet

759 Vgl. KPL 4:3 und KPL 4:4.1 und vgl. Kap. 3.1.5.2 dieser Arbeit.

760 Vgl. KPL 5:5.1. Zu Ansatzkriterien, die zur Aktivierungsfähigkeit führen vgl. Kap. 3.1.5.2 dieser Arbeit.

761 Besonderheiten bei der Behandlung von gewährten Zuschüssen (vgl. Kap. 3.1.6.2.1.3 dieser Arbeit) und von durch Rückstellungen gedeckte Anschaffungs- oder Herstellungskosten (vgl. Kap. 3.1.6.2.1.4 dieser Arbeit.) können auftreten.

762 Vgl. KILA-Anweisung „Planmäßige Abschreibungen“; der KILA verweist zur Behandlung von Ausgaben für eine grundlegende Verbesserung eines Vermögenswertes auf die in IAS 16.24 und IAS 16.25 empfohlene Vorgehensweise.

763 Vgl. Leppiniemi (2000 a), S. 153-154. 
sich das Unternehmen für eine Aktivierung, so ergeben sich für die Folgebehandlung zwei Alternativen. Zum einen können die nachträglichen Anschaffungs- oder Herstellungskosten als selbstständiger Vermögenswert aktiviert und für sie ein eigener Abschreibungsplan erstellt werden; zum anderen können sie den fortgeführten Anschaffungs- oder Herstellungskosten des entsprechenden Vermögenswerts zugerechnet und gemeinsam mit ihm abgeschrieben werden, wobei die Restlaufzeit zu überprüfen ist ${ }^{764}$.

Im Anhang ist grundsätzlich anzugeben, wie die Ausgaben aus Erweiterung oder Verbesserung behandelt worden sind, sofern diese einen wesentlichen Betrag darstellen $^{765}$.

Abnutzbare Vermögenswerte des Sachanlagevermögens sind planmäßig abzuschreiben $^{766}$. Abschreibungsunterschiede zu steuerrechtlichen Regelungen können durch andere als planmäßige Abschreibungen ausgeglichen werden ${ }^{767}$. Jährlich ist bei Erstellung des Abschlusses ein Niederstwerttest durchzuführen, gegebenenfalls sind die fortgeführten Anschaffungs- oder Herstellungskosten außerplanmäBig auf ihren niedrigeren beizulegenden Wert abzuschreiben, sofern die Wertminderung von Dauer ist (gemildertes Niederstwertprinzip) ${ }^{768}$. Unter dem „,niedrigeren beizulegenden Wert" wird hier der Marktwert verstanden. Obwohl wegen des Grundsatzes der Unternehmensfortführung grundsätzlich vom Marktwert als Wiederbeschaffungswert auszugehen ist, kann auch der Veräußerungswert angenommen werden ${ }^{769}$. Existieren die Gründe für außerplanmäßige Abschreibungen in folgenden Geschäftsjahren nicht mehr, ist das Wertaufholungsgebot zu befolgen ${ }^{770}$.

Für Grundstücke und Wasserflächen besteht nach geltendem Recht die Möglichkeit zur Neubewertung ${ }^{771}$. Auf Basis des alten Buchführungsrechts durchgeführte Neubewertungen von Gebäuden und Bauten dürfen beibehalten werden, sofern sie die damals gültigen Neubewertungskriterien weiterhin erfüllen ${ }^{772}$.

Vermögenswerte des Sachanlagevermögens sind in das Umlaufvermögen umzugliedern, sobald sie ihrer Zweckbestimmung nach dem Geschäftsbetrieb nicht mehr dauerhaft dienen sollen. Sie sind auszubuchen, wenn sie veräußert oder stillgelegt werden und im Zusammenhang mit ihnen keine künftigen Einnahmen mehr erwartet werden. Beim Abgang von Vermögenswerten des Sachanlagevermögens

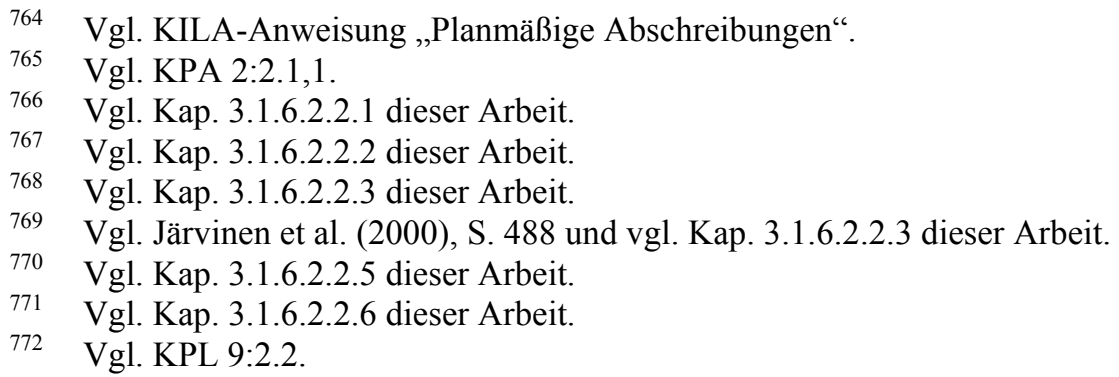


mögens entstehende Gewinne und Verluste sind periodengleich ergebniswirksam zu erfassen.

Folgende Posten sind in der Bilanz gesondert als Sachanlagen auszuweisen ${ }^{773}$ :

- Grundstücke und Wasserflächen

- Gebäude und Bauten

- Maschinen und Geschäftsausstattung

- sonstige materielle Vermögenswerte

- Geleistete Anzahlungen und im Bau befindliche Anschaffungen.

Diese Gliederung weicht von Art. 9 (c) (II) und Art. 10 (c) (II) der 4. EG-Richtlinie $a b$, die eine Unterteilung der Sachanlagen in

- Grundstücke und Gebäude

- Technische Anlagen und Maschinen

- Andere Anlagen, Betriebs- und Geschäftsausstattung

- Anlagen im Bau

vorsehen. Die mit dem Gesetzesentwurf betraute KPL-Arbeitsgruppe 1995 begründet die finnische Untergliederung damit, dass sie die Übersichtlichkeit und auch die Aussagefähigkeit der einzelnen Posten verbessere. Außerdem entspreche diese Systematik derjenigen des finnischen Gewerbesteuerrechts und erleichtere somit die Anwendung der steuerlichen Abschreibungsregeln. Grundstücke und Wasserflächen unterliegen im Gegensatz von Gebäuden und Bauten nicht der Pflicht zur planmäßigen Abschreibung, weshalb eine Trennung dieser Positionen sinnvoll sei. Dem stehe seitens des Richtliniengebers nichts entgegen, da auch in Frankreich Grundstücke und Gebäude nicht in einer Position auszuweisen seien und der zum Thema befragte Vertreter der EU-Kommission keine Einwendungen gehabt habe. Die Arbeitsgruppe lehnt die Übernahme des Postens „Technische Anlagen und Maschinen“ in finnisches Recht ab, weil sie seine Abgrenzung zu Gebäuden - Fabrikanlagen - einerseits und zu „Anderen Anlagen, Betriebs- und Geschäftsausstattung“" andererseits als problematisch ansieht ${ }^{774}$.

Nach Art. 16 der 4. EG-Richtlinie hat die Position „Grundstücke und Gebäude“ auch Rechte an Immobilien und andere ähnliche Rechte, definiert durch den nationalen Gesetzgeber, zu umfassen. Der Kontaktausschuss interpretiert Art. 16 dahingehend, dass als Rechte an Immobilien nur solche Rechte ausgewiesen werden dürfen, welche dem Unternehmenszweck dauerhaft dienen sollen. Unter „Grundstücke und Wasserflächen“ können in Finnland neben den Flächen, die sich im rechtlichen Eigentum des Buchführungspflichtigen befinden, auch im Voraus geleistete Ausgaben aus Pacht gesondert ausgewiesen werden, die wegen ihrer lang anhaltenden Wirkung als immaterielle Vermögenswerte nach KPL 5:11 aktiviert worden sind ${ }^{775}$. Nicht als Grundstücke und Wasserflächen gezeigt

773 Vgl. Bilanzgliederung des KPA 1:6.

774 Vgl. KPL-Arbeitsgruppe 1995, Teil I (1996), S. 185. 
worden sind ${ }^{775}$. Nicht als Grundstücke und Wasserflächen gezeigt werden solche, die auf Grund des Abbaus von Naturschätzen einer regelmäßigen Wertänderung unterliegen. Diese sind Bestandteil der ,sonstigen materiellen Vermögenswerte ${ }^{6776}$. Der Ansatz erfolgt zu Anschaffungskosten, eventuellgekürzt um außerplanmäßige Abschreibungen oder ergänzt um einen auf der Neubewertung basierenden Unterschiedsbetrag.

„Gebäude und Bauten“ enthalten die fortgeführten Anschaffungs- oder Herstellungskosten von Gebäuden und Bauten sowie ihnen zurechenbaren, fest in ihnen integrierte Maschinen wie Heizungsanlagen, Belüftungs- und Klimaanlagen, Stromkabel und Rohrleitungen sowie Aufzüge und Rolltreppen. Diese werden je nach Nutzungsdauer entweder mit dem entsprechenden Bau oder als einzelne Vermögenswerte planmäßig abgeschrieben ${ }^{777}$. Zudem können auch Ausgaben aus Miete oder Pacht ausgewiesen werden, welche die Ansatzkriterien des KPL 5:11 erfüllen. $\mathrm{Zu}$ beachten ist, dass auch auf Basis alter Buchführungsgesetze durchgeführte Neubewertungen hier gezeigt werden können ${ }^{778}$.

Der Posten „Maschinen und Geschäftsausstattung“ umfasst Maschinen und Teile der Betriebsausstattung, die nicht Bestandteil von Gebäuden sind, Fahrzeuge sowie Büromöbel ${ }^{779}$. Sie sind mit ihren fortgeführten Anschaffungs- oder Herstellungskosten zu bewerten.

Dem ausländischen Leser bereitet v. a. der Posten „sonstige materielle Vermögenswerte" Verständnisschwierigkeiten, da ihm sein Inhalt verborgen bleibt und eine in finnischer Sprache verfasste Anhangangabe ihm im Normalfall keine Hilfe bietet. „sonstige materielle Vermögenswerte“ umfassen drei Gruppen von Vermögenswerten:

1. Grundstücke mit Naturschätzen wie Kiesgruben, Steinschläge, Mineralreserven oder Torfmoore,

2. Kunstschätze wie Gemälde oder Skulpturen und

3. lebende Vermögenswerte wie Zuchttiere, Arbeitstiere, milchgebendes Vieh, Obstgärten oder Bepflanzungen, die eine regelmäßige Ernte ermöglichen sollen $^{780}$.

775 Vgl. Järvinen et al. (2000), S. 234.

776 Vgl. Leppiniemi und Leppiniemi (1999), S. 93 und vgl. Järvinen et al. (2000), S. 234.

777 Vgl. Leppiniemi und Leppiniemi (1999), S. 92 und vgl. Järvinen et al. (2000), S. 234.

778 Vgl. KPL 9:2.2.

779 Vgl. Leppiniemi und Leppiniemi (1999), S. 93 und vgl. Järvinen et al. (2000), S. 234.

780 Vgl. KILA:n kuntajaoston tilikarttamalli vom 16. Dezember 1996. KILA gibt öffentlichen Betrieben diese Beispiele als Hilfestellung an die Hand. Nach Meinung der Fachwelt sind sie analog auf privatwirtschaftlich geführte Unternehmen anzuwenden, vgl. Leppiniemi und Leppiniemi (1999), S. 93 und vgl. Järvinen, S. 234-235. 
Mit „geleisteten Anzahlungen und im Bau befindlichen Anschaffungen“ ist zum Zeitpunkt der Bilanzierung (noch) keine Einnahmenerwartung verbunden, sie entsprechen ihrem Charakter nach Forderungen. Sie werden im Sachanlagevermögen gezeigt, weil sie künftige Vermögenswerte dieser Gruppe betreffen. Während geleistete Anzahlungen Zahlungen an Dritte bedeuten, kann es sich bei im Bau befindlichen Anschaffungen auch um Vermögenswerte handeln, die selbst erstellt werden $^{781}$.

\subsection{Geringwertige Wirtschaftsgüter}

Dem Grundsatz nach unterliegen sämtliche materielle Vermögenswerte der Aktivierungspflicht und sind planmäßig über ihre wirtschaftliche Nutzungsdauer abzuschreiben $^{782}$. In der Buchführungspraxis werden jedoch geringwertige Wirtschaftsgüter bereits im Jahr ihrer Anschaffung voll als Aufwand erfasst, obwohl keine entsprechende Vorschrift im Buchführungsgesetz - und auch nicht in der 4. EG-Richtlinie - existiert. Der KILA stimmt aber einer solchen Vorgehensweise zu, da sie dem Prinzip der Wesentlichkeit und auch dem Vorsichtsprinzip entspreche. „Geringwertig“ sei nicht als feststehender Geldbetrag zu definieren, sondern individuell in Abhängigkeit vom Umfang der Geschäftstätigkeit des Unternehmens und der Gesamtsumme seines Anlagevermögens zu bestimmen ${ }^{783}$.

Um gewerbesteuerrechtlich als geringwertiges Wirtschaftsgut anerkannt zu werden, darf die wirtschaftliche Nutzungsdauer eines Vermögenswerts höchstens drei Jahre oder der Anschaffungswert maximal $€ 850,00$ betragen. Die Gesamtsumme abzugsfähiger geringwertiger Wirtschaftsgüter eines Geschäftsjahrs darf $€ 2.500,00$ nicht überschreiten ${ }^{784}$. Die Buchführungspraxis orientiert sich zwar am Gewerbesteuerrecht, Unterschiede zwischen Handelsbilanz und Steuerbilanz sind aber möglich ${ }^{785}$.

781 Vgl. Leppiniemi und Leppiniemi (1999), S. 94 und Leppiniemi (2000 a), S. 253.

782 Vgl. KPL 5:5.1.

783 Vgl. KILA-Anweisung „Planmäßige Abschreibungen“, Abschnitt 2.6.

784 Vgl. EVL 33.

785 Vgl. KILA-Anweisung „Planmäßige Abschreibungen“, Abschnitt 2.6. 


\subsection{Bildung von Festwerten}

Art. 38 der 4. EG-Richtlinie erlaubt unter bestimmten Voraussetzungen die Festbewertung, d. h. den Ansatz von Vermögenswerten mit gleichbleibendem Wert in der Bilanz. Der finnische Gesetzgeber ermöglicht die Festbewertung erstmals im Buchführungsgesetz von 1997, er hat Art. 38 der 4. EG-Richtlinie in KPL 5:5.3 in nationales Recht umgesetzt ${ }^{786}$ :

Zum Sachanlagevermögen gehörende gleichartige und von ihren Anschaffungs- oder Herstellungskosten her geringwertige Vermögenswerte, die vom Buchführungspflichtigen fortlaufend so ersetzt werden, dass ihre Menge und ihr aufaddierter Gesamtwert nur geringfügigen Änderungen unterliegen, dürfen in der Bilanz von einem auf das andere Jahr mit gleichbleibendem Betrag angesetzt werden.

Die Möglichkeit zur Festwertbildung für Vermögenswerte des Sachanlagevermögens wird skeptisch kritisiert. Sie sei lediglich deshalb im Gesetz verankert, weil sie zu den zwingend umzusetzenden Vorschriften der Richtlinie gehöre ${ }^{787}$.

Mit der Festbewertung wird - so die Kritik - der Grundsatz der Einzelbewertung und die sonst gültige Periodisierungspflicht durchbrochen. Hinsichtlich der Auffassung, die Festwertbildung stehe der Periodisierungspflicht entgegen, ist anzumerken, dass Richtliniengeber und finnischer Gesetzgeber von einem gleichbleibenden Betrag in der Bilanz ausgehen, der aber nicht den vollen Anschaffungsoder Herstellungskosten entsprechen muss. Wertigkeitsquoten bei der Festbewertung des abnutzbaren Anlagevermögens sind in Finnland im Fachschrifttum bislang nicht diskutiert worden ${ }^{788}$. In Finnland wird die Möglichkeit zur Festwertbildung regelmäßig nur für geringwertige Wirtschaftsgüter als zulässig erachtet. Beispielhaft werden Bücher einer Handbibliothek, Kleinwerkzeuge, Handies und andere geringwertige Apparate genannt ${ }^{789}$.

Der KILA und das Fachschrifttum äußern die Hoffnung, dass die neu in nationales Recht umgesetzte Regel in der Praxis nur selten Anwendung finde. Es könne auf Grund des Vorsichtsgedankens nicht Ziel sein, solche Vermögenswerte, die bislang als geringwertige Wirtschaftsgüter im Jahr ihrer Anschaffung voll abgeschrieben worden sind, künftig zusammenzufassen und zu aktivieren ${ }^{790}$.

786 Übersetzung ins Deutsche durch die Verfasserin.

787 Vgl. KILA-Anweisung „Planmäßige Abschreibungen“, Abschnitt 2.7 und vgl. Leppiniemi (2000 a), S. 155.

788 Der KILA ist zu diesem Thema bislang nicht befragt worden. Stand September 2001.

789 Vgl. KILA-Anweisung „Planmäßige Abschreibungen“, Abschnitt 2.7, vgl. Järvinen et al. (2000), S. 469.

790 Vgl. KILA-Anweisung „Planmäßige Abschreibungen“, Abschnitt 2.7, vgl. Järvinen et al. (2000), S. 469 und vgl. Leppiniemi (2000 a), S. 155. 


\subsection{Behandlung von Leasingverhältnissen}

Beim Leasingverhältnis handelt es sich um einen Vertrag, bei dem ein Leasinggeber gegen Zahlung eines Entgelts die Nutzungsrechte an einem Vermögenswert (Leasinggegenstand) für einen bestimmten Zeitraum an einen Leasingnehmer überträgt.

Die 4. EG-Richtlinie enthält keine Vorschriften zur bilanziellen Behandlung von Leasingverträgen. Viele Mitgliedstaaten der EU unterscheiden den Regelungen des IAS 17 (revised 1997) und einem Entwurf des Accounting Advisory Forums der EU zu diesem Thema entsprechend in Operating-Leasingverhältnisse und Finanzierungs-Leasingverhältnisse ${ }^{791}$. Eine Unterscheidung in Operating- und Finanzierungs-Leasing kennt das finnische Buchführungsrecht erst seit 1997, und zwar nur für den Konzernabschluss, für den das Finanzierungs-Leasing in Anlehnung an IAS 17 (revised 1997) geregelt ist ${ }^{792}$. Für den finnischen Einzelabschluss hingegen existiert keine das Leasing betreffende Vorschrift, für Sale and Lease Back-Transaktionen sieht der finnische Gesetzgeber ebenfalls keine Regelung $\operatorname{vor}^{793}$.

Beim Operating-Leasing wird nach IAS 17 (revised 1997) der abgeschlossene Leasingvertrag als echter Miet- oder Pachtvertrag klassifiziert, bei dem der Vermieter oder Verpächter rechtlicher und wirtschaftlicher Eigentümer des Leasinggegenstands bleibt. Der Vermieter bzw. Verpächter hat ihn daher in seiner Bilanz als Vermögenswert des Anlagevermögens auszuweisen ${ }^{794}$. Das FinanzierungsLeasing ist nach IAS 17 (revised 1997) dem Charakter nach ein Ratenkauf unter Eigentumsvorbehalt. Das wirtschaftliche Eigentum am Leasinggegenstand ist dem Leasingnehmer zuzurechnen, der deshalb den Leasinggegenstand als Vermö-

791 Vgl. Räty (1998 b), S. 50, die sich auf den von der EU-Kommission herausgegebenen Bericht „Accounting for lease contracts“ des Accounting Advisory Forums von 1995 beruft.

792 Finanzierungs-Leasing für Konzernabschlüsse wird in KPL 6:18 geregelt (Übersetzung ins Deutsche durch die Verfasserin):

„Ist ein Mietvertrag eingegangen worden, wonach zu Beginn des Leasingzeitraumes alle wesentlichen mit dem Eigentum am Leasinggegenstand verbundenen Risiken und Vorteile auf den Leasingnehmer übertragen werden (Finanzierungs-Leasingvertrag), darf der Leasinggeber den Leasinggegenstand in seinem Konzernabschluss so ausweisen, als sei er verkauft worden und der Leasingnehmer so, als habe er ihn gekauft. Über konkrete Voraussetzungen und über die konkrete Behandlung von Finanzierungs-Leasingverträgen entscheidet genauer das Ministerium für Handel und Industrie.

Entscheidet sich ein Buchführungspflichtiger für die Vorgehensweise nach Abs. 1 und nimmt als Leasinggeber den Leasinggegenstand als verkauft an, so ist der aus dem Verkauf entstandene Gewinn oder Verlust über die Laufzeit des Vertrages im Konzernabschluss zu periodisieren."

Die genaue Vorgehensweise bei der Bilanzierung von Finanzierungs-Leasingverhältnissen wird vom Ministerium für Handel und Industrie in der Entscheidung KTM:p 48/1998 geregelt.

793 Vgl. Majala (2001 b), S. 979, Räty (1998 b), S. 50 und Johansson (1998), S. 170.

794 Vgl. IAS 17.3, 17.5, 17.9 und 17.11. 
genswert des Anlagevermögens zu aktivieren und die Leasingverbindlichkeiten zu passivieren hat. Der Leasinggeber weist dagegen in seiner Bilanz nicht den Leasinggegenstand selbst, sondern eine Forderung an den Leasingnehmer aus ${ }^{795}$.

Das Accounting Advisory Forum sieht die Bilanzierung nach rechtlichen oder wirtschaftlichen Aspekten als gleichwertig an und favorisiert weder OperatingLeasing noch Finanzierungs-Leasing. Die Vergleichbarkeit zwischen beiden Ansätzen wird nach Meinung des Forums durch Anhangangaben erreicht ${ }^{796}$.

In Finnland hat es sich als Art guter Buchführung herausgebildet, Leasingverträge wie Miet- oder Pachtverträge zu behandeln. Es ist hierbei unerheblich, ob sie dem Operating- oder dem Finanzierungs-Leasing zuzuordnen sind ${ }^{797}$.

Der Leasinggeber weist in Finnland Leasinggegenstände immer in seinem Anlagevermögen aus und erfasst die Leasingeinnahmen ertragswirksam in seiner Gewinn- und Verlustrechnung. Erfolgt eine Leasingeinnahme in einer Periode und stellt sie einen Ertrag für einen Zeitraum nach dieser Periode dar, wird ein transitorischer Rechnungsabgrenzungsposten gebildet ${ }^{798}$.

Der Leasingnehmer zeigt in Finnland die Leasingzahlungen als Aufwand in seiner Gewinn- und Verlustrechnung oder als Rechnungsabgrenzungsposten. Der Leasingnehmer ist zudem verpflichtet, künftige Verbindlichkeiten aus eingegangenen Leasingverträgen im Anhang anzugeben. Der Nennwert der zu leistenden Zahlungen ist gesondert nach Fälligkeiten mit einer Laufzeit kürzer bzw. länger als einem Geschäftsjahr auszuweisen und Tilgungs- und Zinsanteil gesondert zu zeigen. Im Anhang sind wesentliche Kernpunkte wesentlicher Leasingverträge, Kündigungsfristen und eventuell vereinbarte Kaufoptionen zu nennen ${ }^{799}$.

Fraglich erscheint, ob diese Buchführungspraxis auch in Zukunft Gültigkeit hat, da finnische Konzerne seit In-Kraft-Treten des Buchführungsgesetzes von 1997 das Wahlrecht ausüben können, bei Finanzierungs-Leasingverhältnissen den Leasinggegenstand beim Leasingnehmer zu aktivieren. Es bleibt abzuwarten, ob und wie sich dieses Wahlrecht auf die Einzelabschlüsse der in Konzernabschlüsse einbezogenen Unternehmen auswirkt ${ }^{800}$.

Vgl. IAS 17.3, 17.7 und 17.28.

Vgl. van Hulle und van der Tas (2001), S. 834.

Vgl. KILA 839/1986.

798 Vgl. Majala (2001 b), S. 980 und vgl. Leppiniemi und Leppiniemi (1997), S. 200.

799 Vgl. Leppiniemi und Leppiniemi (1999), S. 197, Järvinen et al. (2000), S. 279 und Majala (2001 b), S. 980.

800 Vgl. Johansson (1998), S. 170. 


\subsubsection{Finanzanlagen}

\subsection{Ansatz, Bewertung und Ausweis}

Unter Finanzanlagen werden solche Finanzinvestitionen verstanden, bei denen seitens des bilanzierenden Unternehmens eine Daueranlageabsicht besteht. Es sollen in mehreren Geschäftsjahren Einnahmen erzielt werden, sei es durch Zinsen, Dividenden oder Wertzuwachs ${ }^{801}$.

Die Bewertung der Finanzanlagen erfolgt bei Zugang zu Anschaffungskosten, wobei das Prinzip der Einzelbewertung zu beachten ist. In der Folge ist der Wertansatz regelmäßig zum Stichtag zu überprüfen, gegebenenfalls sind außerplanmäBige Abschreibungen unter Beachtung des gemilderten Niederstwertprinzips ${ }^{802}$ bzw. Wertaufholungen ${ }^{803}$ vorzunehmen. Auch die Möglichkeit zur Neubewertung besteht ${ }^{804}$.

Verfolgt das Unternehmen mit einer getätigten Finanzinvestition über den Zweck der reinen Kapitalanlage und der damit verbundenen Einnahmenerwartung hinaus auch das Ziel unternehmerischer Einflussnahme, so handelt es sich unter bestimmten Voraussetzungen um eine Finanzanlage bzw. um ein verbundenes Unternehmen oder ein Beteiligungsunternehmen. Im Einzelabschluss sind unter dem Oberbegriff „Finanzanlagen“ in der Bilanz folgende Posten gesondert auszuwei$\operatorname{sen}^{805}$ :

- Anteile an verbundenen Unternehmen

- Forderungen gegenüber verbundenen Unternehmen

- Anteile an Beteiligungsunternehmen

- Forderungen gegenüber Beteiligungsunternehmen

- Andere Aktien und Anteile

- Andere Forderungen

- Eigene Aktien oder Anteile

Aktien oder Anteile am eigenen Unternehmen dürfen nur unter bestimmten Voraussetzungen erworben werden ${ }^{806}$. Sie sind dann bilanzierungsfähig, wenn sie nach Wertpapiermarktgesetz zum öffentlichen Handel zugelassen sind; es kommen somit nur öffentliche Aktiengesellschaften in Betracht. In Höhe der Anschaf-

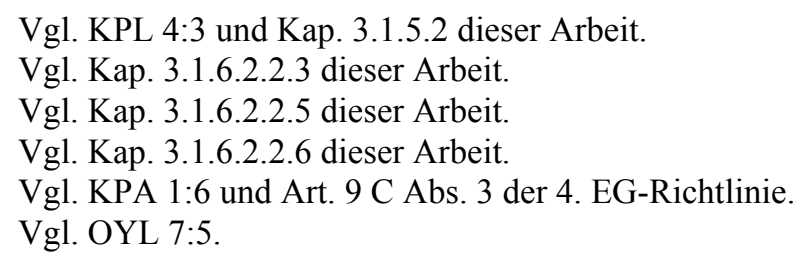


fungskosten ist im Eigenkapital eine Rücklage für eigene Aktien oder Anteile zu bilden, für die eine Ausschüttungssperre besteht ${ }^{807}$.

Kauft eine private Aktiengesellschaft Aktien von ihren Aktionären zurück, hat sie unverzüglich das nicht gebundene Eigenkapital um die Anschaffungskosten dieser Aktien zu korrigieren ${ }^{808}$.

\subsection{Verbundene Unternehmen}

Bei der Beurteilung der Frage, ob eine Finanzanlage als verbundenes Unternehmen oder Beteiligungsunternehmen zu qualifizieren ist, ist das Tatbestandsmerkmal „Bestimmungsrecht“ von zentraler Bedeutung. Der finnische Gesetzgeber hat mit KPL 1:5 die Art. 1 Abs. 1 (a) und Art. 1 Abs. 1 (b) der 7. EG-Richtlinie wie folgt in nationales Recht umgesetzt ${ }^{809}$ :

\section{$\underline{\text { Bestimmungsrecht }}$}

Ein Buchführungspflichtiger hat das Bestimmungsrecht über einen anderen Buchführungspflichtigen oder über ein diesem vergleichbares Unternehmen im Ausland (Zielunternehmen), wenn er innehat:

- mehr als die Hälfte aller aus Aktien oder Gesellschaftsanteilen des Zielunternehmens herrührenden Stimmrechte. Diese Stimmrechtsmehrheit beruht auf Eigentum, Mitgliedschaft, Satzung, Gesellschaftsvertrag oder vergleichbaren Statuten oder anderen Verträgen; oder

- das Recht, die Mitglieder des Vorstands oder vergleichbarer Organe des Zielunternehmens zu benennen oder abzuberufen oder die Mitglieder solcher Organe zu benennen oder abzuberufen, die dieses Recht haben; das Recht beruht auf denselben Faktoren wie die in Nr. 1 gemeinte Stimmrechtsmehrheit.

Bei der Ermittlung des in Abs. 1 gemeinten Stimmrechtsanteils wird eine Abstimmungseinschränkung, die auf dem Gesetz, der Satzung oder dem Gesellschaftsvertrag des Zielunternehmens oder vergleichbaren Statuten beruht, nicht berücksichtigt. Bei der Ermittlung der Gesamtzahl aller Stimmrechte des Zielunternehmens werden diejenigen Stimmrechte abgezogen, die dem Zielunternehmen selbst oder einem seiner Tochterunternehmen i.S.d. KPL 1:6 gehören. Stimmrechte, die einer anderen für Rechnung dieser Unternehmen handelnden Person gehören, werden besagter Person zugerechnet.

Das Bestimmungsrecht beruht entweder auf der Stimmrechtsmehrheit oder auf dem Recht zur Bestellung oder Abberufung der Mehrheit der Organmitglieder.

Die Stimmrechtsmehrheit gründet sich auf rechtliches Eigentum, das Eigentum von Aktien bzw. anderer Gesellschaftsanteile oder Mitgliedschaft. Stimmrechtsmehrheit entsteht aber unter Umständen erst durch Satzung, Gesellschaftsvertrag oder andere abgeschlossene Verträge ${ }^{810}$. Das Recht zur Bestellung bzw. Abberu-

807 Vgl. HE 173/1997, S. 9. Zur Rücklage für eigene Aktien oder Anteile vgl. auch Kap. 3.2.4.1 dieser Arbeit.

808 Vgl. Leppiniemi und Leppiniemi (1999), S. 109-110.

809 Übersetzung ins Deutsche durch die Verfasserin.

810 Vgl. auch Järvinen et al. (2000), S. 78-79. 
fung der Mehrheit der Organmitglieder erfordert hingegen keine Eigentumsrechte am Zielunternehmen ${ }^{811}$.

Die Konzeption des Bestimmungsrechts entspricht dem angelsächsischen ControlKonzept. Es wird auf rechtlich gesicherte Einflussmöglichkeiten abgestellt, unabhängig davon, ob sie genutzt werden oder genutzt werden können. Rechtsform, Sitz und Größe der beteiligten Unternehmen sind für das Bestimmungsrecht irrelevant, lediglich die Unternehmensbeziehung ist von Bedeutung.

Bei der Ermittlung der Stimmrechte sind nach KPL 1:5.2 stimmrechtseinschränkende Tatbestände abzuziehen ${ }^{812}$. Mit dieser Vorschrift sollte Art. 2 Abs. 2 der 7. EG-Richtlinie in finnisches Recht implementiert werden. Zurzeit wird mit KPL 1:6.3 eine neue Vorschrift geschaffen, die Art. 2 Abs. 2 richtlinientreuer, vollständiger und genauer wiedergeben soll ${ }^{813}$.

Von der Gesamtheit aller Stimmrechte der Aktionäre oder Gesellschafter eines Tochterunternehmens sind nach KPL 1:5.3 die Stimmrechte abzuziehen, welche mit Aktien oder Anteilen verbunden sind, die von diesem Unternehmen selbst, von einem seiner Tochterunternehmen oder von einer im eigenen Namen, aber für Rechnung dieser Unternehmen handelnden Person gehalten werden. Der finnische Gesetzgeber hat mit KPL 1:5.3 Art. 2 Abs. 3 der 7. EG-Richtlinie in nationales Recht umgesetzt.

Die Rechtsfolge des Bestimmungsrechts ergibt sich aus KPL 1:6. Der finnische Gesetzgeber definiert Mutter- und Tochterunternehmen, verbundene Unternehmen und den Konzern und übernimmt Art. 41 Abs. 1 der 7. EG-Richtlinie wie folgt $^{814}$ :

811 Eine Stiftung ist in Finnland ein sog. ,autonomes Rechtssubjekt“, an dem keine andere Person das Eigentum haben kann. Organmitglieder einer Stiftung können jedoch Organe anderer Unternehmen sein, z. B. kann in der Satzung einer Stiftung festgelegt sein, dass ihr Vorstand sich aus dem Vorstand einer bestimmten Aktiengesellschaft zusammensetzt. Über solche Organe können andere Unternehmen das Bestellungs-/Abberufungsrecht für die Mehrheit der Mitglieder der Organe einer Stiftung ausüben, sodass gewährleistet ist, dass auch über Stiftungen das Bestimmungsrecht ausgeübt werden kann.

812 Das Aktiengesellschaftsgesetz verlangt dies mit demselbem Wortlaut in OYL 1:3.3 auch für die Aktiengesellschaft.

813 Vgl. auch Järvinen et al. (2000), S. 78-79.

814 Übersetzung ins Deutsche durch die Verfasserin. Die 4. EG-Richtlinie definiert ,verbundene Unternehmen“ nicht. Vorschriften zu „verbundenen Unternehmen“ sind in den Art. 1, 12 und 41 der 7. EG-Richtlinie enthalten. 
Verbundenes Unternehmen, Mutterunternehmen und Tochterunternehmen

Hat ein Buchführungspflichtiger nach KPL 1:5 das Bestimmungsrecht über ein Zielunternehmen, handelt es sich bei ihm um ein Mutterunternehmen und beim Zielunternehmen um ein Tochterunternehmen. Mutter- und Tochterunternehmen bilden zusammen einen Konzern. Ein Mutterunternehmen und seine Töchterunternehmen sind verbundene Unternehmen im Sinne dieses Gesetzes.

Die Vorschrift des Abs. 1 gilt auch, wenn der Buchführungspflichtige mit einem oder mehreren seiner Tochterunternehmen zusammen oder ein Tochterunternehmen allein oder gemeinsam mit anderen Tochterunternehmen das Bestimmungsrecht über ein Zielunternehmen ausübt.

Nach Buchführungsgesetz entstehen Mutter- und Tochterunternehmen unabhängig von ihrer Rechtsform. Das Aktiengesellschaftsgesetz enthält zusätzlich eine identische Regelung für Aktiengesellschaften, die Mutterunternehmen sind ${ }^{815}$.

Im Konzern hält demnach die Mutterunternehmung entweder einen Eigentumsanteil von null bis 100 Prozent am Tochterunternehmen, hat direkt oder indirekt mehr als 50 Prozent der Stimmrechte am Tochterunternehmen inne oder übt auf andere Weise das Bestimmungsrecht aus ${ }^{816}$.

Das seitens des Richtliniengebers in Art. 1 Abs. 2 der 7. EG-Richtlinie gewährte Wahlrecht zum Konzept der einheitlichen Leitung (deutsches bzw. ökonomisches Konzept) hat der finnische Gesetzgeber nicht in finnisches Recht übernommen. Ein Mutter-Tochter-Verhältnis setzt in Finnland ein Weisungsrecht voraus. Eine Mutter-Tochter-Beziehung liegt somit nicht automatisch vor, wenn die Konzernleitung die Geschäftspolitik der Konzerngesellschaften faktisch aufeinander abstimmen kann.

Konsequenz aus einem Mutter-Tochter-Verhältnis im Einzelabschluss ist der gesonderte Ausweis des Anteils am Tochterunternehmen sowie bei allen zum Konzern gehörenden Unternehmen der gesonderte Ausweis von bestimmten Erträgen, Aufwendungen, Forderungen und Verbindlichkeiten.

Vgl. OYL 1:3.1.

Vgl. KHT-yhdistys (1998), S. 61. 


\subsection{Beteiligungen}

Art. 17 der 4. EG-Richtlinie definiert Beteiligungen. Der finnische Gesetzgeber hat diese Vorschrift mit KPL 1:7 in nationales Recht aufgenommen ${ }^{817}$ :

Beteiligungsunternehmen

Ein Beteiligungsunternehmen ist ein nicht zum Konzern des Buchführungspflichtigen gehörendes inländisches oder ausländisches Unternehmen, an dem der Buchführungspflichtige Anteile hält, die eine dauerhafte Verbindung zwischen Buchführungspflichtigen und Beteiligungsunternehmen herstellen, und die dazu bestimmt sind, dem Geschäftsbetrieb des Buchführungspflichtigen oder verbundenen Unternehmen des Buchführungspflichtigen zu dienen. Wenn der Buchführungspflichtige es nicht widerlegt, gilt ein Unternehmen als Beteiligungsunternehmen, wenn der Buchführungspflichtige wenigstens ein Fünftel des gezeichneten Kapitals oder entsprechenden Nennkapitals innehält.

Zwingende Voraussetzung für das Vorliegen einer Beteiligung ist neben einer Daueranlageabsicht auch die Verfolgung von Zielen, die über eine reine Kapitalanlage hinausgehen: unternehmerische Einflussnahme, beispielsweise durch Abstimmung von Produktprogrammen, gemeinsames Marketing oder Kooperation im Bereich Forschung und Entwicklung. Diese Einflussnahme geht aber nicht so weit, dass ein Bestimmungsrecht vorliegt.

Die Absicht unternehmerischer Einflussnahme kann nur schwer nachgewiesen und überprüft werden, sodass Richtlinie und finnisches Buchführungsgesetz als Vereinfachung eine Vermutungsregel vorsehen. Anteile an anderen Unternehmen gelten unabhängig von ihrer Rechtsform als Beteiligung, wenn ihre Höhe 20 Prozent des Nennkapitals übersteigt. Eine auf KPL 1:7 gestützte Widerlegung der Vermutung ist möglich, die Argumentation ist in einem solchen Fall für die Praxis schwierig $^{818}$. Ein Beteiligungsverhältnis kann dann gegeben sein, wenn der an anderen Unternehmen gehaltene Anteil null bis 100 Prozent des Nennkapitals und die Anzahl der Stimmrechte weniger als 50 Prozent der Stimmrechtsgesamtheit beträgt ${ }^{819}$.

Das finnische Buchführungsrecht sieht einen Sonderfall des Beteiligungsunternehmens vor, die Beteiligung im engeren Sinne, in der auf die Anzahl der Stimmrechte mit Einfluss auf Organe, die über Geschäftsführung und Finanzierung entscheiden, abgestellt wird. Sie wird in KPL 1:8 wie folgt definiert ${ }^{820}$ :

817 Übersetzung ins Deutsche durch die Verfasserin.

818 Vgl. Järvinen et al. (2000), S. 79-80. Eine auf ein Gerichtsurteil oder eine KILA-Entscheidung gestützte Widerlegung der Vermutung existiert bislang nicht.

819 Vgl. KHT-yhdistys (1998), S. 61.

820 Übersetzung ins Deutsche durch die Verfasserin. 
Beteiligungsunternehmen im engeren Sinne

Ein Beteiligungsunternehmen im engeren Sinne ist ein Beteiligungsunternehmen, auf das der Buchführungspflichtige einen beträchtlichen Einfluss bei der Führung von Geschäft und Finanzierung hat, das aber nicht zum Konzern des Buchführungspflichtigen gehört. Wenn der Buchführungspflichtige es nicht widerlegt, gilt ein Beteiligungsunternehmen als Beteiligungsunternehmen im engeren Sinne, wenn der Buchführungspflichtige wenigstens ein Fünftel und höchstens die Hälfte der Stimmrechte aus Aktien oder Anteilen an dem Beteiligungsunternehmen hält.

Ein Beteiligungsunternehmen im engeren Sinne wird widerlegbar vermutet, wenn der am anderen Unternehmen gehaltene Anteil null bis 100 Prozent des Nennkapitals und die Anzahl der Stimmrechte 20 bis 50 Prozent der Stimmrechtsgesamtheit umfasst $^{821}$.

Die Unterscheidung in Beteiligungsunternehmen einerseits und Beteiligungsunternehmen im engeren Sinne andererseits ist für den Einzelabschluss nicht relevant $^{822}$. Rechtsfolge einer Beteiligung ist immer der gesonderte Ausweis des Anteils unter Finanzanlagen sowie der gesonderte Ausweis bestimmter Erträge, Aufwendungen, Forderungen und Verbindlichkeiten ${ }^{823}$.

\subsubsection{Umlaufvermögen}

\subsubsection{Ansatz, Bewertung und Ausweis des Vorratsvermögens}

$\mathrm{Zu}$ den Vorräten zählen Vermögenswerte, die im Rohzustand oder in veredeltem Zustand dazu bestimmt sind, an Dritte veräußert oder konsumiert zu werden ${ }^{824}$. Mit ihnen verbunden ist eine Einnahmenerwartung, die sich - in Abgrenzung zu Vermögenswerten des Anlagevermögens - nicht fortlaufend auf mehrere Geschäftsjahre erstreckt ${ }^{825}$.

In der Bilanz sind folgende Posten als Vorräte gesondert auszuweisen ${ }^{826}$ :

- Roh-, Hilfs- und Betriebsstoffe

- Unfertige Erzeugnisse

- Fertige Erzeugnisse / Handelswaren

- Andere Vermögenswerte des Umlaufvermögens

- Geleistete Anzahlungen

$821 \quad$ Vgl. KHT-yhdistys (1998), S. 61.

822 Im Konzernabschluss ist das Vorliegen einer Beteiligung im engeren Sinne deshalb wesentlich, weil für sie umfangreiche Anhangangaben zwingend erforderlich sind, die für Beteiligungen entfallen.

823 Vgl. KPA 1:1 und KPA 1:6.

824 Vgl. KPL 4:4.2 und vgl. auch Kap. 3.1.5.2 dieser Arbeit.

825 Vgl. KPL 4:3 und vgl. auch Kap. 3.1.5.2 dieser Arbeit.

826 Vgl. KPA 1:6. 
Bei Roh-, Hilfs- und Betriebsstoffen handelt es sich sowohl um Vermögenswerte, die zum Verbrauch im Produktionsprozess und zur Erbringung von Dienstleistungen bestimmt sind, als auch um solche, die zur Instandhaltung von Vermögenswerten des Sachanlagevermögens benötigt werden ${ }^{827}$.

Unfertige Erzeugnisse sind materielle und immaterielle Vermögenswerte, die sich noch im Erstellungsvorgang befinden und nach Fertigstellung im Rahmen des gewöhnlichen Geschäftsbetriebs für den Verkauf gedacht sind.

Während fertige Erzeugnisse und Handelswaren zum Verkauf innerhalb des gewöhnlichen Geschäftsbetriebs bereitgehaltene Vermögenswerte umfassen, beinhalten andere Vermögenswerte des Umlaufvermögens solche Vermögenswerte, die außerhalb des gewöhnlichen Geschäftsbetriebs veräußert werden sollen und deren Verkaufszeitpunkt bereits feststeht. Hierbei handelt es sich meist um Vermögenswerte, die ursprünglich zum Anlagevermögen gehörten, wie beispielsweise Grundstücke oder Immobilien ${ }^{828}$.

Vorräte werden mit ihren Anschaffungs- oder Herstellungskosten oder ihrem am Stichtag niedrigeren beizulegenden Wert bewertet. Außerplanmäßige Abschreibungen sind erforderlich, wenn der Wiederbeschaffungs- bzw. Verkaufspreis am Stichtag gegenüber den Anschaffungs- oder Herstellungskosten gesunken ist oder die Vermögenswerte ganz oder teilweise unbrauchbar geworden sind (strenges Niederstwertprinzip) ${ }^{829}$. Der Wertansatz ist jährlich zu überprüfen, Wertaufholungen sind geboten ${ }^{830}$.

Die Anschaffungs- oder Herstellungskosten bestehen aus ihnen zuzuordnenden variablen und, sofern wesentlich, fixen Kosten. Fremdkapitalzinsen dürfen nicht einbezogen werden ${ }^{831}$. Der Grundsatz der Einzelbewertung sieht eine Bewertung der Posten auf der Basis der tatsächlichen Verbrauchsfolge vor. Eine separate Ermittlung der Anschaffungs- oder Herstellungskosten für jeden Vermögenswert erweist sich jedoch dann als nicht praktikabel, wenn es sich um eine große Menge von Vermögenswerten mit hoher Fluktuation handelt oder die Verbrauchsfolge auf Grund der physikalischen Beschaffenheit des Vermögenswerts nicht nachvollzogen werden $\mathrm{kann}^{832}$. Sind Vermögenswerte ihrem Charakter und Verwendungszweck nach so gleichartig, dass sie normalerweise untereinander ausge-

827 Vgl. Leppiniemi und Leppiniemi (1999), S. 98, die auf eine KILA-Anweisung vom 06. April 1999 an öffentliche Betriebe verweisen und die Meinung vertreten, sie sei analog auf private Betriebe anzuwenden.

828 Vgl. Leppiniemi und Leppiniemi (1999), S. 99, die auf ein Kontenrahmen-Beispiel des KILA vom 16. Dezember 1996 für öffentliche Betriebe verweisen und die Meinung vertreten, es sei analog auf private Betriebe anzuwenden. 
tauscht werden können, dürfen Bewertungsvereinfachungen nach KPL 4:5.4 vorgenommen werden ${ }^{833}$ :

$\underline{\text { Anschaffungs- oder Herstellungskosten }}$

[...] (4) Wenn der Buchführungspflichtige es nicht anders ausweist, werden die Anschaffungs- oder Herstellungskosten von gleichartigen Vermögenswerten des Umlaufvermögens unter der Annahme ermittelt, dass sie in der Reihenfolge veräußert werden in der sie auch beschafft werden, oder dass die Reihenfolge bei der VeräuBerung entgegengesetzt zu der der Beschaffung verläuft. Die Anschaffungs- oder Herstellungskosten gleichartiger Vermögenswerte dürfen auch mit gewogenem Durchschnitt bewertet werden. [...]

Der finnische Gesetzgeber hat mit dieser Vorschrift Art. 40 Abs. 1 der 4. EGRichtlinie umgesetzt.

Bei Jahresabschlusserstellung können sich die Unternehmen entscheiden zwischen

- der tatsächlichen Verbrauchsfolge,

- dem „First in, first out“-Verfahren (FIFO),

- dem „Last in, first out“-Verfahren (LIFO) und

- der gewogenen Durchschnittsmethode.

Das finnische Gewerbesteuerrecht sieht neben der tatsächlichen Verbrauchsfolge nur die FIFO-Methode $\operatorname{vor}^{834}$. In der Praxis wird regelmäßig mit gewogenem Durchschnittspreis bewertet, was steuerlich akzeptiert wird, solange das Ergebnis nicht bedeutend von einer FIFO-Bewertung abweicht ${ }^{835}$. Die Anwendung der LIFO-Methode ist erst seit dem In-Kraft-Treten des Buchführungsgesetzes von 1997 gestattet. Die LIFO-Methode ist aber steuerlich weiterhin nicht erlaubt, sodass sie auch deshalb bislang nicht praxisrelevant ist ${ }^{836}$. Das Wahlrecht des Richtliniengebers, neben den genannten zusätzlich noch vergleichbare Verfahren zuzulassen, hat der finnische Gesetzgeber nicht genutzt. Nicht auf zeitlicher Reihenfolge basierende Bewertungsmethoden - wie das „Highest in, first out"-Verfahren (HIFO) oder das „Lowest in, first out“-Verfahren (LOFO) - dürfen nicht unterstellt werden ${ }^{837}$.

833 Übersetzung ins Deutsche durch die Verfasserin.

834 Vgl. EVL 14.2.

835 Vgl. Räty (1998 b), S. 59-60 und vgl. KPL-Arbeitsgruppe 1995, Teil I (1996), S. 64.

836 Vgl. Järvinen et al. (2000), S. 404 und vgl. KPL-Arbeitsgruppe 1995, Teil I (1996), S. 6465. KP 95 empfiehlt an dieser Stelle, die LIFO-Methode nicht zu gestatten, sofern sie nicht auch steuerrechtlich erlaubt werde. Die KPL-Arbeitsgruppe 1995 verweist auf die in der Praxis geringe Akzeptanz der LIFO-Methode in den EU-Mitgliedstaaten und die bessere Vergleichbarkeit von Jahresabschlüssen in Finnland wie europaweit, wenn nicht zu viele Methoden nebeneinander zugelassen werden.

837 Vgl. Järvinen et al. (2000), S. 402. 


\subsubsection{Bildung von Festwerten}

Mit KPL 5:6.3 erlaubt der finnische Gesetzgeber die Festwertbildung in Übereinstimmung mit Art. 38 der 4. EG-Richtlinie ${ }^{838}$ :

Periodisierung der Anschaffungs- oder Herstellungskosten des Umlaufvermögens

[...] (3) Zu den Roh-, Hilfs- und Betriebsstoffen des Umlaufvermögens gehörende gleichartige und von ihren Anschaffungs- oder Herstellungskosten her geringwertige Vermögenswerte, die von Buchführungspflichtigen fortlaufend so ersetzt werden, dass ihre Menge und ihr aufaddierter Gesamtwert nur geringfügigen Änderungen unterliegen, dürfen in der Bilanz von einem aufs andere Jahr mit gleichbleibendem Betrag angesetzt werden.

Ziel dieser Vorschrift ist es, den Unternehmen die Erstellung des Jahresabschlusses zu erleichtern, auch wenn die Arbeitsvereinfachung im Widerspruch zum Grundsatz der Einzelbewertung steht. Der Grundsatz der Wesentlichkeit ist kein vom Richtliniengeber explizit kodifizierter Grundsatz ordnungsmäßiger Buchführung. Er ist in Finnland allgemein anerkannter Bestandteil der Art guter Buchführung. Der Grundsatz der Wesentlichkeit wird dahingehend interpretiert, dass für die Jahresabschlussaussage unwesentliche Positionen nicht unbedingt der theoretisch korrekten Lösung entsprechend bilanziert werden müssen; dies dient einer vereinfachten Handhabung in der Praxis. Das finnische Fachschrifttum vertritt die Auffassung, dass die Implementierung des Art. 38 hinsichtlich der Roh-, Hilfsund Betriebsstoffe in finnisches Recht überflüssig sei, da die Art guter Buchführung eine Festwertbildung erlaube. Festwerte werden in der Praxis allerdings selten gebildet. Ob die explizite Regelung KPL 5:6.3 dieses Verhalten der Unternehmen in der Praxis ändern wird, ist fraglich ${ }^{839}$.

\subsubsection{Langfristige Auftragsfertigung}

Bei langfristiger Auftragsfertigung, die sich über mehr als ein Geschäftsjahr erstreckt, ergibt sich ein bilanzielles Problem aus der Frage nach dem Zeitpunkt der Gewinnrealisierung, nach der Zuordnung von Erträgen und Aufwendungen zu den einzelnen Perioden.

Nach welcher Methode die Unternehmen der EU-Mitgliedstaaten langfristige Auftragsfertigung bilanzieren sollen, wird vom Richtliniengeber nicht geregelt ${ }^{840}$.

838 Übersetzung ins Deutsche durch die Verfasserin.

839 Vgl. Räty (1998 b), S. 62 und vgl. Järvinen et al. (2000), S. 473.

840 Bei Anwendung der Completed Contract-Methode wird die Gewinnrealisierung in derjenigen Rechnungsperiode vollzogen, in der die Abnahme durch den Auftraggeber stattfindet, da das Realisationsprinzip einen Umsatzvorgang und einen abgeschlossenen Übergang des Vertragsobjekts an den Abnehmer voraussetzt. Drohende Verluste sind bereits bei ihrem Bekanntwerden in den Perioden der Herstellung erfolgswirksam zurückzustellen. Diese Inter- 
Während einige Mitgliedstaaten die Completed Contract-Methode mit Hinweis auf Vorsichts- und Realisationsprinzip des Art. 31 der 4. EG-Richtlinie favorisieren $^{841}$, erlauben andere die Percentage of Completion-Methode unter Berufung auf den True and Fair View ${ }^{842}$. Der Kontaktausschuss der EU-Kommission hat einstimmig die Percentage of Completion-Methode unter Beachtung des Vorsichtsprinzips erlaubt ${ }^{843}$. Anwendungsvoraussetzungen sind demnach: Die Gesamteinnahmen aus dem Fertigungsauftrag müssen bekannt, der Fertigstellungsgrad zuverlässig ermittelbar und das Objekt der Auftragsfertigung am Stichtag ausreichend weit fortgeschritten sein. Zudem ist bei vorhersehbaren Verlusten aus dem Gesamtprojekt unverzüglich eine Drohverlustrückstellung zu bilden. Die für die Bilanzierung langfristiger Projekte angewendete Methode und die ihr $\mathrm{zu}$ Grunde gelegten Annahmen sind in Übereinstimmung mit Art. 43 der 4. EG-Richtlinie im Anhang zu nennen ${ }^{844}$.

Die Frage, ob die Anwendung der Percentage of Completion-Methode eine Anwendung der in Art. 31 Abs. 1 der 4. EG-Richtlinie genannten allgemeinen Jahresabschlussgrundsätze oder ein erlaubtes Abweichen von ihnen nach Art. 31 Abs. 2 darstelle, ist von Bedeutung, weil im Fall des Abweichens zusätzliche Angaben im Anhang hinsichtlich der Abweichungsgründe und ihrer Auswirkung auf die Vermögens-, Finanz- und Ertragslage notwendig würden. Die Mitglieder des Kontaktausschusses der EU-Kommission haben 1992 festgestellt, dass die Percentage of Completion-Methode weder eine Abweichung von den Jahresabschlussgrundsätzen noch eine Abweichung von Einzelvorschriften zur Erreichung eines

pretation von Vorsichts-, Realisations- und Imparitätsprinzip kann je nach Größenumfang der langfristigen Aufträge und ihrer Wesentlichkeit für den Hersteller bewirken, dass die Jahresergebnisse im Zeitablauf starken Schwankungen unterliegen. Diese Schwankungen sind nicht durch Unterschiede in der wirtschaftlichen Leistungsfähigkeit des Unternehmens verursacht. Deshalb ist es fraglich, ob durch sie die Jahresabschlussaussage mehrerer Geschäftsjahre zu Gunsten des Gesellschafter- und Gläubigerschutzes verfälscht wird. Sie wird zumindest beeinträchtigt, zudem wird die zwischenbetriebliche und interperiodische Vergleichbarkeit der Jahresabschlüsse erschwert. Vgl. Baetge, Kirsch und Thiele (2000), S. 622. Wird die Percentage of Completion-Methode angewendet, erfolgt eine Umsatzrealisierung nach Fertigstellungsgrad des Auftrags am Stichtag. Hierbei wird im Regelfall angenommen, dass die Entstehung des Gesamterfolgs aus einem Auftrag proportional zum Kostenanfall verläuft (Cost to cost-Methode). Der Fertigstellungsgrad eines Auftrags wird so nach dem Verhältnis der bis zum Bilanzstichtag kumulierten auftragsbezogenen Kosten zu den geschätzten Gesamtkosten eines Auftrags bestimmt (vgl. Baetge, Kirsch und Thiele (2000), S. 625). Eine Teilgewinnrealisierung erfolgt demnach vor endgültiger Abnahme durch den Auftraggeber. Hier stehen nicht die Beachtung des Vorsichts- und des Realisationsprinzips im Vordergrund, sondern die Befolgung des Grundsatzes der periodengerechten Abgrenzung (Matching). (s. auch Kap. 3.1.3.3 dieser Arbeit). Ziel ist eine verbesserte Qualität der Jahresabschlussaussage durch zutreffendere Information als bei der Completed Contract-Methode. Dies ist allerdings kritisch zu sehen, da sowohl Gesamtkosten als auch Gesamterlöse eines Auftrags bis zur Abnahme mit Unsicherheiten behaftet sind (s. auch Kap. 3.1.3.3 dieser Arbeit).

${ }^{841}$ Hierzu zählen beispielsweise Deutschland und Österreich.

842 Das Wahlrecht zwischen der Percentage of Completion-Methode und der Completed Contract-Methode haben Unternehmen beispielsweise in Belgien und Dänemark.

843 Vgl. van Hulle und van der Tas (2001), S. 832.

844 Vgl. EU-Kommission XV/7009/97, S. 8, vgl. Kontaktausschuss (1990), S. 23. 
True and Fair View bedeute, sondern vielmehr im Rahmen der allgemeinen Jahresabschlussgrundsätze liege ${ }^{845}$.

Der finnische Gesetzgeber hat sich dafür entschieden, den Unternehmen das Wahlrecht zwischen der Completed Contract-Methode und der Percentage of Completion-Methode $\mathrm{zu}$ lassen, und die langfristige Auftragsfertigung wie folgt in KPL 5:4 geregelt ${ }^{846}$ :

\title{
Die Buchung von Einnahmen als Ertrag auf Basis des Fertigstellungsgrads
}

\begin{abstract}
Eine aus einer langfristigen Auftragsfertigung entstehende Einnahme darf sukzessive nach dem Fertigstellungsgrad der zu erbringenden Leistung als Ertrag erfasst werden. Der gesamte aus dieser Leistung resultierende Einzeldeckungsbeitrag muss in einem solchen Fall auf verlässliche Weise vorhergesagt werden können. Der Buchführungspflichtige muss auf alle Einnahmen aus langfristiger Auftragsfertigung dieselben Prinzipien und dieselbe Methode bei ihrer erfolgswirksamen Verbuchung anwenden.
\end{abstract}

Der KILA hat diese Vorschrift auf Basis von IAS 11 (revised 1993) in einer allgemeingültigen Anweisung für die Praxis konkretisiert, sachverhaltsrelevante Begriffe definiert sowie Anwendungsvoraussetzungen und Methoden für die Ermittlung der Teilgewinne vorgegeben ${ }^{847}$. Demnach wird von langfristiger Auftragsfertigung in Finnland gesprochen, wenn Fertigungsbeginn (verbindliche Auftragsannahme und Vertragsabschluss) und Fertigungsende (endgültige Abnahme durch den Kunden) in zwei verschiedene Geschäftsjahre fallen. Typischerweise Gegenstand von Fertigungsaufträgen in Finnland sind Großprojekte wie der Bau von Straßen, Brücken, Gebäudesiedlungen, Raffinerien und Industrieanlagen oder auch von einzelnen Objekten wie Schiffen, Papiermaschinen oder Generatoren. Der Umfang solcher Großprojekte wird vom KILA betragsmäßig weder festgelegt noch eine Untergrenze vorgegeben. Um als langfristiger Fertigungsauftrag klassifiziert zu werden, muss das Projekt eine wesentliche Größe im Verhältnis zu den Umsatzerlösen des herstellenden Unternehmens bilden ${ }^{848}$.

Indiz für eine langfristige Auftragsfertigung ist die Existenz einer Einzelprojektüberwachung und einer projektbezogenen Kostenrechnung, mit der Gesamteinnahmen und -ausgaben sowie leistungsabhängig (nach Fertigstellungsgrad) realisierte Ausgaben und ihnen zuzuordnende Einnahmen nach Art guter Buchführung verlässlich ermittelt werden können. Eine solche Kostenrechnung bildet in Finnland die Grundlage für die Erfüllung der Anwendungsvoraussetzungen der Percentage of Completion-Methode ${ }^{849}$ :

845 Vgl. van Hulle und van der Tas (2001), S. 832.

846 Übersetzung ins Deutsche durch die Verfasserin.

847 Vgl. KILA-Anweisung „Teilgewinnrealisierung“. Auch der finnische Gesetzgeber verweist in seiner Regierungsbegründung zum Gesetzesentwurf auf IAS 11; vgl. HE 173/1997, S. 22. Vgl. KILA-Anweisung ,Teilgewinnrealisierung“, Abschnitt 2, in dem KILA auf die Regierungsbegründung zum Gesetzesentwurf 1992 verweist (HE 111/1992 vp.). 
- Die Gesamteinnahmen und Gesamtausgaben eines Fertigungsauftrags können zuverlässig geschätzt werden,

- am Bilanzstichtag können die bislang tatsächlich angefallenen Kosten auf Basis von Kostenrechnung und Buchhaltung verlässlich ermittelt werden und

- der Fertigstellungsgrad am Bilanzstichtag kann zuverlässig gemessen werden.

Wird nur eines der genannten Kriterien nicht erfüllt, darf keine Teilgewinnrealisierung aus laufenden Fertigungsaufträgen erfolgen. Sie müssen gemäß der Completed Contract-Methode bilanziert werden ${ }^{850}$.

Bei Erfüllung der genannten Kriterien dürfen die Unternehmen sowohl die Completed Contract- als auch die Percentage of Completion-Methode anwenden. Gleichgültig, für welche Methode sich das Unternehmen entscheidet, hat es die einmal gewählte Methode stetig und auf alle gleichartigen Sachverhalte anzuwen$\operatorname{den}^{851}$. Wählt ein börsennotiertes Unternehmen mit der Pflicht zur Zwischenberichterstattung die Percentage of Completion-Methode, so hat es die Teilgewinnrealisierung auch unterjährig durchzuführen. Dies gilt auch dann, wenn Fertigungsbeginn und -ende eines Auftrags im selben Geschäftsjahr liegen, aber in unterschiedliche Zwischenberichtszeiträume fallen ${ }^{852}$.

Durch Erfüllung der Anwendungsvoraussetzungen steht nach Ansicht des finnischen Fachschrifttums die Anwendung der Percentage of Completion-Methode nicht im Widerspruch zum geltenden Vorsichts- und Realisationsprinzip. Die Anwendungsvoraussetzungen garantierten vielmehr, dass nur im Geschäftsjahr realisierte Gewinne erfolgswirksam berücksichtigt würden ${ }^{853}$.

Um die Jahresabschlussqualität zu gewährleisten und aus Vereinfachungsgründen, hat der KILA die Gesamteinnahmen, Gesamtausgaben und Fertigstellungsgrad definiert.

Zu den Gesamteinnahmen aus dem Auftrag zählen nach KILA-Anweisung

- der ursprünglich vertraglich fixierte Erlös,

- zusätzliche Erlöse

○ aus nachträglich durch den Kunden bestellten und vom Hersteller erbrachten Leistungen,

850 Vgl. KILA-Anweisung „Teilgewinnrealisierung“, Abschnitt 4.1, in dem KILA sich auf das Vorsichtsprinzip des KPL 3:3.1 beruft.

851 Vgl. KILA-Anweisung „Teilgewinnrealisierung“, Abschnitt 4.2 und vgl. Järvinen et al. (2000), S. 453.

852 Vgl. AML 2:5 und 2:5a.

853 Vgl. Leppiniemi (2000 a), S. 170 und vgl. Järvinen et al. (2000); S. 448-452. Tuokko erläutert die Gefahren der Percentage of Completion-Methode, vgl. (1994), S. 109-110. 
○ aus Kompensationszahlungen für Fehler, die der Kunde zu vertreten hat und

○ aus vertraglich fixiertem Anspruch auf Prämien oder Bonuszahlungen, beispielsweise für Unterschreitung der vereinbarten Bauzeit oder für gute Qualität der geleisteten Arbeit

- Erlösminderungen, z. B. auf Grund zu leistender Konventionalstrafen ${ }^{854}$.

Die Gesamtausgaben aus dem Auftrag setzen sich wie folgt zusammen ${ }^{855}$ :

- dem Auftrag direkt zurechenbare, variable Kosten

- dem Anteil fixer Kosten von Fertigungsaufträgen, die einzelnen Fertigungsaufträgen zugeordnet werden können.

Fixkosten, die nicht einzelnen Aufträgen zugerechnet werden können, dürfen nicht in die Ausgaben langfristiger Fertigung einbezogen werden. Hierzu zählen allgemeine Verwaltungskosten und Vertriebskosten, allgemeine Forschungs- und Entwicklungskosten sowie Abschreibungen solcher Anlagen, die nicht für Fertigungsaufträge genutzt werden. Vor Fertigungsbeginn angefallene Kosten - z. B. für Angebotsabgabe oder Marketing - bilden keinen Bestandteil der Gesamtausgaben. Ebenfalls nicht einbezogen werden dürfen im Rahmen der Kostenrechnung ermittelte kalkulatorische Kosten. Lediglich tatsächlich anfallende Kosten sind relevant ${ }^{856}$.

Sind Gesamteinnahmen und Gesamtausgaben eines Projekts zuverlässig geschätzt, stellt sich in einem nächsten Schritt die Frage nach ihrer Periodisierung. Unabhängig vom Fertigstellungsgrad gilt bei Gesamteinnahmen übersteigenden Gesamtausgaben, dass unverzüglich eine Drohverlustrückstellung in voller Höhe des Unterschiedsbetrags zu bilden ist ${ }^{857}$.

Im seltenen Fall, dass die Endabnahme vor tatsächlichem Fertigungsende erfolgt, wird der Gesamtgewinn unabhängig vom Fertigstellungsgrad des Projekts sofort realisiert. Noch anfallende Kosten für die Beendigung der zu erbringenden Leistung werden als Aufwand ausgewiesen, wenn sie anfallen ${ }^{858}$.

Im Normalfall erfolgt die Periodisierung der Teilgewinne auf Grundlage des Fertigstellungsgrads. Drei verschiedene Verfahren zur Teilgewinnrealisierung können zur Anwendung kommen ${ }^{859}$ :

- Teilgewinne werden entsprechend dem Verhältnis der bis zum Stichtag angefallenen Kosten zu den geschätzten Gesamtkosten realisiert.

- Teilgewinne werden entsprechend dem Anteil der erbrachten Arbeitsleistung an der Gesamt-Arbeitsleistung realisiert.

854 Vgl. KILA-Anweisung „Teilgewinnrealisierung“, Abschnitt 3.

855 Vgl. KILA-Anweisung „Teilgewinnrealisierung“, Abschnitt 3.

856 Vgl. KILA-Anweisung „,Teilgewinnrealisierung“, Abschnitt 3.

857 Vgl. KILA-Anweisung „Teilgewinnrealisierung“, Abschnitt 3.

858 Vgl. KILA-Anweisung „Teilgewinnrealisierung“, Abschnitt 3.

859 Vgl. KILA-Anweisung „Teilgewinnrealisierung“", Abschnitt 5. 
- Teilgewinne werden entsprechend dem vollendeten Anteil am Gesamtprojekt realisiert.

Für langfristige Auftragsfertigung existieren umfangreiche Angabepflichten im Anhang. Neben der gewählten Methode sind Auftragsvolumen langfristiger Aufträge, ihr Anteil am Umlaufvermögen und ihr Anteil an den gebildeten Pflichtrückstellungen sowie der Veränderung der Pflichtrückstellungen im Berichtsjahr aufzuführen. Bei Anwendung der Percentage of Completion-Methode sind zusätzlich die Methode zur Ermittlung des Fertigstellungsgrads, der Anteil des realisierten Teilumsatzes am Gesamtumsatz und an den Forderungen sowie der Anteil der realisierten Teilaufwendungen an den jeweiligen Aufwandsposten der Gewinnund Verlustrechnung zu nennen860.

860 Vgl. KILA-Anweisung „Teilgewinnrealisierung“, Abschnitt 6.3. 


\subsubsection{Finanzumlaufvermögen}

\subsubsection{Forderungen}

\subsection{Ansatz, Bewertung und Ausweis}

Forderungen bilden eine Ausnahme vom Aktivierungsgrundsatz, da mit ihnen keine künftige Einnahmenerwartung verknüpft ist und sie trotzdem zu aktivieren sind. Das Unternehmen hat die Leistung - die Veräußerung eines Produkts, einer Dienstleistung oder die Gewährung eines Darlehens - bereits erbracht und räumt dem Leistungsempfänger einen bestimmten Zeitraum zur Begleichung dieser Leistung ein ${ }^{861}$.

Forderungen werden bei Zugang mit ihrem Nennwert erfasst. Am Stichtag sind sie unter Beachtung des strengen Niederstwertprinzips gegebenenfalls mit ihrem niedrigeren, wahrscheinlich realisierbaren Betrag zu bewerten. Im Zweifelsfall sind Einzelwertberichtigungen in Form von außerplanmäßigen Abschreibungen vorzunehmen $^{862}$. Diese werden direkt von den Forderungen abgesetzt. Drohverlustrückstellungen sind zu bilden, wenn bei Jahresabschlusserstellung ein Forderungsausfall wahrscheinlich wird, der Forderungen des neuen Geschäftsjahrs betrifft. Entfallen die Gründe für eine in der Vergangenheit getätigte Einzelwertberichtigung, hat eine Wertaufholung zu erfolgen ${ }^{863}$.

Pauschalwertberichtigungen hat der finnische Gesetzgeber nicht explizit geregelt. Ist es jedoch wahrscheinlich und anhand von Erfahrungswerten belegbar, dass ein gewisser Prozentsatz des Forderungsbestands ausfallbedroht ist, darf eine freiwillige Drohverlustrückstellung - auszuweisen unter dem steuerlichen Ausgleichsposten - gebildet werden ${ }^{864}$. Kreditversicherungen werden in Finnland selten abgeschlossen, sodass das Ausfallrisiko tendenziell höher als in Deutschland ist.

Eine Abzinsung langfristiger Forderungen ist nicht ausdrücklich vorgesehen; Forderungen zum Barwert der künftigen Nettoeinzahlungen anzusetzen, kann jedoch im Einzelfall mit dem Grundsatz der Vorsicht begründet werden ${ }^{865}$.

Ist der Wert einer Forderung an einen Index oder einen anderen Vergleichswert gebunden, kann er mit Schwankung des Indexes variieren. Steigt der Vergleichswert, wird der Unterschiedsbetrag zum Nennwert grundsätzlich nicht berücksichtigt. Sind die Umstände aber derart, dass der den Nennwert übersteigende Wert

\footnotetext{
861 Vgl. Kap. 3.1.5.2 dieser Arbeit.

862 Vgl. KPL 5:2.1 und Kap. 3.1.6.2.2.4 dieser Arbeit.

863 Vgl. KPL 5:16 und Kap. 3.1.6.2.2.5 dieser Arbeit.

864 Vgl. KPL 5:16.

865 Vgl. Leppiniemi und Leppiniemi (1999), S. 101.
} 
mit Sicherheit realisiert wird, wird der Unterschiedsbetrag als Rechnungsabgrenzungsposten bilanziert und über die Restlaufzeit der Forderung erfolgswirksam aufgelöst $^{866}$.

Eine Saldierung von Forderungen und Verbindlichkeiten, welche dieselben Personen betreffen, ist nicht zulässig ${ }^{867}$.

Sämtliche Forderungen sind in ihren kurzfristigen und ihren langfristigen Anteil zu trennen. Kurzfristig sind sie dann, wenn sie innerhalb eines Jahres fällig wer$\operatorname{den}^{868}$. Aus der Bilanzgliederung des KPA 1:6, mit dem Art. 9 der 4. EG-Richtlinie umgesetzt worden ist, ergibt sich, dass folgende Forderungen gesondert auszuweisen sind ${ }^{869}$ :

- Forderungen aus Lieferungen und Leistungen,

- Forderungen gegenüber verbundenen Unternehmen,

- Forderungen gegenüber Beteiligungsunternehmen,

- Forderungen aus Darlehen,

- Andere Forderungen,

- Ausstehende Einlagen auf das gezeichnete Kapital und

- Rechnungsabgrenzungsposten.

Forderungen aus Lieferungen und Leistungen resultieren aus der Erbringung einer Leistung aus dem gewöhnlichen Geschäftsbetrieb oder aus der Veräußerung von Vermögenswerten des Anlagevermögens. Beim Verkauf solcher Forderungen an andere Unternehmen sind die Forderungen aus der Bilanz auszubuchen, aus der Veräußerung entstehende Gewinne oder Verluste erfolgswirksam zu erfassen und im Finanzergebnis auszuweisen. Verbleibt das Forderungsausfallrisiko beim verkaufenden Unternehmen, so hat es dies im Anhang als Eventualverbindlichkeit anzugeben ${ }^{870}$.

Unter ausstehenden Einlagen auf das gezeichnete Kapital wird eingefordertes, aber noch nicht eingezahltes Kapital ausgewiesen. Dem Charakter nach handelt es sich hierbei um Forderungen an die Gesellschafter ${ }^{871}$. Die Anmeldung zum Handelsregister kann erst dann erfolgen, wenn das gezeichnete Kapital vollständig einbezahlt ist 872 .

Vgl. Leppiniemi (2000 a), S. 267 und vgl. KILA 1523/1998.

Vgl. KPA 1:10.2.

Vgl. KPL 4:7.1.

Vgl. auch KPL-Arbeitsgruppe 1995, Teil I (1996), S. 188-189.

Vgl. KILA 1444/1997, vgl. Leppiniemi (2000 a), S. 257.

Vgl. KILA 1136/1996.

Vgl. OYL 2:9 und vgl. Kap. 2.2.2.2 dieser Arbeit. 


\subsection{Aktiver Rechnungsabgrenzungsposten}

Rechnungsabgrenzungen sind Korrekturposten in der Bilanz, die auf der Grundlage des Prinzips der leistungsabhängigen Buchführung, des Prinzips der Ausgabenperiodisierung und des Realisationsprinzips vorzunehmen sind. Sie werden notwendig, wenn ein zeitraumbezogener Ertrag oder Aufwand und die ihnen jeweils zuzuordnenden Zahlungen in unterschiedliche Perioden fallen ${ }^{873}$.

Der Richtliniengeber hat eine Koordinierung der einzelstaatlichen Gesetzgebung als nicht notwendig angesehen, da es sich bei abzugrenzenden meist um nicht wesentliche Beträge handelt. Um unsachgemäßer Abgrenzung vorzubeugen, fordert der Richtliniengeber eine Anhangangabe dort, wo die Beträge wesentlich $\operatorname{sind}^{874}$. Mit KPL 4:6.1 hat der finnische Gesetzgeber Art. 18 der 4. EG-Richtlinie wie folgt umgesetzt ${ }^{875}$ :

$\underline{\text { Rechnungsabgrenzungsposten }}$

(1) Zur aktiven Rechnungsabgrenzung gehören:

- im Geschäftsjahr oder in früheren Geschäftsjahren geleistete Zahlungen, die erst in zukünftigen Geschäftsjahren auf Basis der leistungsabhängigen Buchung im Aufwand erfasst werden dürfen, sofern sie nicht als geleistete Anzahlungen auszuweisen sind; und

- $\quad$ solche im Geschäftsjahr oder in früheren Geschäftsjahren auf Basis des Prinzips der leistungsabhängigen Buchung realisierte Einnahmen, für die noch keine Einzahlung erfolgt ist, sofern sie nicht unter Forderungen aus Lieferungen und Leistungen auszuweisen sind. $[\ldots]$

Der Definition des KPL 4:6.1 entsprechend umfasst der aktive Rechnungsabgrenzungsposten in Finnland transitorische und antizipative Rechnungsabgrenzungen $^{876}$.

Bei transitorischen Rechnungsabgrenzungen handelt es sich um Ausgaben der abgelaufenen Periode, die Aufwand für eine bestimmte Zeit nach dem AbschlussStichtag darstellen ${ }^{877}$. Zu ihnen zählen typischerweise Mietvorauszahlungen, Versicherungszahlungen sowie Zins- oder Steuervorauszahlungen. Auch geleistete Gehaltsvorschüsse samt zugehöriger Sozialabgaben sind als Rechnungsabgren-

873

Vgl. Baetge, Kirsch und Thiele (2001), S. 461.

Vgl. Art. 18 der 4. EG-Richtlinie, vgl. van Hulle und van der Tas (2001), S. 810.

Übersetzung ins Deutsche durch die Verfasserin.

Hierin unterscheidet sich Finnland von z. B. Deutschland, wo lediglich transitorische Posten als aktiver Rechnungsabgrenzungposten ausgewiesen werden, antizipative Posten hingegen als sonstiger Vermögensgegenstand. Vgl. $§ 250$ Abs. 1 S. 1 und S. 2 HGB.

Noch anders zeigt sich die Situation nach den Standards des IASB. Für Rechnungsabgrenzungsposten ist keine eigenständige Bilanzposition vorgesehen, transitorische wie antizipative Posten sind als „trade and other receivables“ auszuweisen, unterteilt in ,current assets“ und ,non-current assets“. Vgl. IAS 1.66 und IAS 1.53.

Vgl. Baetge, Kirsch und Thiele (2001), S. 464. 
zung auszuweisen ${ }^{878}$. Ein Disagio ist nach finnischer Auffassung nicht als aktive Rechnungsabgrenzung, sondern als immaterieller Vermögenswert - Ausgabe mit lang anhaltender Wirkung - zu behandel ${ }^{879}$.

Antizipative Rechnungsabgrenzungen umfassen Erträge für eine bestimmte Zeit innerhalb der abgelaufenen Periode, die erst nach dem Abschluss-Stichtag zu einer Einnahme führen ${ }^{880}$. $\mathrm{Zu}$ nennen sind beispielsweise Forderungen an Versicherungen aus Schadensfällen, Forderungen aus vereinbarten Jahresboni oder rabatten und Provisionen sowie Forderungen gegenüber dem Finanzamt und Forderungen aus Zinseinnahmen oder Mieteinnahmen ${ }^{881}$.

Auffallend ist, dass der Rechnungsabgrenzungsposten mit seinem transitorischen und antizipativen Bestandteil gesondert unter den Forderungen ausgewiesen wird $^{882}$. Transitorische Posten sind ihrem Charakter nach keine Forderungen. Antizipative Posten stellen ihrem Wesen nach Forderungen dar: Die Leistung ist erbracht, wurde allerdings noch nicht beglichen. Es ist anzunehmen, dass der Ausweis unter den Forderungen für beide Posten darauf zurückzuführen ist, dass sowohl transitorische als auch antizipative Posten den Aktivierungsgrundsatz nicht erfüllen. Beiden liegt keine Ausgabe zu Grunde, mit der eine künftige Einnahmenerwartung verbunden ist. Forderungen bilden nach finnischem Verständnis die einzige Ausnahme vom Aktivierungsgrundsatz.

\subsubsection{Wertpapiere des Finanzumlaufvermögens}

Nach KPL 4:4.3 sind dem Finanzumlaufvermögen Zahlungsmittel, Forderungen und Zahlungsmitteläquivalente zuzurechnen. Unter Zahlungsmitteläquivalenten werden temporär in anderen Formen als Zahlungsmittel oder Forderungen gehaltene Finanzreserven verstanden: Wertpapiere des Finanzumlaufvermögens.

Anders als Wertpapiere des Anlagevermögens werden sie nicht mit dem Zweck gehalten, das Vermögen des Unternehmens durch Zinsen, Dividenden oder Wertzuwachs zu mehren; in diesem Sinn ist keine Einnahmenerwartung mit ihnen verbunden. Wertpapiere des Finanzumlaufvermögens befinden sich im Bestand des Unternehmens, weil im Rahmen der Finanzplanung liquide Mittel mit dem Ziel in Wertpapiere umgewandelt worden sind, in naher Zukunft eine Investition zu finanzieren, oder weil das Unternehmen sie als Zahlungsmittel für erbrachte Leis-

\footnotetext{
878 Vgl. Järvinen et al. (2000), S. 407 und vgl. Leppiniemi und Leppiniemi (1999), S. 102-103.

879 Vgl. Kap. 3.2.1.1.6 dieser Arbeit.

880 Vgl. Baetge, Kirsch und Thiele (2001), S. 464.

881 Vgl. Järvinen et al. (2000), S. 407 und vgl. Leppiniemi und Leppiniemi (1999), S. 102-103.

882 Vgl. KPA 1:6.
} 
tungen entgegengenommen hat ${ }^{883}$. Sie sind jederzeit realisierbar, und es besteht auch die Absicht, sie nur kurzfristig im Portefeuille zu halten.

Wertpapiere des Finanzumlaufvermögens sind mit ihren Anschaffungskosten oder unter Beachtung des strengen Niederstwertprinzips zu bewerten. Am Bilanzstichtag ist gegebenenfalls auf den niedrigeren, wahrscheinlichen Veräußerungspreis abzuschreiben $^{884}$. Das Wertaufholungsgebot ist zu berücksichtigen ${ }^{885}$.

In der Bilanz sind als Wertpapiere des Finanzumlaufvermögens folgende Posten getrennt auszuweisen ${ }^{886}$ :

- Anteile an verbundenen Unternehmen,

- Anteile am eigenen Unternehmen,

- Anteile an anderen Unternehmen und

- andere Wertpapiere

\subsubsection{Eigenkapital}

\subsubsection{Gebundenes und nicht gebundenes Eigenkapital}

Beim Eigenkapital handelt es sich um eine Residualgröße, die nach Abzug aller Schulden und steuerliche Ausgleichsposten von den Vermögenswerten des Unternehmens verbleibt. Ihre Höhe ist indirekt von der Definition, dem Ansatz und der Bewertung der Vermögenswerte, Schulden und steuerliche Ausgleichsposten abhängig ${ }^{887}$.

Das Eigenkapital umfasst die dem Unternehmen von seinen Eigentümern zur Verfügung gestellten Mittel. Diese Mittel sind dem Unternehmen entweder durch Zuführung von außen oder durch Gewinnthesaurierung zugeflossen.

Nach finnischem Verständnis wird zwischen gebundenem, festen und nicht gebundenem, variablen Eigenkapital unterschieden. Der finnische Gesetzgeber definiert diese im Aktiengesellschaftsgesetz OYL 11:6 wie folgt:

Gebundenes Eigenkapital sind das gezeichnete Kapital, die Kapitalrücklage, die gesetzliche Rücklage und die Neubewertungsrücklage. Alle anderen Rücklagen zählen zum nicht gebundenen Eigenkapital. Der Jahresüberschuss und der Gewinnvortrag werden, jeweils gesondert ausgewiesen, dem nicht gebundenen Eigenkapital zuge-

883 Vgl. KILA 1054/1989.

884 Vgl. KPL 5:2.2 und vgl. Kap. 3.1.6.2.2.4 dieser Arbeit.

885 Vgl. KPL 5:16 und vgl. Kap. 3.1.6.2.2.5 dieser Arbeit.

886 Vgl. Bilanzgliederung des KPA 1:6. Zum Begriff „,verbundene Unternehmen“ vgl. Kap. 3.2.1.3.2 dieser Arbeit, zur Bilanzierung eigener Anteile vgl. Kap. 3.2.1.3.1 dieser Arbeit.

887 Vgl. Kap. 3.1.6.3 dieser Arbeit. 
rechnet, der Jahresfehlbetrag und der Verlustvortrag, jeweils gesondert, von ihm abgezogen.

Ein eigenkapitalersetzendes Rangrücktrittsdarlehen wird nach gebundenem und nicht gebundenem Eigenkapital als gesonderter Posten innerhalb des Eigenkapitals ausgewiesen.

Gebundenes Kapital ist dadurch charakterisiert, dass es in seiner Höhe stets voll ausgewiesen wird und so lange unverändert bleibt, bis die Hauptversammlung eine Erhöhung oder Herabsetzung beschließt. Veränderungen des Eigenkapitals, die aus dem Jahresergebnis resultieren, betreffen das nicht gebundene Eigenkapital. Dem Jahresergebnis entsprechend ändert sich der Bestandswert des nicht gebundenen Eigenkapitals mit jedem neuen Jahresabschluss.

Durch bestimmte Kapitalsicherungsvorschriften des Buchführungs- und des Aktiengesellschaftsgesetzes unterliegen das gesamte gebundene sowie Teile des nicht gebundenen Eigenkapitals einer Ausschüttungssperre ${ }^{888}$. Die dauerhafte Bindung an ein Unternehmen ist abhängig von Hauptversammlungsbeschlüssen, Ausschüttungsfähigkeit wird gesetzlich geregelt ${ }^{889}$. Die Art guter Buchführung und der True and Fair View fordern, das Eigenkapital im Anhang gegliedert nach gebundenen und nicht gebundenen Bestandteilen anzugeben ${ }^{890}$. Zusätzlich nennen Aktiengesellschaften die Höhe des ausschüttungsfähigen Eigenkapitals im Anhang ${ }^{891}$.

Es gelten die Gliederungsvorschriften des KPA 1:6:

- Gezeichnetes Kapital

- Kapitalrücklage

- Neubewertungsrücklage

- Sonstige Rücklagen

○ Rücklagen für eigene Aktien oder Anteile

○ Gesetzliche Rücklage

○ Rücklagen 1t. Gesellschaftsvertrag oder Satzung

○ Übrige Rücklagen

- Ergebnisvortrag

- Ergebnis des Geschäftsjahrs

- Eigenkapitalersetzende Rangrücktrittsdarlehen

888 Nicht ausschüttungsfähige Teile des nicht gebundenen Eigenkapitals sind aus freiwilligen Rückstellungen und dem Abschreibungsunterschied in die Rücklagen umgewandelte Kapitalbeträge, Anschaffungskosten eigener Aktien, Währungsumrechnungsdifferenzen, Darlehen und Sicherheiten, die den Mitgliedern des sog. ,inneren Kreises“ einer Aktiengesellschaft gewährt wurden, Forschungskosten und Entwicklungskosten, die nicht den Anforderungen des KTM:p 50/1998 entsprechen. Die das Eigenkapital betreffenden Standards des IASB sind für diejenigen Einzelabschlüsse zu beachten, die in einen nach IAS erstellten Konzernabschluss eingehen. 
Als gezeichnetes Kapital werden die Kapitalbeträge gezeigt, in deren Höhe die Gesellschafter Anteile an der Kapitalgesellschaft übernommen haben und auf welche die Haftung der Gesellschafter beschränkt ist. Das Mindestkapital beträgt bei privaten Aktiengesellschaften $€ 8.000,00$, bei öffentlichen Aktiengesellschaften $€ 80.000,00^{892}$. Es darf ausschließlich der im Handelsregister eingetragene Wert ausgewiesen werden ${ }^{893}$.

In der Kapitalrücklage werden die Beträge erfasst, die dem Unternehmen von aussen zufließen. In sie ist einzustellen ${ }^{894}$

- der Betrag, der bei der Ausgabe von Optionsrechten und Schuldverschreibungen für Wandlungsrechte erzielt wird,

- der Betrag, der bei der Ausgabe von Anteilen über den Nennbetrag hinaus erzielt wird,

- der Betrag, der nach geplanter, aber nicht durchgeführter Neuemission als Schadenersatzzahlung an das Unternehmen fließt,

- der Betrag, der im Zusammenhang mit Effektenanteilssystemen aus dem Verkauf nicht registrierter Aktien herrührt und den die Anteilseigner nicht ausschütten,

- der Betrag, der aus dem Verkauf von Aktien aus einer Kapitalerhöhung herrührt und den die Anteilseigner nicht ausschütten,

- der dem Teil der Herabsetzung des gezeichneten Kapitals entsprechende Betrag, der nicht zur Deckung von Verlusten dient, nicht in die gesetzliche Rücklage eingestellt wird und nicht an die Anteilseigner ausgeschüttet wird,

- der Gewinn aus dem Verkauf eigener Aktien oder Aktien des Mutterunternehmens.

Vor dem 01. September 1997 wurden diese Beträge in die gesetzliche Rücklage eingestellt. Da keine Verpflichtung zur Umgliederung dieser Beträge besteht, kann es sein, dass in der Kapitalrücklage lediglich die nach dem 01. September 1997 angefallenen Beträge berücksichtigt sind, die früheren nach wie vor als gesetzliche Rücklage aufgeführt sind. Die in der Kapitalrücklage enthaltenen Beträge dürfen verwendet werden zur Deckung von Verlusten, sofern das nicht gebundene Eigenkapital hierfür nicht ausreicht, zur Erhöhung des gezeichneten Kapitals ${ }^{895}$, zur Deckung des Unterschiedsbetrags einer Unter-Pari-Emission und zur Herabsetzung der Kapitalrücklage ${ }^{896}$.

892 Vgl. Kap. 2.2.2.2 dieser Arbeit.

893 Das gezeichnete Kapital muss in voller Höhe eingezahlt sein, bevor die Pflicht-Eintragung der Gesellschaft ins Handelsregister möglich ist. Die finnischen Vorschriften sind strenger als Art. 9 der 2. EG-Richtlinie, der lediglich eine Mindest-Einzahlung von 25 Prozent des gezeichneten Kapitals fordert.

895 Eine Kapitalerhöhung setzt einen Hauptversammlungsbeschluss voraus, welcher die Zeichnung der Aktien durch Aktionäre und andere Zeichnungsberechtigte zur Folge hat. Der Haupversammlungsbeschluss bildet noch keine Buchungsgrundlage. Bei Zeichnung wird bi- 
Wird bei Vermögenswerten des Anlagevermögens (Grundstücke und Gewässer sowie Wertpapiere des Finanzanlagevermögens) eine Neubewertung vorgenommen, ist der Unterschiedsbetrag erfolgsneutral in die Neubewertungsrücklage einzustellen $^{897}$. Die Neubewertungsrücklage kann auf Beschluss der Hauptversammlung in gezeichnetes Kapital umgewandelt werden. Sie ist aufzulösen, wenn die in sie eingestellten Beträge entweder vollständig durch den Abgang (erfolgswirksam $^{898}$ ) oder teilweise durch eine Wertminderung des Vermögenswerts (erfolgsneutral) realisiert werden. Reicht wegen zwischenzeitlicher Umwandlung in gezeichnetes Kapital die Neubewertungsrücklage zur Rücknahme der Neubewertung nicht aus, ist an ihrer Stelle das nicht gebundene Eigenkapital in Höhe des Unterschiedsbetrags aufzulösen ${ }^{899}$.

Kauft eine öffentliche Aktiengesellschaft Aktien von ihren Aktionären zurück, sind diese Vermögenswerte in Höhe ihrer Anschaffungskosten in der Bilanz zu aktivieren $^{900}$. Sie können jedoch im Konkursfall nicht - oder nur selten - verwertet werden, da sie dann in der Regel wertlos sind. Aus diesem Grund ist in ihrer Höhe eine Rücklage für eigene Anteile zu bilden, die der Ausschüttungssperre unterliegt und Gläubiger- und Aktionärsschutz bezweckt ${ }^{901}$. Die Zuführung zur Rücklage wird mit aus dem nicht gebundenen Eigenkapital stammenden Mitteln vorgenommen $^{902}$. Sie darf nur aufgelöst werden, soweit die eigenen Anteile wieder veräußert oder auf den niedrigeren Wert am Stichtag abgeschrieben werden ${ }^{903}$.

Die gesetzliche Rücklage hatte nach altem Recht diejenigen Beträge zum Inhalt, die heute die Kapitalrücklage bilden. Vor dem 01. September 1997 in die gesetzliche Rücklage eingestellte Mittel müssen nicht in die Kapitalrücklage umgegliedert werden. Neu der Kapitalrücklage zugeführt werden heutzutage nur noch Ka-

lanziell eine „Forderung aus Aktienemission“ auf der Aktivseite ausgewiesen, auf der Passivseite der gesonderte Posten „Aktienemission“ (nicht Bestandteil des Eigenkapitals). Nach Einzahlung des Kapitals in voller Höhe erfolgt die Meldung an das Handelsregister, das die Kapitalerhöhung einträgt. Erst danach wird die Kapitalerhöhung im Eigenkapital ausgewiesen. Die Einstellung von Kapital in Rücklagen ist keinesfalls erfolgswirksam zu behandeln. Vgl. KILA 1136/1991, Abschnitt 2.3.1.5. Vgl. OYL 12:3.2.

897 Vgl. KPL 5:17 und vgl. Kap. 3.1.6.2.2.6 dieser Arbeit.

898 In einem solchen Fall enthält die Neubewertungsrücklage einen Fremdkapitalanteil aus Steuerstundung. Die steuerliche Behandlung der Neubewertungsrücklage ist nach Art. 33 Abs. 2 (a) der 4. EG-Richtlinie zwingend in der Bilanz oder im Anhang darzustellen. Vgl. hierzu Kap. 3.2.7 dieser Arbeit.

899 Vgl. Leppiniemi und Leppiniemi (1999), S. 108 und vgl. Kap. 3.1.6.2.2.6 dieser Arbeit.

900 Vgl. OYL 11:6a und vgl. Kap. 3.2.1.3.1 dieser Arbeit.

901 Vgl. HE 173/1997, S. 9 und vgl. Järvinen et al. (2000), S. 248. Die Ausschüttungssperre entspricht der Forderung des Art. 22 Abs. 1 (b) der 2. EG-Richtlinie. Vgl. Leppiniemi und Leppiniemi (1999), S. 109. Buchungstechnisch findet eine Passivtausch „Gewinnvortrag an Rücklage für eigene Aktien“ statt.

903 Vgl. Kap. 3.2.1.3.1 dieser Arbeit. Zur Problematik bei der Auflösung der Rücklage für eigene Aktien vgl. Honkamäki (1998), S. 389-393. 
pitalbeträge des nicht gebundenen Eigenkapitals, die auf Beschluss der Hauptversammlung Bestandteil des gebundenen Kapitals werden sollen ${ }^{904}$. Hinsichtlich der Verwendung der gesetzlichen Rücklage gelten die Regeln für die Kapitalrücklage. Anders als in anderen Ländern ${ }^{905}$ ist in Finnland die Dotierung der gesetzlichen Rücklage freiwillig, sodass die Verwendung des Begriffs ,gesetzliche Rücklage“ für den ausländischen Bilanzleser irreführend ist. Die KPL-Arbeitsgruppe 1995 weist in ihrem Bericht darauf hin, dass es eine gesetzliche Rücklage - so wie sie international verstanden wird - in Finnland nicht gebe.

Sehen Gesellschaftsvertrag oder Satzung vor, dass den Gewinnrücklagen bestimmte Mittel aus dem Jahresüberschuss zuzuführen sind, so sind diese als Rücklagen laut Gesellschaftsvertrag oder Satzung auszuweisen. Gesellschaftsvertragsoder Satzungsbestimmungen können auch den Zweck, die Erhöhung oder die Herabsetzung dieser Rücklage vorschreiben.

Auf Beschluss der Hauptversammlung können Kapitalbeträge aus dem Ergebnisvortrag in sonstige Rücklagen umgewandelt werden, die meist zweckgebunden sind: Steuerrücklagen, Rechtsschutzrücklagen, Rücklagen für den allgemeinen Gebrauch oder Gebäuderücklagen $^{906}$.

Der Ergebnisvortrag umfasst die kumulierten Jahresüberschüsse sowie -fehlbeträge früherer Berichtsperioden und bildet somit eine - richtlinienkonforme - Ausnahme vom Saldierungsverbot des KPA 1:10.2.

Der Posten Ergebnis des Geschäftsjahrs entspricht immer dem gleichnamigen Posten in der Gewinn- und Verlustrechnung.

904 Vgl. Leppiniemi und Leppiniemi (1999), S. 110, vgl. Järvinen et al. (2000), S. 248 und vgl. Artsberg (2000), S. 552.

905 Der Entwurf der 5. EG-Richtlinie sieht in Art. 49 Abs. 1 vor, dass fünf Prozent eines jeden Jahresüberschusses in die gesetzliche Rücklage einzustellen sind bis diese zehn Prozent des Werts des gezeichneten Kapitals erreicht.

906 Vgl. KILA 1343/1995, vgl. Leppiniemi und Leppiniemi (1999), S. 111. 


\subsubsection{Eigenkapitalersetzende Rangrücktrittsdarlehen}

Vor 1997 ist eine Überschuldung einer Aktiengesellschaft in Finnland eingetreten, sobald das Eigenkapital in seiner Gesamtsumme ein Drittel des gezeichneten Kapitals unterschritten hatte. Nach Aktiengesellschaftsgesetz 1997 liegt ein Konkursfall bereits dann vor, wenn das Eigenkapital weniger als die Hälfte des gezeichneten Kapitals beträgt ${ }^{907}$. Um die Verschärfung dieser Regelung über den Konkurs auszugleichen, hat der finnische Gesetzgeber eine neue Finanzierungsform für Aktiengesellschaften geschaffen: das in OYL 5:1 kodifizierte eigenkapitalersetzende Rangrücktrittsdarlehen (,„pääomalaina“(908) ${ }^{909}$.

Darlehen, die einer Kapitalgesellschaft von ihren Gesellschaftern oder dritten Gläubigern gewährt werden, sind in der Regel dem Fremdkapital zuzurechnen, da die Darlehensgeber einen Anspruch auf Rückzahlung geltend machen können.

Zur Beseitigung oder Abwendung einer Überschuldung oder auch zur Verbesserung der Eigenkapitalquote können Gläubiger - gleichgültig, ob Gesellschafter oder fremde Dritte - und Schuldner jedoch einen Rangrücktritt vereinbaren, der den Gläubiger im Fall des Konkurses schlechter stellt als andere Gläubiger, ihm aber bei Unternehmensfortführung nicht die Rechte eines Eigentümers verleiht ${ }^{910}$. Werden mit der getroffenen Vereinbarung die in OYL 5:1 vom Gesetzgeber festgelegten Merkmale für ein eigenkapitalersetzendes Rangrücktrittsdarlehen erfüllt, so ist es vom Schuldner als Bestandteil des Eigenkapitals gesondert auszuwei$\operatorname{sen}^{911}$ :

- Der Darlehensgeber tritt mit seinem Anspruch auf Rückzahlung im Fall einer Fusion, einer Abspaltung, einer Schließung einzelner oder ganzer Geschäftsbereiche oder eines Konkurses des Schuldnerunternehmens hinter die Ansprüche anderer Gläubiger zurück.

- Das Darlehen oder auch nur ein Teil des Darlehens dürfen nur dann zurückgezahlt werden, wenn das gezeichnete Kapital die anderen Bestandteile des gebundenen Eigenkapitals sowie die nicht ausschüttungsfähigen Teile des nicht gebundenen Eigenkapitals nach der Rückzahlung vollständig deckt. Als Maßstab dient die zuletzt festgestellte Bilanz. Handelt es sich beim Darlehensnehmer um ein Mutterunternehmen, dann ist als Maß-

907 Vgl. Leppiniemi (1997), S. 12.

908 Wörtlich übersetzt heißt „pääomalaina“ Kapitaldarlehen. In den englischsprachigen Versionen veröffentlichter finnischer Abschlüsse wird das Darlehen „Subordinated Loan“ oder „Capital loan“" genannt.

909 Vgl. Karhu (1998), S. 78.

910 Vgl. Karhu (1998), S. 80

911 Vgl. OYL 5:1, vgl. Karhu (1998), S. 79-80 und 83-88, vgl. Leppiniemi (1997), S. 14 und vgl. Lydman (1998), S. 7-8. 
stab neben dem Einzelabschluss auch der Konzernabschluss heranzuziehen.

- Zinsen auf das Darlehen und andere auf dem Darlehen basierende Vergütungen dürfen nur dann zur Auszahlung kommen, wenn der zu zahlende Betrag - er wird als Ergebnisverwendung betrachtet - auf Basis des zuletzt festgestellten Jahresabschlusses ausschüttungsfähig ist ${ }^{912}$.

- Das Darlehen wird in der Bilanz des Darlehensnehmers als Bestandteil des Eigenkapitals gesondert nach dem gebundenen und dem nicht gebundenen Eigenkapital ausgewiesen ${ }^{913}$.

- Der Gläubiger darf für das Darlehen weder vom Darlehensnehmer noch von verbundenen Unternehmen des Darlehensnehmers eine Sicherheit erhalten. Dieses Verbot beruht auf der Forderung, dass der Gläubiger im Konkursfall schlechter gestellt sein muss als andere Gläubiger.

- Ein schriftlicher Vertrag, mit dem die Erfüllung der geforderten Merkmale beweisbar ist, muss existieren.

Das eigenkapitalersetzende Rangrücktrittsdarlehen weist Fremdkapitalcharakter auf, da sich auf ihm ein Anspruch des Gläubigers auf Rückzahlung begründet. Gleichzeitig ist Eigenkapitalcharakter gegeben, weil durch den Rangrücktritt das Risiko von Kapitalverlusten getragen wird, und zwar auch durch Gläubiger und nicht ausschließlich durch die Gesellschafter.

Mit dieser Finanzierungsform wird das Ziel verfolgt, Unternehmen in wirtschaftlicher Krisensituation vor dem Konkurs zu bewahren. Die Gesellschafter können statt der Zuführung von voll haftendem Eigenkapital ein Darlehen mit Rückzahlungsanspruch gewähren und trotzdem im Konkursfall das Risiko tragen, ohne das aufwendige Verfahren einer Kapitalerhöhung und späterer Kapitalherabsetzung befolgen zu müssen. Die Gläubiger des Unternehmens können Forderungen gegenüber dem Unternehmen in ein eigenkapitalersetzendes Rangrücktrittsdarlehen umwandeln, um dessen Konkurs abzuwenden und einen wichtigen Kunden nicht zu verlieren. Die Gesetzesbegründung nennt aber auch explizit die Vorteile des Darlehens für gesunde Schuldnerunternehmen: Eine verbesserte Eigenkapitalquote erleichtere das Agieren in den internationalen Finanzmärkten und vereinfache die Mittelbeschaffung ${ }^{914}$.

912 Höhe von Zins und Vergütung sind vertraglich frei vereinbar, zur Abgrenzung der Zinsen und Vergütungen über die Laufzeit des Darlehens vgl. KILA 1317/1995 und KILA 959/1988.

913 Vgl. auch KILA-Anweisung „Darstellung von Gewinn- und Verlustrechnung und Bilanz“; im Bilanzgliederungsschema des KPA 1:6 für alle Buchführungspflichtigen ist das nur für Aktiengesellschaften relevante eigenkapitalersetzende Rangrücktrittsdarlehen nicht vorgesehen.

914 Vgl. Karhu (1998), S. 79 und vgl. Leppiniemi (1997), S. 12. 
Der Vorschlag, eigenkapitalersetzende Rangrücktrittsdarlehen als Eigenkapital zu betrachten, hat in der Entstehungsphase des Aktiengesellschaftsgesetzes für eine kontrovers geführte Diskussion in der Fachwelt und im Schrifttum gesorgt. Während die mit dem Entwurf des Aktiengesellschaftsgesetzes betraute Arbeitsgruppe sich dafür ausgesprochen hat, die aus haftungsrechtlicher Sicht eigenkapitalähnlichen Mittel im Eigenkapital auszuweisen ${ }^{915}$, haben der KILA und die KPL-Arbeitsgruppe 1995 dafür plädiert, eigenkapitalersetzende Rangrücktrittsdarlehen dem Fremdkapital zuzuordnen. Der KILA hat darauf hingewiesen, dass das Darlehen weder aus Sicht der Ausgaben-Einnahmen-Theorie der Buchführung noch der Buchführungspraxis die Merkmale von Eigenkapital vorweise und sein Ausweis im Eigenkapital (inländische) Bilanzleser deshalb irreführe ${ }^{916}$. Die KPL-Arbeitsgruppe 1995 hat sich unter Berufung auf die 4. EG-Richtlinie, deren Bilanzgliederung keine eigenkapitalersetzenden Darlehen vorsieht, dafür ausgesprochen, das Darlehen gesondert im Fremdkapital zu zeigen ${ }^{917}$. Die Steuergesetzgebung schließt sich dieser Auffassung an, steuerlich wird das eigenkapitalersetzende Rangrücktrittsdarlehen wie Fremdkapital behandelt ${ }^{918}$.

Das Darlehen widerspricht als Bestandteil des Eigenkapitals der Going ConcernPrämisse des Art. 31 Abs. 1 (a) der 4. EG-Richtlinie und des KPL 3:1.1,1. Unter Zugrundelegung des Grundsatzes der Unternehmensfortführung stellt es Fremdkapital dar, da es lediglich im Konkursfall dem Eigenkapital gleichgestellt wird. Zudem besteht ein Widerspruch zum Vorsichtsprinzip des Art. 31 . Abs. 1 (c) der 4. EG-Richtlinie und des KPL 3:1.1,3. Bei vorsichtiger Interpretation ist das Darlehen dem Fremdkapital zuzurechnen. So hat sich ein Vertreter der EU-Kommission dafür ausgesprochen, das Darlehen als Fremdkapital zu zeigen ${ }^{919}$. Der Kontaktausschuss der EU-Kommission kommt ebenfalls zu der Feststellung, Rangrücktrittsdarlehen - auch wenn diese nicht in allen Punkten der Definition des finnischen Aktiengesellschaftsgesetzes entsprechen - stellten Fremdkapital dar ${ }^{920}$.

Die finnische Gesetzgebung weicht mit der Vorschrift zur Bilanzierung des eigenkapitalersetzenden Rangrücktrittsdarlehens im Eigenkapital erheblich von der Gesetzgebung anderer europäischer Länder und den Standards des IASB ab ${ }^{921}$. Die

915 Vgl. Karhu (1998), S. 82.

916 Vgl. Karhu (1998), S. 81-82.

917 Vgl. KPL-Arbeitsgruppe 1995, Teil I (1996), S. 190-191.

918 Vgl. Lydman (1998), S. 10-11 und vgl. Karhu (1998), S. 86-87.

919 Vgl. KPL-Arbeitsgruppe 1995, Teil I (1996), S. 191.

920 Vgl. KPL-Arbeitsgruppe 1995, Teil I (1996), S. 191, die auf „The Commission of the European Communities: The Accounting Harmonization in the European Communities, Problems of Applying the fourth directive on the annual accounts of limited companies" verweist. Vgl. auch van Hulle und van der Tas (2001), S. 811 und S. 813.

921 Das IASB definiert im Framework Nr. 49 Verbindlichkeiten wie folgt: „A liability is a present obligation of the enterprise arising from past events, the settlement of which is expected to result in an outflow from the enterprise of resources embodying economic benefits." Da mit dem eigenkapitalersetzenden Rangrücktrittsdarlehen eine Rückzahlungsverpflichtung verbunden ist, ist es nach IAS eindeutig dem Fremdkapital zuzuordnen. 
Aussagekraft des Einzelabschlusses des Schuldnerunternehmens sowie des Konzernabschlusses, in den es einbezogen wird, wird hinsichtlich der Vermögensund Finanzlage beeinträchtigt. Auch wirkt sich die Zuordnung des Darlehens zum Eigenkapital auf den Substanzwert des Unternehmens aus. Bei der Ermittlung der Bilanzkennzahlen privater sowie nicht börsennotierter Aktiengesellschaften darf das Eigenkapitaldarlehen dem Eigenkapital zugerechnet werden. Gemäß KILAAnweisung „Erstellung von Jahresabschluss, vorläufigem Abschluss und Zwischenberichten“ müssen börsennotierte Unternehmen das Darlehen für die Berechnung der Kennzahlen dem Fremdkapital zuordnen. Der (ausländische) Bilanzleser sollte eigenkapitalersetzende Rangrücktrittsdarlehen bei der Interpretation finnischer Abschlüsse unbedingt beachten und gegebenenfalls eine gedankliche Umgliederung in Fremdkapital vornehmen.

\subsubsection{Fremdkapital}

\subsubsection{Steuerliche Ausgleichsposten}

\subsection{Abschreibungsunterschied}

In Übereinstimmung mit Art. 35 Abs. 1 (d) der 4. EG-Richtlinie erlaubt der finnische Gesetzgeber in KPL 5:12, steuerlich veranlasste Abschreibungen vorzunehmen, die über die handelsrechtlichen Abschreibungen hinausgehen. Handelsrechtliche und steuerliche Abschreibungen addiert ergeben die erfolgswirksame Jahresabschreibung $^{922}$.

Die steuerlichen Abschreibungen mindern in Finnland als Wertberichtigungen nicht direkt das Anlagevermögen, sondern werden wegen der besseren Aussagefähigkeit der Bilanz passivisch angesetzt. Auf diese Weise wird auf der Aktivseite der handelsrechtlich korrekte Wert des Anlagevermögens ausgewiesen ${ }^{923}$. In der Regel fällt der steuerlich zulässige Abschreibungshöchstsatz bei erlaubter degressiver Abschreibung und kürzerer Nutzungsdauer höher aus als eine handelsrechtliche Abschreibung nach betriebswirtschaftlichen Gesichtspunkten. Fast jedes finnische Unternehmen weist einen Abschreibungsunterschied in seiner Bilanz aus.

Bei dieser Passivierungspflicht für steuerrechtlich veranlasste Abschreibungen handelt es sich um eine finnische Besonderheit. Der Abschreibungsunterschied ist zwar vergleichbar mit dem deutschen Sonderposten mit Rücklageanteil, der nach $\S 281$ Abs. 1 HGB steuerrechtliche Abschreibungen enthalten kann. Allerdings besteht anders als in Finnland nach deutschem Handelsgesetzbuch ein Passivie- 
rungswahlrecht, da in Deutschland steuerrechtliche Abschreibungen auch direkt aktivisch vorgenommen werden können ${ }^{924}$.

In Finnland bilden die passivisch abgesetzten steuerlichen Abschreibungen des Geschäftsjahrs und früherer Geschäftsjahre den kumulierten Abschreibungsunterschied. Was aus steuerlicher Sicht eine passivisch abgesetzte Aufwendung darstellt, ist aus handelsrechtlicher Sicht sowohl zweckgebundene Rücklage mit Eigenkapitalcharakter als auch eine vom Ergebnis abzugsfähige Rückstellung mit Fremdkapitalcharakter. In Höhe des in der Bilanz ausgewiesenen Abschreibungsunterschieds haben steuerliche Abschreibungen zu einer Steuerstundung geführt. Sie führen aber nicht zu einer Steuerfreiheit. Der Abschreibungsunterschied entsteht nicht durch zeitliche Buchungsunterschiede zwischen Handels- und Steuerrecht, im Posten sind keine aktivierungsfähigen latenten Steuern enthalten. Der Posten Abschreibungsunterschied könnte, seinem Charakter entsprechend in Eigenkapital und Fremdkapital aufgeteilt, auch als Rücklagen und Rückstellungen für künftige Steuerzahlungen aus Steuerstundung ausgewiesen werden ${ }^{925}$.

Auf die Umsetzung von Art. 35 und Art. 43 Abs. 1 Nr. 10 der 4. EG-Richtlinie in nationales Recht hat der finnische Gesetzgeber verzichtet. Anhangangaben zur steuerlich veranlassten Abschreibung seien nicht erforderlich, da die in der Richtlinie geforderten Informationen dem Jahresabschlussleser in Bilanz und Gewinnund Verlustrechnung gegeben würden ${ }^{926}$.

Trotz der Forderung nach Vereinheitlichung ist momentan nicht zu erwarten, dass sich Handels- und Steuerrecht einander annähern, sodass damit zu rechnen ist, dass der Abschreibungsunterschied auch künftig ein Merkmal finnischer Abschlüsse bleibt ${ }^{927}$.

924 Wieder anders stellt sich die Situation beispielsweise in Belgien dar, wo die steuerrechtlich veranlasste Abschreibung in der Gewinn- und Verlustrechnung sowie der Bilanz ausgewiesen wird. Der Unterschied zwischen steuerrechtlich veranlasster und handelsrechtlicher Abschreibung wird in Belgien gemäß Art. 43 Abs. 1 Nr. 19 der 4. EG-Richtlinie im Anhang angegeben.

927 Vgl. KPL-Arbeitsgruppe 1995, Teil I (1996), S. 192 und vgl. Räty (1998 c), S. 112. 


\subsection{Freiwillige Rückstellungen mit Rücklageanteil}

In KPL 5:15 räumt der finnische Gesetzgeber allen Buchführungspflichtigen folgendes Wahlrecht ein ${ }^{928}$ :

Freiwillige Rückstellung

Im Jahresabschluss dürfen Investitionsrückstellungen, Rückstellungen für allgemeine Geschäftsrisiken oder andere Rückstellungen dieser Art gebildet werden.

Traditionell sind freiwillige Rückstellungen in Finnland dadurch gekennzeichnet, dass sie weder durch eine Außenverpflichtung (eine Verpflichtung gegenüber Dritten) noch durch eine Innenverpflichtung (Verpflichtung des Bilanzierenden gegenüber sich selbst) begründet sind ${ }^{929}$. Sie werden vielmehr von der Geschäftsführung gebildet, um zu verhindern, dass die entsprechenden Beträge als Bestandteil des versteuerten Jahresüberschusses in das ausschüttungsfähige Eigenkapital fließen. Die Unternehmen verfolgen die Absicht, Geldmittel für künftige, noch nicht näher geplante Investitionen anzusparen oder sich vorsorglich Reserven für unvorhergesehene Ereignisse mit negativem Einfluss auf die Vermögens-, Finanzund Ertragslage zu schaffen ${ }^{930}$. Dem Charakter nach handelt es sich bei freiwilligen Rückstellungen um zweckgebundene Rücklagen.

Der finnische Gesetzgeber verfolgt mit KPL 5:15 die Intention, die Existenz der Unternehmen durch Innenfinanzierung zu sichern. Nach dem Zweiten Weltkrieg ist der Innenfinanzierung in Finnland eine hohe Bedeutung zugekommen, und in der Vergangenheit haben steuerrechtlich gewährte Möglichkeiten der Ergebnisminderung gerade den freiwilligen Rückstellungen eine hohe Akzeptanz in der Praxis verschafft ${ }^{931}$. Der Staat hatte u. a., um hohe Steuersätze bis 65 Prozent zu kompensieren, in den Jahren vor 1992 mit dem Wahlrecht wirtschafts- und sozialpolitische Ziele zu erreichen versucht: Die Sicherung der Unternehmensfortführung durch Reservenbildung sowie die Investitionsförderung zur Verbesserung der internationalen Konkurrenzfähigkeit und letztlich zum Erhalt und zur Schaffung von Arbeitsplätzen sollten gewährleistet werden ${ }^{932}$.

1992 wurden die Steuersätze jedoch auf 28 Prozent gesenkt und gleichzeitig die steuerliche Abzugsfähigkeit freiwilliger Rückstellungen eingeschränkt: Unternehmen nur noch weniger Rechtsformen ist es heute vorbehalten, Investitionsrück-

Übersetzung ins Deutsche durch die Verfasserin.

Vgl. Järvinen et al. (2000), S. 501-502 und vgl. Majala (2001 b), S. 977.

Vgl. Järvinen et al. (2000), S. 501, vgl. Artsberg (2000), S. 553, die ihre Aussage auf Skandinavien ausdehnt, und vgl. Majala (2001 b), S. 977.

931 Vgl. Kap. 2.1.2.2 und 2.1.3.2 dieser Arbeit.

932 Vgl. Artsberg (2000), S. 552 und vgl. Majala (2001 b), S. 977. 
stellungen und Rückstellungen für allgemeine Geschäftsrisiken steuerlich ergebnismindernd zu bilden ${ }^{933}$.

Freiwillige Rückstelllungen werden steuerlich nur dann akzeptiert, sofern sie auch im handelsrechtlichen Abschluss vermerkt sind ${ }^{934}$. Der finnische Gesetzgeber fordert aus Informationsgesichtspunkten, diese auf Grund steuerrechtlicher Vorschriften gebildeten Rückstellungen getrennt in der Bilanz sowie der Gewinn- und Verlustrechnung als ,freiwillige Rückstellungen“ auszuweisen, anstatt ihre Angabe und Begründung lediglich im Anhang zu verlangen ${ }^{935}$.

Die freiwilligen Rückstellungen sind als Mischposten anzusehen, wenn sie steuerlich ergebnismindernd wirken. Sie beinhalten neben dem rücklagenähnlichen Eigenkapitalanteil gleichzeitig einen Fremdkapitalanteil in Höhe der noch zu erwartenden Steuerbelastung bei Auflösung der Rückstellung, da aus der steuerlichen Abzugsfähigkeit keine Steuerfreiheit, sondern lediglich eine Steuerstundung resultiert. Insofern sind sie dem deutschen Sonderposten mit Rücklageanteil vergleichbar.

Kontrovers diskutiert wird im finnischen Fachschrifttum die Frage, ob es einem Unternehmen einer Rechtsform wie der Aktiengesellschaft, die nach Steuerrecht keine Möglichkeit zur Bildung freiwilliger Rückstellungen hat, gestattet sei, diesen Posten handelsrechtlich zu bilden. Befürworter dieser Position betrachten die freiwillige Rückstellung aus steuerlicher Sicht nicht als Mischposten mit Fremdkapitalanteil. Die Rückstellungen seien Teil der Ergebnisverwendung, inhaltlich nicht ausschüttungsfähigen, zweckgebundenen Rücklagen gleichzusetzen. Sie unterschieden sich von Gewinnrücklagen darin, dass sie durch die Geschäftsführung veranlasst seien und nicht auf einem Gesellschafterbeschluss beruhten. Aus handelsrechtlicher Sicht enthielten die freiwilligen Rückstellungen aber einen latenten Steueranteil, der aktivierungsfähig ist.

Die Regierungsbegründung zum Gesetzesentwurf des Buchführungsgesetzes von 1997 betont, dass es verboten sei, Kapitalbeträge freiwillig ohne steuerliche Veranlassung zurückzustellen. Dies entspricht der Forderung des Art. 42 der 4. EGRichtlinie, Rückstellungen nur in Höhe des notwendigen Betrags anzusetzen, und auch Art. 43 Abs. 10, wonach das Ausmaß der Ergebnisbeeinflussung steuerlicher Rückstellungen anzugeben ist. Der Berufsverband der KHT-Wirtschaftsprüfer ist der Ansicht, Aktiengesellschaften dürften keine freiwilligen Rückstellungen bil-

933 Vgl. EVL 46a, der diese Möglichkeit auf folgende Rechtsformen beschränkt: EinzelGewerbetreibende (,liikkeenharjoittaja“), Einzel-Unternehmer (,,ammatinharjoittaja“), Erbengemeinschaften mit ausschließlich natürlichen Personen als Erben (,kuolinpesä“) sowie Vereinigungen (,yhtymä“).

934 Vgl. EVL 54.2.

935 Vgl. KPL-Arbeitsgruppe 1995, Teil I (1996), S. 178-179. 
den. Die KPL-Arbeitsgruppe 1995 stimmt dieser Auffassung indirekt zu, indem sie in ihrem Bericht die Vermutung äußert, dass freiwillige Rückstellungen innerhalb weniger Jahre nach In-Kraft-Treten des Buchführungsgesetzes von 1997 aus den Bilanzen vieler Unternehmen verschwinden würden ${ }^{936}$. In der Praxis zeichnet sich ein entsprechender Trend $a b$, da Unternehmen kaum ein Interesse daran haben, Rückstellungen auf freiwilliger Basis zu bilden, die steuerlich nicht akzeptiert werden ${ }^{937}$.

Kritiker führen an, die Investitionsrückstellung und die Rückstellung für allgemeine Geschäftsrisiken entsprächen steuerrechtlicher Terminologie und seien nach Gewerbesteuerrecht nur gestattet, sofern sie auch handelsrechtlich vorgenommen würden. Der Gesetzgeber verweise in der Gesetzesbegründung zum Buchführungsgesetz zwar auf das Steuerrecht, gestatte aber in KPL 5:15 auch die Bildung von ,,anderen Rückstellungen dieser Art", die das Steuerrecht nicht kenne ${ }^{938}$. Zudem gelte das Buchführungsgesetz und der in ihm enthaltene KPL 5:15 für alle Buchführungspflichtigen. Ein Buchführungspflichtiger sei aber nicht automatisch auch steuerpflichtig. Es sei somit Unternehmen aller Rechtsformen erlaubt, freiwillige Rückstellungen und solche Rückstellungen zu bilden, die das Steuerrecht nicht vorsehe oder aber im Steuerrecht genannte Rückstellungen höher zu dotieren, als das Steuerrecht zulasse ${ }^{939}$. Vertreter dieser Auffassung sehen für alle Buchführungspflichtigen die Möglichkeit zur Bildung freiwilliger Rückstellungen für nicht definierte zukünftige Ereignisse als gegeben $a^{940}$. Teile der Praxis folgen dieser Auffassung bei der Erstellung ihrer Abschlüsse.

Für den Bilanzleser ist es von Bedeutung zu wissen, dass freiwillige Rückstellungen in Konzernabschlüssen - je nach Rechtsform der Konzernunternehmen - sowohl Eigenkapital- als auch Fremdkapitalcharakter aufweisen können und gemäß KPL 6:7 getrennt nach Eigenkapital und Fremdkapitalanteil ausgewiesen werden müssen. Treten sie im Einzelabschluss von Aktiengesellschaften auf, handelt es sich bei ihnen um Rücklagen mit aktivem latenten Steueranteil. 


\subsubsection{Pflichtrückstellungen}

\subsection{Ansatz, Bewertung und Ausweis}

Der Richtliniengeber konkretisiert das Vorsichtsprinzip in Art. 31 Abs. 1 (c) (bb) der 4. EG-Richtlinie dahingehend, dass alle voraussehbaren Risiken und zu vermutenden Verluste, die im Geschäftsjahr oder in früheren Geschäftsjahren entstanden sind, im Jahresabschluss zu berücksichtigen sind. Zusätzlich schreibt er in Art. 20 den Ansatz, in Art. 42 Abs. 1 die Bewertung von Rückstellungen vor. Der finnische Gesetzgeber hat die Intention verfolgt, diese Anforderungen der Richtlinie mit KPL 5:14 in nationales Recht aufzunehmen ${ }^{941}$ :

Zukünftige Aufwendungen und Verluste sowie Pflichtrückstellung

(1) Die Erträge sind um die in Zukunft aus Verbindlichkeiten hervorgehenden Ausgaben und Verluste zu mindern, wenn:

- diese das Geschäftsjahr oder frühere Geschäftsjahre betreffen,

- das Eintreten dieser Ausgaben und Verluste bei Erstellen des Jahresabschlusses als sicher oder wahrscheinlich gilt,

- diesen sicher oder wahrscheinlich keine Einnahme mehr zuzuordnen ist und

- $\quad$ diese auf dem Gesetz oder auf bindenden, vom Buchführungspflichtigen mit Dritten abgeschlossenen Verträgen beruhen.

(2) Von den Erträgen darf auch ein(e) in Zukunft aus einer Verbindlichkeit entstehende Ausgabe oder Verlust abgezogen werden, die bzw. der zweckgebunden ist und die Merkmale erfüllt, die in Abs. $1 \mathrm{Nr}$. 1 bis 3 geregelt sind.

(3) Wenn die genaue Höhe oder der Realisationszeitpunkt einer bzw. eines in Abs. 1 oder 2 gemeinten Ausgabe oder Verlusts nicht bekannt ist, wird die Ausgabe oder der Verlust in der Bilanz als Pflichtrückstellung erfasst.

(4) Die in den Abs. 1 bis 3 gemeinten Verluste und Ausgaben dürfen in Gewinnund Verlustrechnung und Bilanz höchstens mit ihrem wahrscheinlich eintretenden Betrag erfasst werden.

(5) In der Bilanz erfasste Wertberichtigungen der Aktiva dürfen nicht als Pflichtrückstellungen ausgewiesen werden.

KPL 5:14.1 enthält ein Passivierungsgebot für zukünftige Ausgaben und Verluste aus Außenverpflichtungen, deren Ursache im Geschäftsjahr oder früheren Geschäftsjahren liegt und mit denen keine Einnahmenerwartung verbunden ist. Dieses Gebot gilt auch dann, wenn Unsicherheit hinsichtlich Eintreten, Höhe und/oder Wahrscheinlichkeit der Ausgaben und Verluste besteht.

Die Verpflichtungen gegenüber Dritten können zum einen rechtlicher Natur sein, gesetzlich vorgeschrieben - wie die Entsorgung von Altlasten - oder vertraglich vereinbart $^{942}$. Rechtliche Verpflichtungen umfassen auch solche, die aus juristischer Sicht noch nicht eingetreten sind, deren Realisierung aber aus betriebswirtschaftlichem Blickwinkel als sicher oder wahrscheinlich gilt. Zu nennen sind bei-

941 Übersetzung ins Deutsche durch die Verfasserin.

942 Vgl. Räty (1998 b), S. 65, vgl. Luoma (1998), S. 232-233 und vgl. Järvinen et al. (2000), S. 492. 
spielsweise Abfindungszahlungen bei geplanter Restrukturierung, das Einstehen für gegebene Bürgschaften oder sonstige Sicherheiten ${ }^{943}$. Zum anderen können die Verpflichtungen auch wirtschaftlich veranlasst sein, wenn die Allgemeinheit und speziell die Geschäftspartner des Unternehmens eine Leistung erwarten, die erfüllt werden muss, um Image- und Vertrauensverlust abzuwenden: Kulanzzahlungen bei Reklamationen oder Leistungen für den Umweltschutz dort, wo keine öffentlich-rechtliche, sondern eine moralische Verpflichtung vorliegt ${ }^{944}$.

Mit dem Buchführungsgesetz von 1997 wird in KPL 5:14.2 erstmals das Passivierungswahlrecht für künftige Ausgaben oder Verluste eingeräumt, die zweckgebunden sind und auf einer Innenverpflichtung beruhen ${ }^{945}$. Hierzu zählen beispielsweise unterlassene Instandhaltung oder unterlassene Abbruchvorhaben ${ }^{946}$. Betont wird vom Fachschrifttum die notwendige Zweckgebundenheit der Aufwandsrückstellungen: Hier kommt allenfalls eine freiwillige, rücklagenähnliche Rückstellung in Betracht ${ }^{947}$. Es liegt im Ermessen des Unternehmens, eine Verpflichtung gegenüber sich selbst alternativ zur Passivierung in der Bilanz im Anhang anzugeben ${ }^{948}$. Die Art guter Buchführung verlangt hier, das Ansatzwahlrecht bei analogen Sachverhalten einheitlich und stetig auszuüben. JÄRVINEN empfiehlt die Passivierung für den Fall, dass wahrscheinlich oder sicher keine künftige Einnahmen mehr mit dieses oder frühere Geschäftsjahre betreffenden Ausgaben bzw. Verlusten verbunden sind ${ }^{949}$.

Sowohl bei Außen- als auch bei Innenverpflichtungen erweist sich die Zuordnung der erwarteten Ausgaben zu erwarteten Einnahmen bei Ermittlung voraussehbarer Risiken oder vermuteten Verlusten als problematisch. Wenn die Ausgaben die Einnahmen übersteigen, müssen bzw. können sie antizipiert werden - andernfalls dürfen sie keinesfalls vorweggenommen werden.

Fraglich ist, ob hier von Ausgaben bzw. Einnahmen einzelner Sachverhalte, von der Cash-generating unit, von Betriebszweigen oder vom Gesamtunternehmen auszugehen ist.

Für die Praxis am Schwierigsten anzuwenden ist die einzelfallbezogene Betrachtung von Ausgaben und Einnahmen. Sie kann aber gerade im Hinblick auf erwartete außerordentliche Aufwendungen angebracht sein.

943 Vgl. Luoma (1998), S. 237, vgl. Leppiniemi (2000 a), S. 313 und vgl. Leppiniemi und Leppiniemi (1999), S. 115.

944 Vgl. Luoma (1998), S. 236-237, vgl. Leppiniemi (2000 a), S. 313 und vgl. Räty (1998 b), S. 66 .

945 Vgl. Räty (1998 b), S. 65 und vgl. Järvinen et al. (2000), S. 494.

946 Vgl. Luoma (1998), S. 237 und vgl. Räty (1998 b), S. 66.

947 Es darf keine allgemeine Verlustrückstellung gebildet werden, wenn für das kommende Geschäftsjahr ein negatives Jahresergebnis budgetiert ist. Vgl. Luoma (1998), S. 241 und vgl. Järvinen et al. (2000), S. 493. Vgl. auch Kap. 3.2.5.1.2 dieser Arbeit. Vgl. Luoma (1998), S. 233.

949 Vgl. Järvinen et al. (2000), S. 495. 
Die Betrachtung von kundenbezogenen Aufträgen, von Cash-generating units und von Betriebszweigen basierend auf einer aussagefähigen Deckungsbeitragsrechnung findet in der Praxis breite Anwendung.

Bei der bilanziellen Antizipation von Ausgaben und Verlusten ausschließlich vom Gesamtunternehmen auszugehen, ist weder im Sinne des Richtliniengebers noch des finnischen Gesetzgebers. Das Prinzip der Unternehmensfortführung beinhaltet diese Vorgehensweise, sodass die ausschließliche Gesamtunternehmensbetrachtung bei der Rückstellungsbildung nach einhelliger Meinung der Fachwelt abzulehnen ist ${ }^{950}$.

Bilanziert das Unternehmen einen Sachverhalt auf Grund einer Außen- oder Innenverpflichtung, stellt sich die Frage, wo dieser Betrag auszuweisen ist. Bei Unsicherheit hinsichtlich der Eintrittshöhe und/oder des Realisationszeitpunkts ist der Betrag nach KPL 5:14.3 immer den Pflichtrückstellungen zuzuordnen ${ }^{951}$. Sind Betrag und Realisationszeitpunkt bekannt und besteht lediglich Unsicherheit über den Eintritt einer Verpflichtung, so ist die Verpflichtung als Rechnungsabgrenzungsposten oder sonstige Verbindlichkeit auszuweisen, nicht als Rückstellung ${ }^{952}$.

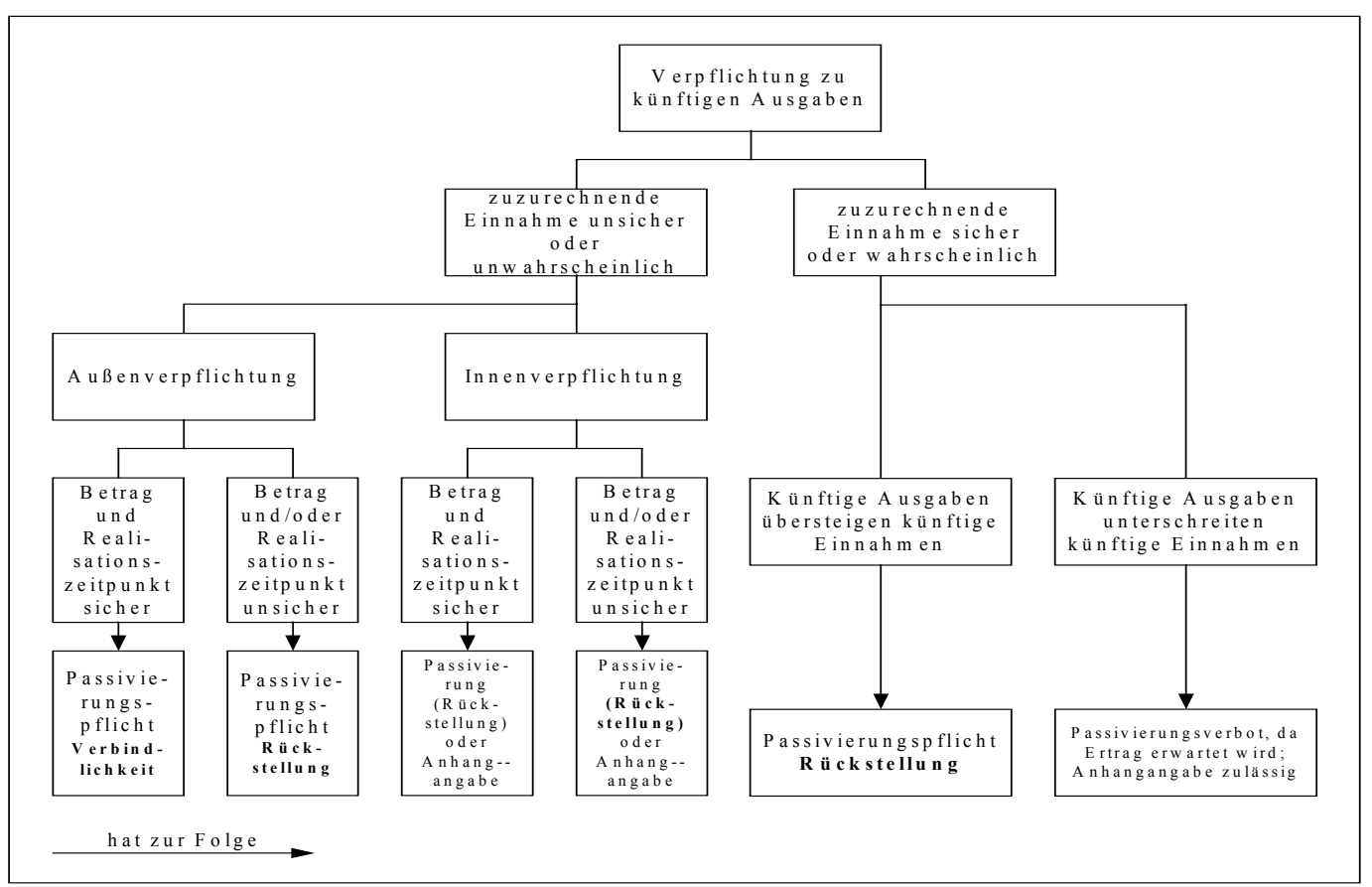

\section{Abb. 9: $\quad$ Abgrenzung von Rückstellungen, Verbindlichkeiten und Even- tualverbindlichkeiten}

950 Vgl. Järvinen et al. (2000), S. 493-494.

951 Vgl. Leppiniemi und Leppiniemi (1999), S. 115, vgl. Majala (2001 b), S. 977, vgl. Luoma (1998), S. 233 und vgl. Järvinen et al. (2000), S. 495.

952 Vgl. Leppiniemi und Leppiniemi (1999), S. 115, vgl. Järvinen et al. (2000), S. 495 und vgl. Luoma (1998), S. 234. Die KPL-Arbeitsgruppe 1995 hat in ihrem Bericht ursprünglich gefordert, erwartete Ausgaben und Verluste nur dann als Verbindlichkeiten auszuweisen, wenn in Bezug auf Eintritt, Höhe und Eintrittsdatum Sicherheit herrsche; bei Unsicherheit hinsichtlich des Eintritts solle eine Rückstellung ausgewiesen werden, selbst wenn Höhe und Eintrittsdatum eindeutig seien. Vgl. hierzu KPL-Arbeitsgruppe 1995, Teil I (1996), S. 66-67 und vgl. Räty (1998 b), S. 64. 
In KPL 5:14.4 fordert der finnische Gesetzgeber in Übereinstimmung mit Art. 42 Abs. 1 der 4. EG-Richtlinie, antizipierte Ausgaben und Verluste höchstens mit ihrem wahrscheinlichen Betrag anzusetzen. Das Vorsichtsprinzip darf nicht überinterpretiert werden, da es sonst bei Bildung und Auflösung der Rückstellung zu einer Verfälschung der Jahresabschlussaussage und des True and Fair View kommen kann. Weder der Gesetzgeber noch der KILA geben vor, nach welcher Methode antizipierte Ausgaben und Verluste zu bewerten sind. Fragen nach der Einbeziehung von Gemeinkosten in Rückstellungen, nach der Einbeziehung künftiger Preissteigerung, nach der Abzinsung der zu antizipierenden Ausgaben oder der Saldierung von künftigen positiven und negativen Erfolgsbeiträgen sind bislang wenig diskutiert worden. Erlaubt ist, was mit der Art guter Buchführung vereinbar ist. Gefordert wird teilweise eine seriöse Schätzung, wie sie Bestandteil jeder Jahresabschlusserstellung ist. Die Ermittlung des Unterschiedsbetrags zwischen künftigen erwarteten Einnahmen und ihnen zuzuordnenden höheren Ausgaben bzw. Verlusten setzt neben Kenntnis der Branche und der branchenspezifischen Gesetzgebung, Erfahrung, Analysefähigkeit und eine funktionierende Kostenrechnung voraus ${ }^{953}$. Mangels nationaler, finnischer Bewertungsregeln ist eine Möglichkeit, die Bemessung der Rückstellung auf Grundlage der Wahrscheinlichkeitsrechnung nach „Best Estimate“, wie sie das IAS Steering Committee vorstellt, durchzuführen und für die Zukunft die Diskussionen um den IAS 10 (reformatted 1994) und den IAS 37 (approved 1998) zu verfolgen ${ }^{954}$.

Mit KPL 5:14.5 hat der Gesetzgeber Art. 20 Abs. 3 der 4. EG-Richtlinie kodifiziert. Die Vorschrift, dass Rückstellungen keine Wertberichtigung zu Aktivposten darstellen dürfen, ist mit dem Buchführungsgesetz von 1997 neu ins Gesetz aufgenommen worden, aber schon früher allgemein anerkannter Bestandteil der Art guter Buchführung gewesen. Die Regelung wird als überflüssig angesehen, da Wertberichtigungen in Form von Abschreibungen und außerplanmäßigen Abschreibungen nicht die Merkmale von Pflichtrückstellungen erfüllen, weil es sich bei ihnen nicht um künftige, sondern um bereits realisierte Ausgaben oder Verluste handele ${ }^{955}$.

In der Bilanz sind folgende Pflichtrückstellungen nach KPA 1:6 gesondert auszuweisen:

- Pensionsrückstellungen

- Steuerrückstellungen

- sonstige Rückstellungen

953 Vgl. Järvinen et al. (2000), S. 496.

954 Vgl. Luoma (1998), S. 241.

955 Vgl. KPL-Arbeitsgruppe 1995, Teil I (1996), S. 69 und vgl. Järvinen et al. (2000), S. 497. 
Passive Rechnungsabgrenzungsposten und sonstige Verbindlichkeiten werden innerhalb der Verbindlichkeiten getrennt aufgeführt.

\subsection{Pensionsrückstellungen}

Der wesentliche Teil der Pensionszahlungen erfolgt in Finnland durch das staatliche Sozialsystem, potenzielle Pensionszahlungen stellen für die meisten Unternehmen keine wesentliche Größe dar. Sofern zwischen einem Unternehmen und seinem Mitarbeiter Einzelvereinbarungen getroffen werden, sind diese von den Unternehmen meist durch entsprechende Versicherungsverträge abgesichert ${ }^{956}$. Existierende unmittelbare wie mittelbare Pensionsverpflichtungen konnten vor 1992 trotz Vorsichtsprinzips als Eventualverbindlichkeiten betrachtet und im Anhang ausgewiesen werden, damit bestand Passivierungswahlrecht. Dieser unvollständige Ansatz von Schulden wurde damit begründet, dass ein Passivierungsgebot Unternehmen davon abhalten könnte, Einzel-Pensionszusagen zu gewähren.

Seit dem Buchführungsgesetz 1992 besteht für sämtliche Pensionszusagen, die nach dem 31. Dezember 1992 eingegangen worden sind, eine Passivierungspflicht. Vorher eingegangene Pensionszusagen waren bis 2001 zwingend erfolgswirksam nachzuholen. Der Buchführungspflichtige hat hierbei entscheiden können, zu welchem Zeitpunkt der Übergangsphase und nach welcher Methode - in einer Summe oder sukzessiv - er die fehlenden Beträge nachgebucht hat ${ }^{957}$.

Heute werden somit alle gewährten Pensionszusagen bilanziell erfasst. Ihr Ausweis erfolgt unter Pflichtrückstellungen, obwohl es sich dem Charakter nach um Verbindlichkeiten handelt, für die eine Gegenleistung bereits erbracht worden ist. $^{958}$

Zur Bewertung existieren keine vom Gesetzgeber oder vom KILA vorgeschriebenen Methoden; Aufschluss geben die Anhangangaben der Unternehmen.

\subsection{Steuerrückstellungen}

Die Rückstellung für Steuern umfasst die tatsächlichen, nicht die latenten Steuern. Bei den tatsächlichen Steuern handelt es sich um den Betrag der geschuldeten oder erstattungsfähigen Ertragsteuern, der aus dem zu versteuernden Einkommen oder dem steuerlichen Verlust der Berichtsperiode resultiert. Dieser Betrag ist

956 Vgl. Artsberg (2000), S. 555-556, die ihre Aussage auf alle Länder Skandinaviens bezieht.

957 Vgl. HE 111/1992 und vgl. KPL-Arbeitsgruppe 1995, Teil I (1996), S. 66-67.

958 Vgl. Järvinen et al. (2000), S. 498. 
unter Berücksichtigung der am Abschluss-Stichtag gültigen Steuervorschriften und Steuersätze zu ermitteln. Der Unterschiedsbetrag zwischen den Steuern des Geschäftsjahrs und den geleisteten Steuervorauszahlungen ist unter aktiver Rechnungsabgrenzung und sonstigen Vermögensgegenständen bzw. unter passiver Rechnungsabgrenzung und sonstigen Verbindlichkeiten auszuweisen ${ }^{959}$.

Die Steuerrückstellung beinhaltet lediglich den Teil der das Geschäftsjahr oder frühere Geschäftsjahre betreffenden tatsächlichen Steuern, deren Höhe oder Realisationszeitpunkt nicht sicher ist. Hierbei handelt es sich um Rückstellungen für wahrscheinlich zu leistende Steuernachzahlungen aus Betriebsprüfung oder aus Rechtsstreitigkeiten mit der Finanzverwaltung ${ }^{960}$.

\subsubsection{Verbindlichkeiten}

\subsection{Ansatz, Bewertung und Ausweis}

Eine Verbindlichkeit liegt vor, wenn

- eine Verpflichtung gegenüber Dritten besteht, der sich der Bilanzierende aus rechtlichen Gründen nicht entziehen kann,

- mit der Verpflichtung eine wirtschaftliche Belastung für das bilanzierende Unternehmen verbunden ist und

- die Höhe der wirtschaftlichen Belastung exakt bemessen werden kann und ihr Realisationszeitpunkt bekannt ist ${ }^{961}$.

Konkretisiert wird ein Teil der Verbindlichkeiten durch den in KPL 3:3.1 Nr. 5 kodifizierten Grundsatz der leistungsbezogenen Abgrenzung. KPL 2:3 ermöglicht unterjährig die zahlungsabhängige Buchung von Geschäftsvorfällen. KPL 3:4 i. V. m. KPL 3:3.1 Nr. 5 fordert von den Bilanzierenden, zahlungsabhängige Buchungen vor Jahresabschlusserstellung zu korrigieren, um das Prinzip der leistungsbezogenen Abgrenzung für den Jahresabschluss einzuhalten. Im Geschäftsjahr angefallene Ausgaben - also Aufwand oder zu aktivierende Beträge - die wegen fehlender Zahlung noch nicht in der Buchhaltung erfasst sind, sind als Verbindlichkeit zu passivieren. Erhaltene Anzahlungen, für die der Buchführungspflichtige noch keine Gegenleistung erbracht hat, sind ebenfalls den Verbindlichkeiten zuzuordnen. Neben diesen Verbindlichkeiten existieren Verbindlichkeiten aus der Finanzierung der Geschäftstätigkeit, wie aufgenommene Darlehen oder Wechselobligationen. Auch sie sind nach Passivierungsgrundsatz und KPL 5:14 zu passivieren.

959 Vgl. Leppiniemi (2000 a), S. 312 und Järvinen et al. (2000), S. 498.

960 Vgl. KPL-Arbeitsgruppe 1995, Teil I (1996), S. 191-192.

961 Vgl. Järvinen et al. (2000), S. 407-408 und S. 431 sowie Majala (2001), S. 975. 
Verbindlichkeiten werden mit ihrem Nennwert in der Bilanz angesetzt. Sind sie an einen Index oder einen sonstigen Vergleichswert gebunden, so sind sie mit dem auf dem Vergleichswert beruhenden höheren Wert als dem Nennwert zu bewerten $^{962}$.

Verbindlichkeiten müssen nach KPL 4:7 ihrer Laufzeit entsprechend unterteilt werden in kurzfristige - kürzer als ein Jahr - und langfristige Verbindlichkeiten, die erst nach Ablauf eines Jahres nach Stichtag fällig werden.

Nach dem Bilanzgliederungsschema des KPA 1:6 sind in Übereinstimmung mit Art. 9 der 4. EG-Richtlinie folgende Posten getrennt auszuweisen:

- Schuldverschreibungen

- Wandelschuldverschreibungen

- Verbindlichkeiten gegenüber Kreditinstituten

- Verbindlichkeiten aus der Rückbeleihung von Pensionsversicherungsbeiträgen

- Erhaltene Anzahlungen

- Verbindlichkeiten aus Lieferungen und Leistungen

- Wechselverbindlichkeiten

- Verbindlichkeiten gegenüber verbundenen Unternehmen

- Verbindlichkeiten gegenüber Beteiligungsunternehmen

- sonstige Verbindlichkeiten

- passive Rechnungsabgrenzungsposten

Nimmt ein Unternehmen ein Darlehen in Form einer Schuldverschreibung - Anleihe, Schuldanerkenntnisschein oder Einlagenzertifikat - auf, verpflichtet es sich zur Zahlung einer bestimmten Geldsumme an den Eigentümer der Schuldverschreibung ${ }^{963}$.

Wandelschuldverschreibungen berechtigen ihren Eigentümer nach OYL 4:1.1 dazu, sie bei einer Aktienemission ganz oder teilweise in Aktien umzutauschen.

Verbindlichkeiten gegenüber Kreditinstituten beinhalten bei Banken, Versicherungen oder Pensionsversicherungsträgern aufgenommene Darlehen. Bereits geleistete Einzahlungen in die Pensionsversicherung, die der Bilanzierende zurückleiht, sind unter Verbindlichkeiten aus der Rückbeleihung von Pensionsversiche-

962 Vgl. KPL 5:2.3.

963 Vgl. KILA:n kuntajaoston tilikarttamalli 16.10.1996. 
$\overline{\text { rungsbeiträgen auszuweisen }}{ }^{964}$. Sie sind nicht gleichzusetzen mit Pensionsrückstellungen $^{965}$.

Erhaltene Anzahlungen betreffen Anzahlungen auf Bestellungen für Gegenstände des Anlagevermögens und des Umlaufvermögens. Bei ihnen handelt es sich um Vorauszahlungen auf typische Leistungsangebote im Rahmen der gewöhnlichen Geschäftstätigkeit. Andere erhaltene Vorauszahlungen sind im Regelfall als Rechnungsabgrenzungsposten auszuweisen ${ }^{966}$.

Verbindlichkeiten aus Lieferungen und Leistungen werden im Nebenbuch geführt. Zu ihnen zählen beispielsweise Verpflichtungen aus dem Kauf von Rohstoffen, Waren und Dienstleistungen ${ }^{967}$.

$\mathrm{Zu}$ Wechselverbindlichkeiten zählen nicht abgezinste, aus der Finanzierungstätigkeit des Buchführungspflichtigen rührende Wechsel, die der Buchführungspflichtige selbst auslösen muss ${ }^{968}$.

\subsection{Sonstige Verbindlichkeiten und passiver Rechnungsabgrenzungs- posten}

Besteht Unsicherheit über den Eintritt einer Verbindlichkeit, so ist diese als ,,sonstige Verbindlichkeit“ oder als „Rechnungsabgrenzungsposten“ auszuweisen ${ }^{969}$. Der KILA hat in den Gutachten 1219/1993 und 1561/1999 den Charakter von sonstigen Verbindlichkeiten und passiven Rechnungsabgrenzungsposten erläutert und diese Begriffe voneinander abgegrenzt ${ }^{970}$.

Der wesentliche Unterschied im Charakter zwischen sonstigen Verbindlichkeiten einerseits und passiven Rechnungsabgrenzungsposten andererseits liegt demanch darin, dass erstere immer auf Basis von Rechnungen oder ähnlicher Dokumentation externer Dritter beruhen, letztere jedoch auch auf interner Kalkulation basieren können. Da die Grenzen zwischen den genannten Posten oft fließend sind, wird

964 Vgl. KILA 1219/1993.

965 Vgl. HE 111/1992.

966 Vgl. KILA 1219/1993.

967 Vgl. KILA 1219/1993.

968 Vgl. KILA 1219/1993.

969 Die KPL-Arbeitsgruppe 1995 hat in ihrem Bericht ursprünglich gefordert, erwartete Ausgaben und Verluste nur dann als Verbindlichkeiten auszuweisen, wenn in Bezug auf Eintritt, Höhe und Eintrittsdatum Sicherheit herrsche; bei Unsicherheit hinsichtlich des Eintritts solle eine Rückstellung ausgewiesen werden, selbst wenn Höhe und Eintrittsdatum eindeutig seien. Vgl. hierzu KPL-Arbeitsgruppe 1995, Teil I (1996), S. 66-67 und vgl. Räty (1998 b), S. 64.

970 Er ist hierbei ausgegangen von der englischsprachigen Version der 4. EG-Richtlinie, die unterscheidet in ,other creditors including tax and social security“ sowie ,accruals and deferred income". 
im finnischen Fachschrifttum der Umstand begrüßt, dass der finnische Gesetzgeber das Ausweiswahlrecht des Art. 9 der 4. EG-Richtlinie dahingehend ausgeübt hat, passive Rechnungsabgrenzungsposten als Bestandteil der Verbindlichkeiten und nicht als gesonderten Posten zu zeigen ${ }^{971}$.

Verbindlichkeiten aus Steuern und Verbindlichkeiten im Rahmen der Sozialen Sicherheit sind immer als sonstige Verbindlichkeiten auszuweisen, auch dann, wenn es sich bei ihnen dem Charakter nach um passive Rechnungsabgrenzungsposten handelt. Begründet wird dies vom Ministerium für Handel und Industrie mit dem in Art. 9 der 4. EG-Richtlinie geforderten Davon-Vermerk solcher Verbindlichkeiten unter den sonstigen Verbindlichkeiten ${ }^{972}$.

Passive Rechnungsabgrenzungsposten definiert der finnische Gesetzgeber zur Umsetzung von Art. 21 der 4. EG-Richtlinie in KPL 4:6.2 wie folgt ${ }^{973}$ :

Zum passiven Rechnungsabgrenzungsposten gehören:

Im Geschäftsjahr oder in früheren Geschäftsjahren erhaltene Anzahlungen für solche Einnahmen, die erst in zukünftigen Geschäftsjahren auf Basis des Grundsatzes der leistungsabhängigen Buchführung realisiert werden, sofern sie nicht als erhaltene Anzahlungen auszuweisen sind;

solche auf Basis des Grundsatzes leistungsabhängiger Buchführung realisierte Ausgaben, für die noch keine Auszahlung erfolgt ist, sofern sie nicht den Verbindlichkeiten aus Lieferungen und Leistungen zuzuordnen sind;

zukünftige Ausgaben und Verluste, sofern sie nicht den in KPL 5:14.3 definierten Pflichtrückstellungen zuzuordnen sind oder direkt von den Bilanzposten abzuziehen sind, in denen der ihnen zu Grunde liegende Sachverhalt enthalten ist.

Passive Rechnungsabgrenzungsposten umfassen die zu passivierenden Ausgaben, die nicht zu den Verbindlichkeiten aus Lieferungen und Leistungen zählen, und die zu passivierenden Einnahmen, die nicht zu den erhaltenen Anzahlungen gehören (Ausschlussprinzip). Passive Rechnungsabgrenzung kann auf Außen- oder Innenverpflichtung beruhen und erfüllt folgende Merkmale ${ }^{974}$ :

- Sie betrifft das Geschäftsjahr oder frühere Geschäftsjahre,

- ihre Realisierung gilt bei Jahresabschlusserstellung als sicher oder wahrscheinlich,

- eine ihr zuzuordnende Einnahme ist weder sicher noch wahrscheinlich,

- sie beruht auf einer gesetzlichen oder vertraglichen Verpflichtung,

- ihre exakte Höhe und ihr genauer Realisationszeitpunkt sind bekannt.

Analog zum aktiven Rechnungsabgrenzungsposten umfasst der passive Rechnungsabgrenzungsposten in Finnland transitorische und antizipative Rechnungsabgrenzungen.

\footnotetext{
971 Vgl. Järvinen et al. (2000), S. 408.

972 Vgl. KTM 20. Dezember 1997, S. 10.

973 Übersetzung ins Deutsche durch die Verfasserin.

974 Vgl. Järvinen et al. (2000), S. 408.
} 
Bei den transitorischen Rechnungabgrenzungen handelt es sich um Einnahmen der abgelaufenen Periode, die einen Ertrag für eine bestimmte Zeit nach dem Abschluss-Stichtag darstellen ${ }^{975}$. Antizipative Rechnungsabgrenzungen bezeichnen bestimmte Aufwendungen für eine bestimmte Zeit nach der abgelaufenen Periode, die erst nach dem Abschluss-Stichtag zu einer Ausgabe führen ${ }^{976}$. Diese antizipativen Rechnungsabgrenzungen stellen ihrem Charakter nach Verbindlichkeiten dar: Es liegt eine Verpflichtung gegenüber Dritten vor, die mit einer wirtschaftlichen Belastung verbunden ist und deren Höhe sowie Realisationszeitpunkt bekannt sind. Ihre Besonderheit besteht darin, dass der Vertragspartner seine Gegenleistung kontinuierlich über einen Zeitraum erbringt, während die eigene Leistung erst erbracht wird, wenn die zeitraumbezogene Gegenleistung vollständig erfüllt ist $^{977}$. Beispielhaft zu nennen sind hier noch nicht gezahlte Lohn- und Gehaltsbestandteile des Geschäftsjahrs (Überstunden, Urlaubsgeld, Tantiemen sowie die zugehörigen Abgaben an Sozialversicherungsträger, Finanzamt und Versicherungen) sowie zu leistende Boni, Skonti und Rabatte des Geschäftsjahres ${ }^{978}$.

\subsubsection{Derivative Finanzinstrumente}

Weder der Richtliniengeber noch der finnische Gesetzgeber regeln explizit, wie ein Unternehmen einen von ihm eingegangen Derivatikontrakt ${ }^{979}$ im Jahresabschluss zu behandeln hat.

Es hat sich als Art guter Buchführung herausgebildet, Derivate nicht bilanziell zu erfassen. Sie werden als schwebende Geschäfte betrachtet, die nicht bilanzierungsfähig sind, gleichgültig, ob sie am Stichtag einen finanziellen Vermögenswert oder eine finanzielle Verbindlichkeit darstellen ${ }^{980}$. Das finnische Finanzministerium schreibt börsennotierten Aktiengesellschaften jedoch vor, aus Derivaten resultierende Zahlungsverpflichtungen und die mit den Derivaten verfolgten Zwecke $^{981}$ im Anhang als Eventualverbindlichkeiten so anzugeben, dass ein den Ver-

Vgl. Baetge, Kirsch und Thiele (2001), S. 464.

Vgl. Baetge, Kirsch und Thiele (2001), S. 336.

Vgl. Baetge, Kirsch und Thiele (2001), S. 336.

Vgl. KILA 1219/1993.

979 Unter einem Derivativkontrakt wird ein Finanzinstrument verstanden oder eine andere vertragliche Vereinbarung mit zwei charakteristischen Merkmalen: ein oder mehrere ,underlyings" (Marktpreis, Index, Wechselkurs, Rating) und ein oder mehrere Nennbeträge bzw. feste oder variable Ausgleichszahlungen. Derivative Finanzinstrumente erzeugen Rechte und Pflichten, sodass Finanzrisiken, die in den zu Grunde liegenden originären Finanzinstrumenten enthalten sind, als separate Rechte und Pflichten zwischen den Vertragsparteien übertragen werden können. Zu Derivativkontrakten zählen beispielsweise Optionsgeschäfte, Futures, Forwards, Zinsswaps und Währungsswaps.

Anders zeigt sich die Situation in Deutschland, wo Derivate zwar grundsätzlich als nicht bilanzierungsfähige schwebende Geschäfte angesehen werden, ein aus ihnen drohender Verlust jedoch bilanziell zurückzustellen ist ( $\$ 252$ Abs. 1 S. 4 HGB).

981 Mit derivativen Finanzinstrumenten können drei Zwecke verfolgt werden: 
trägen gerecht werdender True and Fair View vermittelt wird ${ }^{982}$. Diese Anhangangabe ist zwar nur von börsennotierten Aktiengesellschaften zwingend zu leisten, dennoch informieren viele andere Unternehmen freiwillig auf Basis des Grundsatzes der Vollständigkeit und der Art guter Buchführung im Anhang über von ihnen abgeschlossene derivative Finanzinstrumente.

Hinsichtlich der Bilanzierung von am Abschluss-Stichtag nicht realisierten Währungsdifferenzen aus Derivaten hat der Buchführungspflichtige in Finnland gemäß KPL 5:3 drei Alternativen ${ }^{983}$ :

- Währungsdifferenzen aus Derivaten werden erst in der Periode ihrer Realisierung buchhalterisch erfasst ${ }^{984}$.

- Eine aus einem Derivat entstehende Forderung oder Verbindlichkeit wird mit vertraglich vereinbartem Kurs umgerechnet und so im Anhang vermerkt. Ein Währungsverlust gegenüber dem Stichtagskurs wird auf Grund des Vorsichtsprinzips erfolgswirksam erfasst, ein Währungsgewinn hingegen nicht realisiert. Wird das Derivat als Position derart geschlossen, dass sich Wechselkursänderungen nach der Schließung nicht mehr ergebniswirksam auswirken können, ist die Währungsdifferenz in der Periode der Schließung erfolgswirksam zu buchen ${ }^{985}$.

- Eine aus einem Derivat resultierende Forderung oder Verbindlichkeit wird mit Stichtagskurs umgerechnet und zum so ermittelten Wert im Anhang ausgewiesen. Eine sich aus Unterschieden zwischen Stichtags- und vertraglich vereinbarten Währungskurs ergebende Währungsdifferenz wird am Bilanz-Stichtag als Rechnungsabgrenzungsposten erfasst, der über die Restlaufzeit des Derivativkontrakts ratierlich aufgelöst wird ${ }^{986}$.

In der KILA-Anweisung „Währungsumrechnung“ vom 15. November 1999 wird empfohlen, sich in Zukunft am IAS $39 \mathrm{zu}$ orientieren und die weitere künftige Entwicklung der IAS zu beobachten. Es kann erwartet werden, dass sich die Art guter Buchführung in Finnland dahingehend wandelt, dass IAS 39 mit seinen Ansatz- und Bewertungsvorschriften bei der Behandlung von derivativen Finanzinstrumenten im Jahresabschluss angewendet wird ${ }^{987}$. Demnach würden alle Deriva-

- Handelszweck: Ein Finanzinstrument wird zu dem Zweck gehalten, Gewinn auf Grund von kurzfristigen Marktwertänderungen zu erzielen (vgl. IAS 39.10).

- Held-to-maturity-investment: Ein solches Finanzinstrument mit festen bzw. im Voraus bestimmbaren Zahlungsströmen und gegebener Endfälligkeit soll bis zur Endfälligkeit gehalten werden (vgl. IAS 39.10).

- Sicherungsinstrument: Ein Finanzinstrument wird beispielsweise eingesetzt, um Kursrisiken abzusichern (vgl. IAS 39.10).

Vgl. Järvinen et al. (2000), S. 439, die darauf hinweisen, dass diese Alternative von der Regierung in der Vorlage HE 111/1992 favorisiert wird.

Vgl. Tikka (1999), S. 106. 
te zum Zeitpunkt ihres Vertragsabschlusses buchhalterisch erfasst, es sei denn, ihr Marktwert beläuft sich auf Null ${ }^{988}$.

\subsubsection{Latente Steuerabgrenzung}

Werden erfolgswirksame Sachverhalte auf Grund sich unterscheidender Vorschriften nach Buchführungsgesetz im Jahresabschluss anders erfasst als nach Steuerrecht, weichen das in der Handelsbilanz ausgewiesene Jahresergebnis und das zu versteuernde Jahresergebnis voneinander $\mathrm{ab}$. Bemessungsgrundlage für die $\mathrm{zu}$ leistende Steuerzahlung ist das $\mathrm{zu}$ versteuernde Ergebnis: Unterscheidet sich das zu versteuernde Ergebnis vom handelsbilanziellen Jahresergebnis, weicht die tatsächliche Steuerbelastung von der theoretischen Besteuerung des handelsrechtlichen Ergebnisses (fiktive Steuerbelastung) ab.

Fraglich ist, ob in der handelsbilanziellen Gewinn- und Verlustrechnung unter dem Posten „Steuern vom Ertrag“ die tatsächliche oder fiktive Steuerbelastung gezeigt werden soll. Der finnische Gesetzgeber regelt dieses Problem nicht, sondern überlässt seine Lösung dem allgemeinen Verständnis der Art guter Buchführung. Der KILA hat in 1083/1990 auf Basis des Buchführungsgesetzes von 1973 empfohlen, die tatsächliche Steuerbelastung in der Gewinn- und Verlustrechnung auszuweisen, da diese Vorgehensweise dem Prinzip leistungsabhängiger Buchführung und somit der Art guter Buchführung entspreche. Diese Empfehlung basiert auf der Ausgaben-Einnahmen-Theorie, in der Steuern als Teil der Gewinnverwendung angesehen werden - daher die Konformität mit dem Grundsatz leistungsabhängiger Buchführung. Heute ist es jedoch alternativ auch möglich, die fiktive Steuerbelastung in der Gewinn- und Verlustrechnung zu erfassen, da dieses Vorgehen dem heutigen Verständnis des Grundsatzes leistungsabhängiger Buchführung - Steuerzahlungen werden als Aufwand angesehen, denen die zugehörigen Einnahmen gegenübergestellt werden - entspricht ${ }^{989}$. Die gewählte Variante ist im Anhang anzugeben ${ }^{990}$. In der Literatur wird teilweise gefordert, zum verbesserten Einblick in die Ertragslage die Zeile „Steuern vom Ertrag“ in der Gewinn- und Verlustrechnung je nach Vorgehensweise des Unternehmens umzubenennen in „Steuern vom Ertrag, basierend auf zu versteuerndem Einkommen“ bzw. „Steuern vom Ertrag, basierend auf handelsrechtlichem Jahresergebnis“9991.

Weist ein Unternehmen in seiner Gewinn- und Verlustrechnung - wie allgemein üblich - die tatsächliche Steuerbelastung aus, sieht der finnische Gesetzgeber seit 1997 für die Differenz zwischen tatsächlicher und fiktiver Steuerzahlung unter

\footnotetext{
$988 \quad$ Vgl. IAS 39.27.

989 Vgl. KPL-Arbeitsgruppe 1995, Teil I (1996), S. 131 und vgl. Räty (1998 c), S. 121-122.

990 Vgl. KPA 2:2.1.

991 Vgl. Järvinen et al. (2000), S. 516.
} 
bestimmten Voraussetzungen die Möglichkeit zur Bildung eines Steuerabgrenzungspostens vor. Auf latente Steuern ist KPL 5:18 anzuwenden, mit dem der finnische Gesetzgeber Art. 43 Abs. 1 (11) der 4. EG-Richtlinie in nationales Recht umgesetzt hat ${ }^{992}$ :

Aus Periodisierungsunterschieden resultierende latente Steuerverbindlichkeiten und -forderungen

\begin{abstract}
Aus Periodisierungsunterschieden zwischen Erträgen nach Buchführungsgesetz und ihren entsprechenden zu versteuernden Einnahmen sowie Aufwendungen nach Buchführungsgesetz und ihnen entsprechenden steuerabzugsfähigen Ausgaben resultierende latente Steuerverbindlichkeiten oder Steuerforderungen dürfen unter Beachtung besonderer Vorsicht als eigene Posten in der Gewinn- und Verlustrechnung und der Bilanz vermerkt werden.
\end{abstract}

Für latente Steuern existiert ein Aktivierungs- bzw. Passivierungswahlrecht. Werden sie nicht in der Bilanz erfasst, so ist ihr Wert im Anhang anzugeben, sofern er wesentlich ist ${ }^{993}$.

Bei der Berechnung latenter Steuern dürfen nach KPL 5:18 nur zeitliche Differenzen, d. h. Periodisierungsunterschiede zwischen zu versteuerndem und handelsrechtlichem Ergebnis berücksichtigt werden. Sie entstehen, wenn bei steuerrechtlicher und handelsrechtlicher Gewinnermittlung Erträge und Aufwendungen über einen betrachteten Zeitraum von mehreren Perioden in insgesamt der gleichen Höhe einbezogen werden, allerdings innerhalb dieses Zeitraums in unterschiedlichen Geschäftsjahren berücksichtigt werden ${ }^{994}$.

Zeitliche Differenzen können darauf basieren, dass Erträge in der Handelsbilanz früher als in der Steuerbilanz oder Aufwendungen in der Steuerbilanz früher als in der Handelsbilanz erfasst werden ${ }^{995}$. In diesen Fällen fällt das handelsbilanzielle Ergebnis höher als das steuerbilanzielle Ergebnis aus, es entsteht eine latente Steuerverbindlichkeit, die passiviert werden $\mathrm{kann}^{996}$. Ist indes das steuerbilanzielle Ergebnis höher als das handelsrechtliche, weil Erträge in der Steuerbilanz früher als in der Handelsbilanz oder Aufwendungen in der Handelsbilanz früher als in der Steuerbilanz vermerkt werden, kommt die Bildung einer latenten Steuerforderung in Betracht ${ }^{997}$.

992

993

Übersetzung ins Deutsche durch die Verfasserin.

Vgl. KPL 2:6.1.1 und vgl. KILA-Anweisung „Latente Steuern“, Abschnitt 1.1, Leppiniemi (2000 a), S. 220 und vgl. KPL-Arbeitsgruppe 1995, Teil I (1996), S. 135.

Vgl. KILA-Anweisung „Latente Steuern“, Abschnitt 1.1.

Wegen der umgekehrten Maßgeblichkeit des EVL 54 ist es nach momentan geltendem Steuerrecht äußerst selten der Fall, dass Aufwendungen in der Steuerbilanz früher als in der Handelsbilanz gebucht werden. In der Regel müssen Aufwendungen in der Handelsbilanz erfasst sein, um auch steuerlich abzugsfähig zu sein. Vgl. KILA-Anweisung „Latente Steuern“, Abschnitt 5.1 .

Vgl. KILA-Anweisung „Latente Steuern“, Abschnitt 1.1.

Vgl. KILA-Anweisung „Latente Steuern“, Abschnitt 1.1. 
Der finnische Gesetzgeber gibt nicht explizit an, nach welcher Konzeption die latenten Steuern zu bestimmen seien. Er fordert lediglich, dass beim Ansatz latenter Steuern in Bilanz und Gewinn- und Verlustrechnung besondere Vorsicht geboten sei. Der Richtliniengeber schreibt ebenfalls nicht vor, welche Methode bei der Ermittlung der latenten Steuern anzuwenden ist ${ }^{998}$. Diskutiert werden im finnischen Fachschrifttum die im anglo-amerikanischen Raum entwickelte Liability-Methode (Verbindlichkeitsmethode) und die Deferral-Methode (Abgrenzungsmethode) ${ }^{999}$.

Die Liability-Methode konzentriert sich auf den zutreffenden Vermögens- und Schuldausweis in der Bilanz (statische Bilanztheorie) unter Verwendung desjenigen Steuersatzes, der im späteren Zeitpunkt des Ausgleichs gelten wird. Abgestellt wird auf die wahrscheinliche spätere Einnahme oder Ausgabe, auf künftige Zahlungsverpflichtungen bzw. -ansprüche und nicht auf die Periodenabgrenzung. Die Deferral-Methode zielt, ausgehend von der Gewinn- und Verlustrechnung unter Berücksichtigung aktueller Steuersätze, darauf ab, den Steueraufwand der abgelaufenen Periode korrekt abzubilden ${ }^{1000}$.

Gegen die Deferral-Methode spricht die vom Gesetzgeber geforderte besondere Vorsicht, die durch Anwendung der Liability-Methode mit Verwendung zukünftiger Steuersätze und der Bilanzierung künftiger Zahlungsverpflichtung besser zum Ausdruck kommt ${ }^{1001}$. Die besondere Vorsicht verlangt zudem die Berücksichtigung sämtlicher künftiger latenter Steuern (Comprehensive Application of Tax). Die Nichtbeachtung solcher Periodisierungsunterschiede, die sich erst in ferner Zukunft ausgleichen (Partial Application of Tax), erscheint nicht angebracht ${ }^{1002}$. Gestützt wird die These, bei der Bilanzierung latenter Steuern sei nach LiabilityMethode vorzugehen, durch die Wortwahl des Gesetzgebers. KPL 5:18 gestattet die Bilanzierung von Steuerverbindlichkeiten und Steuerforderungen. Latente Steuerabgrenzungsposten weisen bei der Liability-Methode einen stärkeren Verbindlichkeits- oder Forderungscharakter auf als bei der Deferral-Methode, in der sie eher antizipativen Rechnungsabgrenzungsposten für nicht sichere Zahlungen entsprechen. Auch die Vorschrift des KPA 2:6.1, Steuerverbindlichkeit bzw. -forderung im Anhang anzugeben, sofern sie wesentlich und nicht in der Bilanz vermerkt sind, lässt darauf schließen, dass der Gesetzgeber eher von der Liability-

998 VAN HULLE und VAN DER TAS vertreten die Meinung, sowohl die Deferral-Methode als auch die Liability-Methode, jeweils in Verbindung mit Comprehensive oder Partial Application of Tax, seien richtlinienkonform. Unter Comprehensive Application of Tax wird die Berücksichtigung sämtlicher künftiger latenter Steuern verstanden. Bei Partial Application of Tax werden Periodisierungsunterschiede, die sich erst in ferner Zukunft ausgleichen, nicht beachtet. Vgl. Van Hulle und van der Tas (2001), S. 830.

999 Vgl. Järvinen et al. (2000), S. 519-522, vgl. Nevalainen (1998), S. 263-265, vgl. Räty (1998), S. 122-123 und vgl. KPL-Arbeitsgruppe 1995, Teil I (1996), S. 132-135.

1000 Vgl. Lührmann (1997), S. 102-107.

1001 Vgl. Järvinen et al. (2000), S. 520.

1002 Vgl. Järvinen et al. (2000), S. 521. 
Methode ausgeht ${ }^{1003}$. Zudem gilt die Regel, der Buchführungspflichtige habe sich an den Standards des IASB zu orientieren, falls das finnische Buchführungsrecht und die ihm zu Grunde liegenden Richtlinien Fragen offen lassen. IAS 12 (revised 1996) liegt das Konzept der Liability-Methode zu Grunde. In der Praxis findet aus den genannten Gründen die Liability-Methode breite Anwendung, die Ermittlung latenter Steuern auf Basis der Deferral-Methode steht jedoch nicht im Widerspruch zur Art guter Buchführung und ist deshalb gestattet.

Der KILA hat am 11. Januar 1999 eine allgemeingültige Anweisung veröffentlicht, nach der das bilanzierende Unternehmen bei der Bestimmung der latenten Steuern für den Einzelabschluss zwischen drei Alternativen wählen darf ${ }^{1004}$ :

- Den Mindestanforderungen des Buchführungsgesetzes entsprechend werden die erfolgswirksamen Periodisierungsunterschiede berücksichtigt (Alternative 1).

- Über die Anforderungen des Buchführungsgesetzes hinausgehend werden auf Basis der in IAS 12 (revised 1996) verankerten Grundsätze neben erfolgswirksamen auch nicht erfolgswirksame Periodisierungsunterschiede berücksichtigt (Alternative 2).

- Bei der Ermittlung der latenten Steuern wird IAS 12 (revised 1996) angewendet, wobei allerdings zusätzlich die Vorschriften des Buchführungsgesetzes sowie der 4. EG-Richtlinie beachtet werden müssen (Alternative $3)$.

Die erste und zweite Alternative sind Gegenstand der allgemeingültigen Anweisung vom KILA ${ }^{1005}$. Zur Vorgehensweise bei Alternative 3 wird auf IAS 12 (revised 1996) und die ihn kommentierende Literatur verwiesen ${ }^{1006}$.

Alternative 1 und 2 weichen darin voneinander ab, dass nach Alternative 2 auch zeitliche Unterschiede berücksichtigt werden, die noch nicht zu einem unterschiedlichen Jahresergebnis geführt haben, nämlich nicht erfolgswirksame zeitlich bedingte Ansatzdifferenzen in der Bilanz: die Neubewertung, die nach Steuerrecht nicht durchgeführt werden darf. Ein Ausgleich zwischen Handelsbilanz und Steuerbilanz findet erst bei Verkauf des neubewerteten Vermögenswerts aus dem Anlagevermögen statt. Wird ein Preis über den ursprünglichen Anschaffungskosten realisiert, ist die Neubewertungsrücklage erfolgswirksam aufzulösen. Der Gewinn ist dann steuerpflichtig ${ }^{1007}$. Unter Verkaufsprämisse enthält die Neubewertungs-

1003 Vgl. Järvinen et al. (2000), S. 520.

1004 Vgl. KILA-Anweisung „Latente Steuern“, Abschnitt 3.

1005 Vgl. KILA-Anweisung „Latente Steuern“, Abschnitt 3. Die Ermittlung der latenten Steuern nach Alternative 1 und 2 wird in einzelnen Schritten im Anhang dieser Arbeit dargestellt.

1006 Mit der Forderung, dass IAS 12 (revised 1996) nur unter zusätzlicher Beachtung der im Buchführungsgesetz und der 4. EG-Richtlinie enthaltenen Vorschriften angewendet werden darf, schränkt der KILA gesetzeskonform IAS 12 (revised 1996) ein. Anders als im „Original“-IAS 12 (revised 1996) dürfen nur zeitliche Unterschiede berücksichtigt werden. Alternative 2 und 3 müssen deshalb in etwa zum selben Ergebnis führen. Vgl. KILAAnweisung „Latente Steuern“, Abschnitt 3.1.

1007 Vgl. KILA-Anweisung „Latente Steuern“, Abschnitt 5.1 und Abschnitt 5.4. 
rücklage einen Fremdkapitalanteil. Problematisch ist diese Sichtweise, da das Anlagevermögen seiner Zweckbestimmung nach dem Unternehmen dauerhaft dienen soll. Mit einem Ausgleich zwischen Handelsbilanz und Steuerbilanz ist deshalb voraussichtlich nicht zu rechnen, bei der Neubewertung handelt es sich um eine quasi-permanente Differenz. Dafür, dass diese nach dem Willen des Gesetzgebers bei der Steuerabgrenzung dennoch berücksichtigt werden soll, spricht die in KPL 5:18 geforderte besondere Vorsicht und die daraus resultierende, in KPA 2:6.1.3 enthaltene Vorschrift, im Anhang die mögliche ertragsteuerliche Auswirkung einer Neubewertung anzugeben.

Der KILA verlangt, künftig zu erwartende Steuerminderungen aus Verlustvorträgen in die Ermittlung latenter Steuern einzubeziehen. Bedingung hierfür ist, dass dem Unternehmen wahrscheinlich im betrachteten zukünftigen Zeitraum wieder ausreichend hohe Gewinne zufließen werden, mit denen es die noch nicht genutzten steuerlichen Verlustvorträge verrechnen kann. Bei der Beurteilung der hinreichenden Wahrscheinlichkeit ist besondere Vorsicht geboten ${ }^{1008}$. Verluste dürfen in Finnland über einen Zeitraum von maximal zehn Jahren vorgetragen werden.

$\mathrm{Zu}$ erwartende Steuerminderungen aus Steuergutschriften werden nach finnischem Verständnis nicht als latente Steuern in der Bilanz und Gewinn- und Verlustrechnung erfasst, sondern in den aktiven Rechnungsabgrenzungsposten eingestellt $^{1009}$.

Der Abschreibungsunterschied und die freiwilligen Rückstellungen mit Rücklagencharakter beinhalten gemäß dem KILA keine latenten Steuern. Der im Abschreibungsunterschied enthaltene Fremdkapitalanteil beruhe nicht auf zeitlichen Periodisierungsdifferenzen, da die steuerlichen Abschreibungen wegen der umgekehrten Maßgeblichkeit in der handelsrechtlichen und der steuerrechtlichen Gewinn- und Verlustrechnung in derselben Periode vermerkt würden; er stamme vielmehr aus Steuerstundung. Freiwillige Rückstellungen enthielten - sofern sie steuerlich abzugsfähig sind - ebenfalls keine latenten Steuern aus Periodisierungsdifferenzen. Bildeten jedoch Aktiengesellschaften entgegen der Intention des Gesetzgebers freiwillige Rückstellungen, die nicht steuerlich abzugsfähig seien, beinhalteten diese einen aktivischen latenten Steueranteil. Dieser darf jedoch auf Grund der allgemeingültigen KILA-Anweisung weder aktiviert werden noch muss er im Anhang angegeben werden, da er die Kriterien für latente Steuern nach KILA-Definition nicht erfüllt ${ }^{1010}$. Je nach Wesentlichkeit stellt sich hier die

1008 Vgl. KILA-Anweisung „Latente Steuern“, Abschnitt 5.2 und 7.2.1.

1009 Vgl. KILA-Anweisung „Latente Steuern“, Abschnitt 5.4.

1010 Vgl. KILA-Anweisung „Latente Steuern“, Abschnitt 5.3. 
Frage, ob der Jahresabschluss in einem solchen Fall dem Anspruch des True and Fair View noch genügt ${ }^{1011}$.

Latente Steuerverbindlichkeiten und -forderungen sind mit ihrem Nennwert auszuweisen, sie dürfen nicht abgezinst werden ${ }^{1012}$. Begründet wird dieses Abzinsungsverbot - IAS 12.54 entsprechend - v. a. mit praktischen Problemen einer Abzinsung bei der Jahresabschlusserstellung. Es wären aufwändige Prognosen über die Zeitpunkte der Umkehr temporärer Bilanzdifferenzen notwendig. 


\subsubsection{Umsatzerlöse}

Mit KPL 4:1 hat der finnische Gesetzgeber Art. 28 der 4. EG-Richtlinie wie folgt in nationales Recht umgesetzt ${ }^{1014}$ :

\section{$\underline{\text { Umsatzerlöse }}$}

$\mathrm{Zu}$ den Umsatzerlösen zählen die Erlöse aus der eigentlichen Geschäftstätigkeit des Buchführungspflichtigen nach Abzug von Erlösschmälerungen sowie der Mehrwertsteuer und anderen unmittelbar auf den Umsatz bezogenen Steuern.

Den Umsatzerlösen zuzuordnende Erträge sind dadurch gekennzeichnet, dass sie der Sache nach gewohnheitsmäßig und regelmäßig wiederkehrend sind ${ }^{1015}$. Sie unterscheiden sich hierin deutlich von außerordentlichen Erträgen.

Nicht in die Umsatzerlöse einzubeziehen sind durchlaufende Posten. Diese beeinflussen die Zusammensetzung des Jahresergebnisses nicht, da den Erträgen aus gleichem Grund und in gleicher Höhe Aufwendungen gegenüberstehen. Ebenfalls nicht in die Umsatzerlöse eingerechnet werden dürfen Zuschüsse, Gutschriften und ähnliche Aufwandsminderungen. Sie stellen Korrekturposten zu ihrer jeweiligen Aufwandsbuchung dar ${ }^{1016}$.

Wechselkursdifferenzen aus Umsatzgeschäften dürfen, so ein Vertreter der EUKommission gegenüber der KPL-Arbeitsgruppe 1995, im Sinn des Richtliniengebers direkt den Umsatzerlösen zugerechnet bzw. von ihnen abgezogen werden. Der finnische Gesetzgeber hat sich dafür entschieden, mit Hinblick auf einen verbesserten True and Fair View den Ausweis sämtlicher Währungsdifferenzen im Finanzergebnis zu verlangen ${ }^{1017}$.

Um der Richtlinie zu entsprechen und den True and Fair View zu gewährleisten, ist es nicht mehr gestattet, Frachtkosten, Provisionen und Verluste aus abgeschlossenen Umsatzgeschäften direkt von den Umsatzerlösen abzusetzen. Sie sind vielmehr auf Aufwandskonten zu verbuchen ${ }^{1018}$.

1013 Vorschriften zu einzelnen Posten der Gewinn- und Verlustrechnung sind in Abschnitt 6 der 4. EG-Richtlinie enthalten und umfassen Umsatzerlöse (Art. 28), außerordentliche Erträge und Aufwendungen (Art. 29) sowie Steuern (Art. 30). Die zur Bilanz korrespondierenden Posten der Gewinn- und Verlustrechnung sind bereits bei den Bilanzposten behandelt worden, sodass an dieser Stelle lediglich Umsatzerlöse sowie außerordentliche Posten betrachtet werden.

1014 Übersetzung ins Deutsche durch die Verfasserin.

1015 Vgl. Järvinen et al. (2000), S. 371 und vgl. KILA 1306/1994.

1016 Vgl. KILA 1255/1994.

1017 Vgl. KPL-Arbeitsgruppe 1995, Teil I (1996), S. 100.

1018 Vgl. KPL-Arbeitsgruppe 1995, Teil I (1996), S. 99, vgl. Järvinen et al. (2000), S. 371 und vgl. Räty (1998 c), S. 82. 


\title{
3.3.2 Außerordentliche Erträge und Aufwendungen
}

Nach Art. 29 Abs. 1 der 4. EG-Richtlinie umfassen außerordentliche Erträge und außerordentliche Aufwendungen solche, die außerhalb der normalen Geschäftstätigkeit anfallen. Der finnische Gesetzgeber konkretisiert diese Definition in KPL 4:2 wie folgt ${ }^{1019}$ :

\section{$\underline{\text { Außerordentliche Erträge und Aufwendungen }}$}

\begin{abstract}
Als außerordentlich werden solche Erträge und Aufwendungen bezeichnet, die auf von der normalen Geschäftstätigkeit des Buchführungspflichtigen abweichenden, einzelnen und wesentlichen Vorfällen beruhen.
\end{abstract}

Die Abgrenzung zur „,normalen Geschäftstätigkeit des Buchführungspflichtigen“ erfolgt nach der Art eines Vorfalls im Vergleich zu den Sachverhalten, die typischerweise im Unternehmen auftreten ${ }^{1020}$. Auch die Häufigkeit des Auftretens von Sachverhalten ist für die Beurteilung ihrer Außerordentlichkeit relevant. Diese müssen Einzelfall-Charakter vorweisen ${ }^{1021}$. Ein weiteres Merkmal außerordentlicher Erträge und Aufwendungen ist ihre Wesentlichkeit im Verhältnis zur Größe des betrachteten Unternehmens. Je größer das Unternehmen, desto höher muss ein Geschäftsvorfall betragsmäßig ausfallen, um als außerordentlich gelten zu können $^{1022}$.

Zum außerordentlichen Ergebnis zählen neben Schadensfällen, Verkauf von Tochterunternehmen, Fusionen und Enteignungen auch wesentliche Änderungen in der Buchführungsgesetzgebung, die einen Einmal-Effekt auf das Unternehmensergebnis ausüben. Hier kann beispielsweise die Verpflichtung der Unternehmen angeführt werden, in einer Übergangsphase bis Ende 2000 vorher nicht erfasste Pensionsverpflichtungen nachzubuchen ${ }^{1023}$.

1019 Übersetzung ins Deutsche durch die Verfasserin.

1020 Vgl. HE 173/1997, S. 19, vgl. KPL-Arbeitsgruppe 1995, Teil I (1996), S. 100-102 und vgl. IAS 8, an dem der finnische Gesetzgeber sich bei seiner Definition orientiert hat.

1021 Vgl. Järvinen et al. (2000), S. 376-377.

1022 Vgl. Järvinen et al. (2000), S. 377. Die Forderung nach Wesentlichkeit ist richtlinienkonform, vgl. hierzu van Hulle und van der Tas (2001), S. 818.

1023 Vgl. KILA 1378/1996. 


\subsubsection{Stellung des Lageberichts und Anforderungen an den Lagebe- richt}

Nach KPL 3:1.1.4 ist der Lagebericht - anders als Art. 2 Abs. 1 der 4. EG-Richtlinie es vorsieht - eigenständiger Pflichtbestandteil des Jahresabschlusses. In Art. 2 Abs. 1 der 4. EG-Richtlinie zählt der Richtliniengeber die Bestandteile des Jahresabschlusses erschöpfend auf; der finnische Gesetzgeber versteht diese Aufzählung jedoch als Mindestanforderung.

Gleichzeitig setzt der finnische Gesetzgeber mit KPL 3:1.1.4 Art. 46 Abs. 1 der 4. EG-Richtlinie in nationales Recht um ${ }^{1024}$ :

Inhalt des Jahresabschlusses

Für das Geschäftsjahr ist ein Jahresabschluss mit folgendem Inhalt zu erstellen: $[\ldots]$

4) Lagebericht, in dem Angaben zur Entwicklung der wichtigsten den Geschäftsbetrieb des Buchführungspflichtigen betreffenden Faktoren gemacht werden.

Seit In-Kraft-Treten des Buchführungsgesetzes von 1997 müssen erstmals alle Buchführungspflichtigen einen Lagebericht aufstellen, der denselben Offenlegungs- und Prüfungspflichten unterliegt wie die Gewinn- und Verlustrechnung, die Bilanz und der Anhang ${ }^{1025}$. Die Anforderungen an den Inhalt des Lageberichts konkretisiert der Gesetzgeber, Art. 46 Abs. 2 der 4. EG-Richtlinie umsetzend, in KPA 2: $1^{1026}$ :

$\underline{\text { Im Lagebericht anzugebende Informationen }}$

Im Lagebericht sind neben den bereits in KPL 3:1.1.4 genannten Faktoren folgende darzulegen:

1) Informationen über wesentliche Geschehnisse im Geschäftsjahr und wesentliche Geschehnisse, die sich nach Beendigung des Geschäftsjahrs ereignet haben;

2) Einschätzung der voraussichtlichen zukünftigen Entwicklung; sowie

3) Erklärung über den Umfang des Bereichs Forschung und Entwicklung. $[\ldots]$

Somit sind - da der Lagebericht Bestandteil des Jahresabschlusses ist - Subsequent Events in Finnland nach KPA 2:1,1 in das vergangene Geschäftsjahr rückzubeziehen, wenn auch nicht zahlenmäßig. Hier liegt ein Unterschied in der Auslegung des Stichtagsprinzips zwischen dem finnischen Gesetzgeber und dem Richtliniengeber.

1024 Übersetzung ins Deutsche durch die Verfasserin.

1025 Vgl. KPL-Arbeitsgruppe 1995, Teil I (1996), S. 161.

1026 Übersetzung ins Deutsche durch die Verfasserin. 
Die Buchführungspflichtigen sind gehalten, bei Darstellung von Geschäftsverlauf und Lage ihrer Gesellschaft auch auf die künftige Entwicklung einzugehen, Chancen ebenso wie Risiken aufzuzeigen. Dabei muss der Lagebericht gemeinsam mit den anderen Bestandteilen des Jahresabschlusses den True and Fair View vermitteln.

Die gesetzlichen Vertreter von Aktiengesellschaften müssen zusätzlich darüber informieren, ob ihre Gesellschaft seit dem Geschäftsjahr die Kriterien eines Mutterunternehmens erfüllt und ob Verschmelzungen oder Abspaltungen stattgefunden haben. Sie sind verpflichtet, Name und Standort ausländischer Betriebsstätten und Niederlassungen anzugeben ${ }^{1027}$. Ein Mutterunternehmen ist gezwungen, die Konzernstruktur aufzuzeigen. Ein Tochterunternehmen muss den Namen seines Mutterunternehmens angeben ${ }^{1028}$.

Darüber hinaus müssen Aktiengesellschaften über Kapitalerhöhungen sowie die Bevollmächtigung des Vorstands zur Durchführung von Kapitalerhöhungen berichten ${ }^{1029}$.

Die gesetzlichen Vertreter von Aktiengesellschaften sind aufgefordert, eigene Aktien im Besitz der Gesellschaft und der Mitglieder des inneren Kreises ${ }^{1030}$ sowie die Veränderung dieser Aktienanteile im Geschäftsjahr im Lagebericht zu nennen ${ }^{1031}$. Der Gewinnverwendungsvorschlag des Vorstands ist Teil des Lageberichts.

Zudem sind öffentliche Aktiengesellschaften und solche privaten Aktiengesellschaften, welche die Größenkriterien des KPL 3:9.2 überschreiten, zur Aufstellung einer Kapitalflussrechnung verpflichtet, die dem Lagebericht als Anhang beigefügt wird ${ }^{1032}$.

Es kann sinnvoll sein, bestimmte Sachverhalte oder Risiken nicht im Lagebericht, sondern an geeigneter Stelle im Anhang zu nennen ${ }^{1033}$. Der KILA hat 1999 in einem Fall entschieden, dass ein Ausweisfehler Lagebericht und Anhang betreffend nicht im Widerspruch zum True and Fair View stünde ${ }^{1034}$. Es ist eine deutliche Zunahme der von Unternehmen publizierten und über die gesetzlichen Bestimmungen hinausgehenden Anhangangaben zu beobachten. Tendenziell findet eine Verlagerung der Informationen vom Lagebericht in den Anhang statt ${ }^{1035}$.

\footnotetext{
1027 Vgl. OYL 11:9.2.

1028 Vgl. OYL 11:11.1 und 11:11.3.

1029 Vgl. OYL 11:9.4.

1030 Zur Definition der Mitglieder des „,inneren Kreises” vgl. Fußnote 251 und Kap. 2.2.2.2 dieser Arbeit.

1031 Vgl. OYL 11:9a.

1032 Vgl. OYL 11:9.7. Zur Kapitalflussrechnung vgl. Kap. 3.4.2 dieser Arbeit.

1033 Vgl. Leppiniemi und Leppiniemi (1999), S. 283.

1034 Vgl. KILA 1580/1999.

1035 Vgl. Artsberg (2000), S. 578; sie bezieht ihre Aussage auf alle skandinavischen Länder.
} 


\subsubsection{Kapitalflussrechnung als Bestandteil des Lageberichts}

Der Richtliniengeber sieht in der 4. EG-Richtlinie weder die Erstellung noch die Offenlegung einer Kapitalflussrechnung obligatorisch vor ${ }^{1036}$, sodass in den EUMitgliedstaaten die Anforderungen hinsichtlich der Kapitalflussrechnung stark voneinander abweichen ${ }^{1037}$. Das Accounting Advisory Forum der EU hat 1997 in einer Stellungnahme betont, dass keine Veranlassung dazu bestehe, eine Kapitalflussrechnung von allen Buchführungspflichtigen zu verlangen. Größe des Unternehmens und eine eventuell Börsennotierung können hier ausschlaggebend sein. Fordert der nationale Gesetzgeber indes die Erstellung einer Kapitalflussrechnung, erachtet das Forum hinsichtlich der Ermittlungs- und Darstellungsmethode die Orientierung an Standard 7 (revised 1992) des IASB für sinnvoll ${ }^{1038}$.

In Finnland ist die Kapitalflussrechnung nach OYL 11:9.7 Pflichtbestandteil der Abschlüsse öffentlicher Aktiengesellschaften und solcher privater Aktiengesellschaften, welche die Größenkriterien des KPL 3:9.2 erfüllen ${ }^{1039}$. Der Aufbau, die Grundsätze und die Darstellungsart einer solchen Kapitalflussrechnung sind gesetzlich nicht vorgeschrieben. Der Gesetzgeber fordert lediglich, dass die Kapitalflussrechnung die Herkunft im Geschäftsjahr erfolgter Einstellungen in die Rücklagen und die Verwendung von den Rücklagen entnommenen Beträgen erklä$\mathrm{re}^{1040}$. Der KILA hat am 09. November 1999 eine allgemeingültige Anweisung zur Erstellung von Kapitalflussrechnungen veröffentlicht, die aber mangels gesetzlicher Regelung nicht verbindlich von den Unternehmen anzuwenden ist ${ }^{1041}$.

Die Kapitalflussrechnung ergänzt als Bewegungsrechnung Gewinn- und Verlustrechnung, Bilanz und Anhang um Informationen zur Herkunft und Verwendung liquiditätswirksamer Mittel im Geschäftsjahr. Sie soll dem JahresabschlussAdressaten ein Urteil über die Finanzlage, die Veränderung des Verschuldungsgrads und die Zahlungsbereitschaft des Unternehmens ermöglichen und einen Schluss auf künftige, erwartete Geldströme zulassen. Sie stellt nach Auffassung

1036 Die in der Generalnorm gemeinte Finanzlage zielt nicht auf die Erstellung einer Kapitalflussrechnung ab, sondern vielmehr auf die Liquidität der Unternehmen. Vgl. hierzu van Hulle und van der Tas (2001), S. 797.

1037 Vgl. Räty (2000), S. 38.

1038 Vgl. EU-Kommission XV/6008/94 EN. Eine nach der KILA-Anweisung „Kapitalflussrechnung“ vom 09. November 1999 erstellte Kapitalflussrechnung entspricht grundsätzlich den Anforderungen des IAS 7 (revised 1992). Um jedoch eine völlige Übereinstimmung mit IAS 7 (revised 1992) zu erreichen, sind nach Aussage vom KILA zusätzliche Angaben zu leisten. Vgl. KILA-Anweisung „Kapitalflussrechnung“, Abschnitt 1.3 und vgl. Räty (2000), S. 39.

1039 Die Pflicht zur Aufstellung von Kapitalflussrechnungen ist seit 1980 im Aktiengesellschaftsgesetz verankert. Die Verpflichtung zur Aufstellung von Kapitalflussrechnungen gilt nach Genossenschaftsgesetz (295/1998) § 79 b.2 auch für bestimmte Genossenschaften.

1040 Vgl. OYL 11:9.7.

1041 Vgl. KILA-Anweisung „Kapitalflussrechnung“, Abschnitt 1.3. Das Schema einer Kapitalflussrechnung nach KILA ist im Anhang dieser Arbeit enthalten. 
vom KILA ein brauchbares Instrument bei Unternehmensvergleichen dar, da in ihr - anders als in Bilanz und Gewinn- und Verlustrechnung - Bewertungs- und Periodisierungsprobleme teilweise eliminiert werden. Sie ist als Anhang zum Lagebericht Teil des Jahresabschlusses, der insgesamt den True and Fair View vermitteln soll, und somit prüfungspflichtig ${ }^{1042}$.

Der KILA-Anweisung Folge leistend, muss eine Dreiteilung der Kapitalflussrechnung erfolgen, nach der die Veränderungen der Zahlungsmittel und -äquivalente für die Bereiche laufende Geschäftstätigkeit, Investitionstätigkeit und Finanzierungstätigkeit getrennt ermittelt werden. Die Kapitalflussrechnung kann nach direkter Methode aufgestellt werden, bei der die wesentlichen Mittelzu- und -abflüsse aus laufender Geschäftstätigkeit unmittelbar gezeigt werden. Auch die Anwendung der indirekten Methode, welche die Fondsmittelveränderung aus laufender Geschäftstätigkeit vom Jahresergebnis vor Steuern sowie vor außerordentlichem Ergebnis ableitet, ist möglich ${ }^{1043}$.

Mithilfe beider Methoden wird die Veränderung des Finanzmittelfonds ermittelt, dessen Definition für die Aussagekraft und die internationale Vergleichbarkeit der Kapitalflussrechnung von Bedeutung ist. Nach finnischem Verständnis enthält der Finanzmittelfonds Zahlungsmittel (Kasse und Forderungen gegenüber Kreditinstituten) sowie Zahlungsmitteläquivalente. Die Einbeziehung von Posten in Zahlungsmitteläquivalente erfordert, dass diese zu einem im Voraus bekannten Wert realisiert werden können, das Wertschwankungsrisiko äußerst gering und die Restlaufzeit sehr kurz ist ${ }^{1044}$.

Mittelzu- und -abflüsse aus laufender Geschäftstätigkeit ergeben sich aus der Umsatztätigkeit des Unternehmens. Sie stellen dar, in welchem Ausmaß das Unternehmen im Geschäftsjahr aus seiner Umsatztätigkeit heraus Geldmittel zur Bewahrung der Betriebsbereitschaft erbracht hat, Ertrag in das Eigenkapital der Gesellschafter gezahlt hat, Neu-Investitionen getätigt hat und Darlehen an fremde Dritte zurückgezahlt hat ${ }^{1045}$.

Der gesonderte Ausweis von Zahlungsströmen aus der Investitionstätigkeit soll dem Jahresabschlussleser Informationen darüber vermitteln, in welcher Höhe Zahlungen zur Generierung künftiger Mittelzuflüsse bereitgestellt worden $\operatorname{sind}^{1046}$

1042 Vgl. KILA-Anweisung „Kapitalflussrechnung“, Abschnitt 2 und vgl. KHT-yhdistys (1998), S. 27.

1043 Vgl. KILA-Anweisung „Kapitalflussrechnung“, Abschnitte 5.1 und 5.2. Die vom KILA vorgesehenen Schemata zur direkten und indirekten Methode sind im Anhang dieser Arbeit enthalten.

1044 Vgl. KILA-Anweisung „Kapitalflussrechnung“, Abschnitt 3.

1045 Vgl. KILA-Anweisung „Kapitalflussrechnung“, Abschnitt 2.

1046 Vgl. KILA-Anweisung „Kapitalflussrechnung“, Abschnitt 2. 
Mittelzu- und -abflüsse aus Finanzierungstätigkeit spiegeln die Veränderung von Eigenkapital und Fremdkapital während des Geschäftsjahrs wider ${ }^{1047}$.

Zins- und Dividendenzahlungen sowie außerordentliche Geschäftsvorfälle sind jeweils separat auszuweisen und sachgerecht dem Bereich zuzuordnen, durch den sie veranlasst sind ${ }^{1048}$.

Zahlungsströme aus Kreditaufnahme und -tilgung werden grundsätzlich brutto gezeigt. Ein Nettoausweis kommt dann in Betracht, wenn Darlehen im Geschäftsjahr aufgenommen und vollständig getilgt worden sind. Ein Nettoausweis ist auch dann möglich, wenn ein Darlehen zurückgezahlt und sofort erneuert worden ist $^{1049}$. Veränderungen eigenkapitalersetzender Rangrücktrittsdarlehen sind nicht unter dem Eigenkapital, sondern unter langfristigen Verbindlichkeiten bzw. langfristigen Forderungen auszuweisen ${ }^{1050}$.

In der Kapitalflussrechnung wird die tatsächliche Steuerbelastung ausgewiesen, latente Steuern werden nicht berücksichtigt ${ }^{1051}$.

Unrealisierte Gewinne bzw. Verluste aus der Währungsumrechnung sind nicht Bestandteil der Kapitalflussrechnung, da sie keine Mittelzu- oder -abflüsse darstellen ${ }^{1052}$.

1047 Vgl. KILA-Anweisung „Kapitalflussrechnung“, Abschnitt 2.

1048 Vgl. KILA-Anweisung „Kapitalflussrechnung“", Abschnitte 4.4.1 und 4.4.3.

1049 Vgl. KILA-Anweisung „Kapitalflussrechnung“, Abschnitt 4.4.6. Nach IAS 7 (revised 1992) gelten strengere Saldierungskriterien.

1050 Vgl. KILA-Anweisung „Kapitalflussrechnung“, Abschnitt 4.4.6.

1051 Vgl. KILA-Anweisung „Kapitalflussrechnung“, Abschnitt 4.4.4.

1052 Vgl. KILA-Anweisung „Kapitalflussrechnung“, Abschnitt 4.4.2. 


\subsection{Zusammenfassender Überblick}

In Kap. 3 konnte gezeigt werden, dass der finnische Gesetzgeber die Mindestanforderungen an die dargestellten und untersuchten Bereiche richtlinienkonform umgesetzt hat, auch wenn er teilweise von den in der Richtlinie verbindlich festgelegten Artikeln abweicht. Dies betrifft den Ausweis des Anlagevermögens ${ }^{1053}$ und des steuerlichen Ausgleichspostens ${ }^{1054}$. Diese Abweichungen stehen jedoch nicht im Widerspruch zur Intention des Richtliniengebers und den Grundsätzen ordnungsmäßiger Buchführung, sondern verbessern im Gegenteil die Aussagefähigkeit finnischer Abschlüsse.

Materiell als problematisch erweist sich der den Aktiengesellschaften nach bestimmten, eng auszulegenden Kriterien gestattete Ausweis von kapitalersetzenden Darlehen im Eigenkapital ${ }^{1055}$. Auf die Behandlung solcher Darlehen wird in der Richtlinie nicht explizit eingegangen. Sie im Eigenkapital zu zeigen, steht jedoch im Gegensatz zum Prinzip der Unternehmensfortführung und zum Vorsichtsgedanken. Durch den gesonderten Ausweis innerhalb des Eigenkapitals und das Erfordernis von Darlehensangaben im Anhang stellt der finnische Gesetzgeber aber sicher, dass ein in gesetzmäßiger Weise aufgestellter Jahresabschluss den True and Fair View erfüllt. Der Ausweis kapitalersetzender Darlehen im Eigenkapital sollte deshalb keinen hinreichenden Grund dafür bieten, die Rechtsgültigkeit finnischer Rechnungslegungsvorschriften aus europarechtlicher Sicht zu verneinen.

Der finnische Gesetzgeber hat Normen geschaffen, welche die Regelungslücken der 4. EG-Richtlinie schließen. Diese betreffen für den Einzelabschluss die Fremdwährungsumrechnung ${ }^{1056}$, die Bewertung langfristiger Auftragsfertigung $^{1057}$, die Ermittlung latenter Steuern ${ }^{1058}$ und die Kapitalflussrechnung ${ }^{1059}$. Die Behandlung dieser Sachverhalte wird durch KILA-Anweisungen konkretisiert. Sowohl der Gesetzgeber als auch der KILA haben sich bei der Regelung dieser Sachverhalte an den jeweiligen Standards des IASB orientiert. Da auch das Accounting Advisory Forum der EU eine Orientierung an diesen Standards für sinnvoll erachtet, kann davon ausgegangen werden, dass der finnische Gesetzgeber im Sinne des Richtliniengebers vorgegangen ist.

Mit Kap. 3 ist eine deutschsprachige Basis geschaffen worden, von der aus Finnland mit allen anderen EU-Staaten verglichen werden kann. Im Folgenden werden

\footnotetext{
1053 Vgl. Kap. 3.2.1.2.1 dieser Arbeit.

1054 Vgl. Kap. 3.2.5.1 dieser Arbeit.

1055 Vgl. Kap. 3.2.4.2 dieser Arbeit.

1056 Vgl. Kap. 3.1.7 dieser Arbeit.

1057 Vgl. Kap. 3.2.2.3 dieser Arbeit.

1058 Vgl. Kap. 3.2.7 dieser Arbeit.

1059 Vgl. Kap. 3.4.2 dieser Arbeit.
} 
auf Grundlage der Ergebnisse die Rechnungslegungssysteme Finnlands und Deutschlands miteinander verglichen und der Fortschritt der formalen Harmonisierung zwischen beiden Staaten untersucht. 


\title{
Harmonisierung der Rechnungslegung zwischen Finn- land und Deutschland
}

\author{
Deskriptiv-vergleichende Untersuchung der formalen Harmoni- \\ sierung
}

\subsubsection{Normierung der Rechnungslegung}

\subsubsection{Gesetzgebung mit Rechnungslegungsrelevanz}

\subsection{Buchführungsgesetz und Handelsgesetzbuch}

Bis zum Buchführungsgesetz von 1992 ist der Jahresabschluss in Finnland SAARIOs dynamischer „Ausgaben-Einnahmen-Theorie der Buchführung“ gefolgt ${ }^{1060}$. Heute basiert die Rechnungslegung auf der 4. EG-Richtlinie, die mit dem Buchführungsgesetz KPL von 1992 und 1997 sowie der Buchführungsverordnung KPA von 1992 und 1997 in nationales Recht umgesetzt worden ist ${ }^{1061}$. Wo die 4. EG-Richtlinie die in der vorliegenden Arbeit untersuchten Regelungslücken aufweist, hat sich der finnische Gesetzgeber an den Standards des IASB orientiert und diese an finnische Gegebenheiten angepasst ${ }^{1062}$. Er empfiehlt darüber hinaus, sich bei der Weiterentwicklung der Rechnungslegung an den Standards des IASB zu orientieren ${ }^{1063}$. Die Theorie Martti Saarios hat durch die Transformation der 4. EG-Richtlinie weitgehend an Bedeutung verloren. Sie greift jedoch noch bei der Analyse und Interpretation von Buchführungsregeln ${ }^{1064}$.

Neben Buchführungsgesetz und -verordnung gelten rechtsformspezifische Spezialgesetze und das Wertpapiermarktgesetz ${ }^{1065}$. Rechnungslegungsrelevante Gesetze sind in Finnland traditionell knapper formuliert als in Deutschland, jedoch ausführlicher als in den angelsächsischen Ländern. Sie sind relativ allgemein gehalten und nehmen nicht zu allen Rechnungslegungsproblemen Stellung. Seit Beginn der Internationalisierung der Rechnungslegung Anfang der 1990er Jahre ist ein steigender Detaillierungsgrad zu beobachten, Vorschriften nehmen an Inhalt und Umfang $\mathrm{zu}^{1066}$.

1060 Vgl. Kap. 2.1.3.1 dieser Arbeit.

1061 Vgl. KPL-Arbeitsgruppe 1995, Teil I (1996), S. 13-19.

1062 Dies betrifft folgende Problemstellungen: IAS 11 (revised 1993): Teilgewinnrealisierung langfristiger Projekte; IAS 12 (revised 1996): Behandlung latenter Steuern; IAS 21 (revised 1993): Währungsumrechnung und IAS 7 (revised 1992): Kapitalflussrechnung. Vgl. Majala (2001 b), S. 962.

1063 Vgl. Leppiniemi (2000 a), S. 97-100 und vgl. HE 173/1997, S. 6.

1064 Vgl. Kap. 3.1.3.1 dieser Arbeit.

1065 Vgl. Kap. 2.2.2 dieser Arbeit.

1066 Vgl. Flower (1994), S. 265 und vgl. Majala (2001 b), S. 965. Vgl. auch Kap. 2.2.1.1 und 2.2.1.2 dieser Arbeit. 
Der deutsche Gesetzgeber hat die 4. EG-Richtlinie 1985 mit dem Bilanzrichtlinien-Gesetz in nationales Recht umgesetzt ${ }^{1067}$, in den Jahren 1994 die Mittelstandsrichtlinie und 2000 die KapCo-Richtlinie mit dem KapCoRichtliniegesetz transformiert sowie die Größenklassen angepasst ${ }^{1068}$. Dem deutschen Jahresabschluss nach Handelsgesetzbuch liegt keine bestimmte Bilanzauffassung zu Grunde ${ }^{1069}$.

Rechnungslegungsrelevant sind das Handelsgesetzbuch sowie Spezialgesetze für Unternehmen bestimmter Rechtsformen, Größen und Wirtschaftszweige ${ }^{1070}$. Sie sind - wie in Finnland - relativ allgemein gehalten und nehmen nicht zu allen Rechnungslegungsfragen Stellung ${ }^{1071}$.

\subsection{Steuergesetzgebung}

Sowohl in Finnland als auch in Deutschland ist der handelsrechtliche Abschluss maßgeblich für die Steuerbilanz ${ }^{1072}$. In besonderen Fällen setzen steuerliche Vorschriften eine korrespondierende handelsrechtliche Bilanzierung voraus (umgekehrte Maßgeblichkeit ${ }^{1073}$ ).

Finnland hat 1919 den Grundsatz der Maßgeblichkeit des Jahresabschlusses für die steuerliche Gewinnermittlung und seine Umkehrung aus Deutschland übernommen ${ }^{1074}$. Existiert nach Buchführungsgesetz ein Wahlrecht, richten sich die Jahresabschlussersteller meist nach steuerlichen Vorschriften. Seit 1993 ist in Finnland die Verbindung zwischen Buchführungsgesetz und Steuergesetzgebung gelockert. Es sind zahlreichen Durchbrechungstatbestände eingeräumt worden, sodass Steuervergünstigungen auch geltend gemacht werden dürfen, wenn die steuerlichen Werte nicht im Jahresabschluss enthalten sind ${ }^{1075}$. Steuerliche Aspekte beeinflussen heute in Finnland die Jahresabschlussaussage v. a. bei der Ermittlung der Abschreibungen ${ }^{1076}$ sowie bei der Bildung freiwilliger Rückstellun$\operatorname{gen}^{1077}$.

1067 Das Bilanzrichtliniengesetz trat mit seinen für diese Arbeit wesentlichen Teilen am 01. Januar 1986 in Kraft.

1068 Vgl. KapCoRiLiG in BGBl. I/2000, S. 154 ff. und vgl. BR-Drucksache 458/99, S. 20.

1069 Vgl. 4. EG-Richtlinie und vgl. BeBiKo (1999), § 264 Anm. 35.

1070 Hierzu zählen AktG, GmbHG, GenG, PublG, KWG und VAG.

1071 Vgl. § 243 Abs. 1 HGB, in dem der Gesetzgeber den unbestimmten Rechtsbegriff „Grundsätze ordnungsmäßiger Buchführung" einsetzt.

1072 Vgl. Järvenpää (1996), S. 899-905 und vgl. § 5 Abs. 1 S. 1 EStG.

1073 Vgl. Leppiniemi und Leppiniemi (1997), S. 13-14, vgl. Kap. 2.2.2.3 und vgl. § 5 Abs. 1 S. 2 EStG.

1074 Vgl. Järvenpää (1996), S. 899-905.

1075 Vgl. Kap. 2.2.2.3 dieser Arbeit.

1076 Vgl. EVL 54.2.

1077 Vgl. EVL 46 a. 
In Deutschland richtet sich die Erstellung des Einzelabschlusses generell stark nach den steuerlichen Vorschriften, wenngleich eine Abkopplung der steuerlichen Ansatz- und Bewertungsvorschriften von der handelsrechtlichen Bilanzierung in einigen Gebieten $\mathrm{zu}$ beobachten ist ${ }^{1078}$. Wesentliche Sachverhalte, in denen sich steuerrechtliche Regelungen auf den Jahresabschluss auswirken, umfassen die Abschreibungen sowie die Aktivierung von Material- und Fertigungsgemeinkosten im Vorratsvermögen ${ }^{1079}$.

\subsection{Gesetzgebung betreffend die Wirtschaftsprüfung}

In Finnland werden Zugang, Organisation und Anforderungen an den Berufsstand sowie die Berufsaufsicht im Wirtschaftsprüfungsgesetz und in der Wirtschaftsprüfungsverordnung geregelt. Die Umsetzung der 8. EG-Richtlinie ist im Wirtschaftsprüfungsgesetz vollzogen. Es enthält auch internationale Prüfungsstandards. Das finnische Wirtschaftsprüfungsgesetz wirkt indirekt auf die Jahresabschlusserstellung ein, weil jeder Buchführungspflichtige auch prüfungspflichtig ist.

In Deutschland existiert kein dem finnischen vergleichbares Wirtschaftsprüfungsgesetz. Die 8. EG-Richtlinie hat der Gesetzgeber mit den $\S \S 316-324$ HGB in nationales Recht transformiert. Vorschriften für und über den Berufsstand sind darüber hinaus in der Wirtschaftsprüferordnung und der Berufssatzung der Wirtschaftsprüferkammer enthalten. Dieser Grundsatz der Selbstverwaltung und Deregulierung wird derzeit diskutiert ${ }^{1080}$.

1078 Vgl. BeBiKo (1999), § 243 Anm. 122 a-c, wo Beispiele für Abweichungen zwischen Handels- und Steuerbilanz aufgeführt sind, die sich aus dem Steuerentlastungsgesetz 1999/2000/2002 ergeben.

1079 Vgl. § 7 EStG und vgl. R 33 Abs. 1, Abs. 2 und Abs. 3 EStR.

1080 Vgl. beispielsweise Ernst, Christoph: Peer Review oder Bundesaufsichtsamt: brauchen wir eine Kontrolle der Kontrolleure?, in: Der Wirtschaftsprüfer als Element der Corporate Governance: Vorträge des 12. Bonner Europa-Symposions, Düsseldorf 2001, S. 141-146, vgl. Hommelhoff, ebenda, S. 147-156, vgl. van Hulle, Karel, ebenda, S. 157-162 und vgl. Schmidt, Stefan: Externe Qualitätskontrollen zur Sicherung der Qualität der Abschlussprüfung, Düsseldorf 2002. 


\subsubsection{Buchführungsausschuss und Rechnungslegungsinstitut}

In Finnland ist 1974 als Unterabteilung des Ministeriums für Handel und Industrie der Buchführungsausschuss „Kirjanpitolautakunta“, KILA, gegründet worden ${ }^{1081}$. Seine Aufgaben liegen darin,

- Stellungnahmen und Kommentare zu rechnungslegungsspezifischen Fragen zu liefern,

- bestehende Gesetze zu interpretieren und zu erklären und

- die Handhabung bestehender Vorschriften für die Praxis zu verdeutlichen, mit dem Ziel, die Art guter Buchführung und die Grundsätze ordnungsmäßiger Buchführung zu definieren und weiterzuentwickeln ${ }^{1082}$.

Der KILA veröffentlicht zu diesem Zweck ,allgemeingültige Anweisungen“, die von allen Buchführungspflichtigen verbindlich zu befolgen sind. Er erteilt zudem auf Anfrage von Unternehmen, Verbänden oder Behörden Auskunft zur Lösung von Rechnungslegungsproblemen in Form von

- Gutachten,

- Entscheidungen oder

- Erlaubnissen, in Einzelfragen von Vorschriften abzuweichen ${ }^{1083}$.

Diesen Rechnungslegungshinweisen fehlt zwar die rechtliche Verbindlichkeit, aber die Gerichte orientieren sich bei ihrer Entscheidungsfindung an ihnen ${ }^{1084}$.

In Deutschland wurde im März 1998 das Rechnungslegungsinstitut „Deutsche Rechnungslegungs Standards Committee e.V. - DRSC“" geschaffen ${ }^{1085}$. Sein Verwaltungsrat bestimmt die sieben Mitglieder des Deutschen Standardisierungsrats (DSR), dessen Aufgaben umfassen ${ }^{1086}$ :

- die Entwicklung von Empfehlungen zur Anwendung der Grundsätze über die Konzernrechnungslegung,

- die Beratung des Bundesministeriums der Justiz bei der Fortentwicklung der Rechnungslegung und Gesetzgebungsvorhaben zu Rechnungslegungsvorschriften und

die Vertretung der Bundesrepublik Deutschland in internationalen Standardisierungsgremien.

Die Rollen des DRSC und des Gesetzgebers sind noch nicht klar und juristisch zweifelsfrei abgegrenzt ${ }^{1087}$. Die vom DSR entwickelten Deutschen Rechnungslegungs Standards (DRS) werden vom Bundesministerium der Justiz nach $\S 342$ Abs. 2 HGB veröffentlicht und sind dann nach herrschender Meinung als Grund-

1081 Vgl. KPL (1973) § 34.

1082 Vgl. KPL 8:2 und KPL 8:3.

1083 Vgl. www.vn.fi/ktm/kirjanpitolautakunta, wo alle allgemeingültigen Anweisungen, Gutachten, Entscheidungen sowie Erlaubnisse abrufbar sind.

1084 Vgl. Kap. 2.2.1.2 dieser Arbeit.

1085 Vgl. Zitzelsberger (1998), S. 1018.

1086 Vgl. § 342 Abs. 1 HGB.

1087 Vgl. Pellens (1999), S. 548. 
sätze ordnungsmäßiger Buchführung der Konzernrechnungslegung anzusehen ${ }^{1088}$. Welchen Verpflichtungsgrad die DRS für die Konzernrechnungslegung innehalten, ist noch offen; ihre Anwendung wird für Einzelabschlüsse empfohlen ${ }^{1089}$. Es stellt sich die Frage, ob eine von ihnen abweichende Bilanzierung als GoBkonform gelten kann. Diese Frage kann letztlich nur von den Gerichten beantwortet werden; ein Gerichtsentscheid steht aus ${ }^{1090}$.

Der wesentliche Unterschied in der Arbeit beider Institutionen liegt darin, dass der KILA für sämtliche die Rechnungslegung betreffenden Fragestellungen zuständig ist, während der DSR sich ausschließlich auf die Konzernrechnungslegung konzentriert. Der KILA geht seinen Aufgaben seit 1974 nach, handelt routiniert und wird allgemein anerkannt. Ob das DRSC nationale Anerkennung findet, wird sich zeigen.

\subsubsection{Wirtschaftsprüfende Berufsstände}

In Finnland hat der staatliche Wirtschaftsprüfungsausschuss „Tilintarkastuslautakunta“, TILA, im Ministerium für Handel und Industrie folgende Aufgaben:

- Erteilung von Anweisungen und Stellungnahmen zum Wirtschaftsprüfungsgesetz und zur Wirtschaftsprüfungsverordnung,

- Vorschläge und Kommentare zur Entwicklung prüfungsrelevanter Vorschriften und

- Anleitung, Entwicklung und Beaufsichtigung im Wirtschaftsprüfungswesen.

Der TILA nimmt indirekt Einfluss auf die Rechnungslegung. Die von ihm entwickelten prüfungsrelevanten Vorschriften werden von den Wirtschaftsprüfern umgesetzt, sodass diese in Ausübung ihres Berufs das Bilanzierungsverhalten der Unternehmen beeinflussen ${ }^{1091}$.

Die Berufsverbände der finnischen KHT-Wirtschaftsprüfer und HTM-Wirtschaftsprüfer nehmen mit Veröffentlichungen von erklärendem oder ergänzendem Inhalt aktiv an der Rechnungslegungsentwicklung teil. Sie erstellen keine eigenen Standards, sondern lehnen ihre Verlautbarungen und Empfehlungen überwiegend an die Standards des IASB an. Die Entwicklung der Grundsätze ordnungsmäßiger Abschlussprüfung und Prüfungsstandards erfolgt ebenfalls in Anlehnung an internationale Standards, wie den von der IFAC veröffentlichten ISA. Diese Grundsät-

\footnotetext{
1088 Vgl. $\$ 342$ Abs.2 HGB.

1089 Aus diesem Grund wird in der folgenden Vergleichsanalyse teilweise auf die DRS verwiesen, obwohl sich diese Arbeit nicht mit Konzernabschlüssen, sondern lediglich mit Einzelabschlüssen auseinandersetzt.

1090 Vgl. Pellens (1999), S. 543-544.

1091 Vgl. Kap. 2.2.2.4 dieser Arbeit.
} 
ze ordnungsmäßiger Abschlussprüfung üben indirekt Einflussnahme auf die Rechnungslegung aus ${ }^{1092}$.

In Deutschland gibt das Institut der Wirtschaftsprüfer in Deutschland e.V. (IDW) Hinweise zur Auslegung von Gesetzen. Es veröffentlicht seine Verlautbarungen in Form von Prüfungsstandards, Prüfungshinweisen, Standards, Rechnungslegungshinweisen sowie Stellungnahmen und nimmt so aktiv an der Rechnungslegungsentwicklung teil ${ }^{1093}$.

\subsubsection{Kommentierung zur Rechnungslegung und Meinungsvielfalt}

In Finnland existieren Kommentare und Lehrbücher, verfasst von Wissenschaftlern, Fachleuten aus der Wirtschaft und Wirtschaftsprüfern. Ihnen kommt in der Ausbildung eine große Bedeutung zu. In der Bilanzierungspraxis spiegelt sich die Meinungsvielfalt jedoch kaum wider. Die finnische und deutsche Mentalität unterscheiden sich darin, dass die finnische Gesellschaft tendenziell eher auf Konsens und Kooperation ausgerichtet ist als die deutsche. Kristallisiert sich nicht innerhalb angemessener Zeit eine Meinung als richtungsweisend heraus, wenden sich die Bilanzierenden zu ihrem eigenen Schutz an den KILA und bitten um Lösung des Sachverhalts. Die vom KILA veröffentlichten, für alle Buchführungspflichtigen verbindlichen Verlautbarungen tragen wesentlich zur Vereinheitlichung der Bilanzierung bei ${ }^{1094}$.

In Deutschland werden zahlreiche Kommentare zur Rechnungslegung von Sachverständigen aus Theorie und Praxis veröffentlicht. Mehr- und Mindermeinungen kommen in der Praxis zum Ausdruck.

1092 Vgl. Kap. 2.2.1.3.1 dieser Arbeit.

1093 Vgl. WPH I (2000), Abschnitt B.

1094 Vgl. Artsberg (2000), S. 544 und vgl. Kap. 2.2.1.3.2 dieser Arbeit. 


\subsubsection{Grundlagen der Rechnungslegung}

\subsubsection{Zielsetzung und Adressaten der Rechnungslegung}

Primäres Ziel der finnischen Rechnungslegung ist traditionell die Ermittlung des ausschüttungsfähigen Gewinns ${ }^{1095}$. Dieses Ziel steht in der Praxis im Konflikt mit dem Ziel der Gewinnminimierung aus steuerlichen Überlegungen, der Bildung stiller Reserven unter Hinweis auf das Vorsichtsprinzip ${ }^{1096}$. Mit der Transformation der 4. EG-Richtlinie in nationales Recht sind diese Ziele um die Vermittlung des True and Fair View, den Gesellschafterschutz und den Gläubigerschutz ergänzt worden ${ }^{1097}$. Dennoch wird das Periodenergebnis nach wie vor betont. Deshalb wird das Prinzip der Periodenabgrenzung bzw. leistungsabhängiger Buchführung hervorgehoben ${ }^{1098}$. Zentrale Bedeutung hat nach wie vor die Gewinn- und Verlustrechnung, auch wenn die Bilanz durch Umsetzung der 4. EG-Richtlinie an Bedeutung gewonnen hat ${ }^{1099}$.

Finnische Unternehmen operieren zunehmend auf internationalen Märkten und finanzieren sich auch auf ausländischen Kapitalmärkten. Sie müssen die von ihnen veröffentlichten Informationen an die Erwartungen und Forderungen ausländischer Leser anpassen ${ }^{1100}$. Die Forderung nach einer Anlegerorientierung der Rechnungslegung steigt ${ }^{1101}$.

In Deutschland ist das primäre Ziel der Rechnungslegung traditionell der Gesellschafter- und Gläubigerschutz ${ }^{1102}$. Die Mehrzahl der deutschen Unternehmen finanziert sich durch Fremdkapital, was zu einem Schwerpunkt auf der Bilanz und dem Gläubigerschutzes führt ${ }^{1103}$. Die Betonung liegt auf dem Vorsichtsprinzip ${ }^{1104}$. Die Banken als wichtigste Kapitalgeber haben Zugang zu den finanziellen Informationen der Unternehmen, sodass der Druck auf die Unternehmen relativ gering ist, finanzielle Informationen zu veröffentlichen.

In Deutschland sind nach wie vor verhältnismäßig wenige Unternehmen börsennotiert, auch wenn ihre Anzahl steigt und sich vor allem die Großunternehmen verstärkt an den internationalen Finanzmärkten finanzieren. Dies führt zu einer Orientierung der Rechnungslegung von börsennotierten Unternehmen und Kon-

\footnotetext{
1095 Vgl. KPL-Arbeitsgruppe 1995, Teil I (1996), S. 17.

1096 Vgl. KPL 3:3.1,2.

1097 Vgl. KPL-Arbeitsgruppe 1995, Teil I (1996), S. 19.

1098 Vgl. Artsberg (2000), S. 543-544.

1099 Vgl. Kap. 3.1.1 dieser Arbeit.

1100 Vgl. KPL-Arbeitsgruppe 1995, Teil I (1996), S. 16.

1101 Vgl. HE 173/1997, Abschnitt 1.2.

1102 Vgl. BeBiKo (1999), § 264 Anm. 35.

1103 Vgl. Pellens (1999), S. 28.

1104 Vgl. § 252 Abs. 1 Nr. 4 HGB.
} 
zernen an IAS und US-GAAP ${ }^{1105}$, zumal für am Neuen Markt in Deutschland gehandelte Unternehmen eine Aufstellung des Jahresabschlusses nach einem dieser beiden Rechnungslegungssysteme verpflichtend ist ${ }^{1106}$.

\subsubsection{Instrumente der Rechnungslegung}

\subsection{Bestandteile des Jahresabschlusses}

In Finnland besteht der Jahresabschluss von Unternehmen sämtlicher Rechtsformen und Größen aus

- Bilanz,

- Gewinn- und Verlustrechnung,

- Anhang und

- Lagebericht $^{1107}$.

Dass der Lagebericht eigenständiger und gleichwertiger Bestandteil des Jahresabschlusses ist, hebt die große Bedeutung hervor, die der Gesetzgeber ihm beimisst. Eine Kapitalflussrechnung muss von öffentlichen Aktiengesellschaften und von großen privaten Aktiengesellschaften als Bestandteil des Lageberichts im Einzelabschluss aufgestellt werden ${ }^{1108}$. Aufbau und Gliederung der Kapitalflussrechnung werden in einer allgemeingültigen Anweisung vom KILA geregelt, die sich an IAS 7 (revised 1992) orientiert $^{109}$.

In Deutschland besteht der Jahresabschluss einer Kapitalgesellschaft aus

- Bilanz,

- Gewinn- und Verlustrechnung sowie

- Anhang ${ }^{1110}$.

Zusätzlich müssen mittelgroße und große Kapitalgesellschaften einen Lagebericht aufstellen ${ }^{111}$. Eine Kapitalflussrechnung ist für börsennotierte Mutterunternehmen als Bestandteil des Konzernanhangs vorgeschrieben ${ }^{1112}$. Aufbau und Gliederung von Kapitalflussrechnungen werden in der HFA-Stellungnahme 1/1995 und im DRS 2 des DRSC erläutert.

1105 Vgl. Pellens (1999), S. 7 ff.

1106 Die Europäische Kommission hat zudem entschieden, dass alle europäischen börsennotierten Unternehmen ab 2005 ihre Konzernabschlüsse nach IAS aufzustellen haben.

1107 Vgl. KPL 3:3.1.

1108 Vgl. OYL 11:9.7.

1109 Vgl. KILA-Anweisung „Kapitalflussrechnung“. Vgl. auch Kap. 3.1.2 dieser Arbeit.

1110 Vgl. § 264 Abs. 1 HGB i. V. m. § 242 Abs. 3 HGB.

1111 Vgl. § 264 Abs. 1 HGB.

1112 Vgl. § 287 Abs. 1 S. 2 HGB. 
Sowohl in Finnland als auch in Deutschland muss eine Muttergesellschaft einen eigenständigen Jahresabschluss aufstellen, auch wenn ein Konzernabschluss erstellt wird ${ }^{1113}$.

Freiwillige Angaben im Anhang oder Lagebericht sind in beiden Staaten zulässig, sofern sie die Klarheit und Übersichtlichkeit des Jahresabschlusses nicht beeinträchtigen. Freiwillige Ergänzungen wie Umwelt-, Personal- oder Sozialbilanzen sind ebenfalls erlaubt.

\subsection{Gliederung der Bilanz}

In Finnland sind größenspezifische Bilanzgliederungsschemata in der Buchführungsverordnung auf Basis der 4. EG-Richtlinie vorgegeben ${ }^{114}$. Diese Schemata weichen beim Sachanlagevermögen und bei steuerlichen Ausgleichsposten von der Richtlinie ab, ohne dass hierdurch die Richtlinienkonformität infrage gestellt wird $^{1115}$.

In Deutschland ist die Bilanzgliederung durch $§ 266$ Abs. 2 HGB für Kapitalgesellschaften vorgegeben.

\subsection{Gliederung der Gewinn- und Verlustrechnung}

Sowohl in Finnland als auch in Deutschland sind

- das Gesamtkostenverfahren ${ }^{1116}$ und

- das Umsatzkostenverfahren ${ }^{1117}$

für die Gewinn- und Verlustrechnung zulässig. Das Gesamtkostenverfahren gliedert den Aufwand nach Aufwandsarten, während bei dem Umsatzkostenverfahren die Gliederung umsatzbezogen nach Funktionsbereichen erfolgt. In beiden Staaten ist die Gliederung beider Verfahren durch den Gesetzgeber vorgegeben.

In Finnland sind darüber hinaus rechtsformspezifische Gliederungsschemata für Nicht-Kapitalgesellschaften vorgegeben ${ }^{1118}$.

1113 Vgl. KPL 6:1 bzw. § 264 Abs. 3 HGB.

1114 Vgl. KPA 1:6 und KPA 1:7. Die Gliederungen sind im Anhang dieser Arbeit zu finden.

1115 Vgl. Kap. 3.1.2.1 und Kap. 3.2.5 dieser Arbeit.

1116 Vgl. KPA 1:1 bzw. § 275 Abs. 2 HGB.

1117 Vgl. KPA 1:2. Die Gliederungen des finnischen Gesamt- und Umsatzkostenverfahrens sind im Anhang dieser Arbeit zu finden. Vgl. § 275 Abs. 3 HGB.

1118 Vgl. KPA 1:3 für Stiftungen, KPA 1:4 für Liegenschaften und KPA 1:5 für Einzelunternehmer. 


\subsection{Abweichungen von der gesetzlich vorgegebenen Gliederung}

Ein Abweichen von den Gliederungsschemata der Buchführungsverordnung ist in Finnland nicht gestattet. Die Gliederungsschemata des KPA sind jedoch weiter zu untergliedern, wenn dies der Klarheit und Übersichtlichkeit sowie der Vermittlung eines True and Fair View dient ${ }^{1119}$.

In Deutschland ist von den Gliederungsschemata des HGB abzuweichen, wenn es wegen der Besonderheiten der Gesellschaft zur Aufstellung eines klaren und übersichtlichen Jahresabschlusses erforderlich ist ${ }^{1120}$. Darüber hinaus ist eine weitere Untergliederung der Posten zulässig ${ }^{1121}$.

\subsection{Erleichterungen bei Erstellung des Jahresabschlusses}

In Finnland haben die nach KPL 3:9.2 als klein klassifizierten Unternehmen das Wahlrecht, verkürzte Bilanzen zu erstellen ${ }^{1122}$. In der Bilanz müssen nur die Posten, denen im gesetzlichen Gliederungsschema ein Buchstabe oder eine römische Zahl zugeordnet ist, gezeigt werden ${ }^{1123}$.

Für alle anderen Bestandteile des Jahresabschlusses sind keine Vereinfachungen vorgesehen.

In Deutschland haben die nach $\S 267$ Abs. 1 HGB als klein klassifizierten Unternehmen grundsätzlich die Möglichkeit, verkürzte Jahresabschlüsse zu erstellen und sind berechtigt, diese den Gesellschaftern vorzulegen ${ }^{124}$. Die Vereinfachungen in der Berichterstattung beziehen sich auf die Bilanz, die Gewinn- und Verlustrechnung und den Anhang. Im Wesentlichen handelt es sich um folgende Erleichterungen:

- In der Bilanz müssen nur die Posten, denen im gesetzlichen Gliederungsschema ein Buchstabe oder eine römische Zahl zugeordnet ist, ausgewiesen werden ${ }^{125}$.

- In der Gewinn- und Verlustrechnung dürfen beim Gesamtkostenverfahren die Posten Nr. 1 bis 5 des $\S 275$ Abs. 2 HGB und beim Umsatzkostenverfahren die Posten Nr. 1 bis 3 und 6 des $\S 275$ Abs. 3 HGB zum „Rohergebnis“" zusammengefasst werden ${ }^{1126}$.

\footnotetext{
1119 Vgl. KPA 1:9 und vgl. Kap. 3.1.2.1 dieser Arbeit.

1120 Vgl. § 266 Abs. 1 S. 1 HGB.

1121 Vgl. § 266 Abs. 5 HGB.

1122 Vgl. KPA 1:7.

1123 Vgl. KPA 1:7.

1124 Vgl. § 266 Abs. 1 S. 3 HGB.

1125 Vgl. § 266 Abs. 1 S. 3 HGB.

1126 Vgl. § 276 S. 1 HGB.
} 
- Auf die Aufstellung eines Anlagespiegels darf verzichtet werden ${ }^{1127}$.

- Pflichtangaben im Anhang über Forderungen, Verbindlichkeiten und außerordentliche Posten entfallen ${ }^{128}$.

- Ein Lagebericht braucht nicht erstellt zu werden ${ }^{1129}$.

Die hier zur Gewinn- und Verlustrechnung aufgeführte Erleichterung gilt auch für mittelgroße Gesellschaften im Sinne des $\S 267$ Abs. 2 HGB $^{1130}$.

Die genannten Erleichterungen gelten nicht für Unternehmen, die börsennotiert sind oder deren Anteile im Freiverkehr gehandelt werden, da diese Unternehmen immer als große Gesellschaften im Sinne des HGB eingestuft werden ${ }^{1131}$.

Falls eine Aktiengesellschaft von den Erleichterungen für kleine oder mittelgroße Unternehmen Gebrauch macht, so kann jeder Aktionär verlangen, dass ihm in der Hauptversammlung ein Jahresabschluss vorgelegt wird, in dem diese Erleichterungen nicht angewendet worden sind ${ }^{132}$.

\subsection{Prüfungspflicht}

In Finnland unterliegt jeder Kaufmann, der zur Buchführung verpflichtet ist, der Prüfungspflicht ${ }^{1133}$.

In Deutschland besteht Prüfungspflicht für alle Kapitalgesellschaften, die im Sinne des $\S 267$ Abs. 1 HGB nicht als „klein“ eingestuft sind ${ }^{1134}$. Seit In-Kraft-Treten des Gesetzes zur Kontrolle und Transparenz im Unternehmensbereich (KonTraG) am 28. April 1998 entfällt die Prüfungspflicht von Tochterunternehmen eines zur Aufstellung eines Konzernabschlusses verpflichteten Mutterunternehmens unter den folgenden Voraussetzungen:

- $\quad$ Alle Gesellschafter müssen der Befreiung zugestimmt haben und dieser Beschluss muss offen gelegt werden ${ }^{1135}$,

- das Mutterunternehmen ist zur Verlustübernahme verpflichtet ${ }^{1136}$,

- das Tochterunternehmen ist in den Konzernabschluss einbezogen worden ${ }^{1137}$ und

1127 Vgl. $\$ 274$ a Nr. 1 HGB.

1128 Vgl. $\$ 274$ a Nr. 2 und 3 HGB, § 276 HGB.

1129 Vgl. § 264 Abs. 1 S. 1 HGB.

1130 Vgl. § 276 HGB.

1131 Vgl. § 267 Abs. 3 S. 2 HGB.

1132 Vgl. \$ 131 Abs. 1 S. 3 AktG.

1133 Vgl. TilintL 3:9 i. V. m. rechtsformspezifischer Gesetzgebung und vgl. Kap. 2.2.1.3.1 dieser Arbeit.

1134 Vgl. $\$ 316$ Abs. 1 HGB.

1135 Vgl. § 264 Abs. 3 Nr. 1 HGB und vgl. WPH I (2000), F Anm. 14 ff.

1136 Vgl. $\$ 264$ Abs. 3 Nr. 2 HGB.

1137 Vgl. § 264 Abs. 3 Nr. 3 HGB. 
- $\quad$ die Befreiung des Tochterunternehmens muss im Konzernanhang angegeben werden $^{1138}$.

- Die vom Mutterunternehmen offen zu legenden Unterlagen sind auch beim Handelsregister des befreiten Tochterunternehmens eingereicht worden $^{1139}$.

Die Prüfungspflicht ist auf Personenhandelsgesellschaften, Einzelgesellschaften, Stiftungen, Körperschaften und andere Unternehmen erweitert, die in $\S 3$ Abs. 1 PublG genannt sind ${ }^{1140}$.

\subsection{Offenlegungspflicht}

\subsection{Jährliche Anforderungen}

Grundsätzlich müssen finnische Gesellschaften ihren Abschluss ${ }^{1141}$ mit dem Bestätigungsvermerk des Wirtschaftsprüfers ${ }^{1142}$ bis spätestens sechs Monate nach dem Stichtag beim Handelsregister hinterlegen. Ausgenommen hiervon sind Aktiengesellschaften und Genossenschaften, die sämtliche Unterlagen innerhalb von acht Monaten nach dem Abschluss-Stichtag einreichen müssen, sofern der Abschluss auf der ordentlichen Hauptversammlung festgestellt worden ist. Wird dieser erst auf der Folgeversammlung der ordentlichen Hauptversammlung festgestellt, hat das Unternehmen zehn Monate Zeit, ihn zu hinterlegen.

Beim Handelsregister sind die Unterlagen von jedermann einsehbar, sodass die Offenlegungspflicht mit der Einreichung erfüllt ist ${ }^{1143}$.

In Deutschland haben Kapitalgesellschaften und haftungsbeschränkte Personenhandelsgesellschaften ihren Jahresabschluss und den Lagebericht mit dem Testat des Abschlussprüfers beim Handelsregister einzureichen ${ }^{1144}$. Zusätzlich sind der Gewinnverwendungsvorschlag und der Gewinnverwendungsbeschluss einzureichen, falls diese nicht im Jahresabschluss enthalten sind. Gegebenenfalls ist ein Bericht des Aufsichtsrats einzureichen ${ }^{1145}$.

Große und mittelgroße Gesellschaften müssen die genannten Unterlagen spätestens neun Monate ${ }^{1146}$, kleine Gesellschaften spätestens zwölf Monate nach dem Bilanzstichtag hinterlegen ${ }^{1147}$.

\footnotetext{
1138 Vgl. § 264 Abs. 3 Nr. 4 HGB.

1139 Vgl. § 264 Abs. 3 Nr. 5 HGB.

1140 Vgl. § 3 PublG.

1141 Vgl. KPL 3:9 und vgl. Kap 2.2.2.2.2 und Kap. 2.2.2.2.3 dieser Arbeit.

1142 Vgl. KPL 3:12.

1143 Vgl. KPL 3:10.

1144 Vgl. § 325 Abs. 1 S. 1 HGB.

1145 Vgl. § 325 Abs. 1 S. 1 HGB.

1146 Vgl. § 325 Abs. 1 HGB.
} 
Zuvor haben große Gesellschaften sämtliche Unterlagen im Bundesanzeiger zu veröffentlichen $^{1148}$, während mittelgroße und kleine Gesellschaften nur veröffentlichen müssen, bei welchem Handelsregister und unter welcher Nummer diese Unterlagen hinterlegt worden sind ${ }^{1149}$.

\subsection{Erleichterungen}

In Finnland sind bislang keine Erleichterungen der Offenlegungspflicht kodifiziert. Ein Gesetzesentwurf darüber, dass kleine Unternehmen lediglich Anhang und Bilanz offen zu legen haben, liegt jedoch vor ${ }^{1150}$.

In Deutschland existieren Erleichterungen der Offenlegungspflicht für kleine und mittelgroße Unternehmen.

Kleine Unternehmen müssen lediglich Bilanz und Anhang offen legen ${ }^{1151}$. Der zur Veröffentlichung vorgesehene Anhang muss die Angaben, welche die Gewinnund Verlustrechnung betreffen, nicht enthalten ${ }^{1152}$. Der Gewinnverwendungsvorschlag und der Gewinnverwendungsbeschluss, der den Jahresüberschuss bzw. fehlbetrag zu enthalten hat, sind zusätzlich einzureichen, soweit sie sich nicht aus Bilanz oder Anhang ergeben ${ }^{1153}$.

Mittelgroße Unternehmen dürfen die Bilanz nach verkürztem Gliederungsschema offen legen. Ergänzend müssen sie dann Posten in der Bilanz oder im Anhang angeben, die das Anlagevermögen und die Beziehungen zu beteiligten Unternehmen betreffen. Anhangangaben zu Verbindlichkeiten, zur Ergebnisbeeinflussung auf Grund der Anwendung steuerrechtlicher Vorschriften, zu sonstigen Rückstellungen sowie zum Material- und Personalaufwand bei Anwendung des Umsatzkostenverfahrens brauchen nicht veröffentlicht zu werden ${ }^{1154}$.

Um die Erleichterungen für kleine und mittelgroße Unternehmen wahrnehmen zu können, ist kein besonderer Bericht des Abschlussprüfers erforderlich. Ist der Jahresabschluss von einem Abschlussprüfer auf Grund gesetzlicher Vorschriften geprüft worden, ist der Bestätigungsvermerk oder der Vermerk über dessen Versagung wiederzugeben. Wird der Jahresabschluss wegen der Inanspruchnahme von Erleichterungen nur teilweise offen gelegt und bezieht sich der Bestätigungsvermerk auf den vollständigen Jahresabschluss, so ist hierauf bei der Offenlegung hinzuweisen $^{1155}$.

\footnotetext{
1147 Vgl. § 326 HGB.

1148 Vgl. § 325 Abs. 2 HGB.

1149 Vgl. § 325 Abs. 1 S. 2 HGB.

1150 Vgl. KTM (2000), S. 28-33.

1151 Vgl. § 326 S. 1 HGB.

1152 Vgl. § 326 S. 2 HGB.

1153 Vgl. § 325 Abs. 1 S. 1 HGB.

1154 Vgl. § 327 Abs. 1 HGB.

1155 Vgl. § 328 Abs. 1 Nr. 1 HGB.
} 
Angaben über die Ergebnisverwendung von Gesellschaften mit beschränkter Haftung sind nicht zwingend, sofern sich anhand dieser Angaben die Gewinnanteile von natürlichen Personen feststellen lassen, die Gesellschafter sind ${ }^{1156}$.

\subsection{Sanktionen}

Hat eine finnische Aktiengesellschaft trotz Aufforderung durch den zuständigen Sachbearbeiter ihre Unterlagen nicht innerhalb eines Jahres nach Bilanzstichtag beim Handelsregister eingereicht, bestimmt der Registerrichter die Liquidation $^{1157}$. Die Gesellschaft kann im Handelsregister gelöscht werden, wenn sie ihre Unterlagen über einen Zeitraum von zehn Jahren nicht offen gelegt hat ${ }^{1158}$. Wegen der Härte dieser Sanktionen kommen finnische Unternehmen regelmäßig ihrer Offenlegungspflicht nach.

In Deutschland wurde die Offenlegungspflicht von den meisten Gesellschaften mit beschränkter Haftung bislang $u$. a. deshalb ignoriert, weil die Bußgelder für Nicht-Einhaltung der Einreichungsanforderungen gering sind. Da die meisten Unternehmen in der Rechtsform der $\mathrm{GmbH}$ firmieren, standen der Öffentlichkeit nur wenig Informationen über diese Unternehmen zur Verfügung ${ }^{159}$. Nach

KapCoRiLiG stehen den Registergerichten jetzt das Zwangsgeldverfahren ${ }^{1160}$ und das Ordnungsgeldverfahren ${ }^{1161}$ als Sanktionsmaßnahmen zur Verfügung, wenn Unternehmen ihre Offenlegungspflichten nicht erfüllen. Ob diese Vorschriften die bisher unbefriedigenden Publizitätspraktiken der Unternehmen verbessern, bleibt abzuwarten $^{1162}$.

\footnotetext{
1156 Vgl. § 325 Abs. 1 S. 1 Abs. 3 HGB.

1157 Vgl. OYL 13:4.1,3.

1158 Vgl. OYL 13:18.1.

1159 Vgl. Baetge, Kirsch und Thiele (2001), S. 41.

1160 Vgl. § 335 HGB.

1161 Vgl. § 335 a HGB.

1162 Vgl. Baetge, Kirsch und Thiele (2001), S. 41.
} 


\subsubsection{Grundsätze ordnungsmäßiger Buchführung}

\subsection{Kodifizierte Grundsätze ordnungsmäßiger Buchführung}

Die in der 4. EG-Richtlinie ${ }^{1163}$ namentlich aufgeführten zentralen Jahresabschlussgrundsätze sind sowohl in Finnland als auch in Deutschland explizit in nationales Recht umgesetzt:

- Grundsatz der Fortführung der Unternehmenstätigkeit (Going Concern $)^{1164}$

- Grundsatz der Stetigkeit ${ }^{165}$,

- Grundsatz der Vorsicht mit seinen Ausprägungen Realisationsprinzip, Imparitätsprinzip und Wertaufhellungsprinzip ${ }^{166}$,

- Grundsatz der Bilanzkontinuität ${ }^{1167}$,

- Grundsatz der leistungsbezogenen Abgrenzung (Periodenabgrenzung) $)^{1168}$ und

- Grundsatz der Einzelbewertung ${ }^{1169}$.

Sowohl in Finnland als auch in Deutschland wird bei der Bilanzierung angenommen, dass das Unternehmen seine Tätigkeit in Zukunft fortsetzen wird. Unterschiedlich wird in beiden Ländern nach herrschender Meinung beurteilt, wie lang der Zeitraum sein muss, für den die Unternehmensfortführung noch mit hinreichender Sicherheit erwartet werden kann. In Finnland wird von einer Unternehmensfortführung „bis auf Weiteres“ ausgegangen. Es wird hier ein Zeitraum von mehr als zwölf Monaten zu Grunde gelegt ${ }^{1170}$, während es in Deutschland im Allgemeinen als ausreichend angesehen wird, wenn im Zeitpunkt der Jahresabschlusserstellung der Going Concern für das volle, auf den Stichtag folgende Geschäftsjahr erwartet werden kann ${ }^{1171}$. Abweichungen von diesen Grundsätzen können aber in beiden Ländern unter unternehmensspezifischen Gesichtspunkten geboten $\operatorname{sein}^{1172}$.

Der Grundsatz der Stetigkeit wird in Finnland auf Ansatz und Bewertung von Sachverhalten bezogen, in Deutschland lediglich auf die Bewertung. In beiden Staaten wird er so aufgefasst, dass eine einmal gewählte Bilanzierungs- bzw. Be-

1163 Vgl. Art. 31 der 4. EG-Richtlinie.

1164 Vgl. KPL 3:3.1 Nr. 1, vgl. Kap. 3.1.3.2 dieser Arbeit und vgl. § 252 Abs. 1 Nr. 2 HGB.

1165 Vgl. KPL 3:3.1 Nr. 2, vgl. Kap. 3.1.3.2 dieser Arbeit und vgl. § 252 Abs. 1 Nr. 6 HGB.

1166 Vgl. KPL 3:3.1 Nr. 3 und KPL 3:3.2, vgl. Kap. 3.1.3.2 dieser Arbeit und vgl. § 252 Abs. 1 Nr. 4 HGB.

1167 Vgl. KPL 3:3.1 Nr. 4, vgl. Kap. 3.1.3.2 dieser Arbeit und vgl. § 252 Abs. 1 Nr. 1 HGB.

1168 Vgl. KPL 3:3.1 Nr. 5, vgl. Kap. 3.1.3.2 dieser Arbeit und vgl. § 252 Abs. 1 Nr. 5 HGB.

1169 Vgl. KPL 3:3.1 Nr. 6, vgl. Kap. 3.1.3.2 dieser Arbeit und vgl. § 252 Abs. 1 Nr. 3 HGB.

1170 Vgl. Leppiniemi (2000 a), S. 43 und vgl. Kap. 3.1.3.2 dieser Arbeit.

1171 Vgl. ADS (ab 1995), § 252 Anm. 24 und die dort angegebene Literatur.

1172 Vgl. Leppiniemi (2000 a), S. 43 und vgl. ADS (ab 1995), § 252 Anm. 29. 
wertungsmethode beizubehalten ist, es sei denn, es gibt einen gewichtigen Grund, zu einer anderen Methode zu wechseln. Ein solcher Grund ist beispielsweise eine Änderung von Gesetz oder Rechtsprechung, eine Änderung der Konzernzugehörigkeit, der Gesellschafterstruktur oder eine wesentliche Informationsverbesserung im Hinblick auf den True and Fair View ${ }^{1173}$.

In Finnland wie in Deutschland wird das Vorsichtsprinzip dahingehend interpretiert, dass Vermögenswerte und Erträge nicht überbewertet, Verpflichtungen und Aufwendungen nicht unterbewertet werden dürfen ${ }^{1174}$. In beiden Ländern gleich definiert wird auch der Grundsatz der Periodenabgrenzung: Aufwendungen und Erträge eines Geschäftsjahrs sind unabhängig von den entsprechenden Zahlungen im Jahresabschluss zu berücksichtigen ${ }^{1175}$. Beide Prinzipien werden allerdings in Finnland und Deutschland unterschiedlich stark betont. Kodifizierte GoB werden in Finnland untereinander sowie im Verhältnis zu nicht kodifizierten GoB als gleichwertig angesehen ${ }^{1176}$, während in Deutschland durch Auslegung regelmäßig eine Hierarchie entsteht. Wichtigster Grundsatz in Deutschland ist wegen des Gesellschafter- und Gläubigerschutzgedankens das Vorsichtsprinzip, welches mit seinen Ausprägungen Realisations- und Imparitätsprinzip enger als in Finnland ausgelegt wird. Traditionell kommt in Finnland - wegen der Zielsetzung des Jahresabschlusses, den ausschüttungsfähigen Gewinn zu ermitteln - dem Grundsatz der Periodenabgrenzung eine größere und dem Vorsichtsprinzip eine kleinere Bedeutung zu als in Deutschland ${ }^{1177}$. Gewinne gehen deshalb in Deutschland oft später und Verluste oft früher in die Gewinn- und Verlustrechnung ein als in Finnland. Die unterschiedliche Interpretation von Vorsichtsprinzip und Periodenabgrenzung wird beispielsweise bei folgenden Sachverhalten deutlich:

- Während in Finnland ein Aktivierungswahlrecht für selbst erstellte immaterielle Vermögenswahlrechte existiert ${ }^{1178}$, ist ihre Aktivierung in Deutschland verboten ${ }^{1179}$.

- In Finnland gilt ein Neubewertungswahlrecht für Grundstücke, Wasserflächen und Finanzanlagen ${ }^{1180}$. In Deutschland herrscht wegen des Nominalwertprinzips ein Neubewertungsverbot ${ }^{1181}$.

1173 Vgl. KPL-Arbeitsgruppe 1995, Teil I (1996), S. 44, vgl. Kap. 3.1.3.2 dieser Arbeit und vgl. ADS (ab 1995), § 252 Anm. 112-116.

1174 Vgl. KPL-Arbeitsgruppe 1995, Teil I (1996), S. 44.

1175 Vgl. KPL-Arbeitsgruppe 1995, Teil I (1996), S. 28.

1176 Vgl. KPL-Arbeitsgruppe 1995, Teil I (1996), S. 42.

1177 Das Vorsichtsprinzip wurde in Finnland in der Vergangenheit wegen der mit ihm verbundenen Möglichkeit zur steuerlichen Gewinnminderung stark betont. Heutzutage wird die Bildung stiller Reserven wegen des zu vermittelnden True and Fair View von der Fachwelt kritisch beurteilt. Insofern hat ein Ansichtswechsel stattgefunden. Vgl. Kap. 3.1.3.4 dieser Arbeit.

1178 Vgl. KPL 5:7 bis KPL 5:11.

1179 Vgl. § 248 Abs. 2 HGB.

1180 Vgl. KPL 5:17.

1181 Vgl. $§ 253$ Abs. 1 S.1 HGB. In Deutschland wird derzeit eine Diskussion geführt, in der dieses Nominalwertprinzip zur Disposition gestellt wird. 
- Bei langfristiger Auftragsfertigung kann in Finnland unter bestimmten Voraussetzungen eine Teilgewinnrealisierung vorgenommen werden (Percentage of Completion-Methode) ${ }^{1182}$, während in Deutschland die Gewinnrealisierung im Regelfall erst nach Abschluss des Gesamtprojekts erfolgt (Completed Contract-Methode) ${ }^{1183}$.

- In Deutschland sind Unternehmen zur Bildung von Aufwandsrückstellungen verpflichtet ${ }^{184}$ bzw. berechtigt ${ }^{1185}$. In Finnland kann alternativ zur Rückstellungsbildung eine Anhangangabe gemacht werden ${ }^{1186}$.

In Finnland und in Deutschland wird die Übereinstimmung der Wertansätze der Eröffnungsbilanz mit den Wertansätzen der Schlussbilanz des vorhergehenden Geschäftsjahres verlangt (Bilanzkontinuität).

Nach finnischer wie nach deutscher Auffassung sind im Jahresabschluss ausgewiesene Vermögenswerte und Schulden am Abschluss-Stichtag einzeln zu bewerten. Ausgenommen von diesem Grundsatz der Einzelbewertung sind die Festwertbildung bei Gegenständen des Anlage- und des Umlaufvermögens sowie die Gruppenbewertung bei Gegenständen des Vorratsvermögens (Verbrauchsfolgeverfahren $)^{1187}$. In Deutschland ist darüber hinaus eine Abweichung vom Grundsatz der Einzelbewertung bei der Rückstellungsbildung erlaubt. Hier dürfen Sachverhalte zusammengefasst und anhand von Erfahrungswerten der Vergangenheit entsprechend dem Gesamtvolumen der wahrscheinlichen Risiken bewertet werden. Dies betrifft vor allem Gewährleistungsrückstellungen, Rückstellungen für Rabatte, Boni und Skonti sowie personalbezogene Rückstellungen (Urlaubsanspruch, Überstunden, Tantiemen) ${ }^{1188}$. In Finnland ist es üblich, diese Sachverhalte einzeln zu bewerten. Sie werden ihrem Charakter entsprechend dann nicht als Rückstellungen, sondern als sonstige Verbindlichkeiten oder passiver Rechnungsabgrenzungsposten ausgewiesen $^{1189}$.

\footnotetext{
1182 Vgl. KPL 5:4.

1183 Vgl. $\S 253$ Abs. 1 S. 1 HGB und $\S 255$ Abs. 2 und 3 HGB.

1184 Vgl. § 249 Abs. 1 S. 2 HGB.

1185 Vgl. § 249 Abs. 1 S. 3 und $\S 249$ Abs. 2 HGB.

1186 Vgl. KPL 5:14.2.

1187 Vgl. KPL 5:5.3 und KPL 5:6.3 bzw. vgl. § 240 Abs. 3 HGB, § 240 Abs. 4 HGB und $\S 256$ HGB.

1188 Vgl. BeBiKo (1999), § 252 Anm. 26.

1189 Vgl. KILA 1219/1993 und KILA 1561/1999.
} 


\subsection{True and Fair View}

\subsection{Charakter des True and Fair View}

In Finnland ist der True and Fair View Teil der Grundsätze ordnungsmäßiger Buchführung. Er unterscheidet sich aber von den anderen GoB, da er eine Zielvorstellung ist, welche die anderen GoB ergänzt ${ }^{1190}$. Es ist zu beachten, dass der Lagebericht in Finnland Bestandteil des Jahresabschlusses ist. Er enthält auch solche Informationen, die nicht der Buchhaltung entstammen und nicht den GoB unterliegen. Für diese Informationen ist der True and Fair View von besonderer Bedeutung.

In Deutschland hat der Jahresabschluss einer Kapitalgesellschaft unter Beachtung der GoB ein den tatsächlichen Verhältnissen entsprechendes Bild der Vermögens, Finanz- und Ertragslage zu vermitteln ${ }^{1191}$. Es wird davon ausgegangen, dass ein in Übereinstimmung mit den GoB und den handelsrechtlichen Einzelvorschriften erstellter Jahresabschluss dem True and Fair View entspricht. Der True and Fair View hat die Aufgabe, Regelungslücken zu schließen und Zweifelsfragen zu klären, welche die Einzelvorschriften offen lassen. Er ist den Einzelvorschriften untergeordnet, tritt aber im Konfliktfall insofern nicht hinter die Einzelvorschrift zurück, als dass er eine Zusatzangabe im Anhang bewirkt ${ }^{1192}$.

\subsection{Notwendige Zusatzangaben im Anhang}

In Finnland erfordert ein unter Beachtung der GoB und der Einzelnormen erstellter Jahresabschluss in der Regel keine Zusatzangaben im Anhang, um den True and Fair View zu vermitteln. Erreicht der Jahresabschluss trotz Anwendung gesetzlicher Einzelvorschriften und der GoB nicht die von ihm erwartete Aussagekraft, fordert das finnische Buchführungsgesetz Zusatzangaben im Anhang ${ }^{1193}$. Über die Notwendigkeit von Zusatzangaben hat der Gesetzgeber in 13 kodifizierten Fällen entschieden. Von diesen 13 Vorschriften erlauben zehn die Abweichung von Einzelvorschriften unter der Voraussetzung einer Anhangangabe, in drei weiteren Fällen sind nach Einzelnorm bilanzierte Sachverhalte zwingend um Zusatzangaben zu ergänzen. ${ }^{1194}$.

\footnotetext{
1190 Vgl. KPL 3:2.1.

1191 Vgl. § 264 Abs. 2 HGB. Diese Vorschrift geht zurück auf Art. 2 Abs. 3 der 4. EG-Richtlinie. Vgl. Schruff (1986), S. 28-29.

1192 Vgl. BeBiKo (1999), § 264 Anm. 31.

1193 Vgl. KPL 3:2.1.

1194 Vgl. Kap. 3.1.3.4.4 dieser Arbeit.
} 
Vermittelt in Deutschland der Jahresabschluss auf Grund besonderer Umstände keinen True and Fair View, sind zusätzliche Angaben im Anhang zu machen ${ }^{1195}$. Über die Notwendigkeit von Zusatzangaben entscheiden das den Jahresabschluss erstellende Unternehmen und sein(e) Wirtschaftsprüfer ${ }^{1196}$.

\subsection{True and Fair View-Override}

Da nach finnischer Auffassung ein Widerspruch zwischen GoB bzw. Einzelnormen und dem True and Fair View nicht entstehen kann, bezieht der Gesetzgeber den True and Fair View-Override auf sich selbst und seine eigene Arbeit. Der Gesetzgeber hat das Ministerium für Handel und Industrie bevollmächtigt, darüber zu entscheiden, wann und wie der Override bei bestimmten Sachverhalten von ganzen Branchen oder einzelnen Unternehmen durchgeführt werden darf, um ein besseres Bild der Vermögens-, Finanz- und Ertragslage zu vermitteln ${ }^{1197}$.

Der explizit in nationales Recht umgesetzte True and Fair View-Override entbindet die Unternehmen somit nicht von der Einhaltung der gesetzlichen Vorschriften (strafrechtliche Konsequenzen bei Gesetzesverstößen) ${ }^{1198}$.

Der True and Fair View-Override ist dem deutschen Recht fremd. Es gibt in Deutschland keinen True and Fair View-Override, welcher die Unternehmen von der Einhaltung der gesetzlichen Vorschriften entbindet (strafrechtliche Konsequenzen bei Gesetzesverstößen) ${ }^{1199}$. 


\subsubsection{Ansatzkriterien}

\subsection{Ansatz von Vermögenswerten}

Weder in Finnland noch in Deutschland sind Aktivierungskriterien gesetzlich definiert.

In Finnland sind nach herrschender Meinung Vermögenswerte solche Ausgaben, denen voraussichtlich künftige Einnahmen gegenüberstehen ${ }^{1200}$. Es wird der $\mathrm{Zu}$ fluss eines wirtschaftlichen Nutzens in Form von Zahlungsmitteln oder -äquivalenten erwartet. Als Konsequenz dürfen sämtliche Kosten aktiviert werden, denen eine direkte Einnahmenerwartung zugeordnet werden kann ${ }^{1201}$. Das Abstellen auf die Cash-generating unit ist bei Vermögenswerten des Sachanlagevermögens zulässig $^{1202}$. Wirtschaftlicher Nutzen in Form von Kosteneinsparungen oder anderen unternehmensinternen Vorteilen ist dagegen nicht aktivierungsfähig.

In Deutschland sieht die überwiegende Mehrheit des handelsrechtlichen Schrifttums die selbstständige Verwertbarkeit als notwendiges Kriterium aktivierungsfähiger Vermögensgegenstände an ${ }^{1203}$. Ein aktivierungspflichtiger Vermögensgegenstand liegt dann vor, wenn er außerhalb des bilanzierenden Unternehmens in finanzielle Mittel umgewandelt werden kann. Dies kann durch Veräußerung oder entgeltliche Nutzungsüberlassung geschehen ${ }^{1204}$.

Aus dieser unterschiedlichen Auffassung von Aktivierungsfähigkeit in beiden Ländern wird die unterschiedliche Interpretation des Vorsichtsprinzips ersichtlich, das in Deutschland enger ausgelegt wird als in Finnland.

\subsection{Ansatz von Schulden}

In Finnland und Deutschland sind Schulden anzusetzen,

- wenn eine Verpflichtung des Bilanzierenden vorliegt,

- mit der Verpflichtung eine wirtschaftliche Belastung für den Bilanzierenden verbunden ist,

- die wirtschaftliche Belastung quantifizierbar ist ${ }^{1205}$.

1200 Vgl. KPL 5:1.

1201 Dies betrifft anders als in Deutschland auch Forschungs- und Entwicklungskosten, Schulungskosten und Werbekosten.

1202 Vgl. Kap. 3.1.5.2 dieser Arbeit.

1203 Vgl. Roß (1996), S. 231-253.

1204 Vgl. ADS (ab 1995), § 246 Anm. 26-28 und vgl. Baetge, Kirsch und Thiele (2001), S. 124 127 sowie die dort angegebene Literatur.

1205 Ableitung der Passivierungsgrundsätze aus KPL 5:14.1 bzw. $\$ 249$ Abs. 1 HGB. Vgl. Kap. 3.1.5.3 dieser Arbeit. 


\subsubsection{Bewertung von Aktiva und Passiva}

\subsection{Bewertungsmaßstäbe}

Sowohl in Finnland als auch in Deutschland gilt der Grundsatz der Bewertung zu historischen Anschaffungs- und Herstellungskosten abzüglich planmäßiger und außerplanmäßiger Abschreibungen. Das gemilderte Niederstwertprinzip kommt für das Anlagevermögen ${ }^{1206}$, das strenge Niederstwertprinzip für das Umlaufvermögen $^{1207}$ zur Anwendung. Bewertungsmaßstäbe in beiden Ländern sind neben den Anschaffungs- und Herstellungskosten der Marktwert (Wiederbeschaffungswert oder realisierbarer Betrag), sofern niedriger als die Anschaffungs- und Herstellungskosten, sowie der Barwert ${ }^{1208}$.

In Finnland existieren Ausnahmen zum Bewertungsgrundsatz, die in Deutschland nicht vorgesehen sind ${ }^{1209}$ :

- Die Neubewertung von Grundstücken, Wasserflächen und Finanzanlagen $\mathrm{zu}$ einem die historischen Anschaffungskosten übersteigenden Marktwert ist möglich (Wiederbeschaffungswert bzw. realisierbarer Betrag) ${ }^{1210}$.

- Es erfolgt regelmäßig dann ein Ansatz zum Barwert, wenn der Gesetzgeber in Einzelvorschriften explizit „,besondere Vorsicht“ fordert (Gründungsausgaben $^{1211}$, Entwicklungsausgaben ${ }^{1212}$ ).

\subsection{Bewertung bei Zugang}

\subsection{Anschaffungskosten}

In Finnland setzen sich die Anschaffungskosten als Bewertungsmaßstab für erworbene Vermögenswerte wie folgt zusammen ${ }^{1213}$ :

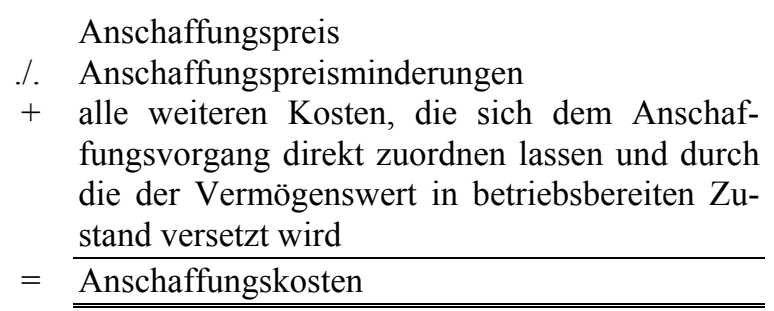

1206 Vgl. KPL 5:6 bzw. $\S 253$ Abs. 2 S. 3 HGB und $\S 279$ Abs. 1 S. 2 HGB.

1207 Vgl. KPL 5:13 bzw. $\S 253$ Abs. 3 S. 1 und 2 HGB.

1208 Vgl. Leppiniemi (2000 a), S. 140-142 und vgl. § 253 Abs. 1, 2 und 3 HGB.

1209 Vgl. Kap. 3.1.6.1 dieser Arbeit.

1210 Vgl. KPL 5:17.

1211 Vgl. KPL 5:7.

1212 Vgl. KPL 5:8.

1213 Vgl. KPL 4:5. 
Für bestimmte nachträgliche Anschaffungskosten existiert ein Aktivierungswahlrecht.

In Deutschland setzen sich Anschaffungskosten als Bewertungsmaßstab für erworbene Vermögensgegenstände wie folgt zusammen ${ }^{1214}$ :

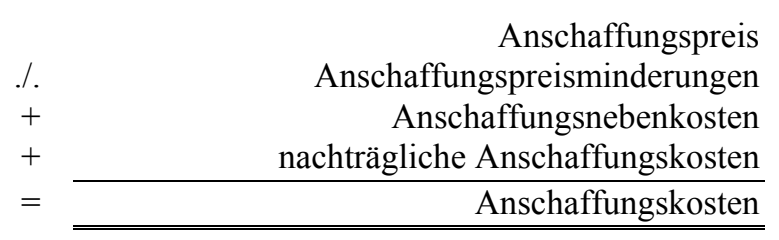

In der Praxis unterscheiden sich die Anschaffungskosten in Finnland und Deutschland dann, wenn ein finnisches Unternehmen das Wahlrecht dahingehend ausübt, nachträgliche Anschaffungskosten nicht zu aktivieren, sondern sie sofort als Aufwand zu erfassen.

\subsection{Herstellungskosten}

In Finnland werden die Herstellungskosten als Bewertungsmaßstab für selbst erstellte Vermögenswerte des Anlagevermögens sowie fertige und unfertige Erzeugnisse des Umlaufvermögens wie folgt gebildet:

\section{Umlaufvermögen}

Einbe-

ziehungs-

pflicht

Einbe-

ziehungs-

wahlrecht
Variable, tatsächlich realisierte Kosten

$$
\begin{aligned}
& \frac{\text { (Teilkostenansatz) }}{\text { Mindestens zu aktivierende Kosten }} \\
& \text { (Teilkostenansatz) }
\end{aligned}
$$

\section{Anlagevermögen}

Variable, tatsächlich realisierte Kosten

(Teilkostenansatz)

Mindestens zu aktivierende Kosten (Teilkostenansatz)

Fixe Kosten (aktivierungsfähig nur, wenn im Verhältnis zu variablen Kosten wesentlich)

Fremdkapitalzinsen, die ohne entsprechende Herstellung vermieden worden wären (aktivierungsfähig nur, wenn Zinsen gemeinsam mit den

fixen Kosten im Verhältnis zu variablen Kosten wesentlich) (Vollkostenansatz)

Maximal zu aktivierende Kosten (Vollkostenansatz) 
In Deutschland setzen sich die Herstellungskosten als Bewertungsmaßstab für selbst erstellte Vermögenswerte des Anlagevermögens sowie fertige und unfertige Erzeugnisse des Umlaufvermögens handelsrechtlich aus folgenden Bestandteilen zusammen $^{1215}$ :

Einbezie-

Anlage- und Umlaufvermögen

\begin{tabular}{|c|c|}
\hline hungspflicht & Materialeinzelkosten \\
\hline+ & Fertigungseinzelkosten \\
\hline+ & Sondereinzelkosten der Fertigung \\
\hline$=$ & Mindestens zu aktivierende Kosten (Teilkostenansatz) \\
\hline $\begin{array}{l}\text { Einbezie- } \\
\text { hungs- }\end{array}$ & \\
\hline wahlrecht & Materialgemeinkosten \\
\hline+ & Fertigungsgemeinkosten \\
\hline+ & Abschreibungen \\
\hline+ & Kosten der allgemeinen Verwaltung \\
\hline+ & Aufwendungen für soziale Einrichtungen des Betriebs \\
\hline+ & Aufwendungen für betriebliche Altersversorgung \\
\hline$=$ & Maximal zu aktivierende Kosten (Vollkostenansatz) \\
\hline
\end{tabular}

Wegen de Grundsatzes der Wirtschaftlichkeit ist es in Deutschland zulässig, Teile der einzeln zurechenbaren Kosten nicht als Einzelkosten, sondern als Gemeinkosten einzubeziehen, wenn die Einzelerfassung einen unangemessen hohen Aufwand verursachen würde (sog. unechte Gemeinkosten) ${ }^{1216}$. Nicht zu den Herstellungskosten gehörig, aber dennoch aktivierungsfähig sind Zinsen für Fremdkapital zur Finanzierung der Herstellung, soweit sie auf den Zeitraum der Herstellung entfallen ${ }^{1217}$.

In der Praxis unterscheiden sich die Herstellungskosten bei unterschiedlicher Ausübung der Wahlrechte durch die Unternehmen innerstaatlich sowie zwischen den Staaten. Je nach Wesentlichkeit des Anlage- und Umlaufvermögens für ein Unternehmen ist der Spielraum groß. Aufschluss über die Zusammensetzung der Herstellungskosten gibt in beiden Ländern der Anhang ${ }^{1218}$.

1215 Vgl. § 255 Abs. 2 HGB.

1216 Vgl. HFA 5/1991 und vgl. Baetge, Kirsch und Thiele (2001), S. 184.

1217 Vgl. § 255 Abs. 3 HGB.

1218 Vgl. KPA 2:2.1 bzw. § 284 Abs. 2 Nr. 1 HGB. 


\subsection{Behandlung von Zuwendungen}

Für die Behandlung von Zuwendungen existiert keine explizite Regelung des finnischen Gesetzgebers. Eine Zuwendung darf nach KILA-Anweisung „Planmäßige Abschreibung“ nicht direkt über die Gewinn- und Verlustrechnung als Ertrag vereinnahmt werden (Grundsatz der Periodenabgrenzung). Der KILA empfiehlt, einen Zuschuss direkt von den Anschaffungs- und Herstellungskosten des Vermögenswerts abzusetzen und so gleichzeitig die Abschreibungsbasis zu verringern $^{1219}$. Alternativ zulässig ist eine Berücksichtigung des Zuschusses als passive Rechnungsabgrenzung, die über die Nutzungsdauer des Vermögenswerts erfolgswirksam aufgelöst wird ${ }^{1220}$.

Auch der deutsche Gesetzgeber hat die Behandlung von Zuwendungen handelsrechtlich nicht geregelt. Es bestehen steuerrechtlich folgende Möglichkeiten:

- Die Zuwendung wird direkt über die Gewinn- und Verlustrechnung als Ertrag vereinnahmt ${ }^{1221}$.

- Die Zuwendung wird über die betriebsgewöhnliche Nutzungsdauer verteilt vereinnahmt. Zwei Vorgehensweisen sind denkbar:

I. Direktes Absetzen der Zuwendung von den Anschaffungs- oder Herstellungskosten des Vermögensgegenstands, wodurch die Abschreibungsbasis verringert wird ${ }^{1222}$.

II. Ausweis der Zuwendung in einem gesonderten Posten zwischen Eigenkapital und Rückstellungen. Die Zuwendung wird planmäßig gemäß Abschreibungsplan für den entsprechenden Gegenstand über die Gewinn- und Verlustrechnung aufgelöst ${ }^{1223}$.

\subsection{Behandlung von Wiederbeschaffungsrückstellungen}

In Finnland ist es unter bestimmten Voraussetzungen gewerbesteuerrechtlich gestattet, Wiederbeschaffungsrückstellungen für Vermögenswerte des Anlagevermögens zu bilden. Wegen des Prinzips der umgekehrten Maßgeblichkeit werden diese Rückstellungen auch handelsrechtlich angesetzt. Die Anschaffungs- oder Herstellungskosten werden in einem solchen Fall bei Zugang des Vermögenswerts in voller Höhe angesetzt und planmäßig über die Nutzungsdauer abgeschrieben. Für die Anschaffung des Vermögenswerts gebildete, zweckgebundene

\footnotetext{
1219 Vgl. KILA-Anweisung „Planmäßige Abschreibung“.

1220 Vgl. KILA-Anweisung „Planmäßige Abschreibung“. Vgl. auch Kap. 3.1.6.2.1.3 dieser Arbeit.

1221 Vgl. R 4 Abs. 2 EStR.

1222 Vgl. R 4 Abs. 2 EStR.

1223 Vgl. HFA 1/1984, S. 612-615.
} 
Wiederbeschaffungsrückstellungen werden, sofort oder über mehrere Jahre verteilt, erfolgswirksam aufgelöst ${ }^{1224}$.

In Deutschland kann in seltenen Fällen eine Rückstellung für künftige Herstellungskosten handelsrechtlich dann in Betracht kommen, wenn die Herstellungskosten eines Vermögensgegenstandes dessen Zeitwert überschreiten (verlustbringende Herstellung) ${ }^{1225}$. Das Steuerentlastungsgesetz 1999/2000/2002 bestimmt ausdrücklich, dass keine Rückstellungen für Aufwendungen gebildet werden dürfen, die Anschaffungs- oder Herstellungskosten für ein Wirtschaftsgut sind ${ }^{1226}$.

\subsection{Bewertung in Folgeperioden}

\subsection{Planmäßige Abschreibungen}

In Finnland und Deutschland umfasst das Abschreibungsvolumen die Anschaffungs- oder Herstellungskosten, vermindert um den erwarteten Restwert ${ }^{1227}$. Ist der Restwert unter Beachtung des Vorsichtsprinzips nicht hinreichend sicher zu beziffern, ist seine Höhe nicht von Bedeutung oder ist der Verkauf am Ende der Nutzungsdauer nicht sichert, darf er bei der Ermittlung des Abschreibungsvolumens nicht berücksichtigt werden ${ }^{1228}$.

Hinsichtlich des Abschreibungsbeginns, der Nutzungsdauer und der Abschreibungsmethode unterscheiden sich Finnland und Deutschland.

In Finnland ist der Abschreibungsbeginn der Zeitpunkt der tatsächlichen Inbetriebnahme des Vermögenswerts im normalen Produktionsprozess. Im Zugangsjahr wird grundsätzlich pro rata temporis abgeschrieben. Halbjahresabschreibungen sind nur dann mit der Art guter Buchführung vereinbar, wenn die planmäßigen Abschreibungen des Geschäftsjahrs in ihrer Gesamtheit nicht verfälscht wer$\operatorname{den}^{1229}$.

In Deutschland beginnt der Abschreibungszeitraum mit dem Zeitpunkt der Betriebsbereitschaft ${ }^{1230}$. Die Abschreibung im Zugangsjahr erfolgt pro rata temporis, sofern nicht folgende Vereinfachungsregel angewendet wird: Entsprechend den

1224 Vgl. EVL 32 und 43 sowie Kap. 3.1.6.2.1.4 und 3.2.5.1.2 dieser Arbeit.

1225 Vgl. BeBiKo (1999), § 249 Anm. 100 Stichwort „Anschaffungs- und Herstellungskosten“.

1226 Vgl. § 5 Abs. 4 b S. 1 EStG.

1227 Vgl. KILA-Anweisung „Planmäßige Abschreibung“, Abschnitt 2.3 und vgl. WPH I (2000), E Anm. 275.

1228 Vgl. KILA-Anweisung „Planmäßige Abschreibung“, Abschnitt 2.3 und vgl. WPH I (2000), E Anm. 275 und vgl. ADS (ab 1995), § 253 Anm. 415.

1229 Vgl. KILA-Anweisung „Planmäßige Abschreibungen“, Abschnitt 3.1.2.

1230 Vgl. ADS (ab 1995), § 253 Anm. 439. 
steuerlichen Vorschriften kann bewegliches Sachanlagevermögen, das im ersten Halbjahr angeschafft worden ist, mit dem vollen Abschreibungssatz abgeschrieben werden. Bei Anschaffung im zweiten Halbjahr kann die halbe Jahresabschreibung angesetzt werden ${ }^{1231}$.

In Finnland umfasst die Nutzungsdauer den Zeitraum, für den die Erzielung von Einnahmen vorausgesehen wird. Die Einnahmenerwartung wird basierend auf dem Grundsatz der Einzelbewertung ${ }^{1232}$ bzw. ausgehend von der Cash-generating unit unter Beachtung des Vorsichtsprinzips ermittelt. Es werden Erfahrungswerte des Unternehmens bzw. seiner Branche herangezogen ${ }^{1233}$.

In Deutschland müssen die Anschaffungs- oder Herstellungskosten planmäßig über die betriebsgewöhnliche Nutzungsdauer der Vermögensgegenstände abgeschrieben werden. Hinweise zur betriebsgewöhnlichen Nutzungsdauer für einzelne Anlagegüter enthalten die von der Finanzverwaltung herausgegebenen AfATabellen ${ }^{1234}$.

In Finnland ist jede Abschreibungsmethode erlaubt, die unter dem Gesichtspunkt der tatsächlichen Nutzungsdauer begründbar ist ${ }^{1235}$. Zu nennen sind die lineare, degressive und auch die progressive Abschreibungsmethode. Breite Anwendung in der Praxis findet die lineare Abschreibungsmethode ${ }^{1236}$.

In Deutschland kann linear oder degressiv abgeschrieben werden. Die progressive Abschreibung ist handelsrechtlich nur in Ausnahmefällen ${ }^{1237}$, steuerrechtlich jedoch nie zulässig ${ }^{1238}$. Das bewegliche Sachanlagevermögen wird in der Praxis üblicherweise in den ersten Jahren nach Anschaffung/Herstellung wegen der damit verbundenen steuerlichen Anerkennung degressiv abgeschrieben. Es wird regelmäßig auf die lineare Abschreibung übergegangen, wenn die Jahresabschreibung höher ist, um die höchstmögliche Minderung der steuerlichen Gewinne zu erreichen $^{1239}$.

1231 Vgl. R 44 Abs. 2 S. 3 EStR.

1232 Vgl. KPL 3:3.1,6.

1233 Vgl. KILA-Anweisung „Planmäßige Abschreibung“, Abschnitt 3.1.1.

1234 Vgl. BStBl. 2000 I, S. 1533, für Anlagegüter, die nach dem 31. Dezember 2000 angeschafft worden sind.

1235 Vgl. KILA-Anweisung „Planmäßige Abschreibung“, Abschnitt 3.2. Vgl. auch Kap. 3.1.6.2.2.1 dieser Arbeit.

1236 Vgl. Leppiniemi (2000 b), S. 22.

1237 Vgl. ADS (ab 1995), § 253 Anm. 402.

1238 Vgl. § 7 EStG.

1239 Vgl. ADS (ab 1995), § 253 Anm. 386 und vgl. § 7 Abs. 3 EStG. 


\subsection{Andere als planmäßige Abschreibungen}

In Finnland werden auf Grund der Umkehrung des Maßgeblichkeitsprinzips erhöhte Abschreibungen nach steuerlichen Vorschriften auch in der Handelsbilanz vorgenommen $^{1240}$, welche verpflichtend als ,,andere als planmäßige Abschreibungen" gesondert ausgewiesen werden ${ }^{1241}$.

In der Bilanz werden sie wie folgt gezeigt:

- Aktivseite: handelsrechtlich korrekter Wert des Anlagevermögens ${ }^{1242}$.

- Passivseite: kumulierter Abschreibungsunterschied zwischen handelsrechtlicher und steuerrechtlicher Abschreibung, der offen vom Anlagevermögen abgesetzt wird $^{1243}$.

In der Gewinn- und Verlustrechnung werden sie wie folgt erfasst:

- Ausweis planmäßiger Abschreibungen im Betriebsergebnis ${ }^{1244}$.

- Ausweis steuerlich veranlasster Abschreibungen als „Änderung des Abschreibungsunterschiedes“ nach dem Jahresergebnis vor Steuerausgleichsposten und Steuerzahlungen $^{1245}$.

Auch in Deutschland werden auf Grund der Umkehrung des Maßgeblichkeitsprinzips steuerlich motivierte Abschreibungen auch in der Handelsbilanz vorgenommen ${ }^{1246}$. Diese steuerlich veranlassten Abschreibungen werden allerdings nicht offen in der Bilanz bzw. der Gewinn- und Verlustrechnung ausgewiesen, sondern sie sind im Anhang angegeben. Es besteht das Wahlrecht, sie in der Bilanz als Sonderposten mit Rücklageanteil vom Anlagevermögen abzusetzen $^{1247}$.

\subsection{Außerplanmäßige Abschreibungen auf das Anlagevermögen}

In Finnland müssen außerplanmäßige Abschreibungen vorgenommen werden, wenn eine voraussichtlich dauernde Wertminderung vorliegt ${ }^{1248}$. Diese Wertminderung kann den einzelnen Vermögenswert oder seine Cash-generating unit betreffen. Dies ist abhängig davon, womit die Einnahmenerwartung verbunden ist. Wird von der Cash-generating unit ausgegangen, dann ist die Wertminderung

\footnotetext{
1240 Vgl. EVL 54.2.

1241 Vgl. KPL 5:12.1.

1242 Vgl. KPA 1:6.

1243 Vgl. KPA 1:6.

1244 Vgl. KPA 1:1.

1245 Vgl. KPA 1:1. Vgl. Kap. 3.1.6.2.2.2 dieser Arbeit.

1246 Vgl. § 279 Abs. 2 HGB.

1247 Vgl. § 281 Abs. 1 HGB.

1248 Vgl. KPL 5:13.
} 
proportional auf die einzelnen Buchwerte der Cash-generating unit zu verteilen ${ }^{1249}$.

Bei einer vorübergehenden Wertminderung besteht ein Abschreibungswahlrecht (gemildertes Niederstwertprinzip). Wertminderungen sind erfolgswirksam in der Gewinn- und Verlustrechnung zu erfassen. Eine Ausnahme bildet die Wertminderung neubewerteter Vermögenswerte. Hier wird die Neubewertungsrücklage erfolgsneutral aufgelöst ${ }^{1250}$. Über sie hinausgehende Abschreibungsbeträge sind erfolgswirksam zu berücksichtigen ${ }^{1251}$.

Auch in Deutschland müssen außerplanmäßige Abschreibungen auf den einzelnen Vermögensgegenstand vorgenommen werden, wenn eine voraussichtlich dauernde Wertminderung vorliegt ${ }^{1252}$. Bei einer vorübergehenden Wertminderung besteht für Kapitalgesellschaften und Personenhandelsgesellschaften - anders als in Finnland - ein Abschreibungsverbot für immaterielle Vermögensgegenstände und Gegenstände des Sachanlagevermögens ${ }^{1253}$. Für Gegenstände des Finanzanlagevermögens gilt in einem solchen Fall ein Abschreibungswahlrecht ${ }^{1254}$. Wertminderungen sind, sofern sie berücksichtigt werden, erfolgswirksam in der Gewinnund Verlustrechnung zu erfassen.

\subsection{Außerplanmäßige Abschreibungen auf das Umlaufvermögen}

In Finnland und Deutschland erfolgt die Bewertung des Umlaufvermögens zu Anschaffungs- bzw. Herstellungskosten oder zu niedrigeren Börsen- oder Marktpreisen (strenges Niederstwertprinzip) ${ }^{1255}$.

Als Marktpreise werden die Wiederbeschaffungs- bzw. Reproduktionskosten oder der niedrigere beizulegende Wert (Netto-Realisationswert) angesehen. Eine Abschreibung auf Wiederbeschaffungs- bzw. Reproduktionskosten ist regelmäßig auch dann erforderlich, wenn ein Veräußerungsgewinn zu erwarten ist. Der Unterschiedsbetrag zwischen Buchwert und niedrigerem Börsen- oder Marktpreis ist erfolgswirksam als Aufwand zu erfassen ${ }^{1256}$.

\footnotetext{
1249 Vgl. KILA-Anweisung „Planmäßige Abschreibung“, Abschnitt 6.

1250 Vgl. KILA-Anweisung „Planmäßige Abschreibung“, Abschnitt 2.2.

1251 Vgl. Kap. 3.1.6.2.2.1 dieser Arbeit.

1252 Vgl. $\S 253$ Abs. 2 und Abs. 3 HGB.

1253 Vgl. § 253 Abs. 1 HGB.

1254 Vgl. § 279 Abs. 1 HGB.

1255 Vgl. KPL 5:6.1 für das Umlaufvermögen, KPL 5:2.1-2 für das Finanzumlaufvermögen bzw. $\S 253$ Abs. 3 HGB.

1256 Vgl. KPL 5:6.1. Vgl. Kap. 3.1.6.2.2.4 dieser Arbeit.
} 


\subsection{Wertaufholung}

In Finnland besteht ein Wertaufholungsgebot für Vermögenswerte des Anlageund Umlaufvermögens, falls der Grund für die außerplanmäßige Abschreibung am Abschluss-Stichtag weggefallen ist ${ }^{1257}$.

Mangels EU-Konkretisierung des Wertaufholungsverfahrens wird in Finnland empfohlen, sich an den Standards des IASB zu orientieren. Die Ermittlung des Wertaufholungsbetrags erfolgt beim Anlagevermögen unter Berücksichtigung derjenigen planmäßigen Abschreibungen, die vorgenommen worden wären, wenn die außerplanmäßige Abschreibung nicht stattgefunden hätte ${ }^{1258}$. Die Wertaufholung ist sofort erfolgswirksam zu erfassen ${ }^{1259}$. Wertaufholungen von Vermögenswerten des Anlagevermögens werden in der Gewinn- und Verlustrechnung grundsätzlich unter dem Posten ausgewiesen, unter dem auch die ursprüngliche Aufwandsbuchung vermerkt worden ist.

Ausnahmen von diesem Grundsatz bilden

- wesentliche Beträge und Korrekturen von Aufwandsbuchungen mehrerer Geschäftsjahre, die Bestandteil des außerordentlichen Ergebnisses sind, und

- Wertaufholungen von Vermögenswerten des Umlaufvermögens, die in der Bestandsveränderung gezeigt werden ${ }^{1260}$.

Auch in Deutschland besteht ein Wertaufholungsgebot für Vermögensgegenstände des Anlage- und des Umlaufvermögens, falls der Grund für die außerplanmäBige Abschreibung weggefallen ist ${ }^{1261}$. Der niedrige Wert konnte in der Vergangenheit beibehalten werden, wenn die Beibehaltung in der Steuerbilanz möglich und nach dem Prinzip der umgekehrten Maßgeblichkeit davon abhängig war, dass der Ansatz ebenfalls in der Handelsbilanz beibehalten wurde ${ }^{1262}$. Mit dem Steueränderungsgesetz 1998 wurde ein steuerliches Wertaufholungsgebot eingeführt, sodass auch das handelsrechtliche Zuschreibungsgebot wirksam wird. Der Aufholungsbetrag kann allerdings in einer Übergangszeit ergebnisneutral in eine Rücklage eingestellt und über fünf Jahre ergebniswirksam verteilt werden.

\footnotetext{
1257 Vgl. KPL 5:16.

1258 Vgl. IAS 16 (revised 1993).

1259 Vgl. IAS 25 (reformatted 1994).

1260 Vgl. Kap. 3.1.6.2.2.5 dieser Arbeit.

1261 Vgl. § 280 Abs. 1 HGB.

1262 Vgl. § 280 Abs. 2 HGB.
} 


\subsection{Neubewertung}

In Finnland existiert ein Wahlrecht zur Neubewertung von Grundstücken und Wasserflächen des Sachanlagevermögens sowie von Wertpapieren des Finanzanlagevermögens ${ }^{1263}$.

Voraussetzung hierfür ist, dass

- der wahrscheinliche Veräußerungspreis des Vermögenswerts über den historischen Anschaffungskosten liegt,

- der Unterschiedsbetrag von Dauer ist und

- der Unterschiedsbetrag wesentlich ist (größer als zehn Prozent der ursprünglichen Anschaffungskosten) ${ }^{1264}$.

Zur Neubewertung wird das Einholen eines Sachverständigen-Gutachtens empfohlen ${ }^{1265}$.

Neubewertungsgewinne werden als nicht realisiert angesehen und erfolgsneutral in eine Neubewertungsrücklage im Eigenkapital eingestellt (Ausschüttungssperre ${ }^{1266}$. Die Neubewertungsrücklage wird bei Wertminderung des Vermögenswerts teilweise oder vollständig erfolgsneutral aufgelöst. Bei Veräußerung des Vermögenswerts und Realisierung der Neubewertungsgewinne ist eine erfolgswirksame Auflösung der Rücklage vorzunehmen ${ }^{1267}$.

In Deutschland sind Neubewertungen nicht gestattet (Nominalwertprinzip ${ }^{1268}$ ).

\subsubsection{Fremdwährungsumrechnung}

Der finnische Gesetzgeber regelt mangels EU-Vorgabe die Währungsumrechnung für den Jahresabschluss im Buchführungsgesetz basierend auf IAS 21 (revised 1993) und IAS 39 (approved 1998) ${ }^{1269}$. Der KILA hat darüber hinaus eine allgemeingültige Anweisung veröffentlicht ${ }^{1270}$.

Die Umrechnung monetärer Bilanzposten erfolgt zum Stichtagsmittelkurs (alternativ zum Geld- bzw. Briefkurs unter Beachtung des Imparitätsprinzips). Ausnahmen sind:

- Geleistete oder erhaltene Anzahlungen werden mit Mittelkurs des Zugangstags bewertet.

\footnotetext{
1263 Vgl. KPL 5:17.

1264 Vgl. Kap. 3.1.6.2.2.6 und 3.2.4.1 dieser Arbeit.

1265 Vgl. KILA 1427/1996 und KILA 1453/1997.

1266 Vgl. OYL 12:3 b.

1267 Vgl. OYL 12:3 b.

1268 Vgl. § 253 Abs. 1 HGB.

1269 Vgl. KPL 5:3.

1270 Vgl. KILA-Anweisung, ,Währungsumrechnung“.
} 
- Nicht monetäre Posten werden zum historischen Kurs am Tag der Zahlung umgerechnet.

In der Gewinn- und Verlustrechnung werden Geschäftsvorfälle zum Entstehungskurs erfasst (Mittelkurs am Zugangstag). Im Anhang sind Eventualverbindlichkeiten zum Stichtagsmittelkurs zu bewerten.

Eine Saldierung der Gewinne und Verluste aus der Währungsumrechnung ist nicht zulässig.

Fremdwährungspositionen, die vertraglich oder anderweitig so fixiert sind, dass sie von Wechselkursänderungen nicht betroffen sind, werden mit fixiertem Kurs bewertet. Wird ein Devisentermingeschäft zum Zweck der Kurssicherung abgeschlossen, so sind am Bilanzstichtag unrealisierte Kursgewinne bzw. -verluste in die Rechnungsabgrenzungsposten einzustellen und über die Restlaufzeit des Geschäfts erfolgswirksam aufzulösen. Wird ein Devisentermingeschäft nicht zum Zweck der Kurssicherung geschlossen, werden aus ihm resultierende nicht realisierte Kursverluste am Bilanzstichtag erfolgswirksam, unrealisierte Kursgewinne hingegen gar nicht erfasst. Bei geschlossenen Positionen gehen Kursgewinne und -verluste in dem Jahr in die Gewinn- und Verlustrechnung ein, in dem die Position geschlossen wird.

Für alle anderen monetären und nicht monetären Posten gilt, dass nicht realisierte Kursverluste erfolgswirksam erfasst werden müssen. Nicht realisierte Fremdwährungsgewinne dürfen dagegen erst dann in die Gewinn- und Verlustrechnung eingehen, wenn ihr Eintritt wahrscheinlich ist ${ }^{1271}$.

In Deutschland gibt der Gesetzgeber keine Regeln für die Währungsumrechnung im Einzelabschluss vor. Da das Imparitätsprinzip zu beachten ist, wird die Umrechnung in der Bilanz zum historischen Kurs oder zum niedrigeren Stichtagskurs für Forderungen bzw. zum höheren Stichtagskurs für Verbindlichkeiten vorgenommen. Nicht monetäre Posten des Anlagevermögens werden zum historischen Kurs am Tag ihres Zugangs umgerechnet. In der Gewinn- und Verlustrechnung werden Geschäftsvorfälle zum Entstehungskurs erfasst. Dabei handelt es sich oft um einen Monatskurs. Im Anhang sind Eventualverbindlichkeiten mit dem Stichtagskurs umzurechnen.

Eine Saldierung der Gewinne und Verluste aus der Währungsumrechnung ist wie in Finnland - nicht zulässig. Fremdwährungspositionen, für die Kurssicherungsgeschäfte abgeschlossen sind, werden zum Sicherungskurs bewertet. Am Bilanzstichtag unrealisierte Fremdwährungsverluste sind stets zu erfassen. Unrealisierte Gewinne können grundsätzlich nicht erfasst werden, es sei denn, es handelt sich um sehr kurzfristige Forderungen.

1271 Vgl. Kap. 3.1.7 dieser Arbeit. 


\subsubsection{Behandlung von Bilanzierungsfehlern aus Vorperioden}

\subsection{Anpassung von Vorjahreszahlen}

Hinsichtlich der Behandlung von Bilanzierungsfehlern aus Vorperioden existiert keine Regelung des finnischen Gesetzgebers. Der KILA hat festgelegt, dass Anpassungen in der Periode, in der ihre Notwendigkeit bekannt wird, erfolgswirksam vorzunehmen sind (Prinzip der Bilanzidentität) ${ }^{1272}$.

Ist die Anpassung im Verhältnis zum Jahresergebnis der laufenden Periode wesentlich, muss sie in der Gewinn- und Verlustrechnung im außerordentlichen Ergebnis ausgewiesen und im Anhang erläutert werden ${ }^{1273}$.

Auch in Deutschland gibt es keine explizite gesetzliche Regelung, unter welchen Umständen Vorjahreszahlen anzupassen sind. Die Änderung von Jahresabschlüssen und die Anpassung der Handelsbilanz an die Steuerbilanz auf Grund einer steuerlichen Außenprüfung werden jedoch in einer Stellungnahme des IDW diskutiert $^{1274}$.

Gemäß HFA 2/1991 dürfen Anpassungen an Vorjahreszahlen nicht erfolgsneutral gebucht werden ${ }^{1275}$. Basierend auf dem Prinzip der Bilanzidentität seien sämtliche Anpassungen erfolgswirksam zu erfassen. Bei Wesentlichkeit müssten die Anpassungen im Anhang erläutert werden.

Eine Anpassung früherer Abschlüsse ist grundsätzlich nur in besonderen Fällen durch Änderung der Abschlüsse der entsprechenden Geschäftsjahre und der Folgeabschlüsse sowie der Neufeststellung der geänderten Abschlüsse möglich ${ }^{1276}$.

\subsubsection{2 Änderung der Bilanzpolitik}

In Finnland ist bei Änderung der Bilanzpolitik, beispielsweise beim Übergang von der Completed Contract-Methode zur Percentage of Completion-Methode, im Anhang auf die Nicht-Vergleichbarkeit zu Vorjahreszahlen hinzuweisen ${ }^{1277}$.

In Deutschland gibt es mit Ausnahme der geforderten Bewertungsstetigkeit ${ }^{1278}$ keine detaillierten handelsrechtlichen Vorschriften hinsichtlich der Zulässigkeit eines Wechsels der Bilanzpolitik. Das IDW hat sich in Form einer Stellungnahme

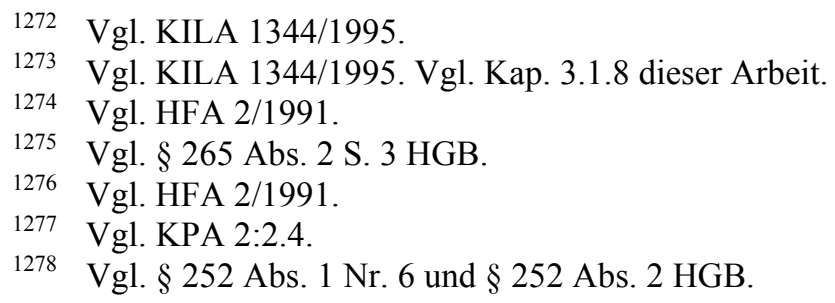


geäußert und eine Abweichung nur in begründeten Ausnahmefällen als zulässig beurteilt ${ }^{1279}$.

Die Auswirkung einer Änderung der Bilanzierungs- und Bewertungsmethoden auf die Vermögens-, Finanz- und Ertragslage ist im Anhang gesondert anzugeben.

\subsubsection{Ansatz und Bewertung einzelner Bilanzposten im Einzelab- schluss $^{1280}$}

\subsubsection{Anlagevermögen}

\subsection{Immaterielle Vermögenswerte}

\subsection{Ansatz und Bewertung}

In Finnland ist ein immaterieller Wert - gleichgültig, ob erworben oder selbst erstellt - dann aktivierungsfähig, wenn

- die erwarteten Einnahmen in Form von Zahlungsmitteln oder -äquivalenten dokumentierbar sind,

- die erwarteten Einnahmen dem Vermögenswert eindeutig zugeordnet werden können (unter Hinweis auf das Vorsichtsprinzip ist kein Abstellen auf die Cash-generating unit erlaubt) und

- die mit seiner Erlangung verbundenen Kosten verlässlich messbar sind.

Selbstständige Verwertbarkeit des Werts und Kontrolle des Unternehmens über den Wert sind nicht erforderlich.

Es existiert ein Aktivierungswahlrecht für immaterielle Vermögenswerte ${ }^{1281}$. Im Falle eines Ansatzes sind diese zu Anschaffungs- oder Herstellungskosten zu aktivieren und planmäßig abzuschreiben.

In Deutschland wird als Aktivierungskriterium häufig auf das Prinzip der selbstständigen Verwertbarkeit verwiesen. Entgeltlich erworbene immaterielle Vermögensgegenstände sind zu Anschaffungskosten zu aktivieren und planmäßig über ihre wirtschaftliche Nutzungsdauer abzuschreiben, auch wenn sie als Teil des Erwerbs eines Geschäftsbetriebs erworben werden (Aktivierungsgebot) ${ }^{1282}$.

Selbst erstellte immaterielle Vermögensgegenstände dürfen - anders als in Finnland - nach $\S 248$ Abs. 2 HGB nicht aktiviert werden ${ }^{1283}$.

\footnotetext{
1279 Vgl. HFA 3/1997.

1280 Analog zur Vorgehensweise in Kap. 4 dieser Arbeit wird im Folgenden deutscher Denkweise entsprechend zunächst auf die Bilanzposten eingegangen, anschließend auf Posten der Gewinn- und Verlustrechnung.

1281 Vgl. Kap. 3.2.1.1.1 dieser Arbeit.

1282 Vgl. $\S 248$ Abs. 2 HGB, § 253 Abs. 1 und Abs. 2 HGB.

1283 Vgl. $\S 248$ Abs. 2 HGB.
} 


\subsection{Gründungsausgaben und Aufwendungen für die Ingangsetzung und Erweiterung des Geschäftsbetriebs}

In Finnland besteht ein Aktivierungswahlrecht für Gründungsausgaben ${ }^{1284}$. Hierbei handelt es sich um Anwalts- und Notargebühren, Ausgaben für die Eintragung ins Handelsregister, Kosten für die Entwicklung der Geschäftsidee, Standortsuche, Planung der Unternehmensstruktur sowie Rekrutierung und Schulung des Basis-Mitarbeiterstamms. Ob Kosten für die Erweiterung des Geschäftsbetriebs, Abspaltung, Umwandlung, Restrukturierung oder Neugründung zu Gründungsausgaben zu zählen und somit aktivierungsfähig sind, ist bislang weder vom Gesetzgeber geregelt noch vom KILA entschieden worden ${ }^{1285}$.

Die Abschreibungsdauer von Gründungsausgaben beträgt maximal fünf Jahre ${ }^{1286}$.

In Deutschland ist eine Aktivierung von Aufwendungen für die Gründung des Unternehmens und die Beschaffung von Eigenkapital nicht erlaubt. Sie werden dem Periodenergebnis belastet ${ }^{1287}$. Aufwendungen für die Ingangsetzung und Erweiterung des Geschäftsbetriebs dürfen als Bilanzierungshilfe aktiviert wer$\operatorname{den}^{1288}$. $\mathrm{Zu}$ ihnen zählen alle Aufwendungen, die während der Anlaufphase des Betriebs durch den erstmaligen Aufbau der Innen- und Außenorganisation und durch die Bereitmachung des Betriebs für die Aufnahme einer geregelten Leistungserbringung gehören. Sie dürfen allerdings weder Anschaffungs- oder Herstellungskosten von aktivierungsfähigen Vermögensgegenständen darstellen noch den Rechnungsabgrenzungsposten zuzurechnen $\operatorname{sein}^{1289}$. In Höhe des Aktivierungsbetrags gilt eine Ausschüttungssperre ${ }^{1290}$. Die Abschreibung der Bilanzierunshilfe erfolgt über maximal vier Jahre ${ }^{1291}$.

\footnotetext{
1284 Vgl. KPL 5:7.

1285 Vgl. Kap. 3.2.1.1.2 dieser Arbeit.

1286 Vgl. KPL 5:7.

1287 Vgl. § 248 Abs. 1 HGB.

1288 Vgl. § 269 S. 1 HGB.

1289 Vgl. BeBiKo (1999), § 269 Anm. 2.

1290 Vgl. § 269 S. 2 HGB.

1291 Vgl. § 282 HGB.
} 


\subsection{Forschungsausgaben}

In Finnland existiert ein Aktivierungswahlrecht für Forschungsausgaben ${ }^{1292}$. Zu ihnen zählen Kosten für die Erlangung neuer Kenntnisse, für das Erforschen ihrer Anwendungsmöglichkeiten, für das Erforschen von Produkt- und Prozessalternativen sowie für die Planung neuer oder verbesserter Produkt- und Prozessalternativen. In Höhe der aktivierten Forschungsausgaben besteht eine Ausschüttungssperre ${ }^{1293}$.

Die Abschreibungsdauer beträgt maximal fünf Jahre, sofern nicht eine längere Abschreibungsdauer (bis zu 20 Jahre) mit der Art guter Buchführung vereinbar ist $^{1294}$.

In Deutschland ist die Aktivierung von Forschungsaufwendungen nicht gestattet $^{1295}$.

\subsection{Entwicklungsausgaben}

In Finnland sind Entwicklungsausgaben in Höhe der Herstellungskosten aktivierungsfähig $^{1296}$, wenn

- eine schriftliche Planung für das Projekt existiert,

- der Vermögenswert klar und eindeutig abgegrenzt werden kann,

- die Kosten verlässlich ermittelt werden können,

- zukünftige Wirtschaftlichkeit und Rentabilität wahrscheinlich sind und

- die aktivierten Beträge nicht höher als der ermittelte künftige kumulierte Deckungsbeitrag sind ${ }^{1297}$

Mit der Aktivierung von Entwicklungsausgaben ist keine Ausschüttungssperre verbunden $^{1298}$.

Die Abschreibungsdauer beträgt maximal fünf Jahre, sofern nicht eine längere Abschreibungsdauer (maximal 20 Jahre) mit der Art guter Buchführung vereinbar ist $^{1299}$.

In Deutschland besteht ein Aktivierungsverbot für Entwicklungsaufwendungen $^{1300}$.

\footnotetext{
1292 Vgl. KPA 1:6.

1293 Vgl. OYL 12:2.1.

1294 Vgl. KPL 5:11. Vgl. Kap. 3.2.1.1.3 dieser Arbeit.

1295 Vgl. § 248 Abs. 2 HGB.

1296 Vgl. KPL 5:8.

1297 Vgl. KTM:p 50/1998 § 4.

1298 Vgl. KTM:p 50/1998.

1299 Vgl. KPL 5:8. Vgl. Kap. 3.2.1.1.3 dieser Arbeit.

1300 Vgl. § 248 Abs. 2 HGB.
} 


\subsection{Geschäfts- oder Firmenwert}

Sowohl in Finnland als auch in Deutschland ist die Aktivierung eines originären Geschäfts- oder Firmenwerts verboten ${ }^{1301}$.

In Finnland und Deutschland wird beim Anteilskauf der derivative Geschäftsoder Firmenwert in der Bilanz des investierenden Unternehmens nicht als solcher ausgewiesen, sondern ist Bestandteil der Beteiligung ${ }^{1302}$.

Bei Übernahme eines Geschäftsbetriebs besteht in Finnland ein Aktivierungswahlrecht für den derivativen Geschäfts- oder Firmenwert. Die Abschreibungsdauer beträgt maximal fünf Jahre oder der Vermögenswert ist über seine wirtschaftliche Nutzungsdauer abzuschreiben. Da diese aber kaum nachweisbar ist, wird in der Praxis regelmäßig linear mit 20 Prozent p. a. abgeschrieben ${ }^{1303}$.

In Deutschland kann der derivative Geschäfts- oder Firmenwert, der bei der Übernahme eines Geschäftsbetriebs anfällt, sofort vollständig oder in jedem folgenden Geschäftsjahr zu mindestens einem Viertel abgeschrieben werden ${ }^{1304}$.

Alternativ kann eine Verteilung über die voraussichtliche Nutzungsdauer erfolgen $^{1305}$. Häufig wird über die geschätzte steuerliche Nutzungsdauer von 15 Jahren abgeschrieben $^{1306}$.

\subsection{Ausgaben mit lang anhaltender Wirkung}

Das finnische Bilanzgliederungsschema sieht als einen Posten der immateriellen Vermögenswerte „Ausgaben mit lang anhaltender Wirkung“ vor. Sie umfassen alle Ausgaben, welche die Voraussetzungen für immaterielle Vermögenswerte erfüllen, aber keinem der anderen immateriellen Vermögenswerte zugeordnet werden können ${ }^{1307}$, beispielsweise

- EDV-Software,

- Werbekosten,

- Schulungskosten,

- Beschaffung von Know-how,

1301 Vgl. Kap. 3.2.1.1.5 dieser Arbeit. Vgl. § 248 Abs. 2 HGB.

1302 Vgl. KPL 5:5 und vgl. BeBiKo (1999), § 255 Anm. $141 \mathrm{ff}$.

1303 Vgl. KPL 5:9. Vgl. Kap. 3.2.1.1.5 dieser Arbeit.

1304 Vgl. § 255 Abs. 4 HGB.

1305 Vgl. § 255 Abs. 4 HGB.

1306 Vgl. § 7 Abs. 1 S. 3 EStG.

1307 Vgl. KPL 5:11. 
- Asphaltierungsarbeiten auf gemieteten oder gepachteten Grundstücken (Arbeiten auf eigenem Grundstück sind dem Sachanlagevermögen zuzuordnen) und

- Disagio.

Für diese Ausgaben besteht nach ein Aktivierungswahlrecht ${ }^{1308}$. Sie sind in maximal fünf Jahren abzuschreiben, sofern die Art guter Buchführung nicht einen längeren Zeitraum, längstens jedoch 20 Jahre, gestattet ${ }^{1309}$.

In Deutschland ist eine solche Bilanzposition nach $\S 266$ Abs. 2 HGB nicht vorgesehen. Software wird in der Praxis regelmäßig unter „,Konzessionen, gewerblichen Schutzrechte und ähnliche Rechte und Werte sowie Lizenzen an solchen Rechten und Werten“ ausgewiesen ${ }^{1310}$. Nach herrschender Meinung gilt in Deutschland das Prinzip der selbstständigen Verwertbarkeit und somit ein Aktivierungsverbot für Werbe- und Schulungskosten sowie für die Beschaffung von Know-how. Asphaltierungsarbeiten, sofern aktivierungsfähig, sind in Deutschland immer Bestandteil des Sachanlagevermögens, auszuweisen unter „Bauten auf fremden Grundstücken“'1311.

Während der finnische Gesetzgeber das Disagio als „Ausgabe mit lang anhaltender Wirkung“ und somit als immateriellen Vermögenswert auffasst ${ }^{1312}$, der planmäßig abzuschreiben ist, geht der deutsche Gesetzgeber davon aus, dass es sich beim Disagio um einen Rechnungsabgrenzungsposten handelt, der ratierlich aufzulösen ist ${ }^{1313}$. In beiden Fällen besteht ein Aktivierungswahlrecht ${ }^{1314}$, sodass es sich letztlich nicht um einen sachlichen Unterschied, sondern um einen Ausweisunterschied zwischen beiden Ländern handelt.

Vgl. KPL 5:11.

Vgl. Kap. 3.2.1.1.6 dieser Arbeit.

Vgl. § 266 Abs. 2 A I 1 HGB.

Vgl. § 266 Abs. 2 A II 1 HGB.

Vgl. KPL 5:10.

1313 Vgl. § 250 Abs. 3 HGB.

1314 Vgl. KPL 5:10 und vgl. § 250 Abs. 3 HGB. 


\subsection{Sachanlagen}

\subsection{Ansatz, Bewertung und Ausweis}

Materielle Vermögenswerte, mit denen über mehrere Jahre eine Einnahmenerwartung verbunden ist $^{1315}$, sind in Finnland aktivierungspflichtig ${ }^{1316}$. Es besteht ein Aktivierungswahlrecht für nachträgliche Anschaffungs- oder Herstellungskosten, die den Vermögenswert erweitern oder verbessern ${ }^{1317}$.

Der Ansatz der Sachanlagen erfolgt zu historischen Anschaffungs- oder Herstellungskosten abzüglich $\mathrm{zu}$ berücksichtigender Abschreibungen ${ }^{1318}$. An jedem Stichtag sind die materiellen Vermögenswerte auf Werthaltigkeit zu überprüfen (gemildertes Niederstwertprinzip $^{1319}$ ). Gegebenenfalls hat eine Wertberichtigung zu erfolgen.

Es besteht ein Wahlrecht zur Neubewertung von Grundstücken und Wasserflächen $^{1320}$.

In Deutschland besteht nach herrschender Meinung eine Aktivierungspflicht für selbstständig verwertbare Vermögensgegenstände, die dem Betriebszweck über mehrere Jahre dienen sollen. Sie werden zu Anschaffungs-/Herstellungskosten abzüglich Abschreibungen bewertet ${ }^{1321}$. Nachträgliche Anschaffungs- oder Herstellungskosten sind aktivierungspflichtig ${ }^{1322}$.

An jedem Stichtag ist eine Überprüfung der Vermögensgegenstände auf Werthaltigkeit durchzuführen. Bei nur vorübergehender Wertminderung ist es Kapitalgesellschaften und Personenhandelsgesellschaften verboten, eine außerplanmäßige Abschreibung vorzunehmen ${ }^{1323}$.

Eine Neubewertung von Gegenständen des Anlagevermögens über ihre ursprünglichen Anschaffungs- und Herstellungskosten hinaus ist in Deutschland nicht zulässig (Nominalwertprinzip) ${ }^{1324}$.

Im Ausweis von Vermögensgegenständen des Sachanlagevermögens liegt ein weiterer Unterschied zwischen finnischem und deutschem Handelsrecht. In Finnland werden Grundstücke und Wasserflächen, Gebäude und Bauten, Maschinen und Geschäftsausstattung, sonstige materielle Vermögenswerte sowie geleistete Anzahlungen und im Bau befindliche Anschaffungen als gesonderte Posten aus-

\footnotetext{
1315 Vgl. KPL 4.3 und 4.4.1 und vgl. Kap. 3.1.5.2 dieser Arbeit.

1316 Vgl. KPL 5:5.1.

1317 Vgl. KILA-Anweisung „Planmäßige Abschreibung“. Vgl. Kap. 3.2.1.2.1 dieser Arbeit.

1318 Vgl. KPL 5:5.1.

1319 Vgl. KPL 5:13.

1320 Vgl. KPL 5:17.

1321 Vgl. § 253 Abs. 2 HGB.

1322 Vgl. § 255 Abs. 1 HGB.

1323 Vgl. § 253 Abs. 2 S. 3 HGB.

1324 Vgl. § 253 Abs. 1 HGB.
} 
gewiesen $^{1325}$. In Deutschland wird untergliedert in Grundstücke und Bauten, technische Anlagen und Maschinen, andere Anlagen, Betriebs- und Geschäftsausstattung sowie Anlagen im $\mathrm{Bau}^{1326}$.

\subsection{Geringwertige Wirtschaftsgüter}

In Finnland dürfen geringwertige Wirtschaftsgüter im Jahr ihrer Anschaffung voll als Aufwand erfasst werden. Diese Vorgehensweise wird aus dem Vorsichtsprinzip und dem Prinzip der Wesentlichkeit abgeleitet. „Geringwertigkeit“" wird handelsrechtlich unternehmensindividuell in Abhängigkeit von Umsatz und Summe des Anlagevermögens bestimmt ${ }^{1327}$.

Gewerbesteuerlich gilt ein Wirtschaftsgut dann als geringwertig, wenn seine tatsächliche Nutzungsdauer geringer als drei Jahre ist oder seine Anschaffungs- oder Herstellungskosten weniger als $€ 850,00$ betragen (steuerlich wird als Summe aller GWG eine Höhe von maximal $€ 2.500,00$ p. a. akzeptiert) ${ }^{1328}$.

Unterschiede zwischen Handels- und Steuerbilanz sind möglich, obwohl die Praxis in Finnland sich oft am Gewerbesteuerrecht orientiert ${ }^{1329}$.

In Deutschland gibt es zwei Möglichkeiten zur Behandlung geringwertiger Wirtschaftsgüter.

Selbstständig verwertbare Vermögensgegenstände des beweglichen abnutzbaren Anlagevermögens von geringem Wert müssen nicht aktiviert werden, sondern dürfen sofort als Aufwand behandelt werden. Sie werden im Anlagespiegel nicht als Zugang aufgezeigt. Geringwertigkeit wird vom Fachschrifttum mit einem Betrag zwischen $€ 750,00$ und $€ 820,00$ beziffert $^{1330}$.

Alternativ dürfen Anlagegegenstände von geringem Wert, deren Anschaffungsoder Herstellungskosten bis zu $€ 410,00$ betragen, steuerlich im Jahr der Anschaffung voll abgeschrieben werden ${ }^{1331}$. Sie werden im Anlagespiegel gleichzeitig als $\mathrm{Zu}$ - und als Abgang erfasst.

Eine identische Behandlung in der Handels- und Steuerbilanz geringwertiger Wirtschaftsgüter ist auf Grund der umgekehrten Maßgeblichkeit erforderlich.

Vgl. KPA 1:6.

Vgl. § 266 Abs. 2 HGB.

Vgl. KILA-Anweisung „Planmäßige Abschreibung“, Abschnitt 2.6.

328 Vgl. EVL 33.

1329 Vgl. Kap. 3.2.1.2.2 dieser Arbeit.

1330 Vgl. BeBiKo (1999), § 253 Anm. 275 und vgl. ADS (ab 1995), § 253 Anm. 412.

1331 Vgl. $§ 6$ Abs. 2 EStG. 


\subsection{Bildung von Festwerten}

In Finnland ist Festwertbildung zwar zulässig ${ }^{1332}$, wird aber in der Praxis de facto kaum durchgeführt. Sie ist dem traditionellen finnischen Verständnis der Einzelbewertung und der Periodisierungspflicht fremd ${ }^{1333}$.

In Deutschland ist eine Festwertbildung ebenfalls erlaubt. Voraussetzung ist die Erfüllung folgender Kriterien:

- Sachanlagevermögen,

- regelmäßiger Ersatz,

- nachrangige Bedeutung des Gesamtwerts (die Summe aller Festwerte ist kleiner als fünf Prozent der Bilanzsumme),

- geringe Veränderung in der Zusammensetzung und

- regelmäßige Bestandsaufnahme, spätestens alle drei Jahre ${ }^{1334}$.

\subsection{Behandlung von Leasingverhältnissen}

In Finnland existiert für den Einzelabschluss keine das Leasing sowie Sale and Lease Back-Transaktionen betreffende Vorschrift. Es hat sich als Art guter Buchführung herausgebildet, dass das rechtliche Eigentum entscheidend für die Bilanzierung ist. Leasingverträge werden wie Miet- oder Pachtverträge behandelt ${ }^{1335}$. Konzerne können seit 1998 wählen, ob sie Finanzierungsleasingverhältnisse beim Leasingnehmer (wirtschaftlicher Eigentümer) aktivieren ${ }^{1336}$. Dieses Wahlrecht wirkt sich künftig eventuellauch auf den Einzelabschluss aus ${ }^{1337}$.

Mangels expliziter handelsrechtlicher Regelung in Deutschland werden in der Praxis die steuerlichen Unterscheidungsmerkmale zur Zurechnung von Leasinggegenständen angewendet ${ }^{1338}$.

Steuerlich ist bei Leasingverträgen, die gemäß ihrem wirtschaftlichen Charakter Finanzierungsgeschäfte nach Art eines Ratenkaufs unter Eigentumsvorbehalt sind (Finanzierungsleasing), das wirtschaftliche Eigentum am Leasinggegenstand dem Leasingnehmer zuzurechnen. Ob der Leasingnehmer oder der Leasinggeber den Gegenstand $\mathrm{zu}$ aktivieren hat, richtet sich nach dem wirtschaftlichen Eigen$\operatorname{tum}^{1339}$.

\footnotetext{
1332 Vgl. KPL 5.5.3.

1333 Vgl. Kap. 3.2.1.2.3 dieser Arbeit.

1334 Vgl. § 240 Abs. 3 HGB.

1335 Vgl. KILA 839/1986.

1336 Vgl. KPL 6:18.

1337 Vgl. Kap. 3.2.1.2.4 dieser Arbeit.

1338 Vgl. BeBiKo (1999), § 246 Anm. 26 und vgl. ADS (ab 1995), § 246 Anm. 342.

1339 Vgl. WPH I (2000), E 25.
} 
Beim Finanzierungsleasing aktiviert der Leasingnehmer den Leasinggegenstand und passiviert die Leasingverbindlichkeit, während der Leasinggeber lediglich eine Forderung an den Leasingnehmer ausweist ${ }^{1340}$.

Da es für den Leasingnehmer wegen des abzugsfähigen Aufwands steuerlich grundsätzlich vorteilhafter ist, den geleasten Vermögensgegenstand nicht zu aktivieren, erfüllen die meisten Leasingverträge nicht die steuerlichen Voraussetzungen für die Annahme des Finanzierungsleasings. Dies führt dazu, dass nur wenige Leasingnehmer ihre geleasten Vermögensgegenstände bilanzieren ${ }^{1341}$.

\subsection{Finanzanlagen}

\subsection{Ansatz und Bewertung}

In beiden Ländern aktivierungspflichtig sind solche Finanzinvestitionen, bei denen seitens des bilanzierenden Unternehmens eine Daueranlageabsicht besteht. Im Einzelabschluss erfolgt die Bewertung der Finanzanlagen zu Anschaffungskosten oder zum niedrigeren beizulegenden Wert ${ }^{1342}$. Es besteht ein Abwertungswahlrecht bei nur vorübergehender Wertminderung ${ }^{1343}$. Eine Wertaufholung ist geboten, falls der Grund für eine außerplanmäßige Abschreibung weggefallen ist ${ }^{1344}$.

In Finnland können Unternehmen nach Buchführungsgesetz das Wahlrecht zur Neubewertung von Wertpapieren des Finanzanlagevermögens ausüben. Diese dürfen zum Marktwert bewertet werden, wenn dieser die historischen Anschaffungskosten überschreitet ${ }^{1345}$. In Höhe des Unterschiedsbetrags ist eine Neubewertungsrücklage zu bilden (Ausschüttungssperre) ${ }^{1346}$.

In Deutschland dürfen die Anschaffungskosten der Finanzanlagen nicht überschritten werden (Nominalwertprinzip) ${ }^{1347}$, eine Neubewertung ist somit nicht zulässig.

\footnotetext{
1340 Vgl. WPH I (2000), E 28 ff.

1341 Vgl. Küting und Figge (1999), S. 363.

1342 Vgl. KPL 5:5.1.

1343 Vgl. KPL 5:13 bzw. § 279 Abs. 1 S. 2 HGB i. V. m. $§ 253$ Abs. 2 HGB.

1344 Vgl. KPL 5:16 bzw. § 280 Abs. 1 und 2 HGB.

1345 Vgl. KPL 5:17.

1346 Vgl. Kap. 3.2.1.3.1 dieser Arbeit.

1347 Vgl. § 253 Abs. 1 HGB.
} 


\subsection{Verbundene Unternehmen}

In Finnland und Deutschland liegen verbundene Unternehmen dann vor, wenn zwischen ihnen ein Mutter-Tochter-Verhältnis besteht. Dieses ist jedoch in beiden Staaten in unterschiedlicher Ausübung des Mitgliedstaatenwahlrechts an unterschiedliche Kriterien geknüpft. Während in Finnland nur das Control-Konzept ${ }^{1348}$ anwendbar ist, kommt in Deutschland zusätzlich das Konzept der einheitlichen Leitung ${ }^{1349}$ in Betracht.

Das Control-Konzept knüpft das Mutter-Tochter-Verhältnis nach finnischem und deutschem Verständnis an die Möglichkeit, das Tochterunternehmen direkt oder indirekt zu beherrschen, und zwar durch

- Mehrheit der Stimmrechte $e^{1350}$

- Organbestellungsrecht ${ }^{1351}$ oder

- Beherrschungsrecht auf Grund von Vertrag oder Satzung ${ }^{1352}$.

Bei Anwendung dieses Konzepts wird in beiden Ländern nicht gefordert, dass dem Mutterunternehmen eine Beteiligung am Tochterunternehmen gehört. Vielmehr wird abgestellt auf die wirtschaftliche Zurechenbarkeit von Rechten ${ }^{1353}$.

Nach dem in Deutschland, aber nicht in Finnland geltenden Konzept der einheitlichen Leitung begründet sich ein Mutter-Tochter-Verhältnis auf

- einheitlicher Leitung (Koordination der Aktivitäten beider Unternehmen) und auf

- einer Beteiligung ${ }^{1354}$.

\subsection{Beteiligungen}

In Finnland wird unterschieden in „Beteiligungen“1355 und in „Beteiligungen im engeren Sinne“61356. Diese Differenzierung ist für den Konzernabschluss, nicht aber für den Einzelabschluss von Bedeutung.

Ein Unternehmen ist in Finnland als Beteiligung anzusehen, wenn dem bilanzierenden Unternehmen null bis 100 Prozent des Nennkapitals und bis zu 50 Prozent der Stimmrechtsgesamtheit an ihm gehören. Es muss sich bei einer Beteiligung nicht um rechtliche Anteile an anderen Unternehmen handeln, es wird auf die

1348 Vgl. KPL 1:6 bzw. § 290 Abs. 2 HGB.

1349 Vgl. § 290 Abs. 1 HGB.

1350 Vgl. KPL 1:6.1 i. V. m. KPL 1:5.1,1 bzw. § 290 Abs. 2 Nr. 1 HGB.

1351 Vgl. KPL 1:6.1 i. V. m. KPL 1:5.1,2 bzw. § 290 Abs. 2 Nr. 2 HGB.

1352 Vgl. KPL 1:6.1 i. V. m. KPL 1:5.1,2 bzw. § 290 Abs. 2 Nr. 3 HGB.

1353 Vgl. Kap. 3.2.1.3.2 dieser Arbeit. Vgl. WPH I (2000), M 37.

1354 Vgl. § 271 Abs. 1 HGB.

1355 Vgl. KPL 1:7.

1356 Vgl. KPL 1:8. 
wirtschaftliche Zurechenbarkeit der Stimmrechte abgestellt. Diese Beteiligungsvermutung ist widerlegbar.

Ein Unternehmen ist widerlegbar als Beteiligung im engeren Sinne anzusehen, wenn dem bilanzierenden Unternehmen null bis 100 Prozent des Nennkapitals und 20 bis 50 Prozent der Stimmrechtsgesamtheit an ihm gehören. Es muss sich bei einer Beteiligung nicht um Anteile an anderen Unternehmen handeln. Wesentliches Kriterium ist die wirtschaftliche Zurechenbarkeit ${ }^{1357}$.

In Deutschland sind Beteiligungen vom Gesetzgeber definiert als ,Anteile an anderen Unternehmen, die bestimmt sind, dem eigenen Geschäftsbetrieb durch Her-

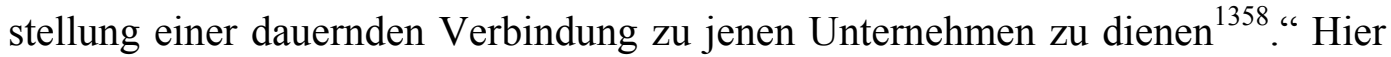
ist anders als in Finnland rechtliches Eigentum Voraussetzung.

Anteile an Kapitalgesellschaften werden widerlegbar dann als Beteiligung vermutet, wenn ihre Höhe 20 bis 50 Prozent des Nennkapitals beträgt ${ }^{1359}$.

\subsection{Phasengleiche Vereinnahmung von Beteiligungserträgen}

Die Vereinnahmung von Beteiligungserträgen ist in Finnland gesetzlich nicht geregelt, sodass deren Erfassung und Ausweis aus den GoB abgeleitet werden. Bei Aktiengesellschaften entspricht es der Art guter Buchführung, erst dann einen Dividendenanspruch zu aktivieren bzw. eine Verpflichtung aus Dividendenzahlungen zu passivieren, wenn die Hauptversammlung den Jahresabschluss festgestellt und über die Gewinnverwendung beschlossen hat. Im Regelfall geschieht dies nicht im Geschäftsjahr, in dem der Gewinn erzielt worden ist, sondern im Folgejahr. Der KILA sieht eine phasengleiche Vereinnahmung jedoch dann als zulässig an, wenn die Hauptversammlung einen realistischen Vorab-Beschluss über die Gewinnverwendung getroffen hat, der mit den Kapitalerhaltungs- und Ausschüttungsvorschriften des Aktiengesellschaftsgesetzes übereinstimmt ${ }^{1360}$.

Grundsätzlich ist in Deutschland ein Dividendenanspruch erst zu dem Zeitpunkt aktivierbar, zu dem ein Gewinnverwendungsbeschluss der Gesellschafter vorliegt. Der Gewinnanspruch ist jedoch zwingend schon im Jahresabschluss des Mutterunternehmens für das Jahr auszuweisen, in dem die Tochtergesellschaft den Gewinn erzielt hat, wenn folgende Voraussetzungen vorliegen ${ }^{1361}$ :

- Das Mutterunternehmen hat die Stimmenmehrheit, um einen entsprechenden Gewinnverwendungsbeschluss herbeizuführen.

1357 Vgl. Kap. 3.2.1.3.3 dieser Arbeit.

1358 Vgl. § 271 Abs. 1 HGB.

1359 Vgl. § 271 Abs. 3 HGB.

1360 Vgl. KILA 1542/1998.

1361 Vgl. BGH vom 12. Januar 1998. 
- Der Feststellungs- und Gewinnverwendungsbeschluss für die Tochtergesellschaft liegt vor Beendigung der Prüfung des Jahresabschlusses des Mutterunternehmens vor.

- Das Geschäftsjahr der Tochtergesellschaft endet nicht nach dem der Muttergesellschaft.

- Der Jahresabschluss der Tochtergesellschaft vermittelt ein den tatsächlichen Verhältnissen entsprechendes Bild der Vermögens-, Finanz- und Ertragslage.

Ein Wahlrecht zur phasengleichen Vereinnahmung liegt vor, wenn bis zum Abschluss der Prüfung des Jahresabschlusses des Mutterunternehmens ein Gewinnverwendungsbeschluss zwar noch nicht vorliegt, der Jahresabschluss der Tochtergesellschaft zu diesem Zeitpunkt jedoch festgestellt worden ist und ein Gewinnverwendungsvorschlag vorliegt ${ }^{1362}$.

\subsubsection{Umlaufvermögen}

\subsection{Ansatz des Vorratsvermögens}

Nach finnischen Buchführungsvorschriften aktivierungspflichtig sind die variablen Anschaffungs- oder Herstellungskosten ${ }^{1363}$.

Für fixe Kosten existiert ein Aktivierungswahlrecht ${ }^{1364}$. Sie sind allerdings nur dann aktivierungsfähig, wenn ihr Betrag im Verhältnis zu den variablen Kosten wesentlich ist ${ }^{1365}$. Fremdkapitalzinsen dürfen bei Vermögenswerten des Umlaufvermögens nicht aktiviert werden ${ }^{1366}$.

In Deutschland aktivierungspflichtig im Sinne des HGB sind die Anschaffungskosten und bei Herstellung die Einzelkosten. Bei Herstellung besteht handelsrechtlich ein Aktivierungswahlrecht für Gemeinkosten und Fremdkapitalzin$\operatorname{sen}^{1367}$.

Steuerlich sind die Einzelkosten sowie die Material- und Fertigungsgemeinkosten und anteilige Abschreibungen des Anlagevermögens aktivierungspflichtig. Vertriebskosten dürfen weder handels- noch steuerrechtlich einbezogen werden.

\footnotetext{
1362 Vgl. BGH vom 03. November 1975.

1363 Vgl. KPL 4:5.1.

1364 Vgl. KPL 4:5.2.

1365 Vgl. KPL 4:5.2.1

1366 Vgl. KPL 4:5.3. Vgl. Kap. 3.2.2.1 dieser Arbeit.

1367 Vgl. $\S 255$ Abs. 2 und Abs. 3 HGB.
} 


\subsection{Bewertung des Vorratsvermögens}

\subsection{Bewertungsmaßstäbe}

In Finnland wie in Deutschland erfolgt die Bewertung der Vorräte zu Anschaffungs- bzw. Herstellungskosten oder zu Börsen- oder Marktpreisen, falls diese niedriger sind (strenges Niederstwertprinzip ${ }^{1368}$ ). Als Marktpreise werden die Wiederbeschaffungs- oder Reproduktionskosten oder der niedrigere beizulegende Wert (Netto-Realisationswert) angesehen. Eine Abschreibung auf Wiederbeschaffungs- bzw. Reproduktionskosten ist in der Regel auch dann erforderlich, wenn ein Veräußerungsgewinn zu erwarten ist.

In Deutschland werden bei der Berechnung des Netto-Realisationswerts von den geschätzten Verkaufserlösen alle noch bis zur Fertigstellung anfallenden Kosten und alle direkten Marketing-, Verkaufs- und Vertriebskosten abgezogen. Da bei der Berechnung des niedrigeren beizulegenden Werts steuerlich die übliche Gewinnspanne abgezogen werden darf, wird dies auch handelsrechtlich akzeptiert $^{1369}$

\subsection{Bewertungsvereinfachungsverfahren}

In Finnland sind nach Buchführungsgesetz explizit erlaubte Bewertungsvereinfachungsmethoden $^{1370}$

- die gewichtete Durchschnittspreismethode,

- das FIFO-Verfahren und

- das LIFO-Verfahren

unter Beachtung des strengen Niederstwertprinzips. Steuerlich ist offiziell nur das FIFO-Verfahren gestattet ${ }^{1371}$. Der gewogene Durchschnittspreis wird jedoch akzeptiert, sofern sein Ergebnis nicht stark von dem der FIFO-Methode abweicht ${ }^{1372}$.

In Deutschland lässt das Handelsrecht Bewertungsvereinfachungsverfahren zu, soweit sie den Grundsätzen ordnungsmäßiger Buchführung entsprechen ${ }^{1373}$. Die gebräuchlichsten Methoden sind handels- und steuerrechtlich

- die Bewertung zu (gewichteten) Durchschnittspreisen und

- die Anwendung des FIFO- sowie

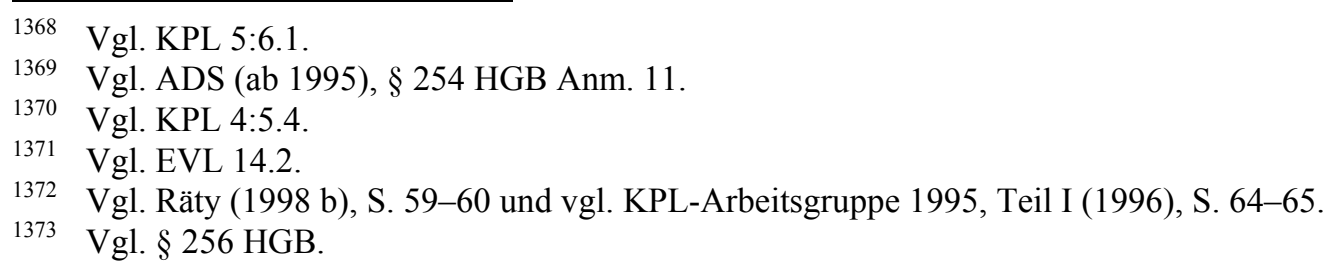


- des LIFO-Verfahrens

unter Beachtung des strengen Niederstwertprinzips.

\subsection{Gängigkeitsabschreibungen}

In Finnland und Deutschland werden Vermögenswerte bzw. Vermögensgegenstände einzelwertberichtigt, sofern diese nicht gängig sind.

In Finnland werden pauschale Abschreibungen wegen mangelnder Gängigkeit in der Praxis selten vorgenommen, da sie dem traditionellen Verständnis des Grundsatzes der Einzelbewertung widersprechen. Sie sind aber wegen des Vorsichtsprinzips mit der Art guter Buchführung vereinbar ${ }^{1374}$.

In Deutschland sind nach herrschender Meinung pauschale Abschreibungen wegen mangelnder Gängigkeit bei einer Vielzahl von Artikelgruppen für Handelsund Fertigungsbetriebe zulässig ${ }^{1375}$. Gängigkeitsabschläge werden steuerlich in begrenztem Umfang für die Ermittlung des niedrigeren Teilwerts wegen gesunkener Wiederbeschaffungskosten oder gesunkener Verkaufserlöse akzeptiert ${ }^{1376}$.

\subsection{Bildung von Festwerten}

In Finnland ist die Festwertbildung im Vorratsvermögen zulässig ${ }^{1377}$. Sie findet jedoch in der Praxis kaum Anwendung, da sie im Widerspruch zum Grundsatz der Einzelbewertung steht ${ }^{1378}$.

In Deutschland ist eine Festwertbildung erlaubt für Roh-, Hilfs- und Betriebsstoffe, wenn folgende Voraussetzungen erfüllt sind ${ }^{1379}$ :

- regelmäßiger Ersatz,

- nachrangige Bedeutung des Gesamtwerts,

- geringe Veränderung in der Zusammensetzung und

- regelmäßige Bestandsaufnahme.

1374 Vgl. Kap. 3.2.2.1 dieser Arbeit.

1375 Vgl. BeBiKo (1999), § 253 Anm. 529 und vgl. ADS (ab 1995), § 253 HGB Anm. 518.

1376 Vgl. BeBiKo (1999), § 253 Anm. 555 ff.

1377 Vgl. KPL 5:6.3.

1378 Vgl. Kap. 3.2.2.2 dieser Arbeit.

1379 Vgl. § 240 Abs. 3 HGB. 


\subsection{Langfristige Auftragsfertigung}

In Finnland und in Deutschland erfolgt die Realisierung von Erträgen aus langfristiger Auftragsfertigung nach der Completed Contract-Methode ${ }^{1380}$. In Finnland ist unter bestimmten Voraussetzungen alternativ die Anwendung der Percentage of Completion-Methode zulässig ${ }^{1381}$.

Ein langfristiger Fertigungsauftrag liegt in Finnland nach einer KILA-Anweisung dann vor, wenn ${ }^{1382}$

- Fertigungsbeginn und -ende in zwei Berichtsperioden fallen und

- das Fertigungsprojekt eine wesentliche Größe im Verhältnis zu den Umsatzerlösen des herstellenden Unternehmens bildet.

Die Voraussetzungen für die Anwendung der Percentage of Completion-Methode lauten:

- Die Gesamteinnahmen und -ausgaben aus dem Auftrag können zuverlässig geschätzt werden,

- die angefallenen Kosten können verlässlich ermittelt werden, und

- der Fertigstellungsgrad kann zuverlässig gemessen werden.

Werden diese Voraussetzungen erfüllt, besteht ein Wahlrecht zur Bilanzierung zwischen der Percentage of Completion-Methode und der Completed ContractMethode. In allen anderen Fällen ist verpflichtend nach Completed ContractMethode vorzugehen ${ }^{1383}$.

Die Gewinnrealisierung erfolgt in Deutschland grundsätzlich erst bei Abrechnung des Gesamtauftrags, sofern nicht von vornherein selbstständige Teilaufträge vertraglich vereinbart werden. Kann bei Anwendung der Completed ContractMethode der True and Fair View nicht vermittelt werden, ist eine Zusatzangabe im Anhang notwendig. Die Percentage of Completion-Methode ist nicht erlaubt.

Werden während der Herstellung drohende Verluste erkennbar, sind in beiden Ländern gemäß Imparitätsprinzip Rückstellungen für drohende Verluste aus schwebenden Geschäften zu bilden ${ }^{1384}$.

1380 Vgl. KPL 5:4 und vgl. BeBiKo (1999), § 255 Anm. 457-464.

1381 Vgl. KPL 5:4 und vgl. Kap. 3.2.2.3 dieser Arbeit.

1382 Vgl. KILA-Anweisung ,Teilgewinnrealisierung“.

1383 Vgl. KPL 5:4.

1384 Vgl. KILA-Anweisung „Teilgewinnrealisierung““. Vgl. Kap. 3.2.2.3 dieser Arbeit. Vgl. § 253 HGB. 


\subsubsection{Finanzumlaufvermögen}

\subsection{Forderungen}

Forderungen werden in Finnland und Deutschland nach denselben Kriterien angesetzt. Es werden Ansprüche aus Leistungen - Lieferung, Dienstleistung oder Darlehensgewährung - aktiviert, welche das bilanzierende Unternehmen erbracht hat und bei denen die Gegenleistung des Vertragspartners noch aussteht. ${ }^{1385}$ Hinsichtlich der Forderungsbewertung bestehen Unterschiede bei Abzinsung, Bindung an Indizes, Pauschalwertberichtigungen und Saldierungsmöglichkeiten.

In Finnland ist eine Abzinsung unverzinslicher Forderungen im Einzelfall zulässig, wenn dies mit dem Vorsichtsprinzip begründbar ist ${ }^{1386}$.

In Deutschland sind unterverzinsliche und unverzinsliche Forderungen mit dem Barwert anzusetzen. In der Praxis werden aus Vereinfachungsgründen lediglich unverzinsliche Forderungen, die nach mehr als einem Jahr fällig werden, abgezinst $^{1387}$.

Ist in Finnland der Wert einer Forderung an einen Index gebunden und steigt dieser Index so, dass der den Nennwert übersteigende Wert sicher realisiert werden kann, dann erfolgt die Bildung eines passiven Rechnungsabgrenzungspostens in Höhe des Unterschiedsbetrags. Der passive Rechnungsabgrenzungsposten wird über die Restlaufzeit der Forderung erfolgswirksam aufgelöst. Drohende Verluste sind sofort zu erfassen ${ }^{1388}$.

In Deutschland erfolgt die Realisierung von Gewinnen aus der Bindung von Forderungen an Indizes erst bei Fälligkeit der Forderungen. Drohende Verluste werden auf Grund des Imparitätsprinzips sofort erfasst ${ }^{1389}$.

In Finnland sind Pauschalwertberichtigungen weder durch den Gesetzgeber noch durch den KILA geregelt. Ist aus Erfahrung belegbar, dass ein gewisser Prozentsatz des Forderungsbestands ausfallbedroht ist, darf eine freiwillige Drohverlustrückstellung zu diesem Zweck gebildet werden, die jedoch steuerlich nicht anerkannt wird ${ }^{1390}$.

Sofern in Deutschland bei einzelnen Forderungen kein konkreter Abschreibungsbedarf besteht, aber allgemein mit Forderungsausfällen oder mit erheblichen Zahlungsverzögerungen gerechnet wird, ist eine Pauschalwertberichtigung zu bilden.

\footnotetext{
1385 Vgl. Järvinen et al. (2000), S. 385 und vgl. Baetge, Kirsch und Thiele (2001), S. 306.

1386 Vgl. Leppiniemi und Leppiniemi (1999), S. 101.

1387 Vgl. BeBiKo (1999), § 253 Anm. 592.

1388 Vgl. Leppiniemi (2000 a), S. 267 und vgl. KILA 1523/1998.

1389 Vgl. § $253 \mathrm{HGB}$.

1390 Vgl. KPL 5:16.
} 
Bereits einzelwertberichtigte Forderungen dürfen in die Ermittlung des Pauschalwertberichtigungsbetrags nicht einbezogen werden. Pauschalwertberichtigungen werden steuerlich in bestimmtem Umfang anerkannt ${ }^{1391}$.

In Finnland ist die Saldierung von Forderungen und Verbindlichkeiten nicht zulässig, auch wenn sie gegenüber derselben Person bestehen ${ }^{1392}$.

In Deutschland hingegen ist die Saldierung von Forderungen und Verbindlichkeiten zwischen denselben Personen zulässig und in der Praxis üblich. Das geltende Verrechnungsverbot ${ }^{1393}$ darf hier wegen der verbesserten Klarheit und Übersichtlichkeit des Jahresabschlusses ${ }^{1394}$ hinsichtlich des haftenden Vermögens und der tatsächlichen Liquiditätslage durchbrochen werden.

\subsection{Sonstige Vermögensgegenstände und aktive Rechnungsabgrenzungsposten}

In Finnland sind sonstige Vermögensgegenstände und aktive Rechnungsabgrenzungsposten Bestandteil der Forderungen ${ }^{1395}$. Dem Rechnungsabgrenzungsposten werden transitorische und antizipative Abgrenzungen zugeordnet ${ }^{1396}$.

In Deutschland zählen sonstige Vermögensgegenstände zu den Forderungen. Der aktive Rechnungsabgrenzungsposten wird gesondert ausgewiesen ${ }^{1397}$. In Deutschland sind antizipative Abgrenzungen Bestandteil der sonstigen Vermögensgegenstände, transitorische Abgrenzungen sind dem Rechnungsabgrenzungsposten zuzuweisen. Zudem können auf Vorräte entfallene Zölle und Verbrauchsteuern abgegrenzt werden, soweit diese nicht in die Anschaffungs- und Herstellungskosten einbezogen wurden ${ }^{1398}$, ebenso die Umsatzsteuer auf enthaltene Anzahlungen ${ }^{1399}$. Ein Disagio kann - anders als in Finnland - in den Rechnungsabgrenzungsposten ausgewiesen werden. In diesem Fall gilt ein planmäßiges Abschreibungsgebot ${ }^{1400}$.

\footnotetext{
1391 Vgl. BeBiKo (1999), § 253 Anm. 578-581.

1392 Vgl. Kap. 3.2.3.1 dieser Arbeit.

1393 Vgl. § 246 Abs. 2 HGB.

1394 Vgl. § 243 Abs. 2 HGB.

1395 Vgl. KPA 1:6.

1396 Vgl. KPL 4:6.1 und vgl. Kap 3.2.3.1.2 dieser Arbeit.

1397 Vgl. § 266 Abs. 2 HGB.

1398 Vgl. § 250 Abs. 1 S. 2 Nr. 1 HGB.

1399 Vgl. $\$ 250$ Abs. 1 S. 2 Nr. 2 HGB.

1400 Vgl. $\S 250$ Abs. 3 HGB und $\S 268$ Abs. 6 HGB.
} 


\subsection{Geleistete Anzahlungen}

In Finnland und Deutschland sind geleistete Anzahlungen höchstens zu ihrem Nennwert anzusetzen ${ }^{1401}$. Sie sind wie Forderungen zu bewerten. Außerplanmäßige Abschreibungen sind vorzunehmen, wenn der wahrscheinliche Wert unter dem Nennwert liegt (strenges Niederstwertprinzip ${ }^{1402}$ ). Ihr Ausweis erfolgt in der Bilanz unter der Position, auf welche die Anzahlungen sich beziehen ${ }^{1403}$.

\subsection{Wertpapiere des Finanzumlaufvermögens}

In Finnland und Deutschland erfolgt die Bewertung von Wertpapieren des Finanzumlaufvermögens zu Anschaffungskosten oder zum niedrigeren Börsen- oder Marktpreisen (strenges Niederstwertprinzip ${ }^{1404}$ ). Dem Wertaufholungsgebot ist Folge $\mathrm{zu}$ leisten ${ }^{1405}$. Bei der Vornahme von Zuschreibungen dürfen nach dem Nominalwertprinzip $^{1406}$ die Anschaffungskosten in beiden Ländern nicht überschritten werden. Die Möglichkeit zur Neubewertung in Finnland betrifft lediglich Wertpapiere des Anlagevermögens ${ }^{1407}$.

\subsection{Eigene Anteile}

In Finnland sind eigene Anteile nur dann bilanzierungsfähig, wenn sie nach Wertpapiermarktgesetz zum öffentlichen Handel zugelassen sind. Je nach Absicht der Anlagedauer erfolgt der Ausweis als „Eigene Anteile“ im Finanzanlage- oder Finanzumlaufvermögen ${ }^{1408}$. In Höhe der eigenen Anteile ist eine Rücklage zu bilden, deren Zweck eine Ausschüttungssperre ist ${ }^{1409}$.

In Deutschland sind eigene Anteile zwingend unter dem Posten „Eigene Anteile“ als Bestandteil der Wertpapiere des Umlaufvermögens auszuweisen ${ }^{1410}$. Mit Hinblick auf den True and Fair View kann eine Zusatzangabe im Anhang erforderlich werden, falls die Absicht besteht, die Anteile auf Dauer im Unternehmen zu halten $^{1411}$. In Höhe der eigenen Anteile im Besitz des Unternehmens ist wie in Finn-

\footnotetext{
1401 Vgl. KPL 5:2.1. Vgl. § 253 Abs. 1 S. 1 HGB.

1402 Vgl. KPL 5:2.2.

1403 Vgl. KPA 1:6. Vgl. § 266 Abs. 2 HGB.

1404 Vgl. KPL 5:2.2. Vgl. § 253 Abs. 3 HGB.

1405 Vgl. KPL 5:16.

1406 Vgl. § 253 Abs. 1 HGB.

1407 Vgl. KPL 5:17. Vgl. Kap. 3.2.3.2 dieser Arbeit.

1408 Vgl. KPA 1:6.

1409 Vgl. Kap. 3.2.3.2 dieser Arbeit.

1410 Vgl. § 265 Abs. 3 S. 2 HGB.

1411 Vgl. § 264 Abs. 2 S. 2 HGB.
} 
land eine Rücklage für eigene Anteile zu bilden, deren Zweck ebenfalls eine Ausschüttungssperre ist ${ }^{1412}$.

\subsubsection{Eigenkapital}

\subsection{Gezeichnetes Kapital}

In Finnland muss das Grundkapital einer privaten Aktiengesellschaft mindestens $€ 8.000,00$, das einer öffentlichen Aktiengesellschaft mindestens $€ 80.000,00$ betragen. Das Grundkapital ist gesondert auszuweisen. Ausstehende Einlagen auf das gezeichnete Kapital sind auf der Aktivseite gesondert unter dem Posten „Forderungen" auszuweisen, da es sich bei ihnen dem Charakter nach um Forderungen gegenüber Gesellschaftern handelt ${ }^{1413}$.

In Deutschland sind das Stammkapital einer Gesellschaft mit beschränkter Haftung, mindestens $€ 25.000,00^{1414}$, und das Grundkapital einer Aktiengesellschaft, das mindestens $€ 50.000,00$ betragen muss ${ }^{1415}$, im Jahresabschluss gesondert auszuweisen. Ausstehende Einlagen auf das gezeichnete Kapital sind auf der Aktivseite vor dem Anlagevermögen auszuweisen ${ }^{1416}$. Dies entspricht ihrem Charakter als Korrekturposten zum Eigenkapital, das auf der Passivseite an erster Stelle gezeigt wird ${ }^{1417}$. Sie bilden damit eine Ausnahme der allgemein anerkannten Auffassung, dass Aktiva keine Korrekturposten zu Passiva darstellen dürfen.

\subsection{Gesetzliche Rücklage}

In Finnland sind gesetzliche Rücklagen in der Bilanzgliederung nach KPA 1:6 vorgesehen. Ihre Dotierung ist freiwillig, sodass es die gesetzliche Rücklage - wie sie international mit dem Ziel des Gläubigerschutzes und der Einschränkung der Eigenkapitalentnahmemöglichkeiten aufgefasst wird - in Finnland nicht gibt ${ }^{1418}$.

Um den Schutz der Gläubiger zu verstärken, müssen Aktiengesellschaften und Kommanditgesellschaften auf Aktien in Deutschland eine gesetzliche Rücklage bilden, der fünf Prozent des Jahresgewinns zuzuführen sind, bis die Kapitalrücklage und die gesetzliche Rücklage zusammen zehn Prozent des Grundkapitals

\footnotetext{
1412 Vgl. § 272 Abs. 4 HGB.

1413 Vgl. KILA 1136/1996. Vgl. Kap. 3.2.4.1 dieser Arbeit.

1414 Vgl. § 5 Abs. 1 GmbHG.

1415 Vgl. $\$ 7$ AktG.

1416 Vgl. § 272 Abs. 1 HGB.

1417 Vgl. BeBiKo (1999), § 266 Anm. 50.

1418 Vgl. Kap. 3.2.4.1 dieser Arbeit.
} 
erreichen $^{1419}$. Die gesetzliche Rücklage ist nicht ausschüttungsfähig. Unter bestimmten Umständen kann sie zum Ausgleich eines Jahresfehlbetrags oder eines Verlustvortrags verwendet werden ${ }^{1420}$.

\subsection{Kapitalrücklage}

In Finnland wie in Deutschland enthält die Kapitalrücklage Agien aus der Ausgabe von Anteilen, Wandelanleihen und Optionsrechten sowie sonstige Zuzahlungen der Gesellschafter ${ }^{1421}$.

In Finnland darf die Kapitalrücklage unter bestimmten Voraussetzungen zur Deckung von Verlusten, zur Erhöhung des gezeichneten Kapitals, zur Deckung des Unterschiedsbetrags einer Unter-pari-Emission sowie zur Herabsetzung der Kapitalrücklage verwendet werden ${ }^{1422}$.

In Deutschland ist diese Rücklage bei Gesellschaften mit beschränkter Haftung in der Regel ausschüttungsfähig ${ }^{1423}$. Bei Aktiengesellschaften darf die Kapitalrücklage nur unter strengen Voraussetzungen ausgeschüttet werden ${ }^{1424}$.

\subsection{Neubewertungsrücklage}

In Finnland wird eine Neubewertungsrücklage gebildet, wenn Grundstücke, Wasserflächen oder Wertpapiere des Finanzanlagevermögens ihrem Marktwert am Stichtag entsprechend über ihren historischen Anschaffungskosten bewertet wer$\operatorname{den}^{1425}$. Der Unterschiedsbetrag zwischen den historischen Anschaffungskosten und dem am Stichtag bilanzierten Marktwert wird erfolgsneutral in diese Rücklage eingestellt ${ }^{1426}$. Er unterliegt einer Ausschüttungssperre.

Entfallen die Gründe für die Aufwertung, so ist sie rückgängig zu machen und die Neubewertungsrücklage erfolgsneutral aufzulösen.

Bei Veräußerung des neubewerteten Vermögenswerts zu einem Betrag über den historischen Anschaffungskosten wird die Neubewertungsrücklage erfolgswirksam aufgelöst, der Unterschiedsbetrag somit realisiert ${ }^{1427}$.

\footnotetext{
1419 Vgl. § 150 Abs. 2 AktG.

1420 Vgl. $\S \S 150$ Abs. 1 und II, 300 AktG.

1421 Vgl. OYL 11:3 a.

1422 Vgl. OYL 12:3.2. Vgl. Kap. 3.2.4.1 dieser Arbeit.

1423 Vgl. § $57 \mathrm{~d}$ GmbHG.

1424 Vgl. § 150 Abs. 3 und 4 AktG.

1425 Vgl. KPL 5:17.

1426 Vgl. KPL 5:17.

1427 Vgl. Kap. 3.2.4.1 dieser Arbeit.
} 
In deutschen Jahresabschlüssen existiert kein der finnischen Praxis entsprechender Posten ${ }^{1428}$, da wegen des geltenden Nominalwertprinzips ${ }^{1429}$ keine Neubewertung vorgenommen werden darf.

\subsection{Rücklage für eigene Anteile}

In Finnland ist eine Rücklage für eigene Anteile in Höhe der Anschaffungskosten dieser Anteile zu bilden, wenn eine öffentliche Aktiengesellschaft ihre Anteile zurückkauft $^{1430}$. Der Zweck der Rücklage ist eine Ausschüttungssperre. Ihre Auflösung ist nur bei Wiederveräußerung der Anteile oder bei außerplanmäßiger Abschreibung auf den niedrigeren Stichtagswert zulässig.

In Deutschland ist eine Rücklage für eigene Anteile zwingend zu bilden, wenn das Unternehmen Anteile von den Aktionären einer Aktiengesellschaft oder Kommanditgesellschaft auf Aktien oder von den Gesellschaftern einer Gesellschaft mit beschränkter Haftung zurückkauft oder unentgeltlich erhält ${ }^{1431}$. Der Zweck der Rücklage für eigene Anteile ist eine Ausschüttungssperre ${ }^{1432}$.

\subsection{Eigenkapitalersetzendes Rangrücktrittsdarlehen}

In Finnland sind einer Aktiengesellschaft von ihren Gesellschaftern oder dritten Gläubigern gewährte Darlehen dem Eigenkapital der Aktiengesellschaft zuzurechnen, wenn ein Rangrücktritt vereinbart worden ist, der bestimmte Anforderungen erfüllt und den Darlehensgeber im Konkursfall schlechter stellt als andere Gläubiger ${ }^{1433}$. Der finnische Gesetzgeber weicht mit dieser Vorschrift deutlich von anderen nationalen Gesetzgebern sowie internationalen Rechnungslegungsgremien $\mathrm{ab}^{1434}$.

Börsennotierte Unternehmen weisen Rangrücktrittsdarlehen im Eigenkapital aus, müssen sie bei der Ermittlung ihrer Bilanzkennzahlen jedoch dem Fremdkapital zuordnen $^{1435}$.

1428 Vgl. § 266 Abs. 3 HGB.

1429 Vgl. § 253 Abs. 1 HGB.

1430 Vgl. KPA 1:6.

1431 Vgl. § 272 Abs. 4 S. 1 HGB i. V. m. § 71 AktG bzw. i. V. m. § 33 GmbHG.

1432 Vgl. § 272 Abs. 4 HGB.

1433 Vgl. OYL 5:1.

1434 Vgl. Karhu (1998), S. 82 und vgl. Kap. 3.2.4.2 dieser Arbeit.

1435 Vgl. KILA-Anweisung „Erstellung von Jahresabschluss, vorläufigem Abschluss und Zwischenberichten“. Vgl. Kap. 3.2.4.2 dieser Arbeit. 
In Deutschland sind Gesellschafterdarlehen mit Rangrücktrittsvereinbarung und kapitalersetzende Darlehen als Fremdkapital unter den Verbindlichkeiten gegenüber Gesellschaftern auszuweisen ${ }^{1436}$.

\subsubsection{Fremdkapital}

\subsection{Steuerlich veranlasste Posten}

Im finnischen Bilanzgliederungsschema existiert auf der Passivseite der Bilanz der steuerliche Ausgleichsposten, den das deutsche HGB so nicht vorsieht.

Dieser Posten umfasst den kumulierten Abschreibungsunterschied (Passivierungspflicht ${ }^{1437}$ ) und freiwillige Rückstellungen mit Rücklageanteil (Passivierungswahlrecht ${ }^{1438}$ ).

Der kumulierte Abschreibungsunterschied enthält den Anteil zulässiger, steuerlich veranlasster anderer als planmäßiger Abschreibungen, der die handelsrechtlichen, planmäßigen Abschreibungen übersteigt. Es besteht eine Passivierungspflicht wegen der damit verbundenen verbesserten Aussagefähigkeit der Bilanz. Auf der Aktivseite wird der handelsrechtlich korrekte Wert des Anlagevermögens ausgewiesen. Der Posten weist sowohl Eigenkapitalcharakter (zweckgebundene Rücklage) als auch Fremdkapitalcharakter (Rückstellung für künftige Steuerzahlung aus Steuerstundung) auf.

Bei den freiwilligen Rückstellungen handelt es sich um Rückstellungen mit Rücklagencharakter (Investitionsrückstellungen oder Rückstellungen für allgemeine Geschäftsrisiken). Sie werden in der Periode ihrer Bildung voll dem Aufwand zugeordnet und in der Periode ihrer Auflösung versteuert. Insofern weist der Posten wegen des Steuerstundungseffekts Eigen- und Fremdkapitalanteile auf, die nach KPL 6:7 im Konzernabschluss getrennt auszuweisen sind. Steuerlich werden freiwillige Rückstellungen regelmäßig nur dann akzeptiert, wenn sie auch handelsrechtlich gebildet werden. Umgekehrt können sie handelsrechtlich unabhängig von steuerlichen Vorschriften gebildet werden mit dem Ziel, die Innenfinanzierungskraft des Unternehmens zu stärken ${ }^{1439}$.

Das deutsche Handelsrecht sieht den „Sonderposten mit Rücklageanteil“ vor, welcher dem finnischen Recht fremd ist. Für bestimmte Posten, die in der Steuerbilanz auf Grund steuerlicher Vorschriften gebildet worden sind, gilt handels-

1436 Vgl. für die GmbH: §§32 a, b GmbHG, für die GmbH \& Co.: §§ 129 a, 172 a HGB, und für die AG: BGH vom 26. März 1984.

1437 Vgl. KPL 5:12.

1438 Vgl. KPL 5:15.

1439 Vgl. KPL 5:15. Vgl. Kap. 3.2.5.1.2 dieser Arbeit. 
rechtlich ein Passivierungswahlrecht ${ }^{1440}$. $\mathrm{Zu}$ nennen sind steuerrechtliche $\mathrm{Ab}$ schreibungen und so genannte steuerfreie Rücklagen.

Steuerrechtliche Abschreibungen sind dadurch gekennzeichnet, dass sie über die handelsrechtlich gebotenen Abschreibungen hinausgehen ${ }^{1441}$.

Steuerfreie Rücklagen werden in der Steuerbilanz schon vor der Ermittlung des steuerpflichtigen Ergebnisses abgesetzt. Sie unterliegen der Besteuerung erst in den Perioden ihrer Auflösung ${ }^{142}$. Wegen dieses Steuerstundungseffekts stellt im Sonderposten mit Rücklageanteil der Anteil der erwarteten künftigen Steuerbelastung Fremdkapital und der nach der künftigen Besteuerung verbleibende Rest Eigenkapital dar ${ }^{1443}$.

\subsection{Rückstellungen}

\subsection{Ansatz}

In Finnland sind Rückstellungen für solche Außenverpflichtungen zu bilden, die wegen der Ungewissheit ihrer Höhe und/oder ihres Realisationszeitpunkts nicht als Verbindlichkeiten oder passive Rechnungsabgrenzungsposten ausgewiesen werden $^{1444}$. Sind Höhe und Fälligkeit sicher, besteht aber Unsicherheit über das Eintreten einer Verpflichtung, erfolgt der Ausweis - anders als in Deutschland nicht als Rückstellung, sondern als Verbindlichkeit oder passiver Rechnungsabgrenzungsposten $^{1445}$.

Es besteht in Finnland ein Passivierungsgebot für künftige Ausgaben und Verluste aus Außenverpflichtungen, die im Geschäftsjahr oder früheren Geschäftsjahren verursacht worden sind und denen keine Einnahmenerwartung mehr gegenübersteht $^{1446}$.

Für künftige Ausgaben und Verluste aus Innenverpflichtungen existiert ein Passivierungswahlrecht. Alternativ können sie im Anhang als Eventualverbindlichkeit genannt werden ${ }^{1447}$.

\footnotetext{
1440 Vgl. $\$ 247$ Abs. 3 HGB.

1441 Vgl. § 254 HGB.

1442 Dies betrifft die Reinvestitionsrücklage $\S 6$ b EStG, die Sanierungsrücklage $\S 6$ d EStG, die Auslandsinvestitionsrücklage $\S 1$ AusInvG, die Rücklage für die künftige Anschaffung oder Herstellung eines Wirtschaftsgutes $\S 7$ g Abs. 3 EStG, die Zuschussrücklage R 34 EstR sowie die Ersatzbeschaffungsrücklage R 35 EStR.

1443 Vgl. Baetge, Kirsch und Thiele (2001), S. 521.

1444 Vgl. KPL 5:14.1.

1445 Vgl. KILA 1219/1993 und KILA 1561/1999.

1446 Vgl. KPL 5:14.1.

1447 Vgl. KPL 5:14.2. Vgl. Kap. 3.2.5.2.1 dieser Arbeit.
} 
In Deutschland sind Rückstellungen zu bilden für Außenverpflichtungen, die wegen der Ungewissheit ihres Bestehens und/oder ihrer Höhe nicht als Verbindlichkeiten ausgewiesen werden, und für drohende Verluste aus schwebenden Geschäften $^{1448}$. Diese Rückstellungen bewirken eine bilanzielle Vorsorge für wahrscheinliche Risiken und werden zu Lasten des Jahresergebnisses und damit der möglichen Gewinnausschüttung gebildet.

Für künftige Ausgaben und Verluste aus Innenverpflichtungen existiert teils ein Passivierungsgebot, teils ein Passivierungswahlrecht. Das Passivierungsgebot greift bei unterlassenen Instandhaltungen, die innerhalb von drei Monaten nachgeholt werden, bei unterlassenen Aufwendungen für Abraumbeseitigung sowie bei zu erwartenden Gewährleistungszahlungen ${ }^{1449}$. Das Passivierungswahlrecht gilt für Instandhaltungsaufwendungen, die nicht innerhalb von vier bis 12 Monaten nachgeholt werden ${ }^{1450}$. Es besteht auch für Aufwendungen, welche genau umschrieben sind, dem oder früheren Geschäftsjahren zuzuordnen sind, am Stichtag wahrscheinlich oder sicher sind, aber hinsichtlich Höheoder Eintrittszeitpunkt unbestimmt sind ${ }^{1451}$.

\subsection{Bewertung}

Mangels nationaler, finnischer Bewertungsregeln (KPL 5:14.4: „Ansatz höchstens zu wahrscheinlichem Betrag") wird im Fachschrifttum empfohlen, die Höhe der Rückstellungen den Regeln des IAS 10 (reformatted 1994) und des IAS 37 (approved 1998) entsprechend zu bemessen ${ }^{1452}$. Daraus folgt:

- Schätzung der Rückstellungen nach „Best Estimate“ (Erwartungswert),

- Ansatz der Rückstellung zum Barwert, wenn die Abzinsung wesentliche Auswirkungen auf die Höhe der zurückzustellenden Verpflichtung hat (IAS 37.45-47), und

- grundsätzlich Einbeziehung künftiger Preissteigerungen.

Nach deutschem HGB sind Rückstellungen nur in Höhe des Betrags anzusetzen, der nach vernünftiger kaufmännischer Beurteilung notwendig ist ${ }^{1453}$. Hierunter wird im Fachschrifttum die wahrscheinliche Inanspruchnahme des Unternehmens unter Berücksichtigung der bestehenden Risiken verstanden. Aus dem Vorsichtsprinzip folgt der Ansatz zu Vollkosten. Eine Abzinsung von Rückstellungen

1448 Vgl. § 249 Abs. 1 HGB. Die derzeitige Regelung des $\S 249$ HGB wurde im Rahmen der Umsetzung der 4. EG-Richtlinie in deutsches Recht durch das Bilanzrichtlinien-Gesetz von 1985 geschaffen und entspricht weitgehend den Vorgaben des umzusetzenden Art. 20 dieser Richtlinie. Vgl. Schruff (1986), S. 94-95.

1449 Vgl. § 249 Abs. 1 S. 1 und 2 HGB.

1450 Vgl. § 249 Abs. 1 S. 3 HGB.

1451 Vgl. § 249 Abs. 2 HGB.

1452 Vgl. Kap. 3.2.5.2.1 dieser Arbeit.

1453 Vgl. § 253 Abs. 1 S. 2 HGB. 
kommt grundsätzlich nicht in Betracht, da sie gegen das Realisationsprinzip verstößt ${ }^{1454}$. Enthält die zu Grunde liegende Verbindlichkeit jedoch einen Zinsanteil, so darf die Rückstellung abgezinst werden ${ }^{1455}$. In Deutschland wird zur Zeit die generelle Abzinsung von Rückstellungen diskutiert ${ }^{1456}$.

Bei der Ermittlung von Drohverlustrückstellungen muss die künftige Preissteigerung in Verpflichtung und Anspruch eingerechnet werden (Imparitätsprinzip) ${ }^{1457}$. Verbindlichkeits-, Kulanz- und Aufwandsrückstellungen werden hingegen zu ihrem Stichtagswert ausgewiesen, da nur Risiken zu berücksichtigen sind, die bis zum Jahresabschluss-Stichtag entstanden sind ${ }^{1458}$.

\subsection{Pensionsrückstellungen}

In Finnland erfolgt der wesentliche Teil der Pensionszahlungen durch das staatliche Sozialsystem. Getroffene Einzelvereinbarungen sind von den Unternehmen meist durch entsprechende Versicherungsverträge abgesichert. Passivierungspflicht besteht für sämtliche mittelbaren und unmittelbaren Zusagen. Pensionsrückstellungen bilden in finnischen Abschlüssen in der Regel keine wesentliche Größe.

Es existieren keine vom Gesetzgeber oder vom KILA vorgeschriebenen Bewertungsgrundsätze. Die Unternehmen sind verpflichtet, die von ihnen zu Grunde gelegten Annahmen für die Bemessung der Rückstellung im Anhang anzugeben ${ }^{1459}$.

In Deutschland ist die betriebliche Altersversorgung üblich.

Die Deckung von Pensionszusagen erfolgt regelmäßig durch Rückstellungsbildung. Die Deckungsverpflichtung besteht für unwiderrufliche unmittelbare Zusagen, bei denen der Pensionsberechtigte seinen Rechtsanspruch nach dem 31. Dezember 1986 erworben hat ${ }^{1460}$. Für davor erteilte unmittelbaren Zusagen und bei Deckung durch eine Unterstützungskasse ist die Bildung einer Rückstellung nicht gesetzlich vorgeschrieben. Die Verpflichtungen müssen zumindest im Anhang angegeben werden (sog. Deckungslücke) ${ }^{1461}$.

1454 Ein Ansatz mit dem Barwert würde in diesem Fall eine ertragsbringende Anlage des im Unternehmen gebundenen Kapitals in Höhe des Kalkulationssatzes unterstellen; insofern handelte es sich bei der Minderung des Rückstellungsbetrags um unrealisierte Erträge. Vgl. Schruff (2001).

1455 Vgl. $§ 253$ Abs. 1 S. 2 HGB. Diese Vorschrift wurde im Rahmen der Umsetzung der Versicherungsbilanzrichtlinie mit dem Versicherungsbilanzrichtlinien-Gesetz vom 24.06.1994 in das HGB eingefügt. Die Kodifizierung diente nach allgemeiner Auffassung lediglich der Klarstellung. Vgl. Schruff (1997), S. 414.

1456 Vgl. Schruff (2001).

1457 Vgl. § 252 Abs. 4 HGB.

1458 Vgl. ADS (ab 1995), § 252 Anm. 38-43.

1459 Vgl. Kap. 3.2.5.2.2 dieser Arbeit.

1460 Vgl. Art. 28 Abs. 1 EGHGB.

1461 Vgl. Art. 28 Abs. 2 EGHGB. 
Änderungen der Pensionszusagen und/oder Berechnungsannahmen werden in vollem Umfang im Jahr der Änderung erfolgswirksam erfasst ${ }^{1462}$.

Wesentliche Berechnungsannahmen werden vom Steuergesetzgeber vorgeschrieben und regelmäßig auch handelsrechtlich beachtet. Folgende Faktoren und biometrischen Rechnungsgrundlagen (Invaliditäts- und Sterbewahrscheinlichkeiten) sind für die versicherungsmathematischen Berechnungen zu berücksichtigen:

- $\quad$ Sterbetafeln von Dr. Klaus Heubeck $1998^{1463}$,

- Barwert bei laufenden Pensionszahlungen ${ }^{1464}$,

- Barwert bei Anwartschaften, wenn keine Gegenleistungen des Arbeitnehmers mehr zu erwarten sind bzw. der Pensionsanspruch in voller Höhe unverfallbar ist ${ }^{1465}$ und

- $\quad$ derzeitiges Gehaltsniveau (zukünftige Steigerungen bleiben außer Betracht).

- Da es kein gesondertes Vermögen gibt, existieren auch keine zukünftigen Erträge, die berücksichtigt werden könnten.

- Um der Fluktuation Rechnung zu tragen, wird für Pensionsanwärter unter 30 Jahre keine Rückstellung gebildet ${ }^{1466}$.

- Es wird im Allgemeinen ein Rechnungszins von sechs Prozent zu Grunde gelegt $^{1467}$.

\subsection{Steuerrückstellungen}

In Finnland enthält die Steuerrückstellung wahrscheinlich zu leistende Steuernachzahlungen aus einer Betriebsprüfung oder aus Rechtsstreitigkeiten mit der Finanzverwaltung. Der am Stichtag ermittelte, nach Abzug etwaiger Vorauszahlungen verbleibende Betrag der Steuerforderung bzw. -schuld für das Geschäftsjahr oder frühere Geschäftsjahre ist unter sonstige Vermögenswerte oder aktiven Rechnungsabgrenzungsposten bzw. sonstigen Verbindlichkeiten oder passiven Rechnungsabgrenzungsposten auszuweisen.

Passive latente Steuern werden in Finnland nicht in die Rückstellungen einbezogen. Für sie existiert ein Passivierungswahlrecht, sodass sie nicht Bestandteil der Pflichtrückstellungen sind, sondern getrennt gezeigt werden ${ }^{1468}$.

\footnotetext{
1462 Vgl. § 249 Abs. 1 HGB.

1463 Vgl. § 6 a EStG.

1464 Vgl. § 253 Abs. 1 S. 2 HGB.

1465 Vgl. § 253 Abs. 1 S. 2 HGB.

1466 Vgl. $\$ 6$ a EStG.

1467 Vgl. $\S 6$ a Abs. 3 EStG und HFA 2/1988.

1468 Vgl. Kap. 3.2.5.2.3 dieser Arbeit.
} 
In Deutschland umfasst die Rückstellung alle ungewissen Verbindlichkeiten aus Steuern. Ungewissheit besteht bis zur Festsetzung der Steuer. Der am Stichtag ermittelte, nach Abzug etwaiger Vorauszahlungen verbleibende Betrag der Steuerschuld für das Geschäftsjahr oder frühere Geschäftsjahre ist als Rückstellung auszuweisen. Nach Erlass des Steuerbescheids ist er in die sonstigen Verbindlichkeiten umzugliedern, wobei ein eventueller Differenzbetrag erfolgswirksam in der Gewinn- und Verlustrechnung zu erfassen ist ${ }^{1469}$.

Passive latente Steuern werden in Deutschland in die Steuerrückstellung einbezo$\operatorname{gen}^{1470}$.

\subsection{Sonstige Rückstellungen}

\subsection{5.2.5.1 Rückstellung für unterlassene Instandhaltungsaufwendungen}

In Finnland gilt für Aufwendungen aus unterlassener Instandhaltung ein Passivierungswahlrecht, wie für alle Rückstellungen aus Innenverpflichtungen ${ }^{1471}$. Alternativ erfolgt eine Angabe im Anhang.

In Deutschland ist eine Rückstellung für im Geschäftsjahr unterlassene Instandhaltung erforderlich für Aufwendungen, die innerhalb von drei Monaten nach dem Bilanzstichtag nachgeholt werden ${ }^{1472}$. Wenn der Aufwand innerhalb von vier bis zwölf Monaten nach diesem Stichtag nachgeholt wird, besteht ein Passivierungswahlrecht ${ }^{1473}$. Erfolgt die Nachholung erst im übernächsten Geschäftsjahr nach Abschluss-Stichtag, ist eine Rückstellungsbildung nicht zulässig.

1469 Vgl. BeBiKo (1999), § 266 Anm. 201.

1470 Vgl. § 274 Abs. 1 i. V. m. § 249 Abs. 1 HGB.

1471 Vgl. KPL 5:14.2. Vgl. Kap. 3.2.5.2.1 dieser Arbeit.

1472 Vgl. § 249 Abs. 1 S. 2 Nr. 1 HGB.

1473 Vgl. § 249 Abs. 1 S. 3 HGB. 


\subsection{2 Drohverlustrückstellung}

Drohende Verluste aus schwebenden Geschäften werden in Finnland und in Deutschland übereinstimmend behandelt.

Für schwebende Beschaffungsgeschäfte gilt in beiden Ländern:

Wenn der Marktpreis am Stichtag eines bestellten, aber erst nach dem Bilanzstichtag gelieferten Vermögensgegenstands des Anlagevermögens unter dem Vertragspreis liegt, ist eine Rückstellung zu bilden, wenn eine dauerhafte Wertminderung vorliegt (gemildertes Niederstwertprinzip).

Bei Gegenständen des Umlaufvermögens ist nicht nur ein bei Weiterveräußerung zu erwartender Verlust, sondern auch die negative Differenz zwischen dem Vertragspreis und dem Marktpreis am Bilanzstichtag (strenges Niederstwertprinzip) rückstellungspflichtig ${ }^{1474}$.

Für schwebende Absatzgeschäfte gilt in beiden Ländern:

Erwartete zukünftige Verluste bei unfertigen Erzeugnissen und Leistungen werden bei der Vorratsbewertung durch Abschreibung der betroffenen Vorräte berücksichtigt. Übersteigt der Verlust den bislang aktivierten Betrag, ist in Höhe des übersteigenden Betrags eine Rückstellung zu bilden, falls es sich um einen wesentlichen Betrag handelt ${ }^{1475}$.

Für Dauerrechtsverhältnisse gilt:

Wenn für die Restlaufzeit eines sich über einen längeren Zeitraum erstreckenden Geschäfts die vom Unternehmen zu erbringenden künftigen Leistungen den Wert der zu empfangenden Gegenleistungen übersteigen, ist eine Rückstellung zu bilden.

\subsection{3 Kulanzrückstellung}

In Finnland und Deutschland sind Rückstellungen für Gewährleistungen zu bilden, die ohne rechtliche Verpflichtung erbracht werden (Kulanzrückstellung) ${ }^{1476}$. Sie werden anhand von Erfahrungswerten bemessen.

1474 Vgl. BeBiKo (1999), § 249 Anm. 69 ff und vgl. Luoma (1998), S. 233-241.

1475 Vgl. BeBiKo (1999), § 249 Anm. 74 f. und vgl. Luoma (1998), S. 233-241.

1476 Vgl. Kap. 3.2.5.2.1 dieser Arbeit. Vgl. § 249 Abs. 1 S. 2 Nr. 2 HGB. 


\subsection{5.2.5.4 Rückstellung für ungewisse Verbindlichkeiten}

Rückstellungen für ungewisse Verbindlichkeiten sind in Finnland und in Deutschland passivierungspflichtig ${ }^{1477}$. Eventualverbindlichkeiten werden in Finnland im Anhang ausgewiesen ${ }^{1478}$, in Deutschland können sie alternativ auch unter der Bilanz aufgeführt werden ${ }^{1479}$.

\subsection{5 Personalbezogene Rückstellungen}

In Finnland werden nicht gezahlte Lohn- und Gehaltsbestandteile aus dem Geschäftsjahr oder früheren Geschäftsjahren als antizipative Rechnungsabgrenzung im passiven Rechnungsabgrenzungsposten ausgewiesen. Hierzu zählen Überstunden, Urlaubsgeld und Tantiemen. Ihre Realisierung ist sicher, da eine Gegenleistung bereits erfolgt ist. Ihre exakte Höhe und ihr Realisationszeitpunkt sind auf Grund interner Kostenrechnung und der Zahlungslauftermine bekannt ${ }^{1480}$. Folglich weisen diese Verpflichtungen keinen Rückstellungscharakter, sondern einen Verbindlichkeitscharakter auf.

In Deutschland werden Erfüllungsrückstände aus nicht gezahlten Lohn- und Gehaltsbestandteilen aus dem Geschäftsjahr oder früheren Geschäftsjahren üblicherweise nicht exakt berechnet, sondern anhand von Erfahrungswerten, Hochrechnungen oder Schätzungen ermittelt. Sie sind daher dem Grund und/oder der Höhe nach unsicher. Verpflichtungen aus Überstunden, Urlaubsgeld und Tantiemen werden deshalb als personalbezogene Rückstellungen ausgewiesen ${ }^{1481}$.

Dieser Sachverhalt verdeutlicht die unterschiedliche Interpretation des Grundsatzes der Einzelbewertung in Finnland und Deutschland.

1477 Vgl. § 249 Abs. 1 S. 1 HGB:

1478 Vgl. Kap. 3.2.5.2.1 dieser Arbeit.

1479 Vgl. § 251 HGB.

1480 Vgl. Kap. 3.2.5.3.2 dieser Arbeit.

1481 Vgl. ADS (ab 1995), § 249 Anm. 60-61. 


\subsection{6 Rückstellung für interne Jahresabschlusskosten}

Interne Jahresabschlusskosten werden in Finnland nicht zurückgestellt ${ }^{1482}$.

In Deutschland wird eine Rückstellung für interne Jahresabschlusskosten auf Grund öffentlich-rechtlicher oder privatrechtlicher Verpflichtung in Handels- und Steuerbilanz gebildet ${ }^{1483}$.

\subsection{7 Jubiläumsrückstellung}

Zahlungen des Arbeitsgebers aus Anlass von Dienstjubiläen sind in Finnland nicht üblich. Daher besteht kein Rückstellungsbedarf ${ }^{1484}$.

In Deutschland werden Rückstellungen für Aufwendungen aus Jubiläumszuwendungen in dem Umfang gebildet, wie die vertraglichen Anspruchsvoraussetzungen durch die vergangene Betriebszugehörigkeit des Arbeitnehmers bis zum Bilanzstichtag erfüllt sind ${ }^{1485}$. Meist sind umfangreiche versicherungsmathematische Berechnungen zur Ermittlung der Rückstellung notwendig ${ }^{1486}$.

\subsection{8 Vertriebsbezogene Rückstellungen}

$\mathrm{Zu}$ leistende Boni, Skonti und Rabatte des Geschäftsjahrs werden in Finnland nicht als Rückstellungen interpretiert, sondern als antizipierte Rechnungsabgrenzungen (Ausweis als passiver Rechnungsabgrenzungsposten). Ihre Realisierung ist sicher, da die Gegenleistung seitens der Kunden bereits erfolgt ist. Auch die exakte Höhe und der Realisationszeitpunkt sind regelmäßig bekannt, da vertragliche Vereinbarungen zu Grunde liegen ${ }^{1487}$.

$\mathrm{Zu}$ leistende Boni, Skonti und Rabatte des Geschäftsjahrs werden in Deutschland regelmäßig als Rückstellungen ausgewiesen, da sie nicht einzeln ermittelt, sondern anhand von Erfahrungswerten, Hochrechnungen oder Schätzungen gebildet werden $^{1488}$.

\footnotetext{
1482 Wie mit internen Jahresabschlusskosten zu verfahren sei wird in der finnischen Fachliteratur nicht diskutiert. In den untersuchten Geschäftsberichten (vgl. Kap. 4.2.2.1 dieser Arbeit) werden interne Jahresabschlusskosten jedoch nicht zurückgestellt.

1483 Vgl. BeBiKo (1999), § 249 Anm. 100.

1484 Vgl. Kap. 3.2.5.2.1 dieser Arbeit.

1485 Vgl. ADS (ab 1995), § 249 Anm. 61.

1486 Vgl. BeBiKo (1999), § 249 Anm. 100.

1487 Vgl. KILA 1219/1993 und vgl. Kap. 3.2.5.3.2 dieser Arbeit.

1488 Vgl. BeBiKo (1999), § 249 Anm. 100.
} 
Wie bei den personalbezogenen Rückstellungen wird auch hier die unterschiedliche Interpretation des Grundsatzes der Einzelbewertung in Finnland und in Deutschland offensichtlich.

\subsection{Verbindlichkeiten}

\subsection{Erhaltene Anzahlungen}

In Finnland besteht ein striktes Saldierungsverbot zwischen erhaltenen Anzahlungen und Aktivsalden ${ }^{1489}$.

In Deutschland dürfen erhaltene Anzahlungen grundsätzlich nicht mit Forderungen saldiert werden (Verrechnungsverbot), es sei denn, sie betreffen denselben Sachverhalt ${ }^{1490}$.

Erhaltene Anzahlungen auf Bestellungen dürfen vom Posten „Vorräte“ offen abgesetzt werden $^{1491}$.

\subsection{Sonstige Verbindlichkeiten und passive Rechnungsabgrenzungs- posten}

In Finnland werden sonstige Verbindlichkeiten und passive Rechnungsabgrenzungsposten unter den Verbindlichkeiten ausgewiesen. Als sonstige Verbindlichkeiten gelten solche Verpflichtungen, die hinsichtlich ihres Eintretens unsicher, aber in Bezug auf Höhe und Realisationszeitpunkt sicher sind. Der Begriff ,,sonstige Verbindlichkeiten“ wird weiter gefasst als in Deutschland, wo solche Verpflichtungen den Rückstellungen zugeordnet werden. Passive Rechnungsabgrenzungsposten enthalten in Finnland sowohl transitorische als auch antizipative Pos$\operatorname{ten}^{1492}$.

In Deutschland werden sonstige Verbindlichkeiten und passive Rechnungsabgrenzungen gesondert ausgewiesen. Antizipative Abgrenzungen werden den sonstigen Verbindlichkeiten zugeordnet, transitorische Abgrenzungen sind im passiven Rechnungsabgrenzungsposten enthalten ${ }^{1493}$.

1489 Vgl. Kap. 3.2.5.3 dieser Arbeit.

1490 Vgl. WPH I (2000), E 52 und vgl. BeBiKo (1999), § 246 Anm. 80-84.

1491 Vgl. § 268 Abs. 5 S. 2 HGB.

1492 Vgl. Kap. 3.2.5.3.2 dieser Arbeit.

1493 Vgl. Baetge, Kirsch und Thiele (2001), S. 336 und S. 464. 


\subsubsection{Derivative Finanzinstrumente}

Sowohl in Finnland als auch in Deutschland haben die Gesetzgeber keine konkreten Vorschriften zur bilanziellen Behandlung derivativer Finanzinstrumente erlassen. Insofern erfolgt die Bilanzierung nach den allgemeinen Vorschriften zum Ansatz, zur Bewertung und zum Ausweis sowie nach den Grundsätzen ordnungsmäßiger Buchführung ${ }^{1494}$.

In Finnland hat sich als Art guter Buchführung herausgebildet, derivative Finanzinstrumente nicht zu bilanzieren. Sie werden als schwebende Geschäfte betrachtet, die nicht bilanzierungsfähig sind. Hierbei ist es unerheblich, ob sie am AbschlussStichtag einen finanziellen Vermögenswert oder eine finanzielle Verbindlichkeit darstellen. Börsennotierte Aktiengesellschaften haben aus Derivaten resultierende Zahlungsverpflichtungen und mit den Derivaten verfolgte Zwecke im Anhang als Eventualverbindlichkeit so anzugeben, dass ein True and Fair View vermittelt wird. Nicht börsennotierte Unternehmen informieren in der Praxis regelmäßig freiwillig über von ihnen gehaltene Derivate und andere Financial Instruments ${ }^{1495}$.

Anderes gilt für die Behandlung derivativer Finanzinstrumente in Deutschland. Auch hier werden derivative Finanzinstrumente zwar grundsätzlich als nicht bilanzierungsfähige schwebende Geschäfte angesehen. Ein drohender Verlust ist jedoch nach dem Imparitätsprinzip zu antizipieren ${ }^{1496}$. Positive Wertentwicklungen gelten vor Veräußerung oder Ausübung der derivativen Finanzinstrumente als nicht realisiert und dürfen nicht ertragswirksam berücksichtigt werden ${ }^{1497}$.

\subsubsection{Latente Steuerabgrenzung}

In Finnland existiert für den Einzelabschluss ein Bilanzierungswahlrecht für aktive und passive latente Steuern ${ }^{1498}$. Werden sie nicht bilanziell erfasst, ist ihr Wert im Anhang anzugeben, sofern er wesentlich ist ${ }^{1499}$. Latente Steuern werden auf alle Timing Differences ermittelt. Alternativ besteht auch die Möglichkeit, sie zusätzlich auf quasi-permanente Differenzen zu berechnen (gegebenenfalls Neubewertungsrücklage) ${ }^{1500}$.

\footnotetext{
1494 Vgl. Järvinen et al. (2000), S. 438, vgl. Kap. 3.2.6 dieser Arbeit und vgl. Baetge, Kirsch und Thiele (2001), S. 650.

1495 Vgl. VM:p 390/1999, Abschnitt 2:5.4.

1496 Vgl. § 249 Abs. 1 HGB.

1497 Vgl. § 252 Abs. 1 Nr. 4 HGB.

1498 Vgl. KPL 5:18.

1499 Vgl. KPL 2:6.1,1 und KILA-Anweisung „Latente Steuern“, Abschnitt 1.1.

1500 Vgl. Kap. 3.2.7 dieser Arbeit.
} 
In Deutschland besteht im Einzelabschluss ein Aktivierungswahlrecht für latente Steuerforderungen ${ }^{1501}$ und eine Passivierungspflicht für latente Steuerverbindlichkeiten $^{1502}$. Wegen des Maßgeblichkeitsprinzips sind latente Steuern im Einzelabschluss selten. Aktive latente Steuern (Bilanzierungshilfe) dürfen im Einzelabschluss nur angesetzt werden, wenn die nach einer Gewinnausschüttung verbleibenden jederzeit auflösbaren Gewinnrücklagen zusammen mit dem Ergebnisvortrag dem aktivierten Betrag mindestens entsprechen ${ }^{1503}$. Passive latente Steuern sind für alle Timing Differences zu bilden ${ }^{1504}$.

\subsubsection{Ausgewählte Posten der Gewinn- und Verlustrechnung ${ }^{1505}$}

\subsubsection{Umsatzerlöse}

Sowohl in Finnland als auch in Deutschland gilt, dass als Umsatzerlöse Erlöse aus der gewöhnlichen Geschäftstätigkeit eines Unternehmens nach Abzug von Erlösschmälerungen sowie Umsatzsteuer auszuweisen sind ${ }^{1506}$. Es ist zu beachten, dass das Vorsichtsprinzip mit seinen Ausprägungen Realisations- und Imparitätsprinzip in Deutschland enger als in Finnland ausgelegt wird. Zudem wird in Finnland das Prinzip der Periodenabgrenzung stärker betont als in Deutschland. Erträge gehen daher in Finnland oft früher in die Gewinn- und Verlustrechnung ein als in Deutschland $^{1507}$.

\subsubsection{Außerordentliche Posten}

Nach finnischem Verständnis weichen außerordentliche Posten von der normalen Geschäftstätigkeit ab, weisen Einzelfall-Charakter auf, und der ihnen zu Grunde liegende Sachverhalt ist für das Unternehmen von wesentlicher Bedeutung. AuBerordentliche Posten enthalten neben Schadensfällen, Verkauf von Tochterunternehmen, Fusionen und Enteignungen auch einmalige Auswirkungen auf das Unternehmensergebnis aus einer wesentlichen Änderung der Rechnungslegungsge-

1501 Vgl. § 274 Abs. 2 HGB.

1502 Vgl. \$2 274 Abs. 1 HGB.

1503 Vgl. § 274 Abs. 2 HGB.

1504 Vgl. § 274 Abs. 1 HGB.

1505 Analog zur Vorgehensweise in Kap. 3.3 dieser Arbeit wird hier lediglich auf die Posten der Gewinn- und Verlustrechnung eingegangen, zu denen explizit in Abschnitt 6 der 4. EGRichtlinie existieren. Weitere Posten der Gewinn- und Verlustrechnung sind bei den korrespondierenden Bilanzposten bereits behandelt worden.

1506 Vgl. KPL 4:1 bzw. § 277 Abs. 1 HGB.

$1507 \mathrm{Zu}$ nennen sind beispielsweise Erträge aus der Währungsumrechnung (vgl. Kap. 4.1.2.6 dieser Arbeit) und Teilgewinne aus langfristiger Auftragsfertigung (vgl. Kap. 4.1.3.2.4 dieser Arbeit). 
setzgebung $^{1508}$. Der Ausweis dieser Posten erfolgt gesondert nach dem Ergebnis aus der gewöhnlichen Geschäftstätigkeit und dem Finanzergebnis.

Außerordentliche Posten sind nach deutscher Auffassung bedeutende Geschäftsvorfälle, die ungewöhnlicher Art sind, außerhalb der gewöhnlichen Geschäftstätigkeit des Unternehmens angefallen sind und sich üblicherweise nicht wiederho$\operatorname{len}^{1509} . \mathrm{Zu}$ nennen sind beispielsweise Restrukturierungsaufwendungen und einmalige Zuschüsse zur Umstrukturierung oder Sanierung. Außerordentliche Posten werden nach dem Ergebnis aus der gewöhnlichen Geschäftstätigkeit ausgewiesen ${ }^{1510}$. Sie sind nach Art und Höhe im Anhang zu erläutern, sofern sie wesentlich für die Beurteilung des True and Fair View sind ${ }^{1511}$.

\subsubsection{Ausgewählte Anhangangaben}

\subsubsection{Segmentinformationen}

Gegenstand der Segmentberichterstattung in Finnland wie in Deutschland sind bislang allein die Umsatzerlöse ${ }^{1512}$.

In Finnland hat jeder Buchführungspflichtige die Umsatzerlöse nach Tätigkeitsbereichen und nach geographischen Märkten aufzugliedern ${ }^{1513}$. Zusätzlich darf der Buchführungspflichtige Aufgliederungen der Umsatzerlöse nach anderen Gesichtspunkten vornehmen, beispielsweise nach Produktions- oder Vertriebsstät$\operatorname{ten}^{1514}$.

In Deutschland haben mittelgroße und große Kapitalgesellschaften eine Aufgliederung der Umsätze nach Tätigkeitsbereichen und geographischen Märkten vorzunehmen $^{1515}$. Bei börsennotierten Mutterunternehmen ist der Konzernanhang seit In-Kraft-Treten des KonTraG um eine sich an internationalen Rechnungslegungsvorschriften orientierende Segmentberichterstattung zu ergänzen ${ }^{1516}$. Weil die Ausgestaltung der Segmentberichterstattung vom Gesetzgeber nicht konkretisiert wird, hat der DSR für Konzernabschlüsse einen Standard zu diesem Thema verabschiedet, dessen Anwendung auch für Einzelabschlüsse empfohlen wird ${ }^{1517}$.

\footnotetext{
1508 Vgl. Kap. 3.3.2 dieser Arbeit.

1509 Vgl. § 277 Abs. 4 HGB.

1510 Vgl. § 275 Abs. 2 Nr. 16-17 HGB und § 275 Abs. 3 Nr. 15-16 HGB.

1511 Vgl. § 271 Abs. 4 S. 2 HGB.

1512 Vgl. KPA 2:3 und vgl. § 285 Nr. 4 HGB.

1513 Vgl. KPA 2:3.1,1.

1514 Vgl. KPL-Arbeitsgruppe (1995), S. 130.

1515 Vgl. § 285 Nr. 4 HGB.

1516 Vgl. § 297 Abs. 1 S. 2 HGB.

1517 Vgl. DRS 3.
} 


\subsubsection{Angaben bezüglich nahestehender Unternehmen und Personen}

Finnische Gesellschaften und deutsche Kapitalgesellschaften müssen Namen und Sitz des Mutterunternehmens der Kapitalgesellschaft, das den Konzernabschluss für den größten Kreis von Unternehmen aufstellt, und ihres Mutterunternehmens, das den Konzernabschluss für den kleinsten Kreis von Unternehmen aufstellt, offen legen ${ }^{1518}$. Auch muss aufgezeigt werden, wo diese Konzernabschlüsse erhältlich sind.

In Finnland wie in Deutschland sind Name und Sitz anderer Unternehmen, an denen die Kapitalgesellschaft mindestens 20 Prozent der Anteile besitzt, unter Angabe des Anteils am gezeichneten Kapital, des Eigenkapitals und des Ergebnisses des letzten Geschäftsjahres, für das ein Jahresabschluss vorliegt, zu nennen $^{1519}$. In Finnland sind diese Informationen zusätzlich auch für diejenigen Gesellschaften zu geben, für die unbegrenzt gehaftet wird ${ }^{1520}$.

Angaben über nahestehende Personen umfassen in Finnland gewährte Darlehen, Bürgschaften sowie Eventualverbindlichkeiten zu Gunsten der Mitglieder des „,inneren Kreises“ einer Aktiengesellschaft ${ }^{1521}$. Zum inneren Kreis zählen Geschäftsführer, Verwaltungs- und Aufsichtsratsmitglieder sowie Wirtschaftsprüfer und diejenigen Personen, die auf Grund von Eigentum, Optionsrechten und Wandelschuldverschreibungen mindestens ein Prozent der Aktien halten, und Angehörige aller genannten Personen ${ }^{1522}$. Darlehen an Geschäftsführer sind in der Bilanz oder im Anhang gesondert auszuweisen ${ }^{1523}$.

Angaben über Beziehungen zu nahestehenden Unternehmen und Personen umfassen in Deutschland Informationen über Konzernsalden, Darlehen und Bezüge von Geschäftsführung, Vorstand und Aufsichtsrat sowie Informationen hinsichtlich Mutter-, Tochter- und assoziierter Unternehmen ${ }^{1524}$.

1518 Vgl. KPA 1:10 und vgl. § 285 Nr. 14 HGB.

1519 Vgl. KPA 1:9.1,1 und vgl. § 285 Nr. 11 HGB.

1520 Vgl. KPL 1:9.1,2.

1521 Vgl. OYL 11:17.

1522 Vgl. OYL 11:4.

1523 Vgl. OYL 11:7.1.

1524 Vgl. § 285 Nr. 9-10 HGB und vgl. Deutscher Corporate Governance Kodex (2002). 


\subsubsection{Ergebnis je Aktie}

Börsennotierte Aktiengesellschaften in Finnland müssen das Ergebnis je Aktie im Verhältnis zum Nennwert der Aktie in Prozent für die letzten fünf Geschäftsjahre angeben $^{1525}$.

Für börsennotierte Unternehmen in Deutschland ist die Offenlegung des Gewinns je Aktie freiwillig. Verbindliche Vorschriften für die Berechnung liegen nicht vor.

\subsubsection{Lagebericht}

Der Lagebericht als Bestandteil des Jahresabschlusses ist in Finnland von allen Buchführungspflichtigen zu erstellen ${ }^{1526}$. In ihm sind Geschäftsverlauf, Lage und künftige Entwicklung so darzustellen, dass sie den True and Fair View vermit$\operatorname{teln}^{1527}$. Es sind zu nennen:

- Die Chancen und Risiken der künftigen Entwicklung ${ }^{1528}$,

- wesentliche Geschehnisse, die sich nach Beendigung des Geschäftsjahrs ereignet haben $^{1529}$,

- $\quad$ eine Einschätzung der künftigen Entwicklung der Gesellschaft ${ }^{1530}$,

- der Umfang von Forschung und Entwicklung ${ }^{1531}$,

- bei Aktiengesellschaften die Veränderung der gesellschaftsrechtlichen Verhältnisse im Geschäftsjahr ${ }^{1532}$ :

- Mutterunternehmen,

- Verschmelzungen,

- Abspaltungen,

- Betriebsstätten und Niederlassungen im Ausland,

- Veränderung der Eigenkapitalstruktur:

- Kapitalerhöhungen,

- $\quad$ eigene Anteile im Besitz der Gesellschaft und der Mitglieder des „,inneren Kreises“,

- Gewinnverwendungsvorschlag.

Bei öffentlichen Aktiengesellschaften und großen privaten Aktiengesellschaften bildet die Kapitalflussrechnung den Anhang zum Lagebericht ${ }^{1533}$.

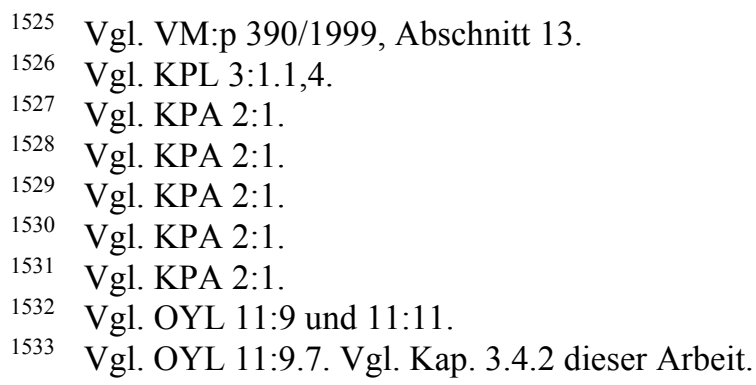


In Deutschland haben mittelgroße und große Kapitalgesellschaften einen Lagebericht aufzustellen ${ }^{1534}$. Er steht eigenständig neben dem Jahresabschluss. Im Lagebericht sind zumindest der Geschäftsverlauf und die Lage der Gesellschaft so darzustellen, dass ein den tatsächlichen Verhältnissen entsprechendes Bild vermittelt wird $^{1535}$. Der Lagebericht soll auch eingehen auf:

- Risiken der künftigen Entwicklung ${ }^{1536}$,

- Vorgänge von besonderer Bedeutung, die nach dem Schluss des Geschäftsjahrs aufgetreten sind ${ }^{1537}$,

- die voraussichtliche Entwicklung der Gesellschaft ${ }^{1538}$,

- den Bereich Forschung und Entwicklung ${ }^{1539}$ und

- bestehende Zweigniederlassungen im In- und Ausland ${ }^{1540}$.

Es ist zulässig - und zuweilen im Sinne des True and Fair View geboten - den Lagebericht um eine über die Mindestanforderungen des $§ 289$ HGB hinausgehende Berichterstattung zu ergänzen. Hier kommen Kapitalflussrechnung, Bewegungsbilanz, Substanzerhaltungsrechnung, Sozialbericht oder Umweltberichterstattung in Betracht. Dieser freiwillige Einblick in die Lage einer Kapitalgesellschaft darf allerdings weder in Form noch Umfang derart gestaltet sein, dass er von den Pflichtangaben ablenkt ${ }^{1541}$.

\subsubsection{Zusammenfassung und kritische Würdigung der Ergebnisse}

In Kap. 4.1 konnte gezeigt werden, dass die formale Harmonisierung zwischen Finnland und Deutschland weit fortgeschritten ist. Dennoch existieren auch nach Umsetzung der 4. EG-Richtlinie Unterschiede zwischen den Rechnungslegungsvorschriften beider Staaten, die sich je nach Sachverhalt beträchtlich auswirken können. Die Differenzen resultieren aus dem unterschiedlichen grundlegenden Verständnis der Rechnungslegung, das sich in beiden Ländern historisch entwickelt hat. Die Geschichte besitzt trotz Transformation der 4. EG-Richtlinie heute noch einen starken, nachhaltigen Einfluss auf die Rechnungslegungsgesetzgebung und -praxis.

Auffallend ist, dass in Finnland viele Regelungslücken durch den Buchführungsausschuss KILA geschlossen werden. Der KILA legt in Zweifelsfragen die Gesetze aus und interpretiert sie verbindlich für die Praxis. Hierin besteht ein wesentlicher Kulturunterschied zwischen den Rechnungslegungssystemen Finnlands und

\footnotetext{
1534 Vgl. § 264 Abs. 1 S. 1 HGB.

1535 Vgl. § 289 Abs. 1 HGB.

1536 Vgl. § 289 Abs. 1 HGB.

1537 Vgl. § 289 Abs. 2 Nr. 1 HGB.

1538 Vgl. § 289 Abs. 2 Nr. 2 HGB.

1539 Vgl. § 289 Abs. 2 Nr. 3 HGB.

1540 Vgl. § 289 Abs. 2 Nr. 4 HGB.

1541 Vgl. BeBiKo (1999), § 289 Anm. 5.
} 
Deutschlands, wo die herrschende Meinung über Literaturbeiträge und Kommentare zur Rechnungslegung von Sachverständigen aus Theorie und Praxis gebildet wird $^{1542}$. Aus diesem Kulturunterschied $\mathrm{zu}$ schließen, bestimmte Sachverhalte seien in Finnland eindeutiger geregelt als in Deutschland, ist nicht korrekt. Auch die Vermutung, die Vergleichbarkeit finnischer Abschlüsse untereinander sei höher als die deutscher Abschlüsse, ist so nicht zutreffend, denn der finnische Gesetzgeber lässt den Unternehmen mehr Wahlrechte als der deutsche. Beispielsweise gilt in Finnland das Aktivierungswahlrecht für immaterielle Vermögenswerte, das Wahlrecht zur Neubewertung, das Wahlrecht zur Anwendung der Percentage of Completion-Methode oder das Ansatzwahlrecht für passive latente Steuern. Zudem hat der KILA die Befugnis, in bestimmten Bereichen einzelnen Unternehmen oder Unternehmen ganzer Branchen Ausnahmen von der geltenden Regel zu erlauben.

In Finnland wie in Deutschland sind die Grundsätze ordnungsmäßiger Buchführung zur Auslegung der gesetzlichen Bestimmungen heranzuziehen. Sie stellen in beiden Staaten Regeln dar, welche die gesamte Rechnungslegung umfassen. Die Unterschiede im grundlegenden Verständnis der Rechnungslegung führen geschichtlich bedingt nach wie vor zu einer unterschiedlichen Betonung in der Anwendung der beiden wesentlichen Rechnungslegungsgrundsätze „Vorsichtsprinzip“ und „Prinzip der Periodenabgrenzung“"1543. Die in Deutschland bedeutendste Regel ist das Vorsichtsprinzip, nach dem alle vorhersehbaren Risiken und Verluste, die bis zum Bilanzstichtag entstanden waren, berücksichtigt werden müssen. Noch nicht realisierte Gewinne dürfen hingegen nicht berücksichtigt werden. Das Vorsichtsprinzip existiert in Finnland ebenfalls seit langem, nur wird es anders interpretiert. Relativ mehr Beachtung findet hier das Prinzip der Periodenabgrenzung. Die finnische Auffassung, was unter einem wahrscheinlichen Verlust oder einem realisierten Gewinn zu verstehen sei, wird deshalb im Einzelfall von der deutschen abweichen.

Sowohl in Deutschland als auch in Finnland führt die umgekehrte Maßgeblichkeit zu einer Beeinflussung der Handelsbilanz durch die Steuergesetzgebung. Die Bindung zwischen Handels- und Steuerbilanz ist in Deutschland heute jedoch enger als in Finnland, wo sie seit 1993 gelockert wird ${ }^{1544}$.

Die stärkere Betonung des Vorsichtsprinzips und die stärkere Berücksichtigung von abweichenden steuerlichen Vorschriften könnten zu der Annahme führen, dass ein nach deutschen Rechnungslegungsvorschriften ermittelter Jahresüberschuss immer niedriger sei als der für ein vergleichbares Unternehmen nach finni-

1542 Vgl. Kap. 4.1.1 dieser Arbeit.

1543 Vgl. Kap. 4.1.2.3.1 dieser Arbeit.

1544 Vgl. Kap. 4.1.1.1.2 dieser Arbeit. 
schen Vorschriften ermittelte. Diese Verallgemeinerung ist so nicht zutreffend. Die Abweichungen ergeben sich vielmehr aus der unterschiedlichen Periodenabgrenzung in beiden Staaten. Gewinne gehen in Deutschland oft später und Verluste oft früher in die Gewinn- und Verlustrechnung ein als in Finnland.

In Finnland können beispielsweise bei langfristiger Auftragsfertigung Umsatzerlöse und Gewinn entsprechend dem Fertigungsgrad realisiert werden, sofern die Beträge mit einiger Wahrscheinlichkeit geschätzt werden können. Nach deutschem Verständnis des Vorsichtsprinzips dürfen Umsatz und Gewinn erst bei Vertragserfüllung in den Jahresabschluss eingehen. Auch können in Finnland Währungsgewinne bei monetären Posten der Bilanz in der Gewinn- und Verlustrechnung erfasst werden, wenn die ihnen zu Grunde liegende Transaktion noch nicht abgeschlossen ist. In Deutschland dürfen Währungsgewinne erst nach Abschluss einer Transaktion erfolgswirksam ausgewiesen werden. Folglich kann in bestimmten Jahren der Gewinn, der nach finnischen Vorschriften ermittelt wurde, höher sein als der nach deutschen Vorschriften errechnete oder umgekehrt. 


\section{Empirische Untersuchung der tatsächlich erreichten Harmonisie- rung anhand ausgewählter Rechnungslegungsaspekte}

\subsubsection{Der I-Index}

Die empirische Messung des tatsächlich realisierten Harmonisierungsgrads innerhalb eines Lands und zwischen einzelnen Ländern kann auf der Basis von Konzentrationsindizes erfolgen ${ }^{1545}$.

Ein Versuch, Rechnungslegungsharmonisierung zu quantifizieren, wurde von VAN DER TAS im Jahr 1988 veröffentlicht und später fortentwickelt ${ }^{1546}$. VAN DER TAS verwendet den Herfindahl-Index, einen speziellen Konzentrationsindex. Er misst mit seiner Hilfe Harmonisierung als Konzentration auf die Anwendung eines bestimmten aus mehreren alternativen Rechnungslegungsverfahren ${ }^{1547}$. Wichtiges Merkmal dieser Messungsmethode ist, dass der Harmonisierungsgrad eines speziellen Rechnungslegungsproblems anhand empirisch ermittelter Anwendungshäufigkeiten alternativer Rechnungslegungsmethoden gemessen wird. Relevant ist die tatsächliche Anwendung von Rechnungslegungsverfahren, nicht die Übereinstimmung des ihnen zu Grunde liegenden Regelwerks ${ }^{1548}$.

VAN DER TAS schlägt vor, den tatsächlich realisierten Harmonisierungsgrad der Rechnungslegung zwischen zwei oder mehreren Staaten mit dem I-Index zu erfassen, den er aus dem Herfindahl-Index entwickelt hat ${ }^{1549}$. Dem I-Index liegt der Gedanke zu Grunde, die Anzahl von Unternehmungen, die jeweils das gleiche alternative Rechnungslegungsverfahren zur Erfassung gleichartiger Geschäftsvorfälle im Jahresabschluss anwenden, als Maß für die Vergleichbarkeit des Inhalts von Jahresabschlussinformationen anzusehen ${ }^{1550}$. Wendet eine große Anzahl von Unternehmungen zweier Staaten die gleiche Rechnungslegungsmethode an, bedeutet dies nach VAN DER TAS, dass die relativen Anwendungshäufigkeiten dieser Methode in beiden Staaten einander intensivieren.

Wissenschaftliche Analysen, die Datenmaterial aus EU-Staaten verwenden, beruhen auf dem I-Index ${ }^{1551}$. Er soll auch in dieser Arbeit zur Feststellung des Harmonisierungsgrads zwischen Deutschland und Finnland herangezogen werden.

1545 Vgl. Pellens (1999), S. 369-372.

1546 Vgl. van der Tas (1988), vgl. van der Tas (1991), vgl. van der Tas (1992 a) und vgl. van der Tas (1992 b).

1547 Vgl. Krisement (1994), S. 26-27.

1548 Vgl. Krisement (1994), S. 27.

1549 Vgl. van der Tas (1988), S. 165.

1550 Vgl. van der Tas (1988), S. 158.

1551 Vgl. beispielsweise die Untersuchungen von Lappalainen (1999), Herrmann und Thomas (1995) sowie Emenyonu und Gray (1992). 
Zum einen ist er relativ einfach zu ermitteln und stellt sich in seiner Aussage klar und eindeutig dar. Zum anderen ermöglicht seine Anwendung eine spätere Ermittlung von Harmonisierungsgraden zwischen Deutschland, Finnland und anderen Staaten, da auf europäischer Ebene bereits entsprechendes Vergleichsmaterial vorhanden ist und auch zukünftig mit Vergleichsmaterial aus anderen Staaten zu rechnen ist.

Der tatsächlich realisierte Harmonisierungsgrad kann gemessen werden, indem die relative Anwendungshäufigkeit einer Methode in Staat A mit der relativen Anwendungshäufigkeit derselben Methode in Staat B multipliziert wird und anschließend die so ermittelten Ergebnisse für alle alternativen Methoden addiert werden. Diese Summe wird I-Index genannt. Bezeichnet also n die Anzahl der alternativen anwendbaren Rechnungslegungsverfahren, $f_{i}^{A}$ die relative Anwendungshäufigkeit der Methode $\mathrm{i}$ in Staat $\mathrm{A}$ und $\mathrm{f}_{\mathrm{i}}^{\mathrm{B}}$ die relative Anwendungshäufigkeit der Methode $\mathrm{i}$ in Staat B und $\mathrm{m}$ die Anzahl der in die Untersuchung einbezogenen Staaten, so lautet der I-Index ${ }^{1552}$

$$
I=\left[\sum_{i=1}^{n}\left(f_{i}^{A} * f_{i}^{B}\right)\right]^{1 /(m-1)}
$$

Das Ergebnis liegt immer zwischen den Werten 0 und 1. Maximale Harmonisierung bedeutet vollständige Konzentration der Anwendung auf ein Rechnungslegungsverfahren.

Im Zustand maximaler Konzentration nimmt der I-Index den Wert 1 an. Der Grad der Harmonisierung wird in der Literatur wie folgt beurteilt ${ }^{1553}$ :

\begin{tabular}{|c|l|}
\hline $\begin{array}{c}\text { Wert des } \\
\text { I-Indexes }\end{array}$ & \multicolumn{1}{|c|}{ Beurteilung des Harmonisierungsgrads } \\
\hline 0,00 & $\begin{array}{l}\text { Vollständig unterschiedliche Anwendung der Rech- } \\
\text { nungslegungsalternativen: } \\
\text { Harmonisierungsgrad ungenügend }\end{array}$ \\
\hline 0,01 bis 0,30 & $\begin{array}{l}\text { Weitgehend unterschiedliche Anwendung der Rech- } \\
\text { nungslegungsalternativen: } \\
\text { Harmonisierungsgrad mangelhaft }\end{array}$ \\
\hline 0,31 bis 0,50 & Harmonisierungsgrad zufriedenstellend \\
\hline 0,51 bis 0,70 & Harmonisierungsgrad befriedigend \\
\hline 0,71 bis 0,97 & $\begin{array}{l}\text { Weitgehend übereinstimmende Anwendung der } \\
\text { Rechnungslegungsalternativen: } \\
\text { Harmonisierungsgrad gut }\end{array}$ \\
\hline 0,98 bis 1,00 & $\begin{array}{l}\text { Identische Anwendung der Rechnungslegungsalter- } \\
\text { nativen: } \\
\text { Harmonisierungsgrad sehr gut }\end{array}$ \\
\hline
\end{tabular}

1552 Vgl. van der Tas (1988), S. 165.

1553 Vgl. Lappalainen (1999), S. 76-77 und Majala (1987), S. 76. 
Zur Veranschaulichung wird angenommen, die relativen Anwendungshäufigkeiten der Methoden 1 und 2 in den Staaten $A$ und B seien wie folgt ${ }^{1554}$ :

\begin{tabular}{|c|c|c|c|}
\cline { 3 - 4 } \multicolumn{1}{c|}{} & \multicolumn{2}{c|}{ Staat } \\
\hline Zeitpunkt t1 & 1 & 0 \\
\hline Methode & 1 & 0 & 1 \\
\hline Methode & 2 & & 0,3 \\
\hline Zeitpunkt t2 & 1 & 0,6 & 0,7 \\
\hline Methode & 1 & 0,4 & \\
\hline Methode & 2 &
\end{tabular}

Der I-Index beträgt dann zum Zeitpunkt $\mathrm{t}_{1}=1 * 0+0 * 1=0$, es kommen vollständig unterschiedliche Rechnungslegungsverfahren zur Anwendung. Zum Zeitpunkt $t_{2}$ beläuft sich der I-Index auf $0,6 * 0,3+0,4 * 0,7=0,46$, der Harmonisierungsgrad ist zufriedenstellend.

Problematisch zeigt sich die Ermittlung des I-Indexes dann, wenn Unternehmungen multiple Berichterstattung anwenden. Diese Unternehmen sind bezüglich des Inhalts bestimmter Jahresabschlussinformationen vergleichbar mit Unternehmen, die ausschließlich ein Verfahren anwenden. Eine Doppelerfassung der Unternehmen mit multipler Berichterstattung muss vermieden werden, um die Aussagefähigkeit des I-Indexes nicht zu beeinträchtigen ${ }^{1555}$. In vorliegender Arbeit wird bei der Untersuchung einzelner Jahresabschlusspositionen zwar aufgezeigt, in welchen Bereichen multiple Berichterstattung erfolgt, bei der Berechnung des entsprechenden I-Indexes wird das betreffende Unternehmen jedoch lediglich einem Verfahren - dem, welches am häufigsten zur Anwendung kommt - zugeordnet.

Als kritisch erweist sich die Anwendung des I-Indexes auch, wenn Unternehmungen nicht angeben, welche Rechnungslegungsalternative sie bei der Bilanzierung gewählt haben. Die Möglichkeit, bei der Ermittlung des I-Indexes die Rechnungslegungsalternative „Keine Angabe“ zu berücksichtigen, würde den I-Index verfälschen ${ }^{1556}$. Deshalb wird in vorliegender empirischer Untersuchung aufgezeigt, in welchen Bereichen keine Angabe erfolgt; das entsprechende Unternehmen wird bei der Berechnung des I-Indexes nicht berücksichtigt.

Der I-Index liefert nicht sachgemäße Ergebnisse, wenn eine Methode in einem der betrachteten Staaten von keinem Unternehmen angewendet wird. Durch Multiplikation mit dem Faktor 0 erhält der I-Index dann nämlich den Wert 0, auch wenn die Unternehmen in ihrer Praxis nicht gänzlich voneinander abweichen.

1554 Vgl. van der Tas (1988), S. 165.

1555 Vgl. Krisement (1994), S. 113-117 und S. 119 und vgl. Lappalainen (1999), S. 95.

1556 Vgl. Lappalainen (1999), S. 94-95. 
Dies ist problematisch, wenn mehr als zwei Methoden oder mehr als zwei Staaten gleichzeitig untersucht werden. Als Lösungsvorschlag wird in der Literatur vorgetragen, den Wert 0,99 anstelle von 1,0 für diejenige Methode einzusetzen, die von allen Unternehmen angewendet wird. Entsprechend ist der Wert 0,01 anstelle von 0,0 der Methode zuzuordnen, die von keinem Unternehmen gewählt wird ${ }^{1557}$.

Folgendes Beispiel soll diese Aussage veranschaulichen ${ }^{1558}$ :

\begin{tabular}{|c|c|c|c|c|c|c|}
\hline & \multicolumn{2}{|c|}{$\begin{array}{c}\text { Absolute Anwen- } \\
\text { dungshäufigkeiten }\end{array}$} & \multicolumn{2}{|c|}{$\begin{array}{c}\text { Relative Anwen- } \\
\text { dungshäufigkeiten bei } \\
\text { I-Index }\end{array}$} & \multicolumn{2}{|c|}{$\begin{array}{c}\text { Relative Anwen- } \\
\text { dungshäufigkeiten bei } \\
\text { korrigiertem I-Index }\end{array}$} \\
\hline & Methode 1 & Methode 2 & Methode 1 & Methode 2 & Methode 1 & Methode 2 \\
\hline \multicolumn{7}{|c|}{ Szenario 1} \\
\hline Staat A & 20 & 0 & 1 & 0 & 0,99 & 0,01 \\
\hline Staat B & 20 & 0 & 1 & 0 & 0,99 & 0,01 \\
\hline Staat C & 0 & 20 & 0 & 1 & 0,01 & 0,99 \\
\hline Staat D & 0 & 20 & 0 & 1 & 0,01 & 0,99 \\
\hline \multicolumn{7}{|c|}{ Szenario 2} \\
\hline Staat A & 20 & 0 & 1 & 0 & 0,99 & 0,01 \\
\hline Staat B & 20 & 0 & 1 & 0 & 0,99 & 0,01 \\
\hline Staat C & 10 & 10 & 0,5 & 0,5 & 0,50 & 0,50 \\
\hline Staat D & 0 & 20 & 0 & 1 & 0,01 & 0,99 \\
\hline \multicolumn{7}{|c|}{ Szenario 3} \\
\hline Staat A & 20 & 0 & 1 & 0 & 0,99 & 0,01 \\
\hline Staat B & 20 & 0 & 1 & 0 & 0,99 & 0,01 \\
\hline Staat C & 20 & 0 & 1 & 0 & 0,99 & 0,01 \\
\hline Staat D & 0 & 20 & 0 & 1 & 0,01 & 0,99 \\
\hline
\end{tabular}

Szenario 1:

I-Index: $(1 * 1 * 0 * 0+0 * 0 * 1 * 1)^{1 / 3}=0$

Korrigierter I-Index: $(0,99 * 0,99 * 0,01 * 0,01+0,01 * 0,01 * 0,99 * 0,99)^{1 / 3}=0,0581$

Szenario 2:

I-Index: $(1 * 1 * 0,5 * 0+0 * 0 * 0,5 * 1)^{1 / 3}=0$

Korrigierter I-Index: $(0,99 * 0,99 * 0,5 * 0,01+0,01 * 0,01 * 0,5 * 0,99)^{1 / 3}=0,1704$

Szenario 3:

I-Index: $(1 * 1 * 1 * 0+0 * 0 * 0 * 1)^{1 / 3}=0$

Korrigierter I-Index: $(0,99 * 0,99 * 0,99 * 0,01+0,01 * 0,01 * 0,01 * 0,99)^{1 / 3}=0,2133$

In vorliegender Analyse soll stets der Korrekturfaktor verwendet werden, auch in den Fällen, in denen er nicht notwendig ist. Vorteilhaft ist die Anwendung des IIndexes wegen seiner einfachen Handhabung und der Eindeutigkeit seiner Aussa-

1557 Vgl. Herrmann und Thomas (1995), S. 256-257.

1558 Vgl. Herrmann und Thomas (1995), S. 256. 
$\mathrm{ge}^{1559}$. Mithilfe des Korrekturfaktors kann er zudem so verändert werden, dass die Ermittlung des Harmonisierungsgrads nicht auf zwei Staaten begrenzt wird, sondern auf viele Staaten ausgedehnt werden kann ${ }^{1560}$. Vorliegende Untersuchung kann in Zukunft um eine beliebige Anzahl von Staaten erweitert werden.

\subsubsection{Messung des Harmonisierungsgrads mithilfe des I-Indexes}

\subsubsection{Datenbasis}

Als Datenbasis der empirischen Untersuchung dienen die veröffentlichten Einzelabschlüsse der größten börsennotierten Unternehmen Finnlands und Deutschlands.

Im Idealfall bezöge diese Untersuchung sämtliche börsennotierten Unternehmen ein. Die Menge der in diesem Fall auszuwertenden Daten kann jedoch in zeitlich angemessenem Rahmen nicht erfasst und verarbeitet werden. Die Stichprobe beschränkt sich auf die zehn größten finnischen und deutschen Unternehmen. Größe wird hier gemessen am Marktkapitalisierungswert per 29. Dezember 2000. Ausschlaggebend sind die von den Börsen in Helsinki - Helsingin Pörssi - und in Frankfurt am Main - Deutsche Börse AG - bekannt gegebenen Ranglisten ${ }^{1561}$.

Banken und Versicherungen werden in dieser Untersuchung nicht berücksichtigt, da für sie sowohl in Finnland als auch in Deutschland branchenspezifische Vorschriften hinsichtlich der Rechnungslegung gelten. Ihre Abschlüsse lassen sich deshalb in beiden Staaten nicht sinnvoll mit denjenigen von Industrie- und Handelsunternehmen vergleichen.

Die 4. EG-Richtlinie wurde in Deutschland mit dem Bilanzrichtlinien-Gesetz von 1985 umgesetzt, in Finnland mit dem Buchführungsgesetz von 1997, das für in 1998 endende Geschäftsjahre erstmals verbindlich anzuwenden war. Sinnvoll erscheint eine Messung des Harmonisierungsgrads zwischen Jahresabschlüssen von Unternehmen beider Staaten deshalb für Geschäftsjahre ab 1998. Da aber davon auszugehen ist, dass im ersten Jahr seiner Gültigkeit in der Praxis noch Unsicherheit im Umgang mit den Regeln des neuen Buchführungsgesetzes herrschte, wird das Jahr 1998 in dieser Untersuchung außer Acht gelassen. Einbezogen werden die Jahre 1999 und 2000 ${ }^{1562}$. Die empirische Betrachtung beider Jahre schließt

1559 Vgl. Lappalainen (1999), S. 93.

1560 Vgl. van der Tas (1988), Appendix 2, S. 168.

1561 Vgl. www.hex.fi Market Data/Monthly Statistics, Table 9 vom 03. Januar 2001 für Finnland und vgl. www.deutschebörse.de für Deutschland.

1562 Zusätzlich zu 1999 und 2000 noch das Jahr 2001 zu berücksichtigen, ist nicht möglich, da zum Zeitpunkt dieser Untersuchung im Frühjahr 2002 noch nicht alle einzubeziehenden Unternehmen ihre Jahresabschlüsse veröffentlicht haben. 
aus, dass einmalige Sondereinflüsse die Aussagefähigkeit der Untersuchung beeinträchtigen.

Verglichen werden aus den genannten Gründen die Einzelabschlüsse der Jahre 1999 und 2000 folgender Unternehmen:

Finnland:

- Nokia Oyj

- Sonera Oyj

- Stora Enso Oyj

- UPM-Kymmene Oyj

- Fortum Oyj

- Elisa Communications Oyj

- Sanoma WSOY Oyj

- Comptel Oyj

- Metso Oyj

- Kone Oyj

Untersucht werden die in finnischer Sprache veröffentlichten Berichte.

Deutschland:

- Deutsche Telekom AG

- Siemens AG

- SAP AG

- DaimlerChrysler AG

- Infineon Technologies AG (nur der Einzelabschluss des Jahres 2000, da das Unternehmen in 2000 erstmals einen Abschluss veröffentlicht hat)

- Bayer AG

- E.ON AG (die E.ON AG ist in 2000 aus der Fusion von Veba AG und Viag AG hervorgegangen; für 1999 wird der Einzelabschluss der Veba AG verwendet)

- BASF AG

- RWE AG

- BMW AG

Untersucht werden die in deutscher Sprache veröffentlichten Berichte.

Angesichts der kleinen Zahl berücksichtigter Unternehmen ist diese Untersuchung nicht repräsentativ, sondern sie dient der Illustration der gewonnenen Erkenntnisse bezüglich der formalen Harmonisierung zwischen Deutschland und Finnland. 


\subsubsection{Empirische Messung des Harmonisierungsgrads ausgesuchter Jahresabschlusspositionen}

\subsection{Vorgehensweise}

Die Rechnungslegungspraxis der in die Untersuchung einbezogenen Unternehmen wird anhand einzelner Positionen der Bilanz sowie der Gewinn- und Verlustrechnung untersucht.

Für Positionen, für welche die Rechnungslegungsvorschriften in Finnland und Deutschland eine einheitliche Vorgehensweise zwingend vorsehen, erübrigt sich eine Messung des Harmonisierungsgrads; hier muss eine hundertprozentige Übereinstimmung in der Bilanzierungspraxis sämtlicher Unternehmen vorliegen ${ }^{1563}$. Diese Fälle gehen aus der deskriptiv-vergleichenden Darstellung in Kap. 4.1 hervor und betreffen beispielsweise das Verbot der Aktivierung originärer Geschäftsoder Firmenwerte und die für Wertpapiere des Finanzumlaufvermögens geltenden Ansatz- und Bewertungskriterien.

Ebenso müssen Sachverhalte, für welche die Gesetze in beiden Staaten zwingend eine unterschiedliche Vorgehensweise hinsichtlich Ansatz oder Bewertung fordern, eine hundertprozentige Abweichung in der Rechnungslegungspraxis in Finnland und Deutschland vorweisen. Auch diese Fälle gehen aus Kap. 4.1 dieser Arbeit hervor. Hier sind beispielsweise Ausweisfragen anzuführen: ein Disagio wird in Finnland stets als immaterieller Vermögenswert gezeigt, in Deutschland als aktiver Rechnungsabgrenzungsposten.

Für beide Extremfälle, hundertprozentige Übereinstimmung wie auch hundertprozentige Divergenz, stellt sich der Stand der formalen Harmonisierung so eindeutig dar, dass auf eine empirische Untersuchung zur Messung des tatsächlich realisierten Harmonisierungsgrads verzichtet werden kann.

Untersucht werden im Folgenden deshalb solche Jahresabschlusspositionen, die durch Ausübung von Wahlrechten in beiden Staaten unterschiedlich behandelt werden können, aber nicht zwangsläufig unterschiedlich behandelt werden müssen. Für diese Positionen stellt sich die Frage, ob eine materielle Harmonisierung erreicht wird. Folgende Sachverhalte, die im Jahresabschluss unterschiedlich erfasst oder bewertet werden können, sind Gegenstand dieser Untersuchung:

Für derivate Geschäfts- oder Firmenwerte existiert in beiden Ländern ein Aktivierungswahlrecht ${ }^{1564}$. Je nach Unternehmenstätigkeit und Wesentlichkeit der Geschäfts- oder Firmenwerte für einen Jahresabschluss können sich hier beträchtli-

1563 Gegenstand dieser Untersuchung ist nicht die Frage, ob die Unternehmen gesetzestreu bilanzieren; dies wird vorausgesetzt.

1564 Vgl. Kap. 4.1.3.1.1.5 dieser Arbeit. 
che Unterschiede mit Auswirkung auf das Jahresergebnis mehrerer Geschäftsjahre ergeben. Es gilt deshalb, den tatsächlich realisierten Harmonisierungsgrad zu ermitteln.

Für Forschungs- und Entwicklungskosten gilt in Finnland unter bestimmten Voraussetzungen ein Aktivierungswahlrecht, in Deutschland gilt immer ein Aktivierungsverbot ${ }^{1565}$. Es stellt sich die Frage nach dem tatsächlich realisierten Harmonisierungsgrad.

Für immaterielle derivative Vermögenswerte, welche die Aktivierungskriterien erfüllen, herrscht in Finnland ein Aktivierungswahlrecht, in Deutschland hingegen ein Aktivierungsgebot ${ }^{1566}$. Fraglich ist, ob die finnische Bilanzierungspraxis das Wahlrecht dahingehend ausübt, dass ein hoher tatsächlich realisierter Harmonisierungsgrad zwischen beiden Staaten erreicht wird.

Finnische Unternehmen haben das Wahlrecht zur Neubewertung von Grundstücken und Wasserflächen des Sachanlagevermögens sowie von Wertpapieren des Finanzanlagevermögens ${ }^{1567}$. In Deutschland sind Neubewertungen nicht gestattet ${ }^{1568}$. Zu untersuchen ist im Hinblick auf den tatsächlich realisierten Harmonisierungsgrad, ob finnische Unternehmen von ihrem Wahlrecht Gebrauch machen.

Zur Folgebewertung des abnutzbaren Anlagevermögens kann in beiden Staaten die lineare oder die degressive Abschreibungsmethode herangezogen werden. Auch kann aus steuerlichen Gründen ein Methodenwechsel von der degressiven auf die lineare Methode vorgenommen werden ${ }^{1569}$. Es gilt festzustellen, wie weit die tatsächlich realisierte Harmonisierung in diesem Bereich fortgeschritten ist. Nicht berücksichtigt werden unterschiedliche Nutzungsdauern. Diese eignen sich kaum für einen Vergleich, da sie abhängig von unternehmensindividuellen Faktoren sind.

Beim Vorratsvermögen stellt sich zum einen die Frage, wie seine Herstellungskosten in der Bilanzierungspraxis ermittelt werden. In Finnland wie in Deutschland sind die variablen bzw. Einzelkosten aktivierungspflichtig. Für Fix- bzw. Gemeinkosten existiert in beiden Staaten ein Aktivierungswahlrecht ${ }^{1570}$. Zum anderen ist zu untersuchen, welche der Bewertungsvereinfachungsmethoden in der Praxis Anwendung finden. Alternativen sind die gewichtete Durchschnittspreis-

\footnotetext{
1565 Vgl. Kap. 3.2.1.1.3, Kap. 4.1.3.1.1.3 und Kap. 4.1.3.1.1.4 dieser Arbeit.

1566 Vgl. Kap. 3.2.1.1.1 und Kap. 4.1.3.1.1.1 dieser Arbeit.

1567 Vgl. Kap. 3.1.6.2.2.6 dieser Arbeit.

1568 Vgl. Kap. 4.1.2.5.3.6 dieser Arbeit.

1569 Vgl. Kap. 4.1.2.5.3.1 dieser Arbeit.

1570 Vgl. Kap. 4.1.3.2.2 dieser Arbeit.
} 
methode, das FIFO-Verfahren und das LIFO-Verfahren. $\mathrm{Zu}$ messen ist der tatsächlich realisierte Harmonisierungsgrad.

Passive latente Steuern können in Finnland wahlweise in der Bilanz angesetzt oder im Anhang angegeben werden ${ }^{1571}$. In Deutschland sind sie zwingend bilanziell zu erfassen. Auch hier ist eine Ermittlung des tatsächlich realisierten Harmonisierungsgrads sinnvoll, da eine unterschiedliche Handhabung sich beträchtlich auf das Unternehmensergebnis mehrerer Perioden auswirken kann.

\subsection{Ansatz eines derivaten Geschäfts- oder Firmenwerts}

Sowohl in Finnland als auch in Deutschland kann der derivative Geschäfts- oder Firmenwert sofort über die Gewinn- und Verlustrechnung als Aufwand erfasst werden. Alternativ darf er aktiviert und in der Folge abgeschrieben werden.

Alle untersuchten Unternehmen, die in den Jahren 1999 und 2000 darüber berichtet haben, wie sie derivative Geschäfts- oder Firmenwerte behandeln, haben diese aktiviert und anschließend planmäßig über die Nutzungsdauer abgeschrieben.

\begin{tabular}{|c|c|c|c|c|c|c|c|c|}
\hline & \multicolumn{4}{|c|}{1999} & \multicolumn{4}{|c|}{2000} \\
\hline & \multicolumn{2}{|c|}{ absolut } & \multicolumn{2}{|c|}{ relativ } & \multicolumn{2}{|c|}{ absolut } & \multicolumn{2}{|c|}{ relativ } \\
\hline & $\begin{array}{l}\text { Auf- } \\
\text { wand }\end{array}$ & Bilanz & $\begin{array}{l}\text { Auf- } \\
\text { wand }\end{array}$ & Bilanz & $\begin{array}{c}\text { Auf- } \\
\text { wand }\end{array}$ & Bilanz & $\begin{array}{l}\text { Auf- } \\
\text { wand }\end{array}$ & Bilanz \\
\hline Finnland & 0 & 5 & 0,01 & 0,99 & 0 & 5 & 0,01 & 0,99 \\
\hline $\begin{array}{l}\text { Deutsch- } \\
\text { land }\end{array}$ & 0 & 8 & 0,01 & 0,99 & 0 & 7 & 0,01 & 0,99 \\
\hline
\end{tabular}

I-Index 1999: $0,01 * 0,01+0,99 * 0,99=0,9802$

I-Index 2000: $0,01 * 0,01+0,99 * 0,99=0,9802$

Der tatsächlich realisierte Harmonisierungsgrad ist somit sehr gut. Wäre bekannt, wie die Unternehmen, welche keine Angaben zu diesem Thema offen legen, den Geschäfts- oder Firmenwert buchhalterisch erfassen, könnte der Harmonisierungsgrad variieren. 


\subsection{Ansatz von Forschungs- und Entwicklungskosten}

\begin{tabular}{|c|c|c|c|c|c|c|c|c|}
\hline & \multicolumn{4}{|c|}{1999} & \multicolumn{4}{c|}{2000} \\
\hline & \multicolumn{2}{|c|}{ absolut } & \multicolumn{2}{c|}{ relativ } & \multicolumn{2}{c|}{ absolut } & \multicolumn{2}{c|}{ relativ } \\
\hline & $\begin{array}{c}\text { Auf- } \\
\text { wand }\end{array}$ & Bilanz & $\begin{array}{c}\text { Auf- } \\
\text { wand }\end{array}$ & Bilanz & $\begin{array}{c}\text { Auf- } \\
\text { wand }\end{array}$ & Bilanz & $\begin{array}{c}\text { Auf- } \\
\text { wand }\end{array}$ & Bilanz \\
\hline Finnland & 8 & 0 & 0,99 & 0,01 & 8 & 0 & 0,99 & 0,01 \\
\hline $\begin{array}{c}\text { Deutsch- } \\
\text { land }\end{array}$ & 10 & 0 & 0,99 & 0,01 & 10 & 0 & 0,99 & 0,01 \\
\hline
\end{tabular}

I-Index 1999: $0,99 * 0,99+0,01 * 0,01=0,9802$

I-Index 2000: $0,99 * 0,99+0,01 * 0,01=0,9802$

In Finnland existiert ein Aktivierungswahlrecht für Forschungsausgaben und bestimmte Entwicklungsausgaben. Von den untersuchten finnischen Unternehmen haben einige die Forschungs- und Entwicklungskosten in vollem Umfang im Aufwand erfasst, aber im Anhang zusätzlich die Information gegeben, in welcher Höhe diese Kosten aktivierungsfähig gewesen wären. Diese Unternehmen sind der Methode „Erfassung im Aufwand“ zugeordnet worden, da sie diese vorrangig anwenden. Insgesamt hat sich bei dieser Untersuchung die Schwierigkeit ergeben, dass viele Unternehmen Forschungs- und Entwicklungskosten in der Gewinn- und Verlustrechnung explizit als gesonderten Posten ausweisen. Immaterielle Vermögenswerte werden von ihnen aber in einer Summe gezeigt und nicht näher erläutert. $\mathrm{Ob}$ sich unter den aktivierten immateriellen Vemögenswerten auch Forschungs- und Entwicklungskosten befinden, ist daher aus dem vorhandenen offen gelegten Datenmaterial nicht immer ersichtlich.

In Deutschland besteht ein Aktivierungsverbot für Forschungs- und Entwicklungskosten, sodass hier alle Unternehmen der Methode „Erfassung im Aufwand“ zugeordnet werden. Dies erfolgt unabhängig davon, ob bei den Unternehmen in den Jahren 1999 oder 2000 tatsächlich Forschungs- und Entwicklungskosten angefallen sind.

Der tatsächlich realisierte Harmonisierungsgrad zwischen beiden Staaten ist sehr gut. Es ist jedoch nicht auszuschließen, dass sich bei detaillierterer Berichterstattung durch die finnischen Unternehmen ein schlechterer Harmonisierungsgrad ergeben würde. 


\subsection{Ansatz von immateriellen Vermögenswerten}

\begin{tabular}{|c|c|c|c|c|c|c|c|c|}
\hline & \multicolumn{4}{|c|}{1999} & \multicolumn{4}{|c|}{2000} \\
\hline & \multicolumn{2}{|c|}{ absolut } & \multicolumn{2}{|c|}{ relativ } & \multicolumn{2}{|c|}{ absolut } & \multicolumn{2}{|c|}{ relativ } \\
\hline & $\begin{array}{l}\text { Auf- } \\
\text { wand }\end{array}$ & Bilanz & $\begin{array}{l}\text { Auf- } \\
\text { wand }\end{array}$ & Bilanz & $\begin{array}{c}\text { Auf- } \\
\text { wand }\end{array}$ & Bilanz & $\begin{array}{l}\text { Auf- } \\
\text { wand }\end{array}$ & Bilanz \\
\hline Finnland & 0 & 10 & 0,01 & 0,99 & 0 & 10 & 0,01 & 0,99 \\
\hline $\begin{array}{l}\text { Deutsch- } \\
\text { land }\end{array}$ & 0 & 10 & 0,01 & 0,99 & 0 & 10 & 0,01 & 0,99 \\
\hline
\end{tabular}

I-Index 1999: $0,01 * 0,01+0,99 * 0,99=0,9802$

I-Index 2000: $0,01 * 0,01+0,99 * 0,99=0,9802$

In Finnland existiert ein Aktivierungswahlrecht für immaterielle Vermögenswerte. Alternativ können sie sofort erfolgswirksam als Aufwand erfasst werden. Alle hier untersuchten Unternehmen üben das Wahlrecht dahingehend aus, dass sie immaterielle Vermögenswerte aktivieren und in der Folge abschreiben. Ob sie allerdings sämtliche aktivierungsfähigen immateriellen Vermögenswerte aktivieren, bleibt offen.

In Deutschland herrscht ein Aktivierungsgebot für aktivierungsfähige immaterielle Vermögensgegenstände. Deshalb wurden hier alle Unternehmen der Methode „Aktivierung“ zugeordnet.

Der tatsächlich realisierte Harmonisierungsgrad zwischen beiden Staaten ist sehr gut.

\subsection{Neubewertung}

In Finnland dürfen Grundstücke und Wasserflächen des Sachanlagevermögens sowie Wertpapiere des Finanzanlagevermögens neubewertet werden. Von den in diese Analyse einbezogenen Unternehmen haben drei das Wahlrecht ausgeübt, Grundstücke über deren ursprüngliche Anschaffungskosten hinaus zu bewerten.

In Deutschland sind Neubewertungen nicht gestattet, alle Unternehmen müssen die Methode „Fortgeführte Anschaffungs- oder Herstellungskosten“ anwenden. 


\begin{tabular}{|c|c|c|c|c|c|c|c|c|}
\hline & \multicolumn{4}{|c|}{1999} & \multicolumn{3}{c|}{2000} \\
\hline & \multicolumn{2}{|c|}{ absolut } & \multicolumn{2}{c|}{ relativ } & \multicolumn{2}{c|}{ absolut } & \multicolumn{2}{c|}{ relativ } \\
\hline & $\begin{array}{c}\text { FHK } \\
\text { AHtgef. }\end{array}$ & $\begin{array}{c}\text { Neube- } \\
\text { wer- } \\
\text { tung }\end{array}$ & $\begin{array}{c}\text { Fortgef. } \\
\text { AHK }\end{array}$ & $\begin{array}{c}\text { Neube- } \\
\text { wer- } \\
\text { tung }\end{array}$ & $\begin{array}{c}\text { Fortgef. } \\
\text { AHK }\end{array}$ & $\begin{array}{c}\text { Neube- } \\
\text { wer- } \\
\text { tung }\end{array}$ & $\begin{array}{c}\text { Fort- } \\
\text { gef. } \\
\text { AHK }\end{array}$ & $\begin{array}{c}\text { Neube- } \\
\text { wer- } \\
\text { tung }\end{array}$ \\
\hline Finnland & 6 & 3 & 0,67 & 0,33 & 6 & 3 & 0,67 & 0,33 \\
\hline $\begin{array}{c}\text { Deutsch- } \\
\text { land }\end{array}$ & 10 & 0 & 0,99 & 0,01 & 10 & 0 & 0,99 & 0,01 \\
\hline
\end{tabular}

I-Index 1999: $0,67 * 0,99+0,33 * 0,01=0,6666$

I-Index 2000: $0,67 * 0,99+0,33 * 0,01=0,6666$

Der tatsächlich realisierte Harmonisierungsgrad stellt sich für die hier untersuchten Unternehmen befriedigend dar.

\subsection{Wahl der Abschreibungsmethode}

In Finnland wie in Deutschland ist es den Unternehmen gestattet, die lineare oder die degressive Abschreibungsmethode anzuwenden. Wegen des Prinzips der umgekehrten Maßgeblichkeit müssen aus steuerlichen Gründen erhöhte Abschreibungen in Finnland auch handelsrechtlich vorgenommen werden; sie sind verbindlich als „Andere als planmäßige Abschreibungen“ gesondert in der Bilanz und Gewinn- und Verlustrechnung auszuweisen. In Deutschland darf unter steuerlichen Gesichtspunkten von der degressiven auf die lineare Abschreibungsmethode übergegangen werden, sobald diese höher ist.

\begin{tabular}{|c|c|c|c|c|c|c|c|c|}
\hline & \multicolumn{4}{|c|}{1999} & \multicolumn{4}{c|}{2000} \\
\hline & \multicolumn{2}{|c|}{ absolut } & \multicolumn{2}{|c|}{ relativ } & \multicolumn{2}{c|}{ absolut } & \multicolumn{2}{c|}{ relativ } \\
\hline & linear & $\begin{array}{c}\text { linear } / \\
\text { degres- } \\
\text { siv }\end{array}$ & linear & $\begin{array}{c}\text { linear } / \\
\text { degres- } \\
\text { siv }\end{array}$ & linear & $\begin{array}{c}\text { linear } / \\
\text { degres- } \\
\text { siv }\end{array}$ & $\begin{array}{c}\text { linear } \\
\text { linear } / \\
\text { de- } \\
\text { gressiv }\end{array}$ \\
\hline Finnland & 0 & 9 & 0,01 & 0,99 & 0 & 9 & 0,01 & 0,99 \\
\hline $\begin{array}{c}\text { Deutsch- } \\
\text { land }\end{array}$ & 0 & 8 & 0,01 & 0,99 & 0 & 8 & 0,01 & 0,99 \\
\hline
\end{tabular}

I-Index 1999: $0,01 * 0,01+0,99 * 0,99=0,9802$

I-Index 2000: $0,01 * 0,01+0,99 * 0,99=0,9802$

Der tatsächlich realisierte Harmonisierungsgrad zwischen Finnland und Deutschland ist somit sehr gut. Alle hier untersuchten Unternehmen nehmen ihre Abschreibungen unter steuerlichen Gesichtspunkten vor. Der erreichte Harmonisierungsgrad darf jedoch nicht darüber hinwegtäuschen, dass Abschreibungssätze und Nutzungsdauern auf Grund der steuerrechtlichen Vorgaben voneinander abweichen.. 
Es ist zudem davon auszugehen, dass die Unternehmen beider Staaten erheblich von denen anderer europäischer Staaten abweichen, welche das Prinzip der umgekehrten Maßgeblichkeit nicht kennen. HERRMANN und THOMAS haben Einzelabschlüsse der Jahre 1992 und 1993 von Unternehmen ausgewählter europäischer Staaten untersucht; Finnland ist nicht in die Studie einbezogen worden. HERRMANN und THOMAS haben festgestellt, dass in Belgien, Dänemark, Frankreich, Irland, den Niederlanden, Portugal und Großbritannien die überwiegende Mehrheit der Unternehmen die lineare Abschreibungsmethode wählt, während einzig in Deutschland aus steuerlichen Gründen der Übergang von der degressiven auf die lineare Abschreibungsmethode favorisiert wird ${ }^{1572}$.

\subsection{Ermittlung der Herstellungskosten beim Vorratsvermögen}

In Finnland und in Deutschland sind die variablen bzw. die Einzelkosten als Bestandteil der Herstellungskosten aktivierungspflichtig. Für Fix- bzw. Gemeinkosten existiert in beiden Staaten handelsrechtlich ein Aktivierungswahlrecht.

\begin{tabular}{|c|c|c|c|c|c|c|c|c|}
\hline & \multicolumn{4}{|c|}{1999} & \multicolumn{4}{c|}{2000} \\
\hline & \multicolumn{2}{|c|}{ absolut } & \multicolumn{2}{c|}{ relativ } & \multicolumn{2}{c|}{ absolut } & \multicolumn{2}{c|}{ relativ } \\
\hline & Aufwand & $\begin{array}{c}\text { Bi- } \\
\text { lanz }\end{array}$ & Aufwand & Bilanz & $\begin{array}{c}\text { Auf- } \\
\text { wand }\end{array}$ & Bilanz & $\begin{array}{c}\text { Auf- } \\
\text { wand }\end{array}$ & Bilanz \\
\hline Finnland & 2 & 4 & 0,33 & 0,67 & 2 & 4 & 0,33 & 0,67 \\
\hline $\begin{array}{c}\text { Deutsch- } \\
\text { land }\end{array}$ & 1 & 9 & 0,10 & 0,90 & 1 & 9 & 0,10 & 0,90 \\
\hline
\end{tabular}

I-Index 1999: $0,33 * 0,10+0,67 * 0,90=0,6360$

I-Index 2000: $0,33 * 0,10+0,67 * 0,90=0,6360$

In Finnland haben vier der untersuchten Unternehmen Fixkosten in die Herstellungskosten einbezogen, zwei nicht. Weitere vier Unternehmen geben nicht an, wie sich ihre Herstellungskosten zusammensetzen. Wünschenswert aus Sicht dieser Untersuchung ist eine qualitative Verbesserung der Anhangangaben. Der tatsächlich realisierte Harmonisierungsgrad könnte anders ausfallen, wäre die Vorgehensweise dieser Unternehmen bekannt.

In Deutschland sind die Material- und Fertigungsgemeinkosten sowie anteilige Abschreibungen auf das Anlagevermögen steuerlich aktivierungspflichtig. Um keine Differenz zwischen Handels- und Steuerbilanz vorzuweisen, bewerten neun von zehn untersuchten Unternehmen ihr Vorratsvermögen nach steuerlichen Vorschriften.

1572 Vgl. Herrmann und Thomas (1995), S. 259. 
Der tatsächlich realisierte Harmonisierungsgrad zwischen beiden Staaten ist befriedigend.

\subsection{Wahl des Bewertungsvereinfachungsverfahrens}

\begin{tabular}{|c|c|c|c|c|c|c|c|c|c|c|c|c|}
\hline & \multicolumn{9}{|c|}{1999} & \multicolumn{1}{c|}{2000} \\
\hline & \multicolumn{4}{|c|}{ absolut } & \multicolumn{3}{c|}{ relativ } & \multicolumn{3}{c|}{ absolut } & \multicolumn{3}{c|}{ relativ } \\
\hline & FIFO & LIFO & $\begin{array}{c}\text { Durch- } \\
\text { schnitt }\end{array}$ & FIFO & LIFO & $\begin{array}{c}\text { Durch- } \\
\text { schnitt }\end{array}$ & FIFO & LIFO & $\begin{array}{c}\text { Durch- } \\
\text { schnitt }\end{array}$ & FIFO & LIFO & $\begin{array}{c}\text { Durch- } \\
\text { schnitt }\end{array}$ \\
\hline Finnland & 4 & 0 & 3 & 0,57 & 0,01 & 0,42 & 3 & 0 & 4 & 0,42 & 0,01 & 0,57 \\
\hline $\begin{array}{c}\text { Deutsch- } \\
\text { land }\end{array}$ & 0 & 6 & 1 & 0,01 & 0,85 & 0,14 & 0 & 6 & 1 & 0,01 & 0,85 & 0,14 \\
\hline
\end{tabular}

I-Index 1999: $0,57 * 0,01+0,01 * 0,85+0,42 * 0,14=0,07$

I-Index 2000: $0,42 * 0,01+0,01 * 0,85+0,57 * 0,14=0,09$

In Finnland sind handelsrechtlich die gewichtete Durchschnittspreismethode, das FIFO-Verfahren und das LIFO-Verfahren erlaubt. Steuerlich ist jedoch offiziell allein das FIFO-Verfahren zulässig, der gewogene Durchschnittspreis wird akzeptiert, sofern er nur geringfügig vom FIFO-Wert abweicht. Die Anwendung steuerlicher Vorschriften kommt in der Praxis klar zum Ausdruck. Von den hier untersuchten Unternehmen wenden in beiden Jahren sieben die FIFO- oder die gewichtete Durchschnittspreismethode an. Drei Unternehmen verzichten auf eine Anhangangabe. Es muss betont werden, dass qualitativ höherwertige Anhangangaben im Hinblick auf die Informationsbedürfnisse der Jahresabschluss-Leser wünschenswert sind.

Gleiches gilt für Deutschland, wo ebenfalls drei der untersuchten Unternehmen eine Anhangangbe für nicht notwendig erachten. In Deutschland üblich ist das LIFO-Verfahren, das von sechs Unternehmen angewendet wird. Nur ein Unternehmen bekennt sich zur gewichteten Durchschnittspreismethode.

Der tatsächlich realisierte Harmonisierungsgrad zwischen beiden Staaten ist mit 0,07 in 1999 und 0,09 in 2000 mangelhaft. $\mathrm{Zu}$ beachten ist seine geringfügige Steigerung von 1999 auf 2000; in allen anderen untersuchten Bereichen ist er in beiden Jahren konstant geblieben. Der Harmonisierungsgrad könnte höher ausfallen, hätten alle untersuchten Unternehmen die von ihnen verfolgte Methode angegeben. Ursache für den niedrigen Harmonisierungsgrad ist - wie schon bei der Wahl der Abschreibungsmethode und der Ermittlung der Herstellungskosten deutlich geworden ist ${ }^{1573}$ - der steuerliche Einfluss durch das Prinzip der umgekehrten Maßgeblichkeit. 


\subsection{Ansatz passiver latenter Steuern}

In Finnland existiert ein Bilanzierungswahlrecht für passive latente Steuern. Bei Verzicht auf die Bilanzierung ist die Höhe der passiven latenten Steuern im Anhang anzugeben, falls diese wesentlich ist. Von den hier untersuchten Unternehmen passivieren drei ihre latenten Steuern, fünf nennen den ermittelten Wert im Anhang. Zwei Unternehmen äußern sich nicht zu den passiven latenten Steuern. $\mathrm{Ob}$ der Grund hierfür in der Unwesentlichkeit der Steuern für den Jahresabschluss liegt oder an anderer Stelle zu suchen ist, kann anhand des vorliegenden Datenmaterials nicht beurteilt werden.

\begin{tabular}{|c|c|c|c|c|c|c|c|c|}
\hline & \multicolumn{4}{|c|}{1999} & \multicolumn{4}{c|}{2000} \\
\hline & \multicolumn{2}{|c|}{ absolut } & \multicolumn{2}{c|}{ relativ } & \multicolumn{2}{c|}{ absolut } & \multicolumn{2}{c|}{ relativ } \\
\hline & Bilanz & Anhang & Bilanz & $\begin{array}{c}\text { An- } \\
\text { hang }\end{array}$ & Bilanz & Anhang & Bilanz & $\begin{array}{c}\text { An- } \\
\text { hang }\end{array}$ \\
\hline Finnland & 3 & 5 & 0,38 & 0,62 & 3 & 5 & 0,38 & 0,62 \\
\hline $\begin{array}{c}\text { Deutsch- } \\
\text { land }\end{array}$ & 10 & 0 & 0,99 & 0,01 & 10 & 0 & 0,99 & 0,01 \\
\hline
\end{tabular}

I-Index 1999: $0,38 * 0,99+0,62 * 0,01=0,3824$

I-Index 2000: $0,38 * 0,99+0,62 * 0,01=0,3824$

In Deutschland besteht eine Passivierungspflicht für latente Steuern, sodass hier alle Unternehmen dem Verfahren „Bilanzierung“ zugeordnet werden. Es ist jedoch nicht aus allen Geschäftsberichten ersichtlich, ob in den Jahren 1999 und 2000 passive latente Steuern ermittelt und bilanziert wurden, da zum Teil die Angabe fehlte.

Der tatsächlich realisierte Harmonisierungsgrad zwischen beiden Staaten ist zufriedenstellend. 


\subsubsection{Zusammenfassung und kritische Würdigung der Untersuchungs- ergebnisse}

Ziel der in Kap. 4.2 durchgeführten empirischen Studie war die Ermittlung des derzeitigen tatsächlich realisierten Harmonisierungsgrads zwischen Finnland und Deutschland. Dieser stellt sich für die Jahre 1999 und 2000 wie folgt dar ${ }^{1574}$ :

\begin{tabular}{|l|l|l|}
\cline { 2 - 3 } \multicolumn{1}{c|}{} & \multicolumn{2}{c|}{$\begin{array}{c}\text { Harmonisierungsgrad zwischen } \\
\text { Finnland und Deutschland }\end{array}$} \\
\cline { 2 - 3 } & \multicolumn{1}{c|}{1999} & \multicolumn{1}{c|}{2000} \\
\hline $\begin{array}{l}\text { Ansatz eines derivativen Ge- } \\
\text { schäfts- oder Firmenwerts }\end{array}$ & Sehr gut $(0,9802)$ & Sehr gut $(0,9802)$ \\
\hline $\begin{array}{l}\text { Ansatz von Forschungs- und } \\
\text { Entwicklungskosten }\end{array}$ & Sehr gut $(0,9802)$ & Sehr gut $(0,9802)$ \\
\hline $\begin{array}{l}\text { Ansatz von immateriellen } \\
\text { Vermögenswerten }\end{array}$ & Sehr gut $(0,9802)$ & Sehr gut $(0,9802)$ \\
\hline Neubewertung & Befriedigend $(0,6666)$ & Befriedigend $(0,6666)$ \\
\hline $\begin{array}{l}\text { Wahl der Abschreibungs- } \\
\text { methode }\end{array}$ & Sehr gut $(0,9802)$ & Sehr gut $(0,9802)$ \\
\hline $\begin{array}{l}\text { Herstellungskosten beim } \\
\text { Vorratsvermögen }\end{array}$ & Befriedigend $(0,6360)$ & Befriedigend $(0,6360)$ \\
\hline $\begin{array}{l}\text { Wahl des Bewertungsverein- } \\
\text { fachungsverfahrens }\end{array}$ & Mangelhaft $(0,07)$ & Mangelhaft $(0,09)$ \\
\hline $\begin{array}{l}\text { Ansatz passiver latenter } \\
\text { Steuern }\end{array}$ & $\begin{array}{l}\text { Zufriedenstellend } \\
(0,3824)\end{array}$ & $\begin{array}{l}\text { Zufriedenstellend } \\
(0,3824)\end{array}$ \\
\hline
\end{tabular}

In vier von acht untersuchten Sachverhalten ist der Stand der Harmonisierung mit „sehr gut“ zu beurteilen. Alle untersuchten finnischen und deutschen Unternehmen, die ihre Vorgehensweise offen legen, aktivieren derivative Geschäfts- oder Firmenwerte und immaterielle Vermögenswerte. Sie erfassen Forschungs- und Entwicklungskosten im Aufwand. Zur planmäßigen Abschreibung wählen sie die degressive Methode mit Übergang zur linearen Methode dann, wenn letztere einen höheren Aufwand generiert. Es wird betont, dass der tatsächlich realisierte Harmonisierungsgrad in den genannten vier Fällen schlechter ausfallen könnte, wenn alle Unternehmen ihre Bilanzierungs- und Bewertungsmethoden offen gelegt hätten.

„Befriedigend“ zeigt sich der tatsächlich realisierte Harmonisierungsgrad bei der Neubewertung, ,zufriedenstellend“ beim Ansatz passiver latenter Steuern. Hier bewirken nationale Wahlrechte in Finnland einen befriedigenden bzw. zufriedenstellenden Harmonisierungsgrad innerhalb des Lands, der dann den Harmonisierungsgrad zwischen Finnland und Deutschland negativ beeinflusst. Ebenfalls „,be-

1574 Vgl. Kap. 4.2.2.2.2 bis Kap. 4.2.2.2.9 dieser Arbeit. 
friedigend" ist der erreichte Grad der tatsächlichen Harmonisierung hinsichtlich der angesetzten Herstellungskosten bei Gegenständen des Vorratsvermögens. Sowohl in Finnland als auch in Deutschland üben die Unternehmen Wahlrechte unterschiedlich aus.

„Mangelhaft" beurteilt wird der tatsächlich realisierte Harmonisierungsgrad bei der Wahl des Bewertungsvereinfachungsverfahrens. In diesem Fall kommen unterschiedliche steuerrechtliche Vorschriften in den Jahresabschlüssen auf Grund des in beiden Staaten existierenden Prinzips der umgekehrten Maßgeblichkeit zum Tragen.

Fraglich ist, wie diese ermittelten Harmonisierungsgrade zu beurteilen sind. Die EU strebt mit der 4. EG-Richtlinie eine Gleichwertigkeit von Jahresabschlüssen dahingehend an, dass Unternehmen der Mitgliedstaaten aus gleichartigen Sachverhalten Informationen bereitstellen, die sich nicht widersprechen. Zudem soll durch Anhangangaben eine Überführung in die jeweils andere Verfahrensweise verstanden werden $^{1575}$.

Unterschiede zwischen den nationalen Rechnungslegungssystemen werden demnach bis zu einem gewissen Grad akzeptiert, wenn sie auch nicht erwünscht sind. Welcher Harmonisierungsgrad ist das Ziel? Ein I-Index mit einem Wert von 0,5 oder besser 0,8? Diese Fragen sind deshalb nicht eindeutig zu beantworten, weil es durchaus denkbar sein kann, dass für einige Sachverhalte ein sehr hoher Harmonisierungsgrad gefordert werden muss, um Vergleichbarkeit zu erreichen. Bei anderen Geschäftsvorfällen kann wegen Unwesentlichkeit ein niedriger Harmonisierungsgrad zugelassen werden, ohne dass die Vergleichbarkeit der Jahresabschlüsse beeinträchtigt wird ${ }^{1576}$.

Das Prinzip der umgekehrten Maßgeblichkeit wirkt sich auf den Harmonisierungsgrad der untersuchten Sachverhalte „Abschreibungsmethode“ - positiv und „Bewertungsvereinfachungsverfahren“ - negativ - aus. Um steuerliche Einflüsse zu Gunsten eines den tatsächlichen Verhältnissen entsprechenden Bildes der Vermögens-, Finanz- und Ertragslage sowie zu Gunsten internationaler Harmonisierung zu unterbinden, müsste in Finnland wie in Deutschland die Beziehung zwischen Handels- und Steuerbilanz weiter gelockert werden. Diese Forderung wird derzeit in beiden Staaten diskutiert.

Ob die ab dem Jahr 2005 auf europäischer Ebene erfolgende Erstellung von Konzernabschlüssen börsennotierter Unternehmen nach den Standards des IASB eine weitere Harmonisierung der Einzelabschlüsse zur Folge hat, bleibt abzuwarten.

1575 Vgl. Art. 54 Abs. 3 (g) EG-Vertrag.

1576 Vgl. Lappalainen (1999), S. 102. 


\section{Zusammenfassung der Ergebnisse und Möglichkeiten einer weiterführenden Betrachtung}

Die finnische Rechnungslegung hat Wurzeln in Deutschland. Trotz enger Wirtschaftsbeziehungen zwischen Finnland und Deutschland und trotz des Bestrebens, die Rechnungslegung international zu harmonisieren, sind die Entwicklung sowie der aktuelle Stand der finnischen Rechnungslegung in Deutschland weitgehend unbekannt.

Ziel vorliegender Untersuchung war es deshalb, die finnischen und deutschen Rechnungslegungsnormen miteinander $\mathrm{zu}$ vergleichen und so den derzeitigen Stand der formalen Harmonisierung zwischen Finnland und Deutschland aufzuzeigen. Auf Basis dieses Rechtsvergleichs sollten zudem Einzelabschlüsse finnischer und deutscher Unternehmen miteinander verglichen und so der tatsächlich realisierte Harmonisierungsgrad zwischen beiden Ländern ermittelt werden. Der Untersuchung wurden vier Vergleichsebenen zu Grunde gelegt: die Kultur-Ebene, die Theorie-Ebene, die Regelwerk-Ebene und die Format-Ebene.

Um einen Vergleich zwischen beiden Ländern aus deutscher Sicht zu ermöglichen, musste Zugang zur Rechnungslegung in Finnland verschafft werden. Soweit für das Verständnis finnischer Rechnungslegung und aktueller Entwicklungen notwendig, führte Kap. 2 den deutschen Leser auf Kultur-Ebene in die Rechnungslegung Finnlands ein.

In Kap. 2.1 wurden die wesentlichen Entwicklungslinien der finnischen Rechnungslegung in chronologischer Reihenfolge dargestellt. Es wurden deutsche, skandinavische und internationale Einflüsse im Zeitablauf aufgezeigt und Denkansätze diskutiert, welche im Zeitablauf maßgebend auf die finnische Rechnungslegung eingewirkt haben und - besonders im Fall der EU und des IASB - auch heute noch einwirken. Hervorgehoben wurde die AusgabenEinnahmen-Theorie Martti Saarios, der die finnische Rechnungslegung von den 50er Jahren des 20. Jahrhunderts bis zum Buchführungsgesetz von 1992 gefolgt ist. Die Prägung der Rechnungslegung durch eine Theorie stellte im internationalen Vergleich eine Besonderheit dar und sorgte im Zeitraum ihrer Gültigkeit für eine große Eigenständigkeit der finnischen Rechnungslegung. Die Darstellung der geschichtlichen Entwicklung in Kap. 2.1 sollte dazu beitragen, das Vorgehen des finnischen Gesetzgebers im Rahmen der europäischen Harmonisierung bei der Transformation der 4. EG-Richtlinie zu erläutern und seine Ausübung von Wahlrechten zu verstehen. Die Theorie hat zwar durch die Transformation der 4. EG-Richtlinie weitgehend an Bedeutung verloren, hat aber den Vorgang der Transformation beeinflusst. Sie ist zudem immer noch gültig für 
formation beeinflusst. Sie ist zudem immer noch gültig für die Analyse und Interpretation von Buchführungsregeln.

Kap. 2.2 ergänzte die geschichtliche Darstellung durch eine Beschreibung der heutigen Regelungsinstitutionen der Rechnungslegung und des heutigen Prozesses zur Normierung der Rechnungslegung. Einflussnehmer und Mittel ihrer Einflussnahme wurden aufgezeigt. Einen Schwerpunkt bildete die Darstellung des Buchführungsausschusses KILA, der Regelungslücken schließt, Gesetze auslegt und sie für die Praxis verbindlich interpretiert.

Aufbauend auf Kap. 2 wurden in Kap. 3 Zielsetzung und Instrumente der finnischen Rechnungslegung, der True and Fair View, Jahresabschlussgrundsätze sowie die Grundsätze ordnungsmäßiger Buchführung, Ansatz und Bewertung von Aktiva und Passiva, wesentliche Bilanzposten, ausgewählter Posten der Gewinnund Verlustrechnung sowie der Lagebericht dargestellt. Untersucht wurde die richtlinienkonforme Transformation von Vorgaben der 4. EG-Richtlinie in die finnische Rechnungslegung, da Richtlinienkonformität eine Voraussetzung für einen sinnvollen Vergleich der Rechnungslegungsregeln von Finnland und Deutschland ist.

Es konnte gezeigt werden, dass der finnische Gesetzgeber die Mindestanforderungen der 4. EG-Richtlinie an die analysierenden Bereiche richtlinienkonform umgesetzt hat, obwohl sein Verständnis des True and Fair View und dessen Overrides nicht mit den - durchaus unterschiedlichen - Vorstellungen anderer europäischer Gesetzgeber übereinstimmt. So kann nach herrschender finnischer Meinung ein Widerspruch zwischen Grundsätzen ordnungsmäßiger Buchführung und True and Fair View nicht entstehen. Daher ist der True and Fair view in erster Linie vom Gesetzgeber und vom Buchführungsausschuss KILA im Normierungsprozess zu berücksichtigen. Der True and Fair View ist v.a. von ihnen bei der Erstellung von Gesetzen, Anweisungen, Gutachten oder Entscheidungen anzuwenden, nicht primär von den Unternehmen. Dieses Verständnis des True and Fair View erklärt auch, dass in Finnland sich nicht der einzelne Jahresabschluss-Ersteller mit der Frage nach der Notwendigkeit von Zusatzangaben im Anhang auseinandersetzt. Vielmehr regelt der Gesetzgeber das Erfordernis von Zusatzangaben für Einzelfälle. Analog bezieht der finnische Gesetzgeber auch den True and Fair ViewOverride auf seine eigene Arbeit. Der Override wird nicht vom einzelnen Jahresabschluss-Ersteller auf den Sachverhalt bezogen angewendet, sondern vom Ministerium für Handel und Industrie gestattet. Ziel dieser Regelung ist es, einer missbräuchlichen Anwendung des Overrides durch einzelne Buchführungspflichtige vorzubeugen. 
Materiell als kritisch erweist sich der ausschließlich den Aktiengesellschaften nach bestimmten, eng auszulegenden Kriterien gestattete Ausweis von kapitalersetzenden Darlehen im Eigenkapital. Die 4. EG-Richtlinie geht zwar nicht explizit auf die Behandlung solcher Darlehen ein; sie im Eigenkapital zu zeigen, steht jedoch im Gegensatz zum Prinzip der Unternehmensfortführung und zum Vorsichtsgedanken. Durch den gesonderten Ausweis innerhalb des Eigenkapitals und zwingend erforderliche Angaben im Anhang stellt der finnische Gesetzgeber aber sicher, dass ein in gesetzmäßiger Weise aufgestellter Jahresabschluss den True and Fair View erfüllt. Der Ausweis kapitalersetzender Darlehen im Eigenkapital sollte daher keinen hinreichenden Grund dafür bieten, die Rechtsgültigkeit finnischer Rechnungslegungsvorschriften für Aktiengesellschaften aus europarechtlicher Sicht zu verneinen.

Der finnische Gesetzgeber weicht zudem hinsichtlich des Ausweises von Anlagevermögen und steuerlichen Ausgleichsposten von den in der Richtlinie verbindlich festgelegten Artikeln ab, erfüllt aber auch in diesen Fällen die Intention des Richtliniengebers; genannte Abweichungen verbessern die Aussagefähigkeit und somit die Qualität finnischer Abschlüsse.

Normen zur Fremdwährungsumrechnung, zur Bewertung langfristiger Auftragsfertigung, zur Ermittlung latenter Steuern und zur Kapitalflussrechnung schließen Regelungslücken der 4. EG-Richtlinie. Gesetzgeber und KILA haben sich bei Erstellung dieser Normen an den jeweiligen Standards des IASB orientiert, was auch das Advisory Forum der EU für sinnvoll erachtet. Es kann daher davon ausgegangen werden, dass der finnische Gesetzgeber im Sinne des Richtliniengebers gehandelt hat.

Diese Auseinandersetzung mit den wesentlichen Denkansätzen und Einzelvorschriften der finnischen Rechnungslegung auf Theorie-Ebene und RegelwerkEbene in Kap. 3 bildet eine Basis, von der aus Finnlands Regelwerk mit denjenigen aller anderen EU-Mitgliedstaaten verglichen werden kann.

In Kap. 4.1 wurde der eigentliche Rechtsvergleich zwischen Finnland und Deutschland erarbeitet. Kulturelle Gemeinsamkeiten und Unterschiede konnten mithilfe der in Kap. 2 gewonnenen Erkenntnisse festgestellt werden. Ein auffallender kultureller Unterschied liegt darin, dass in Finnland viele Regeln durch den Buchführungsausschuss KILA interpretiert und Regelungslücken durch ihn geschlossen werden, während sich in Deutschland aus Literaturbeiträgen und Kommentaren von Sachverständigen aus Theorie und Praxis eine herrschende Meinung herausbildet, die von der Mehrzahl der Jahresabschluss-Ersteller berücksichtigt wird.

Im Wesentlichen konzentrierte sich der Vergleich in Kap. 4.1 auf die Theorieund Regelwerk-Ebene, folgte daher der Struktur von Kap. 3 und verwertete die in 
Kap. 3 erzielten Resultate. Es wurde demonstriert, dass die formale Harmonisierung zwischen Finnland und Deutschland zwar weit fortgeschritten ist, jedoch solche Unterschiede bestehen, die sich auf die Jahresabschlussaussage beträchtlich auswirken können. Insbesondere die unterschiedliche Auslegung von Vorsichts- und Realisationsprinzip und dem Prinzip der Periodenabgrenzung in beiden Staaten muss zwangsläufig zu Abweichungen in der Bilanzierung finnischer und deutscher Unternehmen führen. Gewinne gehen in Deutschland oft später und Verluste oft früher in die Gewinn- und Verlustrechnung ein als in Finnland.

Die formale Harmonisierung wird auch dadurch beeinträchtigt, dass in beiden Staaten die umgekehrte Maßgeblichkeit zur Beeinflussung der Handelsbilanz durch die jeweilige Steuergesetzgebung führt.

Der in Kap. 4.1 erarbeitete Rechtsvergleich diente schließlich dazu, sinnvolle Bereiche zu bestimmen, um an ihnen in Kap. 4.2 empirisch den materiellen, tatsächlich realisierten Harmonisierungsgrad zwischen Abschlüssen finnischer und deutscher Unternehmen mithilfe eines Konzentrationsindexes zu ermitteln. Die Analyse acht ausgewählter Themen anhand von jeweils zehn Abschlüssen börsennotierter Unternehmen der Jahre 1999 und 2000 stellt zwar keine repräsentative Untersuchung dar, zeigt jedoch Trends auf.

Sie führte zu folgenden Ergebnissen: hinsichtlich des Ansatzes derivativer Geschäfts- oder Firmenwerte, des Ansatzes von Forschungs- und Entwicklungskosten und von immateriellen Vermögenswerten sowie der Wahl der Abschreibungsmethode ist der materielle Harmonisierungsgrad ,sehr gut“. Bei Neubewertung und bei der Ermittlung von Herstellungskosten des Vorratsvermögens stellt er sich als „befriedigend“ dar, beim Ansatz passiver latenter Steuern „zufriedenstellend" und bei der Wahl von Bewertungsvereinfachungsverfahren ,mangelhaft"“. Somit ist festzustellen, dass bei mittelmäßiger Harmonisierung der gesetzlichen Vorgaben die Harmonisierung in der Praxis überraschend gut ist. Die Fälle, in denen der tatsächlich realisierte Harmonisierungsgrad noch nicht weit fortgeschritten ist, stammen aus der unterschiedlichen Ausübung von Unternehmenswahlrechten, die auch im Prinzip der umgekehrten Maßgeblichkeit begründet liegt. Die EU-weit angestrebte Gleichwertigkeit der Abschlüsse ist zumindest zwischen Finnland und Deutschland noch nicht in allen Punkten erreicht, die Vergleichbarkeit verbesserungsfähig.

Inwieweit der erreichte Harmonisierungsgrad zwischen Finnland oder Deutschland und anderen EU-Mitgliedstaaten besser oder schlechter ausfällt als der hier ermittelte, kann nicht beurteilt werden. Hier bieten sich für die Zukunft weitere Studien auf Kultur-Ebene, Theorie-Ebene und Regelwerk-Ebene (formaler Har- 
monisierungsgrad) sowie Format-Ebene (tatsächlich realisierter Harmonisierungsgrad) an.

Auch erscheint für weitergehende Studien ein Vergleich mit den an Bedeutung zunehmenden Standards des IASB reizvoll - nicht zuletzt, um das Prinzip der umgekehrten Maßgeblichkeit in beiden Staaten zu diskutieren und Möglichkeiten seiner Vereinbarung mit den IAS zu analysieren.

Steigenden Einfluss genießen derzeit in vielen Staaten die Kapitalflussrechnung und der Lagebericht; beide wurden bislang in internationalen empirischen Studien kaum berücksichtigt. 
Anhang 


\section{Anhang 1: Struktur des finnischen Buchführungsgesetzes ${ }^{1}$}

Kap. 1

Allgemeine Vorschriften

$\S 1 \quad$ Buchführungspflicht

$\S 2$ Doppelte Buchführung

$\S 3$ Art guter Buchführung

$\S 4$ Geschäftsjahr

$\S 5$ Beherrschungsvertrag

$\S 6$ Konzernunternehmen, Mutterunternehmen, Tochterunternehmen

$\S 7$ Beteiligungsunternehmen

$\S 8$ Beteiligungsunternehmen im engeren Sinne

Kap. 2

\section{Buchführung und Buchführungsunterlagen}

$\S 1$ Geschäftsvorfälle

$\S 2$ Buchführungskonten und Kontenrahmen

$\S 3$ Buchungsprinzipien

$\S 4$ Buchungsreihenfolge und -zeitpunkt

$\S 5 \quad$ Beleg

$\S 6 \quad$ Grundsatz der Nachprüfbarkeit

$\$ 7$ Eindeutigkeit

\$ $\quad$ Anwendung von EDV

§ 9 Aufbewahrung der Buchführungsunterlagen im Ausland

$\S 10$ Aufbewahrungszeitraum der Buchführungsunterlagen

Kap. 3 Jahresabschluss

$\S 1 \quad$ Inhalt des Jahresabschlusses

$\S 2$ Richtige und ausreichende Informationen

§ 3 Allgemeine Jahresabschlussgrundsätze

$\S 4$ Korrektur und Ergänzung des Grundsatzes der zahlungsabhängigen Buchung

$\S 5 \quad$ Sprache und Währung

$\S 6$ Erstellungszeitraum

$\S 7$ Datierung und Unterschrift

\& $\quad$ Bilanzbuch

§ 9 Registrierung des Jahresabschlusses

$\S 10 \quad$ Veröffentlichung des Jahresabschlusses

$\S 11$ Herausgabepflicht von Kopien des Jahresabschlusses

$\S 12$ Die Wirtschaftsprüfung betreffende Informationen

$\S 13$ Aufgliederung der Bilanzposten und des Anhangs

Kap. 4

\section{Definition einzelner Jahresabschlussposten}

$\S 1$ Umsatzerlöse

$\S 2$ Außerordentliche Erträge und Aufwendungen

$\S 3$ Langfristige und kurzfristige Aktiva

$\S 4$ Anlage-, Umlauf- und Finanzumlaufvermögen

$\S 5$ Anschaffungs- und Herstellungskosten

$\S 6$ Rechnungsabgrenzungsposten

§ $\quad$ Langfristige Forderungen und Verbindlichkeiten

Kap. 5 Bewertungs- und Periodisierungsvorschriften

$\begin{array}{ll}\S 1 & \text { Periodisierung von Erträgen, Aufwendungen und Verlusten } \\ \S 2 & \text { Forderungen, liquide Mittel und Verbindlichkeiten } \\ \S 3 & \begin{array}{l}\text { Forderungen, Verbindlichkeiten und andere Verpflichtungen in Fremd- } \\ \text { währungen }\end{array} \\ \S 4 & \begin{array}{l}\text { Teilgewinnrealisierung } \\ \S 5\end{array} \\ \begin{array}{l}\text { Periodisierung der Anschaffungs- und Herstellungskosten des Anlage- } \\ \text { vermögens }\end{array} \\ \S 6 & \begin{array}{l}\text { Periodisierung der Anschaffungs- und Herstellungskosten des Umlauf- } \\ \text { vermögens }\end{array} \\ \S 7 & \begin{array}{l}\text { Periodisierung von Gründungsausgaben } \\ \text { Periodisierung von Entwicklungsausgaben }\end{array}\end{array}$

Vgl. KPL 30.12.1997/1336. Übersetzung ins Deutsche durch die Verfasserin. 


\begin{tabular}{|c|c|c|}
\hline & $\S 9$ & Periodisierung des Geschäfts- oder Firmenwerts \\
\hline & $\S 10$ & Eigenkapital-Senkung und Emissionsausgaben für Darlehen \\
\hline & $\S 11$ & Periodisierung anderer Ausgaben mit lang anhaltender Wirkung \\
\hline & $\S 12$ & Andere als planmäßige Abschreibungen \\
\hline & $\S 13$ & Wertminderung \\
\hline & $\S 14$ & Aufwendungen, Verluste und Pflichtrückstellungen \\
\hline & $\S 15$ & Freiwillige Rückstellungen \\
\hline & $\S 16$ & Wertaufholung \\
\hline & $\S 17$ & Neubewertung \\
\hline & $\S 18$ & $\begin{array}{l}\text { Aus Periodisierungsunterschieden resultierende latente Steuerverbind- } \\
\text { lichkeiten und -forderungen }\end{array}$ \\
\hline & $\S 19$ & Transfers zwischen dem Anlage- und dem Umlaufvermögen \\
\hline & $\S 20$ & Eigener Verbrauch \\
\hline Kap. 6 & Kon & abschluss \\
\hline & $\S 1$ & Aufstellungspflicht \\
\hline & $\S 2$ & Konzernabschlussinhalt \\
\hline & $\S 3$ & Ausnahmen von der Einbeziehungspflicht von Tochterunternehmen \\
\hline & $\S 4$ & Aufstellungsgrundsätze \\
\hline & $\S 5$ & Zwischenabschluss von Tochterunternehmen \\
\hline & $\$ 6$ & Jahresabschlussposten ausländischer Tochterunternehmen \\
\hline & $\S 7$ & Konzerninterne Anteile und Minderheitsanteile \\
\hline & $\S 8$ & Bewertungsmethode zu Anschaffungskosten (Past Equity-Methode) \\
\hline & $\S 9$ & Pooling of Interests-Methode \\
\hline & $\S 10$ & $\begin{array}{l}\text { Aktien oder Anteile am Mutterunternehmen im Besitz von Tochter- oder } \\
\text { Mutterunternehmen }\end{array}$ \\
\hline & $\S 11$ & Latente Steuern \\
\hline & $\S 12$ & Konsolidierungspflicht von Beteiligungsunternehmen \\
\hline & $\S 13$ & Konsolidierung von Beteiligungsunternehmen \\
\hline & $\S 14$ & Angabepflicht von Beteiligungsunternehmen \\
\hline & $\S 15$ & Konsolidierung von Gemeinschaftsunternehmen \\
\hline & $\S 16$ & Konzernabschluss von Beteiligungsunternehmen \\
\hline & $\S 17$ & Tochterunternehmen mit abweichendem Geschäftszweck \\
\hline & $\S 18$ & Finanzleasing \\
\hline & $\S 19$ & Aufbewahrung von Konsolidierungsrechnungen \\
\hline Kap. 7 & Bucl & ung von Freiberuflern \\
\hline & $\S 1$ & Anwendung des Gesetzes auf Freiberufler \\
\hline & $\S 2$ & Buchung von Geschäftsvorfällen \\
\hline & $\S 3$ & Geschäftsjahr und Erstellungszeitraum des Jahresabschlusses \\
\hline & $\S 4$ & Erstellung von Gewinn- und Verlustrechnung und Bilanz \\
\hline & $\S 5$ & Aufbewahrungszeitraum von Büchern und Belegen \\
\hline Kap. 8 & Beso & e Vorschriften \\
\hline & $\S 1$ & Kontrolle über die Einhaltung des Gesetzes \\
\hline & $\S 2$ & Buchführungsausschuss KILA \\
\hline & $\S 3$ & Aushändigung von geheimen Informationen \\
\hline & $\S 4$ & Ordnungswidrigkeiten gegen das Buchführungsgesetz \\
\hline & & Die Buchführung betreffende Vorschriften in anderen Gesetzen \\
\hline & & Genauere Vorschriften \\
\hline Kap. 9 & In-K & Treten des Gesetzes und Übergangsvorschriften \\
\hline & & In-Kraft-Treten des Gesetzes \\
\hline & $\S 2$ & Übergangsvorschriften \\
\hline
\end{tabular}




\section{Anhang 2: Finnische Spezialgesetze mit Rechnungslegungsrelevanz}

Für folgende Rechtsformen sind Anforderungen an die Rechnungslegung auch außerhalb des Buchführungsgesetzes geregelt ${ }^{2}$ :

- für Aktiengesellschaften im Aktiengesellschaftsgesetz $\mathrm{OYL}^{3}$ und im Wertpapiermarktgesetz $\mathrm{AML}^{4}$,

- für Genossenschaften im Genossenschaftsgesetz $\mathrm{OKL}^{5}$ und Genossenschaftsbanken im Genossenschaftsbankengesetz ${ }^{6}$,

- für Offene Handelsgesellschaften und Kommanditgesellschaften gemeinsam im Gesetz für Offene Handelsgesellschaften und Kommanditgesellschaften $\mathrm{AKYL}^{7}$,

- für Stiftungen im Stiftungsgesetz ${ }^{8}$,

- für Pensionskassen im Pensionskassengesetz ${ }^{9}$,

- für Versicherungskassen im Versicherungskassengesetz ${ }^{10}$,

- für Versicherungsunternehmen im Versicherungsunternehmensgesetz ${ }^{11}$,

- für Versicherungsvereine im Versicherungsvereinsgesetz ${ }^{12}$,

- für Vereine im Vereinsgesetz ${ }^{13}$, politische Parteien im Parteiengesetz ${ }^{14}$, Wohnrechtsvereine im Gesetz für Wohnrechtsvereine ${ }^{15}$, Fischerei im Fischereigesetz $^{16}$ und im Fischereierlass ${ }^{17}$, Privatstrassen im Gesetz für Privatstras$\operatorname{sen}^{18}$, Handelskammern im Handelskammererlass ${ }^{19}$ und Sparkassen im Sparkassengesetz ${ }^{20}$,

- für Investmentfonds im Gesetz für Investmentfonds ${ }^{21}$,

- für Personalfonds im Personalfondsgesetz ${ }^{22}$ und

- für Effektenanteilssysteme im Effektenanteilsgesetz ${ }^{23}$.

Übersetzung der Gesetzestitel ins Deutsche durch die Verfasserin.

Vgl. Osakeyhtiölaki Nr. 145/1997.

Vgl. Arvopaperimarkkinalaki Nr. 495/1989.

Vgl. Osuuskuntalaki Nr. 247/1954 und Nr. 371/1981.

Vgl. Osuuspankkilaki Nr. 1271/1990.

Vgl. Laki avoimista yhtiöistä ja kommandiittiyhtiöistä Nr. 674/1994,

Vgl. Säätiölaki Nr. 109/1930 und Nr. 349/1987.

Vgl. Eläkesäätiölaki Nr. 1774/1995.

Vgl. Vakuutuskassalaki Nr. 1164/1992.

Vgl. Vakuutusyhtiölaki Nr. 1062/1979.

Vgl. Vakuutusyhdistyslaki Nr. 1250/1987.

Vgl. Yhdistyslaki Nr. 503/1989.

Vgl. Puoluelaki Nr. 10/1969.

Vgl. Asumisoikeusyhdistyksistä annettu laki Nr. 650/1990.

Vgl. Kalastuslaki Nr. 286/1982.

Vgl. Kalastusasetus Nr. 1116/1982.

Vgl. Yksityistielaki Nr. 358/1962.

Vgl. Kauppakamariasetus Nr. 337/1988.

Vgl. Säästöpankkilaki Nr. 1270/1990.

Vgl. Sijoitusrahastolaki Nr. 480/1987.

Vgl. Henkilöstörahastolaki Nr. 814/1989.

Vgl. Arvo-osuusjärjestelmästä annettu laki Nr. 826/1991. 


\section{Anhang 3: Allgemeingültige Anweisungen des KILA}

Der KILA hat seit seiner Gründung im Jahr 1974 folgende allgemeingültige Anweisungen veröffentlicht. Sind im Lauf der Jahre mehrere Anweisungen zum selben Thema erschienen, so hat die jeweils jüngste Anweisung Gültigkeit ${ }^{24}$ :

2000:

- Fixkosten als Bestandteil der Anschaffungs- oder Herstellungskosten („Yleisohje kiinteiden menojen lukemisesta hyödykkeen hankintamenoon")

- Teilgewinnrealisierung (,Yleisohje tulon kirjaamisesta tuotoksi valmistusasteen perusteella“)

- Informationstechnologie in der Buchführung (,Yleisohje koneellisessa kirjanpidossa käytettävistä menetelmistä“)

- Umsatzsteuer (,Yleisohje arvonlisäveron kirjaamisesta“)

- Erstellung von Konzernabschlüssen (,Yleisohje konsernitilinpäätöksen laatimisesta“)

1999:

- Erstellung von Jahresabschluss, vorläufigem Abschluss und Zwischenbericht („Yleisohje valtiovarainministeriön päätöksessä (390/1999) tarkoitetun tilinpäätöksen, tilinpäätöstiedotteen ja osavuosikatsauksen laatimisesta")

- Latente Steuern („Yleisohje laskennallisista veronveloista ja-saamisista“)

- Kapitalflussrechnung (,Yleisohje rahoituslaskelman laatimisesta“)

- Planmäßige Abschreibungen („Yleisohje suunnitelman mukaisista poistoista“)

- Währungsumrechnung (,Yleisohje ulkomaanrahan määräisten saamisten sekä velkojen ja muiden sitoumusten muuttamisesta Suomen rahaksi ${ }^{\text {*) }}$ )

\section{8:}

- Darstellung von Gewinn- und Verlustrechnungen und Bilanz (,Yleisohje tuloslaskelmien ja taseiden esittämisestä“)

1994:

- Umsatzsteuer (,Yleisohje arvonlisäveron kirjaamisesta“)

1993:

- Teilgewinnrealisierung (,Yleisohje pitkän valmistusajan vaativasta suoritteesta syntyvän tulon kirjaamisesta tuotoksi valmistusasteen perusteella")

- Erstellung von Konzernabschlüssen („Yleisohje konsernitilinpäätöksen laatimisesta“)

- Währungsumrechnung („Yleisohje ulkomaanrahan määräisten saamisten sekä velkojen ja muiden sitoumusten muuttamisesta Suomen rahaksi“)

- Planmäßige Abschreibungen („Yleisohje suunnitelman mukaisista poistoista“)

- Fixkosten als Bestandteil der Anschaffungs- oder Herstellungskosten (,Yleisohje hyödykkeen hankintaan ja valmistukseen liittyvien kiinteiden menojen lukemisesta hankintamenoon")

1991:

- Bewertung von Personalfonds („Yleisohje henkilöstörahaston arvonmä̈äritystä koskeva ohje“)

- Jahresabschluss von Personalfonds (,Yleisohje henkilöstörahaston tilinpäätöstä koskevat määräykset ja ohjeet $\left.{ }^{\star \prime}\right)$

\section{0:}

- Informationstechnologie in der Buchführung („Yleisohje koneellisesta kirjanpidosta“)

1983:

- Kapitalflussrechnung (,Yleisohje rahoituslaskelman laadinnasta“)

24 Übersetzung der Titel der allgemeingültigen Anweisungen durch die Verfasserin. 


\section{Anhang 4.1: Finnisches Bilanzgliederungsschema25}

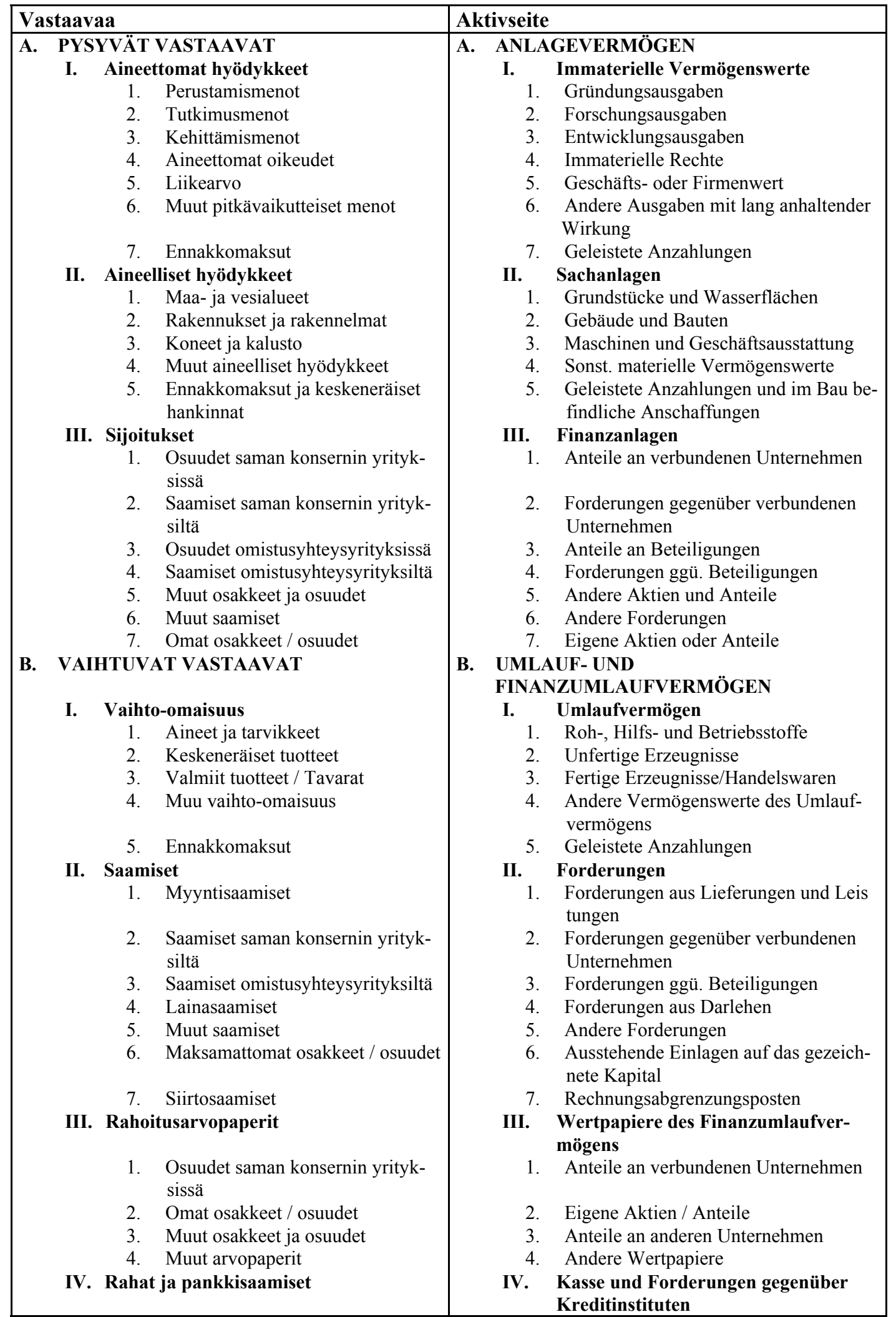

25 Vgl. KPA 1:6.1. Übersetzung ins Deutsche durch die Verfasserin. 


\begin{tabular}{|c|c|}
\hline Vastattavaa & Passivseite \\
\hline $\begin{array}{l}\text { A. OMA PÄÄOMA } \\
\text { I. Osake-, osuus- tai muu vastaava pääo- } \\
\text { ma } \\
\text { II. Ylikurssirahasto } \\
\text { III. Arvonkorotusrahasto } \\
\text { IV. Muut rahastot } \\
\text { 1. Omien osakkeiden / osuuksien rahasto } \\
\text { 2. Vararahasto } \\
\text { 3. Yhtiöjärjestyksen/Sääntöjen mukaiset } \\
\text { 4. Muhtot } \\
\text { V. Edellisten tilikausien voitto (tappio) } \\
\text { VI. Tilikauden voitto (tappio) } \\
\text { BILINPÄÄTÖSSIIRTOJEN KERTYMÄ } \\
\text { 1. Poistoero } \\
\text { 2. Vapaaehtoiset varaukset } \\
\text { PAKOLLISET VARAUKSET } \\
\text { 1. Eläkevaraukset } \\
\text { 2. Verovaraukset } \\
\text { 3. Muut pakolliset varaukset } \\
\text { VIERAS PÄÄOMA } \\
\text { 1. Joukkovelkakirjalainat } \\
\text { 2. Vaihtovelkakirjalainat } \\
\text { 3. Lainat rahoituslaitoksilta } \\
\text { 4. Eläkelainat }\end{array}$ & 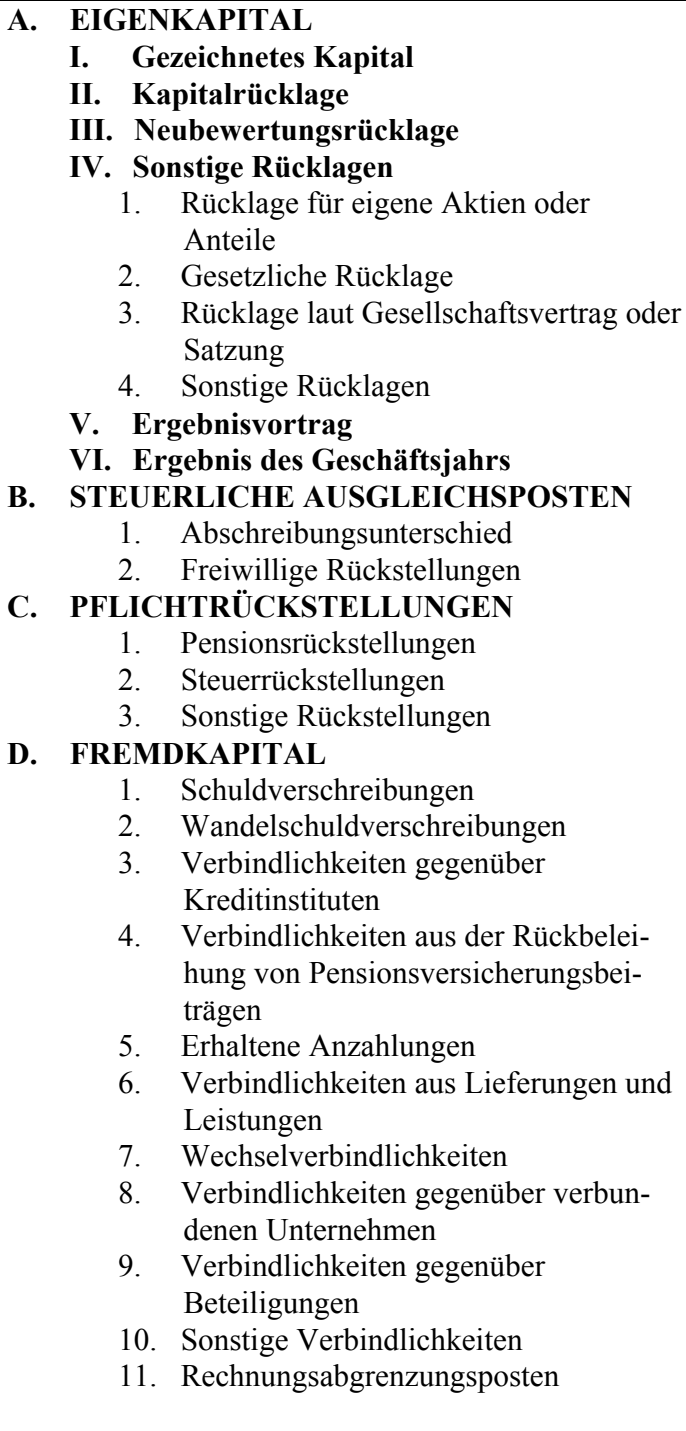 \\
\hline
\end{tabular}




\section{Anhang 4.2: Finnisches vereinfachtes Bilanzgliederungsschema ${ }^{26}$}

Buchführungspflichtige, welche die Größenkriterien des KPL 3:9.2 nicht überschreiten, dürfen das vereinfachte Bilanzgliederungsschema nach KPA 1:7 anwenden. Es umfasst lediglich die Posten des KPA 1:6.1, die mit Buchstaben und römischen Zahlen bezeichnet sind.

\begin{tabular}{|c|c|}
\hline Vastaavaa & Aktivseite \\
\hline $\begin{array}{ll}\text { C. } & \text { PYSYVÄT VASTAAVAT } \\
& \text { I. Aineettomat hyödykkeet } \\
& \text { II. Aineelliset hyödykkeet } \\
\text { III. Sijoitukset } \\
\text { D. VAIHTUVAT VASTAAVAT } \\
\\
\text { I. Vaihto-omaisuus } \\
\text { II. Saamiset } \\
\text { III. Rahat ja pankkisaamiset }\end{array}$ & $\begin{array}{ll}\text { C. } & \text { ANLAGEVERMÖGEN } \\
& \text { I. Immaterielle Vermögenswerte } \\
& \text { II. Sachanlagen } \\
& \text { III. Finanzanlagen } \\
\text { D. } & \text { UMLAUF- UND } \\
& \text { FINANZUMLAUFVERMÖGEN } \\
& \text { I. Umlaufvermögen } \\
\text { II. Forderungen } \\
\text { III. Wertpapiere des Finanzumlaufver- } \\
\\
\text { IV. } \begin{array}{l}\text { Kögens } \\
\end{array} \\
\end{array}$ \\
\hline
\end{tabular}

\begin{tabular}{|c|c|}
\hline Vastattavaa & Passivseite \\
\hline E. ОМА РӒӒОМА & E. EIGENKAPITAL \\
\hline I. Osake-, osuus- tai muu vastaava pääo- & I. Gezeichnetes Kapital \\
\hline ma & II. Kapitalrücklage \\
\hline II. Ylikurssirahasto & III. Neubewertungsrücklage \\
\hline III. Arvonkorotusrahasto & IV. Sonstige Rücklagen \\
\hline IV. Muut rahastot & V. Ergebnisvortrag \\
\hline V. Edellisten tilikausien voitto (tappio) & VI. Ergebnis des Geschäftsjahrs \\
\hline VI. Tilikauden voitto (tappio) & F. STEUERLICHE AUSGLEICHSPOSTEN \\
\hline F. TILINPÄÄTÖSSIIRTOJEN KERTYMÄ & G. PFLICHTRÜCKSTELLUNGEN \\
\hline G. PAKOLLISET VARAUKSET & H. FREMDKAPITAL \\
\hline H. VIERAS PÄÄOMA & \\
\hline
\end{tabular}

26 Vgl. KPA 1:7. Übersetzung ins Deutsche durch die Verfasserin. 


\section{Anhang 5.1: Finnisches Gliederungsschema der Gewinn- und Verlustrech- nung nach Gesamtkostenverfahren ${ }^{27}$}

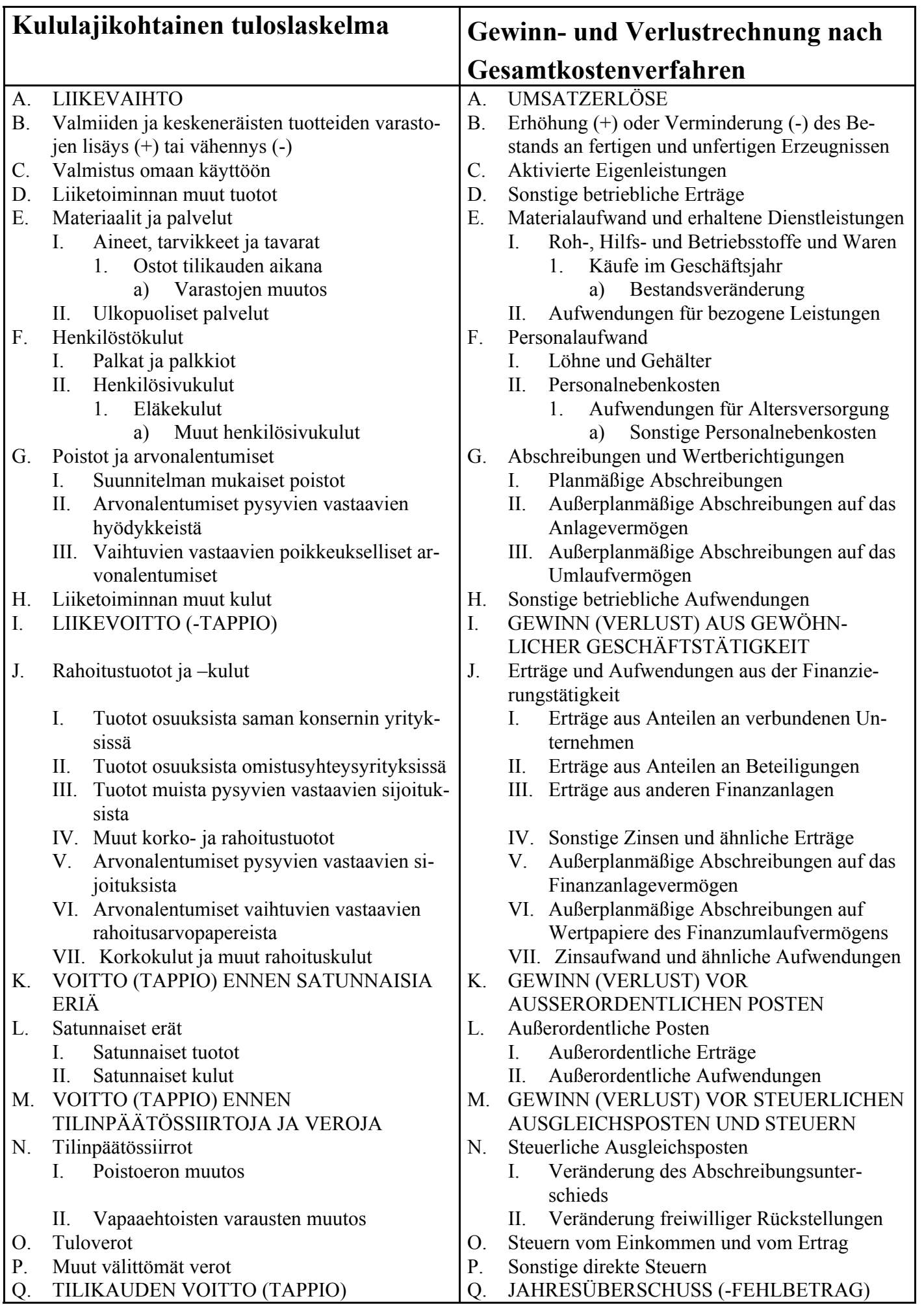

27 Vgl. KPA 1:1. Übersetzung ins Deutsche durch die Verfasserin. 
Anhang 5.2: Finnisches Gliederungsschema der Gewinn- und Verlustrechnung nach Umsatzkostenverfahren ${ }^{28}$

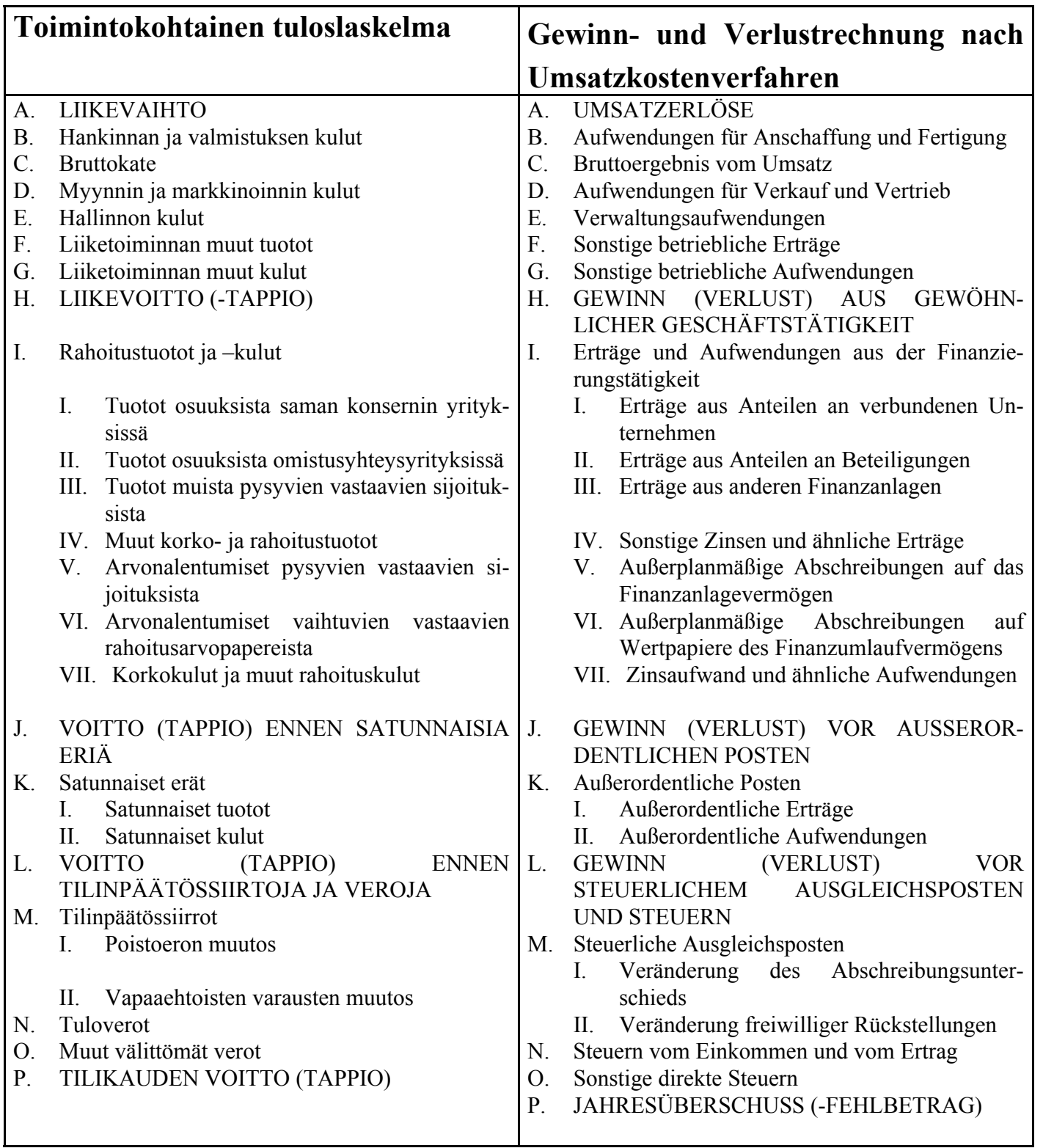

28 Vgl. KPA 1:2. Übersetzung ins Deutsche durch die Verfasserin. 


\section{Anhang 6.1: Gliederungsschema Kapitalflussrechnung nach direkter Metho- de gemäß KILA-Anweisung „Kapitalflussrechnung ${ }^{629}$}

\begin{tabular}{|c|c|c|}
\hline \multicolumn{2}{|c|}{ Suora rahoituslaskelma } & Kapitalflussrechnung - direkte Methode \\
\hline \multicolumn{2}{|c|}{ Liiketoiminnan rahavirta: } & $\begin{array}{l}\text { Mittelzu-/-abfluss aus laufender } \\
\text { Geschäftstätigkeit: }\end{array}$ \\
\hline & Myynnistä saadut maksut & Einzahlungen aus Umsatzerlösen \\
\hline & Liiketoiminnan muista tuotoista saadut maksut & $+\quad \begin{array}{l}\text { Einzahlungen aus Sonstigen betrieblichen Erträ- } \\
\text { gen }\end{array}$ \\
\hline & Maksut liiketoiminnan kuluista & $\begin{array}{l}\text { - } \quad \text { Auszahlungen für Aufwendungen der Geschäfts- } \\
\text { tätigkeit }\end{array}$ \\
\hline$=$ & $\begin{array}{l}\text { Liiketoiminnan rahavirta ennen rahoituseriä ja } \\
\text { veroja }\end{array}$ & $\begin{array}{l}=\quad \text { Mittelzu-/-abfluss aus laufender Geschäftstätig- } \\
\text { keit vor Finanzierungsposten und Steuern }\end{array}$ \\
\hline- & $\begin{array}{l}\text { Maksetut korot ja maksut muista liiketoimin- } \\
\text { nan rahoituskuluista }\end{array}$ & $\begin{array}{l}\text { - Zinszahlungen und Auszahlungen aus anderen } \\
\text { Finanzierungsaufwendungen der Geschäfts- } \\
\text { tätigkeit }\end{array}$ \\
\hline & Saadut korot liiketoiminnasta & $\begin{array}{l}+\quad \begin{array}{l}\text { Erhaltene Zinsen aus laufender Geschäftstätig- } \\
\text { keit }\end{array}\end{array}$ \\
\hline+ & Saadut osingot liiketoiminnasta & $\begin{array}{l}+\quad \begin{array}{l}\text { Erh. Dividenden aus laufender Geschäftstätig- } \\
\text { keit }\end{array}\end{array}$ \\
\hline- & Maksetut välittömät verot & Auszahlungen für direkte Steuern \\
\hline & Rahavirta ennen satunnaisia eriä & Mittelzu-/-abfluss vor außerordentlichen Posten \\
\hline & $\begin{array}{l}\text { Liiketoiminnan satunnaisista eristä johtuva } \\
\text { rahavirta (netto) }\end{array}$ & $\begin{array}{ll}+/- & \begin{array}{l}\text { Außerordentlicher Mittelzu-/-abfluss aus laufen- } \\
\text { der Geschäftstätigkeit (netto) }\end{array}\end{array}$ \\
\hline & Liiketoiminnan rahavirta (A) & $=\begin{array}{ll}\text { Mittelzu-/-abfluss aus laufender } \\
\text { Geschäftstätigkeit (A) }\end{array}$ \\
\hline \multicolumn{2}{|c|}{ Investointien rahavirta: } & \begin{tabular}{|l|} 
Mittelzu-/-abfluss aus Investitionstätigkeit: \\
\end{tabular} \\
\hline & $\begin{array}{l}\text { Investoinnit aineellisiin ja aineettomiin hyö- } \\
\text { dykkeisiin }\end{array}$ & $\begin{array}{ll}- & \text { Investitionen in materielle und immaterielle } \\
& \text { Vermögenswerte }\end{array}$ \\
\hline+ & $\begin{array}{l}\text { Aineellisten ja aineettomien hyödykkeiden } \\
\text { luovutustulot }\end{array}$ & $\begin{array}{l}+\quad \begin{array}{l}\text { Erlöse aus Verkauf von materiellen und immate- } \\
\text { riellen Vermögenswerten }\end{array}\end{array}$ \\
\hline- & Myönnetyt lainat & Auszahlung aus Gewährung von Darlehen \\
\hline- & Investoinnit muihin sijoituksiin & Investitionen in Finanzanlagen \\
\hline+ & Lainasaamisten takaisinmaksut & Einzahlungen aus Darlehensrückzahlung \\
\hline+ & Luovutustulot muista sijoutuksista & Erlöse aus dem Verkauf von Finanzanlagen \\
\hline+ & Saadut korot investoinneista & Erhaltene Zinsen aus Investitionen \\
\hline & Saadut osingot investoinneista & Erhaltene Dividenden aus Investitionen \\
\hline & Investointien rahavirta (B) & Mittelzu-/-abfluss aus Investitionstätigkeit (B) \\
\hline \multicolumn{2}{|c|}{ Rahoituksen rahavirta: } & Mittelzu-/-abfluss aus Finanzierungstätigkeit: \\
\hline+ & Maksullinen osakeanti & Aktienemission \\
\hline & Omien osakkeiden hankkiminen & Kauf eigener Aktien \\
\hline+ & Omien osakkeiden myynti & Verkauf eigener Aktien \\
\hline+ & Lyhytaikaisten lainojen nostot & Aufnahme kurzfristiger Kredite \\
\hline & Lyhytaikaisten lainojen takaisinmaksut & Rückzahlung kurzfristiger Kredite \\
\hline & Pitkäaikaisten lainojen nostot & Aufnahme langfristiger Kredite \\
\hline & Pitkäaikaisten lainojen takaisinmaksut & Rückzahlung langfristiger Kredite \\
\hline & Maksetut osingot ja muu voitonjako & $\begin{array}{l}\text { - Gezahlte Dividenden und andere Ausschüttun- } \\
\text { gen an die Gesellschafter }\end{array}$ \\
\hline \multirow[t]{4}{*}{$=$} & Rahoituksen rahavirta $(\mathrm{C})$ & $\begin{array}{l}\text { Mittelzu-/-abfluss aus Finanzierungstätigkeit } \\
\text { (C) }\end{array}$ \\
\hline & $\begin{array}{l}\text { Rahavarojen muutos }(\mathrm{A}+\mathrm{B}+\mathrm{C}) \text { lisäys }(+) / \\
\text { vähennys }(-)\end{array}$ & $\begin{array}{l}\text { Veränderung der Zahlungsmittel und } \\
\text {-äquivalente }(\mathrm{A}+\mathrm{B}+\mathrm{C}) \text { Erhöhung }(+) / \text { Minde- } \\
\text { rung (-) }\end{array}$ \\
\hline & Rahavarat tilikauden alussa & $\begin{array}{l}\text { Bestand Zahlungsmittel und } \\
\text {-äquivalente am Periodenanfang }\end{array}$ \\
\hline & \begin{tabular}{|l|} 
Rahavarat tilikauden lopussa \\
\end{tabular} & $\begin{array}{l}\text { Bestand Zahlungsmittel und } \\
\text {-äquivalente zu Periodenende }\end{array}$ \\
\hline
\end{tabular}

29 Vgl. KILA-Anweisung „Kapitalflussrechnung“, Abschnitt 5.1.2. Übersetzung ins Deutsche durch die Verfasserin. 


\section{Anhang 6.2: Gliederungsschema Kapitalflussrechnung nach indirekter Me- thode gemäß KILA-Anweisung „Kapitalflussrechnung ${ }^{630}$}

\begin{tabular}{|c|c|c|}
\hline \multicolumn{2}{|c|}{ Epäsuora rahoituslaskelma } & Kapitalflussrechnung - indirekte Methode \\
\hline \multicolumn{2}{|c|}{ Liiketoiminnan rahavirta: } & $\begin{array}{l}\text { Mittelzu-/-abfluss aus laufender Geschäftstätig- } \\
\text { keit: }\end{array}$ \\
\hline & Voitto (tappio) ennen satunnaisia eriä & $\begin{array}{ll}+/- & \text { Jahresüberschuss/-verlust vor außerordentichen } \\
& \text { Posten }\end{array}$ \\
\hline & $\begin{array}{l}\text { Oikaisut: } \\
\text { Suunnitelman mukaiset poistot }\end{array}$ & $\begin{array}{l}\text { Korrekturen: } \\
+\quad \text { Planmäßige Ahschreihunoen }\end{array}$ \\
\hline+ & $\begin{array}{l}\text { Suunnitelman mukaiset poistot } \\
\text { Realisoitumattomat kurssivoitot ia -tapniot }\end{array}$ & $\begin{array}{ll}+ & \text { Planmäßige Abschreibungen } \\
-/+ & \text { Unrealisierte Währungsgewinne und -verluste }\end{array}$ \\
\hline & Muut tuotot ja kulut, joihin ei liity maksua & $\begin{array}{l}-/+ \\
-/+\quad \text { Andere Erträge und Aufwendungen, die nicht } \\
\text { mit einer Zahlung verbunden sind }\end{array}$ \\
\hline$-/+$ & Rahoitustuotot ja-kulut & -/+ Finanzierungserträge/-aufwendungen \\
\hline$+/-$ & Muut oikaisut & $+/-\quad$ Andere Korrekturen \\
\hline & Rahavirta ennen käyttöpääoman muutosta & $=\begin{array}{l}\text { Mittelzu-/-abfluss vor Veränderung des Be- } \\
\text { triebskapitals }\end{array}$ \\
\hline & $\begin{array}{l}\text { Lyhytaikaisten korottomien liikesaamisten } \\
\text { lisäys }(-) \text { / vähennys }(+)\end{array}$ & $\begin{array}{ll}-/+ & \text { Zunahme }(-) / \text { Abnahme }(+) \text { kurzfristiger un- } \\
& \text { verzinslicher Forderungen }\end{array}$ \\
\hline$-/+$ & Vaihto-omaisuuden lisäys (-) / vähennys (+) & $-/+\quad$ Zunahme (-) / Abnahme (+) der Vorräte \\
\hline$+/-$ & $\begin{array}{l}\text { Lyhytaikaisten korottomien velkojen lisäys (+) } \\
\text { / vähennys (-) }\end{array}$ & $\begin{array}{ll}+/- & \text { Zunahme }(+) / \text { Abnahme (-) kurzfristiger un- } \\
& \text { verzinslicher Verbindlichkeiten }\end{array}$ \\
\hline$=$ & $\begin{array}{l}\text { Liiketoiminnan rahavirta ennen rahoituseriä ja } \\
\text { veroja }\end{array}$ & $\begin{array}{l}=\quad \text { Mittelzu-/-abfluss aus laufender Geschäftstä- } \\
\text { tigkeit vor Finanzierungsposten und Steuern }\end{array}$ \\
\hline & $\begin{array}{l}\text { Maksetut korot ja maksut muista liiketoimin- } \\
\text { nan rahoituskuluista }\end{array}$ & $\begin{array}{l}\text { - Zinszahlungen und Auszahlungen aus anderen } \\
\text { Finanzierungsaufwendungen aus laufender } \\
\text { Geschäftstätigkeit }\end{array}$ \\
\hline+ & Saadut osingot liiketoiminnasta & $\begin{array}{l}\text { Erhaltene Dividenden aus laufender Geschäfts- } \\
\text { tätigkeit }\end{array}$ \\
\hline+ & Saadut korot liiketoiminnasta & $\begin{array}{l}+\quad \begin{array}{l}\text { Erhaltene Zinsen aus laufender Geschäftstätig- } \\
\text { keit }\end{array}\end{array}$ \\
\hline- & Maksetut välittömät verot & Auszahlung für direkte Steuern \\
\hline$=$ & Rahavirta ennen satunnaisia eriä & Mittelzu-/-abfluss vor außerordentlichen Posten \\
\hline & $\begin{array}{l}\text { Liiketoiminnan satunnaisista eristä johtuva } \\
\text { rahavirta (netto) }\end{array}$ & \begin{tabular}{|l}
$+/-$ \\
A. o. Mittelzu-/-abfluss aus laufender Ge- \\
schäftstätigkeit (netto)
\end{tabular} \\
\hline & Liiketoiminnan rahavirta (A) & \begin{tabular}{|l}
$=$ \\
$\begin{array}{l}\text { Mittelzu-/-abfluss aus laufender Geschäftstä- } \\
\text { tigkeit (A) }\end{array}$
\end{tabular} \\
\hline Inve & tointien rahavirta: & Mittelzu-/-abfluss aus Investitionstätigkeit: \\
\hline & $\begin{array}{l}\text { Investoinnit aineellisiin ja aineettomiin hyö- } \\
\text { dykkeisiin }\end{array}$ & $\begin{array}{ll}- & \text { Investitionen in materielle und immaterielle } \\
& \text { Vermögenswerte }\end{array}$ \\
\hline+ & $\begin{array}{l}\text { Aineellisten ja aineettomien hyödykkeiden } \\
\text { luovutustulot }\end{array}$ & $\begin{array}{l}+\quad \begin{array}{l}\text { Erlöse aus Verkauf von materiellen und imma- } \\
\text { teriellen Vermögenswerten }\end{array}\end{array}$ \\
\hline- & Myönnetyt lainat & Auszahlung aus Gewährung von Darlehen \\
\hline & Investoinnit muihin sijoituksiin & Investitionen in Finanzanlagen \\
\hline+ & Lainasaamisten takaisinmaksut & Einzahlungen aus Darlehensrückzahlung \\
\hline & Luovutustulot muista sijoutuksista & Erlöse aus Verkauf von Finanzanlagen \\
\hline & Saadut korot investoinneista & Erhaltene Zinsen aus Investitionen \\
\hline & Saadut osingot investoinneista & Erhaltene Dividenden aus Investitionen \\
\hline & Investointien rahavirta $(\mathrm{B})$ & $\begin{array}{l}=\text { Mittelzu-/-abfluss aus Investitionstätigkeit } \\
\text { (B) }\end{array}$ \\
\hline
\end{tabular}

Fortsetzung auf nächster Seite

30 Vgl. KILA-Anweisung „Kapitalflussrechnung“, Abschnitt 5.2.2. Übersetzung ins Deutsche durch die Verfasserin. 


\begin{tabular}{|c|c|c|}
\hline \multicolumn{2}{|c|}{ Rahoituksen rahavirta: } & Mittelzu-/-abfluss aus Finanzierungstätigkeit: \\
\hline & Maksullinen osakeanti & Aktienemission \\
\hline & Omien osakkeiden hankkiminen & Kauf eigener Aktien \\
\hline+ & Omien osakkeiden myynti & Verkauf eigener Aktien \\
\hline+ & Lyhytaikaisten lainojen nostot & Aufnahme kurzfristiger Kredite \\
\hline & Lyhytaikaisten lainojen takaisinmaksut & Rückzahlung kurzfristiger Kredite \\
\hline & Pitkäaikaisten lainojen nostot & Aufnahme langfristiger Kredite \\
\hline & Pitkäaikaisten lainojen takaisinmaksut & Rückzahlung langfristiger Kredite \\
\hline & Maksetut osingot ja muu voitonjako & $\begin{array}{l}\text { - Gezahlte Dividenden und andere Ausschüttun- } \\
\text { gen an die Gesellschafter }\end{array}$ \\
\hline \multirow[t]{4}{*}{$=$} & Rahoituksen rahavirta (C) & $\begin{array}{l}=\quad \text { Mittelzu-/-abfluss aus Finanzierungstätigkeit } \\
\text { (C) }\end{array}$ \\
\hline & $\begin{array}{l}\text { Rahavarojen muutos }(\mathrm{A}+\mathrm{B}+\mathrm{C}) \text { lisäys }(+) / \\
\text { vähennys (-) }\end{array}$ & $\begin{array}{l}\text { Veränderung der Zahlungsmittel und } \\
\text {-äquivalente }(A+B+C) \text { Erhöhung }(+) / \text { Min- } \\
\text { derung }(-)\end{array}$ \\
\hline & Rahavarat tilikauden alussa & $\begin{array}{l}\text { Bestand Zahlungsmittel und } \\
\text {-äquivalente am Periodenanfang }\end{array}$ \\
\hline & \begin{tabular}{|l|} 
Rahavarat tilikauden lopussa \\
\end{tabular} & $\begin{array}{l}\text { Bestand Zahlungsmittel und } \\
\text {-äquivalente zu Periodenende }\end{array}$ \\
\hline
\end{tabular}




\section{Anhang 7: Ermittlung der latenten Steuern nach KILA-Anweisung „Latente Steuern"6}

Um die latenten Steuern zu ermitteln, hat der Buchführungspflichtige nach KILAAnweisung bei Alternative 1 und Alternative 2 wie folgt vorzugehen ${ }^{31}$ :

\begin{tabular}{|c|c|c|}
\hline & Alternative 1 & Alternative 2 \\
\hline 1. & \multicolumn{2}{|c|}{$\begin{array}{l}\text { Auflisten sämtlicher Differenzen zwischen steuerlichem und handelsrechtlichem Jah- } \\
\text { resergebnis. }\end{array}$} \\
\hline 2. & \multicolumn{2}{|c|}{ Streichen der permanenten Differenzen aus der erstellten Liste. } \\
\hline & & $\begin{array}{l}\text { 3. Ermittlung der nicht erfolgswirksamen } \\
\text { Periodisierungsunterschiede. }\end{array}$ \\
\hline 3./4. & \multicolumn{2}{|c|}{$\begin{array}{l}\text { Streichen derjenigen Sachverhalte aus der Liste, für die keine latenten Steuern gebil- } \\
\text { det werden dürfen (Steuergutschriften, Abschreibungsunterschiede, freiwillige Rück- } \\
\text { stellungen). }\end{array}$} \\
\hline 4./5. & \multicolumn{2}{|c|}{ Ermittlung möglicher Verlustvorträge zur künftigen Steuerminderung. } \\
\hline $5 . / 6$. & \multicolumn{2}{|c|}{$\begin{array}{l}\text { Aus den Unterschieden 1.-4. bzw. 1.-5. werden die aktiven bzw. passiven latenten } \\
\text { Steuern berechnet, indem sie mit dem im Zeitpunkt der Umkehr der Differenzen vor- } \\
\text { aussichtlich gültigen Steuersatz multipliziert werden. Herrscht Unsicherheit hinsicht- } \\
\text { lich des Zeitpunkts der Umkehr, ist der aktuelle Steuersatz bzw. der bereits angekün- } \\
\text { digte geänderte Steuersatz der nächsten Berichtsperiode anzuwenden. }\end{array}$} \\
\hline $6 . / 7$. & \multicolumn{2}{|c|}{$\begin{array}{l}\text { Unter Beachtung besonderer Vorsicht muss geprüft werden, ob die ermittelten Werte } \\
\text { in der Bilanz angesetzt werden dürfen. Dürfen einzelne Posten nicht angesetzt wer- } \\
\text { den, sind sie direkt von den anderen Posten abzuziehen. }\end{array}$} \\
\hline & $\begin{array}{l}\text { 7. Werden die ermittelten latenten Steu- } \\
\text { erposten lediglich im Anhang angege- } \\
\text { ben, dann ist die Gesamtsumme (1.-5.), } \\
\text { die abgezogene Summe (6.) und der } \\
\text { Differenzbetrag zu nennen. }\end{array}$ & \\
\hline 8. & \multicolumn{2}{|c|}{$\begin{array}{l}\text { Der in 6./7. ermittelte Posten ist in der Bilanz anzusetzen; eine Saldierung von Forde- } \\
\text { rungen und Verbindlichkeiten ist nur dann gestattet, wenn der Steuerempfänger und } \\
\text { der betrachtete Zeitraum identisch sind. }\end{array}$} \\
\hline 9. & \multicolumn{2}{|c|}{$\begin{array}{l}\text { In der GuV ist der Nettoänderungsbetrag anzugeben, der sich aus der Änderung der } \\
\text { passiven und aktiven latenten Steuern ergibt. }\end{array}$} \\
\hline & & $\begin{array}{l}\text { 9. Beruht der Nettoänderungsbetrag oder } \\
\text { ein Teil von ihm nicht auf erfolgswirksa- } \\
\text { mem Periodenunterschied, sondern auf der } \\
\text { Neubewertung, dann ist dieser auf die } \\
\text { Neubewertung entfallende Änderungsbe- } \\
\text { trag nicht in die GuV, sondern direkt ins } \\
\text { Eigenkapital (Neubewertungsrücklage) zu } \\
\text { buchen. }\end{array}$ \\
\hline
\end{tabular}

31 Vgl. KILA-Anweisung „Latente Steuern“, Abschnitt 7.1.1. Übersetzung ins Deutsche durch die Verfasserin. 


\section{Anhang 8: Glossar}

Im Folgenden werden zentrale rechnungslegungsrelevante Begriffe vom Finnischen ins Deutsche übersetzt und kurz erläutert. Die Übersetzung sämtlicher Posten von Bilanz, Gewinn- und Verlustrechnung sowie Kapitalflussrechnung ist in den Anhängen 4, 5 und 6 zu finden und deshalb nicht Bestandteil dieses Glossars.

\section{Arvonkorotus \\ Neubewertung \\ Wahlrecht zur Neubewertung von Grundstücken, Wasserflächen und Finanzanlagen über die ursprüngli- chen Anschaffungskosten hinaus.}

\section{Arvopaperimarkkinalaki, kurz: AML \\ Wertpapiermarktgesetz \\ Spezialgesetz zum Buchführungsge- setz mit rechnungslegungsrelevanten Vorschriften für börsennotierte öf- fentliche Aktiengesellschaften.}

\section{Asia ennen muotoa-periaate \\ Grundsatz der sachlichen Richtigkeit (Substance over Form)}

Avoimesta yhtiöstä ja kommandiittiyhtiöstä annettu laki,

\section{kurz: AKYL}

Gesetz über die Offene Handelsgesellschaft und die Kommanditgesellschaft

Spezialgesetz zum Buchführungsgesetz mit rechnungslegungsrelevanten Vorschriften für Offene Handelsgesellschaften und Kommanditgesellschaften.

Avoin yhtiö, kurz: Ay

finnische Offene Handelsgesellschaft

\section{Elinkeinoverolaki, kurz: EVL}

Gewerbesteuergesetz

Gesetz mit Einfluss auf die Rechnungslegung wegen des geltenden
Grundsatzes der umgekehrten Maßgeblichkeit.

\section{Emoyritys}

Mutterunternehmen

Unternehmen mit einem oder mehreren Tochterunternehmen. Das Mutter-Tochter-Verhältnis knüpft an das Control-Konzept an.

\section{Entiteettiperiaate}

Grundsatz der Betriebseinheit mit eigener Buchführung

(Entity Principle)

\section{Erillisarvostuksen periaate (netottamiskielto) Grundsatz der Einzelbewertung (Individual Valuation Principle)}

\section{Hallinto \\ Aufsichtsrat einer Aktiengesellschaft}

\section{Hallitus \\ Vorstand einer Aktiengesellschaft}

\section{Hankintameno}

historische Anschaffungs- oder Herstellungskosten

Bewertungsmaßstab für Vermögenswerte bei Erstverbuchung. Das finnische Buchführungsgesetz folgt vorrangig dem Anschaffungs- und Herstellungskostenprinzip, von dem in Einzelfällen abgewichen werden darf bzw. muss. 
HTM tilintarkastajat r.y.

HTM-Wirtschaftsprüferverband

Berufsverband der HTM-Wirtschaftsprüfer, der die Art guter Wirtschaftsprüfung weiterentwickelt, die Arbeitsqualität seiner Mitglieder überwacht und zudem mit zahlreichen Veröffentlichungen an der Rechnungslegungsentwicklung teilnimmt.

\section{HTM-yhteisö}

HTM-Wirtschaftsprüfungsgesellschaft

Von einer lokalen Handelskammer anerkannte Wirtschaftsprüfungsgesellschaft, die für die Prüfung von Unternehmen bestimmter Rechtsformen bis zu einer bestimmten Größe zugelassen ist.

\section{Hyvä kirjanpitotapa \\ Art guter Buchführung}

Generalnorm der Buchführung: Befolgung von Gesetzgebung, Gesetzesmaterialien und Grundsätzen ordnungsmäßiger Buchführung bei der Buchführung

\section{Hyvä tilintarkastustapa \\ Art guter Wirtschaftsprüfung}

\section{Hyväksytty tilimies, kurz: HTM}

HTM-Wirtschaftsprüfer

Von den lokalen Handelskammern anerkannter Wirtschaftsprüfer, der nur Unternehmen vorgeschriebener Rechtsformen prüfen darf, die bestimmte Größenkriterien nicht überschreiten (keine Prüfungszulassung für Kapitalgesellschaften und börsennotierte Gesellschaften).

Jaksotuseroista johtuvat laskennalliset verovelat ja saamiset

Latente Steuern

Latente Steueransprüche und -verbindlichkeiten werden auf zeitliche Unterschiede und wahlweise auch auf quasi-permanente Unterschiede zwischen Handels- und Steuerbilanz ermittelt. Bilanzierungswahlrecht, alternativ Anhangangabe.

\section{Jälleenhankintahinta}

Marktwert im Sinne von Wiederbeschaffungskosten

Betrag, der zur Wiederbeschaffung eines entsprechenden oder gleichartigen Vermögenswerts zum Zeitpunkt der Jahresabschlusserstellung ausgegeben werden müsste.

\section{Jatkuvuuden periaate}

Grundsatz der Fortführung der Unternehmenstätigkeit

(Going Concern)

\section{Julkinen osakeyhtiö, kurz: Oyj} (auf Schwedisch: Aktiebolag publikt, kurz: Abp)

öffentliche Aktiengesellschaft

Börsenzulasssungsfähige Aktiengesellschaft mit einem Mindestkapital von $€ 80.000,00$.

\section{Julkishallinnon tilintarkastaja,} kurz: JHTT

JHTT-Wirtschaftsprüfer

Wirtschaftsprüfer für Unternehmen des öffentlich-rechtlichen Sektors.

Julkisuuden periaate

Grundsatz der Offenlegung

(Disclosure)

\section{Kauppa- ja Teollisuusministeriö,} kurz: KTM

Ministerium für Handel und Industrie

Ministerium, das zuständig ist für den Entwurf von Gesetzen und Verordnungen zur Rechnungslegung, zur Besteuerung und zum Gesellschaftsrecht. Es trifft zudem Entscheidungen, um rechnungslegungsrelevante Gesetze und Verordnungen verbindlich zu konkretisieren. 


\section{Käyttöleasing}

Operate Lease

Als Art guter Buchführung hat sich die Behandlung als Miet- oder Pachtvertrag herausgebildet.

Keskuskauppakamarin hyväksymä tilintarkastaja KHT

KHT-Wirtschaftsprüfer

Autorisierter, von der Zentralhandelskammer zertifizierter Wirtschaftsprüfer, der Unternehmen aller Rechtsformen und Größen prüfen darf

\section{KHT-yhdistys - Föreningen CGR r.y. \\ KHT-Wirtschaftsprüferverband}

Berufsverband der KHT-Wirtschaftsprüfer, der die Art guter Wirtschaftsprüfung weiterentwickelt, die Arbeitsqualität seiner Mitglieder überwacht und zudem mit zahlreichen Veröffentlichungen an der Rechnungslegungsentwicklung teilnimmt.

\section{KHT-yhteisö}

KHT-Wirtschaftsprüfungsgesellschaft

Von der Zentralhandelskammer zertifizierte Wirtschaftsprüfungsgesellschaft, die für die Prüfung von Unternehmen aller Rechtsformen und Größen zugelassen ist.

\section{KILA:n lausunto}

Gutachten des KILA

Gutachten, in dem der KILA auf Anfrage einzelner Unternehmen oder Berufsverbände Stellung zu Einzelfragen nimmt. Am veröffentlichten Gutachten müssen sich alle Buchführungspflichtigen orientieren.

\section{KILA:n päätös}

Entscheidung des KILA

Entscheidung, die der KILA auf Anfrage einzelner Unternehmen oder Berufsverbände hinsichtlich der Fra- ge trifft, ob sie von bestehenden Vorschriften abweichen dürfen.

\section{KILA:n poikkeuslupa}

Erlaubnisse des KILA, von der Regel abzuweichen

Erlaubnis, die der KILA auf Anfrage einzelner Unternehmen oder Berufsverbände erteilt, um ihnen das Abweichen von bestehenden Vorschriften zu ermöglichen. Erlaubnisse gelten nur für den bzw. die Antragsteller und sind zeitlich begrenzt.

\section{KILA:n yleisohje}

Allgemeingültige Anweisung des KILA

Anweisung, in welcher der KILA Gesetze auslegt und anzuwendende Verfahrensweisen zu bestimmten Sachverhalten aufzeigt. Anweisungen sind verbindlich von allen Buchführungspflichtigen zu befolgen.

\section{Kirjanpidon meno-tulo-teoria}

Ausgaben-Einnahmen-Theorie der Buchführung

Von Martti Saario entwickelte Buchführungstheorie, die zwischen 1950 und 1990 die Grundlage der finnischen Rechnungslegung bildete. Die Theorie hat seit dem Buchführungsgesetz von 1992 zunehmend an Einfluss verloren.

\section{Kirjanpitoasetus, kurz: KPA} Buchführungsverordnung

Sie ergänzt das Buchführungsgesetz um Vorschriften zur Gliederung von Gewinn- und Verlustrechnung sowie Bilanz und zu Einzelfragen hinsichtlich Bewertung und Bilanzierung.

\section{Kirjanpitolain uudistamistyöryh- mä 1995}

Arbeitsgruppe von 1995 zur Erneuerung des Buchführungsgesetzes

kurz: KPL-Arbeitsgruppe 1995

Vom Ministerium für Handel und Industrie mit dem Entwurf von Ge- 
setzen zur Transformation der 4. EGRichtlinie in finnisches Recht beauftragte Arbeitsgruppe (Buchführungsgesetz, Gesellschaftsgesetze, Wirtschaftsprüfungsgesetz). Der Bericht der Arbeitsgruppe ist wichtiges Instrument zur Auslegung des Buchführungsgesetzes von 1997.

\section{Kirjanpitolaki, kurz: KPL}

Buchführungsgesetz

Es ist das Mantelgesetz zur Regelung von Buchführungs- und Rechnungslegungsfragen. Mit ihm wurde die 4. EG-Richtlinie in nationales Recht umgesetzt.

\section{Kirjanpitolautakunta, kurz: KILA} staatlicher Buchführungsausschuss

Aufgabe: Lieferung von Stellungnahmen und Kommentaren zu rechnungslegungsspezifischen Fragen, Interpretation bestehender Gesetze und Verdeutlichung von Regeln für die Praxis.

\section{Kirjanpitotoimistojen liitto r.y.,} kurz: KTL

Verband der Buchführungsfirmen

Berufsverband der Buchführungsfirmen, die als Externe die Bücher kleiner und mittelgroßer Unternehmen führen und deren Jahresabschluss erstellen.

\section{Kirjausketju}

Grundsatz der Nachprüfbarkeit, Belegfluss

(Audit trail)

\section{Kommandiittiyhtiö Ky}

Kommanditgesellschaft

\section{Konserniyritys}

Verbundenes Unternehmen

Ein Mutterunternehmen und seine Tochterunternehmen sind sämtlich untereinander verbundene Unternehmen.
Korkein hallinto-oikeus

Oberster finnischer Verwaltungsgerichtshof

\section{Liitetiedot}

Anhang

Pflichtbestandteil eines Jahresabschlusses.

\section{Luotettavuuden periaate}

Grundsatz der Richtigkeit und Willkürfreiheit

(Reliability)

\section{Maksuperuste}

Grundsatz der zahlungsabhängigen Buchführung

\section{Menettelytapojen jatkuvuuden periaate \\ Grundsatz der Stetigkeit \\ (Consistency)}

\section{Meno tulon kohdalle-periaate}

Grundsatz der periodengerechten Abgrenzung

(Matching)

\section{Merkityksellisyyden periaate}

Grundsatz der Bedeutsamkeit (Relevance)

\section{Nopeuden periaate}

Grundsatz der zeitnahen Berichterstattung

(Timeliness)

Oikeat ja riittävät tiedot (oikea ja riittävä kuva)

True and Fair View

Generalnorm des Jahresabschlusses: Darstellung eines richtigen und ausreichenden Bildes der Ertragslage und der ökonomischen Lage eines Buchführungspflichtigen.

Olennaisuuden periaate

Grundsatz der Wesentlichkeit

(Materiality) 


\section{Omistusyhteysyritys \\ Beteiligung \\ Eine Beteiligung wird widerlegbar dann vermutet, wenn ein Unterneh- men null bis 100 Prozent der Anteile am Nennkapital des anderen Unter- nehmens hält und die Anzahl der Stimmrechte null bis 50 Prozent um- fasst. Spezialfall der Beteiligung ist die Beteiligung im engeren Sinne.}

Osakeyhtiö, kurz: Oy (auf Schwedisch: Aktiebolag, kurz: Ab)

private Aktiengesellschaft

Aktiengesellschaft mit einem Mindestkapital von $€ 8.000,00$, die nicht börsenzulassungsfähig ist.

\section{Osakeyhtiölaki, kurz: OYL}

Aktiengesellschaftsgesetz

Spezialgesetz zum Buchführungsgesetz mit rechnungslegungsrelevanten Vorschriften für Aktiengesellschaften.

\section{Osakkuusyritys}

Beteiligung im engeren Sinne

Eine Beteiligung im engeren Sinne wird widerlegbar dann vermutet, wenn ein Unternehmen null bis 100 Prozent der Anteile am Nennkapital des anderen Unternehmens hält und die Anzahl der Stimmrechte 20 bis 50 Prozent umfasst. Sie ist ein Spezialfall der Beteiligung.

\section{Osavuosikatsaus}

Zwischenbericht einer Aktiengesellschaft

Er erfolgt entweder quartalsweise oder für die ersten acht Monate eines Geschäftsjahrs.

\section{Pääkirjanpito}

Hauptbuch

\section{Pääomalaina}

Eigenkapitalersetzendes Rangrücktrittsdarlehen

(Subordinated Loan, Capital Loan)
Rangrücktrittsdarlehen, das bestimmte Voraussetzungen erfüllt und dehalb beim Darlehensnehmer (Aktiengesellschaft) im Eigenkapital ausgewiesen wird.

\section{Pankkitarkastusvirasto}

Bankenaufsicht

Sie trifft auf Anforderung des Gesetzgebers Entscheidungen, die rechnungslegungsrelevante Gesetze und Verordnungen für Banken verbindlich konkretisieren.

\section{Patentti- ja rekisterihallitus \\ Patent- und Registeramt \\ Eine der Aufsichtsbehörden zur Ü- berwachung der Einhaltung von Buchführungsvorschriften (Offenle- gungspflicht).}

\section{Periodisuuden periaate Grundsatz der Periodisierung (Periodicity)}

\section{Peruskirjanpito \\ Grundbuch (Buchführung)}

\section{Rahoituslaskelma}

Kapitalflussrechnung

Pflichtbestandteil eines Jahresabschlusses öffentlicher Aktiengesellschaften und solcher privater Aktiengesellschaften, welche bestimmte Größenkriterien überschreiten. Anhang zum Lagebericht.

\section{Rahoitusleasing}

Finanzierungsleasing (Finance Lease)

Als Art guter Buchführung hat sich die Behandlung als Miet- oder Pachtvertrag herausgebildet.

\section{Rahoitustarkastus}

Aufsichtsamt über die Finanzmärkte Aufsichtsbehörde zur Überwachung der Einhaltung von Buchführungsvorschriften. 


\section{Realisointiperiaate \\ Realisationsprinzip \\ (Realization Principle) \\ Realisointipoisto \\ Realisationsabschreibung \\ Abschreibungsmethode in der Aus- gaben-Einnahmen-Theorie der Buch- führung. Vermögenswerte werden nicht auf Basis ihrer Anschaffungs- oder Herstellungskosten, sondern auf Basis des Periodenergebnisses abge- schrieben.}

Rikoslaki, kurz: RL

Strafgesetzbuch

Samanlajiset ja hankintamenoltaan vähäiset hyödykkeet

Festwert

\section{Sosiaali- ja terveysministeriö}

Sozial- und Gesundheitsministerium

Es trifft auf Anforderung des Gesetzgebers Entscheidungen, die rechnungslegungsrelevante Gesetze und Verordnungen für Versicherungen verbindlich konkretisieren.

\section{Suoriteperiaate}

Grundsatz der leistungsbezogenen Abgrenzung

(Accrual Basis)

\section{Taloudellisuuden periaate}

Grundsatz der vertretbaren Kosten (Balance between Benefit and Costs)

Tase

Bilanz

Pflichtbestandteil eines Jahresabschlusses.

\section{Tasejatkuvuuden periaate}

Grundsatz der Bilanzkontinuität

(Correspondence of Balance Sheets)

Tasekirja

Bilanzbuch
Es enthält Jahresabschluss, Hauptbuch, Grundbuch, das Verzeichnis aller Nebenbücher sowie das Verzeichnis über alle Belegarten des Buchführungspflichtigen.

Täydellisyyden periaate

Grundsatz der Vollständigkeit

(Completeness)

\section{Tilinpäätös}

Jahresabschluss

Er besteht immer aus Gewinn- und Verlustrechnung, Bilanz, Anhang und Lagebericht.

\section{Tilinpäätössiirtojen kertymä}

Steuerliche Ausgleichsposten

Der Einfluss steuerlicher Vorschriften auf den Jahresabschluss wird in diesem Posten isoliert auf der Passivseite der Bilanz ausgewiesen (Passivierungspflicht).

\section{Tilinpäätöstiedote}

vorläufiger Jahresabschluss einer Aktiengesellschaft

Veröffentlichte Vorabmitteilung des Jahresabschlusses und seiner Hauptaussagen.

\section{Tilintarkastusasetus, kurz: TilintA} Wirtschaftsprüfungsverordnung Sie enthält Vorschriften für und über den Berufsstand der Wirtschaftsprüfer, die das Wirtschaftsprüfungsgesetz ergänzen.

\section{Tilintarkastuslaki, kurz: TilintL}

Wirtschaftsprüfungsgesetz

Es enthält Vorschriften für und über den Berufsstand der Wirtschaftsprüfer. Mit ihm sind die Mindestanforderungen der 8. EG-Richtlinie in nationales Recht umgesetzt.

Tilintarkastuslautakunta, kurz: TILA

staatlicher Wirtschaftsprüfungsausschuss im Ministerium für Handel 
und Industrie

Aufgaben: Anweisungen und Stellungnahmen zum Wirtschaftsprüfungsgesetz und zur Wirtschaftsprüfungsverordnung, Entwicklung prüfungsrelevanter Vorschriften und Beaufsichtigung im Wirtschaftsprüfungswesen.

\section{Todennäköinen luovutushinta}

Marktwert im Sinne eines (wahrscheinlich) realisierbaren Betrags

Betrag, der bei Veräußerung des Vermögenswerts zum Zeitpunkt der Jahresabschlusserstellung erzielt werden könnte.

\section{Todennettavuuden periaate}

Grundsatz der Objektivität

(Objectivity)

\section{Toimintakertomus}

Lagebericht

Pflichtbestandteil eines Jahresabschlusses.

\section{Toimitusjohtaja \\ Geschäftsführer}

Tulon kirjaaminen tuotoksi valmistusasteen perusteella

Teilgewinnrealisierung bei langfristiger Auftragsfertigung

Die Gewinnrealisierung bei langfristiger Auftragsfertigung erfolgt nach Completed Contract-Methode. Unter bestimmten Anwendungen ist auch die Percentage of Completion-Methode anwendbar.

Tulonodotuksiin diskontattu perustuva hankinta-arvo

auf einer Einnahmenerwartung beruhender Barwert

Wert aller künftigen Nettoein- bzw. auszahlungen am Bilanzstichtag.

Tuloslaskelma

Gewinn- und Verlustrechnung
Pflichtbestandteil eines Jahresabschlusses.

Tuloverolaki, kurz: TVL

Einkommensteuergesetz

Gesetz mit Einfluss auf die Rechnungslegung wegen des geltenden Grundsatzes der umgekehrten Maßgeblichkeit.

\section{Tytäryritys}

\section{Tochterunternehmen}

Unternehmen, das von einem anderen Unternehmen - dem Mutterunternehmen - beherrscht wird. Das Mutter-Tochter-Verhältnis knüpft an das Control-Konzept an.

\section{Vähäarvoiset käyttöomaisuushan- kinnat}

Geringwertiges Wirtschaftsgut

\section{Vakaan rahanarvon periaate Grundsatz des stabilen Geldwerts (Historical Cost)}

\section{Valtiovarainministeriö VTM}

Finanzministerium

Es trifft auf Anforderung des Gesetzgebers Entscheidungen, welche die rechnungslegungsrelevanten Gesetze und Verordnungen für börsennotierte Aktiengesellschaften und mit Aktien handelnde Unternehmen verbindlich konkretisieren.

Varovaisuuden periaate

Grundsatz der Vorsicht

(Prudence)

Vertailtavuuden periaate

Grundsatz der Vergleichbarkeit

(Comparability)

\section{Yhtiökokous}

Hauptversammlung einer Aktiengesellschaft 
Yhtiön lähipiiri

innerer Kreis einer Aktiengesellschaft

$\mathrm{Zu}$ ihm gehören Geschäftsführer, Verwaltungs- und Aufsichtsratsmitglieder sowie Wirtschaftsprüfer und derjenige, der auf Grund von Eigentum, Optionsrechten und Wandelschuldverschreibungen mindestens ein Prozent der Aktien hält, und Angehörige aller dieser Personen. Zum inneren Kreis sind spezielle Anhangangaben erforderlich.

\section{Yleiset kirjanpidon periaatteet}

Grundsätze ordnungsmäßiger Buchführung $(\mathrm{GoB})$.

Yleislauseke

Generalnorm 


\section{Literaturverzeichnis}

\section{Fachliteratur}

Adams, Carol A., Weetman, Pauline und Gray, Sidney J. [Adams et al. (1993)]:

Reconciling national with international accounting standards - Lessons from a study of Finnish corporate reports,

in: European Accounting Review 1993, 3, S. 471-494.

Adler, Hans; Düring, Walther und Schmaltz, Kurt [ADS (ab 1995)]:

Rechnungslegung und Prüfung der Unternehmen, Kommentar zum HGB, AktG, GmbHG, PublG nach den Vorschriften des Bilanzrichtliniengesetzes, bearbeitet von Karl-Heinz Forster u. a., 6. Aufl., Stuttgart ab 1995.

Alexander, David [Alexander (1993)]:

A European true and fair view?, in: European Accounting Review 1993, 1, S. 59-80.

Alexander, David [Alexander (1996)]:

Truer and fairer. Uninvited comments on invited comments, in: European Accounting Review 1996, 5:3, S. 483-493.

Arovaara, Kaisa; Havukainen, Jari; af Hällström, Carl Gustaf u.v.a. [Arovaara et al. (1998)]:

Mitä uutta kirjanpitolainsäädännössä?, Jyväskylä 1998.

Artsberg, Kristina [Artsberg (2000)]:

Skandinavien, in: Haller, Axel, Raffournier, Bernard und Walton, Peter (Hrsg.) unter Mitarbeit von Eierle, Brigitte, Unternehmenspublizität im internationalen Wettbewerb, Stuttgart 2000, S. 511-560.

Baetge, Jörg; Kirsch, Hans-Jürgen und Thiele, Stefan (2001) [Baetge, Kirsch und Thiele (2001)]:

Bilanzen, 5. überarb. und erw. Aufl., Düsseldorf 2001.

Beine, Frank [Beine (1995)]:

Scheinkonflikte mit dem True and Fair View, in: Die Wirtschaftsprüfung 1995, S. 467-475.

Biener, Herbert und Berneke, Wilhelm [Biener und Berneke (1986)]:

Bilanzrichtlinien-Gesetz, Düsseldorf 1986.

Budde, Wolfgang Dieter (Bearb.) [BeBiKo (1999)]:

Beck'scher Bilanzkommentar: der Jahresabschluß nach Handels- und Steuerrecht und Steuerrecht, Konzernabschluß, Prüfung, Offenlegung; §§ 238 und 339 HGB, 4. neu bearb. Aufl., München 1999.

Burlaud, Alain [Burlaud (1993)]:

Commentaires sur l'article de David Alexander "A European true and fair view?", in: European Accounting Review 1993, 1, S. 91-98. 
Choi, Frederick D. S. und Mueller, Gerhard G. [Choi und Mueller (1992)]: International Accounting, 2. Aufl. Englewood Cliffs 1992.

Döllerer, Georg [Döllerer (1959)]:

Grundsätze ordnungsmäßiger Bilanzierung, deren Entstehung und Ermittlung, in: Betriebsberater 1959, S. 1217-1221.

Ekholm, Bo-Göran und Troberg, Pontus [Ekholm und Troberg (1995)]:

Oikeat ja riittävät tiedot-käsite määriteltynä, in: Tilintarkastus 5/1995, S. 343-346.

Elling, Jens O. [Elling (1993)]:

Financial reporting in the Nordic countries - an introduction, in: European Accounting Review 1993, 3, S. 581-584.

Emenyonu, E. N. und Gray, S. J. [Emenyonu und Gray (1992)]: European Community Accounting Harmonisation: An Empirical Study of Measurement Practices in France, Germany and the United Kingdom, in: Accounting and Business Research, Winter 1992, S. 49-58.

FEE (Hrsg.) [FEE (1992)]:

1992 FEE analysis of European accounting and disclosure practices, Further Analysis of the FEE European Survey of Published Accountants 1991 database, focusing on differences in the practices of listed and unlisted companies and on special accounting issues, London und New York, 1992.

Flower, John (Hrsg.) [Flower (1994)]:

The Regulation of Financial Reporting in the Nordic Countries, Stockholm 1994.

Glaum, Martin und Mandler, Udo [Glaum und Mandler (1996)]:

Rechnungslegung auf globalen Kapitalmärkten: HGB, IAS und US-GAAP, Wiesbaden 1996.

Goerdeler, Reinhard [Goerdeler (1987)]:

Vereinheitlichung des Bilanzwesens, in: Bockelmann, Horst et al. (Hrsg.), Integration der Kapitalmärkte, Frankfurt am Main 1987, S. 119-140.

Gross, Gerhard und Schruff, Lothar [Gross und Schruff (1986)]:

Der Jahresabschluß nach neuem Recht, Aufstellung - Prüfung - Offenlegung, 3. durchgesehene Aufl., Düsseldorf 1986.

Großfeld, Bernhard [Großfeld (1986)]:

Generalnorm (ein den tatsächlichen Verhältnissen entsprechendes Bild der Vermögens-, Finanz- und Ertragslage), in: Leffson, Ulrich; Rückle, Dieter und Großfeld, Bernhard: Handwörterbuch unbestimmter Rechtsbegriffe im Bilanzrecht des HGB, Köln 1986, S. 192-204.

Hagert, Albrecht [Hagert (1997)]:

Das neue finnische Aktiengesetz, in: Internationales Steuerrecht 1997, S. 605-608. 
Heino, Virpi [Heino (1995)]:

Vergleich der finnischen externen Rechnungslegung mit der 4. und 7. EURichtlinie und den deutschen Vorschriften, Arbeitsbericht Nr. 60, RuhrUniversität-Bochum, Institut für Unternehmensführung und Unternehmensforschung, Bochum 1995.

Helenius, Antti [Helenius (1990)]:

Kirjanpidon ja verotuksen erottaminen toisistaan - teoria ja käytäntö, in: Tilintarkastus 3/1990, S. 181-184

Herrmann, Don und Thomas, Wayne [Herrmann und Thomas (1995)]: Harmonisation of Accounting Measurement Practices in the European Community, in: Accounting and Business Research 1995, S. 253-265.

Honkamäki, Tuomas [Honkamäki (1998)]:

Ongelmana omat osakkeet, in: Tilintarkastus 5/1998, S. 389-393.

Hoogendoorn, Martin N. [Hoogendoorn (1996)]:

Accounting and taxation in Europe - A comparative overview, in: European Accounting Review 1996, 5: Supplement, S. 783-794.

IASC (Hrsg.) [IASC (1988)]:

Survey of the use and application of International Accounting Standards, London 1988.

Ilkka, Heikki [Ilkka (1998)]:

Kohti tehokasta kirjanpitoa, in: Arovaara et al. (1998), S. 276-300.

Institut der Wirtschaftsprüfer in Deutschland (Hrsg.) [HFA 1/1984]:

Stellungnahme HFA 1/1984: Bilanzierungsfragen bei Zuwendungen, dargestellt am Beispiel finanzieller Zuwendungen der öffentlichen Hand, in: Die Wirtschaftsprüfung 1984, S. 612-615.

Institut der Wirtschaftsprüfer in Deutschland (Hrsg.) [HFA 2/1988]:

Stellungnahme HFA 2/1988: Pensionsverpflichtungen im Jahresabschluß, in: Die Wirtschaftsprüfung 1988, S. 403-405.

Institut der Wirtschaftsprüfer in Deutschland (Hrsg.) [HFA 2/1991]:

Stellungnahme HFA 2/1991: Änderung von Jahresabschlüssen und Anpassung der Handelsbilanz an die Steuerbilanz.

Institut der Wirtschaftsprüfer in Deutschland (Hrsg.) [HFA 5/1991):

Stellungnahmen HFA 5/1991: Zur Aktivierung von Herstellungskosten.

Institut der Wirtschaftsprüfer in Deutschland (Hrsg.) [HFA 3/1997]:

Stellungnahme HFA 3/1997: Zum Grundsatz der Bewertungsstetigkeit, in: Die Wirtschaftsprüfung 1997, S. 540-544.

Institut der Wirtschaftsprüfer in Deutschland e.V. (Hrsg.) [WPH I (2000)]: Wirtschaftsprüferhandbuch 2000, Handbuch für Rechnungslegung, Prüfung und Beratung, Band I, 12. Aufl., Düsseldorf 2000. 
Ivaska, Helka [Ivaska (2000)]:

Suunnitelman mukaiset poistot - muutoksia ja täsmennyksiä aikaisempaan ohjeistukseen verrattuna, in: Tilintarkastus 4/2000, S. 21-26.

Järvenpää, Marko [Järvenpää (1996)]:

The relationship between taxation and financial accounting in Finland in: European Accounting Review 1996, 5:Supplement, S. 899-914.

Järvinen, Risto, Prepula, Eero, Riistama, Veijo und Tuokko, Yrjö [Järvinen et al. (2000)]:

Kirjanpito ja tilinpäätös - uuden kirjanpitolain mukaan, Porvoo 2000.

Johansson, Per-Olof [Johansson (1998)]:

Rahoitusleasingsopimus tilinpäätöksessä, in: Arovaara et al. (1998), S. 162-171.

Kaisanlahti, Timo [Kaisanlahti (1998)]:

True and fair view-käsite Euroopan Unionissa, in: Kaisanlahti et al. (1998), S. 14-27.

Kaisanlahti, Timo; Räty, Päivi; Tikkanen, Reino; Tuominen, Jorma; Turja, Tom A. [Kaisanlahti et al. (1998)]:

Kirjanpidon erityiskysymyksiä - uuden kirjanpitolain mukaan, Porvoo 1998.

Karhu, Kim [Karhu (1998)]:

Pääomalaina, in: Blummé, Nils; Karhu, Kim und Palvi, Mauri, Osakeyhtiölaki tilinpäätöksessä ja verotuksessa, Porvoo 1997, S. 78-88.

Kettunen, Pertti [Kettunen (1993)]:

Financial accounting and reporting in Finland, in: European Accounting Review 1993, 3, S. 592-602.

KHT-yhdistys - Föreningen CGR r.y. (Hrsg.) [KHT-yhdistys (1995)]:

Tilinpäätöskäytäntö Suomessa, Jyväskylä 1995.

KHT-yhdistys - Föreningen CGR r.y. [KHT-yhdistys (1998)]:

Osakeyhtiön tilinpäätösmalli - Uuden kirjanpitolain mukaan, 11. erneuerte Aufl. Jyväskylä 1998.

KPMG Deutsche Treuhand-Gesellschaft (Hrsg.) [KPMG (1999)]:

International Accounting Standards: eine Einführung in die Rechnungslegung nach den Grundsätzen des IASC, Stuttgart 1999.

Krisement, Vera M. [Krisement (1994)]:

Ansätze zur Messung des Harmonisierungs- und Standardisierungsgrades der externen Rechnungslegung, Frankfurt am Main u. a. 1994.

Küting, Karlheinz und Figge, Horst [Küting und Figge (1999)]:

Mobilien-Leasing in der Handels- und Steuerbilanz, in: Betrieb und Wirtschaft 1999, S. 361-364.

Küting, Karlheinz und Weber, Claus-Peter (Hrsg.) [Küting und Weber (1995)]: 
Handbuch der Rechnungslegung, Kommentar zur Bilanzierung und Prüfung, Band I a, 4., grundlegend überarbeitete und wesentlich erweiterte Aufl., Stuttgart 1995.

Kykkänen, Tapani [Kykkänen (1986)]:

Der Konzernabschluss in Finnland, Arbeitsbericht Nr. 38, Ruhr-Universität Bochum, Institut für Unternehmensführung und Unternehmensforschung, Bochum 1986.

Lappalainen, Niko [Lappalainen (1999)]:

Vuoden 1992 Kirjanpitolain uudistuksen vaikutukset suomalaisten konsernitilinpäätösten harmonisuuteen, Publications of the Turku School of Economics and Business Administration, Series D-3:1999, Turku 1999.

Leppiniemi, Jarmo [Leppiniemi (1997)]:

Pääomalaina, in: Tilisanomat 2/1997, S. 12-19.

Leppiniemi, Jarmo [Leppiniemi (2000 a)]:

Hyvä kirjanpitotapa - Mikä on sallittua, mahdollista, kiellettyä?, 8. erneuerte Aufl. Porvoo 2000.

Leppiniemi, Jarmo [Leppiniemi (2000 b)]:

Poistot, in: Verotus 1/2000, S. 15-27.

Leppiniemi, Jarmo und Leppiniemi, Raili [Leppiniemi und Leppiniemi (1997)]:

Muuttuva kirjanpito - 675 ratkaisua kirjanpidon ongelmiin, Porvoo 1997.

Leppiniemi, Jarmo und Leppiniemi, Raili [Leppiniemi und Leppiniemi (1999)]:

Hyvä tilinpäätöskäytäntö, 3. erneuerte Aufl. Porvoo 1999.

Lührmann, Volker [Lührmann (1997)]:

Latente Steuern im Konzernabschluß, Düsseldorf 1997.

Lukka, Kari und Pihlanto, Pekka [Lukka und Pihlanto (1994)]:

Martti Saario (1906-88): The developer of Finnish accounting theory in: Edwards, John Richard (Hrsg.), Twentieth-century accounting thinkers, London 1994, S. 60-78.

Luoma, Pekka [Luoma (1998)]:

Vastaiset menot ja menetykset sekä pakollinen varaus, in: Arovaara et al. (1998), S. 231-242.

Lydman, Kari [Lydman (1998)]:

Pääomalaina, in: Tilintarkastus 1/1998, S. 7-13.

Määttä, Seppo [Määttä (2000)]:

True and fair view - Ajatuksia oikeista ja riittävistä tiedoista ja kuvasta erityisesti Suomessa käydyn keskustelun valossa, Publications of the Turku School of Economics and Business Administration, Series Discussion and Working Papers 2:2000, Turku 2000. 
Majala, Reino [Majala (1987)]:

Kirjanpitokäytäntöjen vertailevan tutkimuksen käsitteellisen viitekehyksen kehittelyä, Turun Kauppakorkeakoulun julkaisuja, Sarja A-5: 1987, Turku 1987.

Majala, Reino [Majala (1991)]:

Kulttuurierot tilinpäätöskäytäntöjen yhtenäistymisen esteenä, in: Tilintarkastus 3/1991, S. 183-189.

Majala, Reino [Majala (1992)]:

A Conceptual Frame of Reference for Comparative Studies of International Accounting Practices, in: Liiketaloudellinen aikakauskirja - The Finnish Journal of Business Economics, Sonderausgabe 4/1992, S. 325-330.

Majala, Reino [Majala (1994)]:

Finland, in: Flower (1994), S. 65-105.

Majala, Reino [Majala (2001 a)]:

Finland: Group Accounts, in: Ordelheide, Dieter und KPMG (Hrsg.):

Transnational Accounting, 2. Aufl. Stuttgart 2001, S. 993-1008.

Majala, Reino [Majala (2001 b)]:

Finland: Individual Accounts, in: Ordelheide, Dieter und KPMG (Hrsg.):

Transnational Accounting, 2. Aufl., Stuttgart 2001, S. 954-991.

Mäkinen, Lasse [Mäkinen (1996)]:

Väärä tai puutteellinen kuva, in: Tilintarkastus 5/1996.

Mätäsaho, Risto [Mätäsaho (1999)]:

Miten suomalaiset pörssiyritykset raportoivat ympäristöasioidensa taloudellisista vaikutuksista tilinpäätös- ja vuosikertomustiedoissaan?, in: Tilintarkastus 7/1999, S. 509-516.

Näsi, Salme [Näsi (1994)]:

Development of cost accounting in Finland from the last century to the 1960s - a historical review of cost accounting based on accounting literature, in: European Accounting Review 1994, 3:3, S. 489-514.

Näsi, Salme und Näsi, Juha [Näsi und Näsi (1996)]:

Accounting and business economics traditions in Finland - from a practical discipline into a scientific subject and field of research, in: European Accounting Review 1996, 6:2, S. 199-229.

Nieminen, Kauko K. [Nieminen (1995)]:

KHT-yhdistyksen toiminnasta vuosina 1925-1995, in: Tilintarkastus 4/1995, S. 275-283.

Nieminen, Terhi und Niskanen, Jyrki [Nieminen und Niskanen (1998)]:

Suomalaisten pörssiyhtiöiden ympäristöraportoinnin objektiivisuus - Onko ikävistä asioista edelleen vaikea kertoa?, in: Tilintarkastus 7/1998, S. 516-520. 
Nobes, Christopher und Parker, Robert [Nobes und Parker (2000)]:

Comparative international accounting, 6. Aufl. London u. a. 2000.

Ordelheide, Dieter [Ordelheide (1993)]:

True and fair view, A European and a German perspective, in: European Accounting Review 1993, 1, S. 81-90.

Ordelheide, Dieter [Ordelheide (1998)]:

Wettbewerb der Rechnungslegungssysteme IAS, US-GAAP und HGB, in: Börsig, Clemens und Coenenberg, Adolf G. (Hrsg.), Controlling und Rechnungswesen im internationalen Wettbewerb, Kongress-Dokumentation, 51. Deutscher Betriebswirtschafter-Tag 1997, Stuttgart 1998, S. 15-53.

Pärssinen, Harri [Pärssinen (1998)]:

Yleiset tilinpäätösperiaatteet, in: Arovaara et al. (1998), S. 172-184.

Pellens, Bernhard [Pellens (1999)]:

Internationale Rechnungslegung, 3. überarb. und erw. Aufl., Stuttgart 1999.

Pihlanto, Pekka und Lukka, Kari [Pihlanto und Lukka (1993)]:

Martti Saario - suomalaisen laskenta-ajattelun kehittäjä, in: Liiketaloudellinen aikakauskirja 1993, Vol. 41:3, S. 251-275 und S. 277.

Pirinen, Pekka [Pirinen (1996)]:

The 1992 Reform of Finnish Accounting Legislation, Jyväskylä 1996.

Quick, Reiner; Hemeier, Arnd und Parthe, Jens [Quick, Hemeier und Parthe (1999)]:

Wirtschaftsprüfende Berufsstände in Finnland - Zugang, Organisation und Disziplinaraufsicht, in: WPK-Mitteilungen 1999, S. 224-229.

Räty, Päivi [Räty (1992)]:

Reforming Finnish accounting legislation, in: European Accounting Review 1992, 1, S. 413-420.

Räty, Päivi [Räty (1998 a)]:

Onko suomalainen kirjanpitolainsäädäntö nyt kansainvälisesti yhteensopiva?, in: Tilisanomat 2/1998, S. 28-33.

Räty, Päivi [Räty (1998 b)]:

Tilinpäätöksen arvostus- ja jaksotusongelmat, in: Kaisanlahti et al. (1998), S. $40-81$.

Räty, Päivi [Räty (1998c)]:

Tilinpäätösinformaation sisältö, in: Kaisanlahti et al. (1998), S. 82-127.

Räty, Päivi [Räty (2000)]:

Rahoituslaskelman laadinnan uudet ohjeet: liiketoiminnan, investointien ja rahoituksen rahavirrat maksuperusteisiksi, in: Tilintarkastus 2/2000, S. 38-42.

Regierungskommission Corporate Governance [Deutscher Corporate Governance Kodex (2002)]:

Deutscher Corporate Governance Kodex, Düsseldorf 2002. 
Riistama, Veijo [Riistama (1992)]:

"True and fair view"-kirjanpitolaki, in: Tilisanomat 6/1992, S. 6-8.

Riistama, Veijo [Riistama [1996)]:

Oikeat ja riittävät tiedot vai oikea ja riittävä kuva - riittäisikö hyvä kirjanpitotapa?, in: Tilisanomat 5 /1996, S. 15-19.

Roß, Norbert [Roß (1996)]:

Gemeinsamkeiten und Unterschiede handels- und steuerrechlicher Aktivierungskonzeptionen, in: Rechnungslegung und -prüfung 1996, S. 231-253.

Rost, Peter [Rost (1991)]:

Der internationale Harmonisierungsprozeß in der Rechnungslegung, Frankfurt am Main u. a. 1991.

Saarikivi, Maj-Lis [Saarikivi (1999)]:

KHT-tilintarkastajajärjelmä 75 vuotta, in: Tilintarkastus 7/1999, S. 517-523.

Saarikivi, Maj-Lis und Räty, Päivi [Saarikivi und Räty (1996)]:

Kirjanpitolainsäädännön muutosten vaikutukset yrityksille, in: Tilisanomat 3/1996, S. 26-31.

Saario, Martti [Saario (1965)]:

Kirjanpidon meno-tulo-teoria, 2. korr. Aufl., Keuruu 1965.

Schmalenbach, Eugen [Schmalenbach (1933)]:

Grundsätze ordnungsmäßiger Bilanzierung, in: ZfhF 1933, S. 225-233.

Schmalenbach, Eugen [Schmalenbach (1953)]:

Dynamische Bilanz, unter Mitarbeit von Bauer, Richard, 11. Aufl., Köln/Opladen 1953.

Scholtissek, Wolfgang [Scholtissek (1984)]:

Rechnungslegung der Unternehmen in Finnland, Berichte und Dokumente zum ausländischen Wirtschafts- und Steuerrecht Nr. 189/1984.

Schruff, Lothar [Schruff (1986)]:

Entwicklung der 4. EG-Richtlinie, Synoptische Darstellung der Bilanzrichtlinie unter Berücksichtigung der Vorfassungen mit amtlichen Begründungen und weiteren Materialien, Düsseldorf 1986.

Schruff, Lothar [Schruff (1997)]:

Zum Ausweis des Zinsanteils bei der Zuführung zur Pensionsrückstellung, in: Fischer, Thomas R. und Hömberg, Reinhold (Hrsg.): Jahresabschluß und Jahresabschlußprüfung, Probleme, Perspektiven, internationale Einflüsse, Festschrift zum 60. Geburtstag von Jörg Baetge, Düsseldorf 1997, S. 401-426.

Schruff, Lothar [Schruff (2001)]:

Stichtags- und Realisationsprinzip auf den Sperrmüll?, in: Der Betriebsberater 2001, Heft 38, Die erste Seite. 
Sipilä, Pekka [Sipilä (1998)]:

Valmistusaikaisten korkomenojen aktivointi hankintamenoon, in: Arovaara et al. (1998), S. 185-189.

Sipola, Seppo [Sipola (1991)]:

Kirjanpitolainsäädännön uudistukset yritysjohdon näkökulmasta, in: Tilintarkastus 1/1991, S. 13-18.

Sorsa, Eero [Sorsa (1998 a)]:

Hyvän kirjanpitotavan perustana olevat sovinnaissäännöt, in: Tilintarkastus 2/1998, S. 160-163.

Sorsa, Eero [Sorsa (1998 b)]:

Kirjanpitolainsäädännön kehitys Suomessa, in: Tilisanomat 1/1998, S. 32-37.

Suomela, Eero, Palvi, Mauri und Karhu, Kim [Suomela et al. (1995)]:

Tilintarkastuslaki, Jyväskylä 1995.

Tielmann, Sandra [Tielmann (2001)]:

Durchsetzung ordnungsmäßiger Rechnungslegung: ein Beitrag zur aktuellen Enforcement-Diskussion, Düsseldorf 2001.

Tikka, Marja [Tikka (1999)]:

Rahoitusinstrumentit käytännössä - käteisinstrumenttien käsittely

tilinpäätöksessä, in: Tilintarkastus 2/1999; S. 100-106.

Troberg, Pontus [Troberg (1995)]:

Kansainvälisen tilinpäätöksen tulevaisuus, in: Tilintarkastus 2/1995, S. $123-$ 126.

Tuokko, Timo [Tuokko (1996)]:

Oikeat ja riittävät tiedot, in: Tilintarkastus 2/1996.

Tuokko, Yrjö [Tuokko (2000)]:

Vapaa oma pääoma - esitetty, todellinen ja jakokelpoinen, in: Tilintarkastus 1/2000, S. 7-12.

Tuominen, Jorma [Tuominen (1998)]:

Tutkimus- ja kehittämismenojen aktivoiminen, in: Kaisanlahti et al. (1998), S. 28-39.

Turja, Tom A. [Turja (1998)]:

Uuden kirjanpitolain menetelmäsäännökset

in: Kaisanlahti et al. (1998), S. 150-158.

Uusitalo, Uwe und Collan, Matias [Uusitalo und Collan (2000)]:

Finnisches Steuerrecht, in: Beck'sches Steuerberater-Handbuch 2000/2001, München 2000, Kap. G Rz 301-367.

Van der Tas, Leo G. [van der Tas (1988)]:

Measuring Harmonisation of Financial Reporting Practice, Accounting and Business Research, Vol 18, No. 77/1988, S. 157-169. 
Van der Tas, Leo G. [van der Tas (1991)]:

Evidence of the EC Financial Reporting Practice Harmonisation - The Case of Deferred Taxation -, Paper presented at the 14th Annual Congress of the European Accounting Association, Maastricht, April 1991.

Van der Tas, Leo G. [van der Tas (1992 a)]:

Evidence of EC financial reporting practice harmonization - The case of deferred taxation, in: European Accounting Review, 1992, 1, S. 69-104.

Van der Tas, Leo G. [van der Tas (1992 b)]:

Harmonisation of Financial Reporting - With aq special focus on the European Community, Maastricht 1992.

Van Hulle, Karel [van Hulle (1993)]:

Harmonization of accounting standards in the EC - Is it the beginning or is it the end?, in: European Accounting Review 1993, 2, S. 387-396.

Van Hulle, Karel [van Hulle (1994)]:

Implementation of the 4th and 7th EC-Directives in Finland, in: Tilintarkastus 3/1994, S. 233-236.

Van Hulle, Karel [van Hulle (1997)]:

The true and fair view Override in the European Accounting Directives, in: European Accounting Review 1997, 6:4, S. 711-720.

Van Hulle, Karel und van der Tas, Leo G. [van Hulle und van der Tas (2001)]:

European Union: Individual Accounts, in: Ordelheide, Dieter und KPMG (Hrsg.): Transnational Accounting, 2. Aufl., Stuttgart 2001, S. 771-877.

Väyrynen, Jukka [Väyrynen (1993)]:

KILA reivaa rooliaan, in: Tilisanomat 5/1993, S. 6-10.

Zitzelsberger, Siegfried [Zitzelsberger (1998)]:

Aus der Arbeit des IDW, in: Die Wirtschaftsprüfung 1998, S. 1015-1025. 


\section{Internetadressen}

Börse Helsinki (Helsinki Stock Exchange):

www.hex.fi

Datenbank des Amts für amtliche Veröffentlichungen der EU:

www.eudor.com

europäische Institutionen:

www.europa.eu.int

finnische Gesetzestexte:

www.finlex.fi

finnische Zentralhandelskammer:

www.keskuskauppakamari.fi

finnischer Buchführungsausschuss KILA:

www.vn.fi/ktm/kirjanpitolautakunta

Handelsregister Helsinki:

www.prh.fi

OECD:

www.oecd.org

Verband der HTM-Wirtschaftsprüfer:

www.htm.fi/english.html

Verband der KHT-Wirtschaftsprüfer:

www.kht.fi

\section{Wörterbücher}

Hirvensalo, Lauri:

Saksa-Suomi Suursanakirja / Großwörterbuch Deutsch-Finnisch, 7. Aufl., Porvoo/Helsinki/Juva 1994.

Katara, Pekka und Schellbach-Kopra, Ingrid:

Suomi-Saksa Suursanakirja / Großwörterbuch Finnisch-Deutsch, 7. Aufl., Porvoo/Helsinki/Juva 1994.

Katara, Pekka und Schellbach-Kopra, Ingrid:

Suomalais-saksalainen opiskelusanakirja / Finnisch-deutsches Wörterbuch, 5. unveränd. Aufl., Porvoo 1990.

Manninen, Liisa; Pirttioja, Katja; Rintamäki, Titta und Virtanen, Pia: Suomi-Saksa Tekniikan ja Kaupan Sanakirja, Jyväskylä 1996.

Manninen, Liisa; Pirttioja, Katja; Rintamäki, Titta und Virtanen, Pia: Saksa-Suomi Tekniikan ja Kaupan Sanakirja, Jyväskylä 1996.

o. $V$. :

Kirjanpito- ja Tilinpäätössanasto, Helsinki 1995. 


\section{Rechtsquellenverzeichnis}

\section{Richtlinien und offizielle Verkündigungen der Europäischen Union}

4. EG-Richtlinie:

Rat der Europäischen Gemeinschaften, Vierte Richtlinie des Rates vom 25. Juli 1978 auf Grund von Artikel 54 Absatz 3 Buchstabe g) des Vertrages über den Jahresabschluss von Gesellschaften bestimmter Rechtsform (78/660/EWG), in: Amtsblatt der Europäischen Gemeinschaften vom 14. August 1978, Nr. L 222/11--31.

\section{EG-Richtlinie:}

Rat der Europäischen Gemeinschaften, Siebente Richtlinie des Rates vom 13. Juni 1983 auf Grund von Artikel 54 Absatz 3 Buchstabe g) des Vertrages über den konsolidierten Abschluss (83/349/EWG), in: Amtsblatt der Europäischen Gemeinschaften vom 18. Juli 1983, Nr. L 193/1-17.

Mittelstandsrichtlinie:

Rat der Europäischen Gemeinschaften, Richtlinie des Rates vom 08. November 1990 zur Änderung der Richtlinie 78/660/EWG über den Jahresabschluss und der Richtlinie 83/349/EWG über den konsolidierten Abschluss hinsichtlich deer Ausnahmen für kleine und mittlere Gesellschaften sowie der Offenlegung in ECU, in: Amtsblatt der Europäischen Gemeinschaften vom 16. November 1990, Nr. L 317/57-59.

\section{GmbH \& Co-Richtlinie:}

Rat der Europäischen Gemeinschaften, Richtlinie des Rates vom 08. November 1990 zur Änderung der Richtlinien 78/660/EWG und 83/349/EWG über den Jahresabschluss bzw. den konsolidierten Abschluss hinsichtlich ihres Anwendungsbereichs, in: Amtsblatt der Europäischen Gemeinschaften vom 16. November 1990, Nr. L 317/60-62.

Office for Official Publications of the European Community (Hrsg.) [Kontaktausschuss (1990)]:

The Accounting Harmonization in the European Community. Problems of Applying the Fourth Directive on the Annual Accounts of the Limited Companies, Luxembourg 1990.

Richtlinie vom 21. März 1994:

Rat der Europäischen Gemeinschaften, Richtlinie des Rates vom 21. März 1994 zur Änderung der in ECU ausgedrückten Beträge der Richtlinie 78/660/EWG, in: Amtsblatt der Europäischen Gemeinschaften vom 25. März 1994, Nr. L 82/33-34.

EU-Kommission XV/6008/94 EN:

Cash Flow Statements, Document of the Accounting Advisory Forum, $\mathrm{XV} / 6008 / 94 \mathrm{EN}$.

\section{EU-Kommission XV/7009/97:}

Euroopan Union komission tulkitseva tiedonanto neljännen ja seitsemännen tilinpäätösdirektiivin tietyistä artikloista, XV/7009/97. 
EU-Kommission 98/C16/04:

Interpretative Communication of the European Commission (98/C16/04) concerning certain articles of the Fourth and Seventh Council Directives on Accounting, O.J. No. C 16 of 20 January 1998.

\section{Internationale Standards}

International Accounting Standards (IAS):

International Accounting Standards Committee, International Accounting Standards 2000, The full text of all International Accounting Standards and SIC Interpretations extant at 1 January 2000, London 2000.

\section{Finnische nationale Gesetze und Verordnungen}

$A M L$ :

Arvopaperimarkkinalaki 1999/105.

EVL:

Elinkeinoverolaki 17. Januar 1997/75.

KPA:

Kirjanpitoasetus 30.12.1997/1339.

KPL:

Kirjanpitolaki 30.12.1997/1336.

$O Y L$ :

Osakeyhtiölaki 14.02.1997/145.

\section{Regierungsvorlagen an das finnische Parlament über (geplante) Gesetzesän- derungen}

HE 111/1992:

Hallituksen esitys Eduskunnalle laeiksi kirjanpitolain, osakeyhtiölain 11 ja 12 luvun sekä osuuskuntalain muuttamisesta.

HE 89/1996:

Hallituksen esitys Eduskunnalle laiksi osakeyhtiölain ja eräiden siihen liittyvien lakien muuttamisesta.

HE 173/1997:

Hallituksen esitys Eduskunnalle kirjanpitolaeiksi sekä laiksi osakeyhtiölain 11 ja 12 luvun ja osuuskuntalain 79 c $\S:$ muuttamisesta.

\section{Entscheidungen finnischer Ministerien}

Ministerium für Handel und Industrie (Kauppa- ja Teollisuusministeriö)

KTM:p 48/1998.

KTM:p 50/1998. 
KTM:n luonnos kirjanpitolain ja tilintarkastuslain muuttamisesta - yhteenveto lausunnoista, KTM:n tutkimuksia ja raportteja, Helsinki 2000.

\section{Finanzministerium (Valtiovarainministeriö)}

VM:p 905/1994.

VM:p 879/1995.

VM:p 390/1999.

\section{Berichte ministerieller Arbeitsgruppen}

KPL-Arbeitsgruppe 1995, Teil I (1996):

Kirjanpitolain uudistamistyöryhmä 1995:n raportti, kauppa- ja teollisuusministeriön työryhmä- ja toimikuntaraportti 8/1996, osa I.

KPL-Arbeitsgruppe 1995 (Teil II):

Kirjanpitolain uudistamistyöryhmä 1995:n raportti, kauppa- ja teollisuusministeriön työryhmä- ja toimikuntaraportti 8/1996, osa II.

\section{Veröffentlichungen des KILA}

\section{Allgemeingültige KILA-Anweisungen}

KILA-Anweisung ,Darstellung von Gewinn- und Verlustrechnung und Bilanz":

Yleisohje tuloslaskelmien ja taseiden esittämisestä 20.04.1998.

KILA-Anweisung ,Latente Steuern”:

Yleisohje laskennallisista veroveloista ja -saamisista 11.01.1999.

KILA-Anweisung „,Planmäßige Abschreibungen”:

Yleisohje suunnitelman mukaisista poistoista 27.09.1999.

KILA-Anweisung , Kapitalflussrechnung”:

Yleisohje rahoituslaskelman laatimisesta 09.11.1999.

KILA-Anweisung ,,Währungsumrechnung”:

Yleisohje ulkomaanrahan määräisten saamisten sekä velkojen ja muiden sitoumusten muuttamisesta Suomen rahaksi 15.11.1999.

KILA-Anweisung ,Erstellung von Jahresabschluss, vorläufigem Abschluss und Zwischenbericht":

Yleisohje valtiovarainministeriön päätöksessä (390/1999) tarkoitetun tilinpäätökseen, tilinpäätöstiedotteen ja osavuosikatsauksen laatimisesta 17.12.1999.

KILA-Anweisung , Informationstechnologie in der Buchführung”:

Yleisohje koneellisessa kirjanpidossa käytettävistä menetelmistä 22.05.2000. 
KILA-Anweisung „,Fixkosten als Bestandteil der Anschaffungs- oder Herstellungskosten":

Yleisohje kiinteiden menojen lukemisesta hyödykkeen hankintamenoon 04.09.2000.

KILA-Anweisung , Teilgewinnrealisierung”:

Yleisohje tulon kirjaamisesta tuotoksi valmistusasteen perusteella 04.09.2000.

\section{Gutachten und Entscheidungen des KILA}

\begin{tabular}{l|l|l} 
KILA 839/1986 & KILA 1378/1996 & KILA 1534/1998 \\
KILA 886/1986 & KILA 1427/1996 & KILA 1542/1998 \\
KILA 959/1988 & KILA 1444/1997 & KILA 1579/1999 \\
KILA 1054/1989 & KILA 1453/1997 & KILA 1580/1999 \\
KILA 1136/1993 & KILA 1489/1997 & KILA 1588/1999 \\
KILA 1222/1993 & KILA 1520/1998 & KILA 1594/1999 \\
KILA 1306/1994 & KILA 1528/1998 & KILA 1603/2000 \\
KILA 1317/1995 & KILA 1529/1998 & KILA 1604/2000 \\
KILA 1343/1995 & KILA 1532/1998
\end{tabular}

\section{Deutsche nationale Gesetze}

Aktiengesetz [AktG]:

Aktiengesetz vom 06.09.1965 (BGBl. I S. 1089) mit späteren Änderungen.

Bilanzrichtlinien-Gesetz [BiRiLiG]:

Gesetz zur Durchführung der Vierten, Siebenten und Achten Richtlinie des

Rates der Europäischen Gemeinschaften zur Koordinierung des GEsellschaftsrechts (Bilanzrichtlinien-Gesetz) vom 19.12.1985 (BGB1. I S. 2355).

Einkommensteuergesetz [EStG]:

Einkommensteuergesetz (EStG) vom 16.04.1997 (BGBl. I S. 823) mit späteren Änderungen.

Genossenschaftsgesetz [GenG]:

Gesetz betreffend die Erwerbs- und Wirtschaftsgenossenschaften vom 19.08.1994 (BGB1. I S. 2202) mit späteren Änderungen.

Gesetz über die Rechnungslegung von bestimmten Unternehmen und Konzernen (Publizitätsgesetz - PublG) [PublG]:

Gesetz über die Rechnungslegung von bestimmten Unternehmen und Konzernen (Publizitätsgesetz - PublG) vom 15.08.1969 (BGB1. I S. 1189, ber. BGB1. 1970 I S. 1113) mit späteren Änderungen. 
Gesetz zur Kontrolle und Transparenz im Unternehmensbereich [KonTraG]: Gesetz zur Kontrolle und Transparenz im Unternehmensbereich vom 27.04.1998 (BGBl. I S. 786).

GmbH-Gesetz [GmbHG]:

Gesetz betreffend die Gesellschaften mit beschränkter Haftung vom 20.04.1892 (RGB1. S .477) mit späteren Änderungen.

Handelsgesetzbuch [HGB]:

Handelsgesetzbuch vom 10.05.1897 (RGB1. S .219) mit späteren Änderungen.

Kapitalgesellschaften- und Co-Richtlinie-Gesetz [KapCoRiLiG]:

Gesetz zur Durchführung der Richtlinie des Rates der Europäischen Union zur Änderung der Bilanz- und Konzernbilanzrichtlinie hinsichtlich ihres Anwendungsbereichs (90/65/EWG), zur Verbesserung der Offenlegung von Jahresabschlüssen und zur Änderung anderer handelsrechtlicher Bestimmungen vom 24.02.2000 (BGBl. I S. 154).

Steuerentlastungsgesetz 1999/2000/2002:

Steuerentlastungsgesetz 1999/2000/2002 vom 24.03.1999 (BGB1. I S. 402).

\section{Deutsche Rechnungslegungs Standards}

Deutscher Rechnungslegungs Standard Nr. 3 [DRS 3]:

Segmentberichterstattung. Verabschiedung durch den Deutschen Standardisierungsrat am 20.12.1999, Bekanntmachung der deutschsprachigen Fassung gem. $\S 342$ Abs. 2 HGB durch das Bundesministerium der Justiz am 31.05.2000.

\section{Verzeichnis der deutschen Rechtsprechung}

$B G H$ vom 03.11.1975:

BGH, Urteil vom 03.11.1975 - II ZR 67/73, in: Der Betrieb 1976, S. 38-40.

BGH vom 26.03.1984:

BGH, Urteil vom 26.03.1984 - II ZR 171/83, in: Die Aktiengesellschaft 1984, S. 181-188.

$B G H$ vom 12.01.1998:

BGH, Urteil vom 12.01.1998 - II ZR 82/93, in: Der Betrieb 1998, S. 567-569. 